\title{
IntechOpen
}

\section{New Advanced Technologies}

Edited by Aleksandar Lazinica

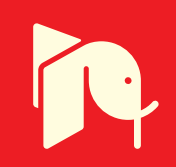



New, Advanced TechnolgGies

Edited by

ALEKSANDAR LAZINICA 


\section{New Advanced Technologies}

http://dx.doi.org/10.5772/227

Edited by Aleksandar Lazinica

\section{(c) The Editor(s) and the Author(s) 2010}

The moral rights of the and the author(s) have been asserted.

All rights to the book as a whole are reserved by INTECH. The book as a whole (compilation) cannot be reproduced, distributed or used for commercial or non-commercial purposes without INTECH's written permission.

Enquiries concerning the use of the book should be directed to INTECH rights and permissions department (permissions@intechopen.com).

Violations are liable to prosecution under the governing Copyright Law.

\section{(cc) BY}

Individual chapters of this publication are distributed under the terms of the Creative Commons Attribution 3.0 Unported License which permits commercial use, distribution and reproduction of the individual chapters, provided the original author(s) and source publication are appropriately acknowledged. If so indicated, certain images may not be included under the Creative Commons license. In such cases users will need to obtain permission from the license holder to reproduce the material. More details and guidelines concerning content reuse and adaptation can be foundat http://www.intechopen.com/copyright-policy.html.

\section{Notice}

Statements and opinions expressed in the chapters are these of the individual contributors and not necessarily those of the editors or publisher. No responsibility is accepted for the accuracy of information contained in the published chapters. The publisher assumes no responsibility for any damage or injury to persons or property arising out of the use of any materials, instructions, methods or ideas contained in the book.

First published in Croatia, 2010 by INTECH d.o.o.

eBook (PDF) Published by IN TECH d.o.o.

Place and year of publication of eBook (PDF): Rijeka, 2019.

IntechOpen is the global imprint of IN TECH d.o.o.

Printed in Croatia

Legal deposit, Croatia: National and University Library in Zagreb

Additional hard and PDF copies can be obtained from orders@intechopen.com

New Advanced Technologies

Edited by Aleksandar Lazinica

p. $\mathrm{cm}$.

ISBN 978-953-307-067-4

eBook (PDF) ISBN 978-953-51-5907-0 


\section{We are IntechOpen, \\ the world's leading publisher of Open Access books}

\section{Built by scientists, for scientists}

\section{$4,400+$}

Open access books available

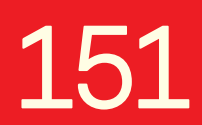

Countries delivered to

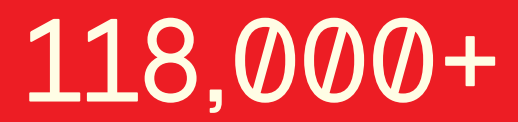

International authors and editors
$130 \mathrm{M}+$

Downloads

Our authors are among the

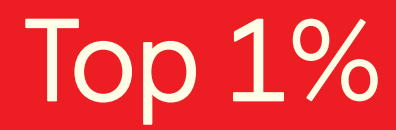

most cited scientists

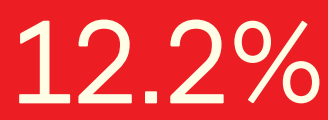

Contributors from top 500 universities

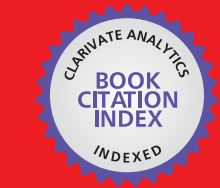

WEB OF SCIENCE ${ }^{\mathrm{TM}}$

Selection of our books indexed in the Book Citation Index in Web of Science ${ }^{\mathrm{TM}}$ Core Collection (BKCI)

Interested in publishing with us?

Contact book.department@intechopen.com

Numbers displayed above are based on latest data collected.

For more information visit www.intechopen.com 



\section{Preface}

This book collects original and innovative research studies concerning advanced technologies in a very wide range of applications.

The book is compiled of 22 chapters written by researchers from different areas and different parts of the world. The book therefore will have international readership of wide spectrum.

Aleksandar Lazinica 



\section{Contents}

Preface

VII

1. A Simplified Deterministic Approach to accurate Modeling of Transfer Function for the Broadband Power Line Communication

001

Abdelali Rennane, Christophe Konaté and Mohamed Machmoum

2. A study of protein structure using amino acid interaction networks

019 Omar Gaci and Stefan Balev

3. Abstraction Hierarchies for Conceptual Engineering Design

047 Esra Aleisa

4. An Ant Colony Optimization algorithm for Flexible Job shop scheduling problem

073 S. G. Ponnambalam, N. Jawahar and B. S. Girish

5. Analytical model of current sheath path based upon shock wave trajectory of plasma layer in a plasma focus device

Reza Amrollahi and Morteza Habibi

6. Authentication with RIPEMD-160 and other alternatives:

A Hardware Design Perspective

H. Michail, A. Gregoriades, V. Kelefouras, G. Athanasiou, A. Kritikakou and C. Goutis

7. Bioinformatics: strategies, trends, and perspectives

Carlos Norberto Fischer and Adriane Beatriz de Souza Serapião

8. Comparative Performance Assessment In Eu Pre-Accession Funds

Arzu Tektas and Asli Deniz Helvacioglu Kuyucu

9. Design and Construction Transceiver Module Using Polymer PLC Hybrid Integration Technology

Vítězslav Jeřábek, Ivan Hüttel, Václav Prajzler and Karel Bušek

10. Design of a self-assembling, repairing and reconfiguring Arithmetic Logic Unit

David Huw Jones, Richard McWilliam and Alan Purvis

11. Discovering Web Server Logs Patterns Using Generalized Association

Rules Algorithm

Mohd Helmy Abd Wahab, Mohd Norzali Haji Mohd

and Mohamad Farhan Mohamad Mohsin 
12. Fuzzy modeling by Active Learning Method Hamid Taheri Shahraiyni

13. Generic Framework for Collaborative Work Environments

Cosmin Porumb, Sanda Porumb, Bogdan Orza and Aurel Vlaicu

14. Integrated medical system using DICOM and HL7 standards

Orza Bogdan, Cordos Alin, Vlaicu Aurel and Meza Serban

15. Modelling Embedded Systems with AADL: A Practical Study

Naeem Muhammad, Yves Vandewoude, Yolande Berbers and Sjir van Loo

16. QuadR-Tree Indexing Selection Engine for Tuning Spatial Database System using Mobile Geographical Information System Technology

Maruto Masserie Sardadi, Daut Daman, Mohd Shafry and Zahabidin Jupri

17. Quran Vibrations in Braille Codes Using the Finite State Machine Technique Abdallah M Abualkishik and Khairuddin Omar

18. Residential Noise Control Requirements for Powerline Communications Channel

Edward Guillen, Julian Lopez and Daniel Padilla

19. Robot Navigation with Speech Commands

Anupam Shukla and Ritu Tiwari

20. Ti indiffused Lithium Niobate $\left(\mathrm{Ti}: \mathrm{LiNbO}_{3}\right)$ Mach-Zehnder interferometer all optical switches: A review

G.Singh, R.P.Yadav and V.Janyani

Roque Mendes Prado Trindade, Benjamín René Callejas Bedregal,

Adrião Duarte Dória Neto and Benedito Melo Acióly

22. Walking Hexapod Robot in Disaster Recovery: Developing Algorithm for Terrain Negotiation and Navigation

Mohiuddin Ahmed, M. R. Khan, Md. Masum Billah and Soheli Farhana 


\title{
A Simplified Deterministic Approach to accurate Modeling of Transfer Function for the Broadband Power Line Communication
}

\author{
Abdelali Rennane, Christophe Konaté and Mohamed Machmoum \\ Nantes of University (Nantes)
}

France

\section{Introduction}

With the deregulation of the telecommunication and electricity markets, great deals of new operators providing some innovative services have appeared. In the telecommunications field, the promise of broad-band internet access with easy installation procedure at low cost may help to gain new customers. The Power Line Communication (PLC) technology is a way to fulfill this function. PLC uses the low voltage (LV) and the medium voltage (MV) networks as communication support (Roka \& Urminsky, 2008; Meng \& Chen, 2004). It consists in an additional signal carrying data information which is superimposed to the $50 / 60 \mathrm{~Hz}$ power wave. As PLC is easy to install, it can be used to extend the internet coverage to areas that are still badly covered by the other broadband technologies, or to provide high speed internet access to every power socket of building. The modeling of the PLC channel is of fundamental importance, since the quality of the transmission is highly influenced by the characteristics of the channel itself. Principally, channel attenuation depends on the characteristics of the cables (length, per-unit-length parameters, and frequency dependence) and of the loads (Roblot, 2007; Barmada et al., 2006). Several techniques have been proposed to properly simulate the PLC channel. A common practice is to consider the transmission path as a black box and to describe the transmission line by a transfer function (Moreno, 2008 ; Philips, 1999). The system parameters are obtained either from measurements and experimental data or from theoretical derivation (Konaté et al. 2007; Anastasiadou \& Antonakoupoulos, 2005). The objective of this chapter is to develop a transfer function model for the LV power line based on the transmission line theory. This model will help the PLC system designer to better understand the channel behavior and to engineer the channel performances under different network configurations and load conditions.

This chapter is organized as follows. We start with a representation of the transmission line as two conductors in section 2. We describe the set up experimental in section 3 . The next section deals with experimental determination of channel impedance and propagation constant. In section 5, the power channel model is established. In section 6 the proposed deterministic method is generalized to network with $\mathrm{N}$ nodes and $\mathrm{M}$ branches. Section 7 
gives simulation results. The influence of branched line, number of branches and impedance matching are discussed. In section 8, we realized experimental measurements for verification of the parametric model for reference channels in the real PLC environment. A concluding recapitulation closes the chapter in the last section.

\section{Transmission Line Parameters of the Cable}

The transmission line model represents the transmission line as an infinite series of two-port elementary components, each representing an infinitesimally short segment of the transmission line. The distributed resistance $R$ of the conductors is represented by a series resistor (expressed in Ohm per unit length). The distributed inductance $L$ is represented by a series inductor (Henrie per unit length). The capacitance $C$ between the two conductors is represented by a shunt capacitor $C$ (Farad per unit length). The conductance $G$ of the dielectric material separating the two conductors is represented by a conductance $G$ shunted between the signal wire and the return wire (Siemens per unit length). The model consists of an infinite series of the elements shown in Fig. 1. The values of the components are specified per unit length so the picture of the component can be derived. The parameters $R, L, C$, and $G$ may also be functions of frequency. An alternative notation is to use $R^{\prime}, L^{\prime}, C^{\prime}$ and $G^{\prime}$ to emphasize that the values are derivatives with respect to length.

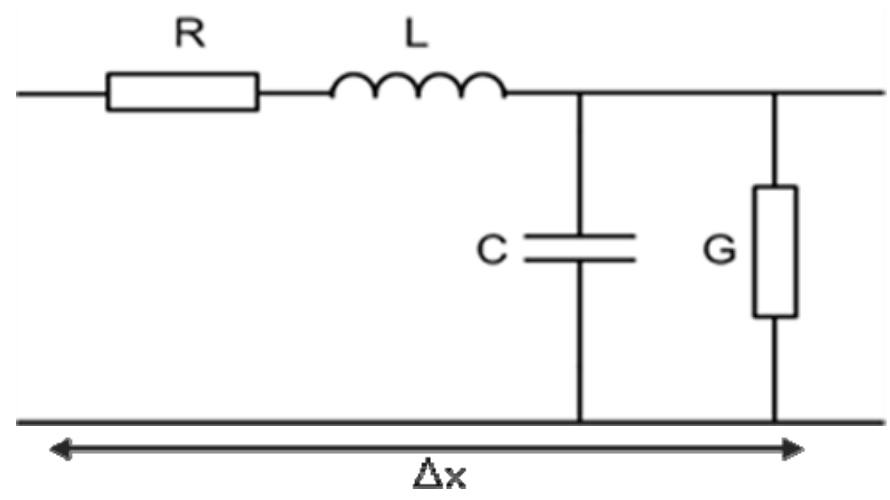

Fig. 1. Distribution network model of a transmission line

The parameters to describe a transmission line are the characteristic impedance $Z_{C}$ and the propagation constant $r$. These parameters are defined as:

$$
\begin{gathered}
\bar{Z}_{C}=\sqrt{\frac{\bar{Z}}{\overline{\bar{Y}}}}=\sqrt{\frac{R^{\prime}+j L^{\prime} \omega}{G^{\prime}+j C^{\prime} \omega}} \\
\gamma=\sqrt{\left(R^{\prime}+j L^{\prime} \omega\right)\left(G^{\prime}+j C^{\prime} \omega\right)}=\alpha+j \beta
\end{gathered}
$$

Where, $\omega=2 \pi \mathrm{f}$ is the angular frequency, $\alpha$ is the attenuation constant and $\beta$ is the phase constant. In our case, considering frequencies in the MHz-range, the resistance per unit length is dominated by the skin effect and the conductance per unit length is mainly 
influenced by the dissipation factor of the dielectric material usually Polyvinyl Chloride (PVC). The result of using geometry and material properties in the above equations results in $R^{\prime} \ll<L^{\prime}$ and $G^{\prime}<<C^{\prime}$ in the frequency range of interest. Hence the cables can be regarded as weakly loss and the characteristic impedance $Z_{C}$ and the propagation constant $\gamma$ can be determined using the following simplified expressions:

$$
\begin{gathered}
\bar{Z}_{C}=\sqrt{\frac{L^{\prime}}{C^{\prime}}} \\
\gamma=\alpha+j \beta=\frac{1}{2}\left(\frac{R^{\prime}}{Z_{C}}+G^{\prime} Z_{C}\right)+j \omega \sqrt{L^{\prime} C^{\prime}}
\end{gathered}
$$

\section{Experimental Set up}

The PLC test environment (Fig. 2) available in the DAL (Digital Applications Laboratory) is realized with a cable type $\mathrm{RO} 2 \mathrm{~V} 3 \mathrm{G}$ of section $2.5 \mathrm{~mm}^{2}$ and powered with three-phase network. It is isolated upstream with a three-phase filter; each phase is also isolated with a single-phase filter. The PLC test environment can simulate various power network topologies like "star" or "trees" topologies. This is enabled by multiple circuits and dedicated switches implemented and drawn from a distribution board which is typical of a real home installation. PLC modems can be tested to check their conformance with the HomePlug AV standard and Interoperability (C\&I). Performance, robustness and digital application tests (Data/Audio/Video flows) tests can also be carried out on this test environment. The PLC test environment represents also a configurable electrical network that can emulate the propagation medium of PLC signals in real home environment. It can be used for $S$ parameters measurements and power line channel measurement, tests of IP services in reproducible electrical propagation conditions, but also for measurements of electrical impedance and noise.

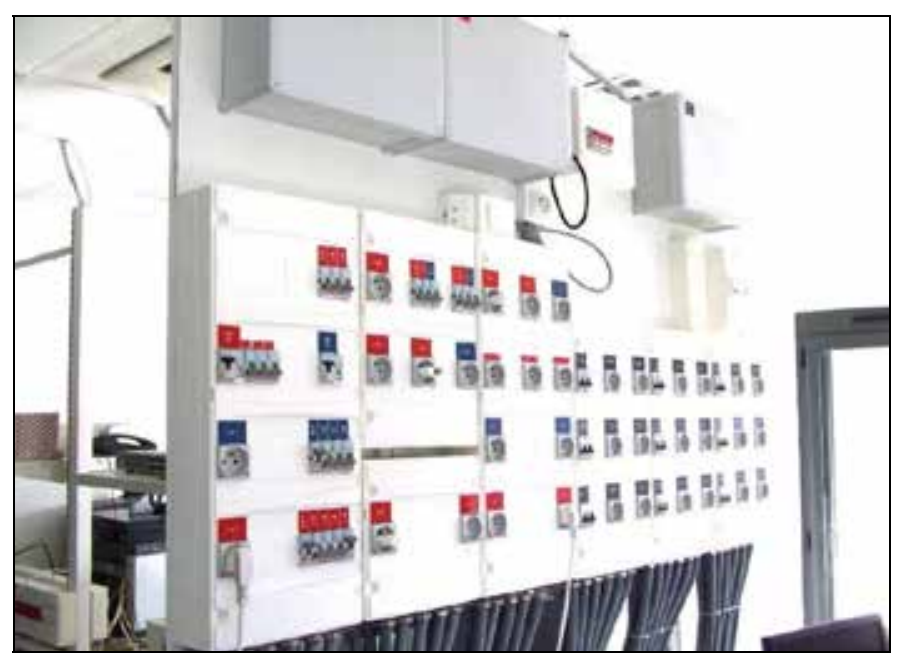

Fig. 2. PLC test environment available in DAL 
When measuring the $S$ parameters as a function of frequency in the range 1-30 MHz, a measurement setup as described in Fig. 3 is used. It consists of the network analyzer (NA) HP4394A (10 Hz-500 MHz), of an Agilent 87513A transmission/reflection set DC-2GHz and of two PLC couplers. These Couplers are designed to reject a $50 \mathrm{~Hz}$ signal and allows to perform measurements in the differential mode. They are connected to the NA HP4395A with two coaxial cables "RG58" and plugged on the electric outlets on their electrical interface.

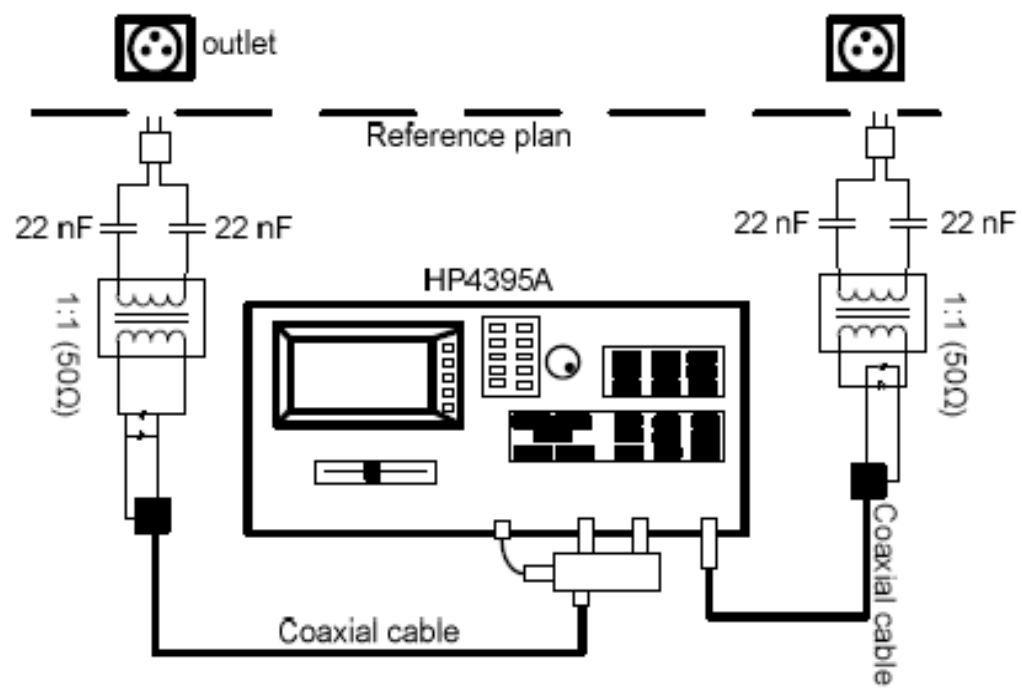

Fig. 3. Transfer function measurement setup

\section{Experimental Determination of ZC and Y}

A transmission line of the length $l$ is kept open at one end, and the impedance at its other end is measured using an impedance bridge. In this chapter the experimental results $\left(Z_{\text {open }}\right.$ and $Z_{\text {short }}$ ) are measured by a network analyzer $4395 \mathrm{~A}$ using in the Impedance analyzer mode. Assume that it is $Z_{\text {open }}$, the process is repeated after placing a short circuit at its open end, and this impedance is recorded as $Z_{\text {short }}$. Using transmission line theory, the impedance at the input of this transmission line, $Z_{\text {in }}(l=0)$ can be determined by:

$$
Z_{\text {in }}=Z_{C} \frac{Z_{L}+Z_{C} \tanh (\gamma \cdot 1)}{Z_{C}+Z_{L} \tanh (\gamma \cdot 1)}
$$

Where

$$
Z_{\text {in }, \text { open }}=Z_{C} \cdot \operatorname{cotanh}(\gamma \cdot 1)
$$

And

$$
\mathrm{Z}_{\text {in, short }}=\mathrm{Z}_{\mathrm{C}} \cdot \tanh (\mathrm{\gamma} \cdot 1)
$$


Combining equations 6 and 7, the characteristic impedance and the propagation constant can deduced:

$$
\begin{aligned}
& Z_{C}=\sqrt{Z_{\text {in,open }} \cdot Z_{\text {in,short }}} \\
& Y=\frac{1}{1} \arg \tanh \left(\sqrt{\frac{Z_{\text {in,short }}}{Z_{\text {in,open }}}}\right)
\end{aligned}
$$

The parameters of the cable used (RO2V $3 \times 2.5 \mathrm{~mm} 2)$ are, $R^{\prime}=0.31 \Omega / m, G^{\prime}=64.7 \mathrm{mS} / \mathrm{m}, L^{\prime}=$ $0.327 \mu \mathrm{H} / \mathrm{m}$ and $C^{\prime}=66.9 \mathrm{pF} / \mathrm{m}$. The mean value of the characteristic impedance $Z_{C}$ is $69.9 \Omega$. The attenuation constant $\alpha$ regarding this cable as well as its attenuation in the examined frequency range $1-30 \mathrm{MHz}$ (for a length of $40 \mathrm{~m}$ ) is shown in Fig. 4. The attenuation constant is modeled by the following relation

$$
\mathrm{a}=\mathrm{a}_{0}+\mathrm{a}_{1} \mathrm{f}^{\mathrm{k}}
$$

Where $a_{0}, a_{1}$ and $k$ (exponent of the attenuation factor) are obtained from measurements (Zimmermann \& Dostert, 2002). Their values are determined using a least-squares fitting method. The obtained attenuation parameters values are $k=0.75, a_{0}=3.11 .10^{-4} m^{-1}, a_{1}=$ $1.76 .10^{-8} \mathrm{~s} / \mathrm{m}$ and the phase is easily deducted based on $L^{\prime}$ and $C^{\prime}$ values.

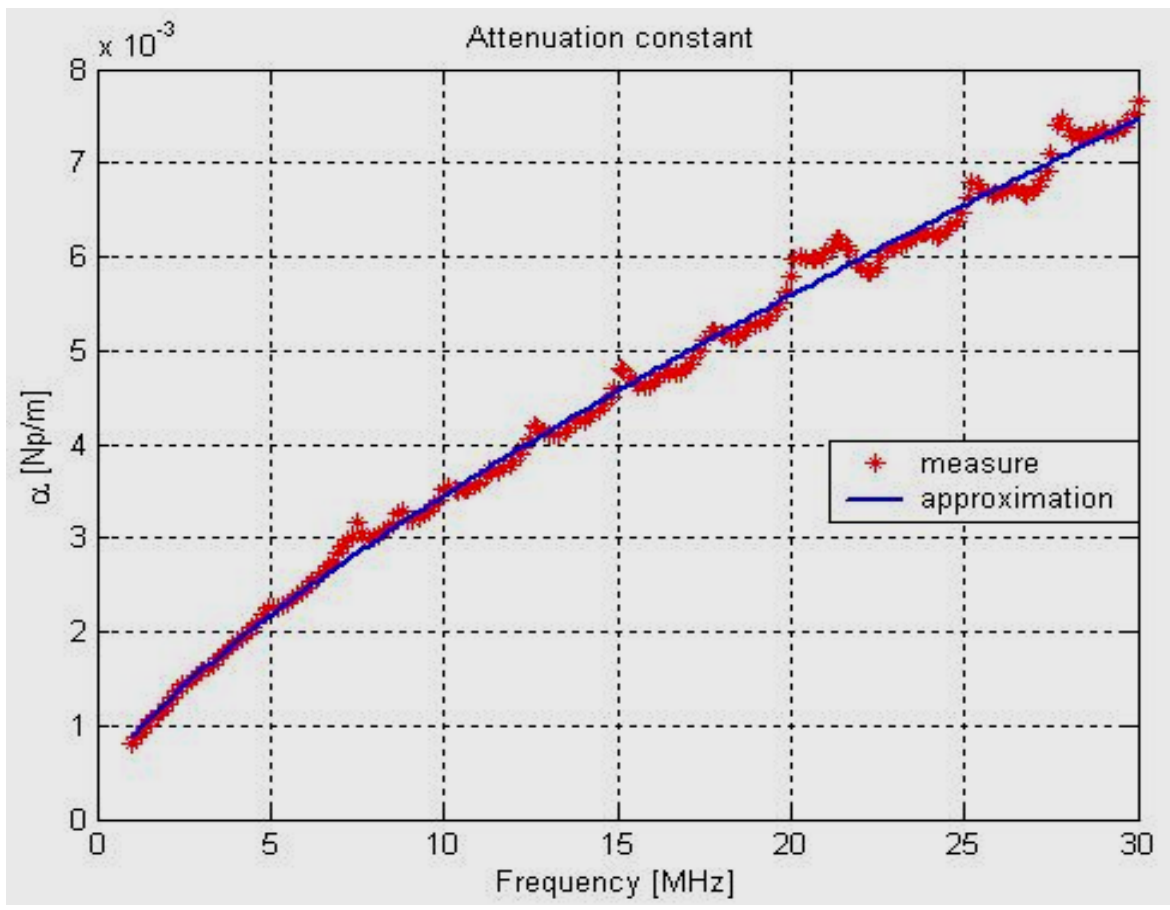

Fig. 4. Attenuation constant of a $40 \mathrm{~m} \mathrm{RO} 2 \mathrm{~V} 3^{*} 2.5 \mathrm{~mm}^{2}$ cable 


\section{Power Line Channel Model}

It has been said that optimization of a transmission system is realizable only when a reasonably accurate channel model is available for investigating the power line network performance in detail (Biglieri et al., 2003). The model proposed by Hensen was straight forward. The attenuation was increasing with frequency and do not take into consideration the multiple phenomenon (Hensen \& Schulz, 2000).

Dostert and Zimmermann say that the only way to model the LV power line network for high frequencies is empirically as like their model. We investigated some deterministic models and implemented simulations using MATLAB software package. This software is readily available and it combines mathematical functions as well as the capability to handle long iterations of complex variables. It allows also graphing and comparing results in a simple manner. The execution time of all simulations (see section 7) does not exceed two minutes. Deterministic model basically means finding the transfer function theoretically without taking actual measurements of the transmission line. These usual models are implemented using consistent techniques based on matriciel calculation to present the complex multi-path network. The model that was followed uses chain matrix theory which is used to compute the transfer function of transmission lines.

In Fig.5, the relation between $V_{1}, V_{2}, I_{1}$ and $I_{2}$ (the input and output voltages and currents of a two port network in the frequency-domain) is represented by:

$$
\left[\begin{array}{l}
\mathrm{V}_{1} \\
\mathrm{I}_{1}
\end{array}\right]=\left[\begin{array}{ll}
\mathrm{A} & \mathrm{B} \\
\mathrm{C} & \mathrm{D}
\end{array}\right]\left[\begin{array}{l}
\mathrm{V}_{2} \\
\mathrm{I}_{2}
\end{array}\right]
$$

Where, the $A B C D$ coefficients are generally complex functions of frequency, and fully characterize the electrical properties of a two port network.

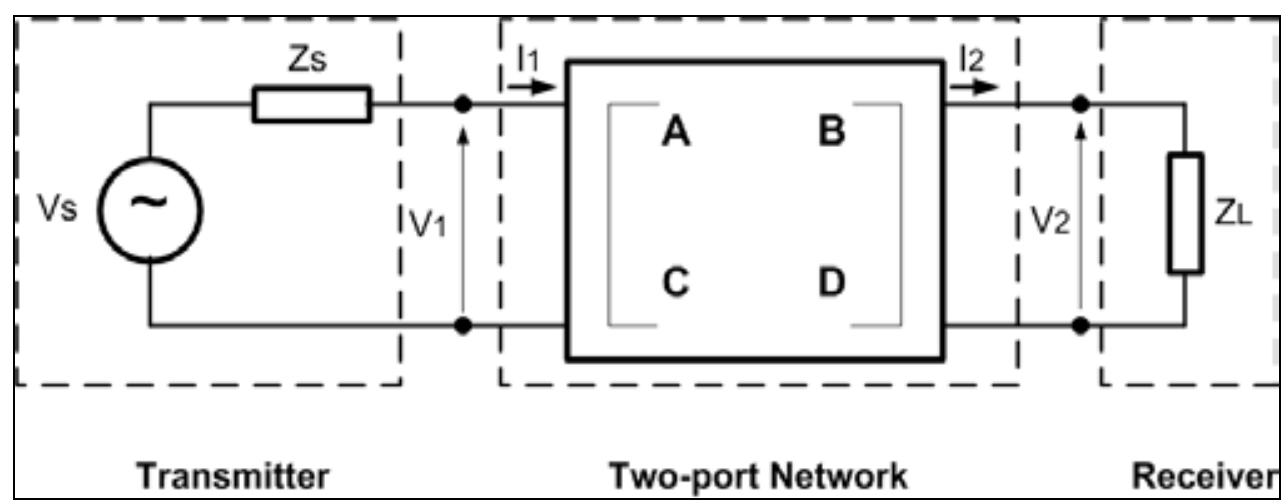

Fig. 5. Block diagram of two port network connected to a Transmitter and Receiver

The $A B C D$ parameters allow us to calculate two important quantities: the transfer function as ratio of the voltage on the load to the source voltage and the input impedance of the network. 


$$
H(f)=\frac{V_{L}}{V_{s}}=\frac{Z_{L}}{A Z_{L}+B+C Z_{S} Z_{L}+D Z_{S}}
$$

With

$$
Z_{\text {in }}=\frac{A Z_{L}+B}{C Z_{L}+D}
$$

Where, $Z_{S}$ and $Z_{L}$ are respectively the source and the load impedance. In the case of a two conductor Transmission Line, the $A B C D$ coefficients and the corresponding transmission matrix $T$ take on the following expression:

$$
\mathrm{T}_{\mathrm{i}}=\left(\begin{array}{cc}
\cosh (\gamma \cdot 1) & \mathrm{Z}_{\mathrm{C}} \cdot \sinh (\gamma \cdot \mathrm{l}) \\
\mathrm{Z}_{\mathrm{C}}^{-1} \cdot \sinh (\gamma \cdot \mathrm{l}) & \cosh (\gamma \cdot \mathrm{l})
\end{array}\right)
$$

Where $l, \gamma$ and $Z_{C}$ are the length, the propagation constant and characteristic impedance of the cable, respectively. These quantities can be either estimated from measurement as done in section 3 .

For information, all parameters $Z_{i n}, r, Z_{C}$ and the quantities $V_{L}, V_{S}, A, B, C$ and $D$ are complex values.

\section{Generalized Determination Method for the Low Voltage PLC Channel}

This deterministic based model has been implemented and tested on the indoor LV PLC network presented by $P$ sections, $N$ derivations and $M$ nodes (see Fig. 6).

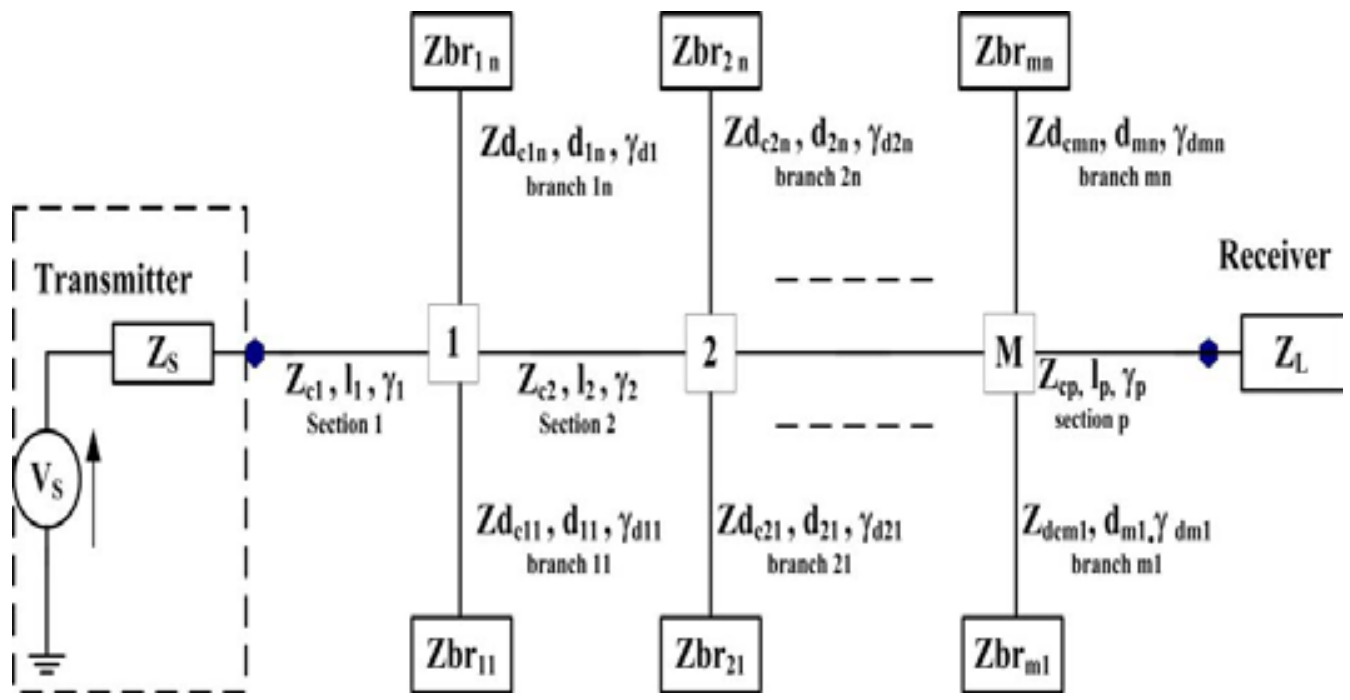

Fig. 6. Power line network with disturbed branches ( $M$ nodes and $N$ derivations) 
The circuit has been partitioned into $2 P$ cascade two-port sub-circuits as shown in Fig. 7. For each sub-circuit it is possible to calculate an $\mathrm{Ai}, \mathrm{Bi}, \mathrm{Ci}$ and $\mathrm{Di}$ coefficients. The total matrix $\mathrm{T}$ is defined as:

$$
\mathrm{T}=\left(\begin{array}{ll}
\mathrm{T}_{11} & \mathrm{~T}_{12} \\
\mathrm{~T}_{21} & \mathrm{~T}_{22}
\end{array}\right)=\mathrm{T}_{0} \cdot \underset{\mathrm{i}=1}{\mathrm{P}-1} \mathrm{~T}_{\mathrm{i}} \cdot \mathrm{T}_{\mathrm{di}} \cdot \mathrm{T}_{\mathrm{P}}
$$

Where,

$$
\mathrm{T}_{0}=\left(\begin{array}{cc}
1 & \mathrm{Z}_{\mathrm{s}} \\
0 & 1
\end{array}\right)
$$

The parameters of matrix $T_{i}$ corresponding to the section $i$ are given as:

$$
\mathrm{T}_{\mathrm{i}}=\left(\begin{array}{cc}
\cosh \left(\mathrm{\gamma}_{\mathrm{i}} \mathrm{l}_{\mathrm{i}}\right) & \mathrm{Z}_{\mathrm{Ci}} \cdot \sinh \left(\mathrm{\gamma}_{\mathrm{i}} \mathrm{l}_{\mathrm{i}}\right) \\
\mathrm{Z}_{\mathrm{Ci}}^{-1} \cdot \sinh \left(\mathrm{\gamma}_{\mathrm{i}} \mathrm{l}_{\mathrm{i}}\right) & \cosh \left(\mathrm{\gamma}_{\mathrm{i}} \mathrm{l}_{\mathrm{i}}\right)
\end{array}\right)
$$

In our study, we have $P$ sections of transmission lines with lengths $l p$ is connected to a node $m, Z b r_{i j}$ is the load at the terminal of each branch and $Z c d_{i j}$ is the characteristic impedance for the node $i$ of the $j$ transmission line branch. $T_{p}$ is the transmission matrix of the last section $P$. $T_{d i}$ is the equivalent transmission matrix of branches connected at node $i$ given by:

$$
\mathrm{T}_{\mathrm{di}}=\left(\begin{array}{cc}
1 & 0 \\
\sum_{\mathrm{j}=1}^{\mathrm{N}} \frac{1}{\overline{\mathrm{Zd}_{\mathrm{eq}}}} & 1
\end{array}\right)
$$

Where, $N$ represents the number of derivations at node $i$, and $Z d_{\text {eqij }}$ is defined as:

$$
\bar{Z} d_{e q i j}=\bar{Z} c d_{i j} \frac{\bar{Z} b r_{i j}+\bar{Z} c d_{i j} \cdot \tanh \left(\overline{\gamma_{d i j}} \cdot d_{i j}\right)}{\bar{Z} b r_{i j} \cdot \tanh \left(\overline{\gamma_{d i j}} \cdot d_{i j}\right)}
$$




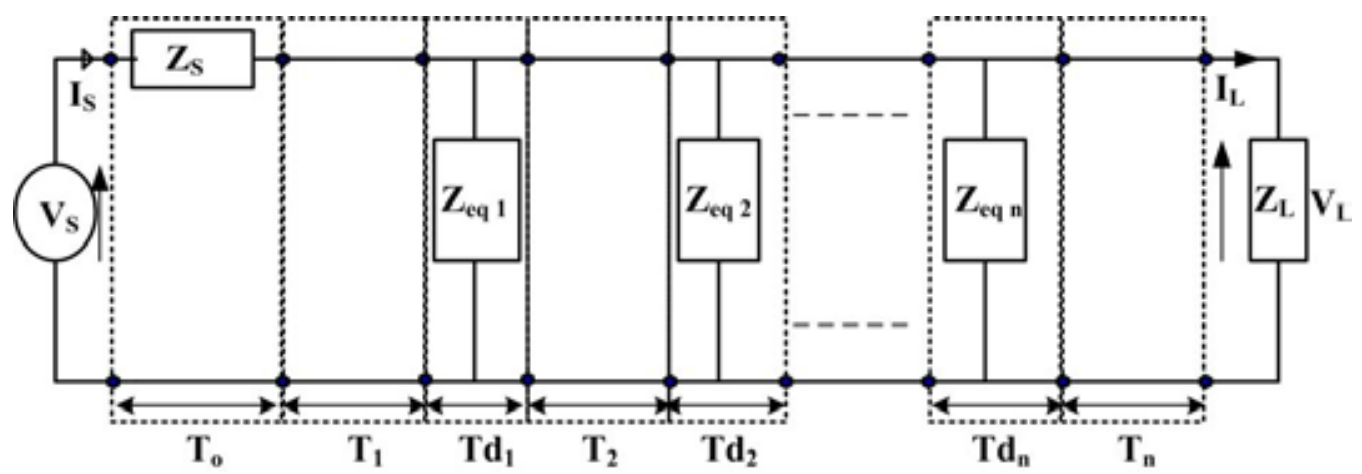

Fig. 7. Basic voltage and current definitions for Two-Port Network with distributed branches

After analyzing the single-branch network in section 5, the remaining task is the determination of the scattering matrix for a cascade of several single-branch networks. Using the microwave theory, there are generally two methods that can be used. The first method is to use the chain scattering matrix (or T-matrix), and the second is to use signal flow graph. For the sake of easier computation, the first method is used in this paper. The relation between the S-matrix parameters (usually measured by a network analyzer) and Tmatrix parameters is given by (Misra, 2004).

$$
\mathrm{T}=\left(\begin{array}{cc}
\mathrm{T}_{11} & \mathrm{~T}_{12} \\
\mathrm{~T}_{21} & \mathrm{~T}_{22}
\end{array}\right)=\left(\begin{array}{cc}
\frac{1}{\mathrm{~S}_{21}} & -\frac{\mathrm{S}_{22}}{\mathrm{~S}_{21}} \\
\frac{\mathrm{S}_{11}}{\mathrm{~S}_{21}} & \mathrm{~S}_{12}-\frac{\mathrm{S}_{11} \cdot \mathrm{S}_{22}}{\mathrm{~S}_{21}}
\end{array}\right)
$$

The S-matrix for the whole network can be obtained by using the following conversion equation:

$$
S=\left(\begin{array}{ll}
S_{11} & S_{12} \\
S_{21} & S_{22}
\end{array}\right)=\left(\begin{array}{cc}
\frac{T_{21}}{T_{11}} & T_{22}-\frac{T_{21} \cdot T_{12}}{T_{11}} \\
\frac{1}{T_{11}} & -\frac{T_{12}}{T_{11}}
\end{array}\right)
$$

Finally, the $\left(T_{11}, T_{12}, T_{21}\right.$ and $\left.T_{22}\right)$ parameters allow us to calculate two important quantities: the generalized transfer function can be represented as ratio of $V_{L}$ to $V_{S}$, and the input impedance of the network with distributed branches.

$$
\begin{gathered}
H(f)=\frac{Z_{L}}{T_{11} Z_{L}+T_{12}+T_{21} Z_{S} Z_{L}+T_{22} Z_{S}} \\
Z_{\text {in }}(f)=\frac{T_{11} Z_{L}+T_{12}}{T_{21} Z_{L}+T_{22}}
\end{gathered}
$$




\section{Simulation Results}

To explore the range of possible PLC, we now consider a typical low voltage line power network. We present the results of our analysis applied to a variety of wire configurations. In all studies, we consider $Z_{L}=Z_{S}=50 \Omega$ and all the lines per unit length parameters are $L^{\prime}=$ $0.327 \mu \mathrm{H} / \mathrm{m}$ and $C^{\prime}=66.9 \mathrm{pF} / \mathrm{m}$.

\subsection{Influence of branch length}

As an example we start study using a channel with one bridge taps. The corresponding configuration is given in Fig. 8. In this figure, $l_{1}=l_{2}=15 \mathrm{~m}\left(l_{1}\right.$ and $l_{2}$ are the lengths of two channel sections), $d_{11}$ is the length of distributed branch which is varied as $5 \mathrm{~m}, 10 \mathrm{~m}, 15 \mathrm{~m}$ and $20 \mathrm{~m}$. The load $Z b r_{11}$ was terminated in open circuit, while $Z_{C 1}, Z_{C 2}$ and $Z d_{C 11}$ are the line characteristic impedance $\left(Z_{C 1}=Z_{C 2}=Z d_{C 11}=69.9 \Omega\right)$.

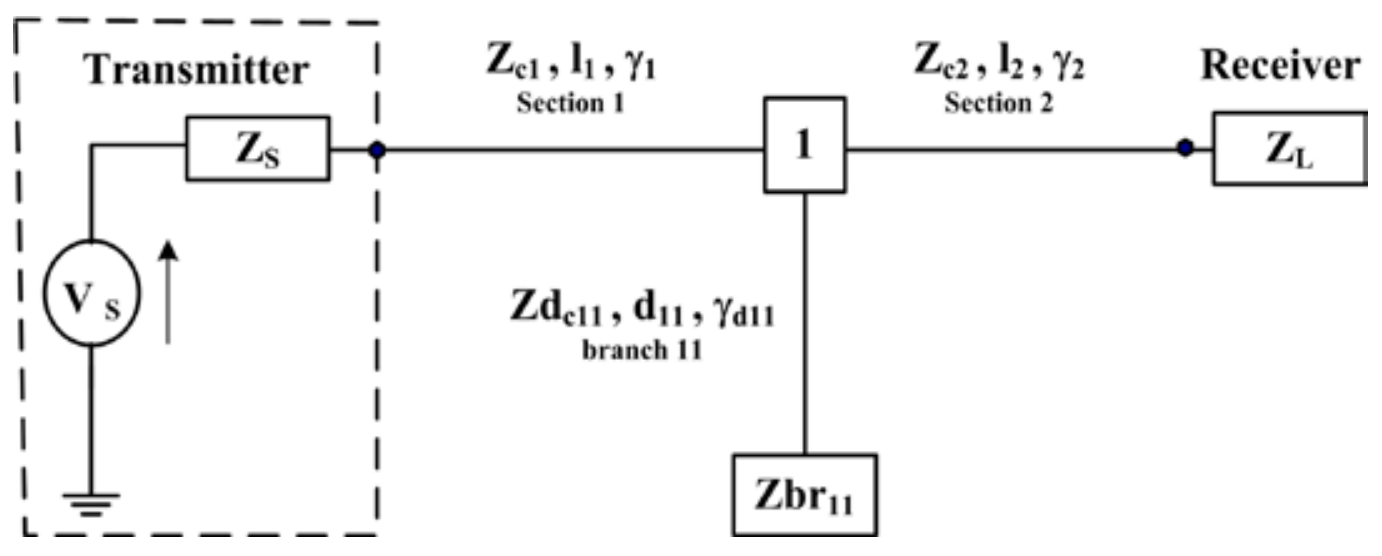

Fig. 8. Configuration of simulated network with a branch

In this case of a two conductor transmission line with two sections, the transmission matrix $T$ takes on the following expression:

$$
\mathrm{T}=\mathrm{T}_{0} \cdot \mathrm{T}_{1} \cdot\left(\begin{array}{cc}
1 & 0 \\
\frac{1}{Z \mathrm{~d}_{\mathrm{eq} 11}} & 1
\end{array}\right) \cdot \mathrm{T}_{2}
$$

Fig. $9(a, b$ and $c)$ show the corresponding frequency responses for various branch line lengths. We observed that in three cases the peaks of frequency responses was not either attenuating with frequencies. As the length of branched line increases the number of notches increases proportionally. 

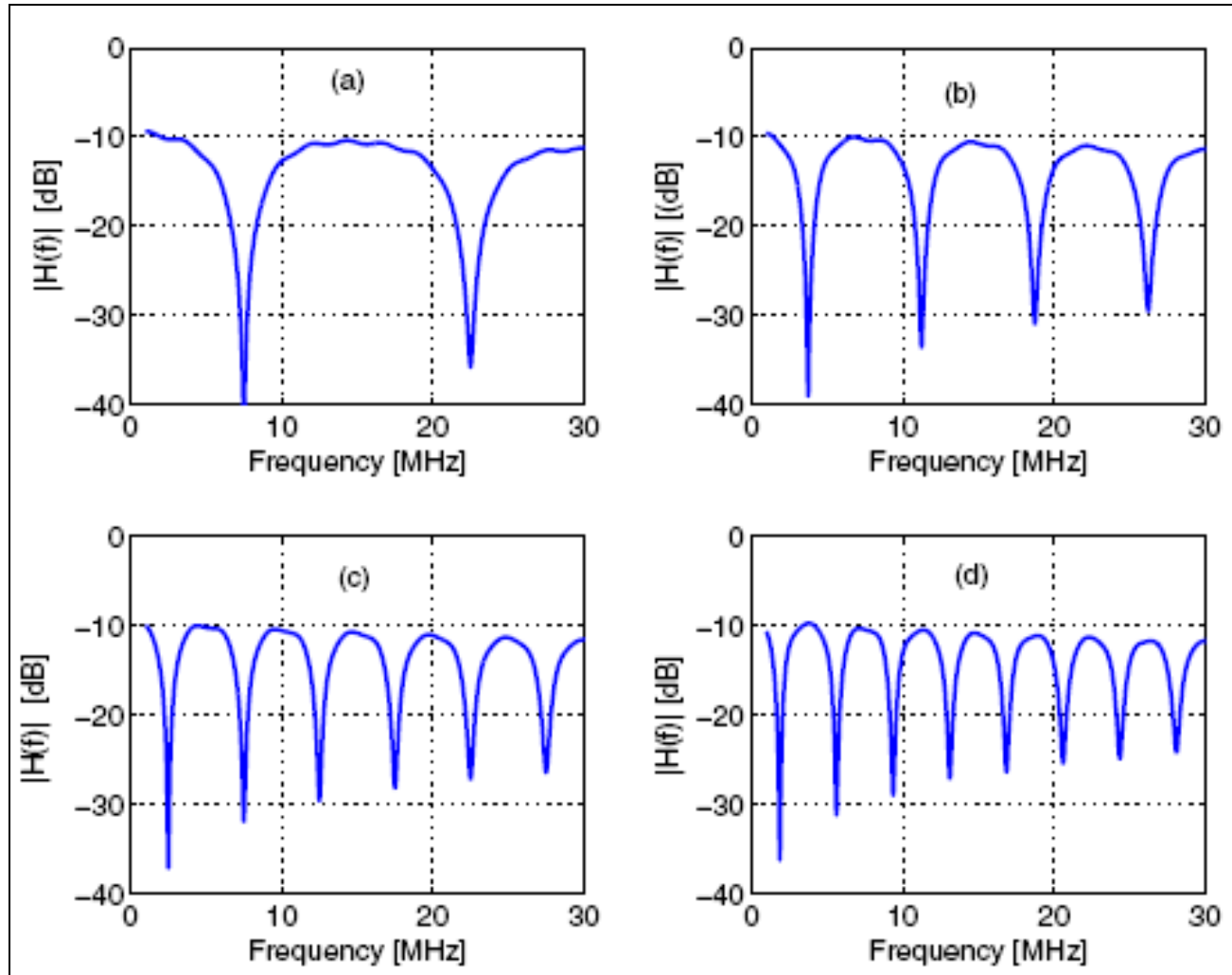

Fig. 9. Simulation results for low channel of power line with one branch of length (a) $5 \mathrm{~m}$, (b) $10 \mathrm{~m},(\mathrm{c}) 15 \mathrm{~m}$ and (d) $20 \mathrm{~m}$

\subsection{Influence of number of branches}

To analyze the effect of number of branches, we consider the configuration shown in Fig. 10. The line length Transmitter- Receiver (Tx-Rx) was kept constant at $60 \mathrm{~m}$; i.e. $l_{1}=l_{2}=l_{3}=l_{4}=$ $15 \mathrm{~m}$, with one, two and three disturbed branches. All branches were $5 \mathrm{~m}$ long $\left(d_{11}=d_{21}=d_{31}\right.$ $=5 \mathrm{~m}$ ) and load $Z b r_{11}, Z b r_{21}$ and $Z b r_{31}$ were terminated in open circuit, while $Z d_{C 11}, Z d_{C 21}$ and $Z d_{C 31}$ are the line characteristic impedances. We repeat the same test as before simulating the transfer function with respect the number of branches.

In this case of a two conductor Transmission Line with four sections, the transmission matrix $T$ takes on the following expression:

$$
\mathrm{T}=\mathrm{T}_{0} \cdot \prod_{\mathrm{i}=1}^{3} \mathrm{~T}_{\mathrm{i}} \cdot\left(\begin{array}{cc}
1 & 0 \\
\frac{1}{\mathrm{Zd_{ \text {eqi } 1 }}} & 1
\end{array}\right) \cdot \mathrm{T}_{4}
$$




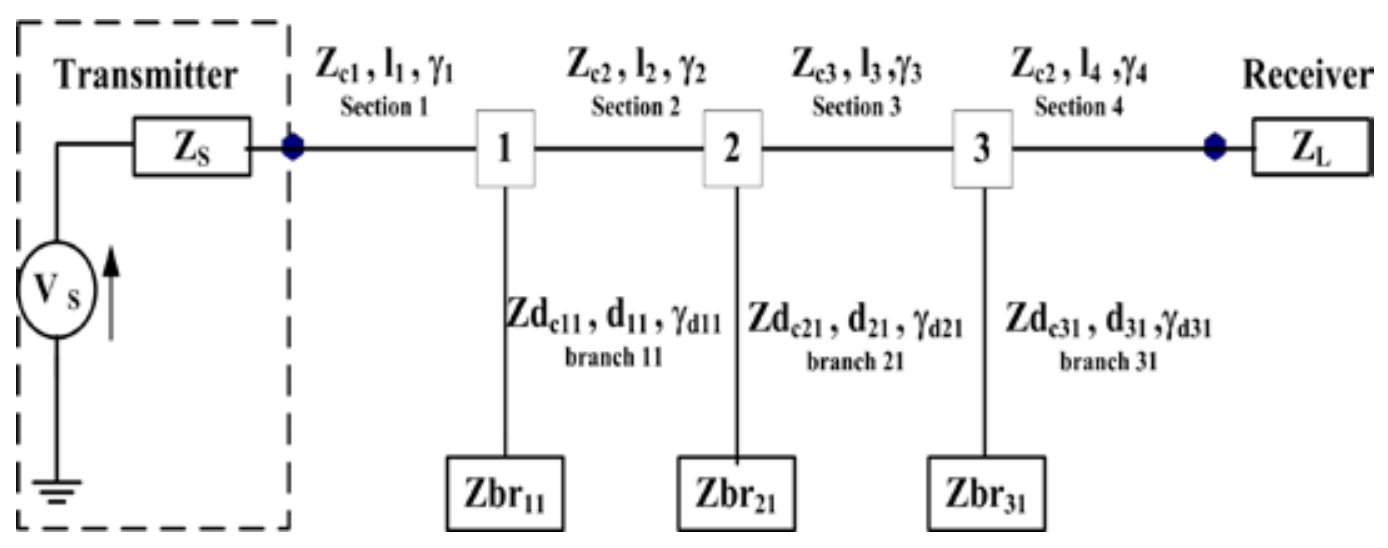

Fig. 10. Power line network with one branch at each node

Fig. 11 shows the transfer function for different number of branches. We noted that the positions of notches are not changed but as the number of branches increases the attenuations of notched point tends to increase.

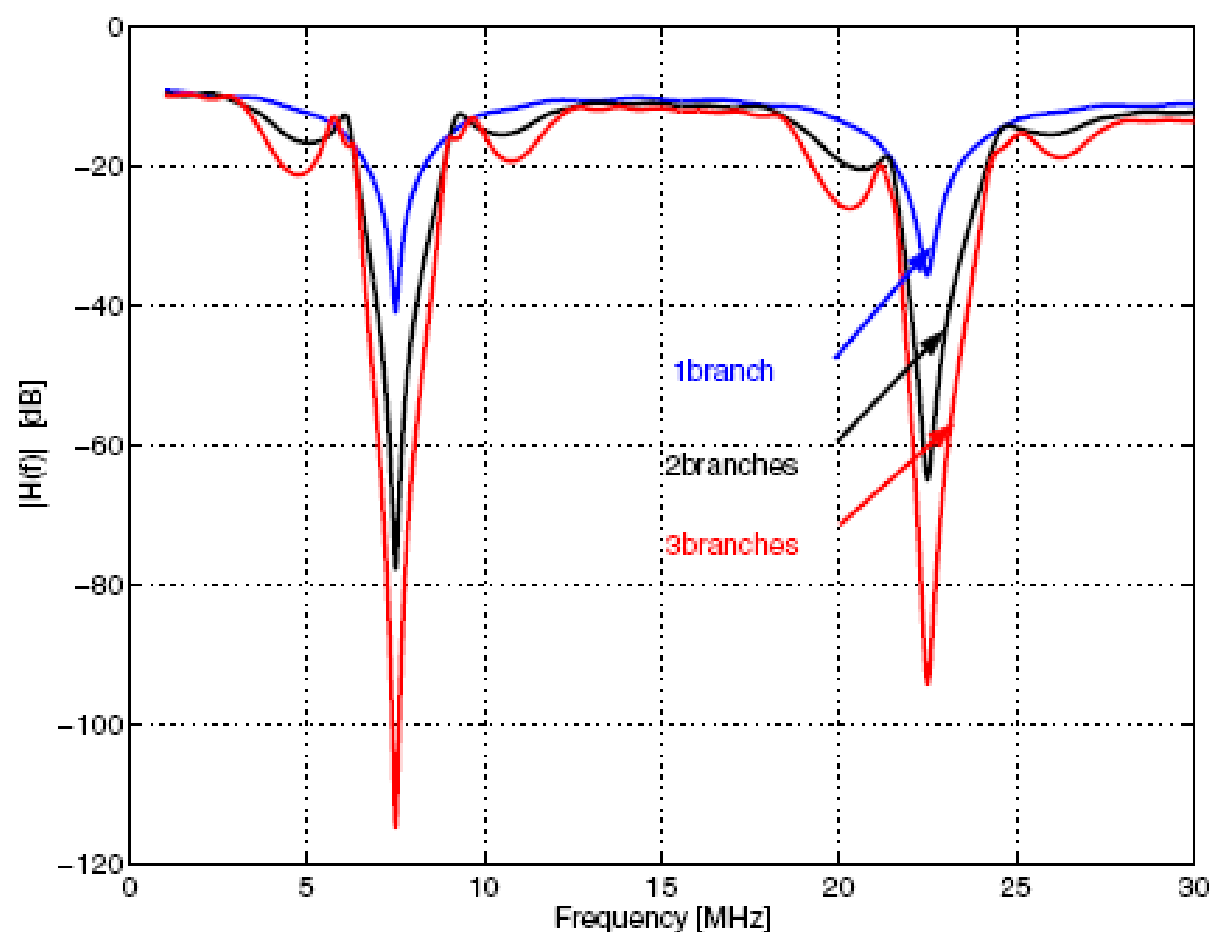

Fig. 11. Simulation results with distributed branches, 1 branch, 2 branches and 3 branches 
We have observed the same result of the power line network with multiple branches at a single node. In fact, the positions of notches are not changed and the number of branches increase allows increasing the amplitude response of notched points.

\subsection{Influence of impedance matching}

This study is accentuated here because it is common that the loads at the termination of branched lines are not only opened circuit. For discussions below we consider the configuration as in Fig. 8. The line length Transmitter-Receiver (Tx-Rx) was kept constant at $30 \mathrm{~m}\left(l_{1}=l_{2}=15 \mathrm{~m}\right)$ while the branch of length $10 \mathrm{~m}$ is connected to the middle of line (Tx$\mathrm{Rx}$ ). The termination $\mathrm{Zbr}_{11}$ was varied according to given load impedance under our investigation. Firstly, we consider the following load impedances with values $69.9 \Omega$, shorted circuit $\left(Z b r_{11}=C . C\right)$ and opened circuit $\left(Z_{b r} r_{11}=C . O\right)$. Remember that $69.9 \Omega$ is the characteristic impedance of the branch. For the load impedance shorted and opened, the position of notches and peaks are shifted in the frequency positions with same attenuation. However for the load characteristic impedance the peeks and notches are disappeared (see Fig. 12).

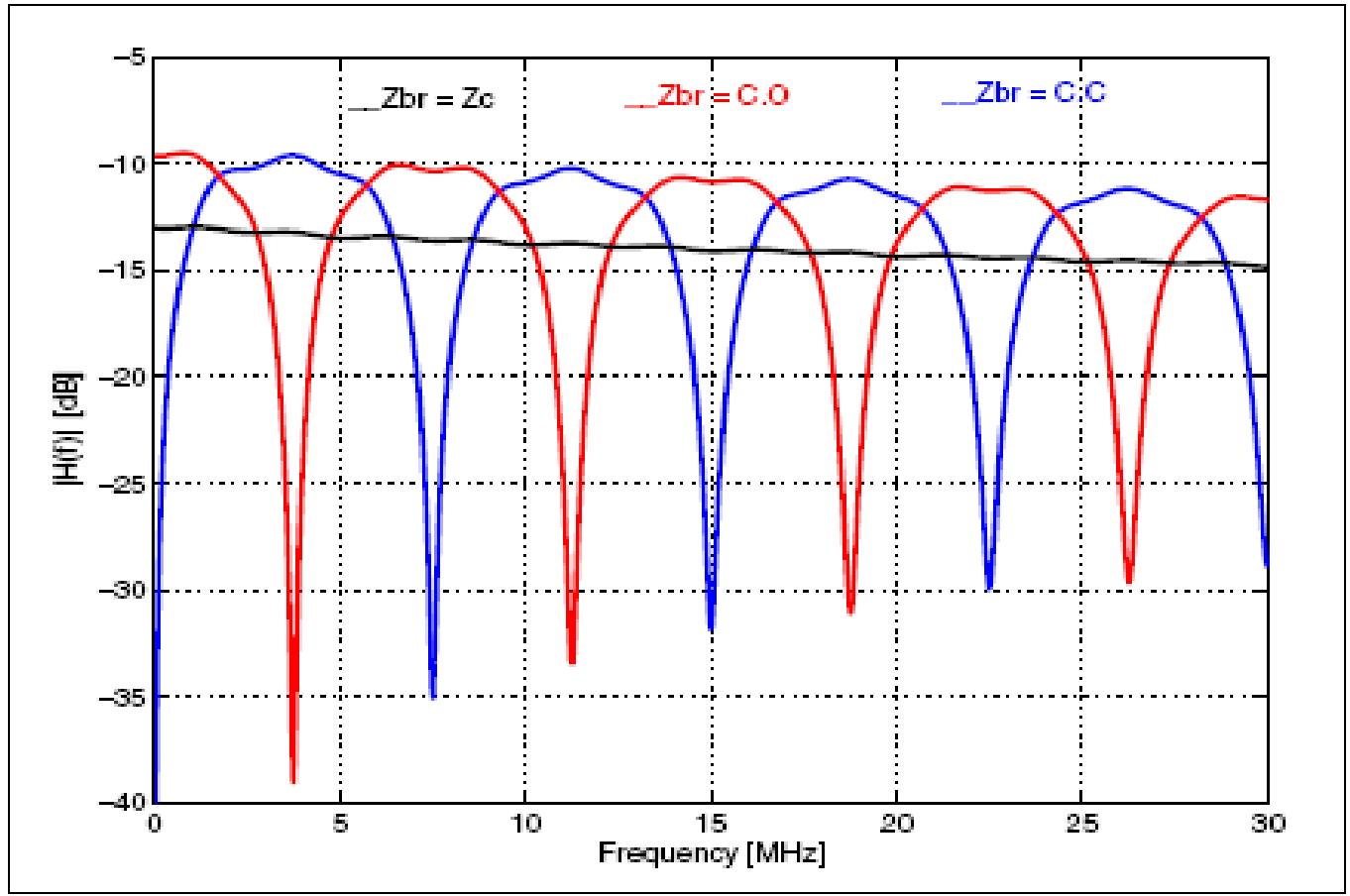

Fig. 12. Simulation results for a LV channel with a branch terminated in open circuit, short circuit and characteristic impedance.

Secondly we propose to analyze the behavior of the characteristic impedance when realistic loads are used. The impedance of proposed realistic loads (TV and microwave) is measured by using the method proposed in (Tang, 2001). The impedance of the power network $Z_{r e f}$ is first measured through a socket when the appliance is not connected to the network. After, 
the appliance is connected near the socket and the network impedance $Z_{\text {tot }}$ is again measured. Therefore, the impedance of the appliance $Z_{a p p}$ can be deducted by:

$$
Z_{\text {tot }}=Z_{\text {app }} / / Z_{\text {ref }}
$$

After rearranging, we get

$$
Z_{\text {app }}=\frac{Z_{\text {ref }} \cdot Z_{\text {tot }}}{Z_{\text {ref }}-Z_{\text {tot }}}
$$

Fig. 13 illustrates the measured impedance of the TV and microwave, always in the frequency range of $1-30 \mathrm{MHz}$. These impedances were used in the derivation of the transfer function of the network under test in Fig. 8.

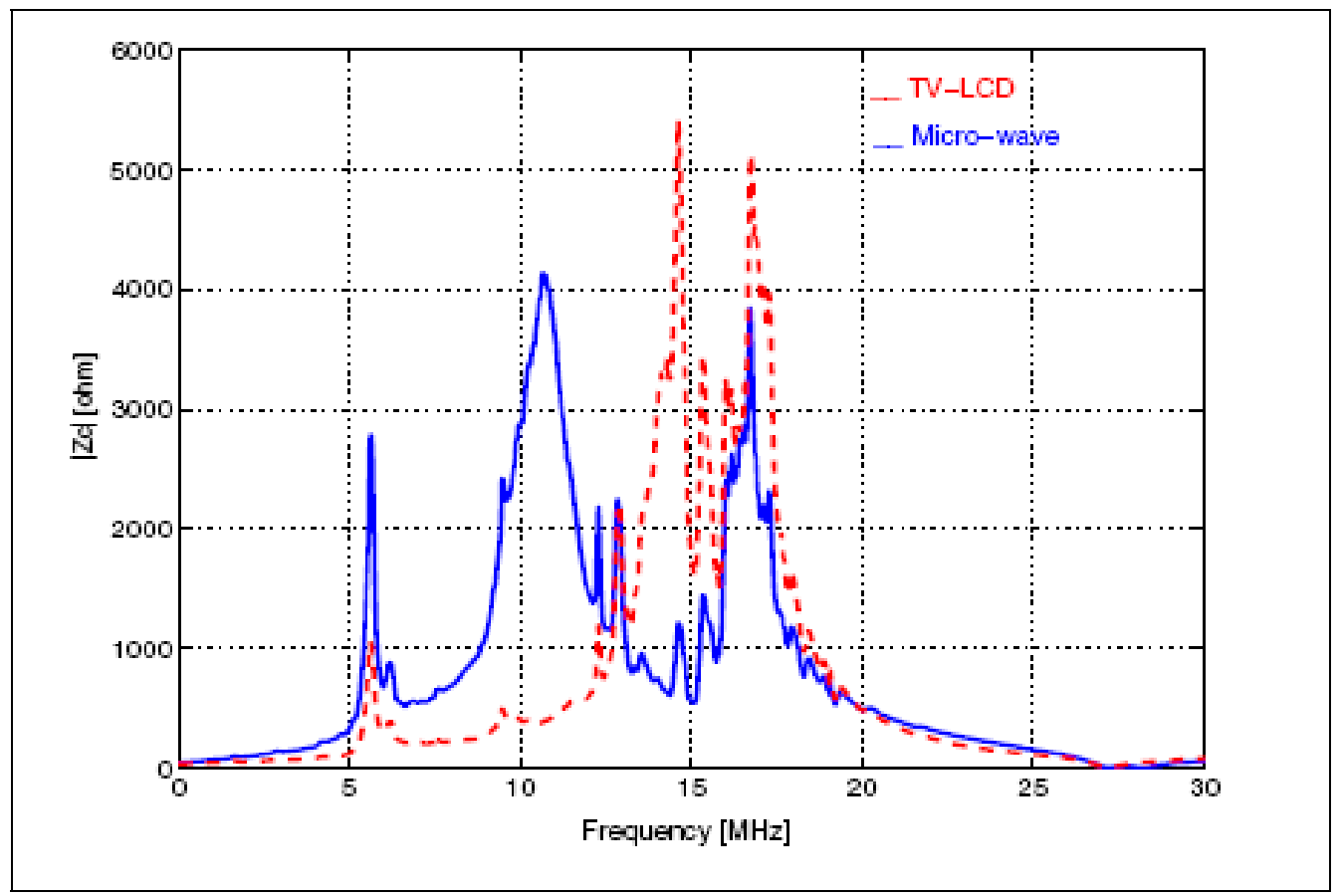

Fig. 13. Impedance of loads TV and Microwave

In this subsection, we have evaluated the response of the magnitude of the derived transfer function for two loads TV and the microwave (see Fig. 14). A strong attenuation notch at about $18 \mathrm{MHz}$, for both cases is mainly caused by the impedances of the branched loads. For $\mathrm{TV}$, the strength of the attenuation at two notches near $10 \mathrm{MHz}$ and $20 \mathrm{MHz}$ is about 40 and $50 \mathrm{~dB}$. For the microwave, we noted an additional notch near $3 \mathrm{MHz}$ attenuated about $40 \mathrm{~dB}$. 


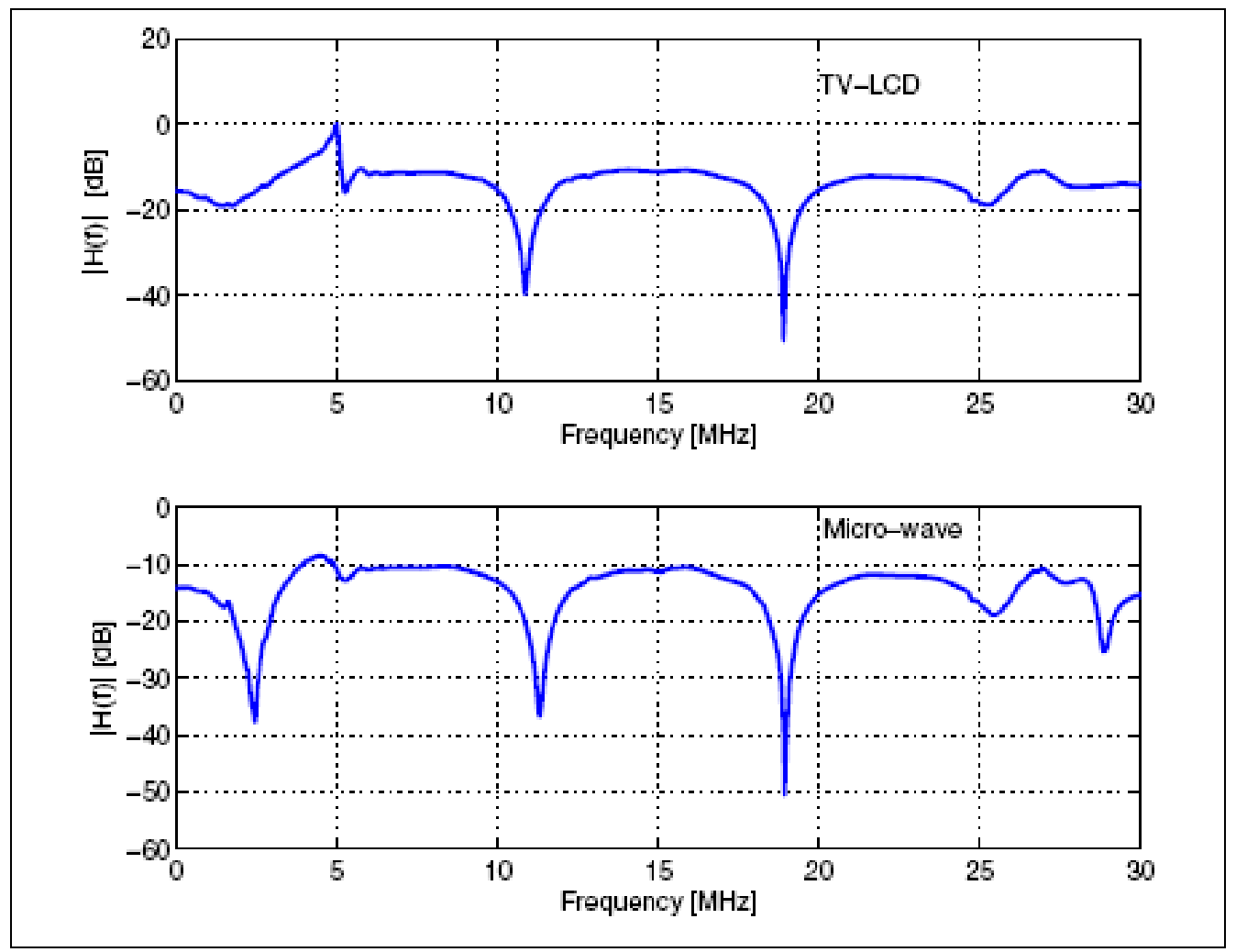

Fig. 14. Simulation results for power line LV network with a branch terminated in TV and Microwave.

\section{Validation of the Model by measurements}

For Validation the results of simulations based on the proposed model are compared with measurements. This was done on the one hand with a sample network with well known topology and geometric dimensions and on the other hand with a real in-home network.

We realized a measurements of the transmission channel characteristics. At the transmitting and the receiving side, an Agilent 4395A network analyzer was located. In addition to this measuring accuracy device, a balun was used to segregate devices from the power distribution network. Data was collected using a network analyzer. The unit was interfaced to a laptop PC, which provided automated control and data archiving. The spectrum analyzer was used in lieu of the regular electronics so that we could continue to work on the while this lengthy measurement ( 3 full days) was underway. The total transfer function including the active balun used in this testing, results shown above are presented in the following. 


\subsection{Power line with one branch}

As an example, we start study using a channel with one bridge taps. The corresponding configuration is given in Fig. 8. In this figure, $l_{1}=l_{2}=15 \mathrm{~m}\left(l_{1}\right.$ and $l_{2}$ are the lengths of two channel sections), $d_{11}$ is the length of distributed branch which is $15 \mathrm{~m}$. The load $Z b_{11}$ was terminated in open circuit, while $Z_{C 1}, Z_{C 2}$ and $Z d_{C 11}$ are the line characteristic impedance $\left(Z_{C 1}=Z_{C 2}=Z d_{C 11}=69.9 \Omega\right)$. The measurement points are refered by $(T x)$ and $(R x)$.

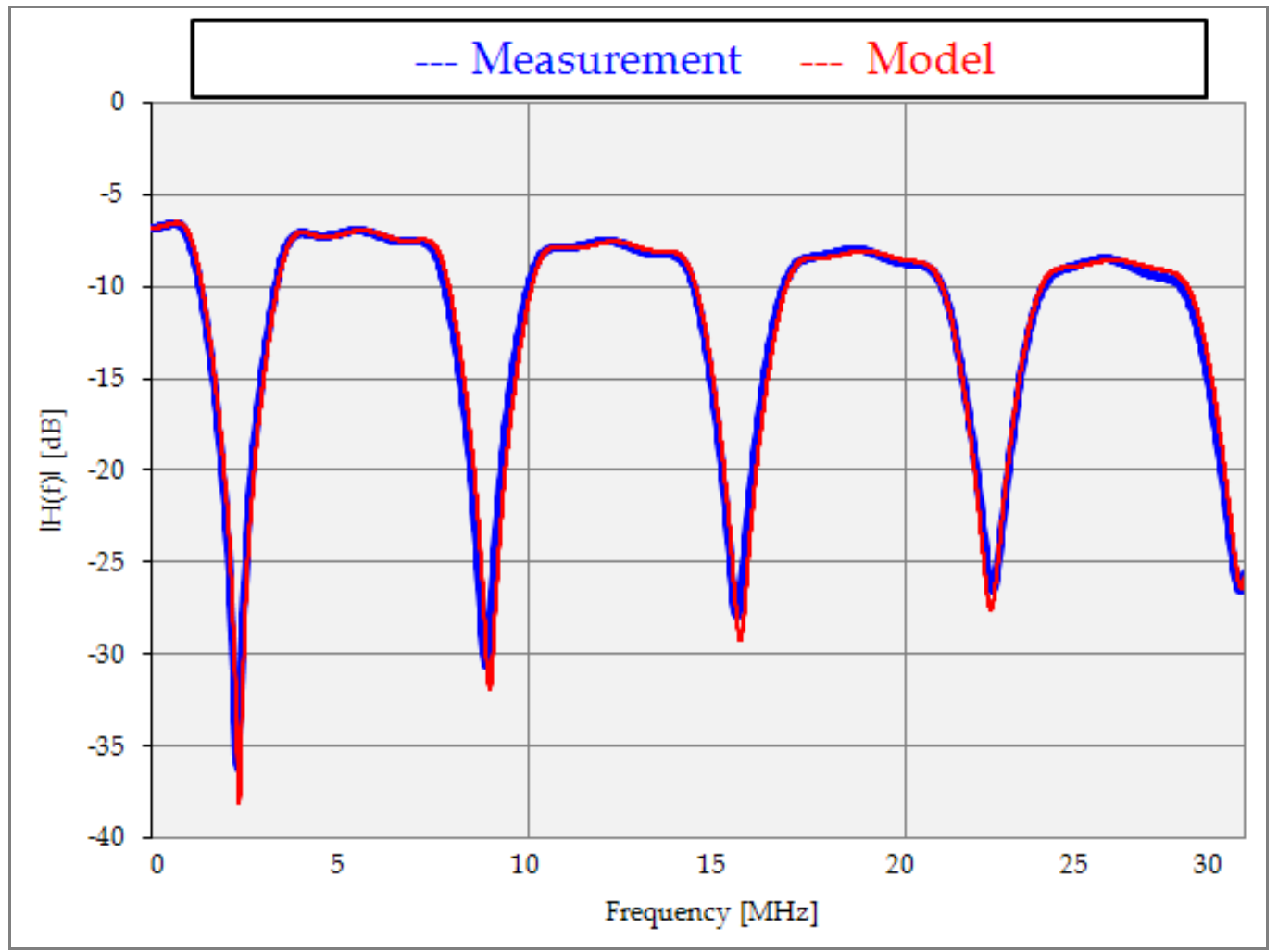

Fig. 15. Comparison between measurement and model of the response of power line channel with one branch

The plots in Fig. 15 illustrate the frequency behavior of transfer function obtained by simulations and measurements. This figure demonstrates that measured transfer function is perfectly close to that simulated by the model.

\subsection{Power line network with two branches}

To analyze the effect of number of branch, we consider the configuration as given in Fig. 8 with two disturbed branches, both branches were $15 \mathrm{~m}$ long $\left(\mathrm{d}_{1}=\mathrm{d}_{2}=15 \mathrm{~m}\right), Z b r_{11}$ and $Z b r_{12}$ were terminated in open circuit, while $Z d c_{1}$ and $Z d c_{2}$ are the line characteristic impedances. Measurements with correct results are presented in Fig. 16. Again, the measurements confirm assumptions that the signal attenuation is directly proportional to the number of 
branches. The measured transfer function is very close to that modeled, also the positions of notches are not changed (compared to the previous case, see Fig.15).

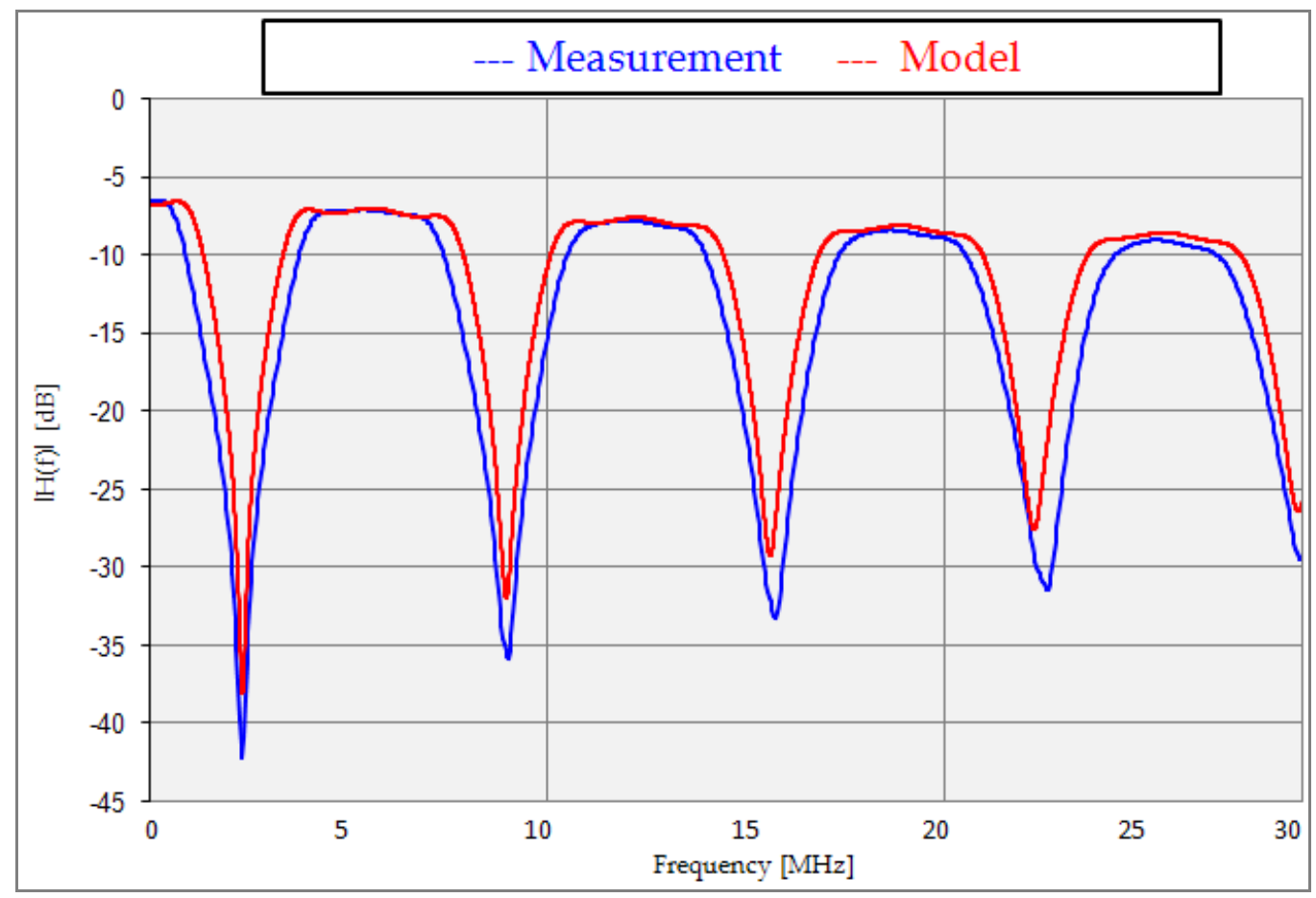

Fig. 16. Comparison between measurement and model of the response of power line channel with two branches

We repeat this test with three branches at each node, we observed that the number of distributed branches increases the amplitude of notched points tends to increase. However, but the position of notches in frequency response depends on the length of branched line, the number of notches increase proportionally with the line length.

\section{Conclusion}

In this chapter, the modeling procedures of power line channel have been presented. The deterministic method uses basic network parameters to derive a transfer function of the channel. The investigated deterministic models were determined from an indoor PLC channel. They are specifically topology dependent. For separate network channels, only the cable parameters, the load impedances and the topology of the network are absolutely needed. The LV network is considered as an $M$ nodes and $N$ branches, which is subdivided into several cascaded two-port of small networks. The transfer function of the channel is later obtained by combining easily the T-matrices of the cascades sub networks. The position of notches in frequency response depends on the length of the branched lines. The increase in branched line length tends to limit the available bandwidth in LV channel, but the 
position of deep notches does not changed with a number of distributed branches. As the number of distributed branches increases the amplitude of notched points tends to increase. The impedance of realistic household's loads is also measured and their influence on the transfer function of indoor channel is analyzed. In power line network, the variation of these impedances with the frequency at the terminations causes a few distortions. The model used has indicated to come up with good results, hence can be used satisfactorily to characterize power line network.

This work has been developed in the frame of the French competitiveness pole "Images \& Réseaux". Sponsor and financial support acknowledgment goes here.

\section{References}

Roka, R. \& Urminsky, J. (2008), “Experimental Measurements for Verification of the Parametric Model for Reference Channels in the Real PLC Environment ", Journal of Electrical Engineering, Vol. 59, No. 3, pp. 146-152.

Meng, H. \& Chen, S. (2004), "Modeling of Transfer Characteristics for The Broadband Power Line communication channel", IEEE Transactions on Power Delivery, Vol. 19, No 3, pp. 529-551. July.

Roblot, S. (2007), "Caractérisation des couplages électromagnétiques dans les réseaux filaires cuivre en vue d'optimiser les transmissions haut débit", Ph.D. the University of Limoges, October.

Barmada, S.; Musolino, A. \& Raugi, M. (2006), "Innovative Model for Time-Varying PLC Response Evaluation", IEEE Journal on Selected Areas in Communications, vol. 24, no. 7, pp. 1317-1326, July.

Moreno-Rodriguez, G. (2008), "An IIR-Filter Approach to Time Variant PLCChannel Modelling", Proc.11 th Int. Symp. on Powerline Communications and Its Applications, pp. 87-92, Jeju Island, Korea, April.

Philips, H. (1999), "Modeling of power line communication channels", Proc.3rd Int. Symp. on Powerline Communications and Its Applications, pp. 14-21, Lancaster, U.K.

Konaté, C.; Machmoum, M. \& Diouris, J. F. (2007), "Multi path Model for Power Line Communication Channel in the frequency Range of 1MHz-30MHz", EUROCON 2007 The International Conference on Computer as a Tool, Warsaw, pp. 984-989, September.

Anastasiadou, D. \& Antonakoupoulos, T. (2005), "Multipath characterization of Indoor Power-Line Networks", IEEE Trans. On Power Delivery, vol. 20, pp. 90-99.

Zimmermann, M. \& Dostert, K. (2002), "A multipath model for the power line channel", IEEE Trans. Commun., vol. 50, pp. 553-559, April.

Biglieri, E. \& al. (2003), "Coding and Modulation for a Horrible Channel", IEEE Communication Magazine, pp. 70-75, May.

Hensen, C. \& Schulz, W. (2000), "Time Dependence of the channel characteristics of Low Voltage Power-Lines and its Effects on Hardware Implementation", AEU Int'1. J. Electronics and Communication, vol. 54, no. 1, pp. 23-32, February.

Devendra Misra, K. (2004),"RF and Microwave Communication circuits, analysis and design", Second Edition, pp. 283-330, ISBN 0-471-47873-3, Wiley Interscience, a John Wiley and Sons, Inc. Publication.

Tang, L.T. (2001), "development of a high-speed PLC system", Master's thesis, Nan yang Technology. Univ., Singapore. 


\title{
A study of protein structure using amino acid interaction networks
}

\author{
Omar Gaci and Stefan Balev \\ University of Le Havre \\ France
}

\section{Introduction}

Proteins are biological macromolecules participating in the large majority of processes which govern organisms. The roles played by proteins are varied and complex. Certain proteins, called enzymes, act as catalysts and increase several orders of magnitude, with a remarkable specificity, the speed of multiple chemical reactions essential to the organism survival. Proteins are also used for storage and transport of small molecules or ions, control the passage of molecules through the cell membranes, etc. Hormones, which transmit information and allow the regulation of complex cellular processes, are also proteins.

Genome sequencing projects generate an ever increasing number of protein sequences. For example, the Human Genome Project has identified over 30,000 genes which may encode about 100,000 proteins. One of the first tasks when annotating a new genome is to assign functions to the proteins produced by the genes. To fully understand the biological functions of proteins, the knowledge of their structure is essential.

In their natural environment, proteins adopt a native compact three-dimensional form. This process is called folding and is not fully understood. The process is a result of interactions between the protein's amino acids which form chemical bonds. In this paper we identify some of the properties of the network of interacting amino acids. We believe that understanding these networks can help to better understand the folding process.

There exist different classifications of proteins according to their structure, such as CATH (Orengo, 1997) and SCOP (Murzin.1995). Proteins from the same class have similar structures and most often, similar functions. In this paper we show that structure classes can also be defined in the terms of the properties of amino acid networks.

\section{Protein Structure}

Unlike other biological macromolecules (e.g., DNA), proteins have complex, irregular structures. They are built up by amino acids that are linked by peptide bonds to form a polypeptide chain. We distinguish four levels of protein structure:

1. The amino acid sequence of a protein's polypeptide chain is called its primary or one- dimensional (1D) structure. It can be considered as a word over the 20-letter letter amino acid alphabet. 
2. Different elements of the sequence form local regular secondary (2D) structures, such as a-helices or $\beta$-strands.

3. The tertiary (3D) structure is formed by packing such structural elements into one or several compact globular units called domains.

4. The final protein may contain several polypeptide chains arranged in a quaternary structure.

By formation of such tertiary and quaternary structure, amino acids far apart in the sequence are brought close together to form functional regions (active sites). The reader can find more on protein structure in (Branden \& Tooze, 1999).

One of the general principles of protein structure is that hydrophobic residues prefer to be inside the protein contributing to form a hydrophobic core and a hydrophilic surface. To maintain a high residue density in the hydrophobic core, proteins adopt regular secondary structures that allow non covalent hydrogen-bond and hold a rigid and stable framework. There are two main classes of secondary structure elements (SSE), a-helices and $\beta$-sheets (see Fig 1).

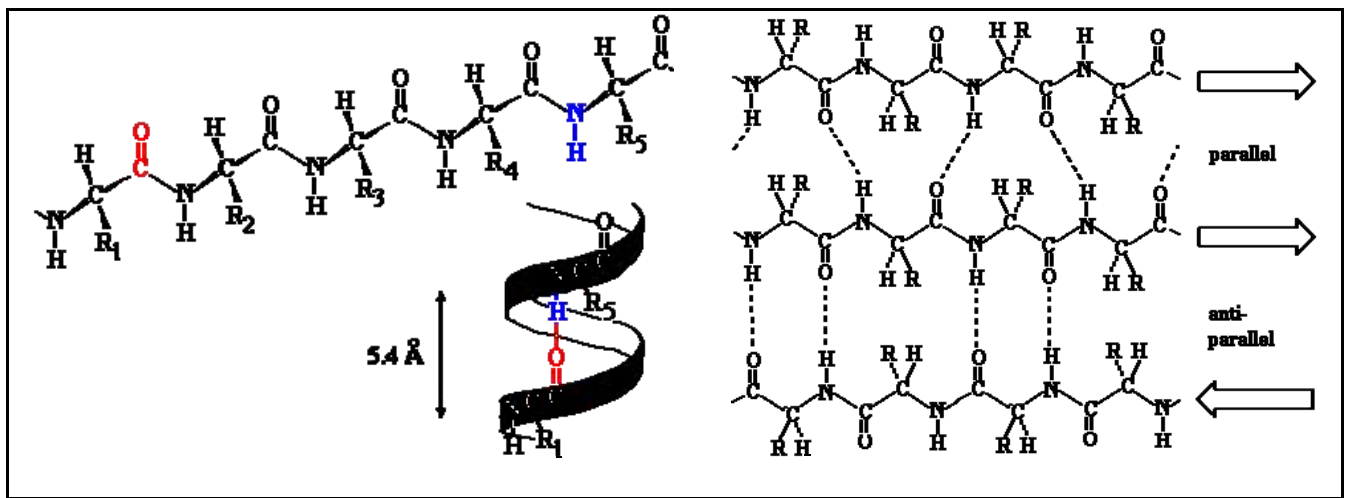

Fig. 1. Left: an a-helix illustrated as ribbon diagram, there are 3.6 residues per turn corresponding to $5.4 \AA$. Right: A $\beta$-sheet composed by three strands.

An a -helix adopts a right-handed helical conformation with 3.6 residues per turn with hydrogen bonds between $\mathrm{C}^{\prime}=\mathrm{O}$ group of residue $n$ and $\mathrm{NH}$ group of residue $n+4$.

A $\beta$-sheet is build up from a combination of several regions of the polypeptide chain where hydrogen bonds can form between $C^{\prime}=O$ groups of one $\beta$ strand and another $\mathrm{NH}$ group parallel to the first strand. There are two kinds of $\beta$-sheet formations, anti-parallel $\beta$-sheets (in which the two strands run in opposite directions) and parallel sheets (in which the two strands run in the same direction).

Based on the local organization of the secondary structure elements (SSE), proteins are divided in the following four classes (Levitt \& Chothia, 1976):

1. All $\alpha$, proteins have only a -helix secondary structure.

2. All $\beta$, proteins have only $\beta$-strand secondary structure.

3. $\alpha / \beta$, proteins have mixed $\alpha$-helix and $\beta$-strand secondary structure.

4. $\alpha+\beta$, proteins have separated $\alpha$-helix and $\beta$-strand secondary structure.

From this first division, a more detailed classification can be done. The most frequently used ones are SCOP, Structural Classification Of Proteins (Murzin et al., 1995), and CATH, Class Architecture Topology Homology (Orengo et al., 1997). They are hierarchical classifications 
of proteins' structural domains. A domain corresponds to a part of a protein which has a hydrophobic core and not much interaction with other parts of the protein.

\section{Models and Methods}

The 3D structure of a protein is determined by the coordinates of its atoms. This information is available in Protein Data Bank (PDB) (Berman et al., 2000), which regroups all experimentally solved protein structures. Using the coordinates of two atoms, one can compute the distance between them. We define the distance between two amino acids as the distance between their Calpha atoms. Considering the Calpha atom as a "center" of the amino acid is an approximation, but it works well enough for our purposes. Let us denote by $N$ the number of amino acids in the protein. A contact map matrix is a $N \times N$ 0-1 matrix, whose element $(i, j)$ is one if there is a contact between amino acids $i$ and $j$ and zero otherwise. It provides useful information about the protein. For example, the secondary structure elements can be identified using this matrix. Indeed, a-helices spread along the main diagonal, while $\beta$-sheets appear as bands parallel or perpendicular to the main diagonal (Ghosh et al., 2007). There are different ways to define the contact between two amino acids. Our notion is based on spacial proximity, so that the contact map can consider non-covalent interactions. We say that two amino acids are in contact iff the distance between them is below a given threshold. A commonly used threshold is $7 \AA$ and this is the value we use.

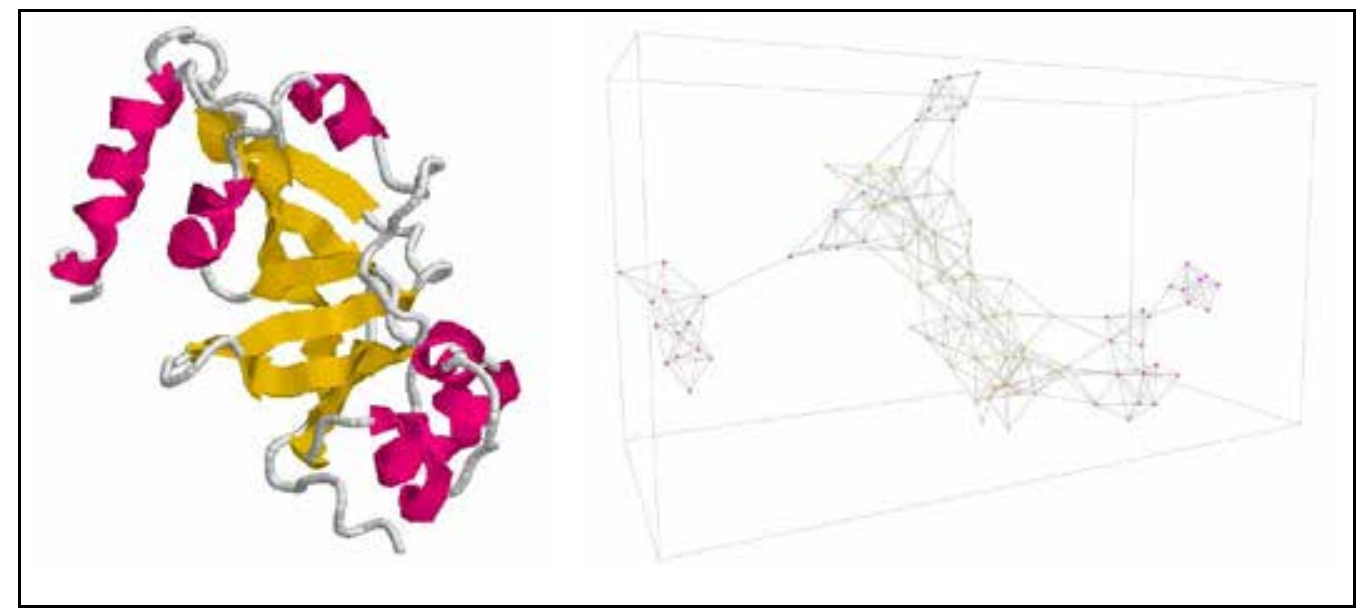

Fig. 2. Protein 1DTP (left) and its SSE-IN (right).

Consider a graph with $N$ vertices (each vertex corresponds to an amino acid) and the contact map matrix as incidence matrix. It is called contact map graph. The contact map graph is an abstract description of the protein structure taking into account only the interactions between the amino acids. Now let us consider the subgraph induced by the set of amino acids participating in SSE. We call this graph SSE interaction network (SSE-IN) and this is the object we study in the present chapter. The reason of ignoring the amino acids not participating in SSE is simple. Evolution tends to preserve the structural core of proteins composed from SSE. In the other hand, the loops (regions between SSE) are not so important to the structure and hence, are subject to more mutations. That is why homologous proteins 
tend to have relatively preserved structural cores and variable loop regions. Thus, the structure determining interactions are those between amino acids belonging to the same SSE on local level and between different SSEs on global level. Fig. 2 gives an example of a protein and its SSE-IN.

In (Muppirala \& Li, 2006) and (Brinda \& Vishveshwara, 2005) the authors rely on similar models of amino acid interaction networks to study some of their properties, in particular concerning the role played by certain nodes or comparing the graph to general interaction networks models. Thanks to this point of view the protein folding problem can be tackled by graph theory approaches.

As we will see in the next section, there are three main models of interaction networks, extensively studied and whose properties are identified. The purpose of our work is to identify specific properties which associate the proteins SSE-IN with a general network model. Based on such a pattern description of SSE-IN, one can plan the study of their formation, dynamics and evolution.

\section{General Models of Interactions Networks}

Many systems, both natural and artificial, can be represented by networks, that is, by sites or vertices bound by links. The study of these networks is interdisciplinary because they appear in scientific fields like physics, biology, computer science or information technology. These studies are lead with the aim to explain how elements interact with each other inside the network and what are the general laws which govern the observed network properties. From physics and computer science to biology and social sciences, researchers have found that a broad variety of systems can be represented as networks, and that there is much to be learned by studying these networks. Indeed, the studies of the Web (Broder et al., 2000), of social networks (Wasserman \& Faust, 1994) or of metabolic networks (Jeong et al., 2000) contribute to put in light common non-trivial properties of these networks which have $a$ priori nothing in common. The ambition is to understand how the large networks are structured, how they evolve and what are the phenomena acting on their constitution and formation.

In this section we present the three main models of interaction networks by describing their specific properties. We also define several measures that we use in the next section in order to study SSE-IN empirically.

\subsection{The Random Graph Model}

The random graph models are one of the oldest network models, introduced in (Solomonoff \& Rapoport, 1951) and further studied in (Erdõs \& Rényi, 1959) and (Erdõs \& Rényi, 1960). These works identify two different classes of random graphs, called Gn,ù and Gn,p and defined by the following connection rules:

- $G_{n, u}$ regroups all graphs with $n$ vertices and $m$ edges. To generate a graph sampled uniformly at random from the set $G_{n, u}$, one has to put $m$ edges between vertex pairs chosen randomly from $n$ initially unconnected vertices.

- $G_{n, p}$ is the set of all graphs consisting of $n$ vertices, where each vertex is connected to others with independent probability $p$. To generate a graph sampled randomly, one has to begin with $n$ initially unconnected vertices and join each pair by an edge with probability $p$. 
In $G_{n, u}$ the number of edges is fixed whereas in $G_{n, p}$ the number of edges can fluctuate but its average is fixed. When $n$ tends to be large the two models are equivalent.

Definition 1 The degree of a vertex $v, k_{v}$, is the number of edges incident to $v$. The mean degree, $z$, of a graph $G$ is defined as follows:

$$
z=\frac{1}{n} \sum_{v \in V} k_{v}=\frac{2 m}{n}=p(n-1)
$$

The degree distribution is one of the important characteristics of this kind of networks because it affects their properties and behavior (Réka \& Barabási, 2000). The random graph $G_{n, p}$ has a binomial degree distribution. The probability $p_{k}$ that a randomly chosen vertex is connected to exactly $\mathrm{k}$ others is (Newman et al., 2001):

$$
p_{k}=\left(\begin{array}{l}
n \\
k
\end{array}\right) p^{k}(1-p)^{n-k}
$$

When $n$ tends to infinity, this becomes:

$$
p_{k}=\lim _{n \rightarrow \infty} \frac{n^{k}}{k !}\left(\frac{p}{1-p}\right)^{k}(1-p)^{n} \approx \frac{z^{k} e^{-z}}{k !}
$$

As we see in Fig. 3, Poisson distributions have different behavior for different mean degrees $z$. Each distribution has a clear peak close to $\mathrm{k}=\mathrm{z}$, followed by a tail that decays as $1 / \mathrm{k}$ ! which is considerably quicker than exponential.

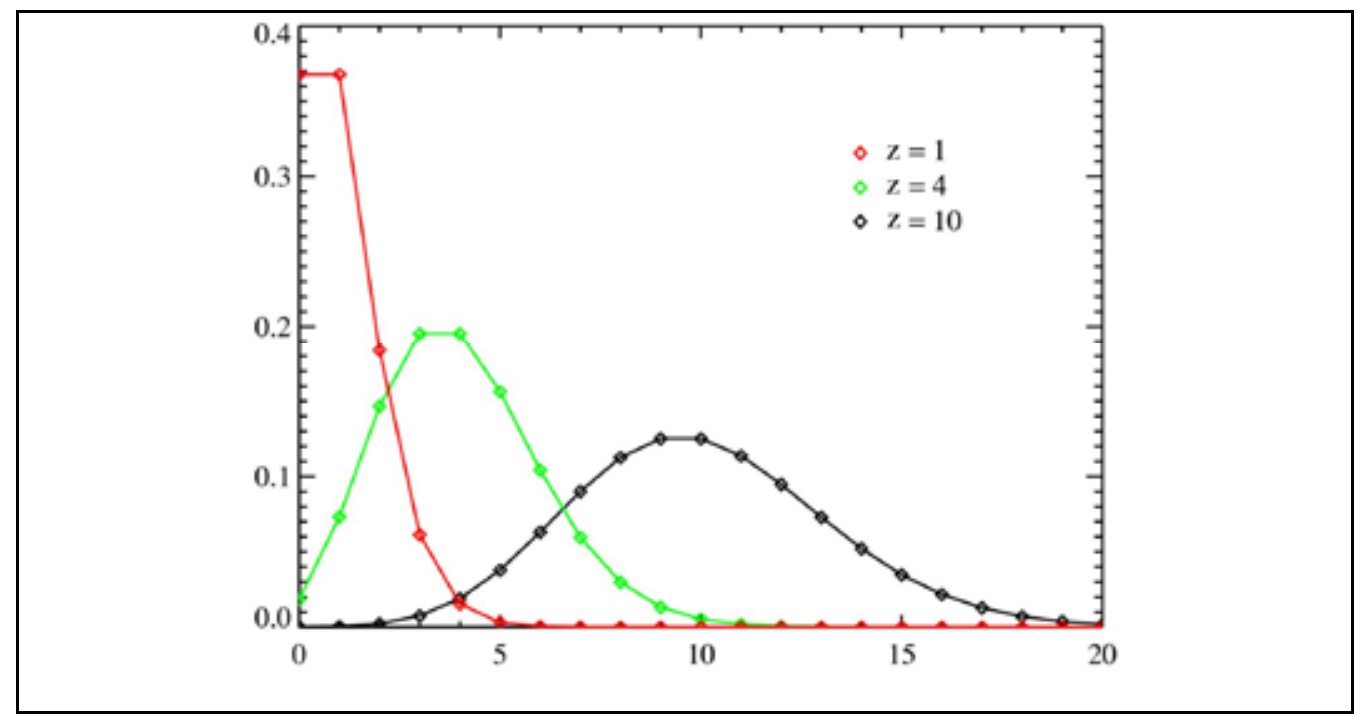

Fig. 3. Poisson distribution $p_{k}=\frac{z^{k} e^{-z}}{k !}$ with $z=1,4$ and 10 . 


\subsection{Small-World Networks}

This network model was introduced in (Watts, 1999) as a model of social networks. It has been since adopted to treat phenomena in physics, computer science or social sciences. The model comes from the observation that many real-world networks have the following two properties:

- The small-world effect, meaning that most pairs of vertices are connected by a short path through the network. This phenomenon has two explanations. First, the concept of "shortcuts" through a network allows to join two distant vertices by a small number of edges (Watts, 1999).

. Second, the concept of "hubs", vertices whose connectivity is higher than others provide bridges between distant vertices because most vertices are linked to them.

- High "clustering'", meaning that there is a high probability that two vertices are connected one to another if they share the same neighbor.

To determine if a network is a small-world, one can use the measures described below and compare them to the corresponding measures of a random graph.

Definition 2 The characteristic path length (Watts, 1999), denoted L, of a graph G is the median of the means of the shortest path lengths connecting each vertex $v$ to all other vertices. More precisely, let $d(v, u)$ be the length of the shortest path between two vertices $v$ and $u$ and let $\overline{d_{v}}$ be the average of $d(v, u)$ over all $u \in V$. Then the characteristic path length is the median of $\left\{\overline{d_{v}}\right\}$.

This definition applies when the graph consists of single connected component. However, the SSE-IN we consider in the next section may have several connected components. In this case, when we calculate the mean of the shortest path lengths $\overline{d(v)}$ we take into account only the vertices $u$ which are in the same connected component as $v$.

Since the mean and the median are practically identical for any reasonably symmetric distribution, the characteristic path length of a random graph is the mean value of the shortest path lengths between any two vertices. The characteristic path length of a random graph with mean degree $z$ is

$$
L_{R G}=\frac{\log n}{\log z}
$$

It increases only logarithmically with the size of the network and remains therefore small even for large systems.

Definition 3 The local clustering coefficient (Watts, 1999), $\mathrm{C}_{\mathrm{v}}$, of a vertex $v$ with $k_{v}$ neighbors measures the density of the links in the neighborhood of $v$.

$$
C_{v}=\frac{\left|E\left(\Gamma_{v}\right)\right|}{\left(\begin{array}{c}
k_{v} \\
2
\end{array}\right)}
$$

where the numerator is the number of edges in the neighborhood of $v$ and the denominator is the number of all possible edges in this neighborhood. The clustering coefficient $C$ of a graph is the average of the local clustering coefficients of all vertices: 


$$
C=\frac{1}{n} \sum_{v \in V} C_{v}
$$

The clustering coefficient of a random graph with mean degree $z$ is

$$
C_{R G}=\frac{z}{n-1}
$$

Watts and Strogatz (Watts, 1999) defined a network to be a small-world if it shows both of the following properties:

$$
\begin{aligned}
& \text { 1. Small world effect: } L \approx L_{R G} \\
& \text { 2. High clustering: } C>>C_{R G}
\end{aligned}
$$

\subsection{Scale-Free Networks}

The most important property of scale-free systems is their invariance to changes in scale. The term "scale-free" refers to a system defined by a functional form $f(x)$ that remains unchanged within a multiplicative factor under rescaling of the independent variable $x$. Indeed, this means power-law forms, since these are the only solutions to $f(a n)=b f(n)$, where $n$ is the number of vertices (Newman, 2002). The scale-invariance property means that any part of the scale-free network is stochastically similar to the whole network and parameters are assumed to be independent of the system size (Jeong et al., 2000).

If $n_{k}$ is the number of vertices having the degree $k$, we define $p_{k}$ as the fraction of vertices that have degree $k$ in the network:

$$
p_{k}=\frac{n_{k}}{n}
$$

The degree distribution can be expressed via the cumulative degree function (Newman, 2002), (Erdõs \& Rényi, 1959):

$$
P_{k}=\sum_{k^{\prime}=k^{\prime}}^{\infty} p_{k^{\prime}}
$$

which is the probabilty for a node to have a degree greater or equal to $k$.

By plotting the cumulative degree function one can observe how its tail evolves, following a power law or an exponential distribution.

The power law distribution is defined as following (Newman, 2002):

$$
P_{k} \approx \sum_{k^{\prime}=k}^{\infty} k^{\prime \alpha} \approx k^{-(\alpha-1)}
$$

and the exponential distribution is defined by the next formula: 


$$
P_{k} \approx \sum_{k^{\prime}=k}^{\infty} e^{-k^{\prime} / \alpha} \approx e^{-k / \alpha}
$$

Between this two distributions, there is a mixture of them where the distribution has a power law regime followed by a sharp cut-off, with an exponential decay of the tail, expressed by the next formula:

$$
P_{k} \approx \sum_{k^{\prime}=k}^{\infty} k^{\prime-\alpha} e^{-k^{\prime} / \alpha} \approx k^{\prime(\alpha-1)} e^{-k / \alpha}
$$

Like a power law distribution, it decreases polynomially, so that the number of vertices with weak degree is important while a reduced proportion of vertices having high degree exists. The last are called "hubs" that is sites with large connectivity through the network, see Fig. 4. The scale-free model depends mainly on the kind of degree distribution, thus a network is defined as a scale-free if:

- The degree distribution is a power law distribution $\mathrm{P}(\mathrm{k}) \approx \mathrm{k}^{-\mathrm{a}}$ over a part of its range.

- The distribution exposant satisfies $2<a \leq 3$ (Goh et al., 2001).

Amaral and al (Amaral et al., 2000) have studied networks whose cumulative degree distribution shape lets appear three kinds of networks. First, scale-free networks whose distribution decays as a power law with an exposant a satisfying bounds seen above. Second, see Fig. 4, broad-scale or truncated scale-free networks whose the degree distribution has a power law regime followed by a sharp cutoff. Third, single-scale networks whose degree distribution decays fast like an exponential.

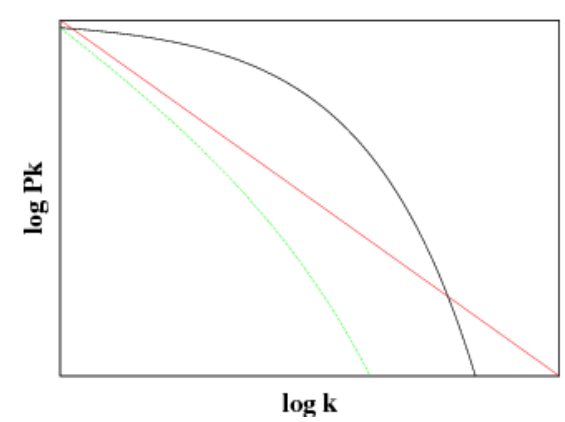

Fig. 4. Degree distribution described in (Amaral et al., 2000). The red line follows a power law, as for scale-free networks. The green line corresponds to truncated scale-free networks. The black curve corresponds to single-scale networks.

\subsection{Topological Measures}

Here, we present some measures that we use to describe proteins' SSE-IN. Among them, there are simple ones, the most frequently used, but also more subtle, which allow a more precise discrimination between interaction networks. 
Diameter and mean distance The distance in a graph $G=(V, E)$ between two vertices $u, v$ $\in V$, denoted by $d(u, v)$, is the length of the shortest path connecting $u$ and $v$ (Diestel, 2000). If there is no path between $u$ and $v$, we suppose that $d(u, v)$ is undefined. A graph diameter, $D$, is the longest shortest path between any two vertices of a graph (Diestel, 2000):

$$
D=\max \{d(u, v) / u, v \in V\}
$$

The mean distance is defined as the average distance between each couple of vertices:

$$
\overline{d_{G}}=\frac{2}{n(n-1)} \sum_{u, v \in V} d(u, v)
$$

Density The density, denoted $\delta$, is defined as the ratio between the number of edges in a graph and the maximum number of edges which it could have:

$$
\delta(G)=\frac{2 m}{n(n-1)} \approx \frac{2 m}{n^{2}}
$$

The density of a graph is a number between 0 and 1 . When the density is close to one, the graph is called dense, when it is close to zero, the graph is called sparse (Coleman \& Moré, 1983).

Clustering coefficients Watts and Strogatz proposed a measure of clustering (Watts, 1999) and defined it as a measure of local vertices density, thus for each node $v$, the local clustering around its neighborhood is defined in the following way:

$$
C_{v}=\frac{1}{2} k_{v}\left(k_{v}-1\right)
$$

The clustering coefficient is a ratio between the number of edges and the maximum number of possible edges in the vertice neighborhood. If we extend the previous defintion to the entire graph, the clustering is given by the expression:

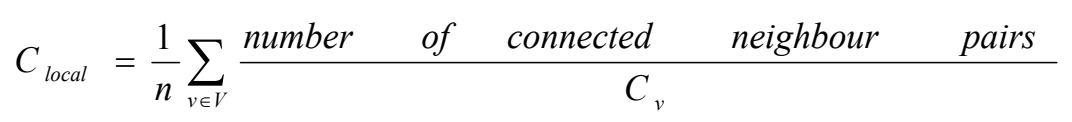

The last defintion is mainly local because for each node, it involves only its neighborhood. The global clustering was studied by Newman et al. (Newman, 2001) and can be mesuared by the following formula:

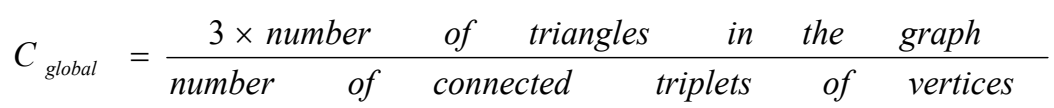

A triangle is formed by three vertices which are all connected and a triplet is constituted by 
three nodes and two edges. The global clustering coefficient $C_{\text {global }}$ is the mean probability that two vertices that are neighbors of the same other vertex will themselves be neighbors.

\section{A First Topological Description}

In this section, we present a publication (Gaci \& Balev, 2008b) where we study the protein SSEIN behavior. We want to observe how proteins from a same structural family provide similar SSE-IN according to their topological properties. To do that, we propose topological measures which we apply on a sample of proteins to put in evidence the existence of equivalence between structural similarity and topological homogeneity in the resulting SSE-IN.

The first step before studying the proteins SSE-IN is to select them according to their SSE arrangements. Thus, a protein belongs to a CATH topology level or a SCOP fold level iff all its domains are the same. We have worked with the CATH v3.1.0 and SCOP 1.7.1 files. We have computed the measures from the previous section for three families of each hierarchical classification, namely SCOP and CATH (see Table 1). We have chosen these three families by classification, in particular because of their huge protein number. Thus, each family provides a broad sample guarantying more general results and avoiding fluctuations. Moreover, these six families contain proteins of very different sizes, varying from several dozens to several thousands amino acids in SSE.

\begin{tabular}{|c|c|c|c|}
\hline Name & Type & Class & Proteins \\
\hline RossmannFold & $\mathrm{CATH}$ & $\alpha \beta$ & 2576 \\
\hline TIM Barrel & CATH & $\frac{1}{\alpha \beta}$ & 1051 \\
\hline Lysozyme & $\mathrm{CATH}$ & Mainly a & 871 \\
\hline Globin-like & SCOP & All a & 733 \\
\hline TIM $\beta$ /a-barrel & SCOP & $\alpha / \beta$ & 896 \\
\hline Lysozyme-like & SCOP & $\overline{\alpha+\beta}$ & 819 \\
\hline
\end{tabular}

Table 1. Families studied, mainly due to their protein number.

\subsection{Diameter and mean distance}

Table 2 shows the average diameter for each one of the studied families. We observe very close diameters between TIM Barrel and TIM beta/alpha-barrel and also between Lysozyme and Lysozyme-like families. This is explained by the fact that each pair of families contains almost the same proteins, in other worlds, Lysozyme topology in CATH is the equivalent of Lysozyme-like fold level in SCOP.

\begin{tabular}{|l|c|}
\hline Name & D \\
\hline RossmannFold & 18.84 \\
\hline TIM Barrel & 19.83 \\
\hline Lysozyme & 12.81 \\
\hline & \\
\hline Globin-like & 15.65 \\
\hline TIM $\beta$ /a-barrel & 20.09 \\
\hline Lysozyme-like & 12.85 \\
\hline
\end{tabular}

Table 2. Average diameter for each family. 
Figure 5 shows the distribution of the diameter values for two of the studied families. We observe that the distribution follows roughly a Poisson law. These results confirm that the mean diameter is a suitable property to discriminate families between them.

The diameter being an upper bound of distances in interaction networks, we expect that the mean distance $z$ will be lower than $D$. Table 3 confirms this. Again, we observe very close values between the equivalent SCOP and CATH families for the reasons discussed above. But we can also see that different families have values which allow discrimination between them based on this parameter. It is interesting to note that the ratio $D / z$ is about 2.5 for all the families. The last property is a characterization of all proteins' SSE-IN.

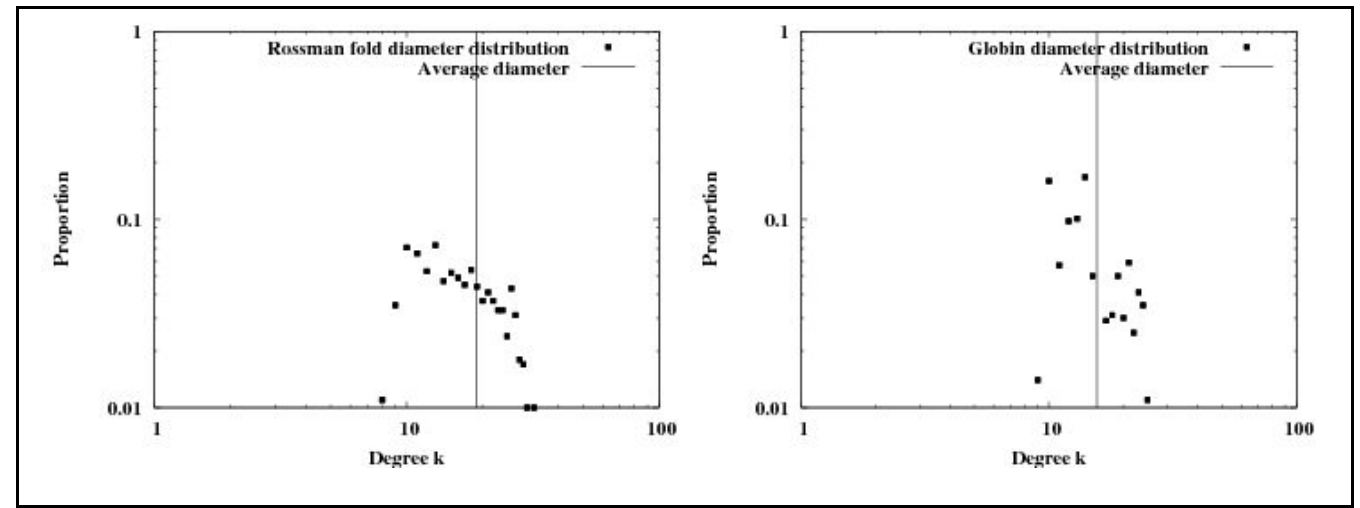

Fig. 5. Average diameter of Rossman fold, left, and beta/alpha-barrel, right.

\begin{tabular}{|l|c|}
\hline Name & $\overline{d_{G}}$ \\
\hline RossmannFold & 7.26 \\
\hline TIM Barrel & 7.79 \\
\hline Lysozyme & 4.99 \\
\hline & \\
\hline Globin-like & 6.64 \\
\hline TIM $\beta$ / a-barrel & 7.86 \\
\hline Lysozyme-like & 5.03 \\
\hline
\end{tabular}

Table 3. Average of mean distances for each family.

\subsection{Density and mean degree}

As defined earlier, the density measures the ratio between the number of available edges and the number of all possible edges. Results presented in Table 4 show that the two families TIM Barrel and TIM beta/alpha-barrel have the minimum density. It has a consequence on their SSE-IN topology. When the density is low, the network is less connected and consequently, the diameter and the average distance are higher. Comparing these results to Tables 2 and 3 one can see the inversely proportional relation between density in one hand, and diameter and average distance on the other. 


\begin{tabular}{|l|c|}
\hline Name & $\delta(G)$ \\
\hline RossmannFold & 0.033 \\
\hline TIM Barrel & 0.03 \\
\hline Lysozyme & 0.038 \\
\hline & \\
\hline Globin-like & 0.034 \\
\hline TIM $\beta$ / a-barrel & 0.029 \\
\hline Lysozyme-like & 0.042 \\
\hline
\end{tabular}

Table 4. Average density for each family.

The mean degree, $z$, is presented in Table 5. The observed values are close enough from one family to another. That is why the mean degree is not discriminating property, but rather a property characterizing all proteins' SSE-IN.

\begin{tabular}{|l|c|}
\hline Name & $z$ \\
\hline RossmannFold & 7.2 \\
\hline TIM Barrel & 7.17 \\
\hline Lysozyme & 6.82 \\
\hline & \\
\hline Globin-like & 7.69 \\
\hline TIM $\beta$ /a-barrel & 7.15 \\
\hline Lysozyme-like & 6.81 \\
\hline
\end{tabular}

Table 5. Average of mean degrees for each family.

\subsection{Degree distribution}

We compute the cumulative degree distribution for all proteins SSE-IN of studied families. A sample of our results is presented on Figure 6. We can remark that the curves follow a power law distribution and can be approximated by the following power-law function:

$$
p_{k}=141.29 k^{-\alpha}, \text { where } \alpha \approx 2.99
$$

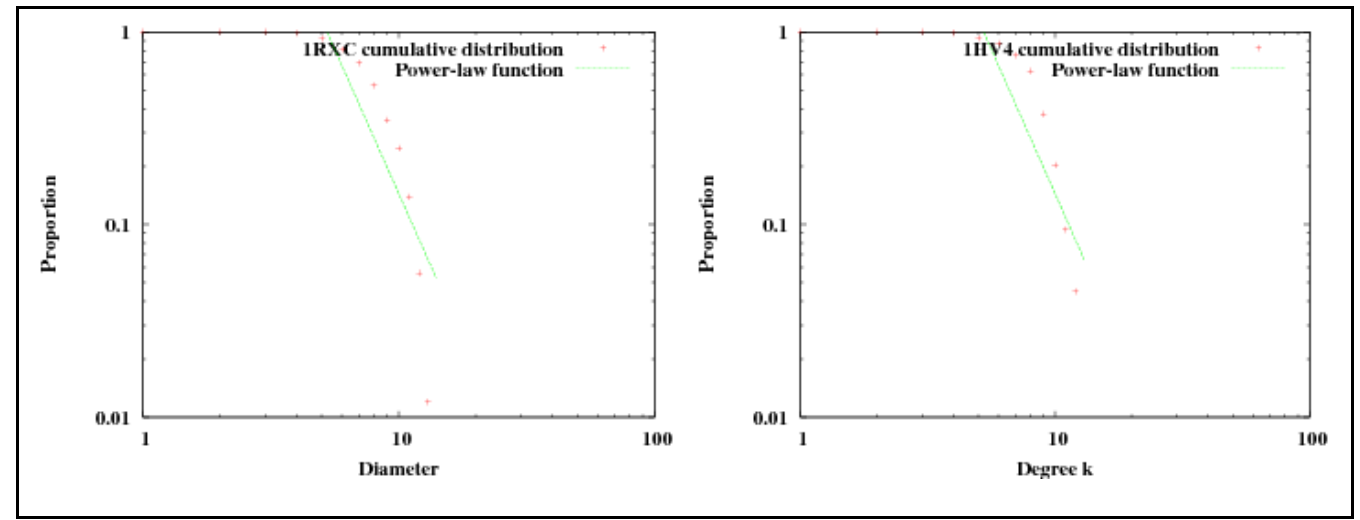

Fig. 6. Cumulative degree distribution for 1RXC from Rossman fold, left, and 1HV4 from TIM beta/alpha-barrel, right. 
We observe the same results for all studied proteins. To explain this phenomenon, we have to rely on two facts. First, the mean degree of all proteins SSE-IN is nearly constant (see Table 5). Second, the degree distribution, see Figure 7, follows a Poisson distribution whose peak is reached for a degree near $z$. These two facts imply that for degree lower than the peak the cumulative degree distribution decreases slowly and after the peak its decrease is fast compared to an exponential one. Consequently, all proteins SSE-IN studied have a similar cumulative degree distribution which can be approximated by a unique power-law function.

\subsection{Clustering Coefficients}

The local clustering Clocal measures the fraction of pairs of a vertex's neighbors and the global clustering Cglobal gives the probability that among three vertices at least two are connected. The results presented in Table 6 show that the clustering coefficients are close for different families and cannot be correlated to density values. Consequently, the neighbour density remains independent of the previously studied properties.

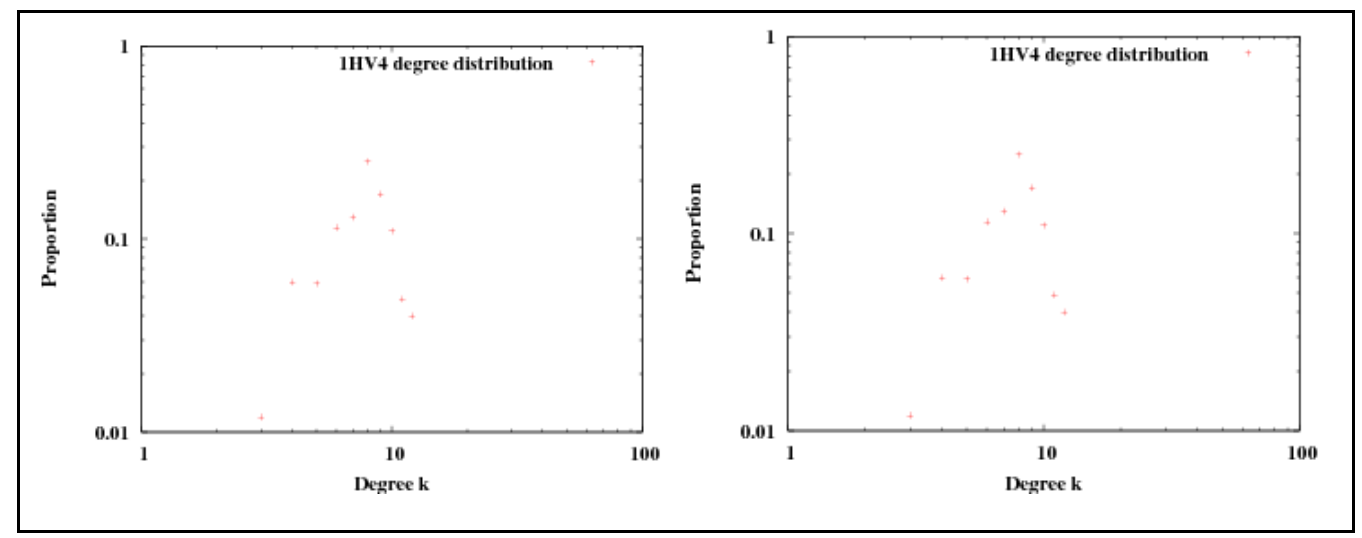

Fig. 7. Degree distribution for 1RXC from Rossman fold, left, and 1HV4 from TIM beta/alpha-barrel, right.

\begin{tabular}{|l|c|c|}
\hline Name & $C_{\text {local }}$ & $C_{\text {global }}$ \\
\hline RossmannFold & 0.63 & 0.56 \\
\hline TIM Barrel & 0.64 & 0.57 \\
\hline Lysozyme & 0.65 & 0.58 \\
\hline & & \\
\hline Globin-like & 0.63 & 0.57 \\
\hline TIM $\beta /$ a-barrel & 0.64 & 0.57 \\
\hline Lysozyme-like & 0.66 & 0.58 \\
\hline
\end{tabular}

Table 6. Clustering coefficients for each family.

\subsection{Consequences}

In this first part we introduce the notion of interaction network of amino acids of a protein (SSE-IN) and study some of the properties of these networks. We give different means to describe a protein structural family by characterizing their SSE-IN. Some of the properties, 
like diameter and density, allow discriminating two distinct families, while others, like mean degree and power law degree distribution, are general properties of all SSE-IN. Thus, proteins having similar structural properties and biological functions will also have similar SSE-IN properties. In this way our model allows us to draw a parallel between biology and graph theory.

\section{Comparisons with the Scale-Free Networks}

We have worked on the entire PDB file available the 18th October 2007, that is 46679 files. We lead our simulations by considering all proteins SSE-IN without limiting us on a particular protein family or basing us on a classification. Indeed, our goal is to identify a general model for proteins SSE-IN and so that we don't discriminate proteins due to their biological function. Thus, despite the heterogeneity we can expect among folded proteins, we assume that a general model of Interaction Networks emerge and can be identified from protein SSE-IN which came from combinaison of the 20 amino acids.

\subsection{General Behavior}

We compute the cumulative degree distribution for all proteins SSE-IN, a sample of our results is presented on Fig 8. We can remark that the curves describe a power law regime followed by the sharp cut-off. The power law function is expressed as following:

$$
p_{k}=213.413 k^{-\alpha}, \text { where } \alpha \approx 3.2
$$

while the distribution is approximated by the next function:

$$
P_{k}=1.48347 k^{0.962514} \exp ^{-k / 2.12615}
$$

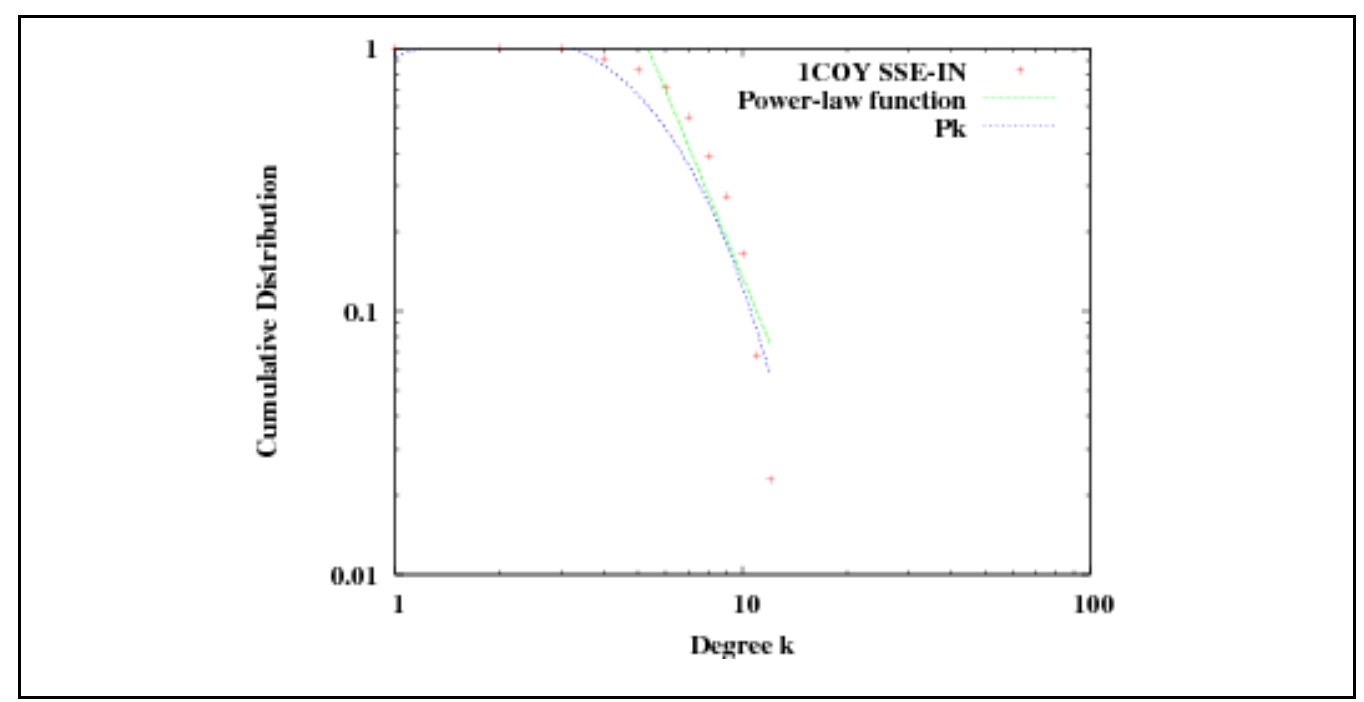

Fig. 8. Cumulative degree distribution for protein 1COY SSE-IN. 
We observe the same result for all studied proteins that is a cumulative degree distribution approximed by the function $P_{k}$. Here, we discuss about characterisitcs or conditions which involve a such behavior.

First, we are interested in the degree distribution and mainly its shape, see Fig 9. We can see that degree distribution follows a Poisson distribution whose peak is reached for a degree near $\mathrm{z}$. This result provides precision about how the vertices are connected within SSE-IN. It implies that the degree of the vertices is homogenous. In other words, a major part of them has a connectivity enough close to the mean degree. Consequently, the cumulative distribution depends on the mean degree value which acts as a threshold beyond which it decreases as an exponential since it's approximed via $P_{k}$.
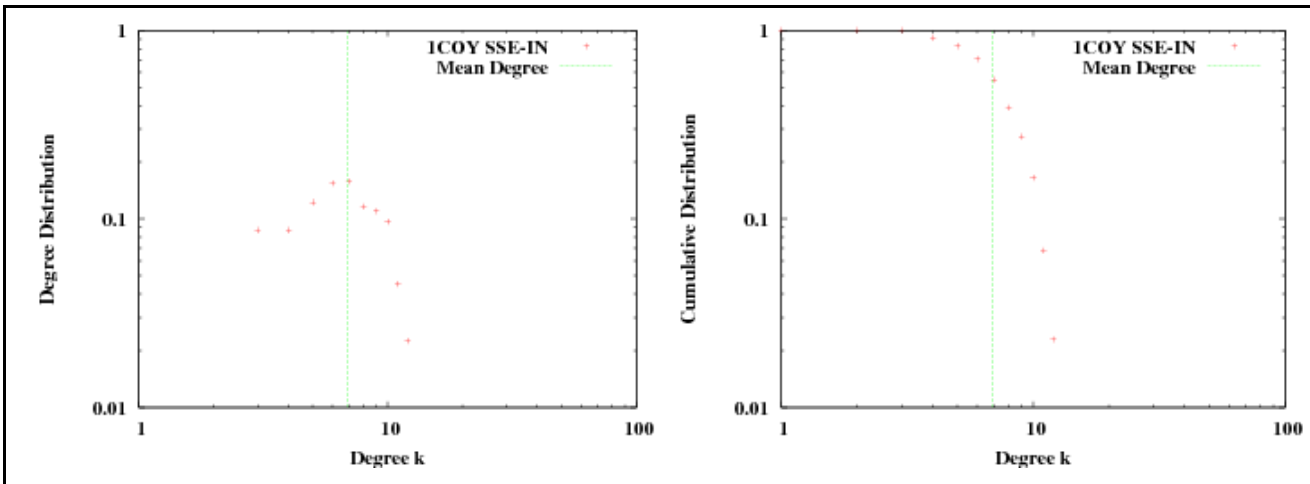

Fig. 9. Degree and cumulative distribution for 1COY SSE-IN. They decrease for degree values greater than the mean degree.

Second, we study how the mean degree evolves through all SSE-IN. Its distribution, see Fig 10 , indicates a relative weak variation according to the size. Even if two protein SSE-IN have size ratio around 10 or 100, their mean degree ratio is esimated to 1.05 or 1.15 and remains in the same scale order.

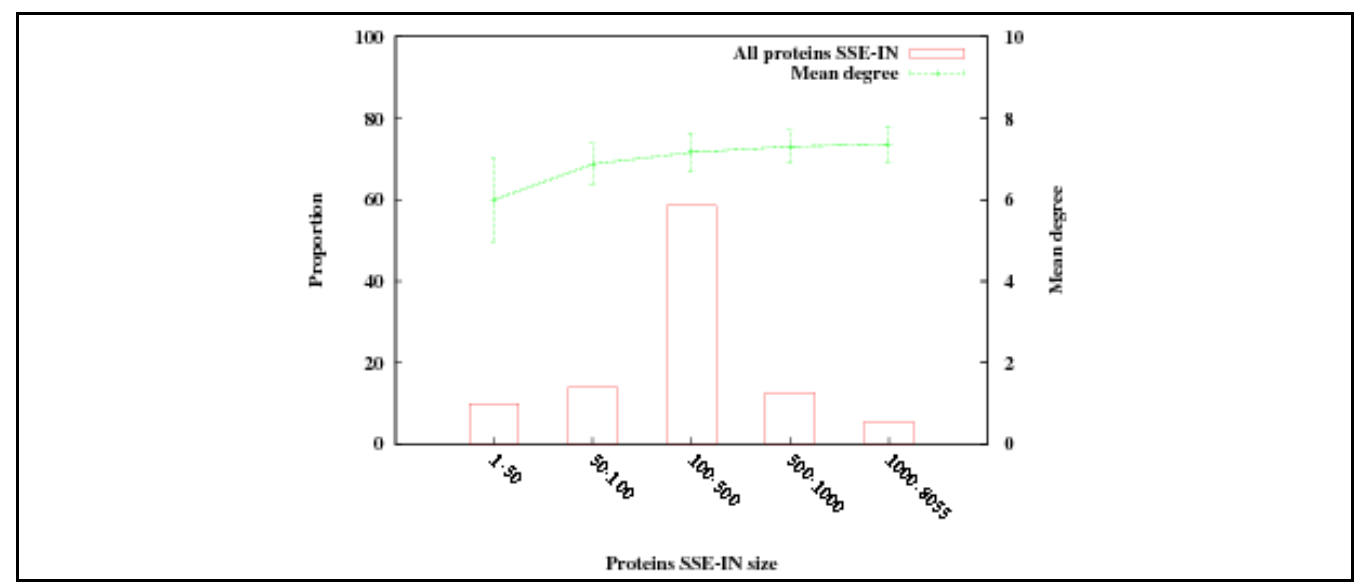

Fig. 10. Mean degree distribution according to protein SSE-IN size. It evolves with values enough close, between 6 and 8 . 
To illustrate the mean degree homogeneity we choose two proteins, namely 1SE9 and 1AON with size respectively 50 and 4988 . Their size ratio is approximately 100. Even if the mean degree are slightly different, the distributions are very similar, see Fig 11.

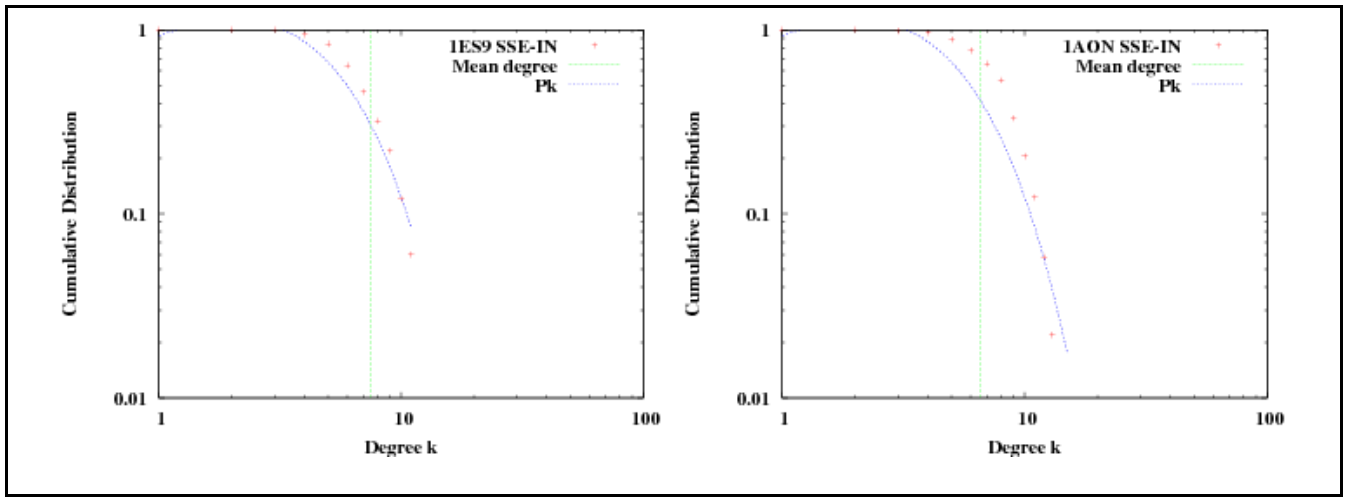

Fig. 11. Cumulative degree distribution of 1SE9 and 1AON SSE-IN whose size equal 50 and and 4988. Despite their important size difference, their mean degree stay close and worth respectively 6.6 and 7.5

To recapitulate, we show that the mean degree values constitute a threshold for protein SSEIN cumulative degree distribution. For degrees lower than the mean degree it decreases slowly and after this threshold its decrease is fast compared to an exponential one, as shown Fig 8, 9 and 10.

Consequently, we find a way to approximate all proteins SSE-IN cumulative degree distribution by the function $P_{k}$ which can be adjusted. This function describes a power law regime followed by a sharp cut-off which arises for degree values exceeding the mean degree. Proteins SSE-IN are so truncated scale-free networks.

Since the degree distribution depends on the mean degree value, we compare for each node its degree as function of $\mathrm{z}$, see Fig 12, to illustrate how nodes interact and in particular to highlight the weak fraction of highly connected nodes, also called hubs, less than $5 \%$ of the total node number.

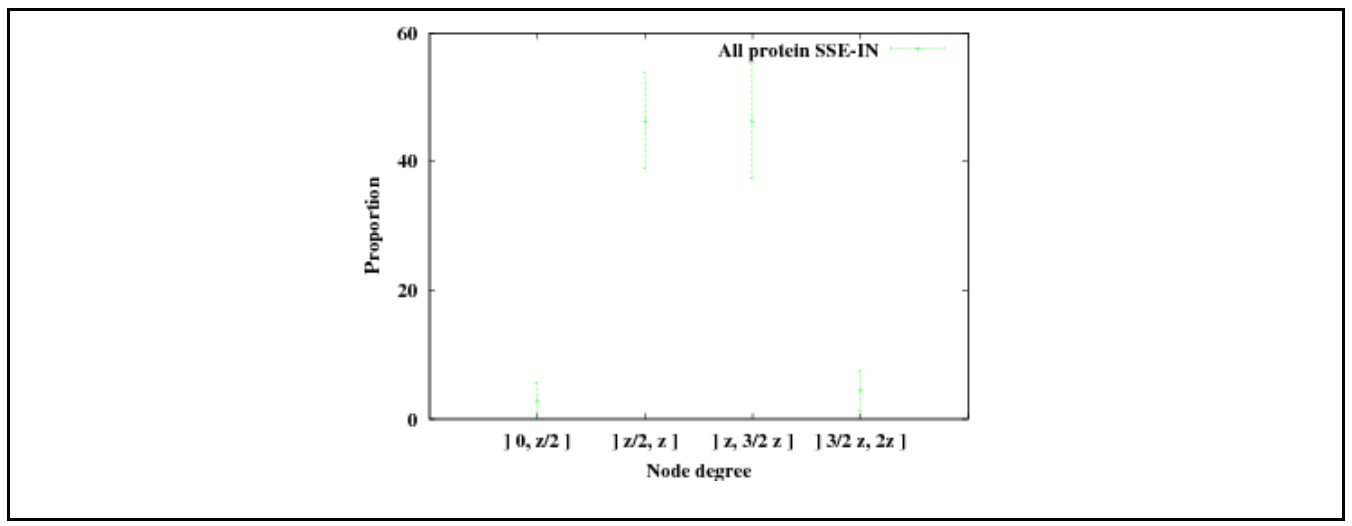

Fig. 12. Degree of nodes in protein SSE-IN as function of $z$. Less than $5 \%$ of nodes are hubs that is having a 'high' connectivity. 
An interesting study is to put in evidence the biological properties of nodes whose connectivity is marginal. Thus, we search to identify nodes which highly interact with their neighbors in the folded proteins. To do that, we have proceeded by grouping the proteins according to their secondary structure. Indeed, we have already shown (Gaci \& Balev, $2008 \mathrm{~b})$ that the protein SSE-IN topologies from structural classifications are homogeneous and established a parallel between structural and topological properties. Based on the SCOP classification and more precisely on the fold families, we have selected a total of 18294 proteins, see Table 7, and studied their SSE-IN to describe the specificities of the hubs.

\begin{tabular}{|l|l|c|}
\hline Class & $\begin{array}{l}\text { Number } \\
\text { of } \\
\text { Families }\end{array}$ & $\begin{array}{l}\text { Number } \\
\text { of } \\
\text { Proteins }\end{array}$ \\
\hline All Alpha & 12 & 2968 \\
\hline All Beta & 17 & 6372 \\
\hline Alpha / Beta & 18 & 5197 \\
\hline Alpha + Beta & 16 & 3757 \\
\hline
\end{tabular}

Table 7. Structural families studied for the Scale-Free properties. We choose only families which count more than 100 proteins, for a total of 18294 proteins.We have worked with the SCOP 1.7.3 classification, a protein belongs to a SCOP fold level if all its domains are the same.

For each protein SSE-IN from a SCOP fold level we identify the nodes whose degree is high, see Fig 12. By grouping nodes as a function of amino acids they represent, we finally obtain the amino acid connectivity score by fold families. By repeating this process at the SCOP class level, we calculate and normalize the cumulated connectivity level of amino acids playing the role of hubs, see Fig 13.

If we consider that the role played by an amino acid inside a folded protein is equivalent to its interaction degree, then these plots show that despite a functional diversity between the 4 SCOP classes, there are globally the same amino acids which interact most, namely the Ala, Cys, Gly, Leu, Val. Therefore, the amino acids having a high connectivity interact independently of the protein biological function, we will explain this behavior further. Thus, the figure shows clearly that the amino acid Ala plays a central role in the studied proteins independently of the classification of their secondary structure.

We also compute the occurrence level of hubs, that is their number of appearances on each protein SSE-IN. We cumulated this score at the SCOP class level to obtain the probability for each amino acid to be a hub having a high connected occurrence in a protein SSE-IN, see Fig 14. 

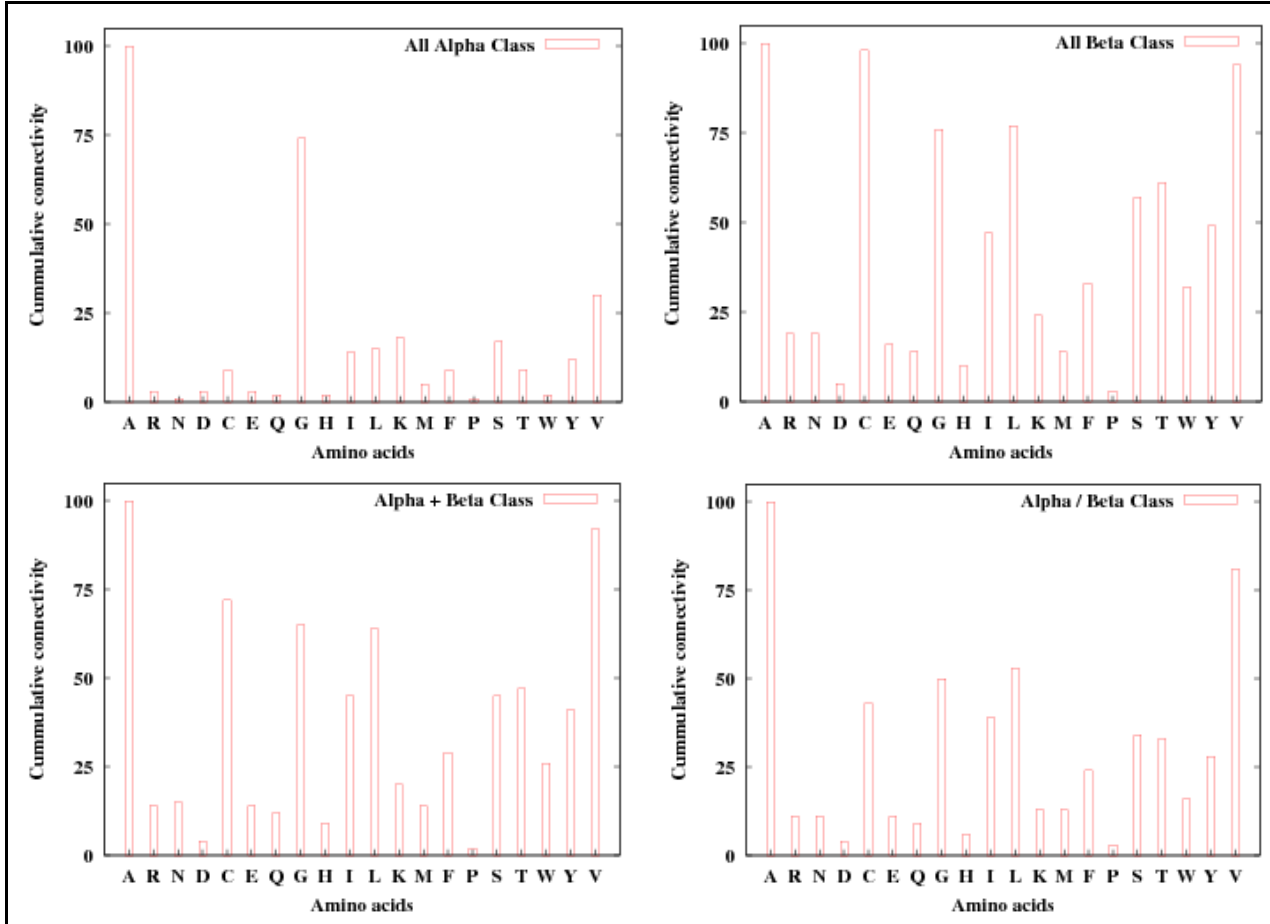

Fig. 13. Normalisation of the amino acid cumulative connectivity when they act as hubs for each SCOP class level. The amino acid Ala interacts higher than the others independently of proteins classification.

We can remark the existence of peaks which show clearly a higly tendency of amino acids Ala, Cys, Gly, Leu and Val to have a high interactivy within the folded protein. Thus, there are the same amino acids which play the role of hubs independently of the structural family, we provide below the cause of this result.

Now, we want to describe the way in which the hubs appear in the folded protein. Indeed, we study the distribution of the hubs position as a function of the structural class of the SSEIN to identify variations dependent or not on the biological function of proteins. To make this study, we attribute an incremental position so that the $\mathrm{H}$ extremity has a position 100 . Then, each time a hub exists in a SSE-IN, we increment its position occurence number and finally normalize by the maximum to obtain the occurence rate of hubs according to their positions in the SSE-IN, see Fig. 15. 


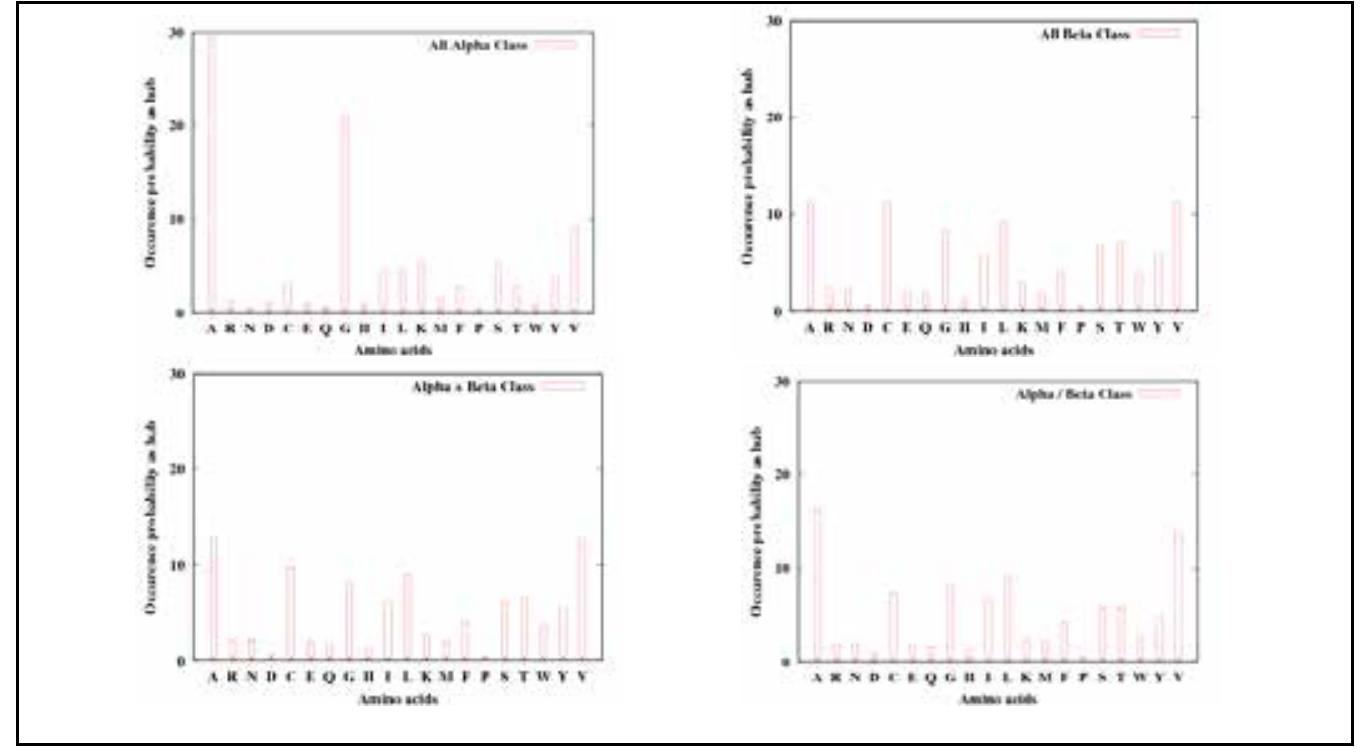

Fig. 14. Occurrence probability of hub amino acids for each SCOP Class level. The probability that an amino acid ALA has a high interaction level is superior than the others independently of proteins classification.

The results show the existence of favorable region in which the hub apparition is higher than somewhere else. This favorable localization is strongly visible for the All Alpha class where the hubs have a tendency to interact around the positions 20, 40 or 80 . The distribution of the hub positions is the most homogenous for the alpha/beta class which involve a dependence to the SSE-IN topology since it not possible to find more than one strong favorable area which appear around the position 60.

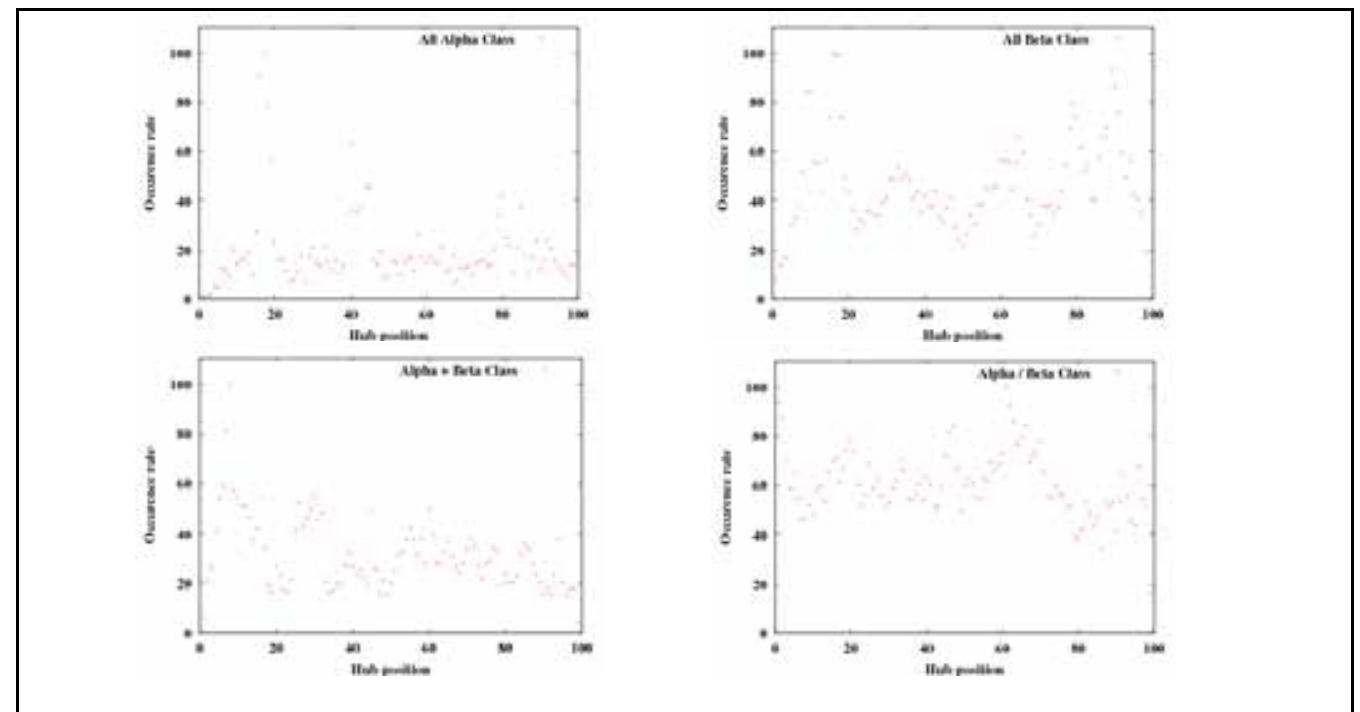

Fig. 15. Occurrence rate of hubs according to their position for each SCOP Class level. 
To illustrate the existence of hubs favorable localization in the SSE-IN, we rely on the richclub phenomenon (Colizza et al., 2006) according to which highly connected nodes have tendancy to be connected to one another. We compute the rich-club connectivity of a hub as the ratio of the number of links to the maximum number of links between nodes belonging to the rich-club.

It appears, see Fig 16, that certain hubs are isolated mainly when the rich-club connectivity is low (position 0, 30, 60 for All Alpha SSE-IN) where as the favorable hub localizations correspond to a high coefficient.

The main observations about hubs behaviour are the following. First, there are four amino acids which have higher probability to have a high connectivity. Second, hubs have tendency to act in particular region in the SSE-IN. These two observations lead us to compute only the occurrence rate of the most frequently encountered hub Ala according to its positions, see Fig 17.

By comparing the figure 15 and 17, it appears clearly that the most highly occurrence rate for the 4 classes correspond to the position of the amino acid Ala. Therefore, we can establish a relation between amino acid position in the primary structure and hub apparition. Then, certain amino acid that is the Ala, Cys, Gly, Leu and Val act as hub because they appear in the protein sequence in favourable region which involve a high interaction within the folded structure.

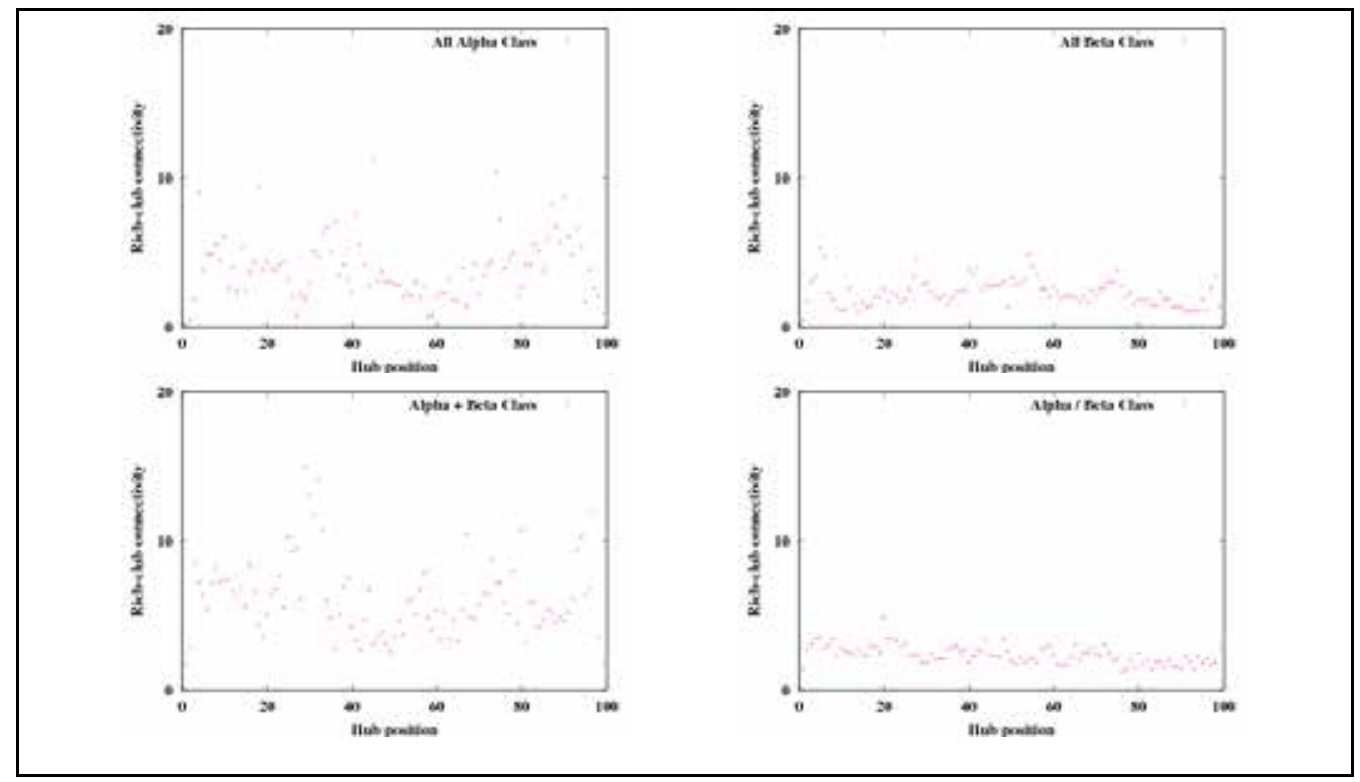

Fig. 16. Hub rich-club connectivity as function of hub position for each SCOP class level.

\subsection{Mean Degree Evolution}

Since the mean degree plays the role of a threshold beyond which the cumulative degree distribution decreases exponentially, it is interesting to study its evolution with the size of the network, see Fig. 10. It appears that the mean degree increases very slightly with the size of the network. Even for networks with size ratio of 100, the mean degree ratio is only 1.15, see Fig. 11. 

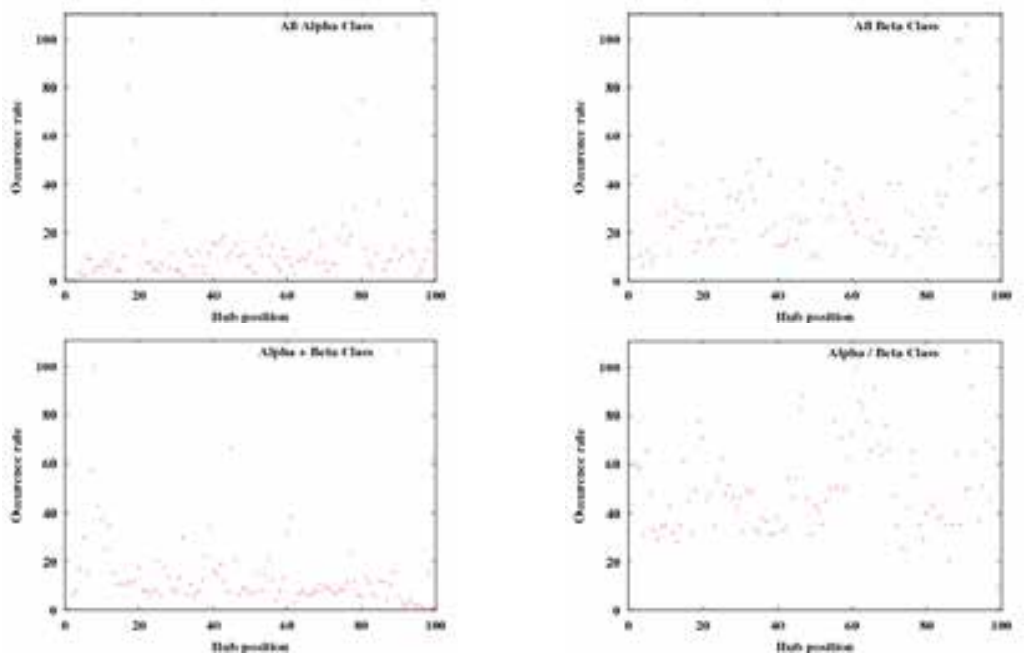

Fig. 17. Occurrence rate of the hub amino acid Ala for each SCOP Class level.

Whatever the size of the network is, we observe that the mean degree is always between 5 and 8 . This mean degree interval is a common property characterizing all SSE-IN. In order to explain this property, let us consider the structure of our networks. They are composed of densely connected subgraphs corresponding to secondary structure elements (see Fig. 18. The number of edges connecting different subgraphs is relatively small, but these edges are the most important, since they correspond to interactions determining the tertiary structure. We start by computing the mean degree in each SSE subgraph. The results are shown on Fig. 19. We can see that the mean degree evolution at microscopic level is almost the same as at macroscopic level (compare to Fig. 10). Independently of the SSE size and type, the mean degree of each SSE subgraph, $z_{S S E}$, is always bounded:

$$
z_{\min }<z_{S S E}<z_{\max }
$$

when the size of the network is more than 10. In the general case $z_{\min }=5$ and $z_{\max }=8$, but when we consider a specific SSE size and type, finer bounds can be found (see Fig. 19).

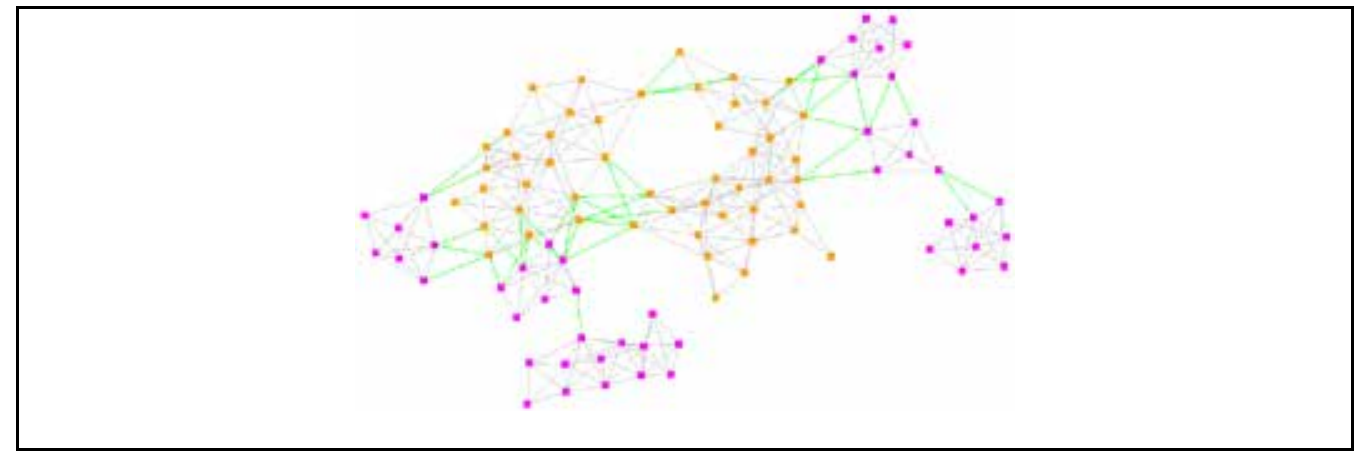

Fig. 18. SSE-IN of 1DTP protein. The edges connecting different SSE are green. 

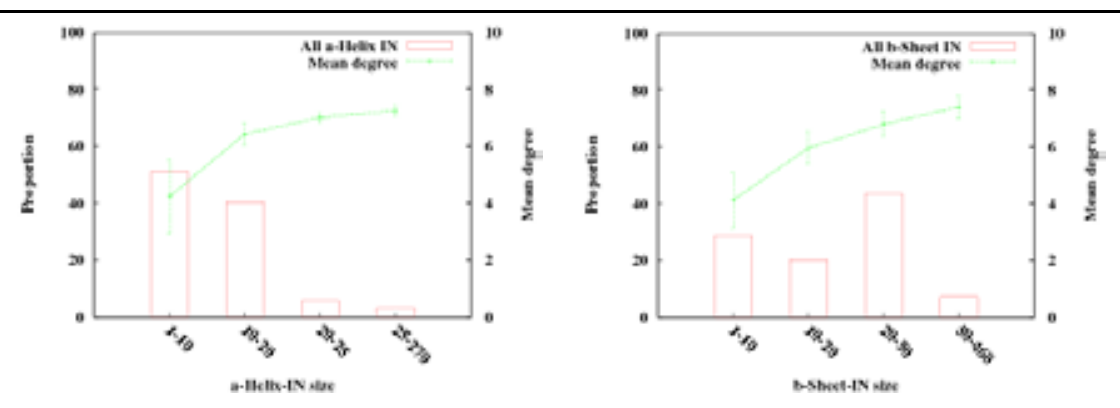

Fig. 19. SSE subgraphs size distribution and mean degree as a function of the size.

Now let us consider a whole protein. Suppose that it contains s secondary structure elements and let the element $i$ has ni vertices and mi edges, $i=1, \ldots, s$. Then the total number of vertices is $n=\sum_{i=1}^{s} n_{i}$ and the total number of edges is $m=\sum_{i=1}^{s} m_{i}+m_{\text {int } e r}$, where minter is the number of edges connecting vertices from different SSEs. Let $r=m_{\text {inter }} / m$ be the ratio of inter-SSE edges. Then:

$$
\frac{m}{n}=\frac{\sum_{i=1}^{s} m_{i}+m_{\text {int } e r}}{\sum_{i=1}^{s} n_{i}}=\frac{\sum_{i=1}^{s} m_{i}}{\sum_{i=1}^{s} n_{i}}+r \frac{m}{n}
$$

and hence for the mean degree $z$ we have

$$
z=\frac{2 m}{n}=\frac{2}{1-r} \frac{\sum_{i=1}^{s} m_{i}}{\sum_{i=1}^{s} n_{i}}
$$

On the other hand, from (1) it follows that

$$
\frac{z_{\min }}{2} n_{i}<m_{i}<\frac{z_{\max }}{2} n_{i}, i=1, \ldots, s
$$

By summing up the last equation we obtain

$$
\frac{z_{\min }}{2}<\frac{\sum_{i=1}^{s} m_{i}}{\sum_{i=1}^{s} n_{i}}<\frac{z_{\max }}{2}
$$

which together with (3) gives

$$
\frac{z_{\min }}{1-r}<z<\frac{z_{\max }}{1-r}
$$


The last equation gives finer bounds on the mean degree. It shows that the bounds on $z$ depend not only on the bounds on $z_{S S E}$, but also on the ratio of inter-SSE edges. A higher proportion of inter-SSE edges shifts up the bounds. Proteins with bigger size have more SSEs and hence more links between different SSEs. This explains the increase of the mean degree with the size of the networks. Fig. 20 shows that the number of inter-SSE edges is quite variable, but is bounded and does not exceed $20 \%$, it's the consequence of the excluded volume effect, since the number of residues that can physically reside within a given radius is limited.

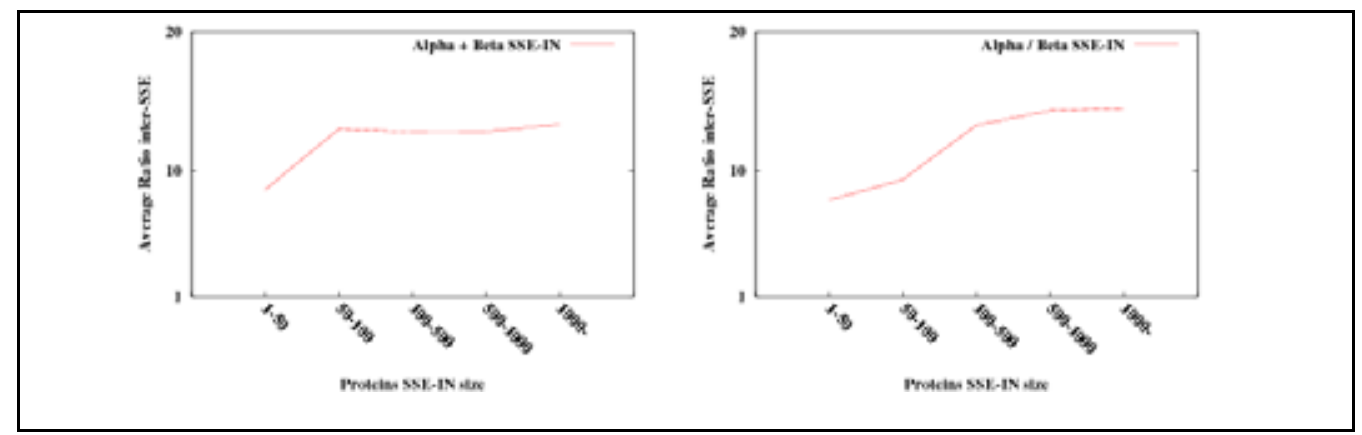

Fig. 20. Ratio of inter-SSE edges $(r)$ as a function of the network size for the four classes of studied proteins.

\subsection{Consequences}

During our study, we have identified a means to approximate the cumulative degree distribution of the SSE-IN. This function describes a power law regime followed by a sharp cut-off. The SSE-In studied are consequently truncated scale-free networks. The study of the hubs shows that certain amino acid play a central role independently of the protein classification. The nature of these hubs depends on their positions in the primary structure.

\section{Comparisons with the Small-World Networks}

In this section we present an empirical characterization of SSE-IN properties. In order to choose our data sample, we have used the SCOP classification. We have worked with the SCOP 1.7.3 files. We have computed the measures from the section (4.2.) for the four mains classes of SCOP (see Table 7). Each class provides a broad sample guarantying more general results and avoiding fluctuations. Moreover, these four classes contain proteins of very different sizes, varying from several dozens to several thousands amino acids in SSE. The results obtained for the different classes are very similar, that is why in the rest of this section we show only the results for

In a previous work (Gaci \& Balev, 2008a), we have studied the two properties of SSE-IN related to the small-world model, namely their $L / L_{R G}$ and $C / C_{R G}$ ratios. Our results show (see Fig. 21) that nearly $60 \%$ of the proteins have SSE-IN consisting of between 100 and 500 amino acids. The small-world properties are satisfied mainly when the size of the network does not exceed 500 amino acids and there are about 15.3\% small-world networks among all SSE-IN. Fig. 4 explains the reason for this low rate. One can see that although highly clustered, most SSE-IN do not satisfy the first small-world property. 
To explain the results presented on Fig. 22, note that the mean degree $z$ is not very different from one SSE-IN to another and is generally independent from the size. When the mean degree is fixed, the characteristic path length of a random graph grows like $\log n$ and its clustering coefficient has $n^{-1}$ behavior. We can see that there is no clear relationship between the characteristic path length and the SSE-IN size. There are proteins with close sizes but very different path lengths. However, in general it grows faster than logarithmically with the size. The figure also shows that SSE-IN are very highly clustered. The $C / C_{R G}$ has clearly linear behavior, hence the clustering coefficient (like the mean degree) is independent from the size.
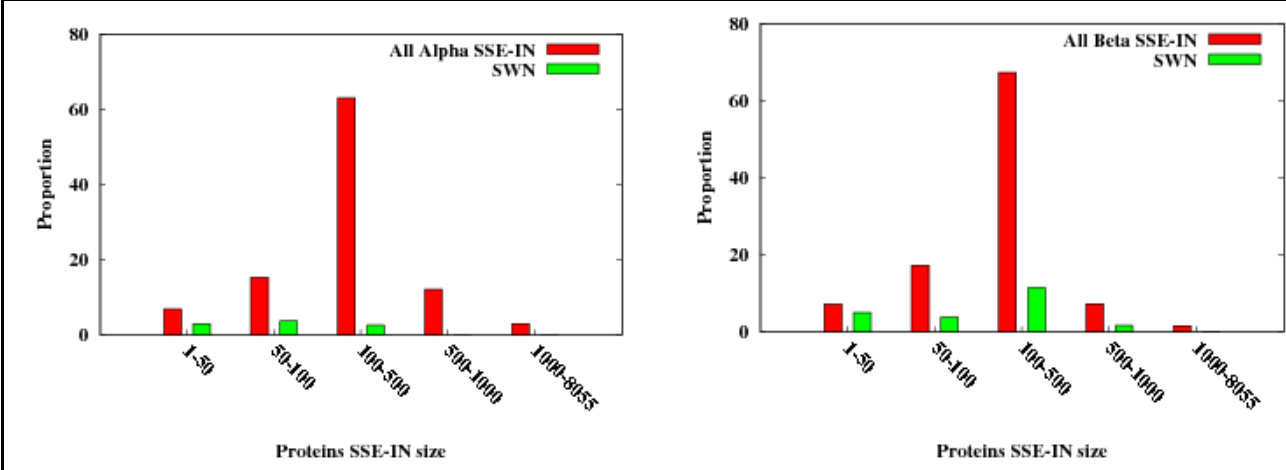

Fig. 21. Size distribution of proteins SSE-IN and small-world networks ratio.

On the other hand, the subnetworks corresponding to single SSEs are almost all small world networks. The last observation brings us to consider the edges whose extremities belong to different SSEs (see Fig. 18). These "shortcuts" represent the interactions between different SSEs and they determine the tertiary protein structure. They provide short paths between different network regions and bigger number of shortcuts implies smaller characteristic path length.

Computing the average connected component size, we observe that this size does not exceed 100 for the smallworld networks (see Fig. 23). Consequently, we identify a necessary condition for SSE-INs to be small-world, their average connected component size has to be less than 100.
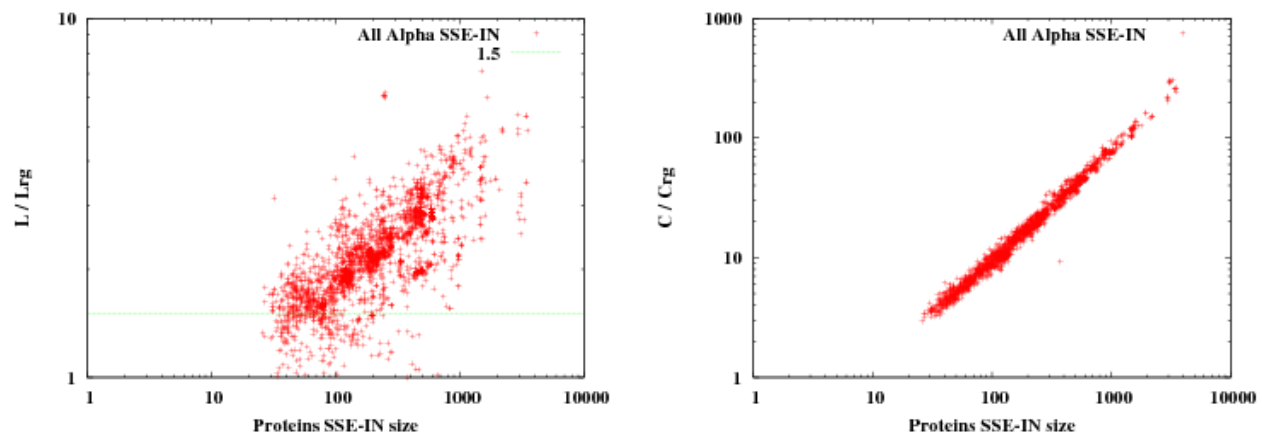

Fig. 22. $L / L_{R G}$ (left) and $C / C_{R G}$ (right) ratios as a function of SSE-IN size. 
In (Gaci \& Balev, 2008a) we show that the protein SSE-IN components interact with each other in the same way, no matter if they are small-world or not, see Fig 23. Then, it is interesting to study not the quantity of interaction inter-SSE but rather their quality, that is we want to put in evidence the role of nodes allowing interaction between different SSEs.

In graph theory, the betweenness of a node is defined as the total number of shortest paths between pairs of nodes that pass through this node. It measures the influence of a node in a network. The betweenness of a node $t$, denoted $B(t)$ is defined as follows:

$$
B(t)=\sum_{u \neq v, u \neq t, v \neq t} \frac{\sigma_{u v}(t)}{\sigma_{u v}}
$$

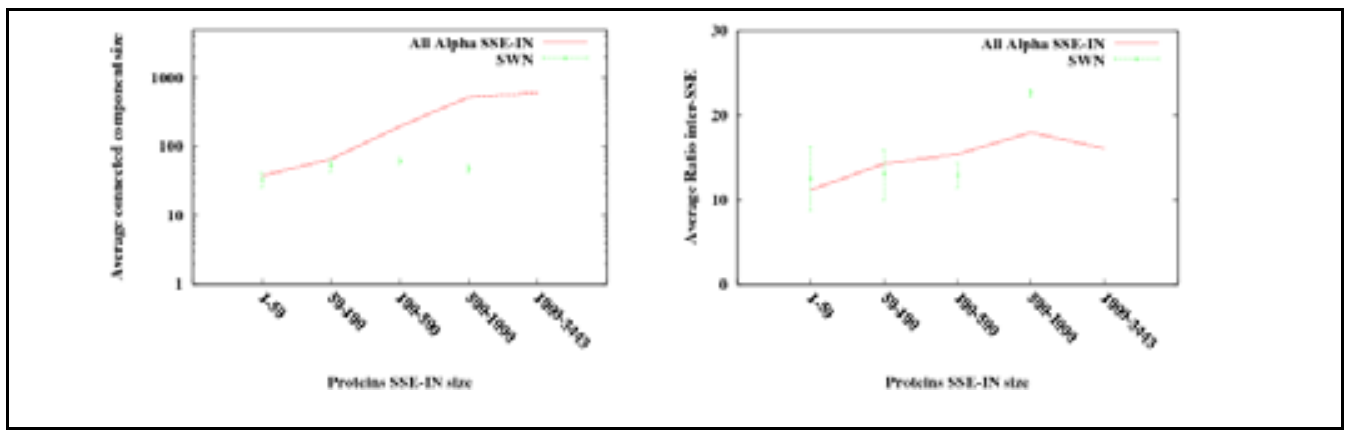

Fig. 23. Average connected component size (left) and shortcut ratio (right) as a function of protein SSE-In size.

where $\sigma_{u v}$ is the number of shortest paths between the nodes $u$ and $v$, and $\sigma_{u v}(t)$ is the number of shortest paths between $u$ to $v$ that pass through $t$.

The average betweenness of nodes interacting with other secondary structure motifs is shown on Fig 24. We can see that the average betweenness of the small-world networks is similar to the average betweenness of the other networks. This means that neither the quantity nor the quality of nodes that link different SSEs guarantees a short characteristic path length.

To better understand the properties of SSE-IN which are small-world, we introduce the secondary structure components interaction network, SSC-IN. This is a network in which each SSE is contracted to a single node. There is an edge between two SSEs $s_{1}$ and $s_{2}$ if theres is at least one interaction between an amino acid belonging to $s_{1}$ and an amino acid

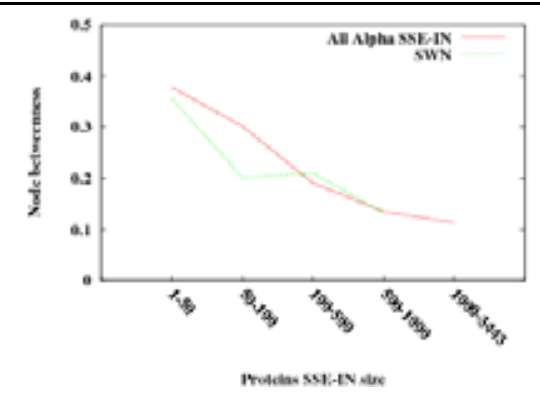

Fig. 24. Betweenness of nodes which link different SSEs. 
is at least one interaction between an amino acid belonging to $s_{1}$ and an amino acid belonging to $s_{2}$. A weight is associated to each edge. The weight of the edge between $s_{1}$ and $s_{2}$ is the average distance (measured in the corresponding SSE-IN) between all pairs of amino acids belonging to $s_{1}$ and $s_{2}$. SSC-IN is an abstraction of SSE-IN which puts in evidence the interactions between SSEs. Fig. 25 shows an example of SSC-IN. We computed the characteristic path lengths and the clustering coefficients for all protein SSC-IN, the results show once again that the small world effect is the discriminant property in the SSCIN. Indeed, for a small-world SSC-IN the characteristic path length grows linearly with the size of the underlying SSE-IN and it never exceeds 4 . The last observation can be correlated with the necessary condition for a SSE-IN to satisfy the small world model. Indeed, the average connected component size is bounded by 100 nodes in small world SSEIN. This has for consequence that the corresponding SSC-IN has bounded average connected component size and therefore a bounded characteristic path length. Then, a protein SSE-IN with size $n>$ 25 is small world if and only if its SSC-IN characteristic path length is on the line shown in Fig 26

\subsection{Consequences}

In this study, we put in evidence in which proportion the SSE-IN satisfy the small-world model. Their constituent that is the subgraphs representing the SSE are in the most majority small-world.

The interactions between these SSE being limiting, it not allows to conserve a short distance when the SSE-IN size increases. Thus, to a SSE-IN belongs to the small-world model, it necessary that the average connected component size doesn't exceed 100 residues. Further, we consider a SSE-IN abstraction, called SSC-IN in which each node represents a SSE. Then, their study provides an alternative way to verify the SSE-IN behavior. Indeed, the characteristic path length in the SSC-IN is enough to infer to deduct if the SSE-IN belongs or not to the small-world model.

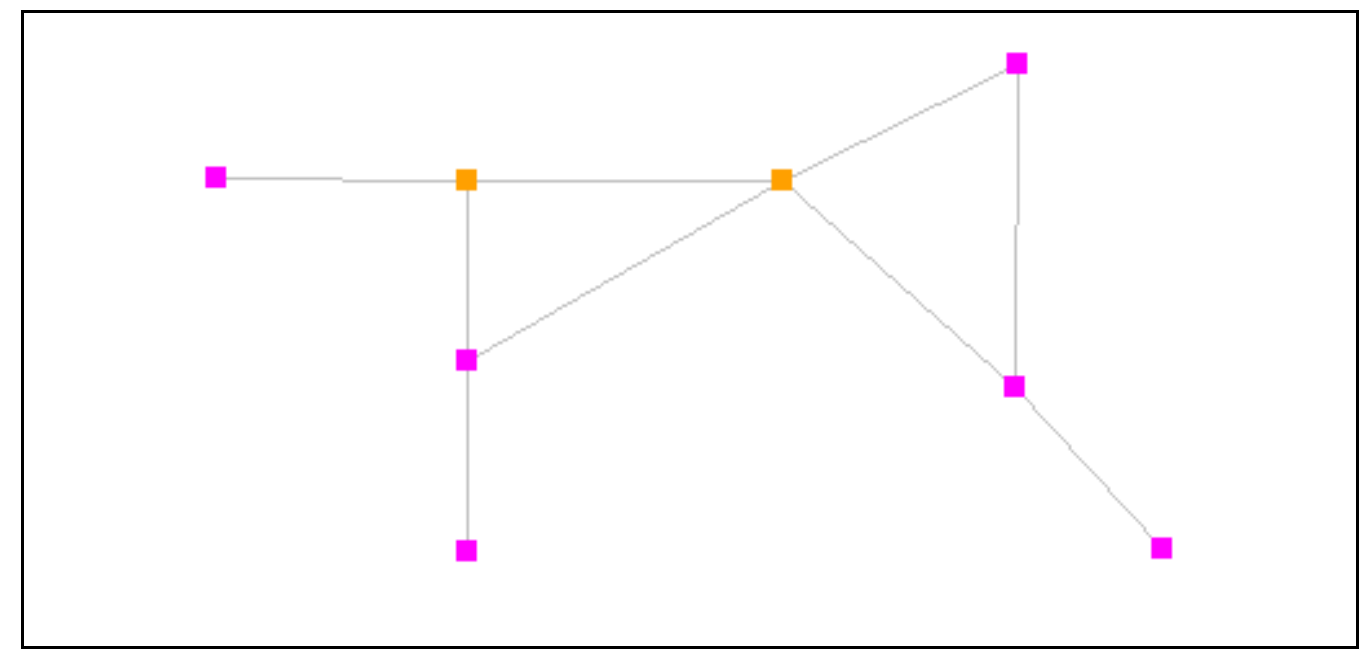

Fig. 25. Protein 1DTP SSC-IN. The edges weight corresponds to the mean distance between the two motifs in the SSE-IN. 


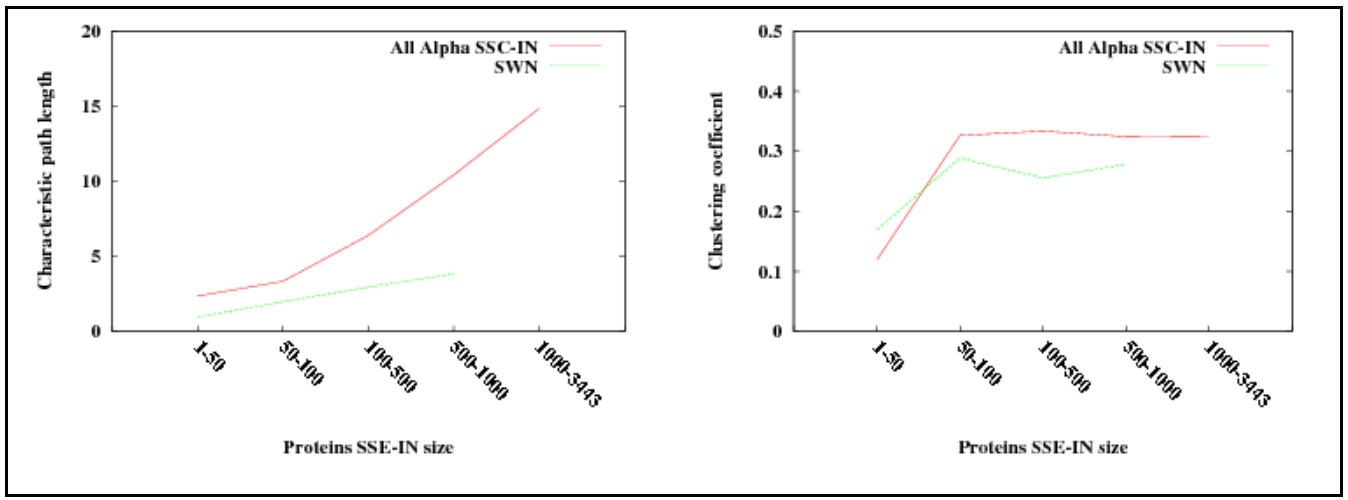

Fig. 26. Characteristic path length in SSC-IN. The distance in SW SSC-IN grows linearly and is bounded by 4 .

\section{Conclusion}

In this chapter, we exploit the possibilities offer by a modelisation using interaction networks. The main hypothesis we use is the conservation from structural toward topological properties. The comparison of the SSE-IN with the general model of interaction networks allows us to describe the consequence of the folding dynamic.

The characterization we propose constitutes a first step of a new approach to the protein folding problem. The properties identified here, but also other properties we plan to study, can give us an insight on the folding process. They can be used to guide a folding simulation in the topological pathway from unfolded to folded state.

\section{References}

Amaral, L. A. N.; Scala, A.; Barthélémy, M. \& Stanley, H. E. (2000). Classes of small-world networks. Proc. Natl. Acad. Sci USA, Vol. 97, No. 21, pp. 11149-11152

Berman, H. M.; Westbrook, J.; Feng, Z.; Gilliland, G.; Bhat, T. N.; Weissig, H.; Shindyalov, I. N. \& Bourne., P. E. (2000). The protein data bank. Nucleic Acids Research, Vol. 28, pp. 235--242

Branden, C. \& Tooze, J. (1999). Introduction to protein structure, Garland Publishing.

Brinda, K. V. \& Vishveshwara, S. (2005). A network representation of protein structures: implications for protein stability. Biophys J, Vol.89, No.6, pp. 4159-4170

Broder, A.; Kumar, R.; Maghoul, F.; Raghavan, P.; Rajagopalan, S.; Stata, R.; Tomkins, A. \& Wiener, J. (2000). Graph structure in the Web. Computer Networks, Vol.33, pp.309320

Coleman, T. F. \& Moré, J. J. (1983). Estimation of sparse jacobian matrices and graph coloring problems. SIAM Journal on Numerical Analysis, Vol.20, pp. 187-209

Colizza, V.; Flammini, A.; Serrano, M. A. \& Vespignani, A. (2006). Detecting rich-club ordering in complex networks. Nature Physics, Vol. 2, pp. 110

Diestel, R. (2000). Graph Theory. Springer Verlag, Princeton, New Jersey

Erdõs, P. \& Rényi, A. (1959). On random graphs \{I\}. Publicationes Mathematicae, Vol. 6, pp. 290-297 
Erdõs, P. \& Rényi, A. (1960). On the evolution of random graphs. Publ. Math. Inst. Hung. Acad. Sci., Vol. 7, pp. 17

Gaci, O. \& Balev, S. (2008a). A general model for amino acid interaction networks. Proceedings of World Academy of Science, Engineering and Technology, Vol. 34, pp. 401405

Gaci, O. \& Balev, S. (2008b). Proteins: From structural classification to amino acid interaction networks. Proceedings of BIOCOMP'08, pp. 728-734, Las Vegas, CSREA Press

Ghosh, A.; Brinda, K. V. \& Vishveshwara, S. (2007). Dynamics of lysozyme structure network: probing the process of unfolding. Biophys J, Vol. 92, No. 7, pp. 2523-2535

Goh, K-.I.; Kahng, B. \& Kim, D. (2001). Universal behavior of load distribution in scale-free networks. Phys. Rev., Vol. 87

Jeong, H.; Tombor, B.; Albert, R.; Oltvai, Z. N. \& Barabási, A.-L. (2000). The large-scale organization of metabolic networks. Nature, Vol. 407, No. 6804, pp. 651-654

Levitt, M. \& Chothia, C. (1976). Structural patterns in globular proteins. Nature, Vol. 261, pp. 552-558

Muppirala, U. K. \& Li, Z. (2006). A simple approach for protein structure discrimination based on the network pattern of conserved hydrophobic residues. Protein Eng Des Sel, Vol. 19, No. 6, pp. 265-275

Murzin, A. G.; Brenner, S. E.; Hubbard, T. \& Chothia, C. (1995). SCOP: a structural classification of the protein database for the investigation of sequence and structures. J. Mol. Biol., Vol. 247, pp. 536-540

Newman, M .E. J. (2001). The strucuture of scientific collaboration networks. Proc. Natl. Acad. Sci USA., Vol. 98, pp. 404-409

Newman, M. E. J. (2002). The structure and function of networks. Computer Physics Communications., Vol. 147, pp. 40-45

Newman, M. E. J.; Strogatz, S. H. \& Watts, D. J. (2001). Random graphs with arbitrary degree distributions and their applications. Physical Review E, Vol. 64

Orengo, C. A.; Michie, A. D.; Jones, S.; Jones, D. T. ; Swindells, M. B. \& Thornton, J. M. (1997). CATH - a hierarchic classification of protein domain structures. Structure, Vol. 5, pp. 1093-1108

Réka, A. \& Barabási, A.-L. (2000). Topology of evolving networks: local events and universality. Physical Review Letters, Vol. 85, No. 24

Solomonoff, R. \& Rapoport, A. (1951). Connectivity of random nets. Bull. Math. Biophys., Vol. 13, pp. 107-111

Wasserman, S. \& Faust, K. (1994). Social network analysis : methods and applications, In Structural analysis in the social sciences, Cambridge University Press, Cambridge, 1994

Watts, D. J. (1999). Small Worlds, Princeton University Press, Princeton, New Jersey, 1999 


\title{
Abstraction Hierarchies for Conceptual Engineering Design
}

\author{
Esra Aleisa, Ph.D. \\ Kuwait University \\ Kuwait
}

\begin{abstract}
Research in Artificial Intelligence has been a forerunner in developing the most detailed and formalized theories that characterize and create consistent abstraction hierarchies for planning and problem solving. However, the representational methods to exploit these theories are complicated, which limit their application into many disciplines, specifically engineering. The objective of this chapter is threefold: to simplify the representation of current AI-based planning, to identify the properties that ensure effective development of abstraction hierarchies, and accordingly, to develop a methodology for effective and consistent generation of abstraction hierarchies. The proposed methodology achieves these objectives by integrating the well-established AI hierarchical abstraction theories with Steward's practical Design Structure Matrices (DSM). The developed methodology consists of three phases. In the first phase, the literal space and its interactions are formally defined and their interactions are represented as a DSM. The second phase involves clustering literals to abstract classes according to the manner by which they communicate. Finally, in the third phase, the abstract literals are stratified into a loop-free abstraction hierarchy. The approach expands the capabilities of AI-based planning and problem solving abstraction hierarchies and improves their functionality by enabling integration and compatibility with practical DSM planning tools. The effectiveness of the developed methodology is demonstrated by applying it to the conceptual design on a high voltage cable manufactring facility.

Keywords: Abstraction; Design Structured Matrix; Hierarchy; Planning; State-Space Representation
\end{abstract}

\section{Introduction}

Abstraction Hierarchies (AHs) are used commonly to represent various large-scale and complex problems (Lam 1996; Holte \& Choueiry 2003; Sebastia, Onaindia et al. 2006). Their values have been realized across a wide spectrum of applications mainly to reduce the complexity of problems and to improve solution efficiency (Holte \& Choueiry 2003; Aleisa 2005). AHs are also used to speed up the development time, save resources, and provide aggregate intelligent output (Goldin \& Klahr 1981; Aleisa 2008). In addition, AH produces designs that are easier to interpret validate and update compared to not using hierarchies. Moreover, AHs help explore design alternatives and produce intelligent decisions at an 
early stage of the design or plan (Sacerdoti 1974; Taylor \& Henderson 1994; Reddy 1996) . Furthermore, AHs assist in focusing on important aspects of the design problem(Hoover \& Rinderle 1994; Sarjoughian, Zeigler et al. 1998; Zeigler, Praehofer et al. 2000). For computational efficiency, AHs have also allows parallel execution of models (Kiran, Cetinkaya et al. 2001), facilitates the utilization of the off-shelf models legacy (McGraw \& MacDonald), and enhances model reusability and rapid prototyping (Zeigler 1987; Lin, Yeh et al. 1996; Pidd 1996; Praehofer 1996; Chen \& Ghosh 1997; Pidd \& Castro 1998; Aleisa \& Lin 2008). However, despite AHs' significant benefits, there is a lack of formal methodologies for hierarchical abstraction generation suitable for engineering design. In fact, hierarchical abstraction in general has been described as a "black art" (Knoblock 1994). In this research, we aim to provide a formal hierarchical abstraction methodology to represent and plan engineering design problems at multiple levels of abstraction. Such that partial design solutions obtained at some abstraction level is preserved while the design is augmented at more detailed levels. The objectives of the methodology are three fold:

(1) to develop a representation for engineering design that supports hierarchical abstraction,

(2) to specify the clustering criteria according to which the abstraction process is defined, and

(3) to develop a layering method, by which clusters of abstracted design parameters should be stratified in a hierarchy, without inducing any backtracking in the design process.

In other words, this research proposes a representation, extracts properties that characterize efficient abstraction methods, and proposes a methodology that utilizes an AI-based analysis of efficient systems but overcomes their complications. The methodology consists of three phases. In Phase I, a literal space representation is proposed to represent planning problems in a DSM-based format. In Phase II, the interactions within the literal space framework are utilized to cluster literals into abstract classes. Finally, in Phase III the abstract classes are stratified to construct loop-free abstraction hierarchies.

The reminder of this chapter is structured as follows: first we provide a brief literature review of some of the most persistent abstraction systems and the reason why they are cumbersome when applied to engineering designs. This necessitates the need for this research. Next we dedicate a separate section to explain each of the three developmental phases of our hierarchical abstraction methodology. Then we provide some analysis on the methodology and theoretically proof that it is loop-free. Finally, we demonstrate the effectiveness of the methodology on the design process of a local high voltage cable manufacturing facility.

\section{Background}

As indicated earlier, AHs have been used to investigate and explore different alternatives earlier in the plan. Moreover, AHs have assisted analysts in focusing on vital aspects of a problem (Hoover \& Rinderle 1994; Sarjoughian, Zeigler et al. 1998), leaving inferior details to be determined later. Despite AHs' benefits, the process of developing hierarchical models is more of an art form (Knoblock 1994). The most detailed analysis of abstraction was conducted by research in Artificial Intelligence, specifically, in the fields of planning and problem solving (Giunchiglia \& Walsh 1992; Armano, Cherchi et al. 2003). ABSTRIPS 
(Sacerdoti 1974; Giunchiglia 1999), one of the earliest abstractions, uses a state-space representation based on a STRIPS (Stanford University Research Institute Planning System) framework. The successors of ABSTRIPS are many, including PRODIGY/EBL (Minton 1988), ABTWEAK (Yang 1990), PABLO (Christensen 1991), ALPINE(Knoblock 1994), HIGHPOINT (Bacchus \& Yang 1992) and more (see (Friske \& Ribeiro 2006; Marie, Priyang et al. 2008)). A comparison of the most persistent abstraction research is provided in Table 1.

\section{The Effectiveness of Abstraction Methods and Applications}

Hierarchical models are a result of an iterative application of some abstraction methods. That is, an ordered sequence of abstraction spaces constitutes the skeleton of an abstraction hierarchy (Knoblock 1994; Giunchiglia 1999). Therefore, since abstraction processes are the building blocks of an $\mathrm{AH}$, the efficiency of the abstraction process directly influences that of the $\mathrm{AH}$. For this reason, the properties of effective abstraction need to be thoroughly investigated, which is the topic of the next section.

\begin{tabular}{|c|c|c|c|c|}
\hline Author(s) & $\begin{array}{c}\text { Measure of } \\
\text { detail }\end{array}$ & Abstraction Approach & $\begin{array}{l}\text { Autom- } \\
\text { atic? }\end{array}$ & $\begin{array}{c}\text { Assumptions/ Notes/ } \\
\text { Contributions }\end{array}$ \\
\hline $\begin{array}{l}\text { Hobbs '85 } \\
\text { Subramanian } \\
\text { ‘89 }\end{array}$ & Piece of data & Reasoning arguments & - & $\begin{array}{l}\text { Suggested abstraction by } \\
\text { proposing arguments without } \\
\text { developing an algorithm }\end{array}$ \\
\hline $\begin{array}{l}\text { Knoblock ‘90 } \\
\text { Ellman ‘93 }\end{array}$ & Piece of data & Relevance reasoning & - & $\begin{array}{l}\text { Showed computational savings } \\
\text { gained by using abstractions both } \\
\text { empirically and theoretically when } \\
\text { applying relevance reasoning } \\
\text { approaches } \\
\text { No backtracking is assumed }\end{array}$ \\
\hline Knoblock '94 & $\begin{array}{l}\text { Domain } \\
\text { dependent }\end{array}$ & $\begin{array}{l}\text { tractable algorithm } \\
\text { that drops irrelevant } \\
\text { literals from original } \\
\text { problem }\end{array}$ & Yes & $\begin{array}{l}\text { Domain independent, only input is } \\
\text { problem formulation, satisfies } \\
\text { monotonicity property }\end{array}$ \\
\hline $\begin{array}{l}\text { Giunchiglia \& } \\
\text { Walsh ‘92 }\end{array}$ & $\begin{array}{l}\text { Elements of the } \\
\text { system or the } \\
\text { language }\end{array}$ & $\begin{array}{l}\text { Mapping between } \\
\text { systems, set theory } \\
\text { and reasoning }\end{array}$ & - & $\begin{array}{l}\text { Established the foundation for } \\
\text { abstraction theory and classified } \\
\text { various types of abstractions }\end{array}$ \\
\hline $\begin{array}{l}\text { Bacchus \& Yang } \\
\text { '92 }\end{array}$ & Piece of data & $\begin{array}{l}\text { Constantly removing } \\
\text { details to simplify the } \\
\text { search space }\end{array}$ & Yes & $\begin{array}{l}\text { Discussed the Downward refinement } \\
\text { property (DRP) and showed how } \\
\text { hierarchical problem solving } \\
\text { techniques that lack this property has } \\
\text { no advantage over nonhierarchical } \\
\text { methods }\end{array}$ \\
\hline Holte et al.'96 & Piece of data & $\begin{array}{l}\text { Used caching } \\
\text { techniques to avoid expanding } \\
\text { the same searches in successive } \\
\text { searches } \\
\end{array}$ & Yes & An admissible $A^{*}$ search technique \\
\hline Lu \& Tcheng ‘91 & $\begin{array}{l}\text { Number of } \\
\text { decision } \\
\text { variable in the } \\
\text { model }\end{array}$ & $\begin{array}{l}\text { Combined inductive learning } \\
\text { approaches with optimization } \\
\text { techniques to evaluate } \\
\text { decisions made at different } \\
\text { levels of abstraction }\end{array}$ & Yes & $\begin{array}{l}\text { Proposed AIMS (Adaptive and } \\
\text { integrative modeling system) } \\
\text { methodology that automatically } \\
\text { abstracts detailed systems using } \\
\text { machine learning approaches } \\
\end{array}$ \\
\hline Pooley ‘91 & $\begin{array}{l}\text { Atomic } \\
\text { processes }\end{array}$ & $\begin{array}{l}\text { Abstract using } \\
\text { graphical } \\
\text { technique } \\
\text { configuration } \\
\text { diagrams } \\
\end{array}$ & No & $\begin{array}{l}\text { Atomic processes from the activity } \\
\text { diagram are coupled to form } \\
\text { configuration diagrams }\end{array}$ \\
\hline
\end{tabular}




\begin{tabular}{|c|c|c|c|c|}
\hline Yager '94 & Piece of data & Neural Networks & Yes & $\begin{array}{l}\text { Can Handle nonnumeric data, } \\
\text { developed a function that } \\
\text { transforms a group of data into a } \\
\text { single data point }\end{array}$ \\
\hline $\begin{array}{l}\text { Hoover \& } \\
\text { Rinderle‘94 }\end{array}$ & $\begin{array}{l}\text { System } \\
\text { parameters/ } \\
\text { variables }\end{array}$ & $\begin{array}{l}\text { Relevance reasoning, concept of } \\
\text { focusing abstractions and } \\
\text { Gröbner bases }\end{array}$ & Yes & $\begin{array}{l}\text { Based on Gröbner bases, assumed } \\
\text { focusing abstractions change the } \\
\text { scope not accuracy, limited to } \\
\text { polynomial equation formulations }\end{array}$ \\
\hline $\begin{array}{l}\text { Kramer \& } \\
\text { Unger'92 }\end{array}$ & $\begin{array}{l}\text { Number of } \\
\text { operators in } \\
\text { each level }\end{array}$ & Subsuming Abstraction & Yes & $\begin{array}{l}\text { The process is type oriented } \\
\text { operator abstracting process that } \\
\text { aims on diminishing the number of } \\
\text { operators in the detailed level }\end{array}$ \\
\hline $\begin{array}{l}\text { Taylor \& } \\
\text { Henderson ‘94 }\end{array}$ & $\begin{array}{l}\text { Features and } \\
\text { forms of a } \\
\text { mechanical } \\
\text { design }\end{array}$ & $\begin{array}{l}\text { Generalization/specialization } \\
\text { and aggregation/ } \\
\text { decomposition }\end{array}$ & No & $\begin{array}{l}\text { Showed the relationships between } \\
\text { forms and features in a mechanical } \\
\text { design and showed how abstraction } \\
\text { could aid the design process }\end{array}$ \\
\hline $\begin{array}{l}\text { Bisantz \& } \\
\text { Vicente '94 }\end{array}$ & $\begin{array}{l}\text { Components } \\
\text { and detailed } \\
\text { functions of a } \\
\text { system }\end{array}$ & $\begin{array}{l}\text { Aggregation/ } \\
\text { decomposition } \\
\text { and a physical/ } \\
\text { functional } \\
\text { abstraction } \\
\text { approaches } \\
\end{array}$ & No & $\begin{array}{l}\text { Presented how to abstract a system } \\
\text { using two orthogonal dimensions } \\
\text { simultaneously, the part/whole and } \\
\text { the physical functional dimension }\end{array}$ \\
\hline Reddy ‘96 & $\begin{array}{l}\text { Details of } \\
\text { system design } \\
\text { specifications }\end{array}$ & $\begin{array}{l}\text { Form empirical models from } \\
\text { training examples using } \\
\text { multiple learning algorithms }\end{array}$ & Yes & $\begin{array}{l}\text { Multiple learning approaches } \\
\text { includes: statistical regression, } \\
\text { neural networks, inductive learning } \\
\text { algorithms }\end{array}$ \\
\hline Fox \& Long ‘95 & Details of a plan & Subsumption abstractions & - & $\begin{array}{l}\text { Discussed how DRP would indicate } \\
\text { if a hierarchical decomposition is } \\
\text { worthwhile }\end{array}$ \\
\hline Sisti \& Farr ‘98 & $\begin{array}{l}\text { Depends on the } \\
\text { model to be } \\
\text { abstracted }\end{array}$ & \begin{tabular}{|l|} 
Abstracted \\
Models \\
using \\
boundary, \\
behavior and \\
form abstractions \\
\end{tabular} & No & $\begin{array}{l}\text { Objective was to improve accuracy } \\
\text { at aggregate level, compared the } \\
\text { terms accuracy, complexity and } \\
\text { level of detail and showed how to } \\
\text { create model hierarchies that can } \\
\text { be interconnected and reused }\end{array}$ \\
\hline
\end{tabular}

Table 1. A comparison of the most persistent research in abstraction

\section{The Seven Desirable Properties of Abstraction Methods}

This section extracts properties that would render an abstraction method to be effective. These include the following characteristics:

(1) Formal. Abstraction methodologies are by large case-dependent, with little to be generalized. Thus, there is a need to develop abstraction methods using well-structured languages and consistent terminology, and to support them with a sound theoretical basis.

(2) Complete. A complete abstraction hierarchy is one that achieves all the steps and preconditions required (Russell \& Norvig 1995). On the other hand, an incomplete abstraction hierarchy is described as a theory-decreasing (TD) abstraction (Giunchiglia \& Walsh 1992). TD abstractions exhibit deficiency by losing information while abstracting, therefore lacking integrity and affecting the quality of obtained abstract solutions. 
(3) Computable. Despite the indispensable need for expertise to articulate effective abstractions, abstraction methods must consist of quantifiable and computable techniques to enable automation(Friske \& Ribeiro 2006) and generalization(Pels 2006).

(4) Produce simpler models. When applied to a problem, an abstraction method should produce simpler models that are easier to understand, handle, and solve compared to the original problem representation (Zeigler 1976; Lu \& Tcheng 1991; Manfaat, Duffy et al. 1998; Kemke \& Walker 2006).

(5) Tractable. Abstraction methods should not involve computational complexities(Gimenez \& Jonsson 2008). If so, then the purpose of abstraction is defeated and abstraction will be futile.

(6) Reduce cost. For abstraction to be effective, the cost of creating an abstract model, solving the problem with the abstract model and mapping the solution back to the original representation should be inexpensive, compared to solving the problem directly using its original (or detailed) representation (Bacchus \& Yang 1992; Levy 1994; Debbie 2003; Zucker 2003)

(7) Produce consistent and cumulative refinement. This is achieved when backtracking is avoided during the exploitation of an abstraction hierarchy. Eliminating backtracking means that there is no need to resolve any established elements from higher abstract levels in the abstraction hierarchy. As this property is particularly important for achieving efficient designs, it is further elaborated in the next section.

\subsection{Consistent and cumulative refinement (ccr) properties}

This chapter uses the term consistent and cumulative refinement (CCR) properties to refer to properties that preserve intermediate solutions or results obtained at abstract levels. The essence of the CCR properties is that already established aspects at higher abstraction levels need not be altered as more details are introduced at lower abstraction levels(Zucker 2003). Among the most formalized CCR properties is the Ordered Monotonicity Property (OMP) of Knoblock (Knoblock 1990; Knoblock 1994). According to Knoblock (Knoblock 1994), OMP guarantees that the structure of an abstract solution is not changed by the process of refining it. For this property to hold, the abstraction hierarchy needs to partition a problem, such that the parts of the problem already solved in an abstract space are maintained while the remaining parts of the problem are solved. OMP has the advantage of being computationally tractable, while it is also able to capture a large class of abstraction problems. However, OMP is a heuristic, and thus does not guarantee a reduction of the search space.

Another CCR property is the Downward Refinement Property (DRP) (Bacchus \& Yang 1992; Helmert 2006). A planning domain is said to possess DRP if all abstract plans can be consistently refined without backtracking across abstraction levels (Fox \& Long 1995). Bacchus and Yang (Bacchus \& Yang 1992) emphasized that when DRP holds, backtracking needs never occur across various levels of the abstraction hierarchy, indicating a hierarchical decomposition is worthwhile(Zucker 2003). However, being a heuristic, DRP encounters difficulties similar to those of OMP. 


\section{Integrating the Design Structured Matrix to Al Hierarchical Abstraction}

Formulated by Steward (Steward 1981), the Design Structure Matrix (DSM) a.k.a. dependency structure matrix, is a project modeling tool to plan, represent and analyze the flow of information among different tasks of complex design projects (McCord 1993; Browning 2001). DSM is a square binary matrix with rows and columns, where is the number of design tasks under consideration (Warfield 1973). If task $i$ is dependent on task $j$, then the entry of the respective column $j$ and row $i$ is unity or marked with an $X$ (Browning 1999; Yassine, Falkenburg et al. 1999). Off-diagonal marks represent coupling between tasks, marks in the upper triangle in DSM represent feedforward coupling, and marks in the lower triangle represent feedback coupling (Rogers 1996). The DSM tasks are rearranged in order to eliminate feedback marks. Then, the DSM is partitioned into blocks of tasks that simultaneously depend on one another. Three different relationships can be identified from a partitioned DSM: sequential, parallel and coupled tasks. A task can be performed sequentially if its row contains a mark just below the diagonal; a task is parallel if there are no marks linking it with other tasks; coupled tasks are ones that hinder a partitioned DSM to be lower triangular (Yassine, Falkenburg et al. 1999). Finally, feedback marks are removed from the DSM in a processes called tearing (Steward 1981) to initiate sequencing within blocks (Eppinger, Whitney et al. 1994).

This research intends to utilize the DSM representational advantage to simplify AI-based abstraction hierarchies.

\section{Hierarchical Abstraction Methodology for Structuring Literal Spaces}

The presented hierarchical abstraction methodology consists of three phases: representation, abstraction and layering. The representation phase were literal spaces are formulated into a transposed DSM. In the second phase, the abstraction phase, the problem literals are clustered into mutually-exclusive abstract equivalence classes (AECs). Finally, in the third phase, the layering phase, the different AECs are stratified into multiple levels of a hierarchy using a level assignment algorithm (LAA). The three phases of the methodology are illustrated in Fig. 1and are discussed in greater detail in the following sections. 


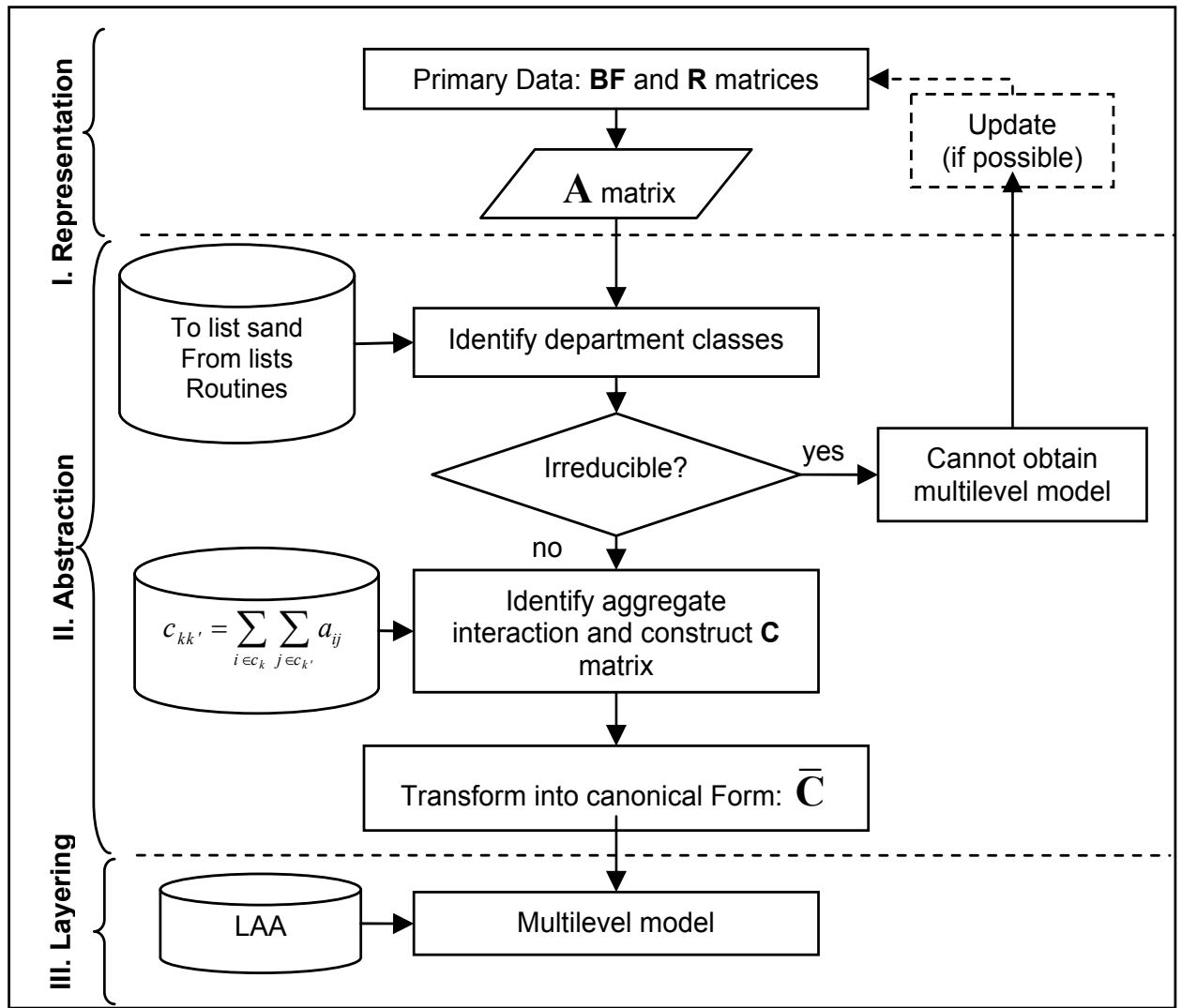

Fig. 1. Hierarchical Abstraction Methodology

\subsection{Phase I: The literal space representation}

Let $l_{i}$ denote a positive or a negative literal $i$, where a literal is defined as an atomic expression or the negation of an atomic expression (Luger 2002). The literal space $\Omega$ is a set that consists of all literals under consideration. Similarly, $O$ denotes the set of operators, $(k=1,2, \ldots, p)$, such that $O=\left\{o_{1}, o_{2}, \ldots, o_{p}\right\}$. Analogous to the STRIPS framework, for each operator, let $p_{k}$ be the set that contains all the preconditions. A precondition set $p_{k}$ is the set of some literals $l_{i} \in \Omega$ that need to be achieved prior to the application of an operator. Similarly, we define $e_{k}$ to be the set of effects of operator $o_{k}$, where an effect is the set of some achieved literals $l_{i} \in \Omega$ that resulted from applying an operator. For that we write each operator a as an ordered tuple of $o(p, e)$. It is possible for $p_{k}$ or to be empty, indicating that a specific operator $k$ does not require preconditions nor result in any effects respectively. As an example, describes an operator $o_{k}$ with no preconditions and literal $l_{i}$ of effects, which is typical for initialization operators. 


\subsubsection{Formalizing Interactions among literals and operators}

The representation of operators in terms of their preconditions and effects indicates a causal relationship between them. This is the result of having some operators $o_{k}, o_{k^{\prime}} \in O \quad\left(k \neq k^{\prime}\right)$ where; hence $o_{k}$ contains among its effects some literal $l \in \Omega$ that is part of the preconditions of $o_{k^{\prime}}$ that are required for it to be applied. If the above holds, then we say that $o_{k}$ establishes some literals for $o_{k^{\prime}}$. Establishment is formally defined below.

- Establishment definition: Let operators $o_{k}, o_{k^{\prime}} \in O$ where $k \neq k^{\prime}$, and literal $l \in \Omega$. Let $e_{k}$ be the set of effects of, and $p_{k^{\prime}}$ be the set of preconditions of $o_{k^{\prime}}$. We say that $o_{k}$ establishes literal $l$ for $o_{k^{\prime}}\left(\right.$ establishes $\left.\left(o_{k}, o_{k^{\prime}},\{l\}\right)\right)$ if and only if

$$
\begin{aligned}
& \exists l \in \Omega, \text { such that } \\
& l \in e_{k} \text {, and }
\end{aligned}
$$

Establishment has been requisitely used in the literature of planning and problem solving within the field of Artificial Intelligence (AI). However, establishment definitions usually impose an additional restriction on the precedence between two operators with respect to a plan. Nevertheless, this restriction is not necessary in this context since it is not intended to produce the shortest possible sequence of operators that transform the initial state to the goal state.

When operators' precedence constraints are not imposed within the establishment definition, establishments can be interpreted as causal links common in engineering applications. In engineering practices, it is customary to represent causality in a matrix representation (Warfield 1973). In this research, we define two types of causal links that result among operators and literals respectively. These causal links are discussed in the following sections.

Operator causality definition: Let $o_{k}, o_{k^{\prime}} \in O$ and $l \in \Omega$; the operator causality link $a_{k k^{\prime}}$ is defined as follows:

$$
a_{k k^{\prime}}= \begin{cases}1 & \text { if establishes }\left(o_{k}, o_{k^{\prime}},\{l\}\right) \text { for some } l \\ 0 & \text { otherwise }\end{cases}
$$

The above definition indicates that if establishes $\left(o_{k}, o_{k^{\prime}},\{l\}\right)$ holds for some operators $o_{k}, o_{k^{\prime}} \in O$ and some $l \in \Omega$, then according to the establishment definition $l$ must be a precondition and an effect in $o_{k}$ and $o_{k^{\prime}}$ respectively (i.e. , $l \in e_{k^{\prime}}$ ). If this is the case, then the operator causal link $a_{k k^{\prime}}$ is greater than zero. Fig. 2 shows a digraph of the operator causality definition.

Fig. 2. Digraph of operator causality

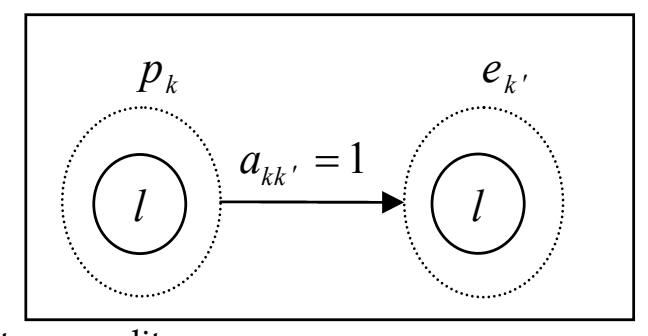




\section{- Causality within Operators}

Operator causality defined earlier identifies the relationships among the different operators. Literal causality however, describes the relationship among literals within operators.

Literal causality definition: Let literals $l_{i}, l_{j} \in \Omega$ and let $p_{k}, e_{k}$ be respectively the preconditions and effects of operator $o_{k} \in O$; the literal causality link is defined as follows:

$$
r_{i j}= \begin{cases}1 & \text { if } \exists o_{k} \in O ; l_{i} \in p_{k}, \text { and } l_{j} \in e_{k} \\ 0 & \text { otherwise }\end{cases}
$$

Therefore, the causality link $r_{i j}$ is nonnegative when literals $l_{i}$ and $l_{j}$ belong to the set of preconditions and effects respectively of any arbitrary operator in $O$. Fig 3 illustrates literal causality among three operators, while Fig. 4 shows the corresponding operator causality and establishment of the former Figure.

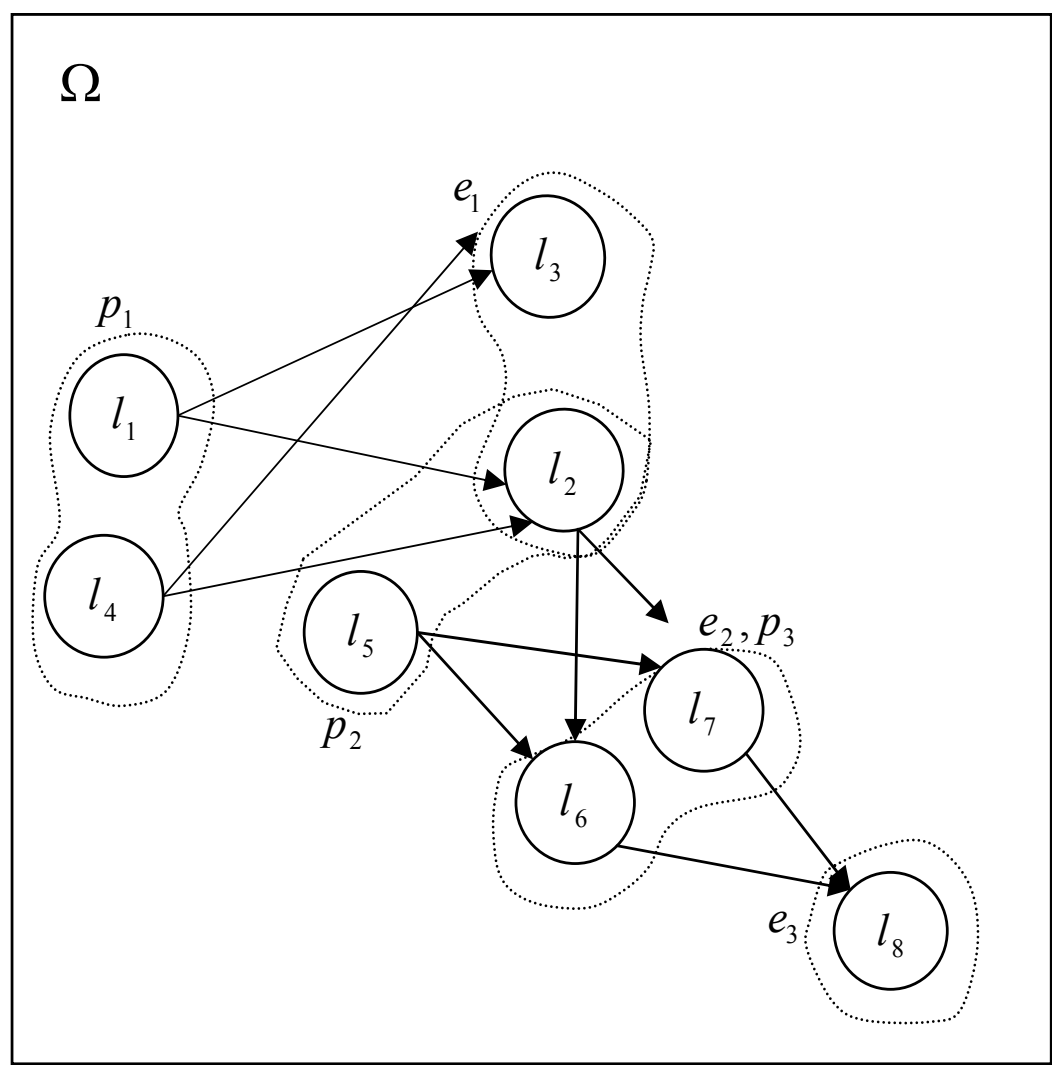

Fig. 3. Literal causality among the literals of three related hypothetical operators 


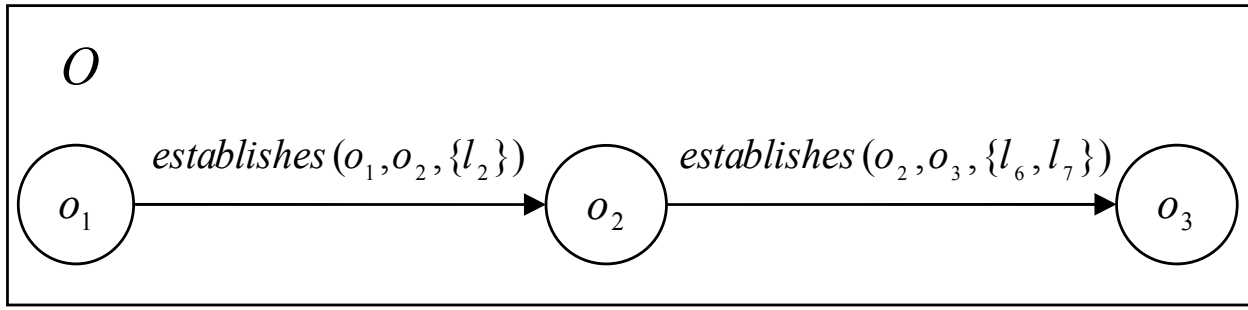

Fig. 4. Establishment resulting from the example given in Fig. 3

Let $\mathbf{R}=\left[r_{i j}\right]$ define a literal causality matrix of size $n \times n$ whose entries follow Eq (2). In graph theory (Deo 1974), $\mathbf{R}$ corresponds to a node-to-node incidence matrix. Moreover, the transposed form of the $\mathbf{R}$ matrix (i.e. $\mathbf{R}^{\mathrm{T}}$ ) has an equivalent structure to that of a DSM(Aleisa \& Lin 2009). This allows us to exploit the well-established methods of DSM to structure literal spaces, while still remaining consistent with the previous literature on statespace literature, the theory of ordered relations (Dartmouth College Writing Group. \& Cogan 1958) and Markov Chains by considering the transposed form.

\subsection{Accessibility and communication among literals}

In this research, we use $\mathbf{R}^{(\mathrm{S})}$ to indicate that the matrix $\mathbf{R}$ is multiplied $s$ times by

itself. Based on matrix theory, we can interpret $r_{i j}^{(S)} \geq 0$ as the ability to reach literal $j$ from $i$, passing through $s$ literals or alternatively through the application of $s$ operators. Note that we shall refer to $r_{i j}^{(1)}$ by $r_{i j}$ for simplicity. Based on the interpretation of $r_{i j}^{(S)} \geq 0$, we define literal accessibility and communication.

Accessibility: We say that $l_{j}$ is accessible from $l_{i}\left(\right.$ accessible $\left.\left(l_{i}, l_{j}\right)\right)$, if and only if $r_{i j}^{(s)}>0$ through a number of operators, $s=1,2, \ldots$.

If there is no operator $o_{k}$ applied on $l_{i}$, then the value of $l_{i}$ is assumed to remain unaffected. Hence, it is legitimate to assume that every literal is accessible at least by itself, therefore:

$$
r_{i j} \geq 0, \forall i=j
$$

Therefore, accessibility has two relational properties:

(1) Reflexive, based on Eq.(3).

(2) Transitive, since:

$$
\begin{aligned}
& \operatorname{accessible}\left(p_{i}, p_{j}\right) \cap \operatorname{accessible}\left(p_{j}, p_{k}\right) \\
& \Rightarrow \operatorname{accessible}\left(p_{i}, p_{k}\right), \\
& \forall p_{i}, p_{j}, p_{k} \in \Omega
\end{aligned},
$$

Communication: Let $l_{i}, l_{j} \in \Omega, l_{i}$ and $l_{j}$ communicate $\left(\operatorname{communicate}\left(l_{i}, l_{j}\right)\right)$ if and only if the following holds: 


$$
\text { accessible }\left(l_{i}, l_{j}\right) \cap \operatorname{accessible}\left(l_{j}, l_{i}\right)
$$

Alternatively, communication between two literals $l_{i}, l_{j} \in \Omega$ implies that the following hold:

$$
r_{i j}^{(s)}>0, r_{j i}^{(s)}>0 \text { for some } s=1,2, \ldots
$$

Let $\mathbf{R}=\left[r_{i j}\right]$ define a literal causality matrix of size $n \times n$. The transposed form of the $\mathbf{R}$ matrix (i.e. ) has an equivalent structure to that of a DSM. This allows us to exploit the wellestablished methods of DSM to structure literal spaces.

\section{Phase II: Abstraction of the Literal Space}

This phase creates an abstract literal space of $\Omega$, denoted by $\omega$ by clustering the literals under consideration into mutually-exclusive partitions.

Eq. (6) shows that communication is a reflexive, symmetric and transitive relation. A relation that exhibits these properties is an equivalence relation (Kemeny \& Snell 1960). Equivalence relations have the ability to partition the universe $\Omega$ upon which it is defined to disjointed partitions (Dartmouth College Writing Group. \& Cogan 1958). Each of these partitions defines a unique cluster of communicating literals, which is referred to as abstract equivalence classes.

\subsection{Abstract equivalence classes}

An abstract equivalence classes (AEC), denoted by $c_{k}(k=1,2, \ldots, m)$, is a set of literals by which all members of this set communicate with one another. $k$ corresponds to the number of $c_{k} \subseteq \omega$. If the abstract literal space $\omega$ consists of a single AEC (i.e. $k=1$ ), is called irreducible to be consistent with the terminology used in Markov Chains (Kao 1997). Therefore, irreducibility implies that the literals of the original literal space $\Omega$ all communicate with one another. Because AECs are developed based on an equivalence relation (i.e. communication), then the following must hold:

$$
\bigcap_{\forall k} c_{k}=\varnothing, \bigcup_{\forall k} c_{k}=\Omega, \forall k
$$

\subsection{The formation of AECS}

The equivalence class formation algorithm (ECFA) is used to abstract the literal space $\Omega$ into $\omega$. In ECFA, $T_{i} \mathrm{~B}$ denotes the to-list of $l_{j}$, such that each $T_{i}$ contains all the literals that $l_{j}$ can access through one or more operators. Similarly a from-list $F_{i}$ is defined to contain all the literals from which $l_{j}$ is accessible through one or more operators. $c_{i}$ is a set of communicating literals that contains $l_{i}$. The codes for constructing a To lists and a From lists are provided in Fig. 5 and Fig. 6 respectively. Detailed steps of these routines are provided in (Gaver \& Thompson 1973). 


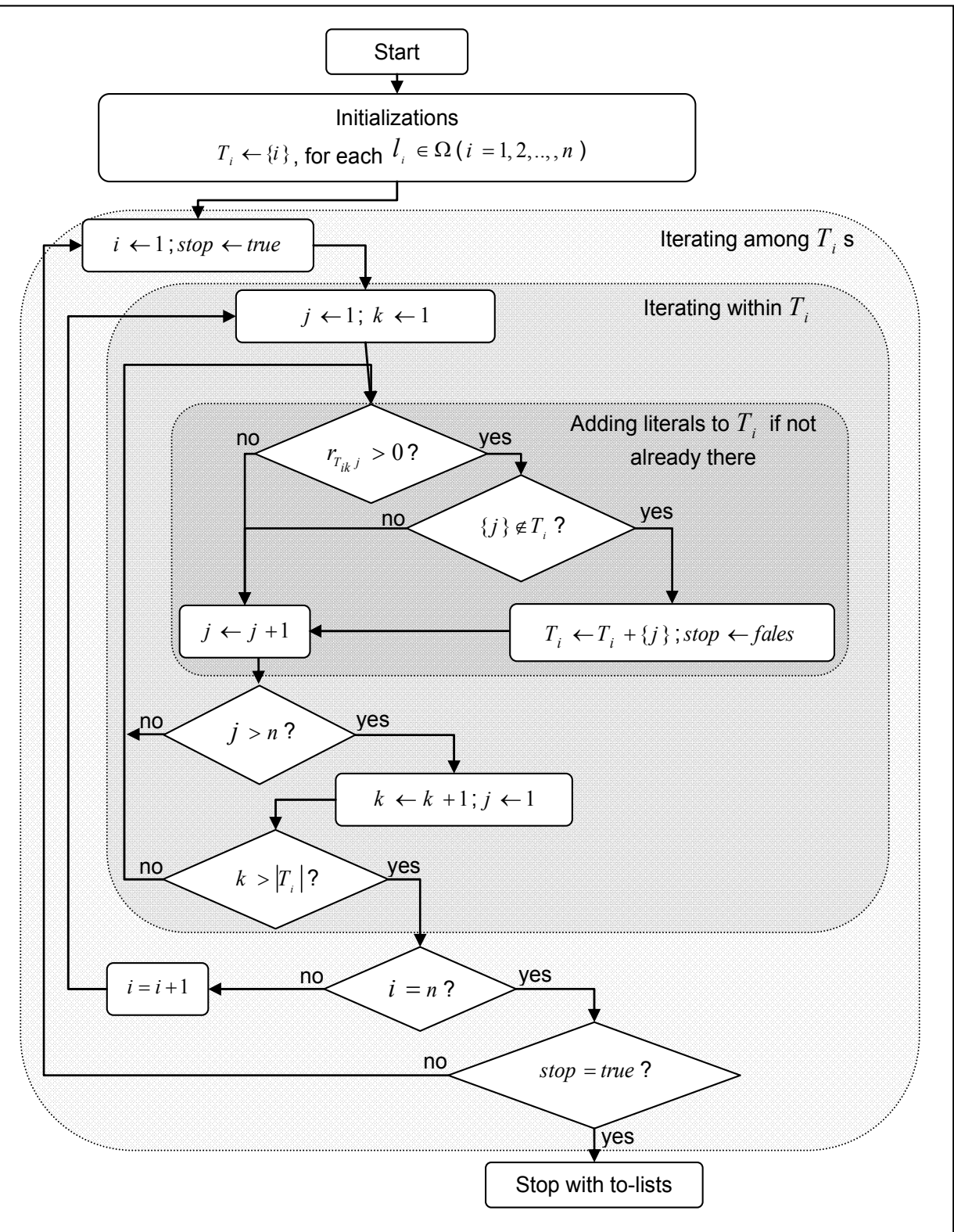

Fig. 5. Routine for constructing to-lists

Constructing equivalence classes of literals: Having obtained the to-list and from-list for each $l_{i}$, AECs can be obtained by intersecting the two sets $T_{i}$ and $F_{i}$ :

$$
c_{i}=T_{i} \cap F_{i}, \forall i
$$




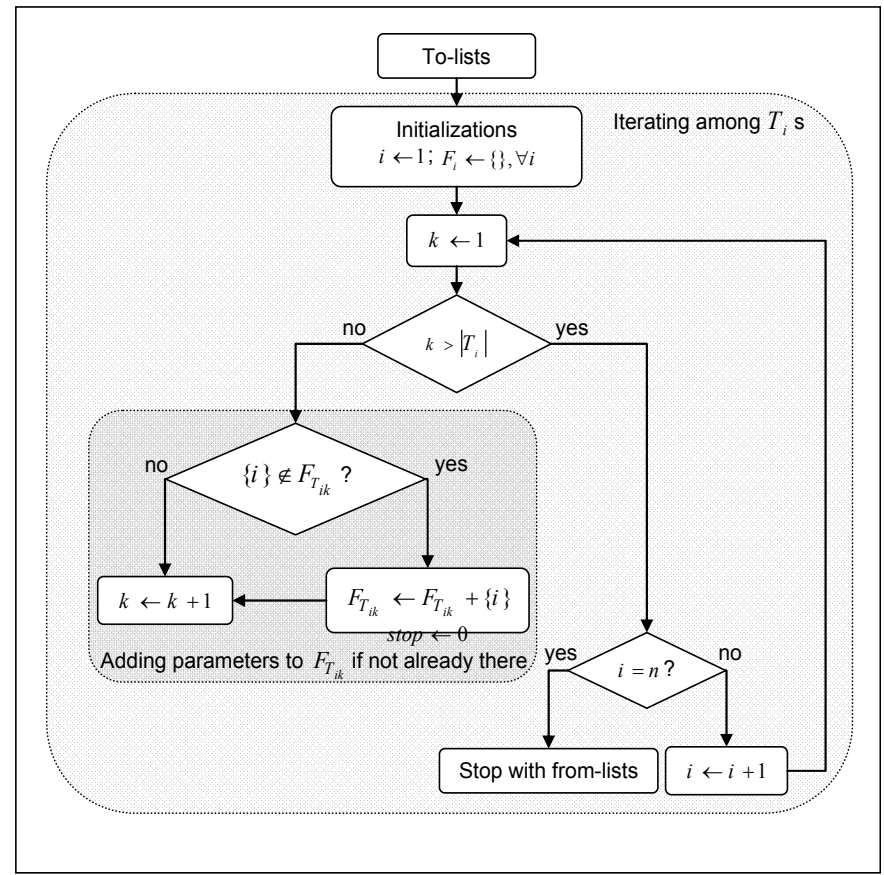

Fig. 6. Routine for constructing From-lists

\subsection{The aggregate interaction matrix}

The classification of literals into AECs leads to the discussion on aggregate interaction or flow that results among them. Let $\mathbf{C}=\left[c_{k k^{\prime}}\right]$ denote the AECs interaction matrix for aggregate flow. Each entry $c_{k k^{\prime}}$ of $\mathbf{C}$ is defined by the Boolean sum of the following equation:

$$
c_{k k^{\prime}}=\sum_{i \in c_{k}} \sum_{j \in c_{k^{\prime}}} r_{i j}
$$

C is a square matrix of size $m \times m$, where $m$ is the number of AECs in $\omega$. Each $c_{k k^{\prime}}$ represents the summation of corresponding rows and columns of the $\mathbf{R}$ matrix. Here, $\mathbf{C}^{(s)}$ denotes the $\mathbf{C}$ matrix multiplied $s$ times by itself. As in the entries of the $\mathbf{R}$ matrix, in $\mathbf{C}$, if $c_{k k^{\prime}}^{(s)}>0$ for some $s=1,2, \ldots$, then there is an interaction between the two AECs $k$ and $k^{\prime}$ passing through $s$ aggregate interactions. Hence AEC $k^{\prime}$ is accessible from AEC $k$. This leads to the definition of AEC accessibility.

\subsection{Classification of AECS:}

Another important characterization of AECs is whether an AEC is absorbing or transient, or maximal transient:

- Absorbing AEC (AAEC): an AEC that does not access any other AEC but itself. Therefore, an AAEC $c_{k} \subseteq \Omega$ is one where:

$$
c_{k k^{\prime}}=0, \forall k \neq k^{\prime}
$$


- $\quad$ Transient AEC (TAEC): an AEC capable of accessing other AECs besides itself. A TAEC satisfies the following:

$$
\exists c_{k k^{\prime}}>0, k \neq k^{\prime}
$$

- Maximal transient AEC (MTAEC): Is TAEC not accessed by any other TAEC beside itself, such that is must satisfy Eq.(9) together with:

$$
\neg \exists c_{k^{\prime \prime} k^{\prime}}>0, k^{\prime \prime} \neq k^{\prime}
$$

\subsection{Canonical form of the $\mathrm{C}$ matrix}

To prepare the $\mathbf{C}$ matrix for the layering phase, Its rows and columns are rearranged, such that the first $m-t$ ones contain the AAECs, while the remaining $t$ ones contain the TAECs. When this segregation is applied to the $\mathbf{C}$ matrix, then it is said to be in canonical form, denoted by $\overline{\mathbf{C}}$. A general structure of a $\overline{\mathbf{C}}$ matrix is given below:

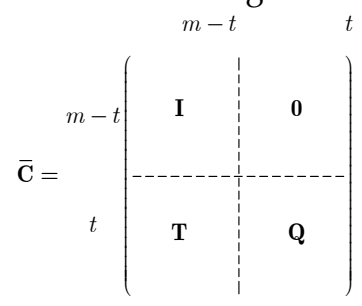

The resultant submatrices of $\overline{\mathbf{C}}$ are as follows:

(1) $\mathbf{I}_{(m-t) \times(m-t)}$ is the identity matrix, because an AAEC has only access to itself.

(2) $\mathbf{0}_{(m-t) \times(t)}$ consists entirely of zeros, since AAECs cannot access TAECs.

(3) $\mathbf{T}_{(t) \times(m-t)}$ represent accessibility from TAECs to each AAEC.

(4) $\mathbf{Q}_{(t) \times(t)}$ depicts accessibility among TAECs.

\section{Phase III: Constructing the Hierarchy}

The construction of an $\mathrm{AH}$ is conducted in a recursive and bottom-up manner, where it starts from the lowest level of detail (level zero) and subsequently building higher levels based on the abstract class accessibility relationships that exist among different AECs. The layering process is designed to eliminate backtracking in the plan.

Level zero is designated to include the details that can be postponed until the end when solving the problem hierarchically. However, level $n$, the highest level of abstraction, includes the details that need to be considered in the beginning. Therefore, the algorithm builds the hierarchy in a bottom-up fashion, but expects it to be executed in a top-down fashion.

\subsection{Constraints for Loop-Free Level Assignments}

The assignment of literals to levels is based on the following constraints to guarantee loop free AHs.

- Constraint 1(Literal Level Assignment Constraint): Let level $\left(l_{i}\right)$ denote the level of the design literal $l_{i}$ in an AH. For all $l_{i}, l_{j} \in \Omega$, if $r_{i j}^{(s)}>0$ for some $s>0$, then $\operatorname{level}\left(l_{i}\right) \geq \operatorname{level}\left(l_{j}\right)$ to avoid backtracking. 
The above constraint indicates that if $l_{i}$ accesses $l_{j}$, then $l_{i}$ should at least be at the same or a higher level than. This confirms findings from previous literature on abstraction hierarchies for planning and problem solving, particularly, Knoblock's (Knoblock 1994) restriction to automatically generate loop-free AHs for planning and problem solving.

- Constraint 2 (Communicating Literals Level Assignment Constraint): Let level $\left(l_{i}\right)$ denote the level of design literal $l_{i}$ in the AH. For all $l_{i}, l_{j} \in \Omega$, if $\operatorname{communicate}\left(l_{i}, l_{j}\right)$, then $\operatorname{level}\left(l_{i}\right)=\operatorname{level}\left(l_{j}\right)$.

If communicate $\left(l_{i}, l_{j}\right)$, then by definition there exists $r_{i j}^{\left(s_{1}\right)}>0$ and $r_{j i}^{\left(s_{2}\right)}>0$ for some $s_{1}, s_{2}>0$. Hence, by Constraint $1, \operatorname{level}\left(l_{i}\right) \geq \operatorname{level}\left(l_{j}\right)$ and $\operatorname{level}\left(l_{i}\right) \leq \operatorname{level}\left(l_{j}\right)$, which implies $\operatorname{level}\left(l_{i}\right)=\operatorname{level}\left(l_{j}\right)$.

- Constraint 3 (AECs Level Assignment Constraint): Let level $\left(c_{k}\right)$ denote the level of $\mathrm{AEC} k$ in an $\mathrm{AH}$. For all $c_{k}, c_{k^{\prime}} \subset \Omega$ where $k \neq k^{\prime}$, if $c_{k k^{\prime}}>0$, then level $\left(c_{k}\right)>\operatorname{level}\left(c_{k^{\prime}}\right)$ to avoid backtracking.

Constraint 3 is a direct result of applying Constraints 1 and 2. Based on the definition of accessibility, if $c_{k k^{\prime}}^{(s)}>0$ then, $\exists l_{i} \in c_{k}$ and $\exists l_{j} \in c_{k^{\prime \prime}}$ such that $r_{i j}^{(s)}>0$ for some $s>0$. Based on Constraint 1 , level $\left(l_{i}\right) \geq \operatorname{level}\left(l_{j}\right)$. Since classes consist of communicating literals, then $\operatorname{level}\left(c_{k}\right)>\operatorname{level}\left(c_{k^{\prime}}\right)$. But classes cannot communicate; therefore, it is not possible to have $\operatorname{level}\left(c_{k}\right)=\operatorname{level}\left(c_{k^{\prime}}\right)$ when $c_{k k^{\prime}}^{(s)}>0$. Therefore, level $\left(c_{k}\right)>\operatorname{level}\left(c_{k^{\prime}}\right)$ for $c_{k k^{\prime}}^{(s)}>0$, and hence $c_{k}$ need to be considered before $c_{k^{\prime}}$ to avoid backtracking. The following theorem shows that applying Constraint 3 will result in loop-free AHs.

\section{Theorem1. : Any AH developed using Constraint 3 is loop-free.}

Proof.

Looping (backtracking) occurs if $\exists c_{k}, c_{k^{\prime}} \subset \omega$, where $\operatorname{classaccessible}\left(c_{k}, c_{k^{\prime}}\right)$ and level $\left(c_{k}\right)>\operatorname{level}\left(c_{k^{\prime}}\right)$. Here it shows that this never occurs, considering the three cases of AAECs, TAECs and MTAECs

- Case I (AAECs): if $c_{k}$ is absorbing, then $\operatorname{level}\left(c_{k}\right)=0 . \quad$ Also $\neg \exists c_{k^{\prime}} \subset \omega$, where classaccessible $\left(c_{k}, c_{k^{\prime}}\right)$; thus level $\left(c_{k^{\prime}}\right)>\operatorname{level}\left(c_{k}\right)$ cannot occur.

- Case II (MTAECs): if $c_{k}$ is a MTAEC, then level $\left(c_{k}\right)=n$. Thus $\neg \exists c_{k^{\prime}} \subset \omega$, where $\operatorname{level}\left(c_{k^{\prime}}\right)>\operatorname{level}\left(c_{k}\right)$.

- Case III (TAECs): if $c_{k}$ is a TAEC, then it must be true that $\exists c_{k^{\prime}, k^{\prime \prime}} \subset \omega$, where classaccessible $\left(c_{k^{\prime}}, c_{k}\right)$ and classaccessible $\left(c_{k}, c_{k^{\prime \prime}}\right)$. Thus, according to Constraint 3 , $\operatorname{level}\left(c_{k^{\prime}}\right)<\operatorname{level}\left(c_{k}\right)<\operatorname{level}\left(c_{k^{\prime \prime}}\right)$, and a reverse order can never occur.

From these three cases, it can be concluded that $\operatorname{level}\left(c_{k^{\prime}}\right)>\operatorname{level}\left(c_{k}\right)$ will never occur for all classaccessible $\left(c_{k}, c_{k^{\prime}}\right)$. Hence the $\mathrm{AH}$ is loop-free.

This proof demonstrates that an AH developed by the methodology in hand will always produce loop-free AHs. 


\section{The Level Assignment Algorithm}

The Level Assignment Algorithm (LAA) generates AHs by assigning AECs to their appropriate level of abstraction. In LAA, the assignments are accomplished on the premises of the preceding developed constraints.

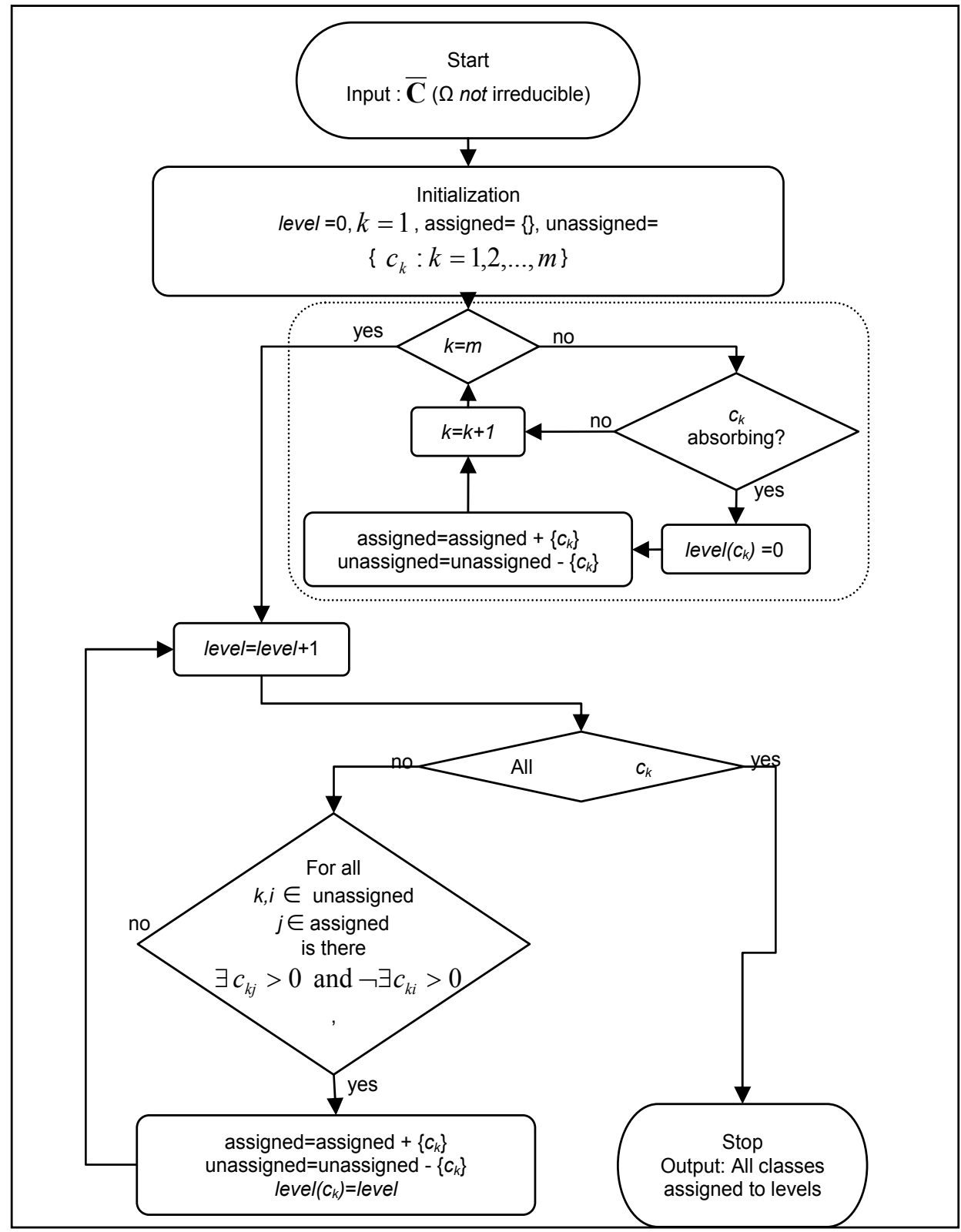

Fig. 7. The level assignment algorithm 


\section{Illustrative Example}

In this section, effectiveness of the developed methodology is demonstrated through the design of a layout for manufacturing plant that produces high voltage power cables. The plant produces a few variations of the high voltage cable shown in Fig. 8, based on customer specifications regarding conductor properties, insulation thickness, cable color coding, armoring metals, and so forth.

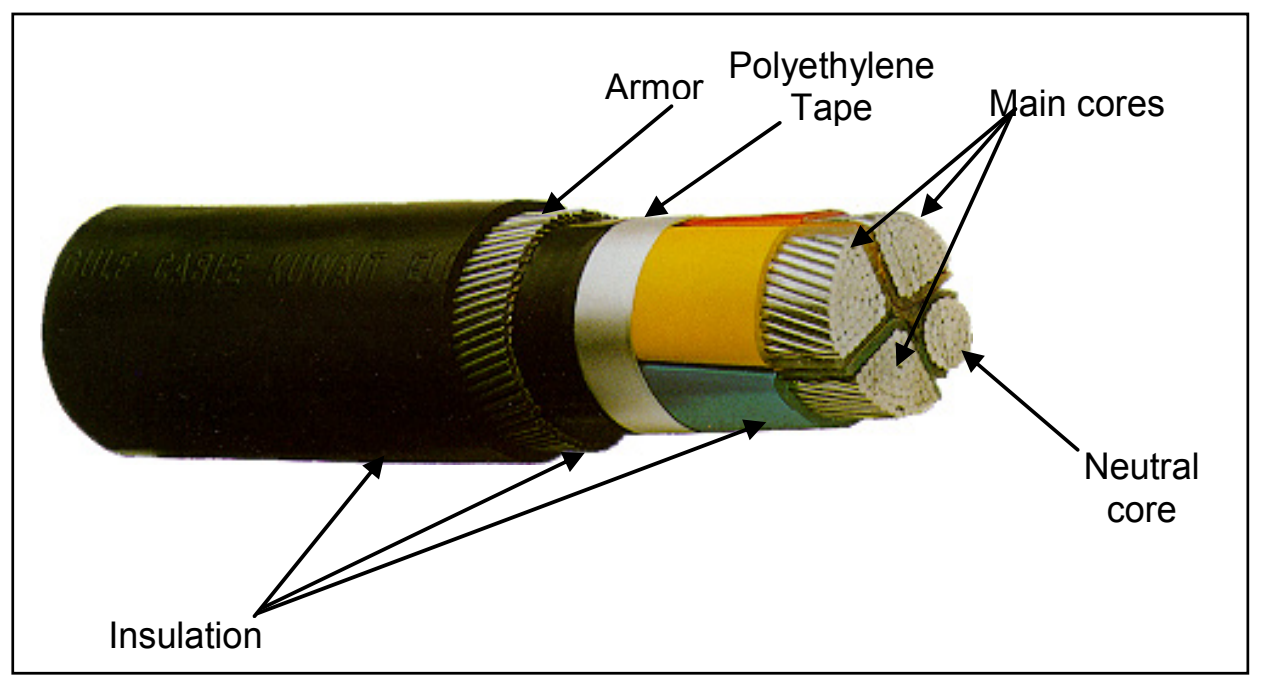

Fig. 8. Components of the high voltage cable

As shown in Fig. 8, the high voltage power cable consists of three main aluminum cores, each of which has a diameter of $300 \mathrm{~mm}$, and a neutral core of $185 \mathrm{~mm}$ diameter. The three main cores and the neutral core consist of 61 and 37 insulated stranded aluminum rods, respectively. The four cores are warped with polyethylene tape that is supported by a layer of insulation. Finally, the cable is armored with steel and wires for protection and is sheathed by an additional layer of insulated. The flow chart shown in Fig. 9, describes the flow of the cable across the different stations. 


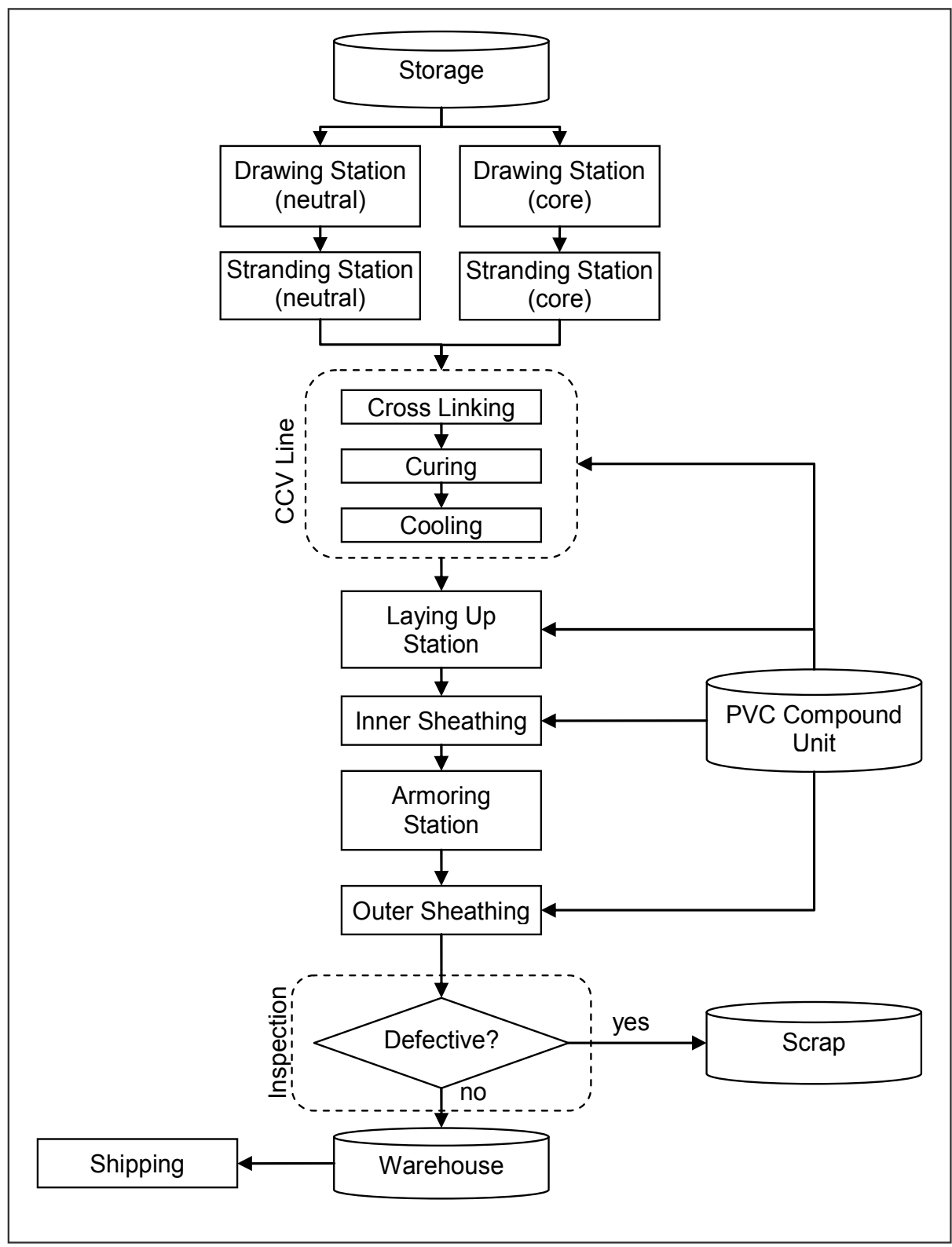

Fig. 9. Flowchart of the manufacturing processes for the high voltage cable

10.1 Phase I: the literal space representation for the cable manufacturing company

The high voltage cable facility consists of 74 machines and areas that are distributed within the cable manufacturing stations shown in Fig. 9 together with WIP areas, forklift parking, storages, warehouses, shop floor offices, lounges, etc. These areas are shown in Table 2. 


\begin{tabular}{|c|c|c|c|c|c|}
\hline $\mathbf{I}_{\mathbf{i}}$ & Code & Name & $\mathbf{I}_{\mathbf{i}}$ & Code & Name \\
\hline 0 & PD1 & Preliminary Drawing Station & 38 & SHS3 & Sheathing Station \\
\hline 1 & PD2 & $"$ & 39 & SHS4 & “ \\
\hline 2 & PD3 & $"$ & 40 & $\mathrm{XL1}$ & Cross-Linking Station \\
\hline 3 & PD4 & $"$ & 41 & $\mathrm{XL2}$ & “ \\
\hline 4 & ID1 & Intermediary Drawing Station & 42 & XL3 & “ \\
\hline 5 & ID2 & “ & 43 & $\mathrm{XL4}$ & $"$ \\
\hline 6 & ID3 & “ & 44 & CU1 & Curing Machinery \\
\hline 7 & ID4 & " & 45 & CU2 & “ \\
\hline 8 & ID5 & “ & 46 & CU3 & “ \\
\hline 9 & ID6 & “ & 47 & CU4 & " \\
\hline 10 & ID7 & “ & 48 & $\mathrm{CO} 1$ & Cooling Station \\
\hline 11 & ID8 & “ & 49 & $\mathrm{CO} 2$ & “ \\
\hline 12 & DD1 & Main Detailed Drawing Station & 50 & $\mathrm{CO} 3$ & “ \\
\hline 13 & DD2 & " & 51 & $\mathrm{CO} 4$ & $"$ \\
\hline 14 & DD3 & “ & 52 & L1 & Lay-up Station \\
\hline 15 & DD4 & “ & 53 & $\mathrm{~L} 2$ & “ \\
\hline 16 & DD5 & “ & 54 & $\mathrm{~A} 1$ & Armoring Station \\
\hline 17 & DD6 & " & 55 & $\mathrm{~A} 2$ & " \\
\hline 18 & DD7 & “ & 56 & PVC & PVC Compound Unit \\
\hline 19 & DD8 & “ & 57 & INS & Inspection \\
\hline 20 & DD9 & “ & 58 & QC & Quality Control Unit \\
\hline 21 & DD10 & “ & 59 & ST & Storage \\
\hline 22 & DD11 & “ & 60 & $\mathrm{WH}$ & Warehouse \\
\hline 23 & DD12 & “ & 61 & WIP1 & Work-In-Process \\
\hline 24 & DD13 & Neutral Detailed Drawing & 62 & WIP2 & “ \\
\hline 25 & DD14 & “ & 63 & WIP3 & “ \\
\hline 26 & DD15 & “ & 64 & WIP4 & “ \\
\hline 27 & DD16 & “ & 65 & FP1 & Forklift Parking \\
\hline 28 & MCS1 & Main Core Stranding & 66 & FP2 & “ \\
\hline 29 & MCS2 & " & 67 & FP3 & " \\
\hline 30 & MCS3 & “ & 68 & $\mathrm{SC}$ & Scrap Center \\
\hline 31 & MCS4 & “ & 69 & OFF & Main Office \\
\hline 32 & MCS5 & “ & 70 & $M C$ & CCV Maintenance \\
\hline 33 & MCS6 & “ & 71 & LOU1 & Employee Lounge \\
\hline 34 & NCS1 & Neutral Core Stranding & 72 & LOU2 & “ \\
\hline 35 & NCS2 & “ & 73 & DOK1 & Docking Station \\
\hline 36 & SHS1 & Sheathing Station & 74 & DOK2 & “ \\
\hline 37 & SHS2 & $"$ & & & \\
\hline
\end{tabular}

Table 2. The machines and support areas for the high voltage cable facility

There are 74 literals ( $n=74$ ) in the literal space $\Omega$ for this problem.

- Interactions among literals: The constraints of the problem define the interactions among the twelve literals listed above. One indicates causality based on accessibility definition between two literals, and zero otherwise. The causality links are depicted in the $\mathbf{R}$ matrix provided in Table 3. 


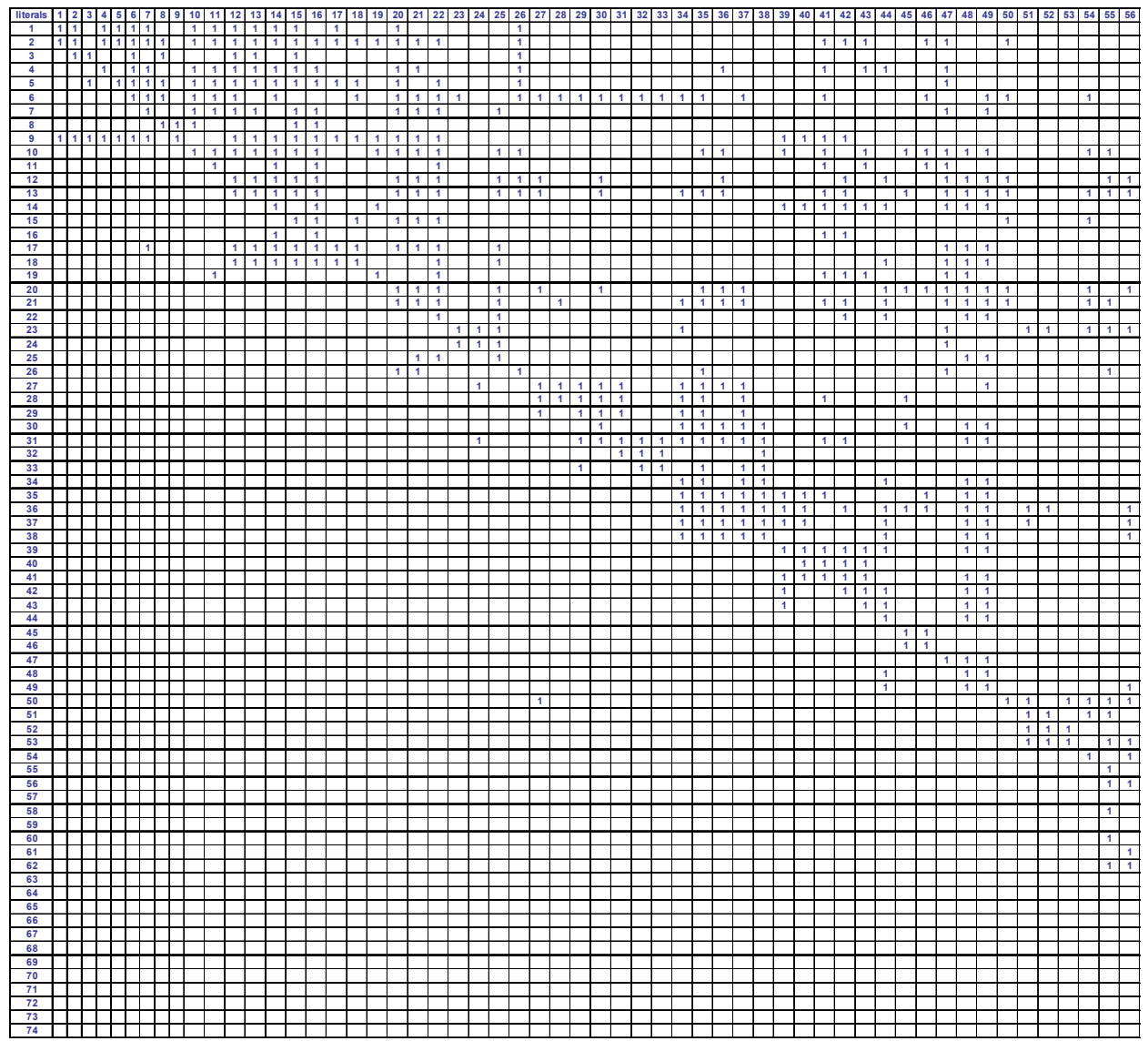

Table 3. The R matrix for the cable manufacturing facility

10.2 Phase II: abstraction of the literal space of the cable manufacturing company Given the $\mathbf{R}$ matrix for the cable manufacturing facility, the literal space is portioned into mutually exclusive AECs using ECFA. Each cluster of AECs constitutes literals that need to be considered simultaneously. AECs obtained from using ECFA are shown in Table 4. 


\begin{tabular}{|c|l|c|c|}
\hline Classes & \multicolumn{1}{|c|}{ Literals $\left(\mathrm{BB}_{\mathrm{iBB}}\right)$} & $\begin{array}{c}\text { Number of Literals } \\
\left(\mathrm{NBB}_{\mathrm{kBB}}\right)\end{array}$ & $\begin{array}{c}\text { Class } \\
\text { Classification }\end{array}$ \\
\hline 0 & $0,1,2,3$, & 4 & Transient \\
\hline 1 & $4,5,6,7,8,9,10,11$ & 8 & Transient \\
\hline 2 & $\begin{array}{l}12,13,14,15,16,17,18,19,20,21,22, \\
23,24,25,26,27,28,29,30,31,32,33, \\
61,65,34,35,62\end{array}$ & 27 & Transient \\
\hline 3 & $\begin{array}{l}36,37,38,39,54,55,56,40,41,42,43, \\
52,53,44,45,46,47,48,49,50,51,70,\end{array}$ & 25 & Transient \\
\hline 4 & $56,63,64,1$ & 7 & Absorbing \\
\hline 5 & $59,71,73$ & 3 & Transient \\
\hline 6 & 69 & 1 & $\begin{array}{l}\text { Maximal } \\
\text { transient }\end{array}$ \\
\hline
\end{tabular}

Table 4. The AECs for the cable manufacturing facility

From Table 4, the abstracted literal space $\omega$ consists of seven AECs. This reduced the problem tremendously to a manageable size.

- Aggregate interactions among AECs: The aggregate interactions among AECs can be obtained using Eq.(9). Accordingly, the $\mathbf{C}$ matrix is constructed and is transformed it to the canonical form $\overline{\mathbf{C}}$ which are provided below.

The entries of $\mathbf{C}$ and $\overline{\mathbf{C}}$ matrix are:

$$
\mathbf{C}=\left(\begin{array}{ccccccc}
1 & 1 & 0 & 0 & 0 & 0 & 0 \\
0 & 1 & 1 & 0 & 0 & 0 & 0 \\
0 & 0 & 1 & 1 & 0 & 0 & 0 \\
0 & 0 & 0 & 1 & 1 & 0 & 0 \\
0 & 0 & 0 & 0 & 1 & 0 & 0 \\
1 & 0 & 0 & 1 & 0 & 1 & 0 \\
0 & 0 & 0 & 1 & 1 & 1 & 1
\end{array}\right) \quad \overline{\mathbf{C}}=\left(\begin{array}{c:cccccc}
1 & 0 & 0 & 0 & 0 & 0 & 0 \\
\hdashline 0 & 1 & 1 & 0 & 0 & 0 & 0 \\
0 & 0 & 1 & 1 & 0 & 0 & 0 \\
0 & 0 & 0 & 1 & 1 & 0 & 0 \\
1 & 0 & 0 & 0 & 1 & 0 & 0 \\
0 & 1 & 0 & 0 & 1 & 1 & 0 \\
1 & 0 & 0 & 0 & 1 & 1 & 1
\end{array}\right)
$$

\subsection{Phase III: constructing the AH for the cable manufacturing case study}

In this phase, the interactions among the different AECs are utilized to recursively develop an $\mathrm{AH}$ to structure the cable manufacturing facility. As indicated in the methodology, AHs are designed to be loop-free. In terms of the problem in hand, obtaining partial solutions at a given abstraction level need not be altered as the process progresses hierarchically to more detailed levels.

Each AEC is assigned to its appropriate abstraction level using LAA as shown in Figure 7. Table 5 illustrates the resultant abstraction hierarchy for the cable manufacturing facility. The levels of the hierarchy indicate the order in which each literal should be introduced to the problem gradually to facilitate loop-free problem execution. 


\begin{tabular}{|c|c|l|c|}
\hline Level & Classes & \multicolumn{1}{|c|}{ Literals $\left(l \mathrm{~B}_{\mathrm{iBB}}\right)$} & $\begin{array}{c}\text { Number of } \\
\text { Literals }\left(\mathrm{NBB}_{k \mathrm{BB}}\right)\end{array}$ \\
\hline 6 & 6 & 69 & 1 \\
\hline 5 & 5 & $59,71,73$ & 3 \\
\hline 4 & 0 & $0,1,2,3$, & 4 \\
\hline 3 & 1 & $4,5,6,7,8,9,10,11$ & 8 \\
\hline 2 & 2 & $\begin{array}{l}12,13,14,15,16,17,18,19,20,21,22,23,24,25, \\
26,27,28,29,30,31,32,33,61,65,34,35,62\end{array}$ \\
\hline 1 & 3 & $\begin{array}{l}36,37,38,39,54,55,56,40,41,42,43,52,53,44, \\
45,46,47,48,49,50,51,70,66,63,64\end{array}$ & 25 \\
\hline 0 & 4 & $57,58,60,67,68,72,74$ & 7 \\
\hline
\end{tabular}

Table 5. The levels of the Abstraction hierarchy for the high voltage cable facility

Executing the abstraction hierarchy top-down and feeding results to a facility layout routine result in the layout provided in Fig 10.

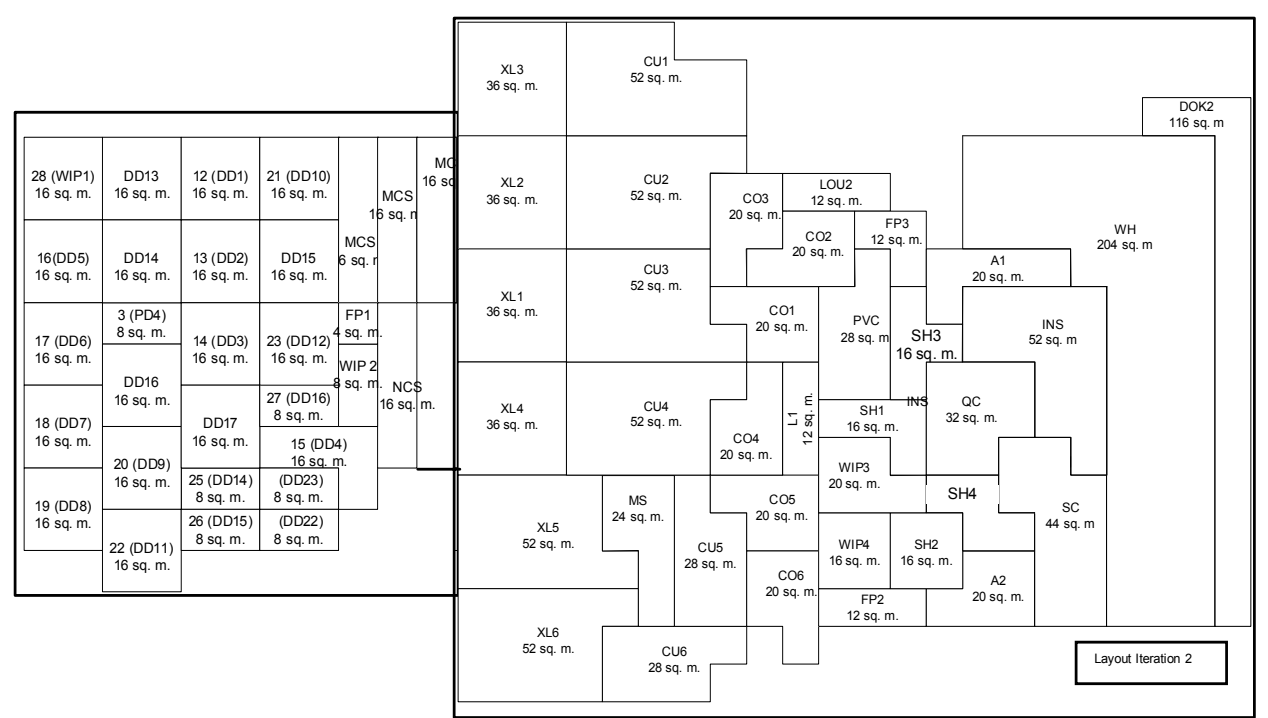

Fig 10. Facility layout for the High voltage cable manufacturing facility

\section{Conclusions}

This research establishes a rigid foundation and a general platform that produces consistent abstraction spaces and hierarchies applicable to various contexts, especially those involving planning and problem solving. The methodology presented adheres to the efficiency measures and specifications prescribed by the latest advances of AI-based abstraction theory. Yet, our hierarchical abstraction methodology exhibits additional practicality as it integrates the theory of abstraction with the convenient representation scheme of Design Structured Matrices. This expands the application of abstraction theories and enhances their 
feasibility to be used in practice. Within the presented methodology, we have also developed several effective methods to efficiently structure and analyze systems to be hierarchically decomposed. These methods were integrated from graph, relation and matrix theories. In addition we have utilized Markov Chains classes' classification methods to identify special behavior in system components and to detect in advance whether or not a system representation is better using hierarchies. The strength of the methodology relies on its ability to structure problems in abstraction hierarchies that result in no backtracking. However, the efficiency of the methodology depends on the context to which it is applied. That is, little gain is expected to be realized when applying the methodology to domains that undergo significant interaction due to the irreducibility problem. The steps of the developed methodology are illustrated in stratifying the design aspects of high voltage cable company into multiple levels of abstraction. This advantageously contributed in introducing the design details of the problem gradually as needed earlier in conceptual stage of planning of the facility. Future research is directed towards quantifying binary relations of literals, developing measures of efficiency and means of eliminating irreducability and inclusion of initial and goal states to the literal space.

\section{References}

Aleisa, E. (2005). Multilevel Integration of Simulation and Facilities Planning for Large-Scale Systems. Department of Industrial Engineering. Buffalo, NY, The State University of New York at Buffalo. Vol., No.

Aleisa, E. (2008). An Overview Of Multilevel And Hierarchical Methods For Discrete-Event Simulation Of Complex Systems. Industrial Simulation Conference (ISC08), Lyon, France.

Aleisa, E. \& L. Lin (2008). Abstraction Hierarchies for Engineering Design. International Journal of Electrical, Computer, and Systems Engineering 2(1): 20-32.

Aleisa, E. \& L. Lin (2009). A Design Structure Matrix Approach For Generating Planning Abstraction Hierarchies. Kuwait Journal of Science and Engineering (KJSE): To appear.

Armano, G.;G. Cherchi \& E. Vargiu (2003). Planning by abstraction using HW[]. Ai(Asterisk)Ia 2003: Advances in Artificial Intelligence, Proceedings. 2829: 349-361.

Bacchus, F. \& Q. Yang (1992). Expected value of hierarchical problem-solving. AAAI-92.

Browning, T. R. (1999). The Design Structure Matrix. Technology Management Handbook. R. C. Dorf. Boca Raton, FL, Chapman \& Hall/CRCnetBASE, : 103-111.

Browning, T. R. (2001). Applying the design structure matrix to system decomposition and integration problems: A review and new directions. IEEE Transactions on Engineering Management 48(3 August): 292-306.

Chen, L.-R. \& S. Ghosh (1997). Modeling and simulation of a hierarchical, distributed, dynamic inventory management scheme. Simulation 68(6 Jun): 340-362.

Christensen, J. (1991). Automatic Abstraction in Planning. Department of Computer Science. Stanford, Ca, Stanford University. Vol., No.: 153.

Dartmouth College Writing Group. \& E. J. Cogan (1958). Modern mathematical methods and models; a book of experimental text materials. Ann Arbor, MI.

Debbie, R. (2003). Knowledge-Based System Explanation: The Ripple-Down Rules Alternative. Knowledge and Information Systems 5(1): 2. 
Deo, N. (1974). Graph theory with applications to engineering and computer science. Englewood Cliffs, N.J., Prentice-Hall.

Eppinger, S. D.;D. E. Whitney;R. P. Smith \& D. A. Gebala (1994). A Model-Based Method for Organizing Tasks in Product Development. Research in Engineering Design-Theory Applications and Concurrent Engineering 6(1): 1-13.

Fox, M. \& D. Long (1995). Hierarchical planning using abstraction. IEE Control Theory and Applications.

Friske, L. M. \& C. H. C. Ribeiro (2006). Planning under uncertainty with abstraction hierarchies. Intelligent Data Engineering and Automated Learning - Ideal 2006, Proceedings. 4224: 1057-1066.

Gaver, D. P. \& G. L. Thompson (1973). Programming and probability models in operations research. Monterey, Ca, Brooks/Cole Pub. Co.

Gimenez, O. \& A. Jonsson (2008). The complexity of planning problems with simple causal graphs. Journal of Artificial Intelligence Research 31: 319-351.

Giunchiglia, F. (1999). Using Abstrips abstractions - Where do we stand? Artificial Intelligence Review 13(3): 201-213.

Giunchiglia, F. \& T. Walsh (1992). A Theory of Abstraction. Artificial Intelligence 57(2-3): 323389.

Goldin, S. E. \& P. Klahr (1981). Learning and Abstraction in Simulation. International Joint Conference on Artificial Intelligence, American Assoc for Artificial Intelligence.

Helmert, M. (2006). The Fast Downward planning system. Journal of Artificial Intelligence Research 26: 191-246.

Holte, R. C. \& B. Y. Choueiry (2003). Abstraction and reformulation in artificial intelligence. Philosophical Transactions of the Royal Society of London Series B-Biological Sciences 358(1435): 1197-1204.

Hoover, S. P. \& J. R. Rinderle (1994). Abstractions, design views and focusing. 6th International Conference on Design Theory and Methodology American Society of Mechanical Engineers, Design Engineering Division (Publication) DE, ASME, New York, NY.

Kao, E. P. C. (1997). An introduction to stochastic processes. Belmont, Calif., USA, Duxbury Press.

Kemeny, J. G. \& J. L. Snell (1960). Finite markov chains. Princeton, N.J., Van Nostrand.

Kemke, C. \& E. Walker (2006). Planning with action abstraction and Plan Decomposition Hierarchies. 2006 Ieee/Wic/Acm International Conference on Intelligent Agent Technology, Proceedings: 447-451.

Kiran, A. S.;T. Cetinkaya \& J. Cabrera (2001). Hierarchical modeling of a shipyard integrated with an external scheduling application. Winter Simulation Conference Proceedings 2: 877-881 (IEEE cat $\mathrm{n}$ 01CH37304).

Knoblock, C. (1990). Learning Abstraction Hierarchies for Problem Solving. AAAI-90, Boston, MA.

Knoblock, C. (1994). Automatically generating abstractions for planning. Artificial Intelligence 68(2 Aug): 243-302.

Knoblock, C. A. (1994). Automatically generating abstractions for planning. Artificial Intelligence 68(2 Aug): 243-302.

Lam, K. P. (1996). Hierarchical Method for Large Scale Two-Dimensional Layout. DET-96, American Society of Mechanical Engineers.

Levy, A. Y. (1994). Creating abstractions using relevance reasoning. AAAI-94, Menlo Park, CA. 
Lin, J. T.;K. C. Yeh \& L. C. Sheu (1996). A context-based object-oriented application framework for discrete event simulation. Computers $\mathcal{E}$ Industrial Engineering 30(4): 579-597.

Lu, S. C. Y. \& D. K. Tcheng (1991). Building layered models to support engineering decision making. A machine learning approach. Journal of Engineering for Industry 113(1): 1-9.

Luger, G. F. (2002). Artificial intelligence : structures and strategies for complex problem solving. Harlow, England ; New York, Pearson Education.

Manfaat, D.;A. H. Duffy \& B. S. Lee (1998). SPIDA: Abstracting and generalizing layout design cases. Artificial Intelligence for Engineering Design, Analysis \& Manufacturing: Aiedam 12(2 Apr): 141-159.

Marie, d.;R. Priyang \& G. Lise (2008). Learning structured Bayesian networks: combining abstraction hierarchies and tree-structured conditional probability tables. Computational Intelligence 24(1): 1.

McCord, K. R. a. E., Steven D. (1993). Managing the Integration Problem in Concurrent Engineering, M.I.T. Sloan School of Management, Cambridge. Vol., No.

McGraw, R. M. \& R. A. MacDonald (2000). Abstract modeling for engineering and engagement level simulations. Winter Simulation Conference Proceedings 1: 326-334.

Minton, S. (1988). Learning Effective Search Control Knowledge: An Explanation-Based Approach, Carnegie-Mellon University. Vol., No.: 231.

Pels, H. J. (2006). Classification hierarchies for product data modelling. Production Planning E Control 17(4): 367.

Pidd, M. (1996). Five simple principles of modelling. Winter Simulation Conference Proceedings, Publ by IEEE, IEEE Service Center, Piscataway, NJ, USA.

Pidd, M. \& R. B. Castro (1998). Hierarchical modular modelling in discrete simulation. Winter Simulation Conference, IEEE, Piscataway, NJ.

Praehofer, H. (1996). An Environment for DEVS-based multi-formalism modeling and simulation in $\mathrm{C}++$. Proceedings of AI, Simulation and Planning in High-Autonomy Systems, Tucson, AZ.

Reddy, S. Y. (1996). Learning abstract models for system design. Ai Edam-Artificial Intelligence for Engineering Design Analysis and Manufacturing 10(2): 167-169.

Rogers, J. L. (1996). DeMAID/GA - An Enhanced Design Manager's Aid for Intelligent Decomposition. 6th AIAA/USAF/NASA/ISSMO Symposium on Multidisciplinary Analysis and Optimization, Seattle, WA.

Russell, S. J. \& P. Norvig (1995). Artificial intelligence : a modern approach. Englewood Cliffs, N.J., Prentice Hall.

Sacerdoti, E. (1974). Planning in a Hierarchy of Abstraction Spaces. Artificial Intelligence 5(2): $115-135$.

Sarjoughian, H. S.;B. P. Zeigler \& F. E. Cellier (1998). Evaluating model abstractions: A quantitative approach. Proceedings of SPIE Enabling Technology for Simulation Science II, Orlando, FL, United States.

Sebastia, L.;E. Onaindia \& E. Marzal (2006). Decomposition of planning problems. Ai Communications 19(1): 49-81.

Steward, D. V. (1981). The Design Structure-System - a Method for Managing the Design of Complex-Systems. Ieee Transactions on Engineering Management 28(3): 71-74. 
Taylor, L. E. \& M. R. Henderson (1994). Roles of features and abstraction in mechanical design. 6th International Conference on Design Theory and Methodology American Society of Mechanical Engineers, Design Engineering Division (Publication) DE, New York, NY, ASME.

Warfield, J. N. (1973). Binary Matrices in System Modeling. Ieee Transactions on Systems Man and Cybernetics SMC3(5): 441-449.

Yang, Q., Tenenberg, J (1990). Abtweak: Abstracting a Nonlinear, Least Commitment Planner. AAAI-90, Boston, MA.

Yassine, A.;D. Falkenburg \& K. Chelst (1999). Engineering design management: an information structure approach. International Journal of Production Research 37(13): 2957-2975.

Zeigler, B. P. (1976). Theory of modelling and simulation. New York, Wiley.

Zeigler, B. P. (1987). Hierarchical, Modular Discrete-Event Modelling in an Object-Oriented Envirionment. Simulation 49(5): 219-230.

Zeigler, B. P.;H. Praehofer \& T. G. Kim (2000). Theory of modeling and simulation : integrating discrete event and continuous complex dynamic systems. San Diego, Academic Press.

Zucker, J. D. (2003). A grounded theory of abstraction in artificial intelligence. Philosophical Transactions of the Royal Society of London Series B-Biological Sciences 358(1435): 12931309. 


\title{
An Ant Colony Optimization algorithm for Flexible Job shop scheduling problem
}

\author{
S. G. Ponnambalam ${ }^{1}$, N. Jawahar ${ }^{2}$ and B. S. Girish ${ }^{3}$ \\ 1School of Engineering, Monash University \\ 2Thiagarajar College of Engineering, Madurai \\ ${ }^{3}$ National Institute of Technology, Calicut \\ ${ }^{1}$ Malaysia \\ 2,3India
}

\section{Introduction}

Scheduling involves the allocation of resources over a period of time to perform a collection of tasks (Baker, 1974). It is a decision-making process that plays an important role in most manufacturing and service industries (Pinedo, 2005). Scheduling in the context of manufacturing systems refers to the determination of the sequence in which jobs are to be processed over the production stages, followed by the determination of the start-time and finish-time of processing of jobs (Conway et al., 1967). An effective schedule enables the industry to utilize its resources effectively and attain the strategic objectives as reflected in its production plan.

The most common manufacturing system worldwide is the job shop. Job shops are associated with the production of small volumes/large variety products and operate in a make-to-order environment (Groover, 2003). Hoitomt et al. (1993) mentions that approximately 50 to $75 \%$ of all manufactured components fall into this category of low volume/high variety and due to the market trends this percentage is likely to increase. Even though flexible manufacturing systems are today's keywords that frequently appear in many research agendas, scheduling of job shops still receive ample attention from both researchers and practitioners due to the reason that job shop scheduling problems exist in many forms in most of the advanced manufacturing systems (Kutanoglu \& Sabuncuoglu, 1999). Besides, analysis of job shop scheduling problems provides important insights into the solution of the scheduling problems encountered in more realistic and complicated systems (Pinedo 2005). In this context, this chapter focuses on scheduling job shops which is an important task for manufacturing industry in terms of improving machine utilization or reducing lead time.

\subsection{Classical Job shop scheduling problem}

The classical job shop scheduling problem (JSP) is the most popular scheduling model in practice (French, 1982; Brucker, 1995; Pinedo, 1995). It has attracted many researchers due to 
its wide applicability and inherent difficulty (Jain \& Meeran, 1999). The formulation of the JSP is based on the assumption that for each part type or production order (job) there is only one processing plan, which prescribes the sequence of operations and the machine on which each operation has to be performed. The $n \times m$ classical JSP involves $n$ jobs and $m$ machines. Each job is to be processed on each machine in a predefined sequence and each machine processing only one job at a time. It is also well known that JSP is NP-hard (Garey et al., 1976).

\subsection{Scheduling Job shops associated with multiple routings}

In practice, the shop-floor setup in a job shop typically consists of multiple copies of the most critical machines so that bottlenecks due to long operations or busy machines can be reduced (Ho et al., 2007). Therefore, an operation may be performed on more than one machine. Job shops also consists of multipurpose machines such as numerically controlled (NC) machines that are loaded with tool magazines and are capable of performing several different types of operations (Vairaktarakis \& Cai, 2003). Due to the overlapping capabilities of these machines, a given operation can be performed by more than one machine. However, in real life it has been a practice that machining operations are assigned to a certain machine tool during the process planning stage and the assignment of machine tools over time to different operations is performed during the scheduling stage. Recently, researchers considered the integration of process planning with scheduling by allowing alternative machine tool routings for operations at the scheduling stage.

Hankins et al. (1984) discussed the advantages of using alternative machine tool routings to improve the productivity. They showed, through an example, that using alternative machine results in reduced lead-time and improves overall machine utilization. Chryssolouris and Chan (1985) discussed the integration of process planning and the decision making process for production resource assignment. They discussed the issue of generating alternative machines/resources based on the process planning information. Wilhelm and Shin (1985) investigated the effectiveness of alternative operations in flexible manufacturing systems. They showed via computational experiments that alternative operations could reduce flow time while increasing machine utilization. However, the consideration of alternative routing option in job shop adds an additional decision of machine allocation during scheduling that increases the complexity of the problem. Therefore, scheduling of job shops that are associated with multiple routings is a much more complex problem than the JSP. Even though the practical applications and advantages of using multiple routings in job shop scheduling are more when compared with fixed routing, still the research focus in this area is very limited. On the above considerations, this chapter addresses a scheduling model of the job shop problem associated with multiple routings.

Two different scheduling models of job shop associated with multiple routings are addressed in the literature. The first model is referred as job shop scheduling with alternative machine tool routings, which was first addressed by Iwata et al. (1978). The same model was later addressed by Brandimarte (1993) as flexible job shop scheduling problem (FJSP). The second model is usually referred as job shop scheduling with multi-purpose machines (MPM-JSP), which was first addressed by Brucker and Schlie (1990). DauzerePeres and Paulli (1997) addressed the MPM-JSP as multiprocessor job shop scheduling problem (MJS). The difference between the two models (FJSP and MPM-JSP/MJS) is that, in the first model the processing time for each operation on its alternative routes differs with 
machine features, whereas in the second model the processing time is same for all the alternative machines of a particular operation. Since the FJSP can be represented as a generalized model of MPM-JSP/MJS, therefore, many recently published research articles refer both the models as FJSP. This chapter, therefore, considers FJSP as the scheduling model of the multiple routing job shop problem and proposes an Ant Colony Optimization based heuristic to solve the problem.

\subsection{Review of solution methodologies}

Since FJSP belongs to the category of NP-hard problems, therefore, heuristic methods had been a predominant choice for the researchers over the traditional mathematical techniques. The heuristic approaches for solving FJSP are generally classified as hierarchical and integrated approaches. In hierarchical approaches, assignment of operations to machines and the sequencing of operations on the machines are treated separately, i.e. assignment and sequencing are considered independently. In integrated approaches assignment and sequencing are not differentiated and considered together.

Iwata et al. (1980) presented two dispatching rules for the FJSP. The first rule is based on EFT (Earliest Finishing Time) rule in which the activity with the earliest finishing time from a set of competing activities is assigned first to a machine tool. The EFT rule is extended to EFTA (Earliest Finishing Time with Alternative Operations) rule to solve the job shop problem with alternative operations. Nasr and Elsayed (1990) investigated the problem of minimizing the mean flow time in a general job shop type machining system with alternative machine tool routings. They developed a Mixed-Integer Linear Programming (MILP) formulation for the problem and proposed two hierarchical approaches to solve the problem. The first heuristic solves the problem by decomposing the problem into subproblems that are easier to solve. The second heuristic called Shortest Finish Time (SFT) rule is an extension of the first heuristic and is based on the Shortest Processing Time (SPT) rule.

During the past two decades there has been much research on the application of metaheuristics to solve the FJSP. The early research on FJSP was focused on development of neighborhood based metaheuristics like Tabu Search (TS) and Simulated Annealing (SA) algorithms. Brandimarte (1993) proposed a hierarchical approach based on TS to solve the FJSP with the objective of minimizing the makespan time. Hurink et al. (1994) represented the FJSP as a disjunctive graph model and proposed a hierarchical approach based on TS to solve the problem. Dauzere-Peres and Paulli (1997) presented a new disjunctive graph model to represent the MJS problem and proposed an integrated approach to solve FJSP. Mastrolilli and Gamberdella (2000) improved the TS approach proposed by Dauzere-Peres and Paulli (1997) and presented two new neighbourhood functions to solve FJSP instances. Najid et al. (2002) proposed a modified SA method for solving FJSP for minimum makespan time criterion. They represented their problem as a disjunctive graph and used the neighbourhood functions developed by Mastrolilli and Gamberdella (2000). Recently, Mehrabad and Fattahi (2005) presented a tabu search algorithm that solves the FJSP with sequence dependent setups to minimize the makespan time. They compared the performance of the algorithm with the optimal solution obtained using a MILP model solved by lingo software. Fattahi et al. (2007) developed a MILP model for the FJSP for minimum makespan time criterion and solved using Lingo software. They showed that solving FJSP using Lingo (Branch and Bound method) is very time consuming and are suitable for solving only smaller size problems. They also proposed two set of heuristics to 
solve the real size problems in which one set of heuristics is based on integrated approach and the other set is based on hierarchical approach. In integrated approach, they used Tabu Search (TS) and Simulated Annealing (SA) heuristics and presented two algorithms. In hierarchical approach, they used TS and SA and proposed four algorithms. Though neighborhood based metaheuristics have been successfully applied to solve FJSP, still the performance of these heuristics depend upon the initial solution and are more susceptible of getting stuck in local optimum. Therefore, most of the recently published research articles on FJSP are focused on developing Population based metaheuristics like Genetic Algorithm (GA), Ant Colony Optimization (ACO), etc. that are useful for any hard optimization problem. Mesghouni et al. (1998) were the first to model GA for FJSP. They proposed a chromosomal representation known as parallel job representation in which a chromosome is represented by a matrix where each row consists of a set of ordered operations of each job. Due to the complexity of decoding the representation, their algorithm incurs significant computational cost. Hussain and Joshi (1998) proposed a two pass GA to solve job shop problem with alternative routing with the objective of minimizing the sum of squared weighted due date deviation for every job. The first pass picks the alternatives using a genetic algorithm and the second pass provides the order and start time of jobs on the selected alternatives by solving a non-linear program. Chen et al. (1999) proposed a GA that uses an A-B string representation to solve FJSP for minimum makespan time criterion. A string contains a list of all operations of all jobs and the machines selected for the corresponding operations while B string contains a list of operations that are processed on each machine. Moon and Lee (2000) developed a mixed integer linear programming (MILP) model and proposed a GA for the job shop scheduling problem with alternative routings. The objective they considered is to minimize the mean flow time. The chromosome representation in their proposed GA consists of two strings, one for machine assignment and the other for schedule generation. Ho and Tay (2004) proposed a GA based tool, namely GENACE, for solving the FJSP for minimum makespan time criterion. The chromosome representation consists of two components, one component for machine selection and the other for operation sequence. Their methodology first generates an initial population using composite dispatching rules. A cultural evolution is then applied to preserve knowledge of schemata and resource allocations learned over each generation. The knowledge or belief spaces in turn influence mutation and selection of individuals. Ho et al. (2007) proposed an architecture for learning and evolving of flexible job shop schedules for minimum makespan criterion called learnable genetic architecture (LEGA), a generalization of their previous approach GENACE (Ho and Tay, 2004). Their proposed LEGA architecture is functionally divided into three modules: namely, a population generator module, Evolutionary algorithm (EA) module and a Schemata Learning (SL) module. The chromosome representation consists of two components, one component for machine selection and the other for operation sequence. The population generator module generates a set of feasible schedules equal to the population size using Composite Dispatching Rules and then encodes it into chromosomes of initial population for subsequent evolution in the EA module. During genetic evolution, the SL module modifies the offspring schedules to improve solution quality and to preserve feasibility based on a memory of conserved schemas resolved from sampled schedules sent dynamically from EA module. Tay and Ho (2008) proposed a genetic programming (GP) based approach for evolving effective composite dispatching rules for solving the multiobjective FJSP. The objective they considered is to minimize the weighted sum of makespan 
time, mean flow time and mean tardiness. They proposed a GP framework in which an individual is composed of terminals (like job release dates, due date, processing time, current time, remaining time, etc.) and algebraic functions. Their GP solves a specific problem by carefully selecting the terminals and functions and generating a composite dispatching rule that satisfies the requirements of that particular problem. They generated five composite dispatching rules using a large training set and compared the results with other popular rules like FIFO, SPT, etc.

In recent years, researchers have shown that $\mathrm{ACO}$, which is a kind of metaheuristic search approach, is competitive with other approaches in terms of performance and CPU requirements in several applications of general and combinatorial optimisation problems. Ant algorithms were first proposed by Dorigo et al. (1992) as a multi-agent heuristic search approach to solve the traveling salesman problem (TSP). Dorigo and Stutzle (1999) suggested various ACO approaches to quadratic assignment problems (QAP), e.g. ant system (AS), ACS, ANTS-QAP, MAX-MIN ant system (MMAS), FANT, and HAS-QAP and compared the results. There is currently considerable activity in the scientific community devoted to extending/applying ant-based algorithms to many different discrete optimisation problems. Recent applications cover problems such as vehicle routing, sequential ordering, graph colouring, routing in communications networks, etc. In the field of scheduling, ACO has been successfully applied to the single machine weighted tardiness problem (den Besten et al., 2000; Liao \& Juan, 2007), flow shop scheduling problem (Rajendran \& Ziegler, 2004; Gajpal \& Rajendran, 2006) and the resource constrained project scheduling problem (Merkle et al., 2002). Colorni et al. (1994) were the first to apply ACO to tackle the job shop scheduling problem (JSP). Blum (2002) proposed an MMAS algorithm for the Group shop scheduling problem which is a generalization of the JSP and the open shop scheduling problem. However, the performances of these algorithms for JSP were far from reaching the state-of-the-art performance. Blum and Sampels (2004) proposed MMAS algorithm with hyper cubic framework for the group shop scheduling problem. They performed experiments with different visibility functions and ranked them based on their performance. They used non-delay schedule generation mechanism for constructing solutions and employed a local search procedure to improve the solutions. Their algorithm performs particularly well for open shop scheduling problems. Heinonen and Pettersson (2007) proposed a hybrid ACO-local search algorithm for solving JSP. They performed experiments with different visibility functions on four different ACO variants and showed that MMAS algorithm performs better than AS, Rank based-AS and ACS. Huang and Liao (2008) presented a hybrid algorithm combining ACO algorithm with a taboo search algorithm for the JSP. Their proposed ACO algorithm employs a decomposition method inspired by the shifting bottleneck procedure, and a mechanism of occasional reoptimizations of partial schedules. The taboo search algorithm is embedded in the ACO algorithm to improve the solution quality. Rossi and Dini (2007) proposed an ACO based software system for solving flexible job shop problem (FJSP) with routing flexibility, sequence-dependent set up and transportation time. They showed the effectiveness of their system by comparing with other alternative approaches (including GA) for various benchmark instances. Girish and Jawahar (2008) proposed MMAS based heuristic for the FJSP for minimum makespan time criterion. Their proposed algorithm outperformed a GA and a constraint programming model solved using ILOG Solver for various benchmark problem instances. 


\subsection{Summary}

The literature review on FJSP reveals that population-based search heuristics such as GA and ACO have emerged as powerful tools for solving FJSP in the recent years. The coding schemes adopted in most of the proposed GAs for FJSP requires repair mechanisms to maintain solution feasibility. A few researchers have incorporated certain schemes to maintain the feasibility of solution which restricts the search to a smaller solution space. Most of the GAs proposed, therefore, have chances of missing the best optimal solution even under extensive searches for larger size problems. The literature review on ACO for scheduling applications reveals that ACO is competitive with other metaheuristic approaches including GA in terms of computational time and solution quality.

In light of the above, this chapter proposes an ACO algorithm to solve the problem under discussion for minimization of makespan time criterion. The proposed ACO algorithm is based on the MMAS algorithm proposed by Stützle and Hoos (2000). The proposed ACO incorporates pheromone trails for both route choice option and active feasible schedule generation and is capable to provide all possible instances that an enumerative search can. The proposed ACO is structured and coded in such a way that it can be easily adapted to generate schedules for any scheduling objective of FJSP.

The rest of the chapter is organized as follows: Section 2 describes the problem; the proposed ACO is explained and illustrated in section 3; Section 4 presents a numerical illustration for the proposed ACO; Section 5 presents the performance study of the proposed ACO for various benchmark instances and Section 6 concludes with scope for future work

\section{Description of the problem}

\subsection{Problem environment}

- $\quad$ There are $m$ machines in the system and $n$ jobs to be processed.

- $\quad$ Each job $i$ requires $J_{i}$ precedence-constrained operations to be performed.

- Each operation $j$ of job $i\left(O_{i j}\right)$ can be processed on a number of alternative (nonidentical) machines and its processing time $t_{i j k}$ differs with the features of machine $k$. This addresses the multiple routings for jobs. An alternative routing could be used if one machine tool is temporarily overloaded while another is idle. The alternative routing is useful where capacity problem arises.

\subsection{Assumptions}

- Jobs are independent and no priorities are assigned to any job type.

- Job pre-emption or cancellation is not allowed.

- Set up and inspection times are included in the processing time.

- All jobs are simultaneously available at time zero.

- After a job is processed on a machine it is transported to the next machine immediately and the transportation time is negligible or included in the operation time.

- Breakdowns are not considered. 


\subsection{Objective}

The objective is to complete all operations at the earliest possible time, which is known as minimum makespan time. This objective would distribute the workload evenly among all processing stations or work centers and all the processing stations would be freed at the makespan time for planning another set of jobs of the next planning horizon.

\subsection{Problem definition}

Scheduling in such an environment requires the following two types of decisions:

- Assignment of each operation to one of its alternative routes (machines) so as to convert the multiple routing job shop problem to a fixed route job shop problem.

- Sequencing the operations on the machines and generating start time and finish time for each operation.

Therefore, the problem can be defined as:

"Determination of optimal or near optimal schedules for the flexible job shop problem by assigning the operations to one of its alternative machines and sequencing the operations on the machines for the objective of minimization of makespan time, given the processing time of operations on all its alternative routes and the precedence relationship between the operations".

\subsection{Problem formulation}

The mathematical formulation for the problem under discussion with the objective of minimizing makespan time is presented below:

Objective:

$$
\operatorname{Minimize}\left[\operatorname{Max}\left(C_{1 J_{1}}, C_{2 J_{2}} \ldots, C_{n J n}\right)\right]
$$

Subject to:

$$
\begin{gathered}
C_{i j}-S_{i j}-\sum_{\left\{k: O i j \in N_{k}\right\}}\left(t_{i j k} \cdot X_{i j k}\right)=0 \quad \forall i, j \\
C_{i^{\prime} j^{\prime}}-C_{i j}+H\left(1-Y_{i j i^{\prime} j^{\prime} k}\right)+H\left(1-X_{i j k}\right)+H\left(1-X_{i^{\prime} j^{\prime} k}\right) \geq t_{i^{\prime} j^{\prime} k}, \\
\forall \quad k,(i, j),\left(i^{\prime}, j^{\prime}\right): O_{i j} \in N_{k}, O_{i^{\prime} j^{\prime}} \in N_{k} \\
C_{i j}-C_{i^{\prime} j^{\prime}}+H\left(Y_{i j i^{\prime} j^{\prime} k}\right)+H\left(1-X_{i j k}\right)+H\left(1-X_{i^{\prime} j^{\prime} k}\right) \geq t_{i j k}, \\
\forall \quad k,(i, j),\left(i^{\prime}, j^{\prime}\right): O_{i j} \in N_{k}, O_{i^{\prime} j^{\prime}} \in N_{k} \\
S_{i j} \geq 0, \quad \forall \quad i, j \\
S_{i j+1}-C_{i j} \geq 0, \quad \forall i, j=1, \ldots, J_{i}-1
\end{gathered}
$$




$$
\begin{gathered}
\sum_{k: O i j \in N_{k}} X_{i j k}=1, \quad \forall i, j \\
X_{i j k}=\left\{\begin{array}{l}
1, \text { if operation } O_{i j} \text { is assigned to machine } k \\
0, \text { otherwise }
\end{array}\right. \\
Y_{i j i^{\prime} j^{\prime} k}=\left\{\begin{array}{l}
1, \text { if operation } O_{i j} \text { precedes } O_{i^{\prime} j^{\prime}} \text { on machine } k \\
0, \text { otherwise }
\end{array}\right.
\end{gathered}
$$

where, $S_{i j}$ and $C_{i j}$ is the start time and completion time of job $i, H$ is a very large positive integer, $N_{k}$ is the set of operations $\left\{O_{i j}\right\}$ that can be loaded on machine $k, X_{i j k}$ is a decision variable for machine selection for operation $O_{i j}$ and $Y_{i j i^{\prime} j^{\prime} k}$ is a decision variable that generates a sequence between the operations $O_{i j}$ and $O_{i^{\prime} j^{\prime}}$ for loading on machine $k$. The constraints set (2) imposes that the difference between the completion time and the starting time of an operation is equal to its processing time on the machine to which it is assigned. This constraint satisfies the assumption that once an operation has started, it cannot be preempted until its completion. Constraints set (3) and (4) ensure that no two operations can be processed simultaneously on the same machine. This disjunctive constraints set (3) becomes inactive when $Y_{i j i^{\prime} j^{\prime} k}=0$ and the disjunctive constraints set (4) becomes inactive when $Y_{i j i^{\prime} j^{\prime} k}=1$. Constraints set (5) ensure that the start time of an operation is always positive. Constraints set (6) represent the precedence relationship among various operations of a job. Constraints set (7) impose that an operation can only be assigned to one machine.

\section{Description of the proposed ACO}

The different modules of the proposed ACO for the flexible job shop problem are outlined as flowchart in Figure 1. The different modules are described below.

Input module: The following data pertaining to the problem are given as input: Number of Jobs $(n)$, number of machines in the shop $(m)$, number of operations $J_{i}$ of each job $i(\forall i)$, number of alternative machines (routes) $R_{i j}$ for operation $j$ of job $i(\forall i, \forall j)$, the machine number $K_{i j r}$ corresponding to the route $r$ of operation $j$ of job $i$ along with its processing time $T_{i j r}(\forall i, \forall j, \forall r)$.

Initialization module: The number of ants (no_ant) is defined, and the pheromone trails used by them for constructing solutions are initialized. This problem uses two pheromone trails: pheromone trail intensity for route selection $\tau_{i j r}(t n)$ gives information about the desirability of choosing route $r$ for operation $O_{i j}$ at iteration $t n$ and pheromone trail intensity $\varepsilon_{k i j i i^{\prime} j^{\prime}}(t n)$, which indicates the desirability of choosing operation $O_{i j}$ directly after the operation $O_{i^{\prime} j^{\prime}}$ is loaded on machine $k$, is used for job conflict resolution while generating feasible schedule using Giffler and Thompson procedure. Thus, $\varepsilon_{k i j i i^{\prime}}(\mathrm{tn})$ indicates the pheromone trail between the operations. The pheromone trails $\tau_{i j r}(1)$ and $\varepsilon_{k i j i} i^{\prime}(1)$ are initialized to the upper trail limit to $\tau_{\max }(1)$ and $\varepsilon_{\max }(1)$, respectively, which causes a higher exploration at the start of the algorithm. 


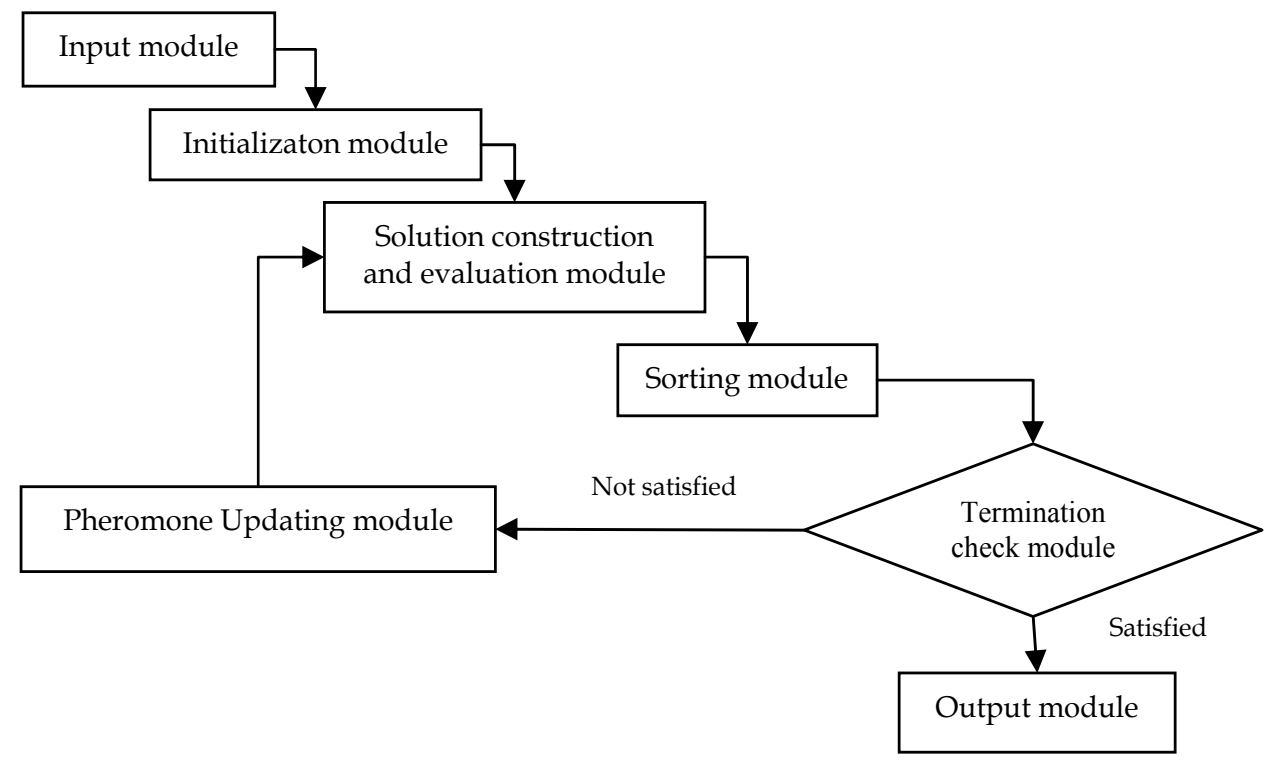

Fig. 1. Procedure of the proposed ACO for FJSP

Solution construction and Evaluation module: Each ant constructs a solution in two stages. In the Ist stage, an ant, at each construction step, allocates an operation of a particular job to one of its available resources. The ants use a probabilistic choice rule which is a function of the pheromone trail $\tau_{i j r}(t n)$ and a heuristic information based on processing time. Ant $z$ chooses to allocate operation $O_{i j}$ to the route $r$ with a probability given by

$$
P_{i j r}^{\prime Z}(t n)=\frac{\left[\tau_{i j r}(t n)\right]^{a} \cdot\left[\eta_{i j r}\right]^{\beta}}{\sum_{l=1}^{R_{i j}}\left[\tau_{i j l}(t n)\right]^{a} \cdot\left[\eta_{i j l}\right]^{\beta}}
$$

where $a$ and $\beta$ are two parameters that control the relative importance of the pheromone trail and the heuristic information (visibility of ant). $\eta_{i j r}$ represents the heuristic information which is a function of the processing time of operation $O_{i j}$ on route $r$ and is given by $\eta_{i j r}=1 / T_{i j r}$. Therefore the probability is a trade-off between visibility (which says that operations requiring lower processing time will have a higher probability to be loaded on the machines, thus implementing a greedy constructive heuristic) and trail intensity at iteration $t n$.

In the IInd stage, on allocation of all operations to the machines, each ant generates a schedule based on Giffler and Thompson (1960) algorithm. Ants, at each construction step s, chooses an operation $O_{i j}$ to allocate to its machine that has the minimum earliest finishing time $\left(E F T_{i}(s)\right)$ among the unassigned operations, provided the chosen operation has no conflict with other operations on the same machine. Ants store the operations that are assigned to its resources in $Q_{k}{ }^{z}(s)$ at each step $s$. Any conflict, if arises in the process of schedule generation is resolved using a probabilistic choice rule which is a function of 
pheromone trail intensity and a heuristic function. If a conflict arises in the construction step $s$ of an ant $z$, then it resolves the conflict by finding the probability of loading operation $O_{i j}$ to its resource $k$ directly after operation $O_{i^{\prime} j^{\prime}}$ loaded on the same machine. If $O_{i j}$ is the first operation in the sequence, then $O_{i^{\prime} j^{\prime}}=0$. The probabilistic rule is given by

$$
\begin{aligned}
& Q_{k}^{z}(s)= \begin{cases}\min \left\{E_{F T}(s)\right\}, & \text { if no conflict exists between jobs } \\
P_{k i j i^{\prime} j^{\prime}}^{\prime \prime}(t n), & \text { if conflict exists }\end{cases} \\
& P_{k i j j^{\prime} j^{\prime}}^{\prime \prime}(t n)=\frac{\left[\varepsilon_{k i j i^{\prime} j^{\prime}}(t n)\right]^{r} \cdot\left[\psi_{i}(s)\right]^{\omega}}{\sum_{(i, j): O i j \in G_{k}(s)}\left[\varepsilon_{k i j i^{\prime} j^{\prime}}(t n)\right]^{r} \cdot\left[\psi_{i}(s)\right]^{\omega}}, \quad \forall(i, j): O_{i j} \in G_{k}(s)
\end{aligned}
$$

where $\psi_{\mathrm{i}}(s)$ represents a heuristic information and is equal to the sum of the processing time of all unassigned operations of job $i$ at step $s, \gamma$ and $\omega$ are the scaling parameters that control the relative importance of the pheromone trail and the heuristic information and $G_{k}$ is the set of contending operations to be loaded on machine $k$. The higher the pheromone intensity $\varepsilon_{k i j i j^{\prime}}(t n)$ and the higher the heuristic information $\psi_{i}(s)$, the higher will be the probability that operation $O_{i j}$ may precede other contending jobs on machine $k$. Each ant generates one feasible schedule and the evaluation parameter makespan time is found from the feasible schedule generated by the ant. This procedure is repeated for all ants.

Sorting module: The best solution of the current iteration (ibest) and the global best (gbest) are sorted and stored separately.

Termination Check module: A specified number of iterations (no_iter) is estimated to terminate the algorithm depending on the size of the problem. Termination directs to the output module; otherwise, continue to the pheromone updating module.

Pheromone updating module: At the end of iteration, the pheromone trails corresponding to only one single ant is updated. This ant may be the one which found the best solution in the current iteration (ibest) or the one which found the best solution from the beginning of the trial (gbest). This pheromone trail update rule is similar to the rule used in Max-Min Ant System proposed by Stützle and Hoos (2000). The pheromone trail update rule corresponding to pheromone trails $\tau_{i j r}(t n)$ and $\varepsilon_{k i j i i^{\prime}}(t n)$ is given as

$$
\begin{gathered}
\tau_{i j r}(t n+1)=\rho \cdot \tau_{i j r}(t n) \quad \forall i, j, r \\
\varepsilon_{k i j i^{\prime} j^{\prime}}(t n+1)=\rho \cdot \varepsilon_{k i j i^{\prime} j^{\prime}}(t n) \quad \forall i, j, i^{\prime}, j^{\prime}, k \\
\tau_{i j r}(t n+1)=\tau_{i j r}(t n+1)+\Delta \tau_{i j r}(\text { best }) \quad \forall i, j, r: O_{i j} \text { is assigned to route } r \\
\varepsilon_{k i j i^{\prime} j^{\prime}}(t n+1)=\varepsilon_{k i j i^{\prime} j^{\prime}}(t n+1)+\Delta \varepsilon_{k i j i^{\prime} j^{\prime}}(\text { best }) \\
\forall k,(i, j),\left(i^{\prime}, j^{\prime}\right), s: O_{i j} \in Q_{k}^{z}(s+1), O_{i^{\prime} j^{\prime}} \in Q_{k}{ }^{z}(s) \\
\Delta \tau_{i j r}(\text { best })=\frac{1}{f(s b e s t)}
\end{gathered}
$$




$$
\Delta \varepsilon_{k i j i^{\prime} j^{\prime}}(\text { best })=\frac{1}{f(\text { sbest })}
$$

where $\rho$ is the evaporation factor in the range [0, 1]. In Equation (16) and Equation (17), $f($ sbest) denotes the makespan time of either the iteration best (ibest) or the global best solution (gbest). When using gbest alone, the search concentrates too fast around this solution, thus getting trapped in poor quality solutions and this danger is reduced when ibest is chosen for the pheromone trail update as ibest solutions may differ from iteration to iteration and a large number of solution components may receive occasional reinforcement. A dynamically mixed strategy of pheromone updating is used where ibest is chosen as default for updating the pheromones and using gbest only every fixed number of iterations. The frequency of using gbest for the pheromone update is increased during the search.

To avoid stagnation of the search the range of possible pheromone trails on each solution component is limited to an interval $\left[\tau_{\max }(t n), \tau_{\min }(t n)\right]$ and $\left[\varepsilon_{\max }(t n), \varepsilon_{\min }(t n)\right]$ corresponding to pheromone trails $\tau_{i j r}(t n)$ and $\varepsilon_{k i j i j^{\prime}}(t n)$ respectively. The pheromone trails are deliberately initialized to $\tau_{\max }(1)$ and $\varepsilon_{\max }(1)$ in order to achieve higher exploration of solutions at the start of the algorithm. In every iteration, it is ensured that pheromone trail respects the limits, i.e., in case of $\tau_{i j r}(t n)>\tau_{\max }(t n)$, or $\tau_{i j r}(t n)<\tau_{\min }(t n)$, then trail intensities are set to $\tau_{i j r}(t n)=\tau_{\max }(t n)$ and $\tau_{i j r}(t n)=\tau_{\min }(t n)$, respectively. The values are determined as follows:

$$
\begin{gathered}
\tau_{\max }(t n+1)=1 /(1-\rho) \cdot f(\text { gbest }) \\
\varepsilon_{\max }(t n+1)=1 /(1-\rho) \cdot f(\text { gbest }) \\
\tau_{\min }(t n+1)=\tau_{\max }(t n+1) / y \\
\varepsilon_{\min }(t n+1)=\varepsilon_{\max }(t n+1) / y
\end{gathered}
$$

where $y$ is a parameter that defines the space of the region between the limits $\left[\tau_{\max }(t n)\right.$, $\left.\tau_{\min }(t n)\right]$ and $\left[\varepsilon_{\max }(t n), \varepsilon_{\min }(t n)\right]$. Hence, each time a new best solution is found, $\tau_{\max }(t n)$ and $\varepsilon_{\max }(t n)$ are updated.

Output Module: This module prints the global best solution of the optimal route choices of all operations and schedule for minimum makespan time criterion.

\section{Numerical Illustration for the proposed ACO}

Table 1 provides the process data of 3 jobs -5 machines problem that is used for illustrating the proposed ACO. 


\begin{tabular}{|c|c|c|c|c|}
\hline \multirow[t]{2}{*}{$\begin{array}{c}\text { Job } \\
i\end{array}$} & \multirow[t]{2}{*}{$\begin{array}{c}\text { Operation } \\
j\end{array}$} & \multirow[t]{2}{*}{$\begin{array}{l}\text { Number of } \\
\text { route choices } \\
R_{i j}\end{array}$} & \multicolumn{2}{|c|}{$\begin{array}{l}\text { Machine No. with Processing time } \\
\qquad K_{i j r}\left(T_{i j r}\right) \\
\text { corresponding to each route } r\end{array}$} \\
\hline & & & $r=1$ & $r=2$ \\
\hline \multirow[t]{3}{*}{1} & 1 & 2 & $2(3)$ & $3(7)$ \\
\hline & 2 & 2 & $1(4)$ & $4(2)$ \\
\hline & 3 & 2 & $1(1)$ & $2(2)$ \\
\hline \multirow[t]{3}{*}{2} & 1 & 2 & $2(5)$ & $5(2)$ \\
\hline & 2 & 2 & $2(3)$ & $3(6)$ \\
\hline & 3 & 2 & $1(3)$ & $5(7)$ \\
\hline \multirow[t]{3}{*}{3} & 1 & 2 & $2(4)$ & $3(5)$ \\
\hline & 2 & 2 & $1(2)$ & $4(3)$ \\
\hline & 3 & 2 & $1(2)$ & $3(3)$ \\
\hline
\end{tabular}

Table 1. Process data of the illustration problem

The above data is given as input in the input module. The number of ants used in this problem is 10 . The pheromone trails, $\tau_{i j r}(1)$ and $\varepsilon_{k i j i j^{\prime}}(1)$ are initialized as $\tau_{\max }(1)=\varepsilon_{\max }(1)=0.1$. In the Ist Stage of solution construction ants reduce the alternate route choice problem into a fixed route problem by allocating operations to the routes using the probabilistic rule given in Equation (10). The parameters used for solution construction are: $a=1, \beta=2$. The Ist stage of solution construction for Ant- 1 is shown in Table 2. After allocating all the operations to the routes, the ants generate feasible schedules using Giffler and Thompson procedure (1960) in the IInd stage. The active feasible schedule formed by ant $z=1$ is shown in Table 3. Conflicts resolved during schedule generation are shown in Table 4 . The parameters set for the probabilistic rule for schedule generation is: $\gamma=1, \omega=2$.

\begin{tabular}{|c|c|c|c|c|c|c|c|c|c|}
\hline $\begin{array}{c}\text { Jobs } \\
i\end{array}$ & $\begin{array}{c}\text { Operations } \\
j\end{array}$ & $\begin{array}{c}\text { Route } \\
r\end{array}$ & \begin{tabular}{|c|} 
Machine \\
$k$
\end{tabular} & $\tau_{i j r}(1)$ & $T_{i j r}$ & $P^{\prime \prime}{ }_{i j r}(1)$ & $\begin{array}{c}\text { Cumulative } \\
P^{\prime \prime} 1_{i j r}(1)\end{array}$ & $\operatorname{rand}()$ & $\begin{array}{c}\text { Assigned } \\
\text { route }\end{array}$ \\
\hline \multirow{6}{*}{1} & \multirow{2}{*}{1} & 1 & 2 & 0.1 & 3 & 0.84 & 0.84 & \multirow{2}{*}{0.041} & \multirow{2}{*}{1} \\
\hline & & 2 & 3 & 0.1 & 7 & 0.16 & 1.0 & & \\
\hline & \multirow{2}{*}{2} & 1 & 1 & 0.1 & 4 & 0.2 & 0.2 & \multirow{2}{*}{0.467} & \multirow{2}{*}{2} \\
\hline & & 2 & 4 & 0.1 & 2 & 0.8 & 1.0 & & \\
\hline & \multirow{2}{*}{3} & 1 & 1 & 0.1 & 1 & 0.8 & 0.8 & \multirow{2}{*}{0.334} & \multirow{2}{*}{1} \\
\hline & & 2 & 2 & 0.1 & 2 & 0.2 & 1.0 & & \\
\hline \multirow{6}{*}{2} & \multirow{2}{*}{1} & 1 & 2 & 0.1 & 5 & 0.13 & 0.13 & \multirow{2}{*}{0.5} & \multirow{2}{*}{2} \\
\hline & & 2 & 5 & 0.1 & 2 & 0.87 & 1.0 & & \\
\hline & \multirow{2}{*}{2} & 1 & 2 & 0.1 & 3 & 0.8 & 0.8 & \multirow{2}{*}{0.169} & \multirow[b]{2}{*}{1} \\
\hline & & 2 & 3 & 0.1 & 6 & 0.2 & 1.0 & & \\
\hline & \multirow{2}{*}{3} & 1 & 1 & 0.1 & 3 & 0.84 & 0.84 & \multirow{2}{*}{0.724} & \multirow[b]{2}{*}{1} \\
\hline & & 2 & 5 & 0.1 & 7 & 0.16 & 1.0 & & \\
\hline \multirow{6}{*}{3} & \multirow{2}{*}{1} & 1 & 2 & 0.1 & 4 & 0.61 & 0.61 & \multirow{2}{*}{0.478} & \multirow{2}{*}{1} \\
\hline & & 2 & 3 & 0.1 & 5 & 0.39 & 1.0 & & \\
\hline & \multirow{2}{*}{2} & 1 & 1 & 0.1 & 2 & 0.69 & 0.69 & \multirow{2}{*}{0.358} & \multirow{2}{*}{1} \\
\hline & & 2 & 4 & 0.1 & 3 & 0.31 & 1.0 & & \\
\hline & \multirow{2}{*}{3} & 1 & 1 & 0.1 & 1 & 0.9 & 0.9 & \multirow{2}{*}{0.962} & \multirow{2}{*}{2} \\
\hline & & 2 & 3 & 0.1 & 3 & 0.1 & 1.0 & & \\
\hline
\end{tabular}

Table 2. Solution construction of ant $z=1$ in stage-I 


\begin{tabular}{|c|c|c|c|c|c|c|c|c|c|c|}
\hline \multirow{2}{*}{$\begin{array}{c}\text { Machine } \\
k\end{array}$} & \multirow{2}{*}{$\begin{array}{c}\text { Job } \\
i\end{array}$} & \multicolumn{9}{|c|}{ Steps of schedule generation $\mathrm{s}$} \\
\hline & & 1 & 2 & 3 & 4 & 5 & 6 & 7 & 8 & 9 \\
\hline \multirow[t]{3}{*}{1} & 1 & & & & & & & & 15 & $15^{*}$ \\
\hline & 2 & & & $8^{*}$ & & & & & & \\
\hline & 3 & & & & & 11 & & & & \\
\hline \multirow[t]{3}{*}{2} & 1 & 3 & 3 & 8 & 8 & 12 & 12 & & & \\
\hline & 2 & & 5 & & & & & & & \\
\hline & 3 & 4 & 4 & 9 & 9 & & & & & \\
\hline \multirow[t]{3}{*}{3} & 1 & & & & & & & & & \\
\hline & 2 & & & & & & & & & \\
\hline & 3 & & & & & & 14 & 14 & $14^{*}$ & \\
\hline \multirow[t]{3}{*}{4} & 1 & & & & & & & 14 & & \\
\hline & 2 & & & & & & & & & \\
\hline & 3 & & & & & & & & & \\
\hline \multirow[t]{3}{*}{5} & 1 & & & & & & & & & \\
\hline & 2 & 2 & & & & & & & & \\
\hline & 3 & & & & & & & & & \\
\hline \multicolumn{2}{|c|}{ Datum Time } & 2 & 3 & 8 & 8 & 11 & 12 & 14 & 14 & $15^{* *}$ \\
\hline \multicolumn{2}{|c|}{ Conflict } & -- & I & -- & II & -- & -- & -- & -- & -- \\
\hline \multicolumn{2}{|c|}{ Sequence of operations $Q_{k}{ }^{z}(s)$} & $\mathrm{O}_{21}$ & $\mathrm{O}_{22}$ & $\mathrm{O}_{23}$ & $\mathrm{O}_{31}$ & $\mathrm{O}_{32}$ & $O_{11}$ & $\mathrm{O}_{12}$ & $\mathrm{O}_{33}$ & $\mathrm{O}_{13}$ \\
\hline
\end{tabular}

*Flow time of jobs $\quad{ }^{* *}$ Makespan time

Table 3. Active feasible schedule generation for solution generated by ant $z=1$

\begin{tabular}{|c|c|c|c|c|c|c|c|c|}
\hline $\begin{array}{c}\text { Conflict } \\
\text { No. }\end{array}$ & $k$ & $\begin{array}{c}\text { Contending } \\
\text { Jobs } \\
i\end{array}$ & $\varepsilon_{k i j i^{\prime} j^{\prime}}(1)$ & $\psi_{i}(s)$ & $P^{\prime \prime} 1_{k i j i i^{\prime}{ }^{\prime}}(1)$ & $\begin{array}{c}\text { Cumulative } \\
P^{\prime \prime 1}{ }_{k i j i j^{\prime}}(1)\end{array}$ & $\operatorname{rand}()$ & $\begin{array}{c}\text { Assigned } \\
\text { Job }\end{array}$ \\
\hline \multirow{3}{*}{ I } & \multirow{3}{*}{2} & 1 & 0.1 & 6 & 0.235 & 0.235 & \multirow{3}{*}{0.464} & \multirow{3}{*}{2} \\
\hline & & 2 & 0.1 & 6 & 0.235 & 0.470 & & \\
\hline & & 3 & 0.1 & 9 & 0.530 & 1.000 & & \\
\hline \multirow{2}{*}{ II } & \multirow{2}{*}{2} & 1 & 0.1 & 6 & 0.308 & 0.308 & \multirow{2}{*}{0.705} & \multirow{2}{*}{3} \\
\hline & & 3 & 0.1 & 9 & 0.692 & 1.000 & & \\
\hline
\end{tabular}

Table 4. Conflict resolved during solution generation by ant $z=1$

After all the ants have generated a feasible schedule, the makespan time is determined and is shown in Table 5. The best solution found in the current iteration $(t n=1)$ is $f($ ibest $)=12$ corresponding to the solution generated by ant $z=2$. The current ibest solution is compared with the gbest solution and if $f(i b e s t)<f($ gbest $)$, then the global best solution is updated with the iteration best solution.

\begin{tabular}{|c|c|c|c|c|c|c|c|c|c|c|}
\hline Ant $z$ & 1 & $2^{*}$ & 3 & 4 & 5 & 6 & 7 & 8 & 9 & 10 \\
\hline $\begin{array}{c}\text { Makespan } \\
\text { time }\end{array}$ & 15 & $12^{* *}$ & 17 & 13 & 17 & 13 & 21 & 12 & 13 & 13 \\
\hline
\end{tabular}

*ibest ant ${ }^{* *}$ f(ibest) solution

Table 5. Solution generated by all ants in first iteration

The dynamic mixed strategy between ibest and gbest for pheromone updating is given below:

fqgbest is the number of iterations for which the ibest solution is used for pheromone updating. A 
gbest solution is used for pheromone updating after fqgbest no. of iterations. This procedure of pheromone updating is repeated until fqgbest $v$ alue changes according to iteration number $t n$. In the first 15 iterations $(t n<=15)$, only ibest solution is used to update the pheromone trails. The value of fqgbest is set at a higher value of 3 for iterations $15<t n<=30$ due to which more number of $i b e s t$ solutions are subjected to pheromone updating. This leads to more exploration of the solution space. The value of $f q^{g b e s t}$ is set at 2 for $30<t n<=50$ and fqgbest=1 for $t n>50$. The parameters set for pheromone update rule are: $\rho=0.9$ and $y=10$. The values of $\tau_{\max }(1), \tau_{\min }(1)$, $\varepsilon_{\max }(1)$ and $\varepsilon_{\min }(1)$ are updated and the pheromone intensities are limited to the interval $\left[\tau_{\max }(2), \tau_{\min }(2)\right]$ and $\left[\varepsilon_{\max }(2), \varepsilon_{\min }(2)\right]$. The updated values are $\tau_{\max }(2)=\varepsilon_{\max }(2)=0.833$ and $\tau_{\min }(2)=\varepsilon_{\min }(2)=0.083$. The pheromone trail intensities for $\tau_{i j r}(t n)$ are updated and is shown in Table 6. The updated pheromone trail intensities for $\varepsilon_{k i j i j^{\prime}}(2)$ obtained with machines 1 to 5 are shown in Tables 7 to 11 respectively. The process of solution construction, evaluation, sorting and pheromone updating is repeated till the termination criterion is reached.

\begin{tabular}{|c|c|c|c|c|c|c|c|c|c|}
\hline \multirow{2}{*}{$\begin{array}{c}\text { Route } \\
r\end{array}$} & \multicolumn{10}{|c|}{ Operation $O_{i j}$} \\
\cline { 2 - 10 } & $O_{11}$ & $O_{12}$ & $O_{13}$ & $O_{21}$ & $O_{22}$ & $O_{23}$ & $O_{31}$ & $O_{32}$ & $O_{33}$ \\
\hline 1 & 0.173 & 0.090 & 0.090 & 0.090 & 0.173 & 0.090 & 0.090 & 0.090 & 0.173 \\
\hline 2 & 0.090 & 0.173 & 0.173 & 0.173 & 0.090 & 0.173 & 0.173 & 0.173 & 0.090 \\
\hline
\end{tabular}

Table 6. Updated pheromone trail intensities for $\tau_{i j r}(2)$

\begin{tabular}{|c|c|c|c|c|c|}
\hline \multirow{2}{*}{$\begin{array}{c}\text { Operation } \\
O_{i^{\prime} j^{\prime}}\end{array}$} & \multicolumn{5}{|c|}{ Operation $O_{i j}$} \\
\cline { 2 - 6 } & $O_{12}$ & $O_{13}$ & $O_{23}$ & $O_{32}$ & $O_{33}$ \\
\hline 0 & 0.090 & 0.173 & 0.090 & 0.090 & 0.090 \\
\hline$O_{12}$ & --- & 0.090 & 0.090 & 0.090 & 0.090 \\
\hline$O_{13}$ & --- & --- & 0.090 & 0.173 & 0.090 \\
\hline$O_{23}$ & 0.090 & 0.090 & --- & 0.090 & 0.090 \\
\hline$O_{32}$ & 0.090 & 0.090 & 0.090 & --- & 0.090 \\
\hline$O_{33}$ & 0.090 & 0.090 & 0.090 & --- & --- \\
\hline
\end{tabular}

Table 7. Updated pheromone trail intensities for $\varepsilon_{k i j i j^{\prime}}(2)$ for machine $k=1$

\begin{tabular}{|c|c|c|c|c|c|}
\hline \multirow{2}{*}{$\begin{array}{c}\text { Operation } \\
O_{i^{\prime} j^{\prime}}\end{array}$} & \multicolumn{5}{|c|}{ Operation $O_{i j}$} \\
\cline { 2 - 6 } & $O_{11}$ & $O_{13}$ & $O_{21}$ & $O_{22}$ & $O_{31}$ \\
\hline 0 & 0.173 & 0.090 & 0.090 & 0.090 & 0.090 \\
\hline$O_{11}$ & --- & 0.090 & 0.090 & 0.173 & 0.090 \\
\hline$O_{13}$ & --- & --- & 0.090 & 0.090 & 0.090 \\
\hline$O_{21}$ & 0.090 & 0.090 & --- & 0.090 & 0.090 \\
\hline$O_{22}$ & 0.090 & 0.090 & --- & 0.090 & 0.090 \\
\hline$O_{31}$ & 0.090 & 0.090 & 0.090 & 0.090 & --- \\
\hline
\end{tabular}

Table 8. Updated pheromone trail intensities for $\varepsilon_{k i j i i^{\prime}}(2)$ for machine $k=2$

\begin{tabular}{|c|c|c|c|c|}
\hline \multirow{2}{*}{ Operation } & \multicolumn{4}{|c|}{ Operation $O_{i j}$} \\
\cline { 2 - 5 }$O_{i{ }^{\prime} j^{\prime}}$ & $O_{11}$ & $O_{22}$ & $O_{31}$ & $O_{33}$ \\
\hline 0 & 0.090 & 0.090 & 0.173 & 0.090 \\
\hline$O_{11}$ & --- & 0.090 & 0.090 & 0.090 \\
\hline$O_{22}$ & 0.090 & --- & 0.090 & 0.090 \\
\hline$O_{31}$ & 0.090 & 0.090 & --- & 0.173 \\
\hline$O_{33}$ & 0.090 & 0.090 & --- & --- \\
\hline
\end{tabular}

Table 9. Updated pheromone trail intensities for $\varepsilon_{k i j i i^{\prime}}(2)$ for machine $k=3$ 


\begin{tabular}{|c|c|c|}
\hline \multirow{2}{*}{$\begin{array}{c}\text { Operation } \\
O_{i^{\prime} j^{\prime}}\end{array}$} & \multicolumn{2}{|c|}{ Operation } \\
\cline { 2 - 3 }$O_{i j}$ \\
\hline 0 & $O_{12}$ & $O_{32}$ \\
\hline$O_{12}$ & 0.173 & 0.090 \\
\hline$O_{32}$ & --- & 0.090 \\
\hline & 0.090 & --- \\
\hline
\end{tabular}

Table 10. Updated pheromone trail intensities for $\varepsilon_{k i j i{ }^{\prime} j}(2)$ for machine $k=4$

\begin{tabular}{|c|c|c|}
\hline \multirow{2}{*}{$\begin{array}{c}\text { Operation } \\
O_{i^{\prime} j^{\prime}}\end{array}$} & \multicolumn{2}{|c|}{ Operation } \\
\cline { 2 - 3 }$O_{i j}$ \\
\hline 0 & $O_{21}$ & $O_{23}$ \\
\hline$O_{21}$ & 0.173 & 0.090 \\
\hline$O_{23}$ & --- & 0.173 \\
\hline & 0.090 & --- \\
\hline
\end{tabular}

Table 11. Updated pheromone trail intensities for $\varepsilon_{k i j i{ }^{\prime} j^{\prime}}(2)$ for machine $k=5$

\section{Performance comparison and results}

The performance of the proposed ACO algorithm is evaluated by comparing its solutions with the Best Known Solutions (BKS) from literature (Mastrolilli and Gamberdella, 2000). A set of benchmark problems is used for the performance evaluation. The first set of benchmark instances are from Thomalla (2001), in which all the problems are flexible job shop instances with total flexibility, i.e., all the operations in each of the problem instances can be performed on all the machines. The second set of benchmark instances are from Brandimarte (1993), in which all the problems are flexible job shop instances with partial flexibility. The results of the proposed ACO algorithm are evolved with the program coded in C language.

Researchers and practitioners are nowadays using constraint programming $(\mathrm{CP})$ techniques, which is a relatively new methodology for solving combinatorial optimization problems (Bockmayr \& Kasper, 1998). Constraint programming can solve an optimization problem by solving a series of decision (or constraint satisfaction) problems resulting from a dichotomy to locate the optimal objective value (Pan \& Shi, 2008). More precisely, each problem is solved through an enumerative search that resembles the tree search of branch-and-bound, except that it involves no bounding and only seeks a feasible solution. However, the overall optimum seeking can be computation-intensive. ILOG Solver, a constraint programming tool marketed by ILOG, has proven to solve scheduling problems in reasonable computational time (Heisig \& Minner, 1999; Pinedo, 2005). Weil et al. (1995) demonstrated the efficiency of ILOG Solver as a modeling and resolution tool for nurse scheduling problem. Quiroga et al. (2005) developed a CP model for a FMS scheduling problem using ILOG OPL and presented its computational efficiency for benchmark problems. In this chapter, a CP model is developed for the FJSP using ILOG OPL language and solved using ILOG OPL Studio. The results of ILOG OPL Studio are used to test the performance of the proposed ACO for the benchmark instances.

The parameters set for the proposed ACO are: $a=1, \beta=2, \gamma=1, \omega=2 \varepsilon_{\max }(1)=\tau_{\max }(1)=0.1, \rho=0.9$ and $y=10$. In the first 100 iterations, only ibest solution is used to update the pheromone trails; fqgbest $=4$, for $100<\mathrm{tn}<=200$; fqgbest $=3$ for $200<\mathrm{tn}<=300$; fqgbest=2 for $300<\mathrm{tn}<=400$; fqgbest $=1$ for $400<\mathrm{tn}<=500$; fqgbest $=0$ for $\mathrm{tn}>500$. The number of ants (no_ant) used in the proposed ACO 
for solution construction is equal to twice the total number of operations. The termination criterion used for ACO is the total number of iterations which is equal to 100 times the total number of operations of all jobs. The parameters for the proposed ACO are obtained by fine tuning through trials. The proposed algorithm is run five times for each problem and the best solution obtained has been taken for comparison. The proposed model in ILOG OPL is run for a pre-specified period of time which is set as the total time required for the proposed ACO to find a solution for a particular problem. Table 12 shows the results of ACO and ILOG OPL Studio that are obtained with a Pentium-IV 2.4GHz processor.

The comparison between the proposed ACO algorithm and the BKS in the literature for the above benchmark problems reveals that the best solution obtained with ACO for seven out of 13 problems is the same as the best known solutions in the literature. For one problem (MK07) the proposed ACO has given a better result than the best known solution. For three problems (MK04, MK05 \& MK06) the results of the proposed ACO is closer to the best known solutions. The proposed algorithm has outperformed ILOG OPL Studio for almost all the Benchmark instances. Therefore, it is inferred from the computational results that the proposed ACO provides better performance than ILOG OPL Studio and is competent with the existing methodologies for FJSP.

\begin{tabular}{|c|c|c|c|c|c|c|}
\hline \multirow{3}{*}{ Reference } & \multirow{3}{*}{$\begin{array}{l}\text { Problem } \\
\text { Name }\end{array}$} & \multirow{3}{*}{$\begin{array}{c}\text { Problem } \\
\text { Size } \\
n \times m\end{array}$} & \multirow{3}{*}{ BKS } & \multicolumn{3}{|c|}{ Makespan time } \\
\hline & & & & \multicolumn{2}{|l|}{ Proposed ACO } & \multirow{2}{*}{$\begin{array}{l}\text { ILOG } \\
\text { OPL } \\
\text { Studio } \\
\end{array}$} \\
\hline & & & & Test runs & Best & \\
\hline \multirow{3}{*}{$\begin{array}{l}\text { Thomalla } \\
\text { (2001) }\end{array}$} & EX1 & $3 \times 3$ & 117 & $117,117,117,117,117$ & 117 & 117 \\
\hline & EX2 & $4 \times 3$ & 109 & $109,109,109,109,109$ & 109 & 109 \\
\hline & EX3 & $6 \times 10$ & 316 & $316,316,316,316,316$ & 316 & 674 \\
\hline \multirow{10}{*}{$\begin{array}{l}\text { Brandimarte } \\
\text { (1993) }\end{array}$} & MK01 & $10 \times 6$ & 40 & $40,40,40,40,40$ & 40 & 52 \\
\hline & MK02 & $10 \times 6$ & 26 & $27,27,27,26,27$ & 26 & 49 \\
\hline & MK03 & $15 \times 8$ & 204 & $204,204,204,204,204$ & 204 & 319 \\
\hline & MK04 & $15 \times 8$ & 60 & $67,66,67,67,67$ & 66 & 67 \\
\hline & MK05 & $15 \times 4$ & 173 & $178,178,174,174,178$ & 174 & 293 \\
\hline & MK06 & $10 \times 15$ & 58 & $77,77,80,77,81$ & 77 & 230 \\
\hline & MK07 & $20 \times 5$ & 144 & $143,144,144,144,144$ & 143 & 223 \\
\hline & MK08 & $20 \times 10$ & 523 & $523,523,523,523,523$ & 523 & 595 \\
\hline & MK09 & $20 \times 10$ & 307 & $328,349,346,340,343$ & 328 & 534 \\
\hline & MK10 & $20 \times 15$ & 198 & $247,258,267,248,254$ & 247 & 385 \\
\hline
\end{tabular}

Table 12. Result obtained with the proposed ACO and ILOG OPL Studio for the set of data from literature

\section{Conclusion}

In this Chapter, we proposed an ACO based heuristic to solve the FJSP for minimum makespan time criterion. The solution construction method used in the proposed ACO makes it capable to rummage through the entire solution space and provide all possible instances that an enumerative search can and is therefore capable of finding the optimal or near-optimal solutions. Since the proposed ACO uses Giffler and Thompson schedule generation procedure for generating active feasible schedules, therefore, the proposed ACO can be easily adapted to generate schedules for any scheduling objective of FJSP. The 
performance of the proposed ACO is analyzed with various benchmark instances, which reveals that the proposed ACO is competent with the existing approaches. The proposed ACO has outperformed the CP model solved using ILOG OPL Studio. A future research issue would be to develop hybrid heuristics by incorporating local search techniques such as Tabu search, Simulated Annealing, etc. to the proposed ACO algorithm.

\section{References}

Baker KR (1974). Introduction to Sequencing and Scheduling. Wiley, New York.

Blum, C. (2002). ACO applied to group shop scheduling: A case study on intensification and diversification. In ANTS 2002, Dorigo M. et al. (ed.), pp. 14-17, Springer-Verlag, Berlin, Germany.

Blum, C. \& Sampels, M. (2004). An ant colony optimization algorithm for shop scheduling problems. Journal of mathematical modeling and algorithms. Vol. 3, No. 3, pp. 285-308.

Bockmayr, A. \& Kasper, T. (1998). Branch and infer: A unifying framework for integer and finite domain constraint programming. INFORMS Journal of Computing, Vol. 10, No. 3, pp.287-300.

Brandimarte, P. (1993). Routing and scheduling in a flexible job shop by tabu Search. Annals of Operations research, Vol. 41, No. 1, pp.157-183.

Brucker, P. (1995). Scheduling algorithms. Springer-Verlag, Berlin-Heidelberg.

Brucker, P. \& Schlie, R. (1990). Job shop scheduling with multi-purpose machines. Computing, Vol.45, pp.369-375.

Chen, H.; Ihlow, J. \& Lehmann, C. (1999). A genetic algorithm for flexible job-shop scheduling. Proceedings of the IEEE International Conference on Robotics and Automation, Vol. 2, pp.1120-1125, IEEE.

Choi, I.C. \& Choi, D.S. (2002). A local search algorithm for job shop scheduling problems with alternative operations and sequence-dependent setups. Computers and Industrial Engineering, Vol. 42, pp. 43-58.

Chryssolouris, G. \& Chan, S. (1985). An integrated approach to process planning and scheduling. Annals of CIRP, Vol. 34, pp.413-417.

Colorni, A.; Dorigo, M.; Maniezzo, V. \& Trubian, M. (1994). Ant System for Job Shop Scheduling. JORBEL - Belgian Journal of Operations Research, Statistics and computer science, Vol. 34, No.1, pp.39-53.

Conway, R.W.; Maxwell, W.L. \& Miller, L.M. (1967). Theory of Scheduling, Addison-Wesley Reading, MA.

Dauzere-Peres, S. \& Paulli, J. (1997). An integrated approach for modeling and solving the general multiprocessor job-shop scheduling problem with tabu search. Annals of Operations Research, Vol. 70, pp.281-306.

den Besten M.; Stützle T. \& Dorigo, M. (2000). An ant colony optimization application to the single machine total weighted tardiness problem. In: Proc. of ANTS'2000, Dorigo, M.; Middendorf, M. \& Stützle, T. (eds), pp. 39-42, Belgium.

Dorigo, M.; Colorni, A. \& Maniezzo, V. (1992). An investigation of some properties of an ant algorithm. In: Proceedings of the conference on parallel problem solving from nature, R. Manner \& B. Manderick (eds), pp 509-520, Elsevier, Brussels, Amsterdam.

Dorigo, M. \& Stutzle, T. (1999). Ant colony algorithms for quadratic assignment problem: new ideas in optimisation. McGraw Hill, New York. 
Fattahi, P.; Mehrabad, M.S. \& Jolai, F. (2007). Mathematical modeling and heuristic approaches to flexible job shop scheduling problems. Journal of Intelligent Manufacturing, Vol. 18, No. 3, pp. 331-342.

French, S. (1982). Sequencing and Scheduling: An introduction to the mathematics of Job-Shop. Ellis Horwood Limited, Chichester.

Gajpal, Y. \& Rajendran, C. (2006). An ant-colony optimization algorithm for minimizing the completion-time variance of jobs in flowshop. International Journal of Production Economics, Vol. 101, No. 2, pp.259-272.

Garey, M.R.; Johnson, D.S. \& Sethi, R. (1976). The complexity of flowshop and jobshop scheduling. Mathematics of Operations Research, Vol. 1, pp.117-129.

Giffler, B. \& Thompson, G.L. (1960). Algorithms for solving production scheduling problems. Operations Research, Vol. 8, pp.487-503.

Girish, B.S. \& Jawahar, N. (2008). Scheduling job shops associated with multiple routings with genetic and ant colony heuristics. International journal of production research, In print, available online DOI:10.1080/00207540701824845.

Groover, M.P. (2003). Automation, production systems, and computer integrated manufacturing. Prentice Hall of India Pvt Ltd, New Delhi, India.

Hankins, S.L.; Wysk, R.A. \& Fox, K.R. (1984). Using a CATS database for alternative machine loading. Journal of Manufacturing Systems, Vol.3, pp.115-120.

Heinonen, J. \& Pettersson, F. (2007). Job-shop scheduling and visibility studies with a hybrid ACO algorithm. In:, Swarm intelligence: Focus on ant and particle swarm optimization, Chan, F. T. S. and Tiwari, M. K. (eds.), pp. 355 - 372, Itech Education and Publishing, Vienna, Austria.

Heisig, G. \& Minner, S. (1999). ILOG OPL Studio. OR Spectrum, Vol. 21, No. 4, pp.419-427.

Ho, N.B. \& Tay, J.C. (2004). GENACE: An Efficient Cultural Algorithm for Solving the Flexible Job-Shop Problem. Proceedings of the IEEE Congress on Evolutionary Computation, Vol.1, pp.1759-1766, IEEE.

Ho, N.B.; Tay, J.C. \& Lai, E.M.K. (2007). An effective architecture for learning and evolving flexible job-shop schedules. European Journal of Operational Research, Vol. 179, pp.316-333.

Hoitomt, D.J.; Luh, P.B. \& Pattipati, K.R. (1993). A practical approach to job shop scheduling problems. IEEE transactions on Robotics and Automation, Vol. 9, No.1, pp.1-13.

Huang, K. \& Liao, C. (2008). Ant colony optimization combined with taboo search for the job shop scheduling problem. Computers and Operations Research, Vol. 35, No. 4, pp.1030-1046.

Hurink, J., Jurisch, B. \& Thole, M. (1994). Tabu search for the job-shop scheduling with multi-purpose machines. OR Spektrum, Vol. 15, pp. 205-215.

Hussain, M.F. and Joshi, S.B. (1998). A Genetic Algorithm for Job Shop Scheduling problems with Alternate Routing. Proceedings of IEEE International conference on systems, man and cybernetics, Vol.3, pp. 2225-2230.

Iwata, K.; Murotsu, Y.; Oba, F. \& Okamura, K. (1980). Solution of large-scale scheduling problems for job-shop type machining systems with alternative machine tools. Annals of the CIRP, Vol. 29, pp. 335-338.

Iwata, K.; Murotsu, Y.; Oba, F. \& Uemura, T. (1978). Optimization of selection of machine tools, loading sequence of parts and machining conditions in job-shop type machining systems. Annals of the CIRP, Vol.27, pp.447-451. 
Jain, A.S. \& Meeran, S. (1999). Deterministic job-shop scheduling: Past, present and future. European Journal of Operational Research, Vol.113, No.2, pp. 390-434.

Kutanoglu, E. \& Sabuncuoglu, I. (1999). An analysis of heuristics in a dynamic job shop with weighted tardiness objectives. International Journal of Production Research, Vol. 37, No.1, pp.165-187.

Liao, C. \& Juan, H. (2007). An ant colony optimization for single-machine tardiness scheduling with sequence-dependent setups. Computers and operations research, Vol. 34, No. 7, pp.1899-1909.

Mastrolilli, M. \& Gamberdella, L.M. (2000). Effective neighbourhood for the flexible job shop problem. Journal of Scheduling, Vol. 3, No.1, pp.3-20.

Mehrabad, M.S. \& Fattahi, P. (2007). Flexible job shop scheduling with tabu search algorithms. The International Journal of Advanced Manufacturing Technology, Vol. 32, No. 5-6, pp. 563-570.

Merkle, D.; Middendorf, M. \& Schmeck, H. (2002). Ant Colony Optimization for resourceconstrained project scheduling. IEEE Transactions on Evolutionary computation, Vol. 6, No. 4, pp.333-345.

Mesghouni, K.; Hammadi, S. \& Borne, P. (1998). Evolution programs for job shop scheduling. Proceedings of the IEEE international conference on computational cybernetics and simulation, Vol. 1, pp.720-725.

Moon, J. \& Lee. J. (2000) Genetic Algorithm Application to the Job Shop Scheduling problem with Alternative Routing. Technical report-Brain Korea 21 logistics Team, Pusan National University.

Najid, N.M., Dauzere-Peres, S. \& Zaidat, A. (2002). A modified simulated annealing method for flexible job shop scheduling problem. Proceedings of the IEEE International Conference on Systems, Man and Cybernetics, Vol. 5, pp. 6-9.

Nasr, N. \& Elsayed, E.A. (1990). Job Shop Scheduling with Alternative Machines. International Journal of Production Research, Vol. 28, pp. 1595-1609.

Pan, Y. \& Shi, L. (2008). New hybrid optimization algorithms for machine scheduling problems. IEEE Transactions on Automation Science and Engineering, Vol. 5, No. 2.

Pinedo, M.L. (1995). Scheduling: theory, algorithms and systems. Englewoodcliffs, New Jersey.

Pinedo, M.L. (2005). Planning and scheduling in manufacturing and services. Springer, New York.

Quiroga, O.; Zeballos, L. \& Henning G.A. (2005). Constraint Programming approach to tool allocation and resource scheduling in FMS. In: Proceedings of the 2005 IEEE International conference on Robotics and automation ICRA-2005, pp. 3715-3720.

Rajendran, C. \& Ziegler, H. (2004). Ant-colony algorithms for permutation flowshop scheduling to minimize makespan/total flowtime of jobs. European Journal of Operational Research, Vol. 155, No. 2, pp.426-438.

Rossi, A. \& Dini, G. (2007). Flexible job-shop scheduling with routing flexibility and separable setup times using ant colony optimization method. Robotics and Computer-Integrated Manufacturing, Vol. 23, No. 5, pp.503-516.

Stutzle, T. \& Hoos, H.H. (2000). Max-min ant system. Future Generation Computer Systems, Vol. 16, pp.889-914.

Tay, J.C. \& Ho, N.B. (2008). Evolving dispatching rules using genetic programming for solving multi-objective flexible job-shop problems. Computers and Industrial Engineering, Vol. 54, pp.453-473. 
Thomalla, C.S. (2001). Job shop scheduling with alternative process plans. International Journal of Production Economics, Vol.74, No.1-3, pp.125-134.

Vairaktarakis, G.L. \& Cai, X. (2003). The value of processing flexibility in multipurpose machines. IIE transactions, Vol. 35, pp.763-774.

Weil, G.; Heus K.; Francois, P. \& Poujade, M. (1995). Constraint programming for nurse scheduling. IEEE Engineering in medicine and biology magazine, Vol. 14, No. 4, pp.417422.

Wilhelm, W. \& Shin, H. (1985). Effectiveness of alternative operations in a flexible manufacturing system. International Journal of Production Research, Vol. 23, pp.65-79. 


\title{
Analytical model of current sheath path based upon shock wave trajectory of plasma layer in a plasma focus device
}

\author{
Reza Amrollahi and Morteza Habibi \\ Amirkabir University of Technology(Tehran Polytechnic)
}

Iran

\section{Introduction}

In plasma focus generators the magnetic energy is stored behind the moving current sheath (Mather,1971). A portion of this energy is converted into plasma energy during the rapid collapse of the current sheath towards the axis beyond the end of the central electrode. Electrical breakdown generates some initial plasma configuration through which the discharge current can flow and at very low pressure a discharge can develop within the whole inter-electrode volume. The current sheath formed at the end of the breakdown phase is accelerated by Lorentz force towards the open end of the inner electrode and then the current sheath sweeps around the end of the anode electrode and finally implodes due to the inward $\mathrm{J} \times \mathrm{B}$ force. When the current sheath reaches the end of the central electrode, it reverses over itself and collapses radially inward, heating the pinching plasma enclosed in it (Mather,1965). The radial compression of CS is open at one end. Hence a gas dynamic shock is propagated ahead of the CS into the undisturbed filling gas (Soto,2005). The snowplow model is used for axial acceleration of CS to obtain axial trajectory, CS speed and current profile. As the CS is assumed to be infinitesimally thin, no information of density is contained in the physics of the equation of motion, although an estimate of density may be obtained by invoking shock wave theory (Lee et al,1988). For a given density and temperature, plasma equilibrium models can be used to calculate the plasma states and emission spectra with the knowledge of the rates of transition and related parameters. The collisional radiative model was developed to fill the gap of several orders of magnitude in electron density where neither the LTE model nor the corona equilibrium is valid. This is a modification of the corona model which takes into account collisional transitions as well as radiative decay from higher bound levels, and three body as well as radiative recombination. The main difficulty with this model is its complication, need of computer time, etc. In the calculations the corona model has been applied as an approximation for simulating the argon plasma in the plasma focus. This could give sufficiently accurate simulation results (Bates et al,1962 \& Yanagidaira,1999). A scheme of a plasma focus system and also the configuration of the 2D cylindrical geometry shock wave at the radial phase of plasma layer are shown in Figure 1. We have developed a new model 
to predict accurately the CS trajectory before the dense plasma pinch formation driven by magnetic force. The path of CS and shock wave are correlated together and if we know the path of the shock front at the radial compression of CS, we can simulate the trajectory of imploding CS. this results can be used for the experimental design of plasma focus and also for stabilizing the hydrodynamic instabilities that affect the implosion of current sheath and the final pinch uniformity. As we will show, the results are in a good agreement with the simulation results of shock wave and CS trajectories which is obtained by the three phase theory of plasma focus performance (Mathuthu et al,1997).
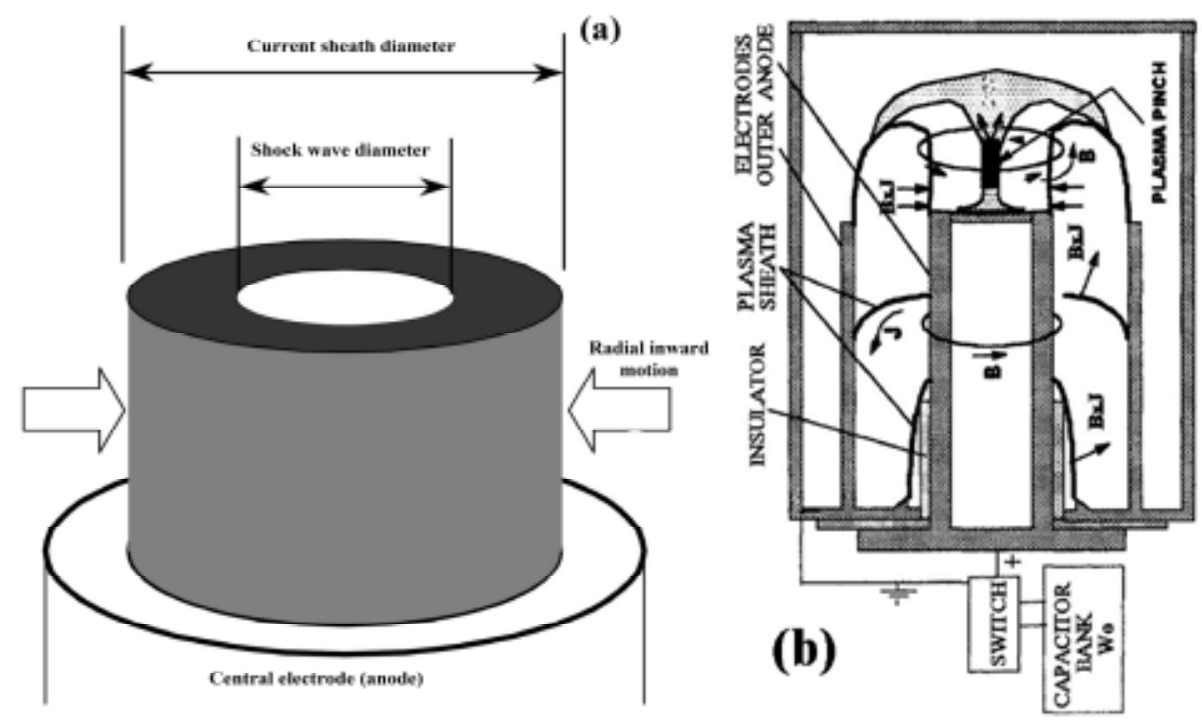

Fig. 1. (a) - conceptual drawing of plasma focus, (b)- configuration of the 2D cylindrical geometry shock wave

\section{Analytical model}

In the plasma focus model a radially implosive plasma slug is formed above the anode in the radial compression of CS (Lindemuth,1982). As it is simplified in Figure 2, plasma slug is formed and compresses radially inward until the shock front meet at the center axis. This slug is driven by the radial inward magnetic piston and plasma column continue compress to a narrow column region and results in a hot and dense plasma. Due to plasma focus geometry and plasma layer dynamic, the motion of the plasma slug can be described by the cylindrical geometry 2D shock wave equations. Conceptual design of the device performed precisely so that the plasma layer moves isentropic between two electrodes. Therefore we can consider an ideal cylindrical magnetic piston of dense plasma produced by electrical discharge between the electrodes of a plasma focus system. At the end of the axial rundown phase, the plasma will form a column at the axis and finally collapse and the period of the radial phase is approximate $50 \sim 200 \mathrm{~ns}$, depending on the plasma focus machine characteristics. Because of such short living plasma we can suppose that the radius of this magnetic piston decreases so rapidly that a strong shock is driven in front of the wall toward the axis of the cylinder. 


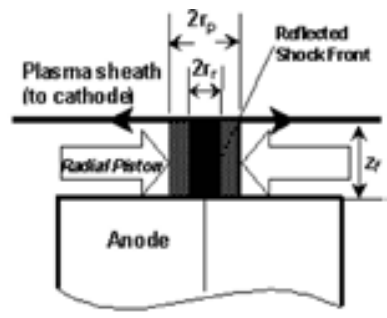

(b)- Reflected Shock Phase

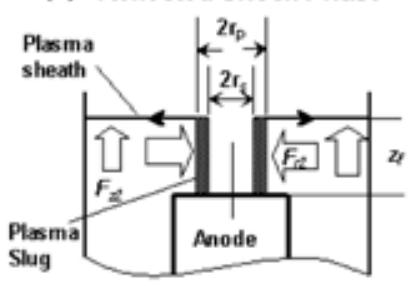

(a)-Radial Inward Shock Phase

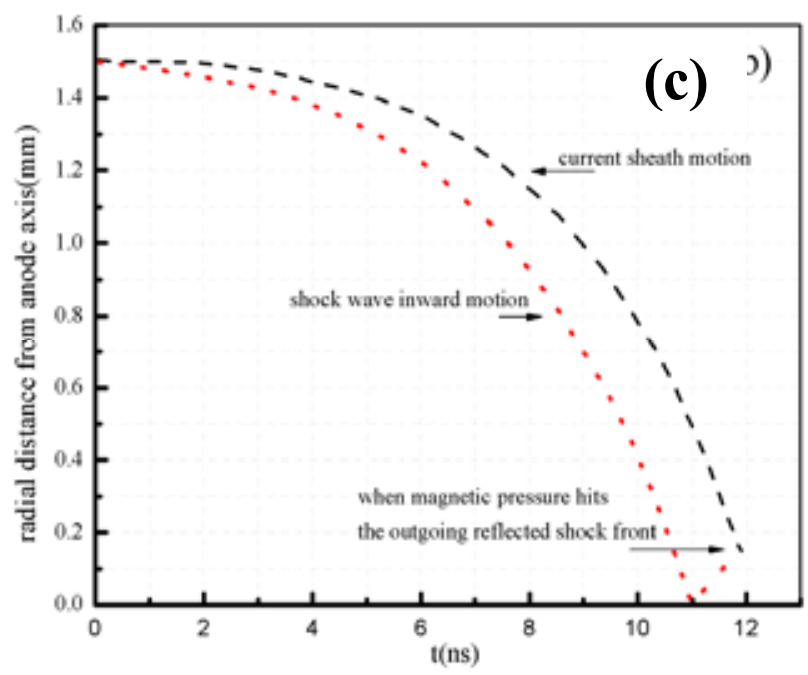

Fig. 2 .a) radial inward shock phase b) reflected shock phase of plasma focus, and c) formation of shock wave driven by CS inward motion

Due to high isentropic behavior of plasma slug we suppose that two particles that are located at different radii in the cylindrical CS, their respective radii will always the same such that the particle initially closer to the axis will always be closer to the axis. This immediately leads to a law for conservation of mass and a method for labeling each particle. In Figure 3 the path of current sheath CS ( $t)$, the path of shock wave $S(t)$, and the path of any particle $\mathrm{P}(\xi, \mathrm{t})$ are plotted.

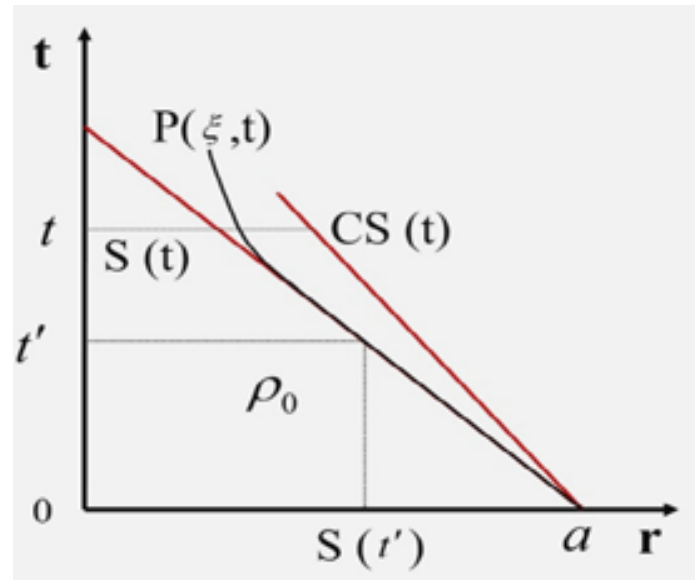

Fig. 3. the path of current sheath CS $(t)$, the path of shock wave $S(t)$, and the path of any particle $\mathrm{P}(\xi, \mathrm{t})$

$\xi$ corresponds to the mass between the CS and considered particle and $t^{\prime}$ refers to the time when the shock passes over the particle. Thus $t^{\prime}$ may be regarded as a function of $\xi$ and 
according to figures 1 , and 2 , we define $\xi=\pi \rho_{0}\left(a^{2}-S\left(t^{\prime}\right)^{2}\right)$ in which $\rho_{0}$ and $a$ are undisturbed gas density and radius of the central electrode respectively (Chernyi,1956, Freeman,1956). The momentum equation is seen to be $\rho \frac{\partial^{2} r}{\partial t^{2}}=-\frac{\partial P}{\partial r}$ where $P$ is the pressure acting on the particle to accelerate it. The process is assumed to be entirely isentropic except fot a jump in entropy as the shock crosses the particle's pass. Therefore the ratio $\frac{P}{\rho^{r}}$ is a constant for each particle as it travels from the shock toward the axis of cylinder. This constant is given by the conditions immediately after the shock. $\gamma$ is the ratio of specific heats(for example $\gamma=1.667$ for Ar as filling gas). The pressure and density of a particle immediately after the shock $\left(P_{i}\right.$ and $\left.\rho_{i}\right)$ can be found by using the shock relations in conjunction with the perfect gas law as $P_{i}\left(t^{\prime}\right)=\rho_{0}(1-\varepsilon) \dot{S}^{2}\left(t^{\prime}\right)$ and $\rho_{i}=\rho_{0} / \varepsilon$ in which $\varepsilon=\frac{\gamma-1}{\gamma+1}$ and $\dot{S}\left(t^{\prime}\right)$ is the velocity of the shock as it crosses the particle $\zeta\left(t^{\prime}\right)$. Thus the isentropic condition is $\frac{P}{\rho^{\gamma}}=\rho_{0}^{1-\gamma} \varepsilon^{\gamma}(1-\varepsilon) \dot{S}^{2}\left(t^{\prime}\right)$. The conservation relation will now be combined into one relationship. At any later time, $\zeta$ may be found by taking the integral $\xi=2 \pi \underset{C S(t)}{\int \rho(\zeta, t)}(r, t) r d r \quad \xi=\pi \rho_{0}\left(a^{2}-S\left(t^{\prime}\right)^{2}\right)$ in which $\rho(r, t)$ is the density at any point on the $(r, t)$ plane. Thus we can conclude that $\left.P^{2}(\zeta, t)=S^{2}(t)+\frac{1}{\pi} \int_{\zeta}^{\zeta_{s}} \rho_{0}^{1 \gamma}(1-\varepsilon) \varepsilon^{\gamma} \dot{S}^{2}\left(t^{\prime}\right)\right\}^{\frac{1}{\gamma}} P^{\frac{1}{\gamma}} d \zeta$ where $\zeta_{s}$ is the value of $\zeta$ at the shock at any time $t$. From the equation of momentum we find that $\frac{\partial P}{\partial r} d r=\frac{1}{2 \pi r} \frac{\partial^{2} r}{\partial t^{2}} d \zeta$. Upon integration from the shock to any particle this becomes $P(\zeta, t)=P_{i}(t)+\frac{1}{2 \pi} \int_{\zeta_{s}(t)}^{\zeta} \frac{\partial^{2} r(\zeta, t)}{\partial t^{2}} \frac{d \zeta}{r(\zeta, t)}$. Let us putting this information back into equation of $P^{2}(\zeta, t)$ and nondimensionalize the quantities appearing in the equation as $x=\frac{r}{a}, x_{s}=\frac{S}{a}, \mathrm{Z}=\frac{\zeta}{\pi a^{2}}, \tau=\frac{t}{t_{0}} . t_{0}$ is chosen so that $\tau$ is in $\mu s$. Due to Schlieren images and visible-radiation pictures taken with a high-speed camera before and after the maximum compression of plasma layer most interesting cases may be covered by assuming a parabolic shock trajectory as $x_{s}=1-\alpha \tau-\beta \tau^{2}$ in which $a$ is nondimensional velocity of the shock and $\beta$ is the shock's constant acceleration or deceleration toward the axis depending upon $\beta$ is positive or negative (Sadowski \& Sholz, 2008). Substituting this information into equation of $P^{2}(\zeta, t)$, we find $x^{2}(Z, \tau)=x_{s}^{2}(\tau)+\varepsilon \int_{Z}^{Z_{s}=1} \int_{x_{s}^{2}(\tau)}\left[\frac{\alpha^{2}+4 \beta(1-\sqrt{1-Z})}{(\alpha+2 \beta)^{2} \frac{1}{2(1-\varepsilon)} \int_{Z}^{1 x_{s}^{2}} \frac{{ }^{2} x}{\tau^{2}} \frac{d Z}{x(Z, \tau)}}\right]^{\frac{1}{\gamma}} d Z$. this 
equation implies that a particle $Z$ is, at a time $\tau$, at a position away from the shock by a distance equal to $\mathcal{E}$ multiplied by an integral, the integrand of which consists of a numerator representing the isentropic condition and a denominator which is a constant fraction of the pressure. $\varepsilon$ varies from 0 for $\gamma=1$ to 0.25 for $\gamma=1.667$. the piston trajectory is $x(0, t)$, that is the piston is always at the particle $Z=0$. Since the right hand side of above equation involves a second derivative of the desired solution, a method of iteration must be used. Due to the difficulty in taking derivatives numerically we should search for an analytic solution that may be placed back into the equation. From this analytic solution a second approximation may be found numerically and compared to the first solution. As a first approximation, it may be assumed that the pressure doesn't change much between the shock wave and CS. In effect we assume $\frac{1}{2(1-\varepsilon)} \int_{z}^{Z_{s}=1-x_{s}^{2}} \frac{\partial^{2} x}{\partial \tau^{2}} \frac{d Z}{x} \prec \prec(\alpha+2 \beta \tau)^{2}$. This relation is good when two conditions are met. First we must not to be too close to the axis. Otherwise $x$ will be small. Secondly the second derivative of $x$ with respect to $\tau$ should be small and the denominator of the integrand of the large integral may approach zero. These conditions are met at least in the beginning stage of piston's propagation. With this assumption, we conclude that $x^{2}(Z, \tau)=x_{s}{ }^{2}(\tau)+\frac{\varepsilon}{(\alpha+2 \beta \tau)^{2 / \gamma}} \int_{z}^{1-x_{s}^{2}(\tau)}(\alpha+4 \beta-4 \beta \sqrt{1-Z})^{\frac{1}{\gamma}} d Z$ which readily integrates to a first approximation for $x$. The equation of CS path for the first approximation becomes $x_{\text {first }}^{2}=x_{s}^{2}+\varepsilon\left(1-x_{s}^{2}\right)-\varepsilon Z$. In order to find the second approximation, we must substitute the second derivative of $x_{\text {first }}(Z, \tau)$ with respect to $\tau$ into the subintegral of $x^{2}(Z, \tau)$ equation. Therefore we have $x_{\text {second }}^{2}=x_{s}^{2}+\varepsilon \int_{Z}^{1-x_{S}^{2}}\left(\frac{2 \varepsilon}{\Omega-\ln (\Gamma-Z)-\Sigma /(\Gamma-Z)}\right)^{\frac{1}{\gamma}} d z$ in which $\Omega=1+\varepsilon+\ln \frac{x_{s}^{2}}{\varepsilon}, \Sigma=\frac{(1-\varepsilon) x_{s}^{2}}{\varepsilon}$ and $\Omega=1+\varepsilon+\ln \frac{x_{s}^{2}}{\varepsilon}, \Sigma=\frac{(1-\varepsilon) x_{s}^{2}}{\varepsilon} \quad \Gamma=\Sigma+1 \quad$ (Drake, $2005 \&$ Lister, 1960).

\section{Simulation results and discussion}

We invoked a program to perform a numerical integration of nondimensional equation obtained for $x^{2}(Z, \tau)$ to simulate path of CS respect to a parabolic strong shock wave trajectory [7]. The program was run for $A=0.999$ and $B=0.001$ and was also run for $A=1$ and $B=0$. The general philosophy of the program is first to determine what time steps to use, and then when the time steps are known, to calculate all quantities that are dependent only upon time for the first $\Delta \tau$. Then at the time under consideration the quantities that depend on $Z$ are calculated. Specifically the value of the integrand for $Z=Z_{s}$ is first calculated. Then the values of $Z$ for which we want the particle positions spelled out are determined. The program does this by taking $Z_{S^{\prime}}$ rounding it off to the next 
lowest 0.05 and then using in steps of 0.05 , the steps for which the two integrations are performed. The subintegral is found for the interval from $Z_{S}$ to the next lower $Z$. With this value of the subintegral, the total integral may be found giving $x$ for the rounded off $Z$ and the time $\Delta \tau$. Using the next lower value of $Z$, the next portion of the subintegral is added to the value obtained above. Similarly the next portion of entire integral is added on to the part already found. This process is carried on until we reach the value $Z=0$ which is the piston. At this time we proceed to the next time and repeat the entire process.

Figure 4 shows a constant velocity shock $(\beta=0)$ with the CS path computed for $\gamma=1.667$ and $\gamma=1.1$.
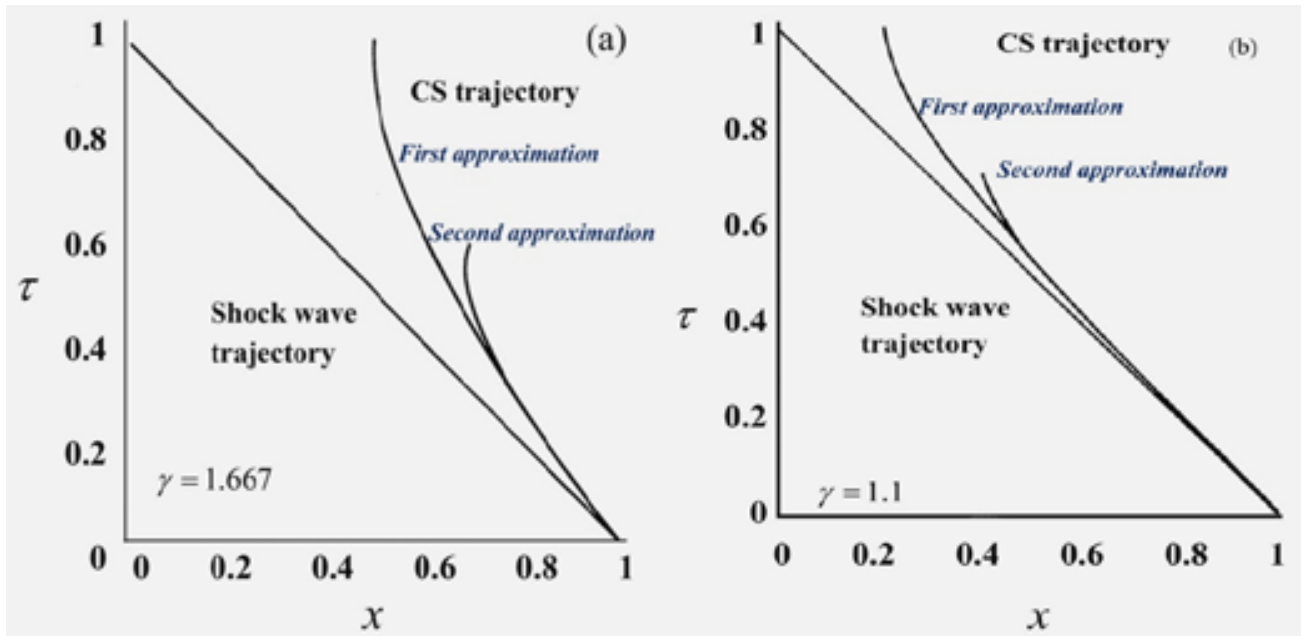

Fig. 4. CS trajectory for constant velocity shock

In Figure 5, CS trajectory for accelerating shock and in Figure 6, CS trajectory for decelerating shock $(\gamma=1.667$, and 1.1$)$ simulated.
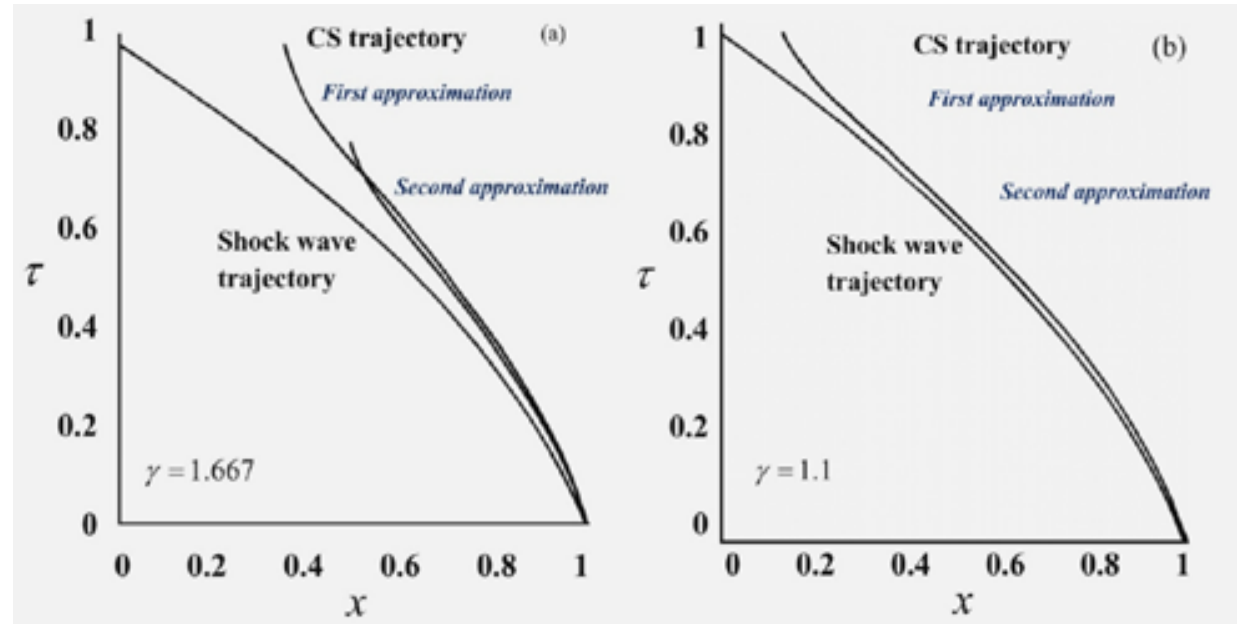

Fig. 5. CS trajectory for accelerating velocity shock 
In the pinch phase of focused plasma much of the energy available is absorbed in the ionization process (Shan et al,2000). Here the real value of $\gamma$ when argon is used as working gas may be expected would be closer to 1.1 than to 1.667 . As it shown in Figure 4, for $r=1.667$ it is seen that the first and second approximations for the CS trajectory are very close together until the CS reaches a radial position 0.75 . At this point the second approximation diverges from the first approximation and ultimately turns back toward its initial position. Physically a decrease in the denominator corresponds to a decrease in pressure at the CS. It is logical the pressure decrease from the shock to the CS at a given time because in this region of the flow, there is quasi-steady supersonic flow into a converging channel which implies a decrease in velocity and a corresponding adverse pressure gradient [12]. Since the conditions behind the shock are fixed, the pressure at the CS must be steadily decreasing as the gap between the shock and CS widens. As we see in Figure 5, for $r=1.1$ there is no difference large enough to be seen between the first and second approximations until the second approximation reaches the zero pressure limit. This occurs at $x(0, \tau)=0.27$ that is much smaller than the final radius of the CS for the constant shock.

This fact implies that the CS pushing an accelerating shock has control over the shock for a longer time than the CS pushing a constant velocity shock. For $\gamma=1.667$, the accelerating shock has a piston path given by the second approximation that is closer to the center than the first approximation. That is, in order to accelerate the flow, the pressure at the piston must be greater than the pressure at the shock. For the first approximation, this pressure difference is neglected. In the second approximation it is included. This effect is also present for the $\gamma=1.1$ case; however, it is so small that it cannot be seen on the scale of Figure 5 . Figure 6 shows the case of a decelerating shock. There is little new on this graph except that the piston turns back even sooner than it does for the constant velocity shock.
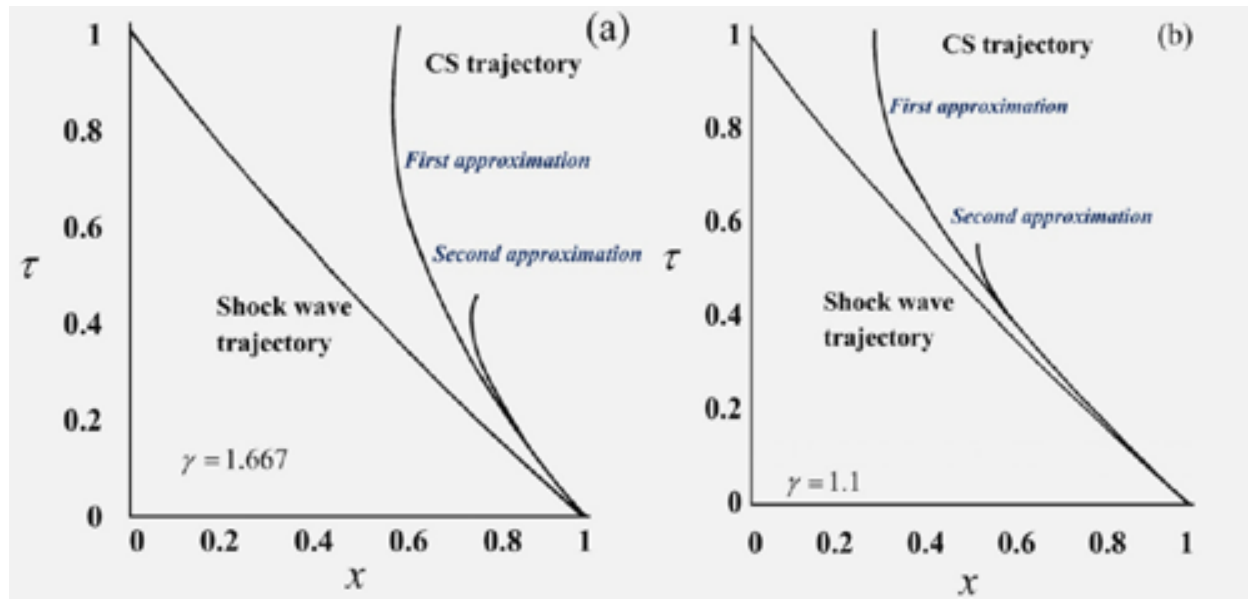

Fig. 6. CS trajectory for decelerating velocity shock

To compare the results with the three phase theory of plasma focus performance We solved the axial and radial phases' current sheath equations coupled with equivalent plasma focus 
circuit equations (Habibi et al,2008). In Figure 7, shock wave and CS trajectories obtained by the three phase theory have been compared with the simulation result of the analytical model. As we see, there is a good agreement between two models especially when the CS moved toward the axis of central electrode and similarity of curves obtained by these models can illustrate validity of presented analytical model.

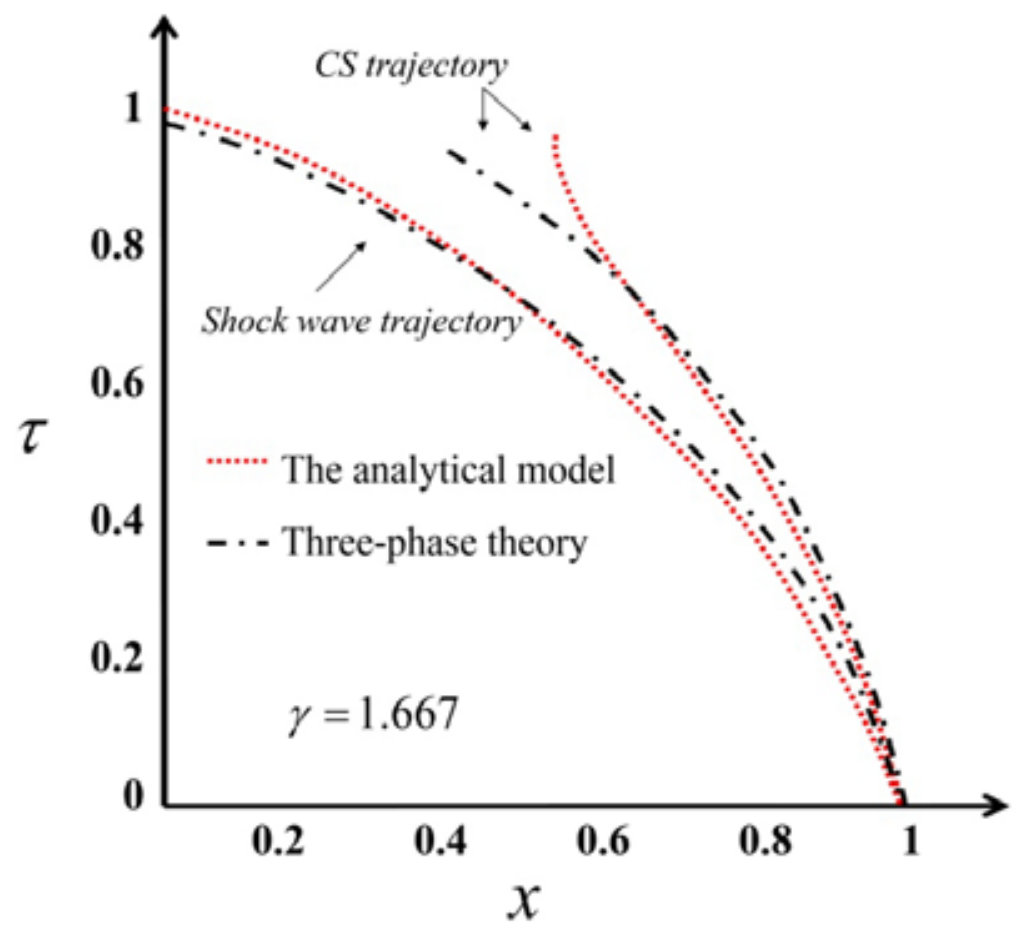

Fig. 7. trajectory of shock wave and CS based upon proposed analytical model and the three phase theory

\section{Conclusion}

In this paper we presented an analytical model to describe the CS path based upon the shock wave trajectory before dense pinch formation in a plasma focus system. When the results from the analytical method are compared to the results obtained by the three phase theory of plasma focus, it is seen that the trajectory of CS and shock wave were almost the same for accelerating velocity of shock wave. The results of first and second approximations for the straight shock wave don't have physical relevance. When this procedure is extended to the accelerating and decelerating shock trajectories, it would seem to imply that the first and second approximation are very close together and also an accelerating shock is controlled more by its piston than is a decelerating shock wave. Because of complexity of pinched plasma behavior, this approach can be applied to investigate the dynamic of accelerating plasma layer. 


\section{References}

Bates, D.R. , A.E. Kingston and R.W.P. McWhirter, (1962) Porc. Roy. Soc., A 267-297

Drake, P., (2005) High-Energy-Density Physics, ISBN-10 3-540-29314-0 Springer Berlin Heidelberg New York

Habibi M., Amrollahi R. , Attaran M., and Etaati R.,(2008) Design, construction and the first experiments on the Amirkabir Plasma Focus (APF) facility, Plasma Devices and operations, Vol. 16, No. 3, 163-169

Lee S., T. Y. Tou, S. P. Moo, et al, (1988) a simple facility for the teaching of plasma dynamics and plasma nuclear fusion, Am. J. Phys. 56,1,62-68

Leopoldo Soto, (2005)New trends and future perspectives on plasma focus research, Plasma Physics and Control Fusion, 47,361-381

Lindemuth, I. R. , B. C. Freeman,( 1982) Shock dynamics and neutron production in an explosive generator driven dense plasma focus, Appl. Phys. Lett. 40(6)

Lister, M. (1960) "The Numerical Solutions of Hyperbolic Partial Differential Equations by the Method of Characteristics," Chap. 15 of Mathematical Methods for Disital Computers, John Wiley \& Sons, Inc.

Mather J. W., (1971) Methods of Experimental Physics, P. 187, 9B, Academic Press, New York

Mather J. W., (1965)Formation of high density deuterium plasma focus, the physics of fluid, V.8, N.2

Mathuthu M., Zengeni G., Gholap A. V. , (1997) The Three-Phase Theory for Plasma Focus Devices, IEEE TRANSACTIONS ON PLASMA SCIENCE, VOL. 25, NO. 6

Sadowski, M. D. , M. Sholz, (2008) The main issues of research on dense magnetized plasmas in PF discharges, Plasma Sources Sci. Technol. $17-024001$

Shan.B., M.H. Liu, R. S. Rawat, and S. Lee, X-ray Emission Properties of Neon and Argon Plasmas in Conventional and Gas-puff Plasma Focus Devices, Proceedings of Regional Conference on Plasma Research in 21st Century, Thailand, 75-79 (2000)

Yanagidaira, T. , K. Shimoda, Y. Ono, and K. Hirano, (1999)"Soft x-ray emission in the neon gas-puff plasma focus", Phys. of Plasmas, 6-4679-4684 


\title{
Authentication with RIPEMD-160 and other alternatives: A Hardware Design Perspective
}

\author{
H. Michail(1) , A. Gregoriades (2), V. Kelefouras(1), \\ G. Athanasiou $(1)$, A. Kritikakou ${ }^{(1)}$ and C. Goutis(1), \\ (1)Electrical \& Computer Engineering Department, University of Patras, Greece \\ (2) Computer Science \& Engineering Department, European University, Cyprus
}

\section{Abstract}

Taking into consideration the rapid evolution of communication standards that include message authentication and integrity verification, it is realized that constructions like MAC and HMAC, are widely used in the most popular cryptographic schemes since provision of a way to check the integrity of information transmitted over or stored in an unreliable medium is a prime necessity in the world of open computing and communications.

MACs are used so as to protect both a message's integrity as well as its authenticity, by allowing verifiers (who also possess the secret key) to detect any changes to the message content. In every modern cryptographic scheme that is used to secure a crucial application that calls for security, a keyed-hash message authentication code, or HMAC, is incorporated. Beyond HMAC, a block cipher algorithm is also incorporated (i.e like AES), thus resulting to the whole security scheme. The proposed hardware design invokes a number of optimizing techniques like pipeline, evaluation-based partial unrolling, certain algorithmic transformations in space and time and computational re-ordering, leading to a highthroughput and low-power design for the whole HMAC construction. Finally, a new algorithm, CMAC, for producing message authenticating codes (MACs) which was recently proposed by NIST, is also described. The proposed security scheme incorporates a FIPS approved and a secure block cipher algorithm (that might have already been deployed in the security scheme) and was standardized by NIST in May, 2005. This work concludes with an efficient hardware implementation of the CMAC standard.

\section{Introduction}

Security issues are crucial as long as the further spread of internet applications is concerned. This is true particularly today considering the fact that sensitive data are stored in networked computers and transferred via several types of networks. It is critical that this sensitive data are protected from eavesdroppers, thieves or generally anyone who tries to illegally acquire the sensitive data. Thus it is important to store the data safely and in such 
way that will discourage anyone from getting into them. Beliefs and claims for lack of security in our internet transactions could lead someone to assume that is the main reason for many people to avoid transactions via internet.

Cryptography is the scientific field that among other has to do with offering security over internet. Cryptography is a significant weapon in our quest to protect our treasure: the sensitive data. Seen as a process, we can assume that cryptography is nothing more than a simple mathematic function that transforms one set of numbers (or characters, objects etc) to another. This means that it is used to change comprehensible data (messages) to a seemingly corrupted group of numbers (characters). Cryptography services can be conducted by a wide range of applications offering specific security requirements like authentication, integrity, non-repudiation, etc. Cryptographic features are incorporated in almost every application that has to do with transactions that handle personal and sensitive data.

Applications like the Public Key Infrastracture (PKI) (NIST, 2001 b), IPSec (NIST, 2005 a), Secure Electronic Transactions (SET) (Loeb, 1998), and the 802.16 (Johnston \& Walker, 2004) standard for Local and Metropolitan Area Networks incorporate authenticating and other services. These other services often include encryption but in this chapter we will focus on ways to achieve authentication efficiently through different mechanisms and on how to achieve high performance implementations.

All the pre-reffered applications presuppose that an authenticating module that includes a certain cryptographic function called "hash function" is nested in the implementation of the application. Hash function $\mathrm{H}(\mathrm{M})$ is a transformation that takes an input message $\mathrm{M}$ and returns a fixed-size string, which is called the hash value $h$ (that is, $h=H(M)$ ). Hash functions with just this property have a variety of general computational uses, but when employed in cryptography, the hash functions are usually chosen to have some additional properties. The basic requirements for a cryptographic hash function are as follows.

- $\quad$ The input can be of any length.

- $\quad$ The output has a fixed length.

- $\mathrm{H}(\mathrm{x})$ is relatively easy to compute for any given $\mathrm{x}$.

- $\mathrm{H}(\mathrm{x})$ is one-way.

- $\quad \mathrm{H}(\mathrm{x})$ is collision-free.

A hash function $\mathrm{H}$ is said to be one-way if it is hard to invert, where "hard to invert" means that given a hash value $h$, it is computationally infeasible to find some input $x$ such that $H(x)$ $=h$. If, given a message $x$, it is computationally infeasible to find a message $y$ not equal to $x$ such that $\mathrm{H}(\mathrm{x})=\mathrm{H}(\mathrm{y})$, then $\mathrm{H}$ is said to be a weakly collision-free hash function. A strongly collision-free hash function $\mathrm{H}$ is one for which it is computationally infeasible to find any two messages $x$ and $y$ such that $H(x)=H(y)$. The hash value represents concisely the longer message or document from which it was computed meaning that the target of a hash function is to provide a "signature" of $M$ that is unique. One can think of the hash value as a "digital fingerprint" of the larger document.

From the algorithmic part of view, hash functions have a lot of resemblances to compression algorithms. Hash functions are iterative algorithms, which in order to compute the final hash value, perform a number of identical or slightly different operations. Each operation is based on a special block that receives as inputs, a message block from the initial message $\mathrm{M}$ of arbitrary length and the outputs of the previous blocks of the message. It is common to reference the final hash value as Message Digest (MD) as a tribute to Professor Ronald Rivest who authored the MD family hash functions. 
Other applications that require the usage of a hash function for authentication are the Virtual Private Networks (VPN's) (NIST, 2005 a) that companies are establishing in order to exploit on-line collaboration. Moreover digital signature algorithms like DSA (NIST, 1994) that are used for authenticating services like electronic mail, electronic funds transfer, electronic data interchange, software distribution, data storage etc are based on using a critical cryptographic primitive like hash functions. Hashes are also used to identify files on peer-to-peer file sharing networks. For example, in ed2k links which are links used by the eDonkey2000 family of P2P programs, such as eDonkey2000 itself, e-Mule, Hybrid MLDonkey, and others. These links are primarily used to provide a unique identifier for file without referring to a specific location, like in FTP and HTTP links. In an ed2k link the hash is combined with the file size, providing sufficient information for locating file sources, downloading the file and verifying its contents.

Furthermore cryptographically secure hashes are used in signed code systems such as Microsoft's Windows Update (Microsoft, 2005) to ensure the integrity and authenticity of downloaded programs and patches. Hashes are also used to periodically check on-disk code integrity in systems such as Tripwire (Kim \& Spafford, 1994). Last but not least US federal on 2004 came up with a new plan to help secure electronic voting (Biever, 2004), employing a hash function so as to achieve higher level of security and therefore more confidence in evoting than ever before.

Hash functions serve a dual role in practical signature schemes: they expand the domain of messages that can be signed by a scheme and they are an essential element of the scheme's security (Mironov, 2005). Hashing cores are also critical for security in networks and mobile services, as in SSL (Stephen, 2000), which is a Web protocol for establishing authenticated and encrypted sessions between Web Servers and Web clients. A common method for client authentication is to require the client to present password previously registered with the server. Storing passwords of all users on the server poses an obvious security risk. Fortunately the server need not know the passwords - it may store their hashes (together with some "salt" to frustrate dictionary attacks) and use the information to match it with the hashes of alleged passwords (Morris \& Thompson, 1979).

\section{MACs: HMAC and CMAC}

Hashing functions are also the main modules that exist in the HMAC (NIST, 2007 a) algorithm that produces Message Authentication Codes (MACs). A cryptographic message authentication code (MAC) is a short piece of information used to authenticate a message. A MAC algorithm accepts as input a secret key and an arbitrary-length message to be authenticated, and outputs a MAC (sometimes known as a tag). The MAC value protects both a message's integrity as well as its authenticity, by allowing verifiers (who also possess the secret key) to detect any changes to the message content.

A keyed-hash message authentication code, or HMAC, is a type of message authentication code (MAC) calculated using a cryptographic hash function in combination with a secret key. As with any MAC, it may be used to simultaneously verify both the data integrity and the authenticity of a message. Any iterative cryptographic hash function may be used in the calculation of an HMAC. The cryptographic strength of the HMAC depends upon the cryptographic strength of the underlying hash function, on the size and quality of the key and the size of the hash output length in bits. 
Taking into consideration the rapid evolution of the communication standards that include message authentication and integrity verification, we realize that constructions like HMAC, are widely used in the most popular cryptographic schemes since provision of a way to check the integrity of information transmitted over or stored in an unreliable medium is a prime necessity in the world of open computing and communications. Typically, message authentication codes are used between two parties that share a secret key in order to authenticate information transmitted between these parties.

MACs differ from digital signatures, as MAC values are both generated and verified using the same secret key. This implies that the sender and receiver of a message must agree on keys before initiating communications, as is the case with symmetric encryption. For the same reason, MACs do not provide the property of non-repudiation offered by signatures: any user who can verify a MAC is also capable of generating MACs for other messages.

The most common way of producing a MAC is based on the incorporation of a cryptographic hash function, thus resulting to keyed-hash message authentication code, or HMAC. Besides authentication with hash functions, another very popular service that is also incorporated in most applications is encryption/decryption process which is based on the usage of a block cipher algorithm. So it seems that is also useful to have a method for producing MACs using such block cipher algorithms in cases where we want to avoid producing MACs using hash functions.

It was only on May 2005 when NIST (National Institute of Standards and Technology) decided to standardize a new algorithm for producing message authenticating codes (MACs) using secure block cipher algorithms. This new method is the CMAC standard (NIST, 2005 b). This algorithm was standardized recently and thus no many implementations have been presented concerning the CMAC standard. Moreover it has not been widely adopted by industry since its advantages are major but only in certain cases.

Another reason for the non-development of hardware CMAC implementations is that the functionality of a CMAC can be incorporated in a security scheme with an HMAC which uses a hash function and this is the case in most of today's applications. Industry must be persuaded for the necessity of this algorithm if it is to start introducing CMAC hardware implementations, which are costly comparing to software implementations. Later in this section a specific case of an application (e-voting) that can be benefited a great deal from the usage of CMAC instead of HMAC is going to be commented so as to bring out this necessity.

This necessity mainly has to do with the numerous applications that do not call for high throughput implementations (as long as the security part is concerned) but for small-sized or low-power implementations. In these cases the usage of CMAC instead of HMAC is ideal since there is no need to incorporate both a hash function and a cipher block but only a block cipher algorithm like AES (NIST, 2001 a) that can handle all aspects of security in the certain security scheme.

Such security applications are those used in e-voting where there is a lot of time available for the transaction to be performed and thus for the sake of small-sized and low power implementations CMAC seems to be a very good solution. The whole application of evoting consists of numerous widespread portable clients located at places where people vote and few central servers which collect data from all distributed clients. It is obvious that these sessions must be secured so some kind of security scheme should be used. Because the voter has, relatively speaking, a lot of time to interact with the voting device there is no great need 
for high throughput implementations as long as the portable clients are concerned. Instead it is a prior necessity that these portable voting machines are small, have reduced power dissipation and are cheap due to the great number of these devices that are needed in evoting application. However it has to be mentioned that there is a need for high throughput implementation for the corresponding server of the application.

So in this case it seems that the best solution is to use CMAC for authentication instead of HMAC and fully exploit the incorporated cipher block algorithm that is nested in the numerous clients and few servers to encrypt the transmitted data. This way the cost is dramatically reduced since only a block cipher is used instead of a block cipher and a hash function. The same goes for power dissipation and integration area of the portable voting devices. As long as the corresponding servers are concerned multiple instances of the block cipher can be incorporated and certain design techniques can be used so as to achieve high throughput implementations.

Moreover it has to be noticed that this is a trend in our days, considering many standards are either under consideration or published by NIST in 2007 like XTS (NIST, 2007 b) and GCM (NIST, 2008) where security schemes tend to use a cipher block like AES in order to handle all security aspects in an application. It seems that there is a tendency to use only one basic cryptographic component i.e block cipher algorithm AES and based on that to broaden offered security services over storage devices, wireless networks and so on.

Another great advantage of this perspective is that the time-to-market constraint is shorter, besides the reduced cost. The need of providing all kind of security services with the usage of only one basic cryptographic component has emerged, as it is clearly seen especially during the last year with the hot debate about XTS and GCM .

CMAC like XTS and GCM focuses on the service that intends to offer ensuring the security level and simplifying the whole implementation without using sophisticated algorithms for each case. This way we manage to save a significant amount of integration area and power consumption, reduce the design and implementation complexity and offer the chance to concentrate only on the optimization of the incorporated block cipher algorithm in order to improve the performance of the whole security scheme.

Thus in applications where there are a few servers (for centralized data process) and a great number of distributed portable clients (for data collection), that need to be cheap, small and have minor power consumption without great concern about throughput, like in e-voting, the adoption of CMAC for MAC generation seems to be ideal since it will result to efficient solutions with great reliability.

At the end of this chapter CMAC mechanism will be analysed and finally implementation details concerning CMAC will be provided in brief considering the incorporated block cipher algorithm as an installable component.

\section{Current Status and Design Issues}

It is quite clear that all the pre-referred applications that incorporate security mechanisms producing MACs either with the usage of a hash function (HMAC) or a block cipher (CMAC) are addressing more users/clients and thus, the increase of their throughput is a prior necessity, particularly for the corresponding server of these services. This is because the cryptographic system, especially the server, has to reach the highest degree of throughput in order to satisfy immediately all requests for service from all users-clients. In 
some of these cryptographic schemes, where a hash function is used for the authentication part, the throughput of the incorporated hash functions determines the throughput of the whole security scheme.

Many leading companies offer and also improve hardware implementations of hash functions. Although software encryption is becoming more prevalent today, hardware is the embodiment of choice for military and serious commercial applications (Schneier, 1996). The NSA, for example, authorizes only encryption in hardware.

The first reason to do so is speed. Many cryptographers have tried to make their algorithms more suitable for software implementations; however specialized hardware will always win the speed race. Additionally, hashing is often a computation-intensive task. Tying up the computer's primary processor for this is inefficient. Moving hashing tasks to another chip, even if that chip is just another processor, makes the whole system faster.

Efficient software implementations of hash functions have been extensively studied in the literature (McCurley, 1994), (Nakajima \& Matsui, 2002) and (Ballard, 2004). All these studies verify that hardware implementations are much faster than the corresponding software implementations.

The second reason is security. An encryption algorithm running on a generalized computer has no physical protection. A malicious attacker is able to go in with various debugging tools and surreptitiously modify the algorithm without anyone ever realizing it. Hardware encryption devices can be securely encapsulated to prevent this. In (Van Oorschot et al., 2005) the security problems of software implementations were proven for a great variety of different general-purpose processors (including UltraSparc, x86, PowerPC, AMD64, Alpha, and ARM).

The latter mentioned facts were strong motivation to propose some novel techniques and correspondingly designs and implementations for a certain hash function like RIPEMD-160 which was developed in the framework of the EU project RIPE (Race Integrity Primitives Evaluation).The proposed designs introduce a small area penalty but manage to increase significantly the overall throughput of the hash core. Our future goal is to manage to combine these techniques and propose an ultra high throughput design of the RIPEMD-160 hashing algorithm.

This high throughput is essential for certain cryptographic applications such as HMAC mechanism in security schemes of IPSec and SSL/TLS. The security schemes for these applications incorporate encryption and authenticating modules. Lately many implementations of the AES encryption modules have been designed that exceed or approach the limit of 20 Gbps of Throughput (Hodjat \& Verbauwhede, 2004) and operate at very high operating frequencies. So it is crucial to design hash functions that also achieve high throughput and this can be done with the proposed techniques.

However it has to be noticed that RIPEMD-160 is not widely adopted and thus there has not been conducted thorough research in antithesis to MD-5, SHA-1, SHA-256 etc. This chapter intends to be a guide for alternatives ways to achieve authentication such as HMACs with RIPEMD-160, and CMACs with the usage of a block cipher algorithm. Above that, it also intends to propose optimizations for the corresponding hardware designs and implementations as it has already been achieved for more popular hash functions that are also used for authenticating purposes. 


\section{HMAC RIPEMD-160: A Hardware Design Perspective}

\subsection{RIPEMD-160 hash function design architecture}

In this section an efficient pipelined design and implementation of the RIPEMD-160 (Dobbertin et al., 1996) hash function is going to be presented. Then in the following section novel techniques are going to be presented and used in order to optimize the presented implementation so as to achieve higher througput that is essential for servers in security schemes of IPSec, SSL/TLS etc. Finally at the end of this section the whole HMAC-RIPEMD160 system architecture is going to be presented incorporating the optimized version of RIPEMD-160 that will be demonstrated.

The fixed length of each of the processed blocks in RIPEMD-160 is 512 bits. As it is described in (Dobbertin et al., 1996) Ripemd-160 hash function has a bitsize of the hash-result and chaining variable of 160 bits (five 32-bit words). Ripemd-160 uses two parallel processes of five rounds, with sixteen operations for each round $(5 \times 16$ operations for the whole process). The input in every round is five bit words of data, the message word $X_{i}$ and a 32 bit constant $\mathrm{K}_{\mathrm{i}}$.

Unfolding the expressions of $a_{t}, b_{t}, c_{t}, d_{t}, e_{t}$ that describe the five input words, it is observed that $b_{t-1}, c_{t-1}, d_{t-1}, e_{t-1}$ values are assigned directly to outputs $c_{t}, d_{t}, e_{t}$, at respectively. In Eq. (1) the expressions of $a_{t}, b_{t}, c_{t}, d_{t}$ and $e_{t}$ are defined.

$$
\begin{gathered}
e_{t}=d_{t-1} \\
d_{t}=\operatorname{ROL}_{10}\left(c_{t-1}\right) \\
c_{t}=b_{t-1} \\
b_{t}=e_{t-1}+\operatorname{ROL}_{s}\left[f_{t}\left(b_{t-1,} c_{t-1}, d_{t-1}\right)+a_{t-1}+X_{i}+K_{j}\right] \\
a_{t}=e_{t-1}
\end{gathered}
$$

where $\operatorname{ROL}_{x}(y)$ represents cyclic shift (rotation) of word $y$ to the left by $x$ bits and $f_{t}(z, w, v)$ denotes the non-linear function which depends on the round being in process. From Eq.(1), it can be derived that the maximum delay is observed on the calculation of the $b_{t}$, value.

The operation block of RIPEMD-160 is illustarted in Figure 1 where it can be observed that the critical path consists of three addition stages and a multiplexer via which the values pass each time to/and feed the operation block.

The Ripemd-160 hash function consists of two parallel processes of five rounds, with sixteen transformations for each round ( $5 \times 16$ transformations for the process). All the rounds in the processes are similar but each one performs a different operation on five 32-bit inputs. The data (input message) are processed 16 times in each transformation round resulting in 80 transformations performed in total per process. Each operation is performed in every clock cycle and a certain 32-bit word $X_{i}$ has to be supplied to the transformation round. This 32-bit $X_{i}$ results from a specific process on the 512-bit input block_in through appropriate permutations on the initial $16 X_{i}$ word blocks that are exported from 512-bit input block.

The computation of each $X_{i}$ (beyond the first $16 X_{i}$ that are computed by a simple split of the 512-bit input block_in) takes place in a specially designed unit named " $\mathrm{Xi}^{\prime}$ permutation" unit.

The implemented Ripemd-160 core has five primary inputs h0, h1, h2, h3, h4 where the initial values are supplied. These can be the ones specified by the standard or others that have occurred from process of the former block of the message in case that the input message consists of more than one block. However in most cases the input intended to be 
authenticated is just one block. In the unlikely event of messages that consist of many blocks a scheduler is needed in order to apply pipelines stages. This chapter will not focus on this scheduler, as it is sure that it will go beyond the scope of the book.

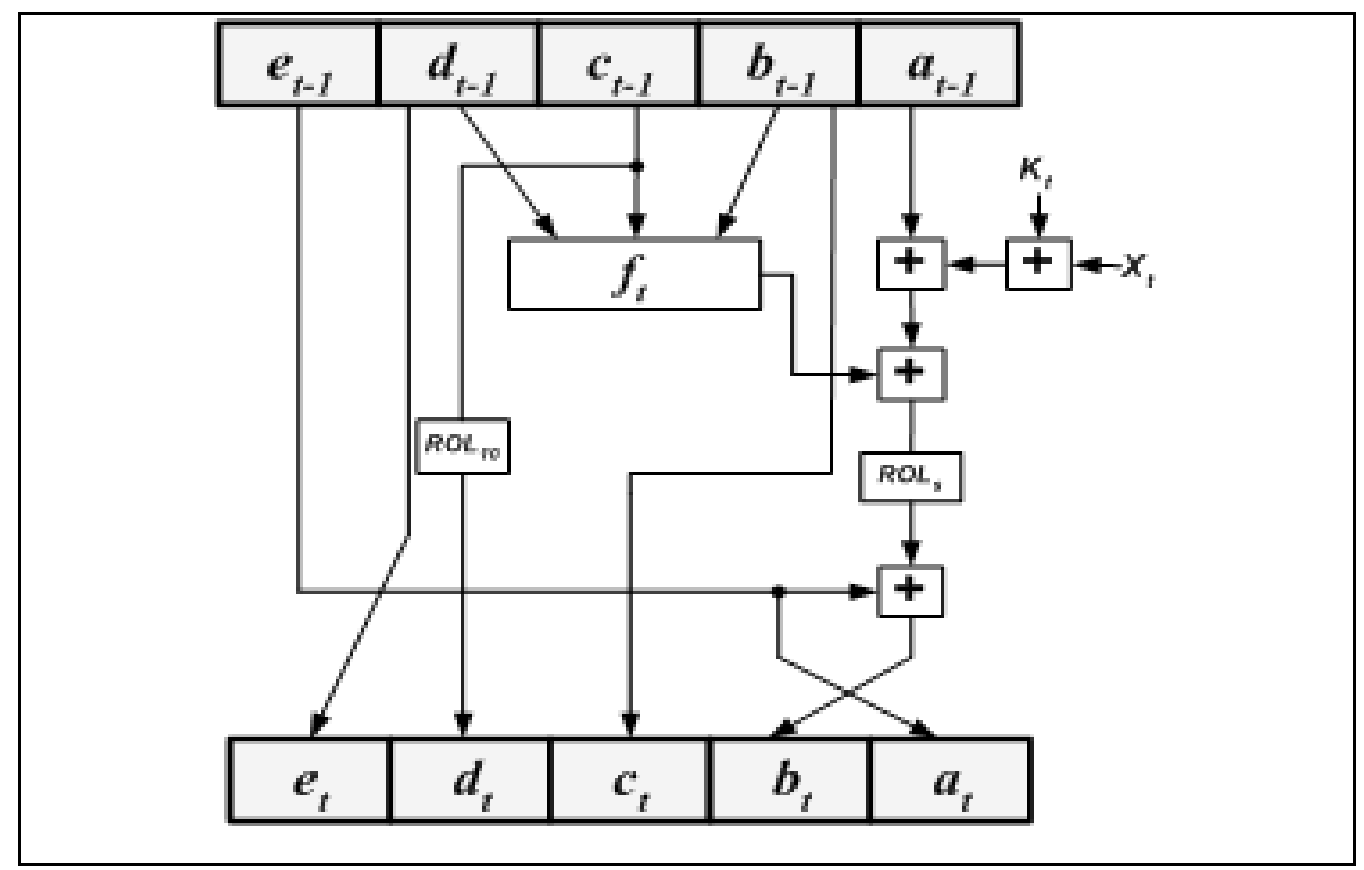

Fig. 1. RIPEMD - 160 operation block (also mentioned as "Round")

The proposed system architecture is illustrated in the following Figure 2. This implementation is suitable for any system that uses the Ripemd-160 hash function, constrained only by the assumption that the start_counter1 signal is applied one clock cycle before the final padded message is available in the 512-bit input block_in of Ripemd-160 core. Concurrently to the arrival of the 512-bit input block_in the signal start_round1 has to be applied and 80 clock cycles later the hash value is pending on the 160-bit output ripemd160 hash value of the Ripemd-160 core.

In order to apply pipeline stages to the Ripemd-160 core, ten 160-bit registers have to be used between the data transformation rounds. In Figure 2, these registers are implanted at the end of each transformation round and this technique ensures that five 512-bit data blocks can be processed at the same time and finally a 160-bit message digest is produced every 16 clock cycles. All multiplexers are controlled by the Count_16 component. The Count_16 component also arranges when the $16 \mathrm{X}_{\mathrm{i}}^{\prime} \mathrm{s}$ - that are pending in the input of the register and are related to the process in the next round - will be stored in the register. Taking in consideration that five messages can be concurrently processed, $2 * 5 * 16 X_{i}^{\prime} s$ (160 $X_{i}^{\prime}$ s for every message) must be computed and stored. Instead of this, for every message only ${ }^{*} 16 \mathrm{X}_{\mathrm{i}}^{\prime} \mathrm{s}$ are computed and saved, those used in the currently processed transformation round for the two processes. So, this way only $2{ }^{*} 5^{\star} 16 X_{i}^{\prime} s$ ( $32 X_{i}^{\prime}$ s for every message) must be computed and stored. The $\mathrm{Xi}^{\prime} \mathrm{s}$ for the next transformation round of every message are computed and pending in the " $\mathrm{Xi}^{\prime}$ permutation" units. When a message proceeds to the next 
transformation round, the $\mathrm{Xi}^{\prime}$ s needed in the new round are stored in the corresponding registers. These registers supply the new round with the $16 X_{i}^{\prime} s$ and at the same time the 16 $X_{i}^{\prime} s$ required for the next round, for the same message, are computed and pending on the corresponding "Xi' permutation" unit.

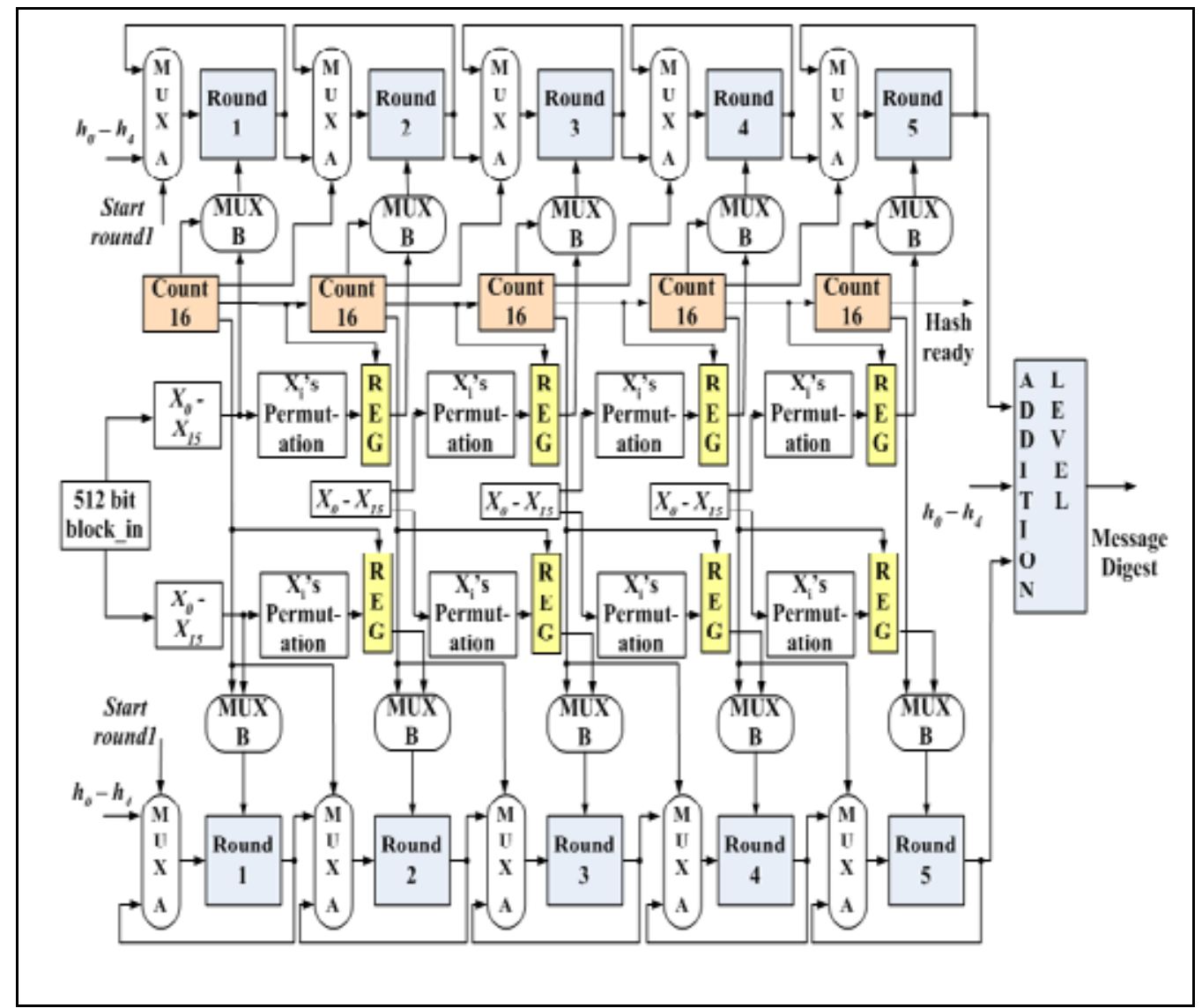

Fig. 2. Ripemd-160 Core

Implanting registers at the end of every transformation round and considering the fact that the process in every round lasts 16 clock cycles leads to the following conclusion; the implemented Ripemd-160 core can be supplied with a new 512-bit input block_in if only 16 clock cycles have past from the last time instance that the Ripemd-160 core was again supplied. This leads to a maximum throughput limit for the Ripemd-160 core which is determined by the fact that at each processing instance only 5 different messages at most can be concurrently at process. During this 16 clock cycles the 512-bit input block_in has to remain stable in order for the Ripemd-160 core to function properly. This way we save up time ( 1 clock cycle) and hardware that would be spent if it was decided to store in registers the 512-bit input block_in. This wouldn't improve at all the proposed implementation and it doesn't incur any design or functional problems.

The 512-bit input block_in is kept stable to the output of the unit that is responsible for the 
padding procedure and will be mentioned to the next section. The five Count_16 components are responsible for the synchronization of all procedures that have to be done in order to obtain the hash value. Each one is enabled only when in the corresponding transformation round there is a message that is being processed. When the process in that round reaches the end the Count_16 component arranges that the process will continue to the next round. The five Mux_A multiplexers for each process ensure that every time the process of a message ends in one round, the process will be continued in the next round. The five Mux_B multiplexers also for each process, ensure that, at any time instance, the correct $X_{i}$ is supplied to each operation of the round during the process of a message.

The presented implementation illustrates all the necessary implementation details and it is also indented to serve as a reference implementation to compare with in order to propose some improvements in its architecture which correspondingly are going to increase the performance of the whole RIPEMD hashing core as well as the corresponding HMAC implementation.

The latter presented hashing core was captured in VHDL and was fully simulated and verified using the Model Technology's ModelSim Simulator. Verification of the designs' operation was achieved using a large set of test vectors, apart from the test example proposed by the standards. The synthesis tool used to port VHDL to the targeted technologies was Synplicity's Synplify Pro Synthesis Tool. Simulation of the designs was also performed after synthesis, exploiting the back annotated information that was extracted from the synthesis tool. Further evaluation of the designs was performed using the prototype board for the Xilinx Virtex device family. The achieved operating frequency, the required integration area and the corresponding throughput in three different FPGA families are pictured in Table 1.

\begin{tabular}{|c|c|c|c|}
\hline Technology & Area (CLBs) & Frequency (MHz) & Throughput (Gbps) \\
\hline Virtex & 1798 & 51.6 & 1.65 \\
\hline Virtex-E & 1856 & 64.6 & 2.08 \\
\hline Virtex-II & 1985 & 74.6 & 2.38 \\
\hline
\end{tabular}

Table 1. RIPEMD-160 implementation synthesis result

The throughput it is calculated from Eq.2.

$$
\text { Throughput= \#bits * } \mathrm{f}_{\mathrm{operation}} / \text { \#operations }
$$

where \#bits is equal to the number of bits processed by the hash function, \#operations corresponds to the required clock cycles between successive messages to generate each Message Digest and $\mathrm{f}_{\text {operation }}$ indicates the maximum operating frequency of the circuit.

The results of Table 1 give a rough estimation of the performance of the proposed implementation in various FPGA platform boards. When placement and routing takes place, using vendor's tools, a more realistic performance of the RIPEMD implementation arises which slightly varies from the reported results in Table 1 . The implementation is synthesizable in most FPGA families, resulting in a reusable, general purpose implementation. 


\subsection{Optimized design of RIPEMD-160 hash function}

There is a big variety of choices that can be made at early stages of the design/development process to help us achieve increased performance. Although the need for high throughput is recognized, the performance of all hardware implementations concerning hash functions is degraded because not much effort has been paid on optimizing the inner logic of the transformation rounds. Instead academia and industry has been focused on using system level techniques like loop unrolling, pipeline etc, but they have not develop techniques able to optimize the critical path of these hash functions that is located in the transformation round as it analyzed previously. In this section two different techniques aiming to achieve this, are going to be presented. In each case the achieved optimization will also be stated. The next step of our research team is to combine these techniques with others in a certain methodology aiming to optimize the RIPEMD-160 hash function. This goal has already been achieved by our research team regarding other hash functions like MD-5, SHA-1, SHA-256 etc. [Michail et al, 2008].

The proposed technique to increase the operation block's frequency is based on algorithmic transformations allowing simultaneous spatial and temporal transformations. Spatial transformations are those that manipulate and re-order the position of the available resources in order to generate the same output, gaining either in performance or in power dissipation. Temporal transformations are those that manage values highly dependant from time and sequence of appearing. Applying a set of transformations on the algorithms of RIPEMD-160 it is possible to increase the hash core's performance significantly. A major advantage of applying a transformation on the algorithm is the capability to know explicitly the penalty this action requires to be paid.

In particular these two techniques are:

1) Spatial Pre-computation of additions contributing to the critical path.

2) Temporal Pre-computation of some values that are needed in following operations.

Examining the expressions described in (1) and represented in Figure 1, it is observed that some input values are assigned directly to some output values respectively. From Figure 1, it is derived that the maximum delay is observed on the calculation of the $b_{t}$, value. Obviously the critical path consists of three addition stages and a multiplexer that feeds back the operation block.

A notice that can be made by observing Figure 2 is that some outputs are derived directly from some inputs values respectively. So we can assume that it is possible during one operation to pre-calculate some intermediate values that will be used in the next operation. Therefore, while the main calculations are in progress, at the same time some values that are going to be needed in the next operation can also be in progress of calculation. Furthermore, moving the pipeline stage to an appropriate intermediate point we can store these intermediate calculated values, the critical path is divided resulting in a decrease of the maximum delay without paying any worth-mentioning area penalty.

These pre-calculations are applied only to those output values that derive directly (hardwired) from inputs. So while the main calculations are in progress, some intermediate values needed at the next operation can also be in progress of calculation. Further benefits from the pre-calculation process can be achieved by re-ordering in space the registers. Moving the pipeline stage to an appropriate intermediate point to store these intermediate calculated values, the critical path is divided resulting in a decrease of the maximum delay with negligible, if any, area penalty. 
It should be pointed out that the existing pipeline registers are moved somewhere in the middle of each operation block so as to shorten the critical path and no extra inner-loop pipeline stage is introduced. This means that the existing four pipeline registers (for outerroute pipelining) are re-arranged in space, and it is not the case that more pipeline registers (for inner-round pipelining) are applied. Re-ordering of existing registers results in disjoining dependencies of the outputs from input signals. Although the operation block was changed, the logic it implemented was the same, and at the same time the critical path was shortened.

Thus, the RIPEMD-160 equations representing Figure 1 is transformed to generate the intermediate values $\mathrm{a}_{\mathrm{t}-1}{ }^{*}, \mathrm{~b}_{\mathrm{t}-1}{ }^{*}, \mathrm{c}_{\mathrm{t}-1}{ }^{*}, \mathrm{~d}_{\mathrm{t}-1}{ }^{*}, \mathrm{e}_{\mathrm{t}-1}{ }^{*}$ and $\mathrm{g}_{\mathrm{t}-1}$ as illustrated in Figure 3 . In Figure 3 the pre-computation technique applied in RIPEMD-160 hash function is illustrated. Each operation block now consists of two units the "Pre-Computation" unit which is responsible for the pre-computation of the values that are needed in the next operation and the "FinalCalculation" unit which is responsible for the final computations of each operation.

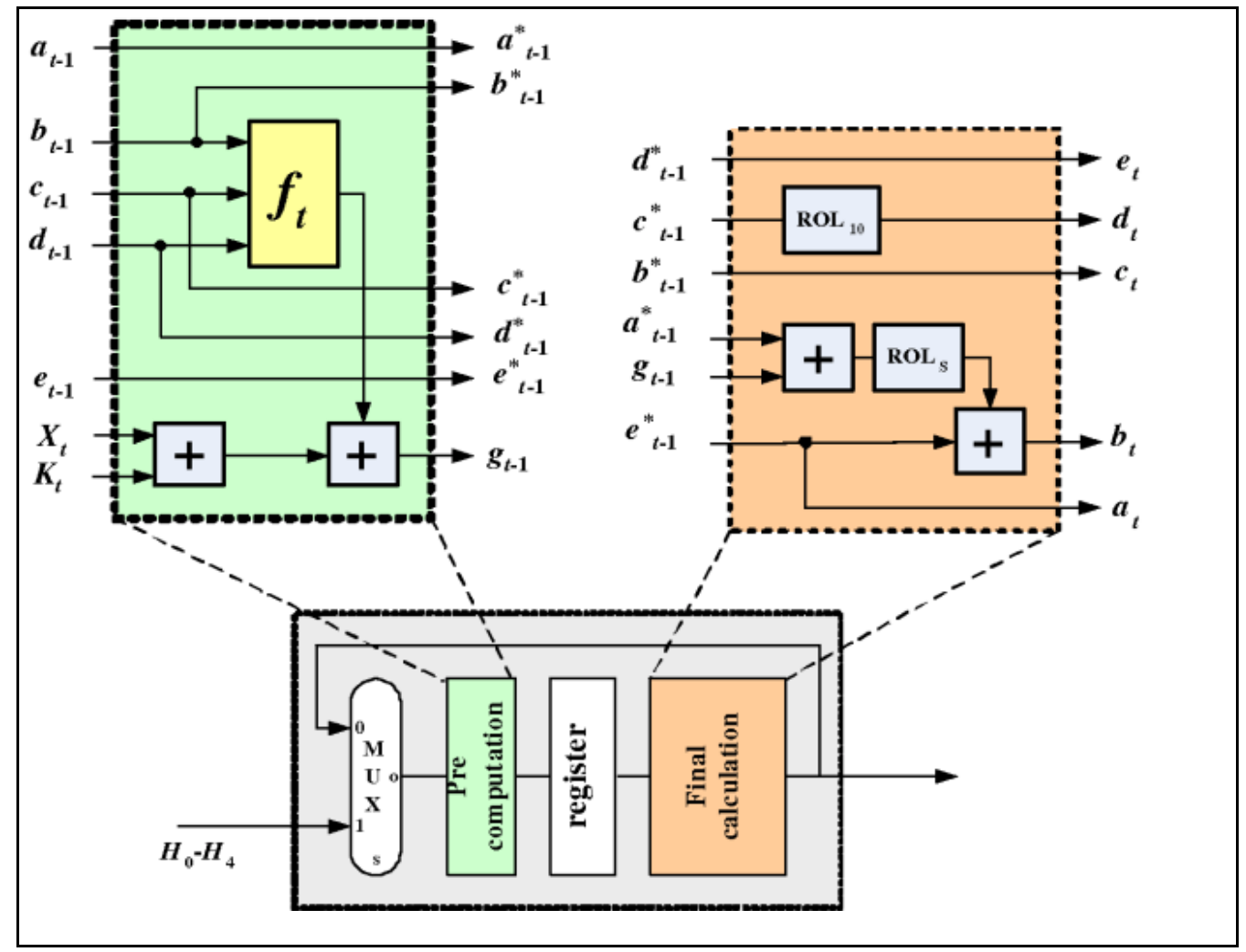

Fig. 3. Spatial pre-computation applied on the operation block of RIPEMD-160

Notice that in Figure 3 output $b_{t}$ enters the multiplexer and feeds a no-load wire $b_{t-1}$ which stores its value to the register as $b_{t-1}{ }^{*}$. Also notice at the "Pre-Computation" unit that the inputs $a_{t-1}, c_{t-1}, d_{t-1}, e_{t-1}$, which is equal with the values $a_{t-1}{ }^{*}, c_{t-1}{ }^{*}, d_{t-1}{ }^{*}, e_{t-1}$ * respectively, are fed through the multiplexer from the intermediate register outputs $e_{t-1}{ }^{*}, b_{t-1}{ }^{*}, c_{t-1}{ }^{*}, d_{t-1}{ }^{*}$ respectively. The introduced area penalty is small, only a single register for each "round", 
that stores the intermediate value $\mathrm{g}_{\mathrm{t}-1}$. In order to reduce the critical path by one addition level, we will continue with the application of the second technique, which introduces a temporal pre-computation of the values.

From the "Final-Calculation" stage of Figure 3, one can observe that in every operation, from the current value of $d_{t-1}$, derives directly the value of $e_{t}$ (at the next operation). Also, from the current value of $e_{t}$, derives directly the value of $a_{t+1}$. Consequently, the value of $a$, is the same as the value of was two operations earlier.

So it is valid to write the following equation:

$$
a_{t+1}=e_{t}=d_{t-1}
$$

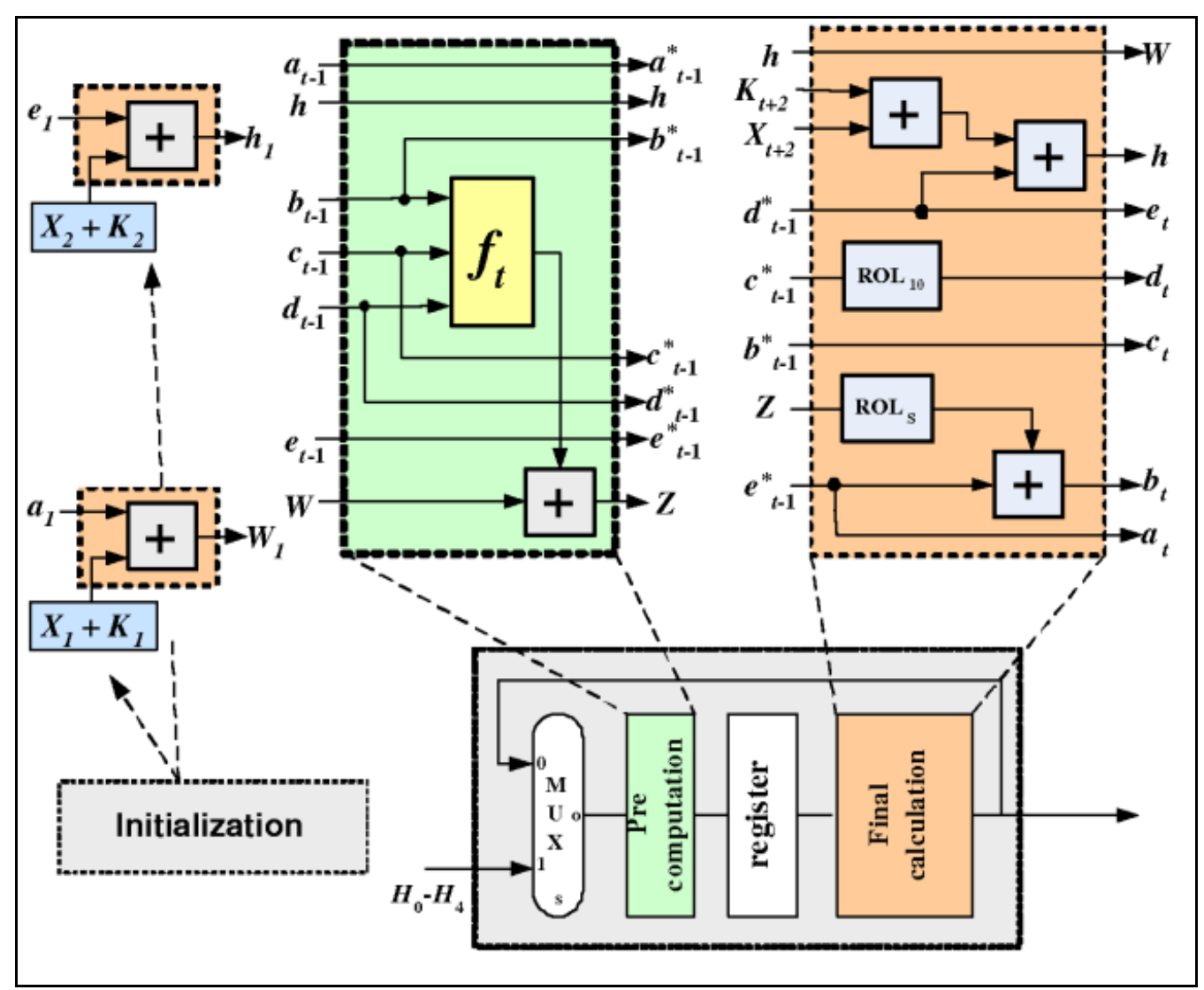

Fig. 4. The proposed RIPEMD-160 operation block after applying temporal transformation

Thus, we perform the temporal pre-computation of the sum $\left(X_{t+2}+K_{t+2}\right)+a_{t+1}$ two operations before it is used, by calculating the sum $\left(\mathrm{X}_{\mathrm{t}+2}+\mathrm{K}_{\mathrm{t}+2}\right)+\mathrm{d}_{\mathrm{t}-1}$ at the "FinalCalculation" unit, when the operation $t$ is being executed. Then this sum at the "PreComputation" stage of the next operation $(t+1)$ saved into the register $h$ and represent the sum $\left(X_{t+2}+K_{t+2}\right)+e_{t}$. At the "Final-Calculation" unit of the same operation, the value of $W$ derives directly from the value of $h$. The computed sum now of the value $W$ represents the $\operatorname{sum}\left(X_{t+2}+K_{t+2}\right)+a_{t+1}$. 
Finally at the "Pre-Computation" unit on the next operation (which is the operation $t+2$ ) the sum $Z=W+f_{t}$ is calculated. The computed sum now represents the value $\left(X_{t+2}+K_{t+2}\right)+a_{t+1}$ $+f_{t}$. This sum is part of the computations needed for the calculation of $b_{t+2}$ value. What remains for the computation of the value $b_{t+2}$ is the rotation (Rols) of the value $Z$ and then the addition in this result of the value $e_{t+2}$, as is performed in the "Final-Calculation" in Figure 4.

Observing Figure 4 we see that the critical path is not located any more in the computation of the $b_{t}$ value but in the computation of the value of $Z$. This means that the critical path in Figure 4 has been reduced, from three addition stages, a Non Linear Function $f_{t}$ and a multiplexer in Figure 1, to two addition stages, a Non Linear Function $\mathrm{f}_{\mathrm{t}}$ and multiplexer. Thus, the critical path is shortened by one adder level, which contributes approximately $30 \%$ to the overall maximum delay.

However, we must notice that an initialization of the values of $W$ and $h$ is needed as it is illustrated in Figure 4. The introduced area penalty is only two 32-bit registers, which are used for storing the intermediate results of the values $W$ and $h$ that are required. This area penalty sure enough is worth paying for an increase of throughput at about $35 \%$.

The proposed technique to improve the throughput of RIPEMD-160 needs also to be verified. Thus the optimized design of RIPEMD-160 was developed and captured in VHDL and was fully simulated and verified using the Model Technology's ModelSim Simulator. In Table 2, the experimental results are offered. From the experimental results, it was verified that RIPEMD-160 proposed implementation was about 35\% faster than the conventional implementation.

\begin{tabular}{|c|c|c|c|}
\hline Technology & Area (CLBs) & Frequency (MHz) & Throughput (Gbps) \\
\hline Virtex & 2057 & 68.2 & 2.18 \\
\hline Virtex-E & 2256 & $86 . .6$ & 2.75 \\
\hline Virtex-II & 2548 & 94.6 & 3.02 \\
\hline
\end{tabular}

Table 2. RIPEMD-160 optimized implementation synthesis result

It is noticed that researchers are moving on to optimize the inner operational block so as to achieve higher throughputs for their implementations. In order to make fair comparisons the proposed RIPEMD-160 implementation was evaluated for a medium performing FPGA technology as it is practically impossible to integrate it in all the necessary technologies. Then it is compared to competitive implementations as they are given in Table 3. In Table 3, reference is given to other implementations of RIPEMD-160 as they were reported in the technical literature by other researchers. These implementations are highly optimized, depending on the design approach the designer has selected to follow.

The implementations of (Bosselaers et al., 1996), (Ng et al., 2004) present low throughput as they are software based. The highest throughput is reported by (Sklavos \& Koufopavlou, 2005), which achieves a throughput of 2 Gbps. This is about 35\% less than the throughput that is achieved for the implementation of RIPEMD-160 that is presented in this work. Furthermore, comparing the implementations of other researchers to the proposed one, it 
can be observed that all of them fall short in throughput, in a range that varies from $2-41$ times less than the proposed implementation. In the following Table, it is worth mentioning that the proposed implementation outperform even ASIC implementations, exhibiting the collateral benefit of the application of the proposed design technique.

\begin{tabular}{|c|c|c|c|}
\hline Implementation & Technology & $\begin{array}{l}\text { Frequency } \\
\qquad(\mathrm{MHz})\end{array}$ & $\begin{array}{c}\text { Throughput } \\
\text { (Mbps) }\end{array}$ \\
\hline Proposed & $\begin{array}{c}\text { FPGA } \\
\text { Virtex xcv300E }\end{array}$ & 86.7 & 2752 \\
\hline (Bosselaers et al., 1996) & $\begin{array}{l}\text { Software } \\
\text { (Pentium) }\end{array}$ & 90 & 45.5 \\
\hline (Ng et al., 2004) & $\begin{array}{l}\text { FPGA } \\
\text { Altera }\end{array}$ & 26.6 & 84 \\
\hline $\begin{array}{c}\text { (Sklavos \& } \\
\text { Koufopavlou, 2003) }\end{array}$ & $\begin{array}{c}\text { FPGA } \\
\text { Virtex xcv300E }\end{array}$ & 65 & 2000 \\
\hline (Satoh \& Inoue, 2005) & $\begin{array}{c}\text { ASIC } \\
(0.13 \text { um CMOS) }\end{array}$ & - & 1442 \\
\hline (Dominikus, 2002) & $\begin{array}{c}\text { ASIC } \\
(0.6 \mathrm{um})\end{array}$ & - & 89 \\
\hline (Dominikus, 2002) & $\begin{array}{c}\text { FPGA } \\
\text { Virtex xcv300E }\end{array}$ & 42.9 & 65 \\
\hline (Khan et al., 2005) & $\begin{array}{c}\text { FPGA } \\
\text { Virtex xcv300E }\end{array}$ & 37 & 117 \\
\hline
\end{tabular}

Table 3. Comparison of the proposed implementation of RIPEMD-160 with the competition

Finally, it also has to be mentioned that more implementations exist but with much lower achieved throughput and most of them are outdated. These were not considered for comparisons since it would be rather unfair not only because these were the first attempts to optimize hash functions but also because at that time the existing implementation tools were not that sophisticated like the ones that exist nowadays. It should also be pointed out that there are some implementations that focus on area requirements, without applying any pipeline stage. In these implementations effort has been paid only for minimizing area. Those implementations are designated for use in mobile and portable devices where area requirements and power dissipation is the major factor. Throughput is not such a dramatic factor in these cases because the clients can wait a few seconds for the transaction to be carried out successfully

\subsection{HMAC mechanism design architecture}

The scope of an HMAC implementation is to authenticate both the source of a message and its integrity without the use of any additional mechanisms. HMACs have two functionally 
distinct parameters, a message input and a secret key known only to the message originator and intended receiver(s). HMAC is used not as a cipher, but rather as a mechanism for signing a packet with a key at one end of the connection, and then verifying the signature at the other end using the same key. Without the key it is infeasible to generate a packet with the correct signature.

HMAC mechanism is mainly consisted of the utilized hash function which is the factor that determines the throughput and the operating frequency of the HMAC mechanism. So it can be assumed that implementing hashing cores with very high throughput will result in high throughput implementation of HMAC mechanism which will in his turn result in high throughput implementation of the whole security scheme, whish is essential in IPSec, SSL/TLS and elsewhere. In this work the usage of the RIPEMD-160 hash function is adopted for reasons that have already been presented, presenting the alternative HMACRIPEMD-160 implemntation.

In this section the HMAC hardware architecture is going to be presented incorporating the optimized version of RIPEMD-160 hash function that has already been presented .The whole HMAC system architecture is illustrated in Figure 5 according to the standard that describes its functionality. As it can be seen two hashing cores have to be incorporated in the HMAC imlementations regardless of the selected hash function. So, the architecture in Figure 5 is quite generic and another hashing function can also be incorporated. In this work two pipelined implementations of RIPEMD-160 hasing core with optimized operation block is going to be used for the implementation of the whole HMAC mechanism, thus resulting to a pipelined HMAC implementation.

Analyzing the illstrated system design in Figure 5, we should begin from the the MS RAM where all message schedules $\mathrm{Wt}$ of the padded message are stored. The Constants Array is a hardwired array that provides the constant values $\mathrm{Kt}$ and the constant initialization values. Additionally, it includes the $\mathrm{Wt}$ generators and the Key Generation unit that is required at the HMAC mechanism.

As it was previously mentioned this components are essential for the hashing cores to function properly. The HMAC must firstly be initialized before processing any message. The initialization procedure corresponds to computing the hash values of two certain 512-bit blocks, which are the corresponding keys, and this is performed independently in the two RIPEMD-160 cores. When the initialization procedure is completed, the hash values from the outputs of the two RIPEMD-160 cores are stored in the Constants Array unit and are then used continuously in the two RIPEMD-160 cores are the new initial values. These values as well as the corresponding keys shall be protected and this is the reason why they are stored in registers in the Constants Array unit and not in the RAM memory which is considered as vulnerable.

This is the first time that a message can be supplied for process to the first RIPEMD-160 core as long as it has been padded, while the initialization procedure was in progress. After being processed in the first RIPEMD-160 core the message will be padded and forwarded for process in the second RIPEMD-160 core that exists in the HMAC mechanism and is illustrated in Figure 5 with the notation "HASHING CORE". As it has already been explained 1 , in the proposed hardware implementation of RIPEMD-160 five pipeline stages have been applied.This means that in the presented implementation ten pipeline registers are available and thus ten different messages can be concurrently processed.

In the proposed implementation each hmac value is computed after 161 clock cycles ( 80 for 
each one of the two RIPEMD-160 cores and one clock cycle for the intermediate paddingregister).The method of intermediate storing the values that have arise from the processing of the two keys at the RIPEMD-160 cores, saves the time of processing two 512-bit blocks for every message and also allows pipeline technique to be applied, increasing this way both speed and throughput of the HMAC implementation. These stored intermediate values shall be treated and protected in the same way as the secret keys.

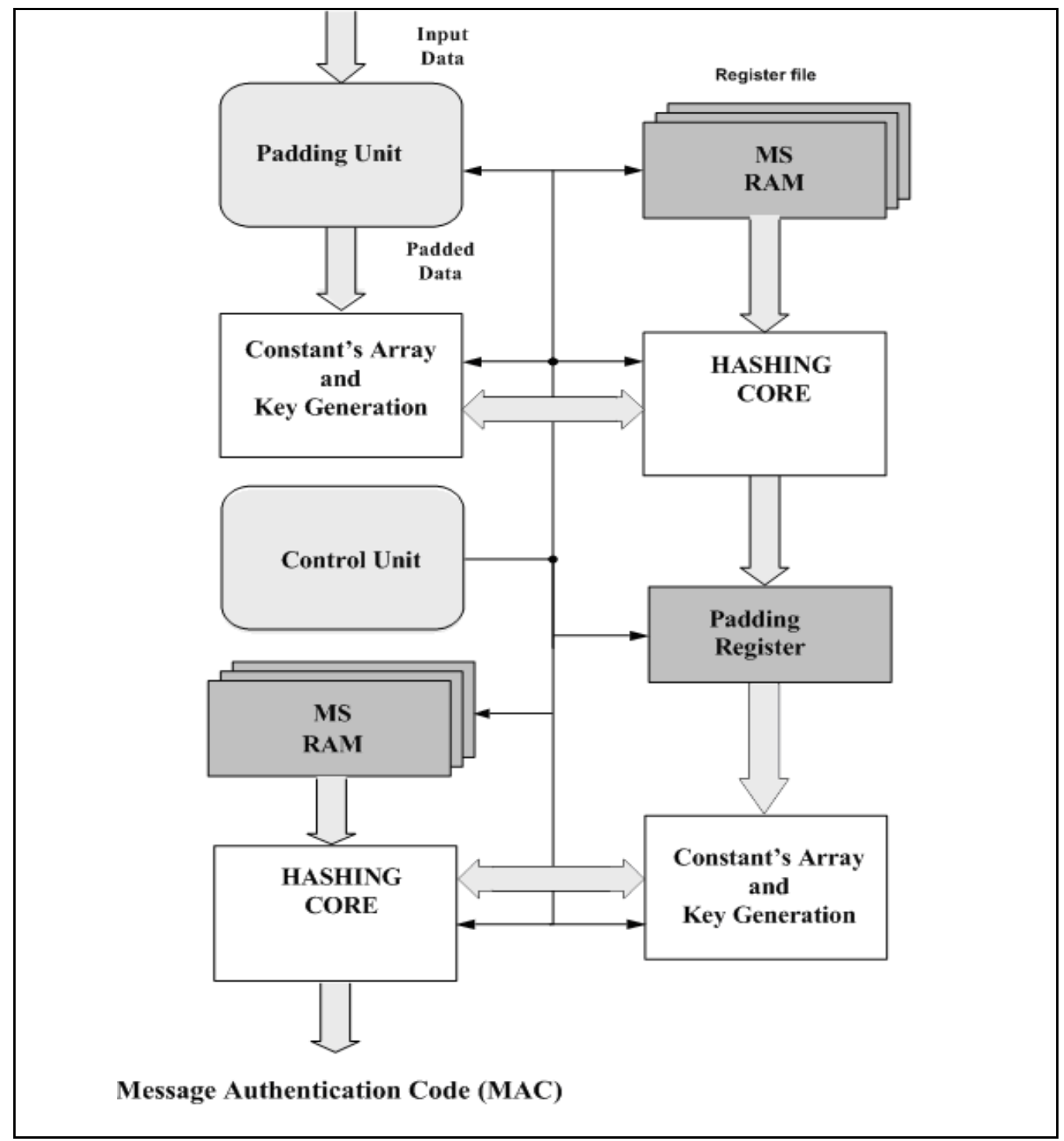

Fig. 5. HMAC system architecture 


\section{CMAC: A Reliable Alternative}

In previous sections we have already outlined the importance of CMAC as an alternative way to provide authentication services in certain applications. This necessity mainly has to do with the numerous applications that do not call for high throughput implementations (as long as the security part is concerned) but for small-sized or low-power implementations. In these cases the usage of CMAC instead of HMAC is ideal since there is no need to incorporate both a hash function and a cipher block but only a block cipher algorithm like AES that can handle all aspects of security in the certain security scheme. In this section implementation details concerning CMAC will be provided.

It has to be noticed that, to the best of our knowledge, there is no other hardware implementations concerning CMAC (CMAC neural network has no relation to CMAC for producing MACs for integrity). This is partially due to the fact that this algorithm was recently proposed and industry has not yet introduced to the market related hardware products. However some software CMAC implementations do exist but obviously these can be neither commented nor compared to our work which proposes a hardware implementation.

The CMAC algorithm incorporates a symmetric key block cipher like AES (Advanced Encryption Standard), TDEA or any other FIPS (Federal Information Processing standards) approved block cipher algorithm. Block ciphers consist of two operations one for encryption and one for decryption. However in the implementation of the CMAC algorithm only one of these operations is needed, and usually the encryption process is selected. The cipher encryption process is a permutation on bit strings of a fixed length; the strings are called blocks and their size varies depending on the used block cipher. For the AES it is 128 bits whereas for TDEA is 64 bits. The CMAC algorithm implies the usage of a secret key which is the block cipher key. The corresponding key shall be protected for its secrecy and used exclusively for the CMAC mode of the chosen block cipher.

In (NIST, $2005 \mathrm{~b}$ ), the authentication procedure using a cipher block is described. There all the prerequisites are mentioned and thoroughly described. This process has some similarities with the procedure that is followed in order to produce a MAC using a hash function.The sender produces the MAC using the eight steps described in (NIST, $2005 \mathrm{~b}$ ) and thus producing the CMAC that is the MAC with the usage of a cipher block like AES. The CMAC standard implies the usage of a FIPS approved cipher block and the most widely currently used block cipher algorithm is the AES which is considered as absolutely secure. For implementation's shake this block cipher was designed in order to be used in the whole CMAC structure according to its standard without any sophisticated optimization technique. However the implementation of the block cipher will not be presented in this chapter since our intention is to provide an abstract knowledge about an alternative way to offer authenticating servises, thus we shall focus on CMAC and not on AES.

However it has to be stressed that only the encryption process was developed since the CMAC uses either the encryption or the decryption process of the selected block cipher algorithm. However in a real's world security scheme both processes exist for encrypting and decrypting the exchanged data between the communicating parts and at the computation of a CMAC value simply only the one process is used. Other block cipher algorithms that can be used in the CMAC mechanism are all FIPS approved block ciphers such as TDEA. However we decided to use the encryption process of the AES block cipher because it is the most widespread worldwide. 
As it will be referred the critical path of the whole CMAC mechanism is located at the AES core and so at a later time effort can be paid in optimizing the used cipher block (i.e AES) and thus optimize the CMAC mechanism. In the implementation scheme in this section the block cipher algorithm that has been designed and implemented is noted as "Block Cipher". This is illustrated in Figure 6 where the whole CMAC architecture is outlined.

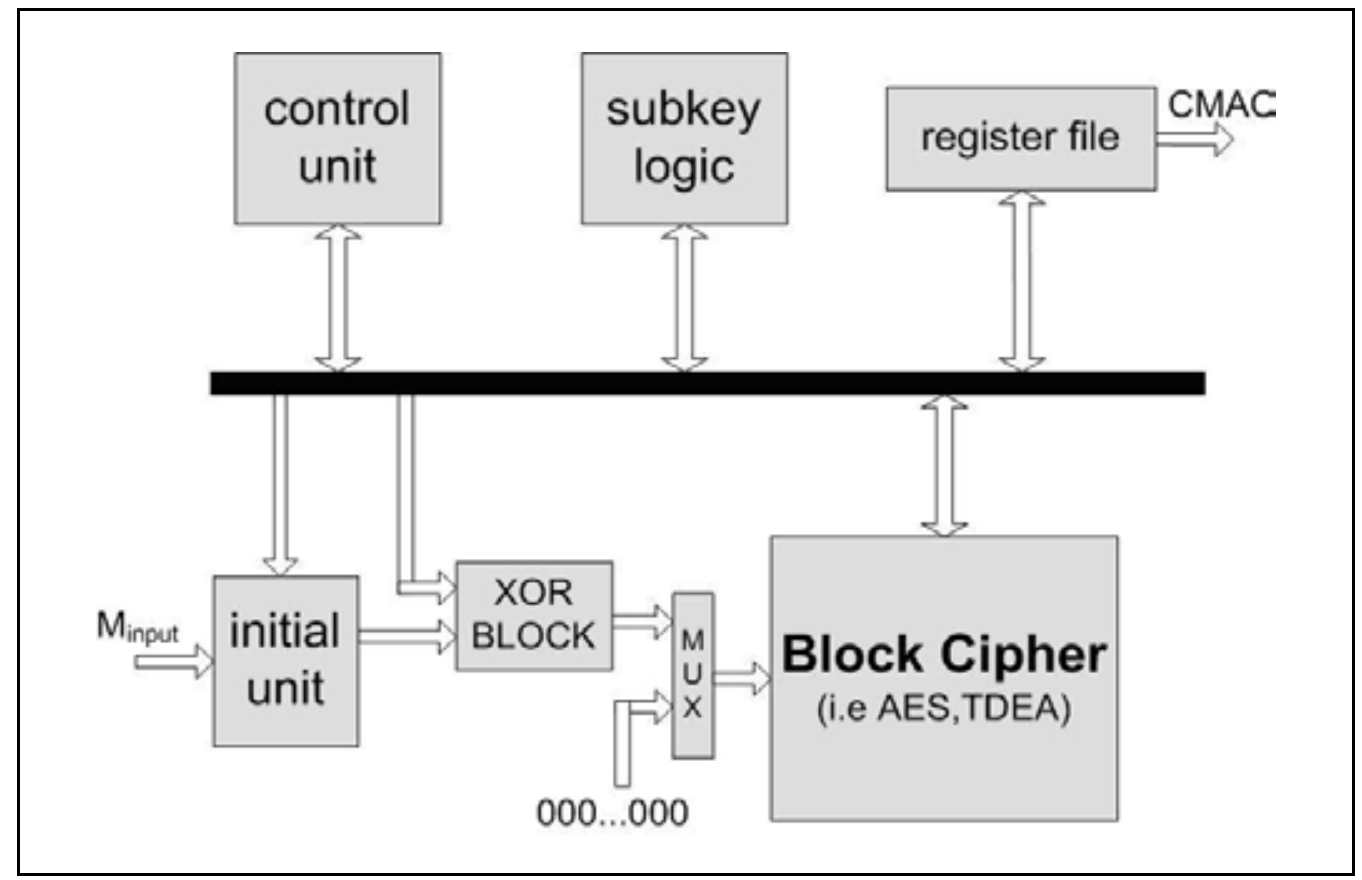

Fig. 6. CMAC Implementation

Any FIPS approved cipher block algorithm can be used instead of AES and TDEA in the same way as it is shown in the previous figure. The interface for the communication of the different components will remain the same. The CMAC implementation consists of several parts.

The overlying software-based system feeds the CMAC scheme (implemented in hardware) with the properly padded (when needed) input blocks $M_{\text {input }}$ which are parts of the whole message that is intended to be protected for authentication. The padding process is a quite simple process that can be carried out by the software. This does not imply security problems since there is no secrecy or keys information that should be protected at this point. The initial unit part is the hardware component responsible for the conditional bitwise operation xor between the $\mathrm{M}_{\text {input }}$ and one of the subkeys $\mathrm{K}_{1}$ or $\mathrm{K}_{2}$.

This part must be implemented in hardware although it could easily be carried out in software and is illustrated at the left in Figure 7. The necessity for hardware implementation of the specific parts arises from the fact that in this unit certain operations concerning the two subkeys exist. If this process is carried out in software then the whole security system is vulnerable since the two subkeys can easily be retrieved. Hardware implementation increases dramatically security since the $K_{1}$ and $K_{2}$ values are stored in registers. Furthermore in order to ensure an even more higher level of security in our implementation 
the key $\mathrm{K}$ and the two subkeys $\mathrm{K}_{1}$ and $\mathrm{K}_{2}$ are not stored in a RAM memory but in shadow registers. This way we avoid the danger someone who manages to access and read the security system's memory (RAM) to reveal our keys and subkeys that are stored there.

At the initialization phase the two subkeys $K_{1}$ and $K_{2}$ must be computed from the main key $\mathrm{K}$. This procedure is carried out by the hardware component "subkey logic" that is illustrated at the right in Figure 7. This component loads the key $\mathrm{K}$ from the register file and an already available output of the used block cipher algorithm and in two clock cycles it computes the subkeys $\mathrm{K}_{1}$ and $\mathrm{K}_{2}$ that are then saved in the register file.

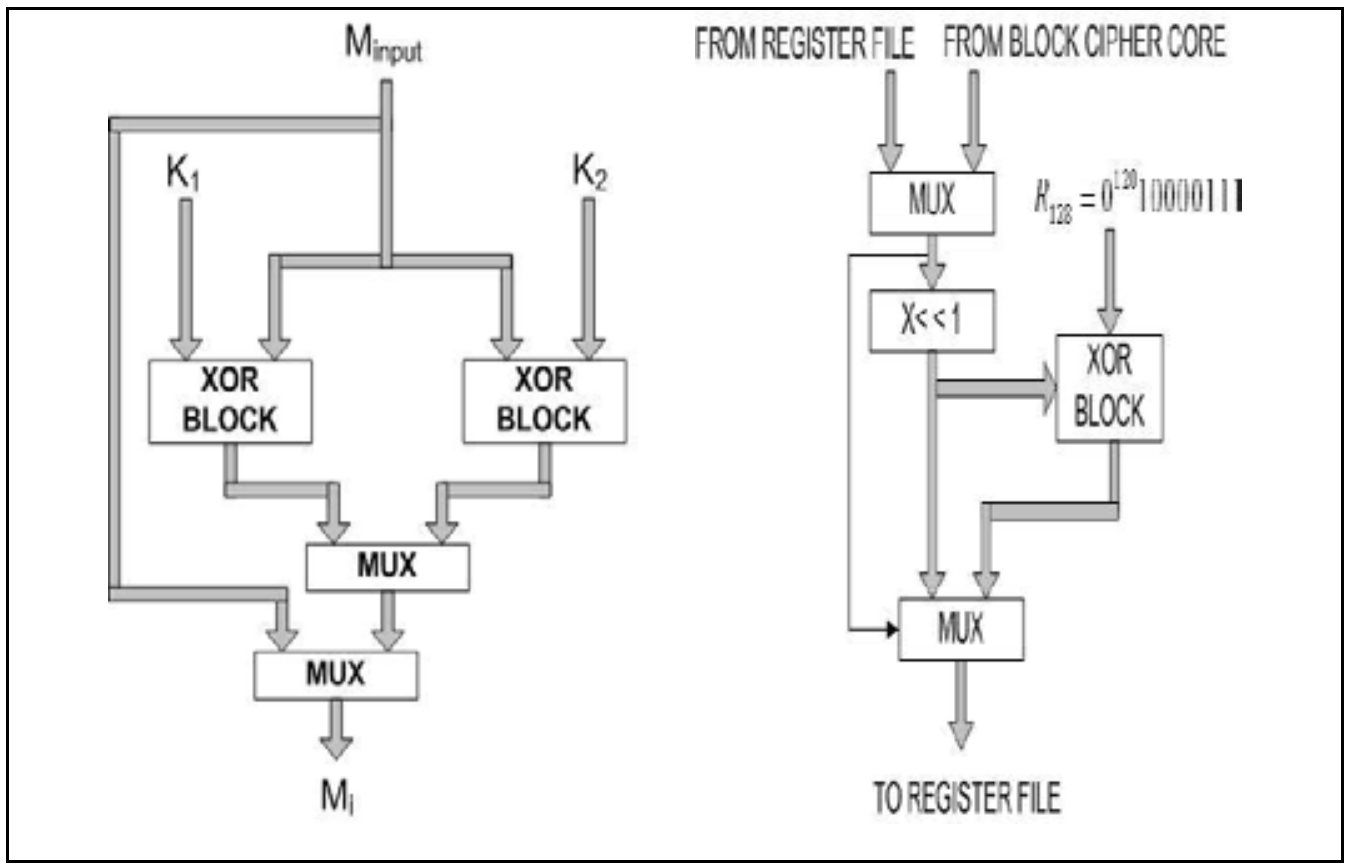

Fig. 7. Units "Initial Unit" (left) and "Subkey Logic"(right)

The selected output from the cipher block is the so before $K_{1}$ and $K_{2}$ computations take place the must be first computed. Thus this component is enabled only after has been computed and is disabled after two clock cycles resulting to a low power operation. This component is enabled again only if the used main key $\mathrm{K}$ has changed for some reason.

The "XOR BLOCK" component is a simple component performing the bitwise xor operation. The "Register File" consists of some registers that are used for storing keys, subkeys, the final CMAC value as well as some useful intermediate values.

Finally the control unit consists of some small counters and is responsible for the correct synchronization of all the components that exist in the CMAC scheme. It is also responsible for the enabling of the several components just when they are needed and disabling them just after that.

So incorporating any FIPS approved secure block cipher algorithm in the presented design will result in an efficient implementation of CMAC mechanism. It can be seen that CMAC is simpler, smaller and thus less costly comparing to HMAC. So it is obvious that in cases that were described and are eligible to incorporate CMAC, this selection is advantageous. 


\section{Conclusion}

In this chapter two ways of providing authentication services (HMAC and CMAC) have been presented. Certain applications' criteria that have to be taken into consideration to choose between CMAC and HMAC were stated. Implementation details and system architecture were provided for both alternatives focusing on the manageability of these designs so as to be easy to incorporate any secure hash function in case of HMAC or any secure block cipher algorithm in case of CMAC. We focused on the design and implementation of RIPEMD-160 hash function that can be used for authentication in an HMAC mechanism and two techniques were presented that resulted to the optimization of the RIPEMD-160 hash function by 35\% in throughput with a minor area penalty.

\section{Acknowledgement}

This work was partially supported by the project PENED 2003 No 03ED507, which is funded in $80 \%$ by the European Union- European Social fund and in $20 \%$ by the Greek stateGreek Secretariat for Research and Technology.

\section{References}

Ballard, K. (2004). http:/ / digestit.ken-nethballard.com/blog/index.php?/archives/P2.html

Biever C. (2004). US boosts e-voting software security, NewScientist.com news, http://www.newscientist.com/article.ns?id=dn6593

Bosselaers, A., Govaerts, R. and Vandewalle, J. (1996). Fast hashing on the Pentium, Proceedings of the Advances in Cryptology (Crypto1996), pp. 298-312

Dobbertin H., Bosselaers A., Preneel B. (1996). RIPEMD-160: A Strengthened Version of RIPEMD

Dominikus, S. (2002). A Hardware Implementation of MD4-Family Hash Algorithms. Proceedings of 9th IEEE International Conference on Electronics, Circuits and Systems (ICECS 2002), Vol. III, pp. 1143-1146, Sep. 2002.

Hodjat A. and Verbauwhede I., A 21.54 Gbits/s Fully Pipelined AES Processor on FPGA. IEEE Symposium on Field-Programmable Custom Computing Machines Systems, (FCCM '04) pp. 308-309, 2004

Johnston D. and Walker J.,(2004). Overview of IEEE802.16 Security, IEEE Security and Privacy

Khan, E., El-Kharashi, M.W., Gebali, F. and Abd-El-Barr, M. (2005). An FPGA Design of a Unified Hash Engine for IPSec Authentication. Proceedings of the Fifth international Workshop on System-on-Chip For Real-Time Applications (IWSOC2005), pp. 450-453

Kim G.H. and Spafford E.H., (1994). The Design and Implementation of Tripwire: A File System Integrity Checker, 2nd ACM Conf. Computer and Communications Security, pp. 18-29

Loeb L. (1998). Secure Electronic Transactions: Introduction and Technical Reference, Artech House Publishers

McCurley K.S. (1994). A Fast Portable Implementation of the Secure Hash Algorithm, Sandia National Laboratories Technical Report SAND93-2591. 
Michail H.E, Kakarountas A. P., Milidonis A. S. and Goutis C. E., (2008). A Top-Down Design Methodology for Implementing Ultra High-Speed Hashing Cores, IEEE Transactions on Dependable and Secure Computing, accepted for publication

Microsoft, (2005). Internet Explorer 6: Digital Certificates, http://www.microsoft.com/windows/ie/ie6/using/howto/security/digitalcert/using.mspx.

Mironov I. (2005), Hash functions: "Theory, attacks, and applications", Microsoft Research, Silicon Valley Campus

Morris R. and Thompson K. (1979)., Password security: A case history, Communications of ACM, vol. 22(11), pp. 594-597

Nakajima, J. and Matsui, M. (2002). Performance Analysis and Parallel Implementation of Dedicated Hash Functions, Lecture Notes in Computer Science (LNCS), vol. 2332, pp. 165-180. Springer.

Ng, C.-W., Ng, T.-S. and Yip, K.-W. (2004) .A unified architecture of MD5 and RIPEMD-160 hash algorithms. Proceedings of IEEE International Symposium on Circuits and Systems (ISCAS 2004). Vol. 2, pp. 889-892.

NIST, FIPS 186, (DSS), (1994). Digital Signature Standard (DSS) , US Dept of Commerce

NIST, (2001) a. Advanced Encryption Standard (AES) Home Page , US Dept of Commerce http://csrc.nist.gov/encryption/aes

NIST, SP 800-32., (2001) b. Introduction to Public Key Technology and the Federal PKI Infrastructure, US Dept of Commerce

NIST, SP800-77, (2005) a .Guide to IPSec VPN's, US Dept of Commerce

NIST, (2005) b. Recommendation for Block Cipher Modes of Operation: The CMAC Mode for Authentication, US Dept of Commerce http://csrc.nist.gov/publications/nistpubs/ index.html\#sp800-38B1998

NIST, FIPS 198-1, (2007) a. The Keyed-Hash Message Authentication Code (HMAC), US Dept of Commerce

NIST, (2007) b. XTS-AES, IEEE 1609 approved, under consideration from NIST for FIPS 1402 , US Dept of Commerce http://grouper.ieee.org/groups/1619tmp/1619-2007NIST-Submission.pdf

NIST, (2008). Recommendation for Block Cipher Modes of Operation: Galois/Counter Mode (GCM) and GMAC, FIPS PUB 800-38D, US Dept of Commerce http://csrc.nist.gov/publications/nistpubs/800-38D/SP-800-38D.pdf

Satoh, A. and Inoue, T. (2005). ASIC-Hardware-Focused Comparison for Hash Functions MD5, RIPEMD-160, and SHS. Proceedings of International Conference on Information Technology: Coding and Computing (ITCC2005), Vol. I, pp. 532-537.

Schneier, B. (1996). Applied Cryptography - Protocols, Algorithms and Source Code in C, Second Edition, John Wiley and Sons.

Sklavos, N. and Koufopavlou, O. (2005). On the hardware implementation of RIPEMD processor: Networking high speed hashing, up to 2 Gbps, Computers \& Electrical Engineering, Vol. 31, Is. 6, pp. 361-379.

Stephen T., (2000). SSL \& TLS Essentials: Securing the Web, John Wiley and sons Publications

Van Oorschot, P.C., Somayaji, A. and Wurster, G. (2005). Hardware-Assisted Circumvention of Self-Hashing Software Tamper Resistance. IEEE Transactions on Dependable and Secure Computing, Vol. 02, No. 2, pp. 82-92. 


\title{
Bioinformatics: strategies, trends, and perspectives
}

\author{
Carlos Norberto Fischer and Adriane Beatriz de Souza Serapião \\ São Paulo State University - UNESP - Rio Claro, S.P.
}

Brazil

\section{Introduction}

With the advances in the genome area, new techniques and automation processes for DNA sequencing, the amount of data produced has increased exponentially. Analyzing this data, in order to identify interesting biological features, is an enormous challenge, especially if it would be done manually. Think about trying to find a specific word in a book, say Don Quixote, and we have to search word by word. How long it would take?

Bioinformatics has played an important role trying to help specialists to analyze data of a specific genome. The application of information technology, associated with techniques from applied mathematics, informatics, statistics, and computer science, has allowed the discovering of interesting and important characteristics in genomes, allowing to understand and solve several biological problems, or even to generate more knowledge or insight about the problem and its involved biological processes, what can bring advances in the used techniques.

In Computing area, for example, an ordinary type of task is to process texts. There are several problems involving strings, like trying to find a specific word (we could say "to align words") or a similar one (considering a particular pattern of characters) in a text. When processing genomic data, if it is desired to search for a specific pattern (and its approximations) in DNA sequences, the natural way is to use solutions already implemented. Thus, for pattern (exact or not) search and similar problems, bioinformaticians have developed computational tools that apply techniques and algorithms well-known in Computing area in order to solve these important genomic problems. Sometimes, they need to adapt algorithms for considering specific features of the biological problem. Two good examples of this case are Sequence Aligning and Sequence Assembly, processes resulting of adaptations in algorithms in order to consider insertion, deletion, and substitution of nucleotides in DNA sequences.

Some statistical and computational techniques, such as Hidden Markov Models (HMMs), Stochastic Grammars, and Conditional Random Fields (CRFs) have been successfully applied for modeling, analysis, discovery, classification, and alignment of biological sequences (Yoon \& Vaidyanathan, 2004, 2005). HMMs (Rabiner, 1989) and Stochastic Grammars (Sakakibara et al., 1994) are forms of generative models to label sequences, assigning a joint probability distribution of, for example, the gene hidden structure $y$ and the 
observed nucleotide sequence $x$. The parameters of these methods are trained to maximize the joint likelihood of training examples. CRFs (Lafferty et al., 2001) are probabilistic frameworks for labelling and segmenting sequential data. They consist of an undirected graphical model that defines a single distribution over label sequences given a particular observation sequence.

An example of a very interesting biological problem is the one related to Transposable Elements (TEs). Computational techniques have been developed for systematic genome annotation of TE families, such as homology-based, structure-based, de novo, and comparative genomic methods (Bergman \& Quesneville, 2007). Homology-based methods are supported on detecting similarity to known TE protein-coding sequences using prior knowledge acquired based on previously identified TE sequences (Agarwal \& States, 1994; Kurtz et al., 2000). Structure-based methods bear prior knowledge about the common structural features shared by different TEs that are required in their mobility processes (Andrieu et al., 2004; McCarthy \& McDonald, 2003). These two methods can include HMM, stochastic grammars, and CRF approaches. De novo method essays to find TEs using repeat regions in genome without considering prior information about TE structure or similarity (Bao \& Eddy, 2002; Campagna et al., 2005; Edgar \& Myers, 2005; Pevzner et al., 2004). Comparative genomic methods (Caspi \& Pachter, 2006) seek for insertion regions where multiple alignments of genome sequences are disrupted by a large insertion in one or more species. Applying filtering and concatenating, insertion regions are then locally aligned by similarity with all other insertion regions to identify repeat insertion regions. However, all of these methods have presented limited success. Some available computational resources for TE discovery and TE detection are summarized in (Bergman \& Quesneville, 2007).

We intend to present in the following sections a few examples of biological problems and solutions that can be used to attack them, from simple strategies to alternative ones.

\section{Bioinformatics: Simple Solutions and Challenges}

Depending on the features of the biological problem being treated, relatively simple solutions from, for example, Computing area can be sufficient for helping to solve it. Thus, in some situations, it is interesting, more immediate, and simpler to think in strategies that can consider the use of existent bioinformatics tools created for other purposes and not to solve the specific problem under investigation, avoiding the development of a new tool with functionalities similar to others. Next, we present two examples of this situation.

\subsection{An Immediate Approach for Polymorphism Identification}

Polymorphisms are related to the insertion, deletion, and substitution of one or more nucleotides in a DNA strand (Bell, 2002; Bentley, 2000), events that can occur during the duplication process of these strands. Two important and common types of polymorphism are the so called SNP - Single Nucleotide Polymorphism, which is related to only one nucleotide, and the InDels, when there are insertions and deletions of more than one nucleotide.

SNPs constitute until 90\% of all human genetic variations and occur, in average, each 1000 pairs of nucleotide bases in human genome (Bentley, 2000). SNPs are very important genomic elements and can be related to several genetic diseases. Polymorphisms, in general, have received much interest from the specialists in genetic enhancement researches aimed at 
plants and animals. They can be used as molecular markers and for species evolution studies.

When verifying alterations in sequences from different genomes, the natural way to detect SNPs/InDels is to do this in two separate steps: (a) to align the DNA sequences of interest and (b) to verify possible differences between them. A new bioinformatics tool can be developed for executing both steps in order to make this detection process more accurate and, mainly, faster.

However, a problem associated with the development of a new algorithm and its related computational tool is that all the implementation process can take a long period of time and the created tool can produce initially suspicious or unexpected results, demanding new hours of test execution and coding maintenance before the tool can be considered useful and trustful.

Thus, instead of developing a new computational program for aligning sequences (the first step inside the process described above for SNP/InDel identification), an immediate solution is to use already implemented and well-established computational tools aimed at aligning sequences. There are several bioinformatics tools for aligning sequences that can be used in processes of SNP/InDel detection; two examples are CAP3 (Huang \& Madan, 1999) and Phrap (Phrap, 2009) assembler programs, well-known and largely used in several genomic projects, that align sequences and join them in groups making easier and faster the analysis process.

It is important to say that similar tools aimed to analyze the alignments must be developed in both cases, whether either a new tool is implemented for doing the alignments or an already existent assembler program is used.

This strategy of using an existent assembler for doing sequence alignments has been used for several research groups and inside complete applications aimed at SNP identification (for example, Matukumalli et al., 2006; Savage et al., 2005; Tang et al., 2006).

The Group of Bioinformatics of Rio Claro (GBIRC) has developed a computational system aimed to help specialists involved in SNPs (and InDels) analysis processes. We also have used the approach described above. For this, the system uses CAP3 for sequence alignment and our Perl scripts parse the results verifying possible differences between sequences. These scripts also extract all the information of interest from the results and insert them in a relational database (the use of a relational database facilitates information recovery and analysis of the results). Whether the number of putative polymorphisms detected in the process is great, the system can provide ways to do a selection of part of them based on specific criteria (for example, type of polymorphism, size, and location in a sequence), reducing the number of SNPs candidates to be analyzed. All this process is executed in an automated way. The system was implemented for the web platform, allowing collaborative work between remote researchers. The system and its DEMO can be found at http://gbirc.rc.unesp.br/snpcomp/.

\subsection{Transposable Elements: A Challenge in Genomics}

Repeat elements are a significant part of almost any genome. Repetitive sequences can be primary divided into three classes: local repeats (tandem repeats and simple sequence repeats), families of dispersed repeats (mostly Transposable Elements (TEs) and retrotransposed cellular genes), and segmental duplications (duplicated genomic fragments). 
TEs are a heterogeneous class of genetic elements that vary in structure, mechanism of transposition, and choice of target sites. They are known by a variety of names, including controlling elements, cassettes, jumping genes, roving genes, mobile genes, and mobile genetic elements.

In recent years, much evidence has been found that repetitive sequences play a crucial role in various biological processes of eukaryotic genomes (Craig et al., 2002). Thus, the computational identification of repeats is currently receiving much attention. However, because of the mobile character, the traditional repeater finders cannot give satisfactory results in TE analysis and it is necessary more complex and robust models for this purpose.

The great importance of TEs for molecular mechanisms and genome evolution has generated increasing interest in developing methods and tools for more efficient, sensitive, and accurate discovering, classifying, detecting, and visualizing workflows of TEs in gene sequences. The amount and variety of TEs that can be found in a genome boast fundamental and huge challenges to genome sequencing, assembly, alignment, and annotation processes. The problem of repeat type classification is not well-defined (Kapitonov \& Jurka, 2008; Wicker et al., 2007) and the development of computational algorithms to deal with it is still a hard task, despite some heuristic approaches have been attempted with some results. The main challenges for these approaches are to distinguish TEs from all other repeat classes and to identify distinct TE families. By turn, TE discovery and detection are influenced by other types of genomic repeats (simple repeats and segmental duplications).

Despite the TE classification used in projects around the world, some classes of TEs have remarkable features in their structures. Two examples are LTR class (TEs that present a Long Terminal Repeats) and TIR class (TEs presenting Terminal Inverted Repeats). For these cases, we could say, it is not difficult to produce computational applications for detecting these repeats - all is need is to search for repetitions, inverted or not, of character strings. But, for several other TE classes there is little (or even no) evident characteristic in their composition that could be used properly to construct computational tools.

However, for all TE classes, a simple solution can be used in order to overcome the lack of more evident characteristics. In some public databases, such as the NCBI GenBank (GenBank, 2009), the TIGR (Ouyang \& Buell, 2004), and Repbase (RepBase, 2009), we can find a lot of sequences that have been characterized as TEs. These sequences can be downloaded and separated into classes. Thus, a simple approach to identify TEs can be considered: to align new (target) sequences against the known TE sequences, searching for similarity between them and classifying the target sequences as possible members of one of these classes.

In a similar way to SNP identification, we can develop a new bioinformatics tool for doing sequence alignments and verification of similarities. But, as described before, we have to consider the problems associated with the development of a new algorithm and its computational tool. Again, an immediate solution is to use already existent and wellestablished computational applications for doing both tasks. For this, a very useful tool is BLAST (Altschul et al., 1990). BLAST (Basic Local Alignment Search Tool) is a set of programs, largely used in so many genomic projects, that aligns a query sequence against known sequences of an available genomic database and reports their similarities using two metrics, called e-value and score, showing the alignments. Thus, BLAST can also be used for the identification of TEs inside a new set of genomic sequences. Another very useful bioinformatics tool that can be used for this purpose is RepeatMasker (Smit et al., 2009), an 
application that searches for repeats using its associate database of sequences, including of TEs.

Several projects that intent to identify TEs have used these applications (BLAST and RepeatMasker). It is the case of our group. GBIRC has implemented SATEComp, a computational system aimed at helping specialists to analyze TEs. Initially, SATEComp used only BLAST. Although BLAST and RepeatMasker use similarities for identification of TEs, due to some reasons (preference of use, for completing the results produced by BLAST, etc.) we have opted for including the execution of RepeatMasker in our environment as well. Perl scripts developed by our group parse the results of both applications, extract all the information of interest and insert them in a relational database. The results are presented to the user in a tabular format facilitating the visualization and comparison of them. All this process (alignments with BLAST and RepeatMasker, extraction of information from the resulting files and insertion in the database) is executed automatically by SATEComp. This automatic computational pipeline allows a faster and more reliable identification of TEs in several genomes. Thus, our system can be used as a tool for performing comparative studies considering different organisms, task that would be quite complex and laborious if it was made by manual annotation procedures. The system was primarily constructed for the web platform. Thus, researchers remotely located can work in a collaborative way inside a same project. More about SATEComp can be found in (Fischer et al., 2008) and on Internet at http:/ /gbirc.rc.unesp.br/satecomp/, that includes a DEMO of the system.

Another possible way to detect TEs in genomic sequences is through the use of Hidden Markov Models (HMMs). An HMM (Sean, 1998; Krogh, 1998) is a finite set of states, each of which is associated with a (generally multidimensional) probability distribution. Transitions among the states are governed by a set of probabilities called Transition Probabilities. In a particular state, an outcome or observation can be generated, according to the associated probability distribution.

HMMs have helped to solve some problems in molecular biology, in special as probabilistic profiles of protein families. An HMM can be used in order to search possible members of a particular protein family inside a new genomic database. One form of constructing an HMM for this purpose is to align known sequences of the family and capture information about each type of amino acid considering each column of the alignment (in other words, counting the number for each amino acid present in each position of the alignment). Thus, the HMM generated could be compared with each target biosequence.

Since HMMs can be used for identification of members of protein families, it is natural to think in applying them for detection of members of TE classes. Several groups have considered applying HMMs for doing this work (for example, Andrieu et al., 2004; Juretic, 2004; Urgi, 2009), again, including our group.

HMMER (Hmmer, 2009) is a computational application that has been successfully used for constructing HMMs and searching for known proteins in a new database. All that is necessary is to provide HMMER with the sequence alignments. Our group has executed several tests using HMMER with representative sequences of TEs. We observed that in some cases the results produced by HMMER were complementary to the ones generated by SATEComp. Thus, we decided to incorporate the HMM approach in our system in order to have more precise and reliable results inside the same computational environment.

However, there is a problem with this approach where we need representative sequences: new TE sequences can be lost in the analysis process due to the lack or weakness of 
similarity between the target sequences and the sequences included presently into a class. Moreover, discovering of a new class of TEs is not possible using this approach because we must have the representative sequences of TEs for the alignments. Thus, other solutions for the construction of auxiliary tools in analysis processes of TEs, and to help in several other types of biological problem, must be proposed in order to obtain results more complete and reliable.

\section{Recent Development of Natural Computing Techniques in Bioinformatics}

Despite the great contributions Genomics and Proteomics have received from using algorithms and techniques developed in other areas, for many biological problems, the consideration of simple techniques is not enough due to the involved characteristics and conditions or the absence of additional knowledge about the problems. For these cases, it is necessary to develop new approaches or consider the application of more sophisticate already existent ones such as some solutions from Computational Intelligence area. Also, even for problems that have been already treated in Bioinformatics, there is a lack of more efficient, sensitive, and accurate tools. So, it is necessary to propose alternatives to treat a problem in a more efficient manner, developing strategies to attack it properly.

In last years, Natural Computing methods have been applied to many fields of Bioinformatics (Fogel, 2008; Hassanien et al., 2008; Masulli \& Mitra, 2009), such as protein structure prediction, protein folding simulation, microarray data analysis, and gene regulatory networks modeling. Natural Computing is a branch of the Computational Intelligence area that extracts ideas from nature to develop computational systems for problem solving which is related to optimization, data processing, and analysis techniques. Among the many approaches within computing inspired by natural and biological principles, the most well-known ones are Neural Networks (Haykin, 1999), Fuzzy Systems (Pedrycz \& Gomide, 2007), Evolutionary Computing (Michalewicz, 1998), Swarm Intelligence (Bonabeau et al., 1999), Immunocomputing (de Castro \& Timmis, 2002), and Simulated Annealing (Kirkpatrick, 1983).

Neural Networks are motivated by the highly interconnected neural structures in the brain and the nervous system. Fuzzy Systems are based on an extension of traditional logic in order to represent uncertainty and qualitative reasoning. Evolutionary Computing (i.e., genetic algorithms, genetic programming, and grammatical evolution) uses concepts of mutation, recombination and natural selection from biology. Swarm Intelligence (i.e., including particle swarm optimization (Eberhart et al., 2001), ant colony systems (Dorigo \& Blum, 2005), and bee hive algorithms (Baykasoglu et al., 2007)) mimics the emergent collective behaviour of groups of simple agents (like social insects, birds and fishes), that result from the local interactions of the agents with each other and with their environment. Immunocomputing (or artificial immune systems) is inspired by the principles and processes of the vertebrate immune system. Simulated Annealing stems from an analogy between the way in which a metal cools and freezes into a minimum energy crystalline structure. The main applications of these methods refer to tasks as signal processing, classification, clustering, feature selection, optimization, data visualization, data mining, and information fusion.

Protein structure prediction (PSP) and protein folding (PF) problems are currently some of the most interesting problems in molecular biology. Intelligent techniques have been 
applied to these problems, like as Neural Networks (Pollastri et al., 2002), Evolutionary Computing (Pedersen \& Moult, 1997; Cutello et al., 2006), Fuzzy Systems (Blanco et al., 2002), Immune Systems (Nicosia, 2004), Swarm Intelligence (Bahamish et al., 2008), and Simulated Annealing (Simons et al., 1997).

DNA sequence alignment is an important operation in Genomics and Proteomics that consists of finding similarity between genome segments. It is very useful, for instance, to study functional, structural or evolutionary relationships between organisms, to investigate gene regulation, and to predict the function of novel genes within any species. This task has also received contribution of these innovative techniques as Ant Colony Systems (Chen et al., 2007; Zhao et al., 2008; Chen et al., 2009) and Genetic Algorithms (Nguyen et al., 2002; Shyu et al., 2004; Jangam \& Chakraborti, 2007).

The automatic motif discovery problem is a multiple sequence local alignment problem that involves the search for approximate matches. Motifs are conservative sequence patterns among the regulatory regions of correlated genes. A number of natural computing approaches have been proposed to handle the problem, including Neural Networks (Mahony et al., 2006), Genetic Algorithms (Hemalatha \& Vivekanandan, 2008; Kaya, 2009; Venugopal, 2009), and Swarm Intelligence (Lei \& Ruan, 2008).

Pattern discovery, DNA mapping, gene identification, and sequence labeling form challenging computational problems in Bioinformatics. Such problems can be treated with methods for data classification, detection, segmentation, clustering, or prediction. Again, Natural Computing approaches demonstrate to be useful for such purposes. Different techniques were used to accomplish these tasks, such as Neural Networks (Li et al., 2003; Mateos et al., 2003; You \& Liu, 2007), Genetic Algorithms (Quesneville \& Anxolabéhère, 2001; Pereira et al., 2009; Jacob et al., 2009), Immunocomputing (Wang et al., 2008a-b), Swarm Intelligence (Zhang et al., 2007; Greene et al., 2008), and Simulated Annealing (Filippone et al, 2005, 2006).

Detecting repetition by sequence alignment methods is relatively easy. However, TE identification problem is notoriously difficult because the processes of TE evolution remain questionable. The moving ability, coding capacity, duplicating process, and mutation dynamics of TEs are not well understood and are still debated. The integration of diverse computational tools and techniques for a comprehensive analysis of TEs is still an open challenge in Bioinformatics.

The problem of automated TE detection and classification seems to be deranged and jumbled. A systematic algorithmic procedure using the traditional methods appears to be unable to perform the covering of the variety of TEs' sequences and families. Thus, more adaptive procedures, including intelligent and versatile strategies, can furnish further improvements in accuracy of computational tools because different repeat compositions may demand adjusting of parameters in genome analysis.

Natural Computing is a promising framework for TE prediction, providing ability to capture dependencies and to incorporate non-probabilistic evidences and heterogeneous data. Nevertheless, despite of the great number of intelligent methods and approaches being used in several problems of the Bioinformatics field, the application of natural computing in TEs is still only bashfully investigated (Quesneville \& Anxolabéhère, 2001; Langdon \& Banzhaf, 2008). Our explanation makes contribution to Bioinformatics by calling attention to this important issue. More systematic studies of promoting natural computing as a whole for handling genome repeats are desirable. 
On the other hand, traditional HMM can also take benefit of intelligent computing techniques to increase the model capability and improve its performance in different genomic problems. Some hybrid models were already developed incorporating Fuzzy Logic (Collyda et al., 2006, 2007; Bidargaddi et al., 2008), particle Swarm Optimization (Rasmussen \& Krink, 2003), and Genetic Algorithms (Won et al., 2004) approaches.

There are several general concepts underlying many approaches in natural computing, like parallelism, adaptation, distributivity, interactivity, emergence, self-organization, feedback, etc. Yet, the employment of natural computing in genome problems goes far beyond such features. Regardless of applying a specific nature inspired computing method or using hybrid strategies with traditional methods to solve biological problems, there are other aspects related to this approach that can contribute to obtain models to both represent biological knowledge and predict the characteristics of biological systems, enhancing the quality and accuracy of solutions. Other aspect is the ability to capture interactions among data and variables. For these reasons, we believe that natural computing seems to offer fruitful contributions for this field and even to be extended to address problems not considered here.

\section{Innovative Hardware and Architecture Solutions for Bioinformatics}

Several research groups have dedicated much effort in trying to develop bioinformatics tools that would be executed faster than similar existent ones. There is no doubt that it is very important to develop new "faster" software.

However, it is necessary to propose solutions in terms of hardware as well, be as a dedicate and complex computational architecture or as a relatively simple but specialized device, in order to treat specifically genomic problems intending to reduce the processing time - for example a hardware aimed at DNA sequence comparison.

In the same way we described before for software, we present below some hardware and architecture solutions that can be considered with the objective of reducing the processing time and, consequently, the response time for tasks related to genomic analysis.

Maybe the most natural way to aim that objective is to execute several processes simultaneously, a very common form of obtaining faster answers. One example of hardware solution for reducing the response time is the one used by the National Center for Biotechnology Information - NCBI, USA. NCBI provides a public service where users can request searches in its molecular sequence databases with the BLAST tool. Daily, thousands of requests must be served, requiring high availability and high performance from the NCBI computers. A "simple" solution used by that center was to distribute BLAST searches across multiple worker nodes (as they say): each node is responsible for searches in only part of a database, reducing significantly the response time for the requests (Blast, 2009). Several other groups have applied known computational approaches similar to that used by NCBI, in special, the ones involving computer clusters and parallel processing.

On the other hand, some non-traditional computing approaches have been presented by other groups. For example, consider the following. The information generated in sequencing processes has been stored in computer files as sequences of nucleotides. Each sequenced DNA nucleotide can be represented as a character (A, C, G, T) and inside a computer as one byte, a set of eight bits. Since every bit can assume one of two values (zero or one), using eight bits, we can obtain 256 different combinations, that is, 256 distinct characters. 
However, for a strand of DNA, it is necessary only four combinations (four characters), what can be represented using only two bits. With this approach, it is possible to fit four characters in each byte, reducing the size of each sequence file to one fourth of the original size. This approach can be used as a simple form of reducing the size of DNA databases and the space necessary in storage devices. Also, for several types of biological problems, less space in computer main memory will be required for processing data in that format. Moreover, since all the data set is smaller, a greater number of data (nucleotides) can be transferred between storage devices and main memory in each access, reducing the number of accesses necessary to those devices (that are considered slow) and, consequently, the transfer time.

However, probably the main benefit of this approach is that it can help in reducing the processing time of biosequences. Certainly, the most common operation when treating DNA sequences is comparison of nucleotides, which is used in searches for patterns, in sequence assembly processes, when aligning sequences, and in several other activities related to genomic sequences. In the current model of comparing two sequences, one nucleotide of a sequence is compared to one nucleotide of the other sequence. It means that one byte with one nucleotide is considered at a time in each comparison. Using the approach described above, four nucleotides into each byte of each sequence will be considered in each comparison, allowing to compare a greater quantity of nucleotides in each operation, reducing the total processing time.

Some works have been presented considering approaches similar to the described one. For example, Krishnamurthy and colleagues in (Krishnamurthy et al., 2007) describe about the design of BLASTN (the version of BLAST for nucleotides) for their architecture Mercury.

However, some (more) complex architectures have been proposed for reducing the processing time. For example, Hasan and colleagues in (Hasan et al., 2007) present an overview of various global and local sequence alignment methods describing about some considered architectures.

\section{Conclusion}

In this chapter we intended to present indications of forms to solve, or at least to help to solve, some important problems in Genomics. Depending on the characteristics of the biological problem being treated, simple and traditional algorithms from, for example, Computing area can be very useful to solve it. However, in many other situations, more sophisticate techniques must be considered in order to help to understand more complex problems and then to try to propose solutions, both in terms of software and dedicate hardware.

\section{References}

Agarwal, P. \& States, D.J. (1994). The Repeat Pattern Toolkit (RPT): analyzing the structure and evolution of the C. elegans genome, Proc Int Conf Intell Syst Mol Biol, 2:1-9.

Altschul, S.F.; Madden, T.L.; Schäffer, A.A.; Zhang, J.; Zhang, Z.; Miller, W. \& Lipman, D.J. (1997). Gapped BLAST and PSI-BLAST: a new generation of protein database search programs, Nucleic Acids Res., Vol. 25, pp.3389-3402. 
Andrieu, O.; Fiston, A-S.; Anxolabéhère, D. \& Quesneville, H. (2004). Detection of transposable elements by their compositional bias, BMC Bioinformatics, Vol. 5:94.

Bahamish, H.A.A.; Abdullah, R. \& Salam, R.A. (2008). Protein Conformational Search Using Bees Algorithm, Proceedings of the 2th Asia International Conference on Modelling $\mathcal{E}$ Simulation, pp. 911-916.

Bell, J.I. (2002). Single nucleotide polymorphisms and disease gene mapping, Arthritis Res., Vol. 4 (Suppl. 3), pp. S273-S278.

Blanco, A.; Pelta, D.A. \& Verdegay, J.-L. (2002). Applying a fuzzy sets-based heuristic to the protein structure prediction problem, International Journal of Intelligent Systems, Vol. 17, pp. 629-643.

Bao, Z. \& Eddy, S.R. (2002). Automated de novo identification of repeat sequence families in sequenced genomes, Genome Res., Vol. 112, pp. 1269-1276.

Baykasoglu, A.; Özbakır, L. \& Tapkan, P. (2007). Artificial Bee Colony Algorithm and Its Application to Generalized Assignment Problem. In: Swarm Intelligence: Focus on Ant and Particle Swarm Optimization, F.T.S. Chan and M.K. Tiwari (Eds.), Itech Education and Publishing, Vienna, Austria, pp. 113-144.

Bentley, D.R. (2000). The Human Genome Project - An Overview, Medicinal Research Reviews, Vol. 20, pp. 189-196.

Bergman, C.M. \& Quesneville, H. (2007). Discovering and detecting transposable elements in genome sequences, Briefings in Bioinformatics, Vol. 8, No. 6, pp. 382-392.

Bidargaddi, N.P.; Chetty, M. \& Kamruzzaman, J. (2008). Hidden Markov models incorporating fuzzy measures and integrals for protein sequence identification and alignment, Genomics Proteomics Bioinformatics, Vol. 6, No. 2, pp. 98-110.

BLAST Documentation (2009). Available at: ftp://ftp.ncbi.nlm.nih.gov/blast/documents/ blast-sc2004.pdf . Last access: May 2009.

Bonabeau, E.; Dorigo, M. \& Theraulaz, G. (1999). Swarm Intelligence: From Natural to Artificial Systems, Oxford University Press.

Campagna, D.; Romualdi, C.; Vitulo, N. et al. (2005). RAP: a new computer program for de novo identification of repeated sequences in whole genomes, Bioinformatics, Vol. 21, pp. 582-588.

Caspi, A.; Pachter, L. (2006). Identification of transposable elements using multiple alignments of related genomes, Genome Res., Vol. 16, pp. 260-270.

Chen, L.; Liu, W. \& Chen, J. (2007). Ant Colony Optimization Method for Multiple Sequence Alignment, Proceedings of the 2007 International Conference on Machine Learning and Cybernetics, Vol. 2, pp. 914-919.

Chen, W.; Liao, B.; Zhu, W.; Liu, H. \& Zeng, Q. (2009). An Ant Colony Pairwise Alignment Based on the Dot Plots, Journal of Comput Chem, Vol. 30, pp. 93-97.

Collyda, C.; Diplaris, S.; Mitkas, P.A.; Maglaveras, N. \& Pappas, C. (2006). Fuzzy Hidden Markov Models: a new approach in multiple sequence alignment, Studies in Health Technology and Informatics, Vol. 129, pp. 1245-1249.

Collyda, C.; Diplaris, S.; Mitkas, P.A.; Maglaveras, N. \& Pappas, C. (2007). Enhancing the quality of phylogenetic analysis using fuzzy hidden Markov model alignments, Studies in Health Technology and Informatics, Vol. 124, pp. 99-104.

Craig, N.L.; Craigie, R.; Gellert, M. \& Lambowitz, A.M. (2002). Mobile DNA II. American Society for Microbiology Press, Washington, D.C. 
Cutello, V.; Narzisi, G. \& Nicosia, G. (2006). A multi-objective evolutionary approach to the protein structure prediction problem, Journal of The Royal Society Interface, Vol. 3, pp. 139-151.

de Castro, L. \& Timmis, J. (2002). Artificial immune systems: A new computational approach. Springer-Verlag, London. UK.

Dorigo, M. \& Blum, C. (2005). Ant colony optimization theory: A survey, Theoretical Computer Science, Vol. 344, No. 2-3, pp. 243-278.

Eberhart, R.C.; Kennedy, J. \& Shi, Y. (2001). Swarm Intelligence, Elsevier Science.

Edgar, R.C. \& Myers, E.W. (2005). PILER: Identification and classification of genomic repeats, Bioinformatics, Vol. 21, pp. i152-i158.

Filippone, M.; Masulli, F. \& Rovetta, S. (2005). Unsupervised gene selection and clustering using simulated annealing. In: Isabelle Bloch, Alfredo Petrosino, and Andrea Tettamanzi (eds.), WILF, Lecture Notes in Computer Science, Vol. 3849, Springer, pp. 229-235.

Filippone, M.; Masulli, F. \& Rovetta, S. (2006). Supervised classification and gene selection using simulated annealing, Proceedings of the Int. Joint Conf. on Neural Networks, IEEE, pp. 3566-3571.

Fischer, C.N.; Cerri, R.; Costa, E.P. \& Bacci Jr., M. (2008). SATEComp: a Tool for in Silico Analysis of Transposable Elements, Proceedings of World Academy of Science, Engineering and Technology, Vol. 34, October, pp. 878-882.

Fogel, G.F. (2008). Computational intelligence approaches for pattern discovery in biological systems, Briefings in Bioinformatics, Vol. 9, pp. 307-316.

GenBank - NCBI (2009). Available at: http://www.ncbi.nlm.nih.gov/Genbank/ . Last access: May 2009.

Greene, C.S.; White, B.C. \& Moore, J.H. (2008). Ant colony optimization for genome-wide genetic analysis, Lecture Notes in Computer Science, Vol. 5217, pp. 37-47.

HMMER Documentation (2009). Available at: http://hmmer.janelia.org/ . Last access: May 2009.

Hasan, L.; Al-Ars, Z. \& Vassiliadis, S. (2007). Hardware Acceleration of Sequence Alignment Algorithms - An Overview, IEEE Xplore, Sept., pp.92-97.

Hassanien, A.-E.; Milanova, M.G.; Smolinski, T.G. \& Abraham, A. (2008). Computational intelligence in solving bioinformatics problems: Reviews, perspectives, and challenges, Studies in Computational Intelligence, Vol. 151, pp. 3-47.

Haykin, S. (1999). Neural Networks. A Comprehensive Foundation, second ed., Prentice-Hall, Upper Saddle River, NJ.

Hemalatha, M. \& Vivekanandan, K. (2008). Genetic algorithm based probabilistic motif discovery in unaligned biological sequences, Journal of Computer Science, Vol. 4, No. 8, pp. 625-630.

Huang, X. \& Madan, A. (1999). CAP3: A DNA Sequence Assembly Program, Genome Research, Vol.9, n.9, pp. 868-877.

Jacob, E.; Nair, K.N.R. \& Sasikumar, R. (2009). A fuzzy-driven genetic algorithm for sequence segmentation applied to genomic sequences, Applied Soft Computing Journal, Vol. 9, No. 2, pp. 488-496.

Jangam, S.R. \& Chakraborti, N. (2007). A novel method for alignment of two nucleic acid sequences using ant colony optimization and genetic algorithms, Applied Soft Computing, Vol. 7, No. 3, pp. 1121-1130. 
Juretic, N.; Bureau, T.E. \& Bruskiewich, R.M. (2004). Transposable element annotation of the rice genome, Bioinformatics, Vol. 20, n.2, pp. 155-160.

Kapitonov, V.V. \& Jurka, J. (2008). A universal classification of eukaryotic transposable elements implemented in RepBase, Nature Reviews Genetics, Vol. 9, pp. 411-412, May.

Kaya, M. (2009). MOGAMOD: Multi-objective genetic algorithm for motif discovery, Expert Systems with Applications, Vol. 36 (2 PART 1), pp. 1039-1047.

Kirkpatrick, S.; Gelatt, C.D. \& Vecchi, M.P. (1983). Optimization by simulated annealing, Science, Vol. 220, pp. 661-680.

Krishnamurthy, P.; Buhler, J.; Chamberlain, R.; Franklin, M.; Gyang, K.; Jacob, J. \& Lancaster, J. (2007). Biosequence Similarity Search on the Mercury System, Journal of VLSI Signal Processing Systems, Vol. 49, pp. 101-121.

Krogh, A. (1998). An Introduction to Hidden Markov Models for Biological Sequences, In: SALZBERG, Steven; SEARLS, David; KASIF, Simon (Comp.). Computational Methods in Molecular Biology. [s.i.]: Elsevier, Chap. 4, pp. 45-63.

Kurtz, S.; Ohlebusch, F.; Schleiermacher, C.; Stoye, J. \& Giegerich, R. (2000). Computation and visualization of degenerate repeats in complete genomes, Proc. Int. Conf. Intel. Syst. Mol. Biol. 8: 228-238.

Lafferty, J.; McCallum, A. \& Pereira, F. (2001). Conditional random fields: probabilistic models for segmenting and labeling sequence data. In: International Conference on Machine Learning.

Langdon, W.B. \& Banzhaf, W. (2008). Repeated patterns in genetic programming, Natural Computing, Vol. 7, pp. 589-613.

Lei, C. \& Ruan, J. (2008). A particle swarm optimization algorithm for finding DNA sequence motifs, Proceedings of the 2008 IEEE International Conference on Bioinformatics and Biomedicine Workshops (BIBMW), art. no. 4686231, PA, USA, Nov., pp. 166-173.

Li, Y.; Yang, C. \& Zhang, W. (2003). The Neural Network Method of DNA Sequence Classification, Compute Simulation, Vol. 20, No. 2, pp.65-68.

Mahony, S.; Benos, P.V.; Smith, T.J. \& Golden, A. (2006). Self-organizing neural networks to support the discovery of DNA-binding motifs, Neural Networks, Vol. 19, pp. 950962.

Masulli, F. \& Mitra, S. (2009). Natural computing methods in bioinformatics: A survey, Information Fusion, Vol. 10, pp. 211-216.

Mateos, A.; Dopazo J.; Jansen, R.; Tu, Y.; Gerstein, M. \& Stolovitzky, G. (2002). Systematic learning of gene functional classes from DNA array expression data by using multilayer perceptrons, Genome Res. Vol. 12, pp. 1703-1715.

Matukumalli, K.L; Grefenstette, J.J; , Hyten, D.L; Choi, I-Y; Cregan, P.B \& Van Tassell, C.P. (2006). SNP-PHAGE - High throughput SNP discovery pipeline, BMC Bioinformatics, Vol. 7:468, Available from: http://www.biomedcentral.com/14712105/7/468.

McCarthy, E.M. \& McDonald, J.F. (2003). LTR_STRUC: a novel search and identification program for LTR retrotransposons, Bioinformatics, Vol. 19, pp. 362-7.

Michalewicz, Z. (1998). Genetic Algorithms + Data Structures = Evolution Programs, Springer. 
Nguyen, H.D.; Yoshihara, I.; Yamamori, K. \& Yasunaga, M. (2002). Aligning multiple protein sequences by parallel hybrid genetic algorithm. Genome Inform., Vol. 13, pp. $123-132$.

Nicosia, G. (2004). Immune algorithms for optimization and protein structure prediction, Ph.D. thesis, University of Catania, Italy.

Ouyang, S. \& Buell, C.R. (2004). The TIGR Plant Repeat Databases: a collective resource for the identification of repetitive sequences in plants, Nucleic Acids Research, Vol. 32, Database issue, pp. 360-363.

Pedersen, J.T. \& Moult, J. (1997). Protein Folding Simulations with Genetic Algorithms and a Detailed Molecular Description, Journal of Molecular Biology, Vol. 269, pp. 240-259.

Pedrycz, W. \& Gomide, F. (2007). Fuzzy Systems Engineering: Toward Human-Centric Computing, IEEE/Wiley Interscience.

Pereira, P.; Silva, F. \& Fonseca, N.A. (2009). BIORED - A genetic algorithm for pattern detection in biosequences, Advances in Soft Computing, Vol. 49, pp. 156-165.

Pevzner, P.A.; Tang, H. \& Tesler, G. (2004). De novo repeat classification and fragment assembly, Genome Res., Vol. 14, pp. 1786-1796.

Phrap Documentation (2009). Available at: http:/ / www.phrap.org/ . Last access: May 2009.

Pollastri, G.; Przybylski, D.; Rost, B. \& Baldi, P. (2002). Improving the prediction of protein secondary structure in three and eight classes using recurrent neural networks and profiles, Proteins, Vol. 47, pp. 228-235.

Quesneville, H. \& Anxolabéhère, D. (2001). Genetic algorithm-based model of evolutionary dynamics of class II transposable elements, J. Theor. Biol., Vol. 213, pp. 21-30.

Rabiner, L.R. (1989). A tutorial on hidden Markov models and selected applications in speech recognition, Proceedings of the IEEE, Vol. 77, No. 2, pp. 257-285.

Rasmussen T.K. \& Krink, T. (2003). Improved Hidden Markov Model training for multiple sequence alignment by a particle swarm optimization-evolutionary algorithm hybrid, Bio Systems, Vol. 72, No. 1-2, pp. 5-17.

RepBase (2009). Available at: http://www.girinst.org/server/RepBase/ . Last access: May 2009.

Sakakibara, Y.; Brown, M.; Hughey, R.; Mian, I.S.; Sjölander, K.; Underwood, R.C. \& Haussler, D. (1994). Stochastic context-free grammars for tRNA modeling, Nucleic Acids Res., Vol. 22, pp. 5112-5120.

Savage, D.; Batley, J.; Erwin, T.; Logan, E.; Love, C.G; Lim, G.A.C.; Mongin, E.; Barker, G.; Spangenberg, G.C.; Edwards, D. (2005). SNPServer: a real-time SNP discovery tool, Nucleic Acids Research, Vol. 33, pp. 493-495.

Sean, R. E. (1998). Profile Hidden Markov Models. Bioinformatics Review, [s.i.], Vol. 14, n. 9, pp. 755-763.

Shyu, C.; Sheneman, L. \& Foster, J.A. (2004). Multiple sequence alignment with evolutionary computation, Genet. Prog. Evol. Mach., Vol. 5, pp. 121-144.

Simons, K.T.; Kooperberg, C.; Huang, E. \& Baker, D. (1997). Assembly of protein tertiary structures from fragments with similar local sequences using simulated annealing and Bayesian scoring functions, Journal of Molecular Biology, Vol. 268, pp. 209-225.

Smit, A.F.A.; Hubley, R.; \& Green, P. (2009) - RepeatMasker Documentation. Available at: http://www.repeatmasker.org . Last access: May 2009.

Szklarczyk, R. \& Heringa, J. (2004). Tracking repeats using significance and transitivity, Bioinformatics, Vol. 20 (Suppl 1), pp. i311-i317. 
Tang, J.; Vosman, B.; Voorrips, R.E.; van der Linden, C.G. \& Leunissen, J.A.M. (2006). QualitySNP: a pipeline for detecting single nucleotide polymorphisms and insertions/deletions in EST data from diploid and polyploid species, BMC Bioinformatics, Vol. 7:438, Available from: http://www.biomedcentral.com/14712105/7/438.

Venugopal, K.R.; Srinivasa, K.G. \& Patnaik, L.M. (2009). Merge based genetic algorithm for motif discovery, Studies in Computational Intelligence, Vol. 190, pp. 331-341.

Urgi (2009). Available at: http://urgi.versailles.inra.fr/research/index.php. Last access: May 2009.

Wang, L.; Zhang, J.; Huang, X.; Gong, G. (2008a). DNA sequences classification based on immune evolution network, Proceedings of the 5th International Conference on Fuzzy Systems and Knowledge Discovery (FSKD 2008), art. no. 4666157, pp. 448-452.

Wang, L.; Zhang, J.; Gong, G.; Peng, M. (2008b). Application of immune classifier based on increment of diversity in the model species genomes identification, Proceedings of the International Conference on Intelligent Computation Technology and Automation (ICICTA 2008), art. no. 4659437, pp. 30-35.

Wicker, T; Sabot, F.; Hua-Van, A.; Bennetzenm, J.L., Capy, P.; Chalhoub, B.; Flavell, A.; Leroy, P.; Morgante, M.; Panaud, O.; Paux, E.; SanMiguel, P. \& Schulman, A.H. (2007). A unified classification system for eukaryotic transposable elements, Nature Reviews Genetics, Vol. 8, pp. 973-982, May.

Won, K.-J.; Prügel-Bennett, A. \& Krogh, A. (2004). Training HMM structure with genetic algorithm for biological sequence analysis, Bioinformatics, Vol. 20, No. 18, pp. 36133619.

Yoon, B.-J. \& Vaidyanathan, P.P. (2004). HMM with auxiliary memory: A new tool for modeling RNA secondary structures, Proceedings of the 38th Asilomar Conference on Signals, Systems, and Computers, Monterey, CA.

Yoon, B.-J. \& Vaidyanathan, P.P. (2005). Optimal alignment algorithm for context-sensitive hidden Markov models, Proceedings of the 30th International Conference on Acoustics, Speech, and Signal Processing, Philadelphia.

You, W. \& Liu, Y. (2007). Classifying DNA sequences by artificial neural network model, Science and Technology Information, Vol. 25, pp. 89-90.

Zhang, H.; Song, X. \& Wang, H. (2007). Feature gene selection base on binary particle swarm optimization and support vector machine, Computer and Applied Chemistry, Vol. 24, No. 9, pp.1159-1162.

Zhao, Y.; Ma, P.; Lan, J.; Liang, C. \& Ji, G. (2008). An improved ant colony algorithm for DNA sequence alignment, Proceedings of the 2008 International Symposium on Information Science and Engineering (ISISE 2008), Vol. 2, pp. 683-688. 


\title{
Comparative Performance Assessment In EU Pre-Accession Funds
}

\author{
Arzu Tektas and Asli Deniz Helvacioglu Kuyucu \\ Bogazici University \\ Turkey
}

\section{Introduction}

European Union accession process designates candidate countries to adopt EU policies and laws to achieve concerted economic integration and social cohesion with the European system. Pre-accession funds are offered as the main instruments of EU enlargement programs in the course of harmonization. Each pioneered in different policy areas in line with the priorities of the development schemes of the candidate country; those funds motivate regional development, enhance civil participation and most importantly facilitate the Europeanization of the candidate country. The major challenge of pre-accession fund schemes derives from the ability of the candidate country to utilize those financial assistance programs in order to attain the most beneficial outcomes for all of the interest groups in the society. This certainly needs the configuration of the institutional templates and administrative capacity in the context of the requirements of the EU policies as well as the right establishment of fund allocation architectures that fit with the dynamics of the candidate country.

This study aims at developing an analytical framework to assess the comparative performance and analyze the efficiency of the EU programs in the candidate countries, particularly in Turkey, in attaining the objectives of regional development policies. Such an analysis would contribute both to ascertain the absorption capacity of the regions for further EU funding and to identify the best practices in the three regional development grant schemes in Turkey, established and allocated under the pre-accession process. Comparative performance measurement approach can involve parametric or nonparametric methods. Major problem with a parametric approach is the requirement for a priori estimation of the type of method such as linear, non-linear etc. which may lead to a misspecified model. It might also get difficult to deal with multiple inputs and multiple outputs simultaneously. These problems can be overcome utilizing the non-parametric methods such as the Data Envelopment Analysis (DEA). DEA constructs an efficient boundary using the methods of linear programming and measures the distance of any unit from the boundary. It computes the relative efficiency of a unit and derives a variety of information about comparative

Authors thank Bogazici University Research Fund for supporting the Project (08HN201) titled "Ex-Post Efficiency Analysis of European and Internationally Funded Projects". 
performance assessment. Benchmark units that may be used as role models for other units are identified. Based on factor utilization levels of benchmark units, information is derived on the marginal rates of substitution between the factors of production of inefficient units. It thus becomes possible to know which mix of resources, scope of activities each inefficient unit may adopt to improve their performance.

The paper proceeds with a discussion of the absorption capacity analysis in the context of comparative performance analysis. The following section is about Data Envelopment Analysis with specific emphasis on the CCR Model. Section four presents the absorption capacity analysis of NUTS II regions in Turkey and discusses the results of the DEA model with respect to macroeconomic, administrative and financial dimensions of absorption capacity. The paper ends with the conclusion section.

The study contributes to the literature by introducing and implementing an analytical approach for comparative performance assessment of absorption capacities. The approach The approach may be utilized as a common pattern of efficiency analysis for the candidate countries. Implementing the adopted DEA model to the regional development grant schemes in Turkey, a coherent strategy can be proposed to improve the efficiency of the preaccession funds for regional development in Turkey.

\section{Absorption Capacity Analysis in the Context of Comparative Performance Assessment}

Comparative performance assessment in the EU pre-accession process stands as one of the major areas of structural fund management. It provides decision-makers the required information about the consequences of projects, plans, policies and regulations with regard to the objectives designated to be achieved at the first hand. Therefore it could be noted that comparative performance assessment is more likely a strategic tool in the EU accession process. European Union proposes a unique system of funding for the management of the deepening of the economic integration and introduces specially designed funding schemes nearly for all of the policy areas to promote economic and social cohesion among the candidate countries. The current European system of funding is based on more rigorous systems of management, monitoring, control and evaluation. European Commission (2004), states that most of the effects of cohesion policy cannot be expressed in quantitative terms. Accordingly, beyond the net impact of policy on GDP or employment, its added value arises from other aspects like the contribution made to regional development by factors such as strategic planning, integrated development policies, partnership evaluation and the exchange of experience, know-how and the good practices between regions.

In the literature there are several studies focus on the efficiency assessment of the funds being allocated and their impact upon growth in the context of comparative performance assessment. Some econometric analyses state that funds have a negligible or even a negative impact on convergence, while others imply a significant positive impact. Related studies propose a number of different models and approaches to calculate the efficiency and the impact. The studies may be outlined under four titles; Europeanization studies, absorption capacity studies, added value studies and impact assessment studies. Even though they are categorized in accordance with their starting point whether to find out the level of Europeanization, the absorption capacity for further EU funding or to identify the added 
value resulting from the Community assistance, they all tend to explore the relation between the funds and their impacts on the region.

OECD defines absorption capacity as the accumulation of adequate information dissemination, capacity building in local governance and civil society for the conception and implementation of development projects (OECD, 2006). Absorption capacity leads to a strong performance of the EU funds only if the economy, efficiency and effectiveness are taken fully into account (Sumpikova et.al, 2004). Sumpikova et.al. (2004), define absorption capacity as the extent to which a state is able to fully spend the allocated financial resources from the EU. The literature on absorption capacity of the EU funds in the Candidate Countries uses the absorption classification of the EU providing three main definitions (Zerbirati 2004; Sumpikova et. al. 2004; Oprescu et.al. 2005, Georgescu, 2008).

- Macroeconomic absorption capacity, which can be defined and measured in terms of the ratio of GDP levels to Structural Funds allocated. (the upper limit for the Structural and Cohesion Funds was generally set at 4 percent of the GDP of the respective Member State)

-Administrative absorption capacity, which can be defined as the ability and skills of central, regional and local authorities to prepare acceptable plans, programs, and projects in due time, to decide on programs and projects, to arrange co-ordination among the principal partners, to cope with the vast amount of administrative and reporting work required by the Commission, and to finance and supervise implementation properly, avoiding fraud as much as possible.

- Financial absorption capacity, which means the ability to co-finance the EU-supported programs and projects, to plan and guarantee these national contributions in multiannual budgets, and to collect these contributions from several partners (public and private), interested in a program or project.

\section{Data Envelopment Analysis}

Data Envelopment Analysis (DEA) is a nonparametric, deterministic performance analysis tool. DEA is a "data oriented" approach for evaluating the performance of a set of peer units called Decision Making Units (DMUs) which convert multiple inputs into multiple outputs (Cooper et al., 2000). DEA is among the highly preferred methods of performance or efficiency analysis basically due to a number of advantages over parametric methods. Unlike most other approaches like regression analysis that need a priori assumptions, DEA requires very few assumptions. It does not attempt to explain the nature of the relations between the multiple inputs and multiple outputs that belong to the analysis units.

Within DEA context, the relative efficiency of any DMU is calculated as the weighted sum of outputs to weighted sum of inputs. The efficiency of a DMU is a scalar ranging between zero and one, which is evaluated through a linear programming model. By calculating the efficiency of each DMU, DEA forms a production possibility frontier from the most efficient DMUs. DEA connects the points of efficient DMUs with each other by interpolation to form the frontier. The new lines and points on this frontier are also relatively efficient. Inefficient production is also possible and inefficient DMUs are enveloped by the production possibility frontier (Thanassoulis, 2001). A DMU is to be rated as fully (100\%) efficient on the basis of available evidence if and only if the performances of other DMUs do not show that some of its inputs or outputs can be improved without worsening some of its other 
inputs or outputs (Cooper et al., 2004). In other words, a DMU is efficient if and only if it is not dominated by some other DMU(s) with which it can be compared. This definition deals with the "technical" aspects of efficiency. Properties such as, economies of scale, piecewise linearity, categorical variables, ordinal relationships can also be treated through DEA.

As pointed out in Cooper et al. (2000), DEA has also been used to supply new insights into entities that have previously been evaluated by other methods. Benchmarking with DEA has identified sources of inefficiency in some of the most profitable benchmark firms and this has made it possible to identify better benchmarks in many applied studies.

DEA is used not only to evaluate the efficiency scores of each DMU and then to rank them, but also to realize improvement opportunities for the relatively inefficient DMUs. For this purpose, DEA specifies one or more efficient peers for each inefficient DMU in order to emulate their operating practices to improve performance. It also estimates the target levels of the input-output variables which the DMU should attain to operate efficiently.

It is also possible to retain extra information related to efficient units. DEA identifies the reference frequencies and weights of efficient DMUs as being role models for inefficient units. If the reference frequency and weight of an efficient DMU is high, this generally implies that it is a well performing unit because it outperforms many other units. It is most probably a better role model for less efficient units to emulate because its operating practices match them more closely than a unit that is rarely an efficient peer (Thanassoulis, 2003)".

Identification of the above mentioned functions of DEA becomes possible by solving the primal linear programming problem as well as utilizing the primal-dual relationships of linear programming models.

DEA includes a number of alternative (but related) approaches for evaluating performance. In the following section, the basic model that is also used in this study is presented in detail.

\subsection{The CCR Model of Data Envelopment Analysis}

The basic DEA model form, CCR is developed by Charnes, Cooper, and Rhodes (1978). According to Charnes et al. (1978), the efficiency of any DMU is calculated as the maximum of a ratio of weighted outputs to weighted inputs. The weights of virtual inputs and virtual outputs are calculated for each decision making unit by using linear programming. The objective function for the DMU that is being evaluated includes maximization of its virtual output to virtual input ratio. The accompanying $\mathrm{n}$ constraints impose that the virtual output to virtual input ratio of every DMU, including the unit being evaluated, must be less than or equal to one. For modeling purposes, number of units to be evaluated is taken as n. Each unit utilizes varying amounts of $\mathrm{m}$ different inputs to produce $\mathrm{s}$ different outputs. Specifically, $j^{\text {th }}$ unit consumes $x_{i j}$ amount of input $i$ and produces $y_{r j}$ amount of output $r$. In this case, the input matrix and the output matrix can be defined as $X=\left(x_{i j}\right) \in R^{m \times n}$ and $Y=$ $\left(\mathrm{y}_{\mathrm{ij}}\right) \in \mathrm{R}^{\mathrm{s} x}$ respectively. In the following formulations, $\lambda$ is a non-negative vector in $\mathrm{R}$; the vectors $\mathrm{s}^{-} \in \mathrm{R}^{\mathrm{m}}$ and $\mathrm{s}^{+} \in \mathrm{R}^{\mathrm{s}}$ indicate the input excess and output shortfall respectively. This nonlinear programming problem of Charnes et al. (1978) is formulated as

$$
\max h_{0}(u, v)=\frac{\sum_{r} u_{r} y_{r o}}{\sum_{i} v_{i} x_{i o}}
$$

subject to: 


$$
\begin{gathered}
\frac{\sum_{r} u_{r} y_{r j}}{\sum_{i} v_{i} x_{i j}} \leq 1 \quad j=1, \ldots, n \\
u_{r}, v_{i} \geq 0 \text { for all } \mathrm{i} \text { and } \mathrm{r}
\end{gathered}
$$

where

$\mathrm{h}_{0}$ : maximum efficiency ratio for $\mathrm{DMU}_{\mathrm{o}}$.

$\mathrm{n}$ : number of DMUs to be analyzed

$\mathrm{v}_{\mathrm{i}}$ : weight of input i for $\mathrm{DMU}_{\mathrm{o}}$

$\mathrm{u}_{\mathrm{r}}$ : weight of output $\mathrm{r}$ for $\mathrm{DMU}_{\mathrm{o}}$

$\mathrm{x}_{\mathrm{io}}$ : input value of $\mathrm{x}_{\mathrm{i}}$ for $\mathrm{DMU}_{\mathrm{o}}$

$\mathrm{y}_{\mathrm{ro}}$ : output value of $\mathrm{y}_{\mathrm{r}}$ for $\mathrm{DMU}_{\mathrm{o}}$

The $v_{i}$ and $u_{r}$ are the decision variables indicating the weights assigned to each related input or output in order to reach the maximum possible efficiency score; $x_{\text {io }}$ and $y_{\text {ro }}$ are the observed input and output variables for the rated unit 0 .

As explained by Cook and Zhu (2005), the non-linear model yields infinite number of solutions. This is due to the fact that if $\left(u^{*}, v^{*}\right)$ is optimal, then $\left(\alpha u^{*}, \alpha v^{*}\right)$ is also optimal for $\alpha>0$. The non-linear formula can be converted to the equivalent linear programming problem via the transformation developed by Charnes and Cooper (1962) for linear fractional programming, which selects the solution $(\mathrm{u}, \mathrm{v})$ for which $\sum_{i=1}^{m} v_{i} x_{i o}=1$.

The equivalent linear programming problem is presented below. (the output and input weights are indicated as $(\mu, v))$.

$$
\begin{gathered}
\operatorname{Max} z=\sum_{r=1}^{s} \mu_{r} y_{r o} \\
\text { subject to } \\
\sum_{r=1}^{s} \mu_{r} y_{r j}-\sum_{i=1}^{m} v_{i} x_{i j} \leq 0 \\
\sum_{i=1}^{m} v_{i} x_{i o}=1 \\
\mu_{r}, v_{i} \geq 0
\end{gathered}
$$

The linear programming problem is run for each DMU to determine the optimal $\left(\mu^{*}, \mathrm{v}^{*}\right)$ values and the relative efficiency scores of each DMU. The linear dual model of the basic CCR model is presented below.

$$
\begin{gathered}
\theta^{*}=\min \theta \\
\text { subject to } \\
\sum_{j=1}^{n} x_{i j} \lambda_{j} \leq \theta x_{i o} \quad i=1,2, \ldots . m
\end{gathered}
$$




$$
\begin{gathered}
\sum_{j=1}^{n} y_{r j} \lambda_{j} \geq y_{r o} \quad r=1,2, \ldots . . s \\
\lambda_{j} \geq 0 \quad j=1,2, \ldots . n ;
\end{gathered}
$$

The optimal solution $\theta^{*}$ yields the maximum efficiency score of the rated DMU. The process is repeated for each DMU $j$. If $\theta^{*}=1$, then the current input level cannot be reduced, indicating that $\mathrm{DMU}_{\mathrm{o}}$ is on the frontier and it's efficient. Otherwise, if $\theta^{*}<1$, then $\mathrm{DMU}_{\mathrm{o}}$ is dominated by the frontier and is not efficient. Based on the dual theorem of linear programming $\mathrm{z}^{*}=\theta^{*}$, the optimal solutions of the dual and primal problems are equal. Hence either problem may be used to obtain an efficiency score.

In DEA methodology, the efficient frontier can be estimated by using output-oriented or input-oriented models. Output-oriented models aim to increase the outputs proportionally given a certain amount of input. On the other hand, input-oriented models find out the amount that the inputs can be decreased proportionally given a certain level of output.

The DEA model presented below aims to minimize the amount of resources used for the given output level.

$$
\begin{aligned}
& \operatorname{Min} h_{0}(u, v)=\frac{\sum_{i} v_{i} x_{i o}}{\sum_{r} u_{r} y_{r o}} \\
& \text { subject to } \\
& \frac{\sum_{i} v_{i} x_{i o}}{\sum_{r} u_{r} y_{r o}} \leq 1 ; j=1, \ldots, n, \\
& u_{r}, v_{i} \geq \varepsilon>0 \text { for all i and } \mathrm{r} .
\end{aligned}
$$

Transformation of input oriented model to linear model along with its dual model is presented in the following pair (Cook and Zhu, 2005).

$$
\begin{gathered}
\min q=\sum_{i=1}^{m} v_{i} x_{i o} \\
\text { subject to } \\
\sum_{i=1}^{m} v_{i} x_{i j}-\sum_{r=1}^{s} \mu_{r} y_{r j} \geq 0 \\
\sum_{r=1}^{s} \mu_{r} y_{r o}=1 \\
\mu_{r}, v_{i} \geq \varepsilon>0 \quad \forall r, i
\end{gathered}
$$

for which the linear dual model is defined below.

$$
\max \phi+\varepsilon\left(\sum_{\substack{i=1 \\ \text { subject to }}}^{m}{s_{i}^{-}}^{-}+\sum_{r=1}^{s} s_{i}^{+}\right)
$$




$$
\begin{array}{ll}
\sum_{j=1}^{n} x_{i j} \lambda_{j}+s_{i}^{-}=x_{i o} & i=1,2, \ldots ., m ; \\
\sum_{j=1}^{n} y_{i j} \lambda_{j}-s_{i}^{+}=\phi y_{r o} & r=1,2, \ldots ., s ; \\
\lambda_{j} \geq 0 & j=1,2, \ldots \ldots, n .
\end{array}
$$

\section{Absorption Capacity Analysis of NUTS II Regions in Turkey}

In 2002, Turkey adopted the NUTS-IBBS (The Nomenclature of territorial Units for Statistics), a system of regional statistical data collection that is used in the EU. This system provides a new regional mapping of three levels. NUTS I and NUTS II levels cover 12 regions and 26 regions respectively; whereas NUTS III level represents all of the 81 cities in Turkey.

Table 1 summarizes related information on the NUTS II regions that have already enjoyed specific regional development fund schemes. Those funds are established under the preaccession assistance to harmonize regional development policy and practices with EU and activate local development potentials and initiatives at identified priority regions, through special regional development programs. (Pre-Accession Economic Program, 2007).

The three development fund calls cover eight regions and 27 cities in Turkey as classified in Table 1 . These programs are supported by a joint monitoring system that is formed by the coordination of State Planning Organization and the Central Finance and Contracts Unit (CFCU). In this context, 1,200 projects in 8 NUTS II regions and 27 provinces are implemented (State Planning Organization, 2008). The Calls are designed under three broad priorities; improvement of infrastructure, increasing the competitiveness of SME's located in the periphery, fostering local development initiatives that serve to advance human resource and entrepreneurship. Being the first calls in Turkey along with the regional diversities resulted in varying levels of efficiencies. These first calls have turned into an experimental phase for most of the beneficiaries rather than generating direct influence on regional development. Furthermore, these calls provided the first actual data on the potential absorption capacity of the regions. With these developments, the evaluation of post call efficiencies emerged as a significant issue for analyzing the three dimensions of absorption capacity.

\subsection{Discussion of DEA Model Results}

Application of the DEA model requires defining the related input output variables. Literature review and data availability are the major determinants in the choice of the variables. Availability of detailed and standardized data has been a major issue since the regional development calls analyzed in this study, are among the first in Turkey. Based on the previous studies (Helvacioglu Kuyucu and Tektas, 2008), output variable is taken as the annual percentage change in the level of tax for city $i$, where percentage change is the percentage difference between the pre and post project periods. The variable can be justified by the fact that the development projects result in an increase in infrastructure, increase in new business opportunities, decrease in costs, and an increase in income as well as the level of tax. Input variables are the total budget allocated to the projects, number of projects, average duration of the projects and a weighted indicator of project types. Including a project-type related variable seems meaningful due to the significant structural differences 
among the infrastructure and development projects. The indicator reflects the strength of the project type in creating an impact on factors such as the number of potential beneficiaries, duration of the impact, geographical coverage. In this aspect, the weight of infrastructure projects is ten times the weight of development projects. The related knowledge is gathered by interviewing project coordinators and by reviewing project reports (Helvacioglu Kuyucu and Tektas, 2008)

\begin{tabular}{|c|c|c|c|c|c|c|c|c|c|c|}
\hline Region/City & Population & $\begin{array}{l}\text { Area } \\
\left(\mathrm{km}^{2}\right)\end{array}$ & Budget & \begin{tabular}{|l|} 
No. of \\
Projects
\end{tabular} & $\begin{array}{l}\text { No. of } \\
\text { Development } \\
\text { Projects }\end{array}$ & $\begin{array}{l}\text { No. of SME } \\
\text { Projects }\end{array}$ & \begin{tabular}{|l|} 
No. \\
Infrastructure \\
Projects
\end{tabular} & $\begin{array}{l}\text { Average } \\
\text { Duration }\end{array}$ & $\begin{array}{l}\% \text { Tax } \\
\text { Change }\end{array}$ & \begin{tabular}{|l} 
Calculated \\
DEA \\
Efficiency \\
Scores \\
\end{tabular} \\
\hline TRA2 & & & & & & & & & & $\mathrm{E}_{\mathrm{avr}}=0.95$ \\
\hline Ağr1 & 530.879 & 11.499 & 4131913 & 16 & 3 & 9 & 3 & 1,00 & 40,07 & 1 \\
\hline Ardahan & 112.721 & 4.968 & 3214088 & 14 & 5 & 5 & 4 & 8,50 & 34,44 & 1 \\
\hline Iğdır & 181.866 & 3.588 & 5142957 & 9 & 1 & 6 & 4 & 9,82 & 30,29 & 1 \\
\hline Kars & 312.205 & 10.139 & 4188614 & 17 & 6 & 5 & 6 & 10 & 33,77 & 0.81 \\
\hline TR72 & & & & & & & & & & $\mathrm{E}_{\text {avr }}=0.77$ \\
\hline Sivas & 638.464 & 28.567 & 6282743 & 49 & 22 & 22 & 5 & 10,04 & 41,89 & 0.82 \\
\hline Kayseri & 1.165 .088 & 17.109 & 12606459 & 86 & 40 & 43 & 4 & 10,19 & 34,63 & 0.65 \\
\hline Yozgat & 492.127 & 14.074 & 4413498 & 20 & 10 & 8 & 2 & 10,00 & 33,16 & 0.85 \\
\hline TR52 & & & & & & & & & & $\mathrm{E}_{\mathrm{avr}}=0.62$ \\
\hline Karaman & 226.049 & 8.869 & \begin{tabular}{|l|}
7714239 \\
\end{tabular} & 39 & 13 & 21 & 5 & 9,59 & 19,75 & 0.42 \\
\hline Konya & \begin{tabular}{|l}
1.959 .082 \\
\end{tabular} & 40.813 & 16692522 & 166 & 28 & 131 & 7 & 8,83 & 38,42 & \begin{tabular}{|l|}
0.81 \\
\end{tabular} \\
\hline TRB1 & & & & & & & & & & $\mathrm{E}_{\text {avr }}=0.78$ \\
\hline Bingöl & 251.552 & 8.254 & 5355701 & 23 & 10 & 7 & 6 & 8,43 & 45,52 & 1 \\
\hline Elazı̆g & 541.258 & 9.281 & 6984615 & 33 & 11 & 17 & 5 & 9,97 & 29,87 & 0.62 \\
\hline Malatya & 722.065 & 12.103 & 6657161 & 38 & 16 & 12 & 10 & 10,13 & 28,80 & 0.53 \\
\hline Tunceli & 804.022 & 7.686 & 3198211 & 14 & 9 & 1 & 4 & 9,21 & 33,69 & 0.98 \\
\hline TRB2 & & & & & & & & & & $E_{\text {avr }}=0.73$ \\
\hline Bitlis & 327.886 & 7.094 & 4099691 & 55 & 30 & 20 & 5 & 9,91 & 35,15 & 0.82 \\
\hline Hakkari & 246.469 & 7.179 & 2714611 & 30 & 17 & 8 & 5 & 10,83 & 25,73 & 0.67 \\
\hline Muș & 405.509 & 8.067 & 4580464 & 38 & 19 & 8 & 11 & 10,87 & 34,26 & 0.72 \\
\hline Van & 979.671 & 22.984 & 13394514 & 142 & 82 & 32 & 28 & 10,92 & 41,51 & 0.70 \\
\hline TR82 & & & & & & & & & & $\mathrm{E}_{\mathrm{avr}}=0.91$ \\
\hline Çankırı & 174.012 & 7.492 & 4713213 & 19 & 6 & 11 & 2 & 10,00 & 37,63 & 1 \\
\hline Kastamonu & 360.366 & 13.158 & 6815135 & 47 & 18 & 21 & 8 & 9,51 & 38,29 & 0.75 \\
\hline Sinop & 198.412 & 28.567 & 1950060 & 19 & 9 & 8 & 2 & 9,32 & 29,16 & 1 \\
\hline TR83 & & & & & & & & & & $\mathrm{E}_{\mathrm{avr}}=0.79$ \\
\hline Amasya & 328.674 & 5.731 & 1170425 & 26 & 6 & 18 & 2 & 8,35 & 24,84 & 1 \\
\hline Çorum & 549.828 & 12.796 & 5852773 & 44 & 9 & 30 & 5 & 9,62 & 31,28 & 0.67 \\
\hline Samsun & 1.228 .959 & 9.364 & 7796566 & 82 & 27 & 47 & 8 & 9,51 & 40,22 & 0.78 \\
\hline Tokat & 620.722 & 10.073 & 7245120 & 42 & 17 & 21 & 4 & 9,93 & 33,90 & 0.72 \\
\hline TRA1 & & & & & & & & & & $\mathrm{E}_{\text {avr }}=0.87$ \\
\hline Bayburt & 76.609 & 3.739 & 4266303 & 26 & 15 & 3 & 8 & 9,42 & 38,04 & 0.90 \\
\hline Erzincan & 213.538 & 11.728 & 3606552 & 30 & 10 & 14 & 6 & 10,13 & 35,83 & 0.88 \\
\hline Erzurum & 784.941 & 25.330 & 3972528 & 62 & 31 & 31 & 0 & 9,71 & 33,10 & 0.86 \\
\hline
\end{tabular}

Data are compiled using statistics of TUIK (Turkish Statistics Institute) and reports of regional development fund calls for pre and post call years.

Table 1. Regional Efficiency Ratings 
Macroeconomic absorption capacity is analyzed in three dimensions. In the Candidate Countries, it is measured as the ratio of GDP levels to Structural Funds allocated. In general, the EU system foresees the upper limit for the Structural and Cohesion Funds as 4 percent of the GDP of the respective Member State. This principle is also being applied to Candidate Countries even though the funds allocated to the Candidate Countries usually remain significantly limited compared to the volume of the funds provided to the Member States. In order to evaluate the macroeconomic absorption capacity in Turkey, one of the two regional development funding mechanisms of the EU might be considered. The first alternative is to use the total value of the EU funds distributed under the regional development fund schemes for the period 2005-2007, which amounts to $€ 263$ million. Applying this approach, it is seen that the regional development fund schemes reach only $0.06 \%$ of the GDP of Turkey as of 2007. The second alternative is to use the IPA (Instrument for Pre-Accession) financial assistance program's annual strand for regional development which is $€ 167.5$ million as the absorption capacity in 2007 . This would only equal to $0.04 \%$ of GDP. Both of the approaches, clearly show that the macroeconomic absorption capacity in Turkey bears great potential as the available funds are very limited, even negligible when compared to the EU average. The two major reasons for this may be explained as the novelty and the limited amount of the funds allocated to the regional development in Turkey. On the other hand, operating structures have been designated for the Regional Development of IPA in 2008. This would mean that the structural and cohesion funds are expected to have greater budgets in the near future.

Administrative absorption capacity includes management, planning and coordination ability and skills of central, regional and local authorities. The cities with higher administrative capability can successfully manage the information flow, training programs and local networking of potential partners which in turn lead to formation of more functional and effective project teams with higher project management skills.

In analysis of the DEA model results in terms of the administrative capacities of cities, the emphasis should be given to efficient cities. Within DEA context, efficient cities which form benchmarks (references) to remaining inefficient ones deserve special attention. Table 2 presents the efficient cities along with their reference frequencies and their average weights on the suggested improvements of the inefficient cities that take them as reference.

\begin{tabular}{|l|c|c|}
\hline City & $\begin{array}{l}\text { Reference frequency to other } \\
\text { cities }\end{array}$ & $\begin{array}{l}\text { Average impact on the efficiency } \\
\text { score }\end{array}$ \\
\hline Bingol & 17 & 0.556 \\
\hline Agr1 & 9 & 0.302 \\
\hline Amasya & 7 & 0.353 \\
\hline Ardahan & 4 & 0.431 \\
\hline Cankır1 & 2 & 0.278 \\
\hline Sinop & 1 & 0.006 \\
\hline Igdır & 0 & 0.000 \\
\hline
\end{tabular}

Table 2. Reference frequencies and weights of efficient cities

Bingol emerges as the city with the highest reference frequency as well as weight, followed by Agri and Amasya. Bingol and Agri benefit from the highest annual percentage change in tax which may be a crucial factor in their high efficiency score and their selection as 
reference cities. On the other hand, Amasya is a noteworthy reference city despite its relatively low percentage change in tax value. Amasya is the only efficient city in its region while others have lower than average efficiency scores. Amasya is actually a high level benchmark within the region which makes the remaining cities relatively less efficient. Amasya owns the first development office in Turkey which is established in compliance with the Yeşilırmak Development Plan; the first regional development strategy paper ever designed parallel to the EU schemes. This scheme foresees higher allocation of government funds to the regional actors such as municipalities, NGOs and related institutions. The technical assistance provided by an English technical consultant group has also increased the regional development agency's level of expertise on project development and management. Furthermore, Yeşilırmak Project has a significant impact on the objectives of this region's call which create a perfect balance between the Call's and Amasya's objectives. This fact emerges as another reason for Amasya's efficiency level.

Financial absorption capacity. The average budget allocation and the contribution of the partners to the budget under the additionality principle present a general outlook for the financial absorption capacity. Table 3 presents the contribution of budget and other variables to the efficiency scores. The DEA analysis shows that total budget allocated to the regional development projects at each city emerges as a significant variable in determining the efficiency scores. In 14 of the 27 cities, the average weight of budget on the efficiency score is found as $49 \%$. Analyses further depict that in 5 of the 7 efficient cities, budget stands as the most significant variable affecting the efficiency scores. In those 5 cities with the highest efficiency scores, the average weight of budget on the efficiency score attained is quite significant with $42 \%$. This may be due to the high importance of the budget in the achievement of efficient regional development programs. In most of the cities classified under the grant schemes, budget is considered as the lacking element of novel and tailormade regional development projects. Once the budget is guaranteed the local administrations have become much more capable of undertaking projects that serve to regional priorities. Parallel to this finding, in the cities where the budget does not have a weight on the efficiency score, the weight is replaced by the variable that shows average project duration at each city. This shift can be explained under the scope of administrative absorption capacity. The cities with higher administrative capability have better managed the information flow, training programs and formed a local network of potential partners which lead to the establishment of more functional and effective project teams with higher project management skills. It is observed that annual percentage change in tax level influences the average weight of total budget allocated to the regional development projects at each city on the efficiency score. The annual percentage change in tax level varies between $42 \%$ and $20 \%$ for the cities in the sample set. In the three cities with the highest percentage increase ( $>40 \%)$; Sivas, Bingol and Van, the budget has no weight on the efficiency score. Among the eight cities which have an increase greater than $37.5 \%$, efficiency scores of only two cities are influenced by the budget. These results support the fact that the cities with higher tax revenues are less dependent on the project budget and rely on administrative absorption capacity rather than financial absorption capacity. 


\begin{tabular}{|c|c|c|c|c|c|}
\hline Region/City & Efficiency & \multicolumn{4}{|c|}{ Contribution of each input variable to the efficiency score } \\
\hline CALL 1 & & Budget & Number & Duration & TI Impact \\
\hline TRA2 & $E_{\text {avg }}=0.95$ & & & & \\
\hline Agr1 & 1 & 0.48 & 0.52 & & \\
\hline Ardahan & 1 & 0.68 & 0.32 & & \\
\hline Igdır & 1 & 0.07 & 0.93 & & \\
\hline Kars & 0.81 & 0.28 & 0.24 & 0.48 & \\
\hline TR72 & $E_{\text {avg }}=0.88$ & & & & \\
\hline Sivas & 0.82 & & & 0.63 & 0.37 \\
\hline Kayseri & 0.65 & & & 0.61 & 0.39 \\
\hline Yozgat & 0.85 & 0.39 & & 0.11 & 0.50 \\
\hline TR52 & $E_{\text {avg }}=0.62$ & & & & \\
\hline Karaman & 0.42 & & & 0.65 & 0.35 \\
\hline Konya & 0.81 & & & 1 & \\
\hline TRB1 & $E_{\text {avg }}=0.78$ & & & & \\
\hline Bingoöl & 1 & & 0.55 & 0.45 & 0 \\
\hline Elazığ & 0.62 & & & 0.67 & 0.33 \\
\hline Malatya & 0.53 & & & 1 & \\
\hline Tunceli & 0.98 & 0.67 & 0.33 & & \\
\hline \multicolumn{6}{|l|}{ CALL 2} \\
\hline TRB2 & $E_{\text {avg }}=0.73$ & & & & \\
\hline Bitlis & 0.82 & 0.47 & & 0.53 & \\
\hline Hakkari & 0.67 & 0.35 & 0.06 & 0.59 & \\
\hline Muş & 0.72 & 0.47 & & 0.53 & \\
\hline Van & 0.70 & & & 1 & \\
\hline \multicolumn{6}{|l|}{ CALL 3} \\
\hline TR82 & $E_{\text {avg }}=0.91$ & & & & \\
\hline Cankırı & 1 & & & 0.69 & 0.31 \\
\hline Kastamonu & 0.75 & & & 1 & \\
\hline Sinop & 1 & 0.67 & 0.33 & & \\
\hline TR83 & $E_{\text {avg }}=0.79$ & & & & \\
\hline Amasya & 1 & 1 & & & \\
\hline Corum & 0.67 & & & 0.67 & 0.33 \\
\hline Samsun & 0.78 & & & 1 & \\
\hline Tokat & 0.72 & & & 0.68 & 0.32 \\
\hline TRA1 & $E_{\text {avg }}=0.86$ & & & & \\
\hline Bayburt & 0.90 & 0.50 & 0.04 & 0.46 & \\
\hline Erzincan & 0.88 & 0.43 & & 0.57 & \\
\hline Erzurum & 0.86 & 0.35 & & 0.11 & 0.54 \\
\hline
\end{tabular}

Table 3. Contribution of each input variable to the efficiency score (vi * xi)

\section{Conclusion}

The study focuses on the comparative performance evaluation of EU pre-accession funds from the point of absorption capacity. The absorption capacity concept is undertaken in three major dimensions; namely, macroeconomic, administrative and financial. Relating to this objective, an analytical approach is proposed utilizing the data envelopment analysis. The method is implemented on the NUTS II regions in Turkey that have already enjoyed 
specific regional development fund schemes. The analysis covers 1,200 projects in 8 NUTS II regions and 27 provinces in Turkey.

Research results depict that the macroeconomic absorption capacity in Turkey bears great potential due to the fact that the available funds are very limited, even negligible when compared to the EU average. The analysis results pertaining to the administrative capacities of cities cite significant differences. The administrations that have provided strategies to integrate with the EU funding mechanisms and that have created special networks for partnership and knowledge flow achieved a critical competitive edge and benefited more form the EU harmonization process. The DEA results show that total budget allocated to the regional development projects at each city emerges as a significant variable in determining the performance levels and financial absorption capacity remains as the key factor for the cities where the administrative absorption capacity is weak. It is noteworthy that all these funding schemes are very new in Turkey and the regional development programs used in this study form the very first examples of EU model funding.

The study contributes to the literature by introducing and implementing an analytical approach for comparing the performance of EU pre-accession funds in terms of the absorption capacities. The approach may be utilized as a common pattern of efficiency analysis for the candidate countries. Implementing the adopted DEA model to the regional development grant schemes, a coherent strategy can be proposed to improve the efficiency of the pre-accession funds for regional development in Turkey. Further research could be possible by integrating the EU-originated cohesion funds which would be available in Turkey in the near future along with the recently approved national regional development state aid system.

\section{References}

Charnes, A., Cooper, W. (1962). Programming with linear fractional functionals, Naval Research Logistics Quarterly, vol. 9, pp. 181-185.

Charnes, A., Cooper, W., Rhodes, E. (1978). Measuring the efficiency of decision making units, European Journal of Operational Research, vol. 2, pp. 429-44.

Cook, W. and Zhu, J. (2005). Modeling Performance Measurement. Applications and Implementation Isues in DEA, Springer Science+Business Media, New York.

Cooper, W.W., Seiford, L.M. \& Tone, K. (2000). Data Envelopment Analysis: A Comprehensive Text with Models, Applications, References and DEA-Solver Software, Kluwer Academic Publishers, Boston.

Cooper, W.W., Seiford, L.M. \& Zhu, J. (2004). Data envelopment analysis history, models and interpretations, Handbook of Data Envelopment Analysis, W.W. Cooper, L.M. Seiford \& J. Zhu (ed.) pp.1-39. Kluwer Academic Publishers, Boston.

European Commission (2004). A New Partnership for Cohesion, Convergence, Competitiveness, Cooperation. Third Report on Economic and Social Cohesion, Brussels.

Georgescui G. (2008). Determinants of Increasing EU Funds Absorption Capacity in Romania, Annales Universitatis Apulensis Series Oeconomica, 2008, vol. 2, issue 10, pages 16-24.

Helvacioglu Kuyucu, A. D. and Tektas, A., (2008). The Impact of Local Decision-Making in Regional Development Schemes on the Achievement of Efficiency in EU Funds, International Journal of Social Sciences, Vol.3, No. 3, pp. 180-188. 
OECD (2006). Czech Republic: Partnerships for Administrative and Absorption Capacity. Retrieved: 02.05.2008. http://www.oecd.org/dataoecd/7/35/37729375

Oprescu G., Lumini D., Illie F \& Pislaru D. (2005). Analysis of Absorption Capacity of the EU Funds in Romania, European Institute of Romania, Pre-Accession Impact Studies, Study No.1.

Pre-Accession Economic Program of Turkey, Ankara, 2007.

Reiner, G. \& Hoffman, P. (2006). Efficiency analysis of supply chain processes. International Journal of Production Research, vol. 44, pp. 5065-5087.

State Planning Organization, NUTS II Regions in Turkey, Retrieved: 14.07.2008, www.dpt.gov.tr

Sumpikova M, Pavel, J., \& Klazar, S. (2004). EU Funds: Absorption Capacity and Effectiveness of Their Use with Focus on Regional Level in the Czech Republic, Conference on Problems Facing the New Member States. Retrieved: 02.05.2008.

http://www.nispa.sk/_portal/files/conferences/2004/papers/200404061630450.S umplikova-Pavel-Klazar.doc

Thanassoulis, E. (2001). Introduction to the theory and application of Data Envelopment Analysis: A Foundation text with integrated software, Kluwer Academic Publishers, Boston.

Zerbinati, S. (2004). Europeanization and EU Funding in Italy and England: A Comparative Local Perspective, Journal of European Public Policy, vol. 11. No.6, pp. 1000-1019. 


\title{
Design and Construction Transceiver Module Using Polymer PLC Hybrid Integration Technology
}

\author{
Vítězslav Jeřábek ${ }^{1}$, Ivan Hüttel ${ }^{2}$, Václav Prajzler ${ }^{1}$ and Karel Bušek ${ }^{1}$ \\ 1. Czech Technical University in Prague \\ 2. Institute of Chemical Technology, Prague \\ Czech Republic
}

\section{Introduction}

Planar lightwave circuit (PLC) hybrid integration technology enables us to construct component by combining PLC with passive function (fiber and planar optical waveguides) and active optoelectronics devices (laser diodes, optical amplifiers and photodiodes) hybridized on a PLC (Hashimoto, T. \& Nakasuga, Y., 1998). Recently, in foreign sources optical waveguides and hybrid circuits based on the polymeric materials such as the polymetametacrylate (PMMA), deutered ethylene glycoldimetacrylate, (for the wave length of $1300 \mathrm{~nm}$ ) or pentafluoro-phenylmetacrylate (for the wave length of $1550 \mathrm{~nm}$ ) or some type of the epoxy polymers and others optical materials including some glasses were investigated. (Eldada, L. \& Shacklette, L.W., 2000).

The optical bidirectional transceiver module (TRx) is the key component for subscriber part of the passive optical networks (PON) for the fiber to the home (FTTH) topology. Our optical triplex TRx module has been designed with $\mathrm{R}$ Soft simulation programs and constructed by using a polymer PLC hybrid integration technology. The transceiver module consists of a planar polymer waveguides with a volume holographic grating triplex (VHGT) filter, surfaceilluminated photodiode (SI-PD) and spot-size converted Fabry-Pérot laser diode (SSC-LD) in SMD package. An optical TRx module transmits a $1310 \mathrm{~nm}$ radiation upload and receives a $1490 \mathrm{~nm}$ download data as well as a $1550 \mathrm{~nm}$ download digital video signals for wavelength division multiplexing WDM cable TV application (Young-Tak, Yoon-Jung Park, 2006).

The optics TRx module was built on the polymeric material technological solution (PMMA and SU-8 2000 epoxy polymer) and the diagnostics of optical waveguiding structures for the integrated optics project and wants to develop the processes of the design of active photonic integrated structures further. As for selected theoretical issues dealing with the preparation of planar photonic hybrid integrated circuits, the works take up previously discovered major dependence of the index of refraction of selected polymeric thin layers on the electric field (Švorčík, V., Hüttel, I., Paláček, P.,2007) and using of this phenomenon at the production of planar channel optical waveguides. 
First stage of the PLC design was to show how the radiation from the single mode optical fiber can be coupled to the planar optical waveguide. It allows making optical circuits on a planar substrate. The highly power efficient optical coupling between these devices is significantly important for determination of the planar waveguide attenuation.

The second stage will be a design and fabrication of the platform technology for hybrid PLC to match numerical aperture the waveguide made by organic polymer and SI-PD receivers or SSC-LD transmitter of the microwave optoelectronic transceiver TRx, which some subsection has been designed and simulated previously.

\section{Design and results}

\subsection{Measuring and simulation of the optical elements}

The first stage of our project was concentrated on design and coupling of the optical fiber to the channel optical waveguide and measuring attenuation. For this purpose, the $650 \mathrm{~nm}$ and $1550 \mathrm{~nm}$ wavelength semiconductor lasers were used. The radiation was coupled into a single mode optical fiber (SMF) which had a thickness core of $3.5 \mu \mathrm{m}$ with a thickness cladding layer of $125 \mu \mathrm{m}$, the NA $=0.14$ and its refractive index difference of $0.36 \%$; this fiber was matched to the channel waveguides by a piezoelectric driven micromanipulator to acquire sufficient position accuracy in the alignment process. When the desired alignment was achieved, the optical power meter for the measuring of the transmitted optical power across the waveguides was used. The optical waveguides were made from polymer Su- 8 2000, lithium niobate or glass BK7. For fabrication of the channel waveguides in polymer was used spin - counting and UV exposition. The lithium niobate channel was fabricated by diffusion of Ti metal layers, the samples were cut to rectangular parallelepipeds and the channels were made by a conventional lithographic method. The samples of the bulk glasses were fabricated mixing the major components of glass $\left(\mathrm{SiO}_{2}\right.$, with $\left.\mathrm{Al}_{2} \underline{\mathrm{O}}_{3}, \mathrm{Na}_{2} \underline{\mathrm{O}_{3}}\right)$ with the network intermediates $(\mathrm{MgO}, \mathrm{CaO}$ and/or $\mathrm{ZnO})$. The channels waveguides for this glass were fabricated by $\mathrm{Ag}^{+} \leftrightarrow \mathrm{Na}^{+}$and $\mathrm{K}^{+} \leftrightarrow \mathrm{Na}^{+}$ion exchange. The most important characteristic of the samples is the attenuation. This attenuation is attributable to scattering, absorption and radiation. The attenuation of polymer SU-8 2000, glasses and lithium niobate optical waveguides were measured by the method end-fire coupling with waveguide of different length. The measurement is repeat for the large number of waveguide samples (in our case, we measured ten samples). The attenuation on to the waveguide can be determined from the relation (1) and the results of the measurement are given in Table 1.

\begin{tabular}{|c|c|c|c|c|}
\hline $\begin{array}{c}\text { Channel } \\
\text { waveguide }\end{array}$ & $\begin{array}{c}\lambda \\
(\mu \mathrm{m})\end{array}$ & $\begin{array}{c}\text { Width } \\
(\mu \mathrm{m})\end{array}$ & $\begin{array}{c}\text { Height } \\
(\mu \mathrm{m})\end{array}$ & $\begin{array}{c}\text { Attenuation } \\
(\mathrm{dB} / \mathrm{cm})\end{array}$ \\
\hline $\mathrm{Su} 8$ & 650 & 200 & 10 & 4.15 \\
\hline $\mathrm{Su} 8$ & 1550 & 200 & 10 & 2.35 \\
\hline $\mathrm{BK} 7$ & 650 & 6.8 & 7.0 & 0.499 \\
\hline $\mathrm{LiNbO}_{3}$ & 650 & 5.1 & 6.8 & 1.13 \\
\hline
\end{tabular}

Table 1. Parameters of the optical waveguides losses 


$$
n=\frac{10 \log \left(P_{2} / P_{1}\right)}{Z_{1}-Z_{2}}\left[\frac{d B}{c m}\right]
$$

Where $P 1$ is output power sample with length $Z_{1}$ and $P_{2}$ is output power same sample with length $Z_{2}$ and $Z_{1}>Z_{2}$.

The second stage of our research will be the design and fabrication of the platform technology for hybrid PLC to match the waveguide and microwave optoelectronic receiver, transmitter with SI-PD and SSC-LD element, which has been designed and simulated previously. The hybrid PLC has the two parts. The first part is the planar optical waveguide with a VHGT filter and collimation lenses, the second part is optoelectronic system with electrical microwave section. The optical part was realized by polymer layers optical waveguides or by microoptics. The polymer optical waveguides was deposited by using spin coating method on silica on silicon or GaAs substrate. As polymer we choose epoxy polymer NANOTM SU-8 2000 (SU-8 2000) from MicroChem Corp with cover layer PMMA. The optoelectronic element was fixed on a special metallic or alumina submount on the composite substrate. The VHGT filter is diffractive element with volume holographic gratings, which containing volume periodic changes index of refraction. The VHGT filter diffracts the $1550 \mathrm{~nm}$ and $1490 \mathrm{~nm}$ wavelength radiation into separate directions, while transmitting the $1310 \mathrm{~nm}$ radiation. When optical radiation incidents on holographic grating and satisfy Bragg phase condition, then happen to diffraction optical radiation for wavelength $\lambda_{B}$ of the transmission grating (2)

$$
\lambda_{B}=2 \Lambda \sin \theta
$$

where $\lambda_{B}$ is Bragg wavelength, $\Lambda$ is grating period and $\theta$ is angle of the diffraction. The simple schema of the VHGT filter is given on the Fig.1

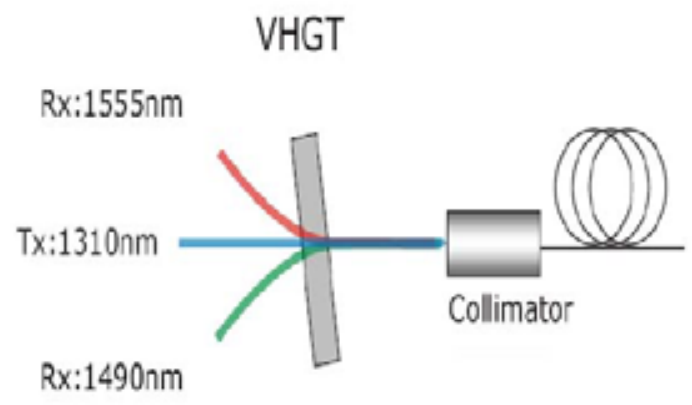

Fig. 1. Simple schema of the VHGT filtre arrangement

The diffraction angle of the VHGT filter was measured by the beam analyzing system Beam Profiler BP 104-IR from Thor Labs, see Fig.2. 

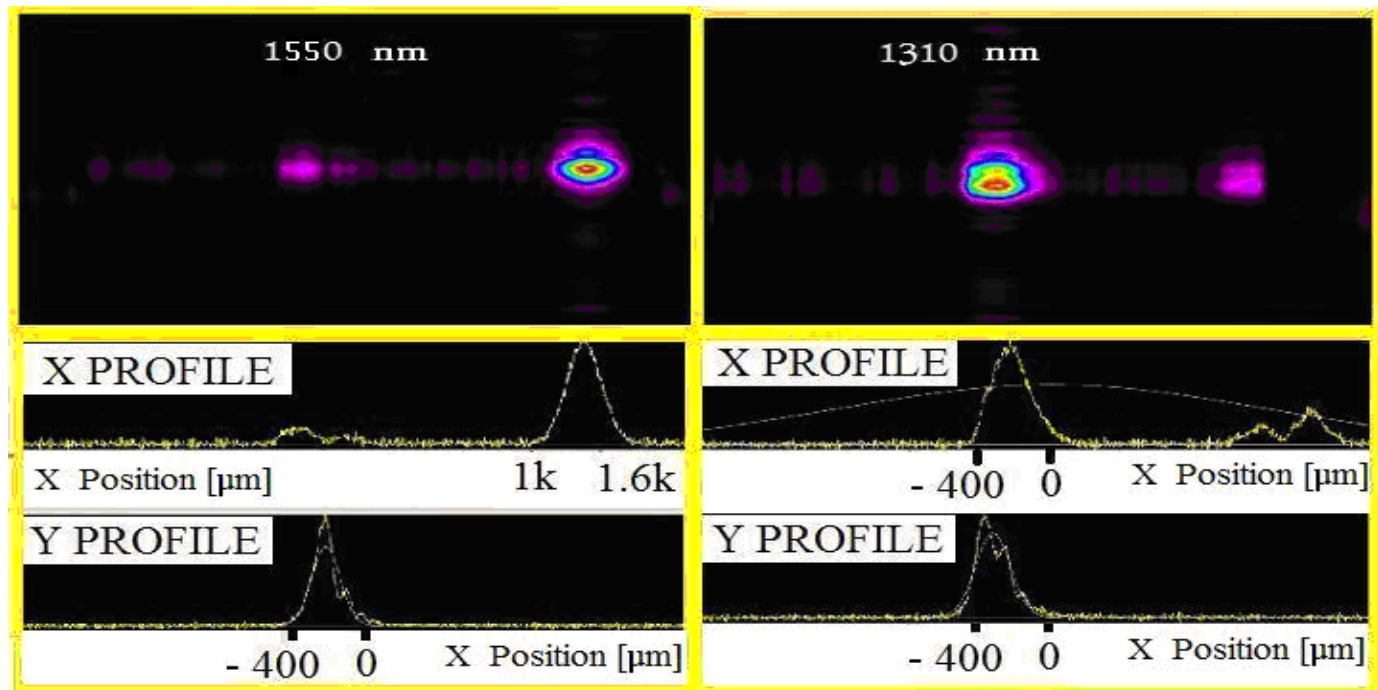

Fig. 2. The diagram of the diffraction angle measuring, which was determind as the radiation spot deflection in $X$-axis for the wavelength $1310 \mathrm{~nm}$ and $1550 \mathrm{~nm}$ as well as for $1490 \mathrm{~nm}$. (The difference in Z-axis is $8 \mathrm{~mm}$, difference in X-axis is $1.31 \mathrm{~mm}$, diffraction angle is 19.3 degree for wavelength $1550 \mathrm{~nm}$ and zero degree for $1310 \mathrm{~nm}$ )

On the basis formula (2) was derived grating period $\Lambda=2.388 \mu \mathrm{m}$. Further parameters of the VHGT filter were the temperature sensitivity $0.01 \mathrm{~nm} /{ }^{\circ} \mathrm{C}$ and insert losses max. $0.1 \mathrm{~dB}$.

The optoelectronic part was created by two receivers with SI-PD PIN InGaAs photodiode chips on the alumina submount and transmitter with SSC-LD InGaAsP laser diode on metallic submount. The PIN photodiode and laser was placed in the groove for elimination height offset. The optimum distance among optical waveguides facet on base polymer SU82000 and optical fiber or PIN photodiodes in the receiving part was specified by BMP program with the waveguide facet width $100 \mu \mathrm{m}$ and thickness $2 \mu \mathrm{m}$, buffer layer thickness was also $2 \mu \mathrm{m}$, refractive index waveguide layer $\mathrm{n}_{F}=1.596$, refractive index buffer layer $\mathrm{n}_{B}=1.46$, refractive index substrate $\mathrm{n}_{S}=3.5$, wavelength $1550 \mathrm{~nm}$. The cover layer of the optical waveguides was PMMA with thickness $5 \mu \mathrm{m}$ and refractive index $\mathrm{n}_{C}=1,49$. On the basis of the simulations in the program Beam Prop was derived the optimal distance of output facet optical waveguide and detection area photodiode $220 \mu \mathrm{m}$. The PIN photodiodes had antireflection coating (index of refraction $n=1.5$ at $1550 \mathrm{~nm}$ ).

The realization of the hybrid microwave integrated circuit of the photonic receiver was composed of a substrate from composite HF material Rodgers, polymer SU8-2000 optical waveguides with the ridge width $100 \mu \mathrm{m}$, thickness $2 \mu \mathrm{m}$ and fast PIN photodiode in SMD package. The optimal distance measuring assessment between detector optical radiation and output facet optical polymeric waveguide placed in micromanipulator is shown in Fig.3. InGaAs PIN photodiode with the diameter active detection area $50 \mu \mathrm{m}$ was used. The movement of PIN photodiode was controlled by handle micromanipulator with clamp clip. The optimal distance of PIN photodiode and output facet optical polymer waveguide $160 \mu \mathrm{m}$ was measured. The differences of the simulated and measured value were given especially by error of the PIN photodiode and output facet waveguide distance measurement and accuracy of the multimode model used for simulation. 


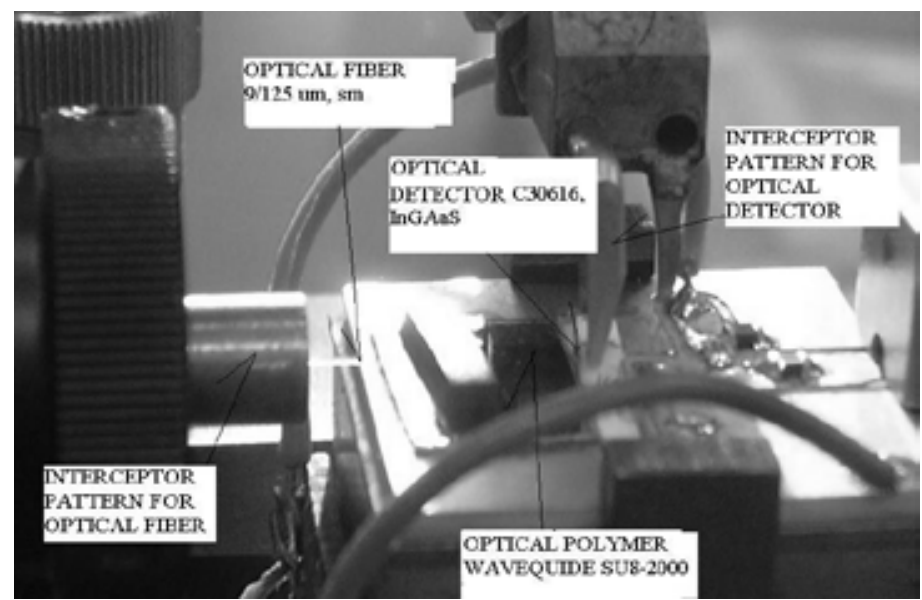

Fig. 3. Detail measuring setup for put optimal distance between optical PIN photodiode in SMD package and output facet of the planar optical polymer waveguide

The adjustment of the matching output PIN photodiode and the input microwave amplifier is necessary to achieve maximal responsivity and minimal losses in the electric transmission paths of the photonic receiver. The electrical part is made by thin layer hybrid microwave electrical integrated circuit. Our work was concentrated on design and construction of a microwave hybrid optoelectronic receiver (Jeřábek V.,Arciniega J.A., 2009), where the PIN photodiode was connected by microstripe line to input of the HBT amplifier. The all parts are placed on the composite material substrate.

\subsection{Calculation}

The theoretical analysis describes the microstrip connection between the PIN photodiode and the input of the HBT amplifier by the small signal equivalent circuit. For frequency response analysis we used the small signal equivalent circuit of the OE receiver input Fig. 4.

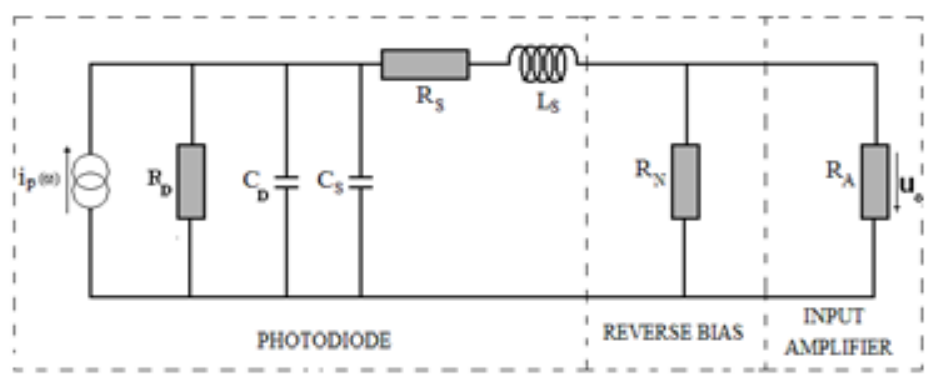

Fig. 4. The small signal equivalent circuit of the PIN photodiode with SMD assembly and input of the HBT amplifier 
where

$i_{p}(\omega)$ - PIN photodiode photocurrent source

$C_{D}$ - capacitance of depletion layer $0.5 \mathrm{pF}$

$C_{S}$ - stray capacitance $1 \mathrm{pF}$

$R_{S}$ - series resistance $10 \Omega$

$R_{D}$ - dynamic resistance of the PIN photodiode $1 \mathrm{k} \Omega$

$L$ - inductance generated by the photodiode SMD carrier

$R_{N}$ - thin film resistor for reverse bias voltage $2 \mathrm{k} \Omega$

$R_{A}$ - input impedance $50 \Omega$ of the ideal amplifier

The PIN photodiode (C30606ECER from Judson Technologies) chip connection to the microstrip waveguide is composed from compensation inductance made by gold microstrip on the alumina carrier $L_{1}$ and gold wire connection $L_{2}$. For calculation frequency response limit it was counted inductance $L$ given by (3).

$$
L=L_{1}+L_{2}
$$

For the cutoff angle frequency $\omega_{\mathrm{T}}$ of the module complex transition $Z_{\mathrm{T}}$ impedance characteristic (4) was derived

$$
\left|Z_{T}\right|=\frac{R_{D} R_{P}}{\left[\left(R_{D}+R_{S}+R_{P}-\omega^{2} L C_{D} R_{D}\right)^{2}+\omega^{2}\left(L+R_{D} R_{S} C_{D}+R_{D} R_{P} C_{D}\right)^{2}\right]^{/ 2}}
$$

The cut off angle frequency $\omega_{\mathrm{T}}$ was derived as root of the transcendent equation (5)

$$
\left|Z_{T}\left(\omega_{T}\right)\right|=\left|Z_{T}(0)\right| / 2^{1 / 2}
$$

where $\left|Z_{T}(0)\right|$ is module of the impedance for $\omega=0, R_{P}$ is parallel $R_{A}$ and $R_{N}$ combination.

The limit frequency $\mathrm{f}_{\mathrm{T}}$ received by solve equation (5) was $\mathrm{f}_{\mathrm{T}}=2,78 \mathrm{GHz}$. The module $\left|Z_{T}(0)\right|=46,07 \Omega, \quad C_{T}=1,5 \mathrm{pF}$ and $\mathrm{L}=4,5 \mathrm{nH}$.

The optimum value of the inductance $L_{\text {opt }}$ for maximally flat transition impedance $Z_{T}$ characteristic is given after (Sackinger E., 2005) as (6)

$$
L_{\text {opt }}=0,4 R_{T}^{2} C_{T}=1,85 n H
$$

The cut off frequency for optimum value $L_{\text {opt }}$ was calculated as $\mathrm{f}_{\mathrm{T}}=3,25 \mathrm{GHz}$.

The small signal equivalent circuit presented in the Fig. 4 was implemented for simulation in Win Mide program. The capacity of depletion layer $C_{D}$ is a function of reverse bias voltage and for $5 \mathrm{~V}$ is catalog value $0,50 \mathrm{pF}$. $C_{s}$ is stray capacity signal connection PIN photodiode SMD assembly. For good high frequency response it is essential to be $C_{D}$ and $C_{s}$ kept as low as possible. After that it is necessary to reduce $R_{A}$ or to provide high-frequency equalization. The inductance and capacity generated by the photodiode SMD carrier was simulated to analyze its influence on the device. The measured and simulated results $S_{21}$ frequency characteristic at the frequency range $0,1-3,5 \mathrm{GHz}$ is shown in the Fig. 5 . 


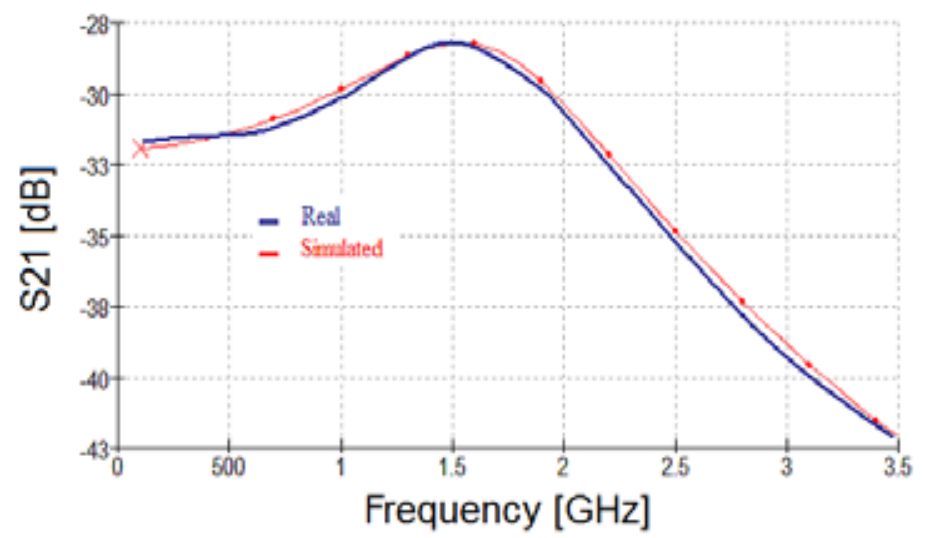

Fig. 5. The measured and simulated $S_{21}$ frequency characteristic of the OE receiver

The resonance of the lumped inductance $L$ in the signal way make the peaking effect in the $S_{21}$ frequency characteristic shift the bandwidth of the WDM receiver to 2,.5 GHz with acceptable ripple. For the transmitter was used spot-size converted Fabry-Perot laser diode (SSC-LD) for $1300 \mathrm{~nm}$ wavelength and monitoring surface-illuminated photodetector (SIPD) for average optical power stabilization of LD in one SMD package. The optical bidirectional transceiver module TRx is given on Fig. 6 . The transceiver module consists of a planar polymer waveguides with a VHGT filter with a collimation lens, SI-PD photodetectors and SSC-LD laser diode in SMD package. In the separate substrate of the bidirectional transceiver module electrical part was situated LD driver and optical power stabilized integrated circuits (IC) as well as IC HBT amplifier of the OE receiver.

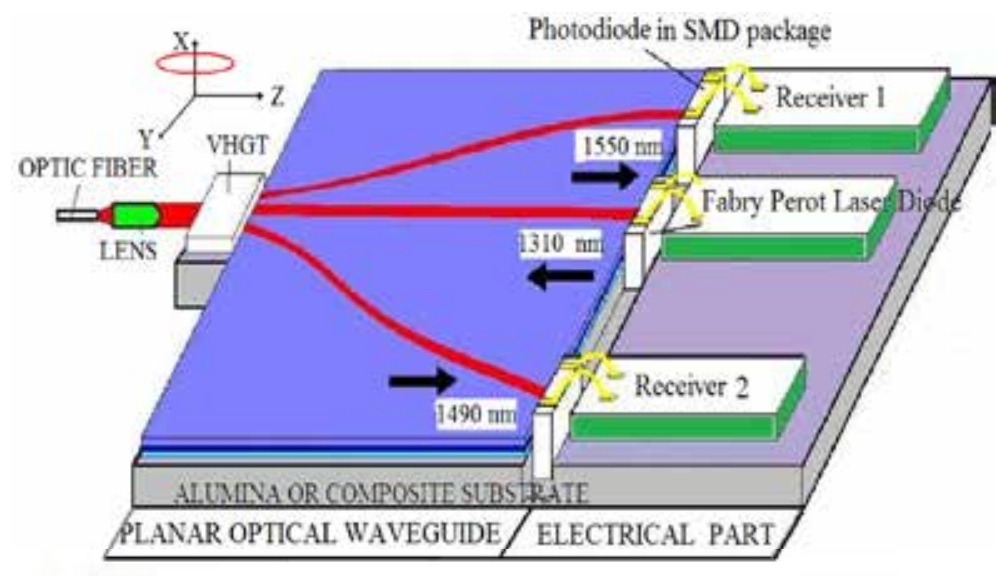

Fig. 6. The optical bidirectional transceiver module TRx

\section{Conclusion}

In this paper were presented first steps, which guide to the design and construction of a VHGT attached WDM triplex transceiver module TRx using polymer PLC hybrid integration technology. We report on the results measuring of the optical attenuation of the 
SU-8 2000 epoxy polymer optical waveguides. The average attenuation of the waveguides made by polymer SU-8 was $4.15 \mathrm{~dB} / \mathrm{cm}$ for $650 \mathrm{~nm}$ and $2.35 \mathrm{~dB} / \mathrm{cm}$ for $1550 \mathrm{~nm}$ radiation, which is slightly higher than glasses and lithium niobate waveguides samples. We presented the diffraction angle measuring results of the VHGT filter between 1310 and 1550 $\mathrm{nm}$ wavelength which was 19.3 degree as well as $1490 \mathrm{~nm}$.

The second step was design of the hybrid photonic planar integrated receiver with a polymer optical waveguide, InGaAs p-i-n photodiode and integrated HBT amplifier. The calculated coupling efficiency, for direct coupling to the input facet of the optical polymer waveguide (SU8-2000) with the SMF laser source optical radiation $1550 \mathrm{~nm}$ was $39 \%$, for fundamental mode $\mathrm{TE}_{0}$. The difference among calculated and measuring results was given lower quality of the waveguide facets. The optimal distance between optical polymeric waveguide facet and pi-n photodiode was simulate in program Beam Prop. The simulation was optimized to maximum transfer optical power. The optimal distance of the output facet optical waveguide and the detection area photodetector was $220 \mu \mathrm{m}$. The verification of the optimal distance was made by measuring with seven axis system micromanipulator at $160 \mu \mathrm{m}$. The measurement differences are given by the error of the measuring system and multimode model used for simulation. The above mentioned results were used for the design fundamental masks of the photonic receiver. Further work will be concentrated on design and construction of optoelectronic transmitting part, which will be fabricated by use existing technology with bandwidth $2.5 \mathrm{GHz}$ as the main optoelectronic part of the hybrid PLC integrated microwave transceiver TRx for the FTTH topology optical PON networks.

\section{Acknowledgements}

Our research is supported by the Grant Agency of the Czech Republic under grant number 102/06/0424 and the research program MSM6840770014 of the Czech Technical University in Prague.

\section{References}

Hashimoto, T. \& Nakasuga, Y. (1998). Optical Hybrid Integration Technique with Planar Lightwave Circuit Platform, J. of Lightwave Technology, Volume 16, Number 7, (1998), pp. 1246-1257, ISSN: 0733-8724.

Eldada, L. \& Shacklette, L.W. (2000). Advances in Polymer Integrated Optics, IEEE Journal of Selected Topics in Quantum Electronics, Volume 6, Number 1, (2000), pp. 54-68, ISSN: 1077-260X

Young-Tak \& Yoon-Jung P. (2006). Fabrication of a TFF-Attached WDM-Type Triplex Transceiver Module Using Silica Habrid Integration Technology, Journal of Lightwave Technology, Volume 24, Number 12, (2006), pp. 5031-5038, ISSN: 0733-8724.

Švorčík, V., Hüttel, I. \& Paláček, P. (2007). Polymethylmethacrylate Optical Waveguides Prepared in Electrical Field, Materials Letters, Volume 61, Number 1-4, (2007), pp. 953-955, ISSN: 0167-577X.

Jeřábek, V. \& Arciniega, J.A. (2009). Hybrid Optoelectronic Receiver with Gigahertz Bandwidth, Electronic Horizon, to be published.

Sackinger E. (2005). Broadband Circuits for Optical Fiber Communication, pp.193-194, ISBN 0471-71233-7, John Wiley \& Sons Inc, New Jersey. 


\title{
Design of a self-assembling, repairing and reconfiguring Arithmetic Logic Unit
}

\author{
David Huw Jones, Richard McWilliam and Alan Purvis \\ University of Durham \\ England
}

\section{Introduction}

The consumer will not tolerate products that are prone to failure: cars such as the Skoda Felicia or the Ford Pinto, motorbikes such as the BSA Dandy scooter, computers such as the Apple III - these devices were all commercially unsuccessful simply because of their high failure rate. For a product to succeed, the designer must consider the intended lifespan of the device and then maximise the probability of its survival for that period.

Within the systems upon which we depend, or choose to trust, for our survival - be they aeroplanes, trains, armaments or systems critical to a country's infrastructure - failure cannot be tolerated without adequate notice and provision for repair or replacement. 500 people died when Pfizer's replacement heart valves failed, costing the company upwards of \$200 million. The structural failures of the de Havilland Comet jet aeroplane caused five crashes before commercial flights were cancelled. A designer must therefore consider the significance of a component failure and make provisions for repair or replacement.

Components that are too costly to repair or replace, because of limited accessibility or limited resources, cannot be subject to failure. The early operational failures of the Hubble telescope were due to a flawed mirror. A space mission costing $\$ 8$ million was required to fix the telescope with correcting lenses. Thus for a system to succeed, a designer must consider the cost of repair when deciding on the required level of reliability.

The trend is for devices to be smaller and more functional than their predecessors. Modular redundancy, the use of extra copies of failure-prone hardware that can mask, or take the place of its damaged counterparts, has costs in both size and functionality. If this trend continues, the price of modular redundancy will become greater and an alternative will need to be considered.

Aside from demanding applications, reliability engineers face a challenge from systems formed on unreliable mediums. Plastic electronics systems are formed on flexible substrates, typically by the sequential deposition of conductive or semi-conductive organic polymers (such as Poly-3,4-ethylenedioxythiophene) using analogue or digital graphic printing technologies. Potential applications include flexible displays, photovoltaic's that can cover non-planar surfaces, and smart packaging, including battery testers, flexible batteries and RFID tags. However, flexible substrates are prone to distortion, potentially compromising the deposited system and causing its failure. Redundancy is particularly well equipped to 
cope with the failure of known unreliable sub-systems, but is much less well suited to coping with a system of which any part, or combination of parts, is likely to fail.

Since 2002 NASA have been running a series of workshops under the title "Ultra reliability"; this with the goal of increasing systems reliability by an order of magnitude across complex systems, hardware (including aircraft, aerospace craft and launch vehicles), software, human interactions, long life missions, infrastructure development, and cross cutting technologies (Shapiro, 2006). It is not difficult to see the difficulties standard redundancy techniques will face in long life missions (a manned mission to Mars will take upwards of six months, the $\$ 720$ million Mars Reconnaissance Orbiter has a planned life of at least four years) that are vulnerable to cosmic rays.

\section{Morphogenesis-inspired ultra reliability}

Morphogenesis provides biological systems with a robust framework for the differentiation of undeveloped or partially developed cells. Remarkable examples of self-repair co-ordinated by morphogenesis include:

- The human liver: This organ is capable of withstanding and repairing damage to up to two-thirds of its constituent cells.

- The Salamander: If bisected from its tail, the tail will often grow back.

- Ascidians (marine filter feeders), whose blood cells alone have been reported to give rise to a fully functional organism (Berrill and Cohen, 1936).

Morphogens are soluble proteins that diffuse about source cells within developing tissue. These chemical messages co-ordinate the differentiation of cells, determining what type of cell belongs where in the tissue. This self-assembly, self-repair mechanism can be modelled on cellular automata.

Cellular automata (CA) are systems in which space and time are discrete. CA consist of an array of cells, each of which determines its next state (typically an integer or a colour) from the state of its immediate neighbours using a next-state rule that is common to every cell. The analysis presented in (Jones et al., 2008) showed that for the array of cells to converge to one particular pattern of states regardless of its initial conditions, the next-state rule cannot use its current state as an input, nor can it use the state of more than one neighbour per axis as an input. That is, if the array is two-dimensional, in determining its next state a cell can only use inputs from neighbours either above or below itself, either to the left or to the right of itself. The paper goes on to demonstrate a deterministic algorithm for the design of CA that converge to a specific pattern of states.

If each state maps to a particular logic function, the CA will converge toward a pattern of logic units that could ultimately form any effective electronic system. Such a CA will converge to this form regardless of its initial conditions, effectively making it intrinsically self-repairing. Furthermore, if the boundary conditions of this CA are altered the CA will converge to a different pattern. If this is considered during the design process, the CA becomes able to self-reconfigure.

To be of any use, a reliability mechanism must be less prone to failure than that which it is trying to protect. However, as each cell must be identical, achieving this homogeneity for large system blocks adds a significant cost in replicated function to the design. Thus the optimum scale at which to discretise the design into a cellular architecture is found at the minima of total system complexity (see figure 1). 


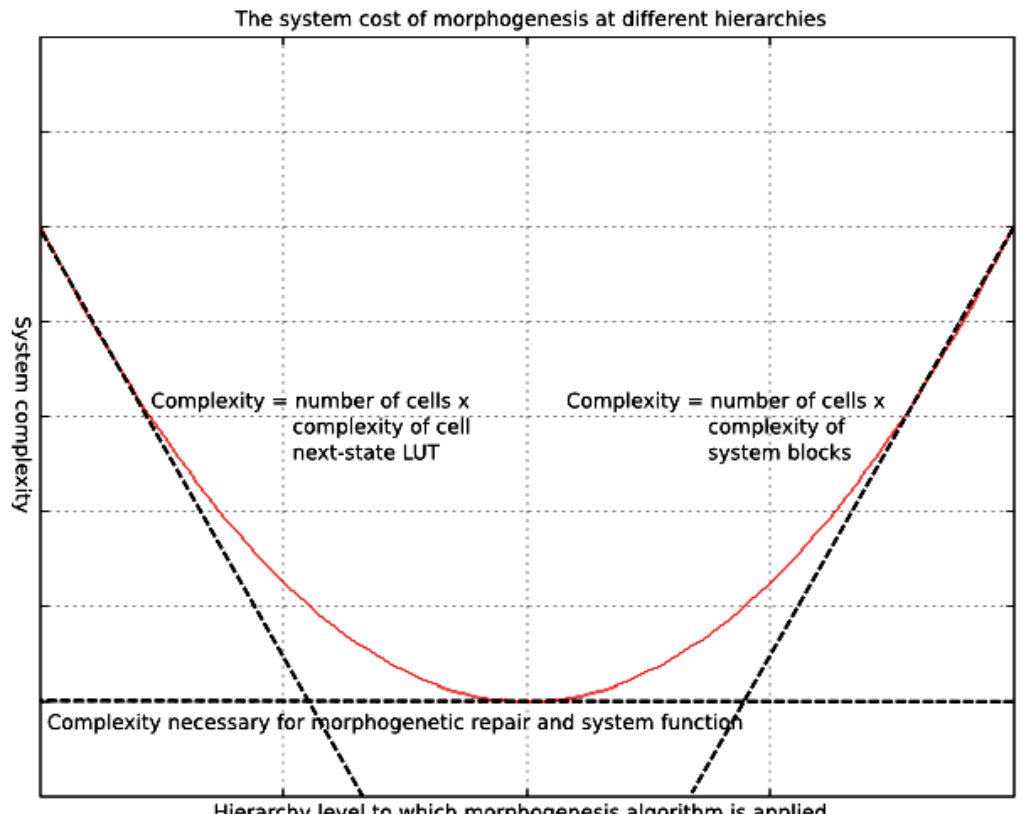

Fig. 1. The system cost of morphogenesis at different hierarchies

Existing reprogrammable devices (PLA, PAL or FPGA) are not optimised for the fine-grained self-reprogrammable logic required for this self-assembling algorithm. An appropriate custom Application Specific Integrated Circuit (ASIC) could be optimised to use fewer units of logic than an equivalent FPGA implementation. One possible embodiment of a cell within an ASIC is shown in figure 2.

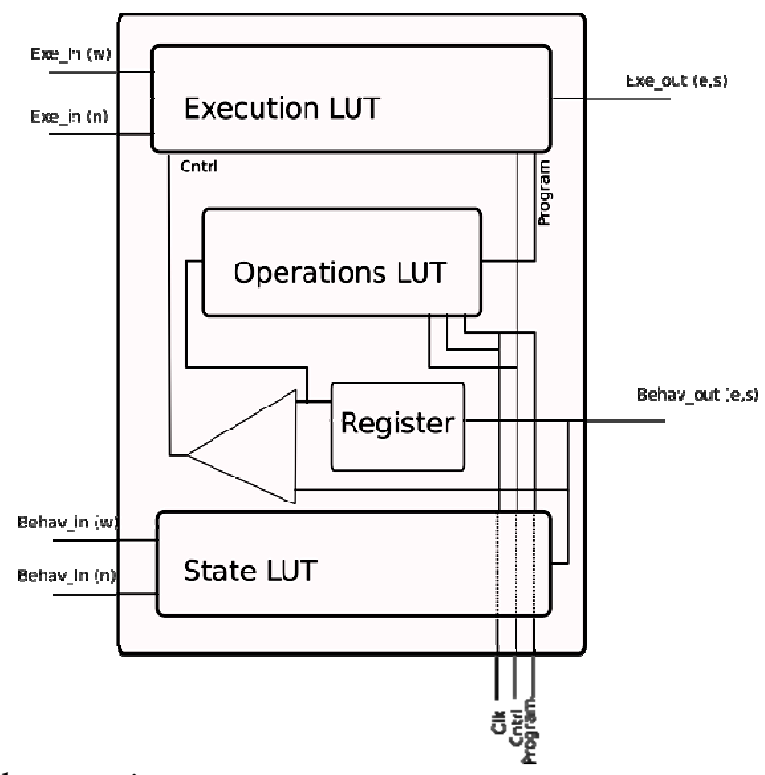

Fig. 2. An ASIC implementation 
The state of each cell must map to a component function, coded in the form of a bitstream that can be written to a look-up table and executed. Every bitstream required for the automata is stored in the function look-up table. The bitstream is selected by the cell state, loaded into the execution look-up table and executed. Every time the cell configuration changes (as detected by the cell comparator), the execution look-up table is reloaded with a new bitstream.

Another consideration is the use of redundant cells. Biological implementations of morphogenesis have an advantage over any electronic implementations: in the event of a cell being permanently damaged, biology can grow a replacement. This is something that is currently not possible in electronic devices. Thus, redundant cells - cells that can take the place of any other in the event of permanent failure - must be an integral part of the design. In order for a redundant cell to take the place of any damaged cell, every cell would have to be directly connected to every other cell. An alternative is to place every cell on a shared bus and provide each cell with an appropriate interface. Dynamic, in-situ re-programmable routing is another possibility. There are already various algorithms (Thoma, 2003) for managing dynamic routing.

\section{Design of a self-assembling self-repairing one-bit full-adder}

The schematic for a one-bit full-adder can be seen in figure 3 .

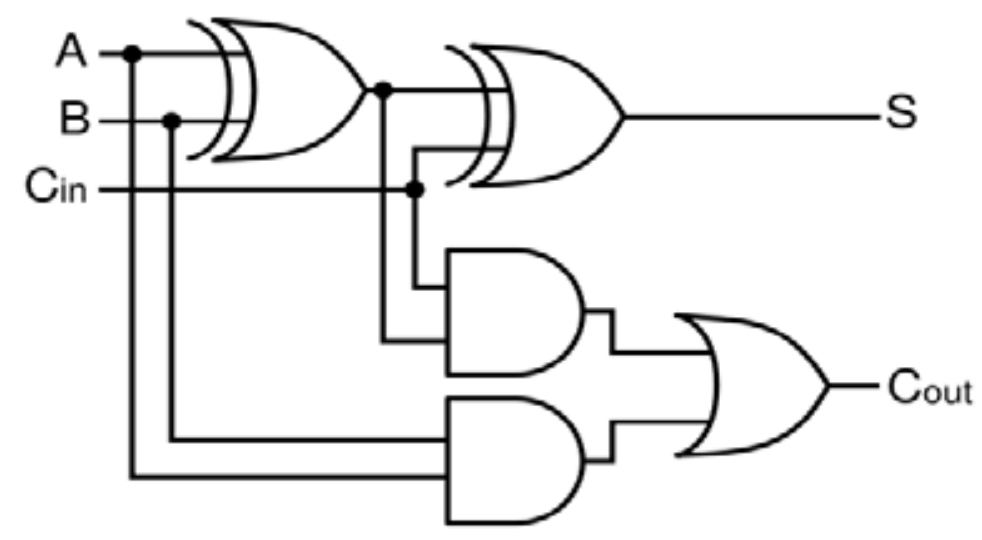

Fig. 3. A 1-bit full-adder schematic

In order to minimise the component count (and therefore the number of components that contribute to the device failure rate) there are a few constraints to the schematic design:

- Every cell determines its state from two of its immediate neighbours. Other than power and clock lines there are no global connections required for the CA to converge to its correct state. If a full-adder is to be implemented on such a platform, it would ideally not require any global connections either.

- The cell has no bi-directional communications: it relies on two-inputs and two-output lines in a feed-forward arrangement in order to converge. Likewise an ideal full-adder design should be built on this arrangement. This means each component cell cannot feedback data to a cell that lies earlier on on the data path. 
- $\quad$ Each cell has two output lines, but the state-output is common to both. Again, the most appropriate implementation of a full-adder design will piggy-back these existing communications lines and not require additional networking. One consequence of this is that no two data lines can cross.

- Because we want this full-adder to be scalable, the one-bit full-adder modules should be stackable, that is, if the modules are arranged one on top of one another, the carry-out lines should connect to the carry-in line beneath it.

- In order for the full-adder to be scalable, the CA state pattern must repeat until it uses all the available cells.

- There should be as few different cell-types (equivalent to the size of the CA alphabet) as is necessary, and there should be as few cells per one-bit module as is necessary.

Figure 4 shows how the schematic of figure 1 has been revised to ensure there are no crossed data lines. Figure 5 shows the different cell operations and their corresponding state assignments. Figure 6 shows the schematic laid out over 16 cells. Figure 7 shows the design (implemented on an ALTERA FPGA) self-assembling. Note that this design requires the following boundary conditions:

- $\quad$ The cell connected to input 'A' of the full-adder must be to below a state '7'.

- The cell connected to input 'B' of the full-adder must be to the right of a state '2'.

- The top-left cell of the first bit of the full-adder must be below a state ' 1 '.

- $\quad$ The cell to the right of the top-left cell of the first bit must be below a state '2'. Since the bottom row of each 16 cell design starts with the states ' 1 ' and ' 2 ', these boundary conditions propagate to the subsequent bits and the design repeats until it runs out of cells.

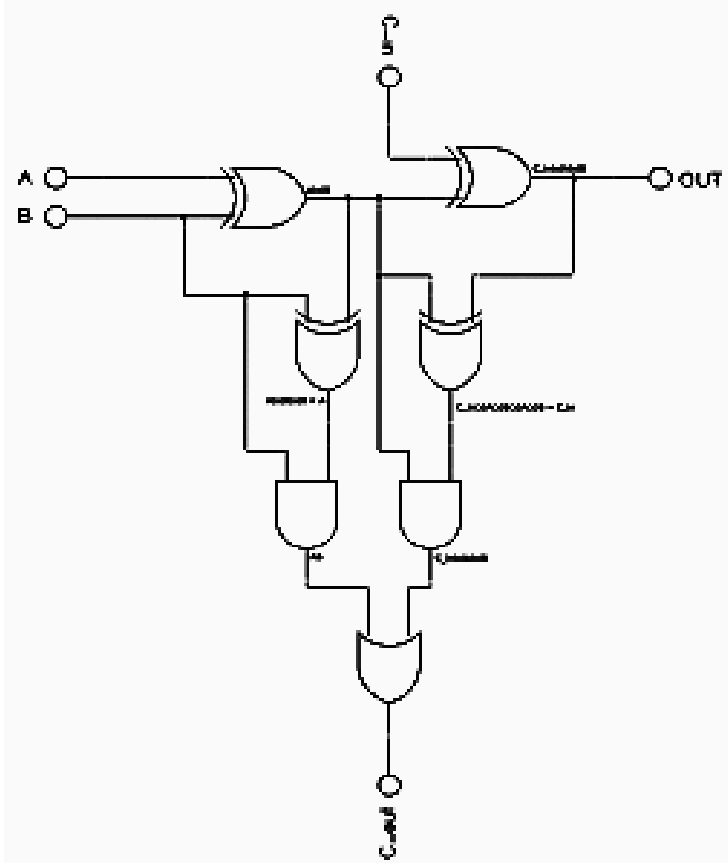

Fig. 4. An alternative one-bit full-adder schematic 


\begin{tabular}{|c|c|c|c|c|c|}
\hline Cell function & OR & & XOR & $\downarrow$ & $\begin{array}{l}\text { XOR/ } \\
\text { AND }\end{array}$ \\
\hline Cell states & 0 & 1,7 & 2 & 3,4 & 5 \\
\hline
\end{tabular}

Fig. 5. Different types of cell

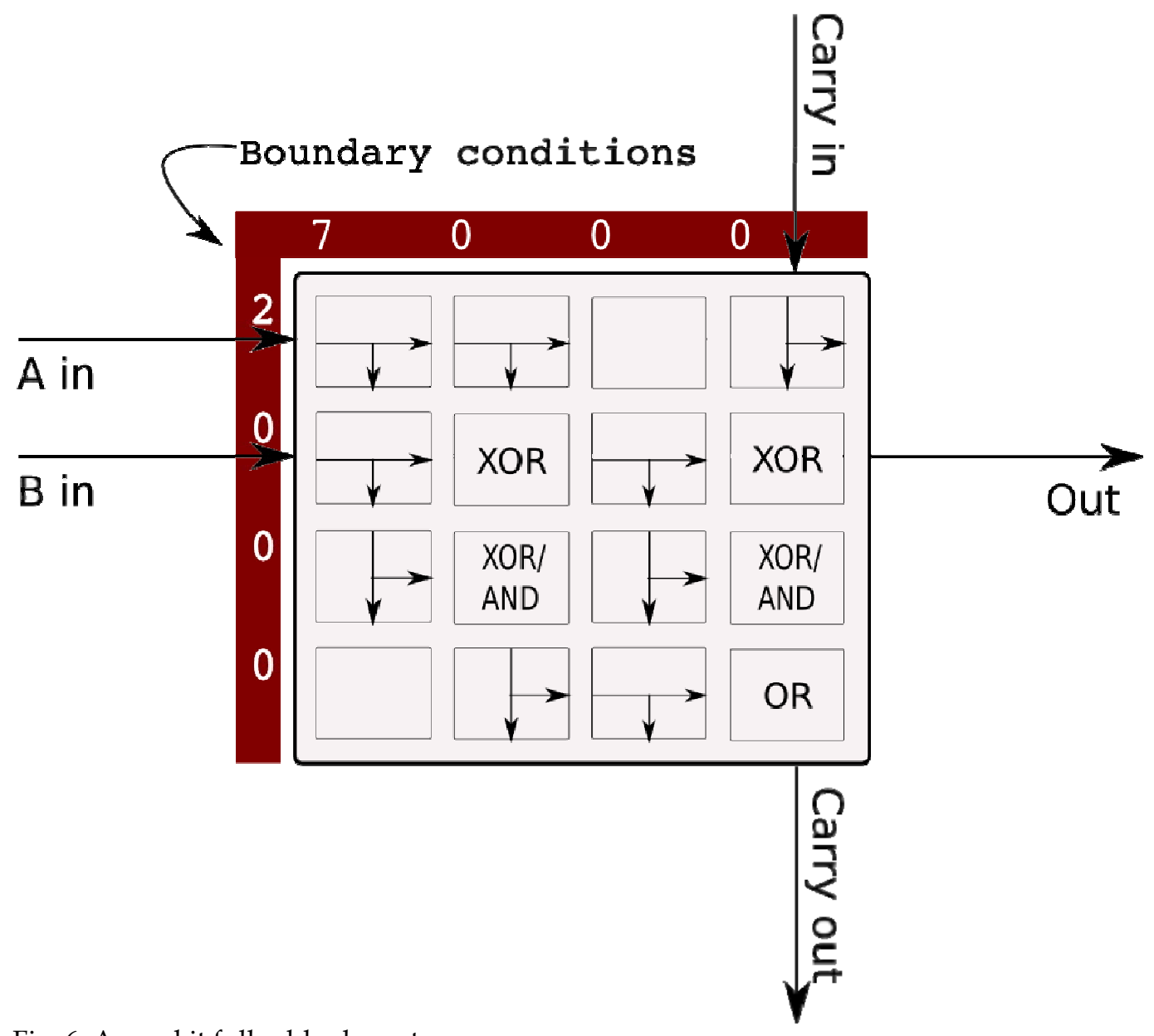

Fig. 6. A one-bit full-adder layout 


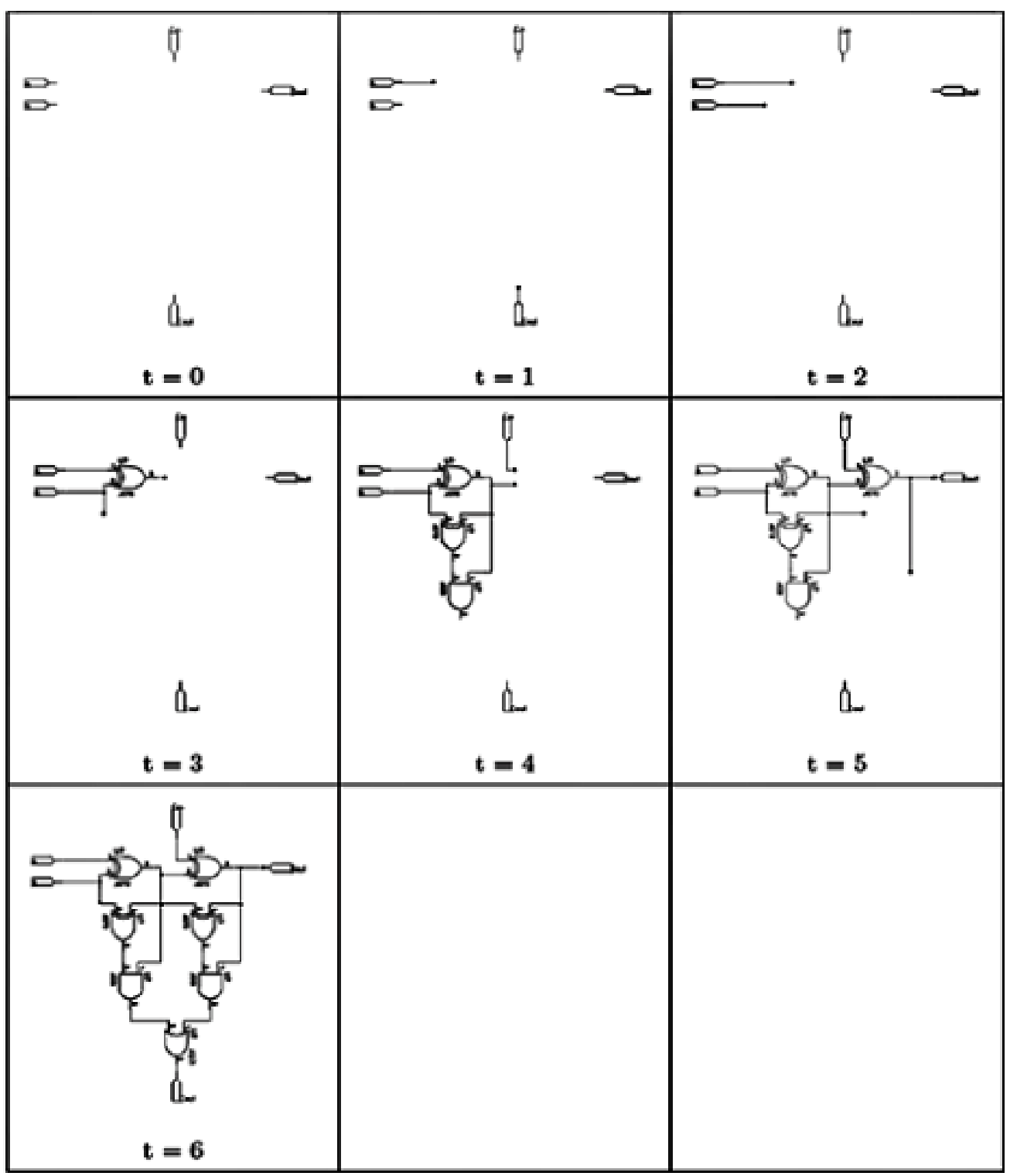

Fig. 8. A one-bit full-adder self-assembling

\section{Design of a self-assembling self-repairing one-bit Arithmetic Logic Unit}

In order for the full-adder design to correctly self-assemble, the boundary conditions of the array must be precisely set. If these are changed, the arrangement of cell types will change. This effect can be taken advantage of by designing the cell array to respond to changes in the boundary conditions with desired alternative arrangements. Thus, the full-adder could be converted into a full-subtracter by changing one of the boundary conditions. Likewise, the 
array can be programmed to perform other functions of an arithmetic logic unit (ALU) (e.g. AND, OR and NOT gates) with different boundary conditions.

Since we want the design to scale, these boundary condition changes need to propagate to the bottom of the 16 cell arrangement so that the subsequent 16 cells can also re-configure to perform the requested function. This requirement is responsible for some of the more esoteric logic arrangements (for instance a NOT gate being built from two XOR gates and a NOT gate) present in the designs. Figure 9 shows the logic arrangements and boundary conditions for the ALU functions, AND, OR, NOT and SUBTRACT.

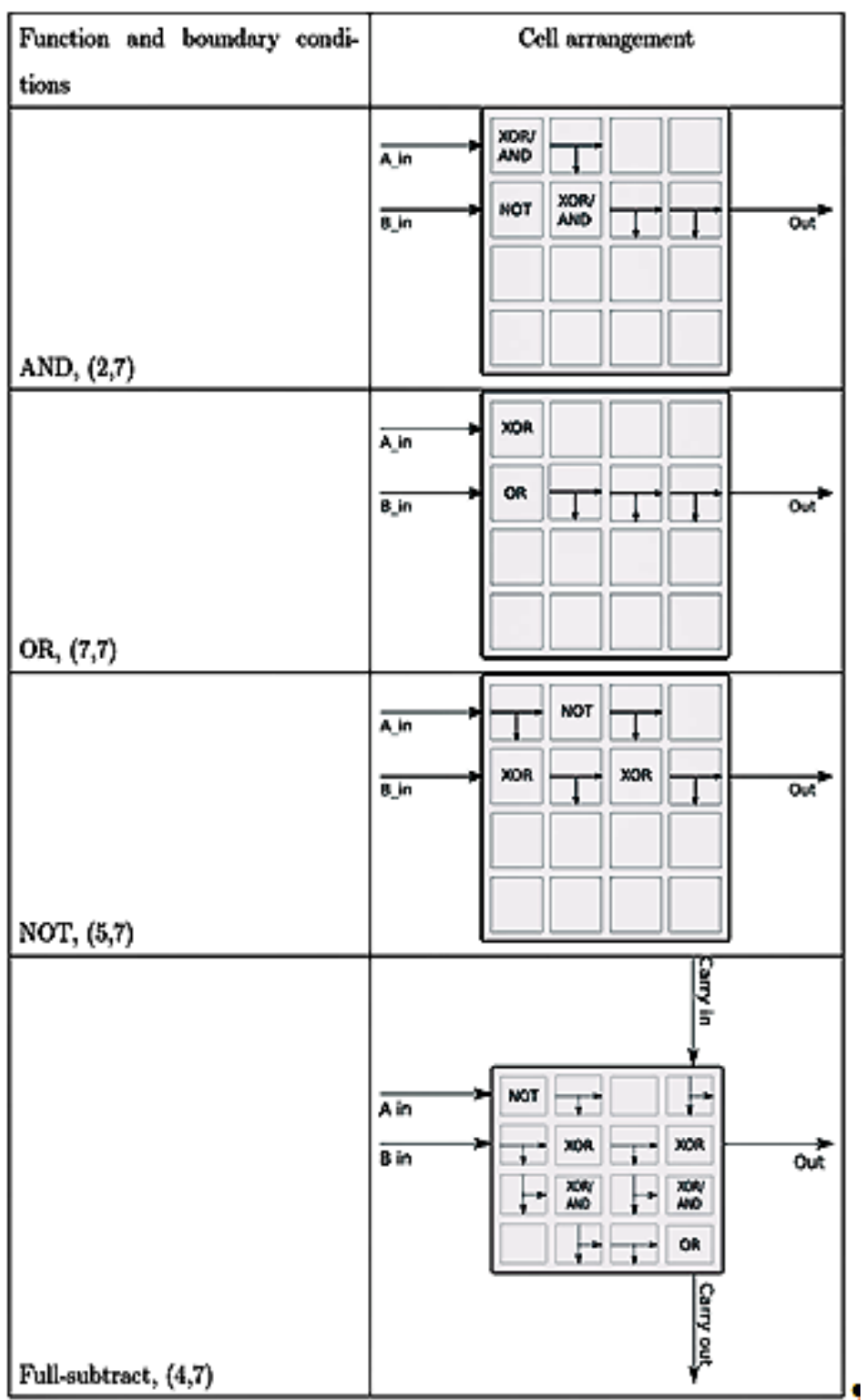

Fig. 9. Cell arrangements and boundary conditions for ALU 


\section{Assessing the reliability of the ALU}

The mechanisms of CA and morphogenesis require a homogenous array of 'stem' cells that are capable of becoming any type of cell during system development and repair. The LUT of FPGAs are conceptually just such a cell, but while some FPGAs are capable of partitioning and reprogramming a small portion of their entire configuration, the bit-stream configuration data must be provided from a source external to the FPGA. This means that without a significant re-design of the FPGA infrastructure, the application of mitosis (replication of cell function) is not possible. As a result it is no longer enough for each cell to be capable of becoming any other type of cell; instead, each cell must be capable of performing the function of any other type of cell without any in-situ re-programming.

In order to assess the reliability of this self-repairing ALU and compare it to the reliability of a standard ALU design and an N-modular redundant ALU design, each will be implemented on a field programmable gate array (FPGA). The number of logic blocks used by each component of each design and the systems dependence on each will be used with the reliability analysis techniques described in the previous chapter in order to assess the MTBF of both.

There are a number of assumptions in this analysis:

- $\quad$ The FPGA is an ALTERA Stratix II, the design software is QUARTUS. The fitting process used by Quartus to convert the VHDL design into a netlist will not necessarily create a netlist that uses the fewest number of logic units. As the self-repairing ALU is more complicated than a standard ALU, if the conversion does not create the smallest design possible the self-repairing ALU will be affected to a greater extent. However, for this analysis it is necessary to assume they are both affected equally.

- $\quad$ Each design scales linearly. Thus an eight-bit ALU will use eight times the number of logic units as a single-bit ALU. Also a triple-modular redundant system will use three times the number of logic units (plus more for the voter) as the single ALU.

- $\quad$ The MTBF of the design can be assessed by considering the MTBF of the FPGA and multiplying by the fraction of it used by the design. While this ignores single points of failure (clocks, power lines, etc) it is not unreasonable as the most common failure-mode of an FPGA are temporary, localised degredation, typically due to stuck-at faults on interconnects (Touba, 1999).

- The ALTERA does not support partial reprogramming. Thus while the state of each cell, their arrangement and interconnections are intrinsically self-repairing, there is no way for the function that each cell performs to be corrected in the event of a failure. However, for the purposes of this analysis it will be assumed that the hardware does support reprogramming and the necessary logic is built into the BIST.

The cell component can be seen in figure 10 and a one-bit ALU comprised of 16 cells can be seen in figure 11 . 


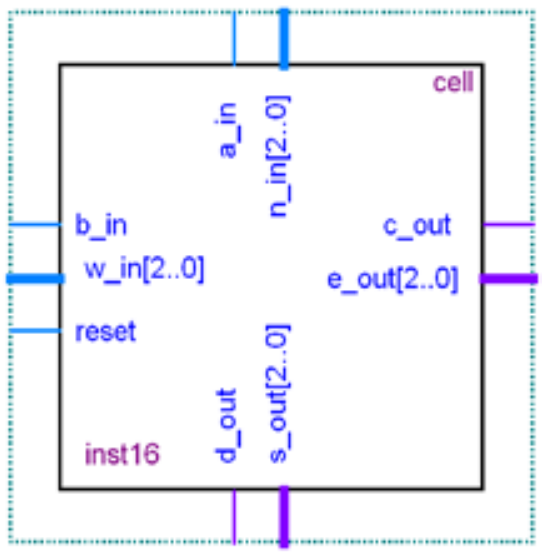

\begin{tabular}{|l|l|}
\hline $\begin{array}{l}\text { a_in } \\
\text { b_in }\end{array}$ & Logic inputs \\
\hline c_out & Logic outputs \\
d_out & \\
\hline n_in & State inputs \\
w_in & \\
\hline $\begin{array}{l}\text { e_out } \\
\text { s_out }\end{array}$ & State outputs \\
\hline reset & Global reset \\
\hline
\end{tabular}

Fig. 10. A cell of the ALU design

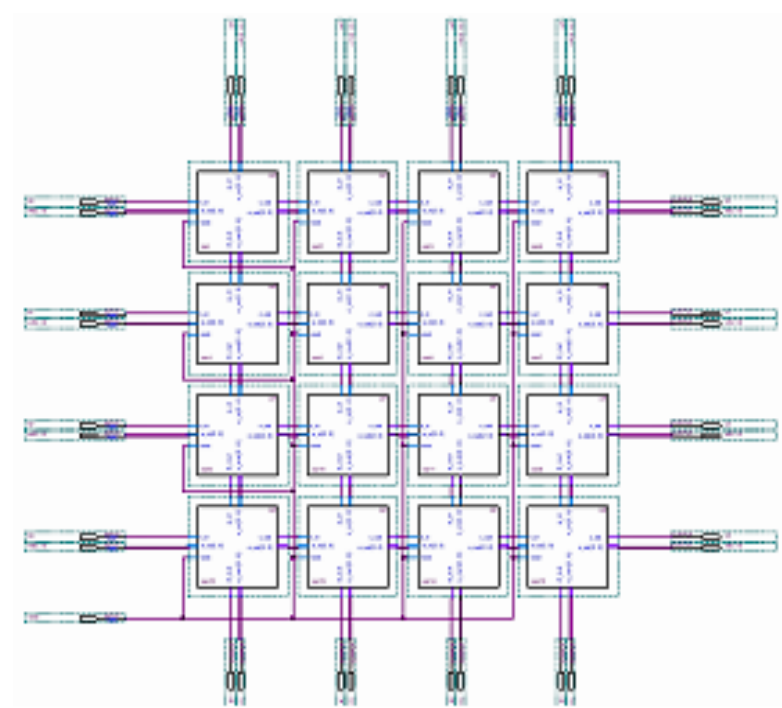

Fig. 11. A 1-bit ALU made of 16 cells

Below are the results of compiling these designs for the Stratix II FPGA.

\begin{tabular}{|l|l|l|}
\hline Component & Logic Units & \% of FPGA \\
\hline 8-bit ALU & 48 & $<1 \%$ \\
\hline 3-Mod 8-bit ALU & 152 & $2 \%$ \\
\hline$\bullet$ ALU & 48 & $<1 \%$ \\
\hline$\bullet$ Voter & 8 & $<1 \%$ \\
\hline 162-Mod 8-bit ALU & 9120 & $100 \%$ \\
\hline 8-bit self-assembling ALU & 9120 & $100 \%$ \\
\hline$\bullet$ Cell & 87 & $1 \%$ \\
\hline$\bullet$ BIST & 1 & $<1 \%$ \\
\hline
\end{tabular}

Table 1. Comparative logic unit usage of different ALU designs 
A 162-modular redundant system uses the same resources as the self-assembling self-repairing design. A simple analysis shows the self-assembling solution can tolerate a greater number of failures than its static-redundancy equivalent.

The 162-modular system can tolerate $\frac{N}{2}-1=80$ module failures without a system failure.

The self-assembling system consists of 128 identical cells. Provided the self-repair code is still working, the system can tolerate 127 cell failures at any one time because each failed cell can copy the operating code from the one remaining cell.

The table below shows the reliability parameters appropriate to the ALTERA Stratix II FPGA. Using the MIL-HDBK-217F standard and a part-stress analysis, the failure rate of the FPGA can be determined as $\lambda_{p}=0.32$ failures per $10^{6}$ hours. Using this and the table above the failure rates for individual components of the design can be determined.

\begin{tabular}{|l|l|l|l|}
\hline Variable & Nomenclature & Category & Value \\
\hline Base failure rate & $\mathrm{C}_{1}$ & PLA $>5000$ gates & 0.042 \\
\hline Operating temperature & $\pi_{t}$ & $25^{\circ} \mathrm{C}$ & 0.1 \\
\hline Package failure rate & $\mathrm{C}_{2}$ & $>300$ Pins & 0.16 \\
\hline Environment factor & $\pi_{E}$ & Ground, Fixed & 2 \\
\hline Quality factor & $\pi_{Q}$ & Undocumented & 1 \\
\hline Learning factor & $\pi_{L}$ & $>$ 2 years & 1 \\
\hline
\end{tabular}

Table 2. Logic unit reliability parameters for an FPGA

While it is possible to form a fault-tree model of the ALU, this would be inappropriate because of its state-dependent failure modes. A more appropriate approach is the use of a Markov model (see figure 12) of the three operating states.

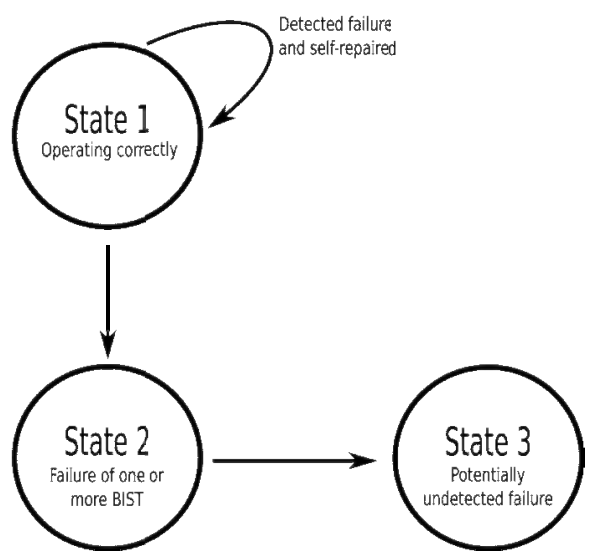

Fig. 12. Markov model of the ALU, memory and processor systems

Using the Chapman-Kolmogorov equation (1) for Markov analysis, it is possible to analyse this model in order to determine the probabilities, $P(t)$, of each failure mode. 


$$
\begin{gathered}
\frac{d}{d t} P(t)=P(t) Q(t) \\
P(0)=[1,0,0,0]
\end{gathered}
$$

$\mathrm{Q}(\mathrm{t})$, the transition matrix, is derived from the Markov model. Each failure rate, $\lambda$, refers to a transition between states.

$$
Q(t)=\left(\begin{array}{ccc}
-\lambda_{1} & 0 & 0 \\
\lambda_{1} & -\lambda_{2} & 0 \\
0 & \lambda_{2} & 0
\end{array}\right)
$$

From (2) and (3) it is possible to form three coupled differential equations, (4), (5) and (6).

$$
\begin{gathered}
\frac{d}{d t} P_{1}(t)=-\lambda_{1} P_{1}(t) \\
\frac{d}{d t} P_{2}(t)=\lambda_{1} P_{1}(t)-\lambda_{2} P_{2}(t) \\
\frac{d}{d t} P_{3}(t)=\lambda_{2} P_{2}(t)
\end{gathered}
$$

The solution to (4) that meets the initial conditions (2), is (7).

$$
P_{1}(t)=e^{-\lambda_{1} t}
$$

Equation (5) can be rearranged to form (8).

$$
\frac{d}{d t} P_{2}(t)+\lambda_{2} P_{2}(t)=\lambda_{1} P_{1}(t)
$$

By multiplying (8) by the integrating factor $e^{\lambda_{2} t}$ we obtain equations (9) and (10).

$$
\begin{gathered}
e^{\lambda_{2} t} \frac{d}{d t} P_{2}(t)+e^{\lambda_{2} t} \lambda_{2} P_{2}(t)=e^{\lambda_{2} t} \lambda_{1} P_{1}(t) \\
\frac{d}{d t}\left[e^{\lambda_{2} t} P_{2}(t)\right]=e^{\lambda_{2} t} \lambda_{1} P_{1}(t)
\end{gathered}
$$

Substituting (7) into (10) gives (11) and (12).

$$
\begin{aligned}
& \frac{d}{d t}\left[e^{\lambda_{2} t} P_{2}(t)\right]=e^{\lambda_{2} t} \lambda_{1} e^{-\lambda_{1} t} \\
& \frac{d}{d t}\left[e^{\lambda_{2} t} P_{2}(t)\right]=e^{\left(\lambda_{2}-\lambda_{1}\right) t} \lambda_{1}
\end{aligned}
$$


Integrating both sides of (12) with respect to $t$ gives:

$$
e^{\lambda_{2} t} P_{2}(t)=\frac{\lambda_{1}}{\lambda_{2}-\lambda_{1}} e^{\left(\lambda_{2}-\lambda_{1}\right) t}+C
$$

By dividing both sides of (13) by $e^{\lambda_{2} t}$ we obtain:

$$
\begin{gathered}
P_{2}(t)=\frac{\lambda_{1}}{\left(\lambda_{2}-\lambda_{1}\right) e^{\lambda_{2} t}} e^{\left(\lambda_{2}-\lambda_{1}\right) t}+\frac{c}{e^{\lambda_{2} t}} \\
P_{2}(t)=\frac{\lambda_{1}}{\lambda_{2}-\lambda_{1}} e^{-\lambda_{1} t}+C e^{-\lambda_{2} t}
\end{gathered}
$$

Substituting the initial conditions (2) into (15) we can determine $C$.

$$
\begin{gathered}
0=\frac{\lambda_{1}}{\lambda_{2}-\lambda_{1}} e^{-\lambda_{1} .0}+C e^{-\lambda_{2} .0} \\
0=\frac{\lambda_{1}}{\lambda_{2}-\lambda_{1}}+C
\end{gathered}
$$

Substituting (17) into (15) we get a final answer for $P_{2}(t)$.

$$
P_{2}(t)=\frac{\lambda_{1}}{\lambda_{2}-\lambda_{1}}\left(e^{-\lambda_{1} t}-e^{-\lambda_{2} t}\right)
$$

The sum of the state probabilities must be one, because the system must reside in one of the three states.

$$
\sum_{i} P_{i}(t)=1
$$

Thus the solution to (6) is found by subtracting the results from the previous two results.

$$
P_{3}(t)=1-P_{1}(t)-P_{2}(t)
$$

Figure 13 shows the reliability $\left(P_{1}(t)+P_{2}(t)\right)$ versus time of the self-repairing ALU and an $\mathrm{N}$-modular redundant ALU of an equivalent size. 


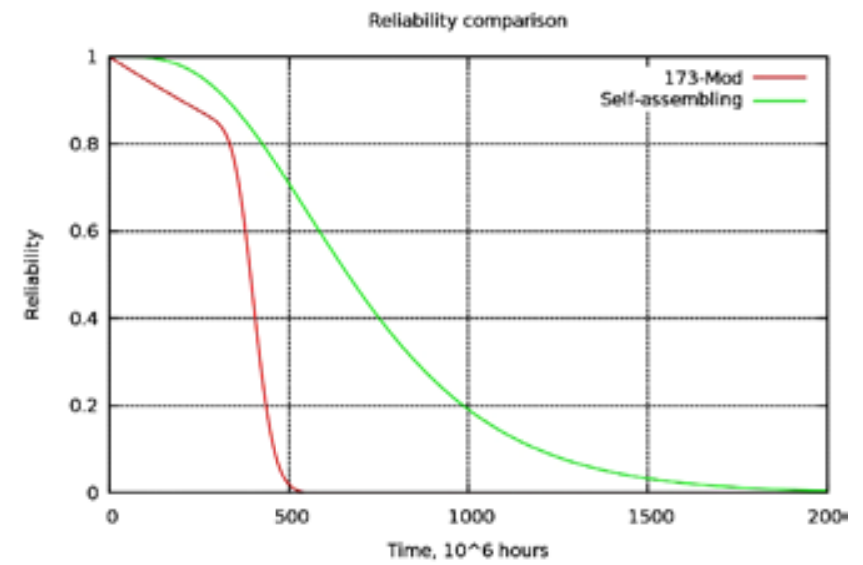

Fig. 13. Reliability comparison (No redundant cells)

The reliability demonstrated by this self-repairing design is due to it being able to tolerate those failure modes that can be corrected by reconfiguring the device. The reliability of the self-repairing ALU can be further increased by introducing small numbers of redundant cells to the design to replace irreparably broken cells. Figure 14 shows the reliability of a self-repairing ALU with 2 redundant cells as well as the reliability of an N-modular redundant system of equivalent size. Figure 15 shows the reliability of a system with 5 redundant cells, figure 16 shows the reliability of a system with 10 redundant cells.

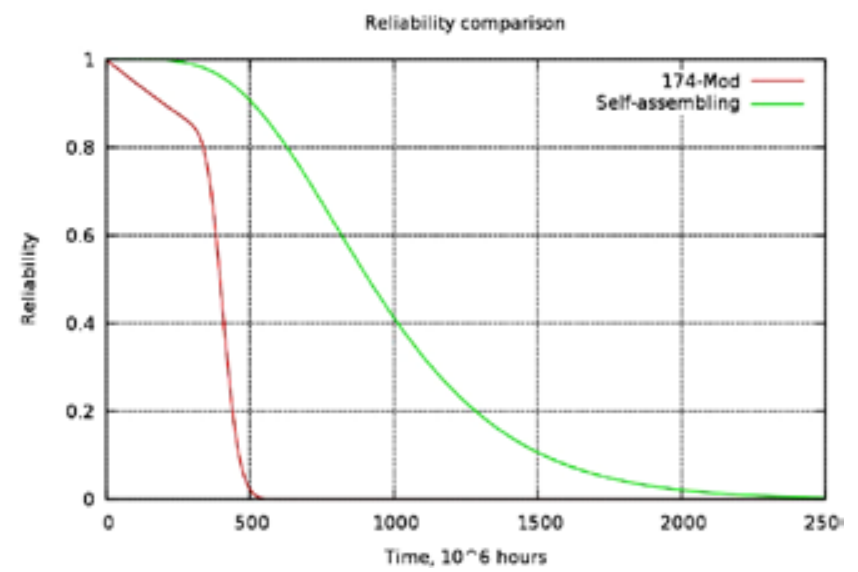

Fig. 14. Reliability comparison (2 redundant cells) 


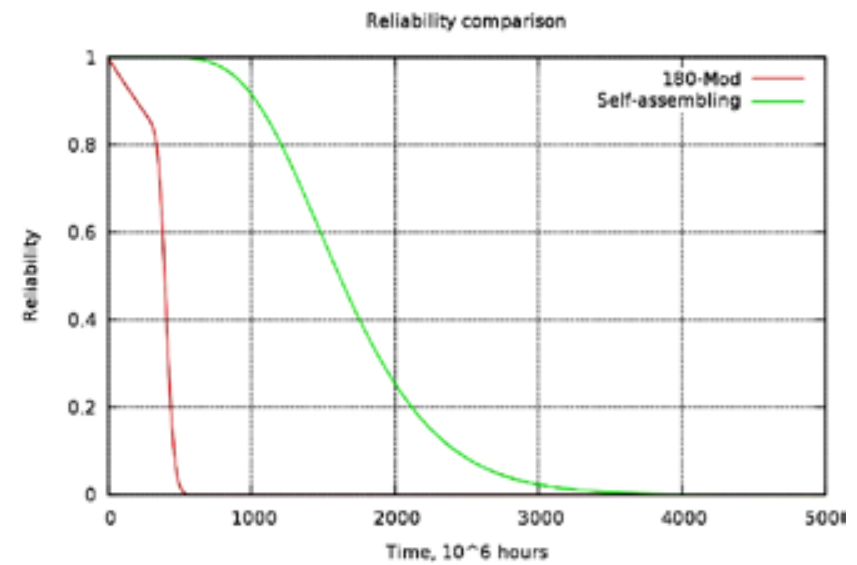

Fig. 15. Reliability comparison (5 redundant cells)

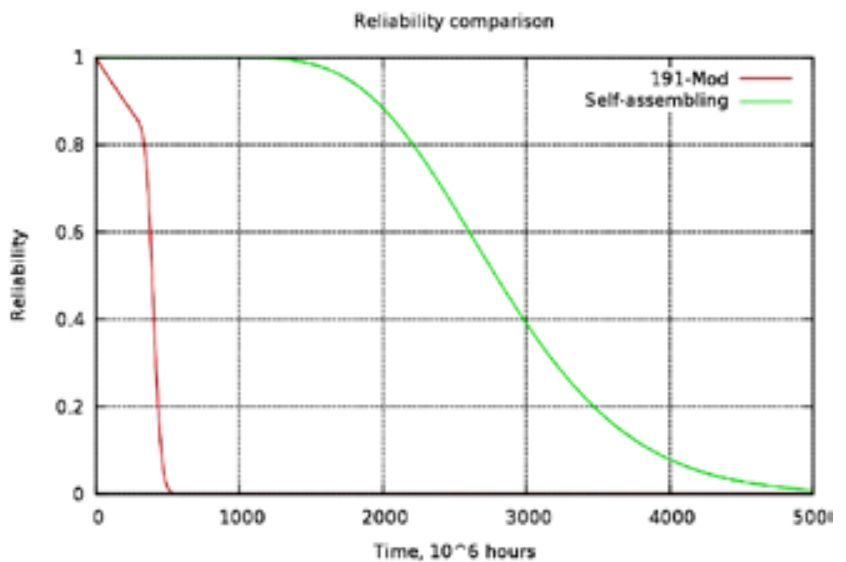

Fig. 16. Reliability comparison (10 redundant cells)

In order to quantify the reliability improvement shown in figures 13-16 the mean time to failure (MTTF) of each system will be calculated at $400.10^{6}$ hours.

$$
M T T F=\frac{400}{\ln \left(\frac{\lambda_{1}}{\lambda_{2}-\lambda_{1}}\left(e^{-400 \cdot \lambda_{1}}-e^{-400 \cdot \lambda_{2}}\right)+e^{-400 \cdot \lambda_{1}}\right)}
$$

\begin{tabular}{|l|l|l|}
\hline Redundant cells & $\begin{array}{l}\text { MTTF (106hrs) of } \\
\text { self-assembling technique }\end{array}$ & $\begin{array}{l}\text { MTTF (106hrs) of N-mod } \\
\text { technique }\end{array}$ \\
\hline 0 & $5.730 \times 10^{2}$ & $5.004 \times 10^{2}$ \\
\hline 1 & $2.144 \times 10^{3}$ & $5.009 \times 10^{2}$ \\
\hline 2 & $1.007 \times 10^{4}$ & $5.362 \times 10^{2}$ \\
\hline 5 & $3.560 \times 10^{6}$ & $5.367 \times 10^{2}$ \\
\hline 10 & $1.240 \times 10^{12}$ & $5.040 \times 10^{2}$ \\
\hline
\end{tabular}


With no redundant cells available to the self-assembling design, the MTTF is slightly longer than that of its N-modular equivalent. However making available small numbers of cells significantly increases its MTTF (by a factor of $10^{10}$ hours in the case of 10 redundant cells) performance over its equivalent $\mathrm{N}$-modular design.

\section{Conclusions}

This technique, whilst demonstrating an improved reliability over an equivalent N-modular redundant system, is not without cost:

- $\quad$ The minimum size of the self-assembling ALU is a factor of a hundred times greater than that of a single ALU and over thirty times that of a triple modular redundant ALU.

- The design algorithm is not trivial. Modular redundancy can be easily applied to any system without a significant schematic re-design. This is not true of the self-assembling approach. Firstly an appropriate level for cellular discretisation must be selected. Next, the system function must be divided between cells such that short, preferably not overlapping, buses between nearest neighbour cells are the primary means of data transmission. Lastly the rules necessary to co-ordinate the assembly and differentiation of these must be generated.

For the reasons above, this approach may not be particularly applicable to the design of reliable, commercial projects. However it certainly fits into the 'Ultra reliability' category. Any system that must operate in harsh environments (e.g. satellites and space shuttles), or that cannot tolerate failure, could benefit from this technique.

The self-assembling, self-repairing capabilities of morphogenesis can be mimicked in the design of self-assembling, self-repairing electronic circuits. These circuits are formed on an array of discrete, identical cells. To achieve correct system performance, each cell determines its state then uses this to determine its component type. Furthermore, these arrays can be designed to metamorphosise into different circuits depending on the boundary conditions of the array. This design approach has been demonstrated with an ALU design on an FPGA and its reliability assessed using state-based models and a stochastic analysis. Subsequently, this reliability was compared to the reliability of an N-modular ALU of equivalent FPGA logic unit use, and found to perform significantly better.

\section{References}

Berrill N.J, \& Cohen, A. (1936). Regeneration in Clavellina lepadiformis, Journal of experimental biology, 13.

Jones, D. H; McWilliam, R. P. \& Purvis, A. (2009), Designing convergent cellular automata, Biosystems.

Shapiro, A. (2006), Ultra-Reliability at NASA, 44th AIAA Aerospace Sciences Meeting and Exhibit.

Thoma, Y; Sanchez, E; Arostegui, J. M. \& Tempesti, G. (2003), A dynamic routing algorithm for a bio-inspired reconfigurable circuit, Lecture notes in computer science.

Touba, D. N. A. (1999), A low cost approach to detecting, locating and avoiding interconnect faults in $\{\mathrm{FPGA}\}$-based reconfigurable systems, Proceedings of the International conference VLSI design. 


\title{
Discovering Web Server Logs Patterns Using Generalized Association Rules Algorithm
}

\author{
Mohd Helmy Abd Wahab르. Mohd Norzali Haji Mohd² \\ and Mohamad Farhan Mohamad Mohsin ${ }^{3}$ \\ 1,2Universiti Tun Hussein Onn Malaysia \\ ${ }^{3}$ Universiti Utara Malaysia \\ Malaysia
}

\section{Introduction}

With the explosive growth of data available on the World Wide Web (WWW), discovery and analysis of useful information from the World Wide Web becomes a practical necessity. Data Mining is primarily concerned with the discovery of knowledge and aims to provide answers to questions that people do not know how to ask. It is not an automatic process but one that exhaustively explores very large volumes of data to determine otherwise hidden relationships. The process extracts high quality information that can be used to draw conclusions based on relationships or patterns within the data.

Using the techniques used in Data Mining, Web Mining applies the techniques to the Internet by analyzing server logs and other personalized data collected from customers to provide meaningful information and knowledge. Web access pattern, which is the sequence of accesses pursued by users frequently, is a kind of interesting and useful knowledge in practice (Pei, 2000). Today web browsers provide easy access to myriad sources of text and multimedia data. With approximately 4.3 billion documents online and 20 million new web pages published each day (Tanasa and Trousse, 2004), more than 1000000000 pages are indexed by search engines, and finding the desired information is not an easy task (Pal et al., 2002). Web Mining is now a popular term of techniques to analyze the data from World Wide Web (Pramudiono, 2004). A widely accepted definition of the web mining is the application of data mining techniques to web data. With regard to the type of web data, web mining can be classified into three types: Web Content Mining, Web Structure Mining and Web Usage Mining.

As an important extension of data mining, Web mining is an integrated technology of various research fields including computational linguistics, statistics, informatics, artificial intelligence (AI) and knowledge discovery (Fayyad et al., 1996; Lee and Liu, 2001). Srivastava et al. (2002) classified Web Mining into three categories: Web Content Mining, Web Structure Mining, and Web Usage Mining (see Figure 1). 


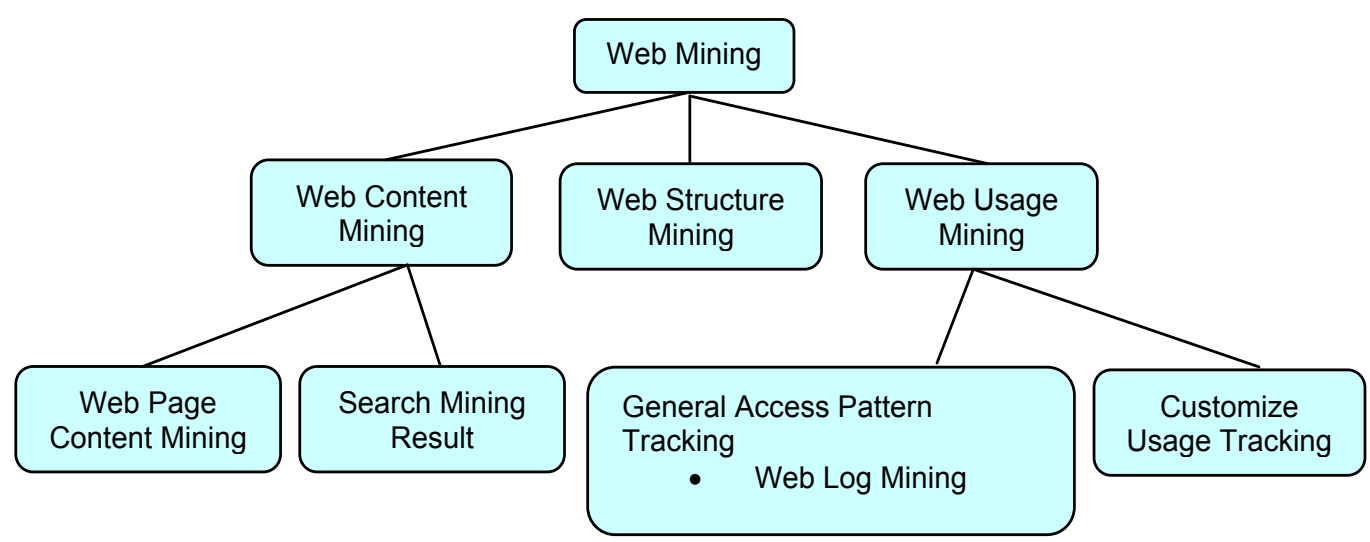

Fig. 1. Taxonomy of Web Mining

Content Mining involves mining web data contents (Madria, 1999) ranging from the HTML based document and XML-based documents found in the web servers to the mining of data and knowledge from the data source. Content Mining consists of two domain areas: Web Page Content Mining and Search Mining Result. Content data corresponds to the collection of facts from a Web page that was designed to convey information to the users (Srivastava et al., 2000). It may consist of text, images, audio, video, or structured records such as lists and tables.

Web Structure Mining (Chakrabarti et al., 1999) used to discover the model underlying the link structures of the Web. This type of mining can be further divided into two types based on the structural data used.

- Hyperlinks: A Hyperlink is a structural unit that connects a Web page to different location, either within the same Web page or to a different Web page. A Hyperlink that connects to a different part of the same Web page is called an Intra-Document Hyperlink, and a hyperlink that connects two different pages is called InterDocument Hyperlink. There has been a significant body of work on hyperlink analysis of which provides an up-to-date survey (Desikan et al., 2002).

- Document Structure: In addition, the content within a Web page can also be organized in a tree structure format, based on the various HTML and XML tags within the page. Mining efforts have focused on automatically extracting document object model (DOM) structures out of documents (Wang and Lui, 1998; Moh et al., 2000).

The amount of information available on World Wide Web (WWW) and databases has increased and is still rapidly increasing (Mohamadian, 2001). For a particular website, normally hundred thousand of users will be accessing a particular site. The administrator of a system has an access to the server log. However, the pattern of site usage cannot be analyzed without the use of a tool. Therefore, Data Mining method would ease the System Administrator to mine the usage patterns of a particular site. The work applied on Education Portal organized by Utusan Malaysia as one of the most popular web portal that offer a variety of services such as question bank, quiz, notes, games and etc.

The findings from this study provide an overview of the usage pattern of Tutor.com Portal. The study also demonstrates the use of Generalized Association Rules in Web Usage 
Mining. The outcome of this study can be used by the Tutor.com's System administrator as a guideline in enhancing the use of Tutor.com Web Portals.

\section{Association Rule}

Today, there are several efficient algorithms that cope with the popular and computationally expensive task of association rule mining. One of data mining techniques that are commonly used in web mining is association rules. Since its introduction in 1993 (Agrawal et al., 1994), the task of association rule mining has received a great deal of attention. In brief, an association rule is an expression $X \rightarrow Y$, where $X$ and $Y$ are sets of items. The meaning of such rules is quite intuitive: Given a database $D$ of transactions where each transaction $T \in D$ is a set of items, $X \rightarrow Y$ expresses that whenever a transaction $T$ contains $X$ than $T$ probably contains $Y$ also. The probability or rule confidence is defined as the percentage of transactions containing $Y$ in addition to $X$ with regard to the overall number of transactions containing $X$. That is, the rule confidence can be understood as the conditional probability $p(Y \subseteq T \mid X \subseteq T)$.

Different methods for building association-rule based prediction models using the web logs have been proposed by (Yang et al., 2000) and the proposed model had empirically tested on real web logs. However, there has been no systematic study on the relative merits of these methods. While Borgelt and Kruse (2002) describe an implementation of the well-known apriori algorithm for the induction of association rules that is based on the concept of a prefix tree. A traditional model of association rule mining (Tao et al., 2003) is adapted to handle weighted association rule mining problems where each item is allowed to have a weight. This research goal is to steer the web mining focus to those significant relationships involving items with significant weights rather than being flooded in the combinatorial explosion of insignificant relationships.

In practices, users are often interested in subset of association rules. For example, they may only want rules that contains a specific item or rules that contains children of specific item in hierarchy. While such constraint can be applied as a post-processing step, integrating them into the mining algorithm can dramatically reduce the execution time. Several fast algorithms for mining association rules have been developed by Srikant et al. (1997) to discover this problem.

Web mining using Association rules is one of the most commonly used techniques so far. Since this study is exploratory in nature, the association rules technique as employed. To this end, a generalized association rule is selected due to its better performance when compared with the standard association rule (Xue et al. (2001; Dunham, 2002).

\section{Related Work}

\subsection{The Challenge}

The challenge to discover valuable knowledge from chaotic WWW has become a challenging task to research community. Explosive growth in size and usage of the World Wide Web has made it necessary for Web site administrators to track and analyze the navigation patterns of Web site visitors. The scale of the web data exceeds any conventional databases, and therefore there is a need to analyze the data available on the web. There are also needs from the users of the web and business built around the web to benefit more 
from the web data. For example many users still complain from the poor performance of the websites and the difficulty to obtain their goal in the current websites because of the poor site structure or mismatches between site design and user needs (Pramudiono, 2004). However, data mining techniques are not easily applicable to Web data due to problems both related with the technology underlying the Web and the lack of standards in the design and implementation of Web pages.

Research in web mining is at cross road of several research communities such as database, information retrieval, and within artificial intelligence (AI), especially in sub areas of machine learning, natural language processing (Kosala and Blockeel, 2000) and in business and e-commerce domain areas (Mobasher et al., 1996). Web mining can be broadly defined as the discovery and analysis of useful information from the WWW (Mobasher et al., 1996). In general, web mining can be classified into web structure mining, web content mining and web usage mining. This study focuses on web usage mining, which analyzes the history of user behavior in the form of access patterns recorded in web access logs of web server. Organizations often generate and collect large volume of data in their daily operations. Most of this information is usually greeted automatically by web servers and collected in server access logs. Other sources of user information include referrer logs which contains information about the referring pages for each page reference, and user registration or survey data gathered via tools such as CGI scripts.

\subsection{Web Usage Mining}

Web usage mining is a research field that focuses on the development of techniques and tools to study users' web navigation behavior. Understanding the visitors' navigation preferences is an essential step in the study of the quality of an electronic commerce site. In fact, understanding the most likely access patterns of users allows the service provider to customise and adapt the site's interface for the individual user (Perkowitz and Etzioni, 1997), and to improve the site's static structure within the underlying hypertext system, (Rosenfeld and Morville, 1998).

When web users interact with a site, data recording their behavior is stored in web server logs. These log files may contain invaluable information characterizing the users' experience in the site. In addition, since in a medium size site log files amount to several megabytes a day, there is a necessity of techniques and tools to help take advantage of their content.

Currently, several commercial log analysis tools are available (Stout, 1997). However, these tools have limited analysis capabilities producing only results such as summary statistics and frequency counts of page visits. Statistical analysis is the most common method to extract knowledge about visitors to a Web site (Srivastava, 2000). By analyzing the session file, one can perform different kinds of descriptive statistical analyses (frequency, mean, median, etc.) on variables such as page views, viewing time and length of navigational path while Nakayama et al. (2000) also try to discover the gap between the website designer's expectations and visitor behavior. Nakayama et al. (2000) uses the inter-page conceptual relevance to estimate the former, and the inter-page access co-occurrence to estimate the latter. The approach focused on website design improvement by using multiple regressions to predict hyperlink traversal from page layout features.

In the meantime the research community has been studying techniques to take full advantage of the information available in log files and the more relevant of such techniques are presented. Various techniques have been used in web usage mining such as adaptive 
neural network to visualize the Website usage patterns (Perotti, 2003) and these visual representations support the identification of clusters of Web page that are frequently visited together by users.

\section{Steps in Manipulating Log Files}

This section presents the step involved in the server log process. The section also discusses the preprocessing of the server logs and pattern mining. In addition, the algorithm of generalized association rules is also presented. However, the lack of standardizing the data preprocessing, we adopting methodology for intersite Web Usage Mining as proposed by Tanasa and Trousse (2004).

\subsection{Raw Log Files}

The log files are text files that can range in size from $1 \mathrm{~KB}$ to $100 \mathrm{MB}$, depending on the traffic at a given a website (Novak and Hoffman, 1996). In determining the amount of traffic a site receives during a specified period of time, it is important to understand what exactly; the $\log$ files are counting and tracking. In particular, there is a critical distinction between a hit and access, wherein: -

- A hit is any file from web site that a user downloads. A Hit can be text document, image, movie, or a sound file. If a user downloads a web pages that has 6 images on it, then that user "hit" the web site seven times (6 images +1 text page).

- An access, or sometimes called a page hit, is an entire page download by a user regardless of the number of images, sounds, or movies. If a user downloads a web page that has 6 images on it, then that user just accessed one page of the web site.

In this study, raw log file were collected from Portal Pendidikan Utusan Malaysia or known as Tutor.com. This portal focuses on education and provides more information related to education purposes such as Tutorials, Question Banks, Teaching Guidelines, and etc. For the analysis purposes, data that consists of 82683 records was retrieved from the server and needed to be preprocessed.

The raw log files consists of 19 attributes such as Date, Time, Client IP, AuthUser, ServerName, ServerIP, ServerPort, Request Method, URI-Stem, URI-Query, Protocol Status, Time Taken, Bytes Sent, Bytes Received, Protocol Version, Host, User Agent, Cookies, Referer. One of the main problems encountered when dealing with the log files is the amount of data needs to be preprocessed (Drott, 1998). A sample of a single entry log file is displayed in Figure 4.2

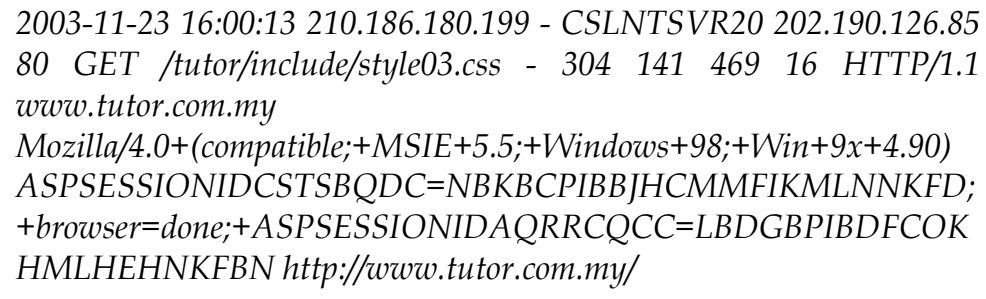

Figure 4.1: Single entry of raw log file 


\subsection{Preprocessing}

From the technical point of view, Web usage mining is the application of data mining techniques to usage logs of large data repositories. The purpose of it is to produce result that can be used to improve and optimize the content of a site (Drott, 1998). In this phase, the starting point and critical point for successful log mining is data preprocessing. The required tasks are data cleaning, user identification and session identification. One of the most popular tools for data preprocessing is Log Data Processor (LODAP) which designed to preprocessing the log data (Castellano, Fanelli, and Torsello, 2007). However, we prefer by writing our own algorithm to perform data cleaning which specifically based on the requirement for generalized association rules. The algorithm can be illustrated below (Mohd Helmy Abd Wahab et. al., 2008)

$1 \quad$ Const ForReading $=1$

2 Const ForWriting $=2$

4 Sub ReadLog( Physical-Path, ModeFile-1, TypeOfLogFile, ModeFile

5 2,StrTypeOfLogFormat)

6

$7 \quad$ RecordCounter $=0$

8 Set LogReader $=$ Server.CreateObject ("IISLog")

16 Retrieve Log Attributes

$17 \quad \ldots . .$.

$18 \quad \ldots .$.

19 RecordCounter $=$ RecordCounter +1

20 LogReader.ReadLogRecord

21 Loop

23 LogReader.CloseLogFile

24 End Sub

An entry of Web server log contains the time stamp of a traversal from a source to a target page, the IP address of the originating host, the type of request (GET and POST) and other data. Many entries that are considered uninteresting for mining were removed from the data files. The filtering is an application dependent. While in most cases accesses to embedded content such as image and scripts are filtered out. However, before applying data mining algorithm, data preprocessing must be performed to convert the raw data into data abstraction necessary for the further processing (see Table 1.). 


\begin{tabular}{|l|l|l|l|l|l|l|}
\hline Trans & ClientIP & Datetime & Method & ServerIP & Port & URI Stem \\
\hline 0 & 202.185 .122 .151 & $\begin{array}{l}11 / 23 / 2003 \\
4: 00: 01 \text { PM }\end{array}$ & GET & 202.190 .126 .85 & 80 & $/$ index.asp \\
\hline 1 & 202.185 .122 .151 & $\begin{array}{l}11 / 23 / 2003 \\
4: 00: 08 ~ P M\end{array}$ & GET & 202.190 .126 .85 & 80 & $/$ index.asp \\
\hline 2 & 210.186 .180 .199 & $\begin{array}{l}11 / 23 / 2003 \\
4: 00: 10 \text { PM }\end{array}$ & GET & 202.190 .126 .85 & 80 & /index.asp \\
\hline 3 & 210.186 .180 .199 & $\begin{array}{l}11 / 23 / 2003 \\
4: 00: 13 \text { PM }\end{array}$ & GET & 202.190 .126 .85 & 80 & $\begin{array}{l}\text { /tutor/include/ } \\
\text { style03.css }\end{array}$ \\
\hline 4 & 210.186 .180 .199 & $\begin{array}{l}11 / 23 / 2003 \\
4: 00: 13 \text { PM }\end{array}$ & GET & 202.190 .126 .85 & 80 & $\begin{array}{l}\text { /tutor/include/ } \\
\text { detectBrowser } \\
\text { cookie.js }\end{array}$ \\
\hline
\end{tabular}

Table 1. Preprocessed Log File

Table 1 exhibits the sample of preprocessed log file. All attributes after preprocessing cannot be shown in the table above due to the space restriction. After preprocessing completed, the pattern mining was performed to mine the access pattern.

\subsection{Generalized Association Rules}

In this phase, Generalized Association Rules is used to mine the data in order to obtain the support and confidence for each rule. Generalized association rule is one of the commonly used web usage mining technique. Concept hierarchy is used to illustrate the relationship between options provided by Tutor.com. Concept hierarchy shows the set of relationship between different items, generalized association rules allow rules at different levels (Dunham, 2002). For the exploratory purpose, this study uses the generalized association rules in mining patterns from server logs of Tutor.com due to the hierarchical structure of the options provided. From the log file, a structure of the web site is shown in Figure 2.

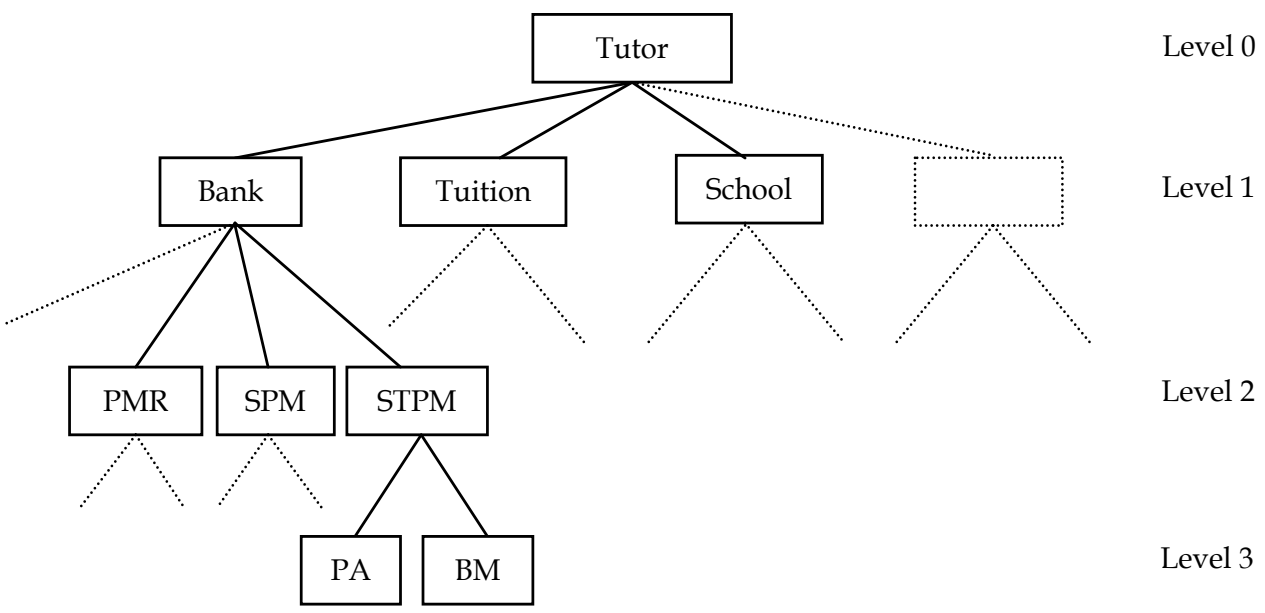

Fig. 2. Hierarchy structure for Portal Pendidikan Utusan Malaysia

Figure 2 depicts the hierarchy structure for Portal Pendidikan Utusan Malaysia. The structure was captured from the Server logs. This study only expands the structure until 
level 3, specifically focusing on Question Banks and Tuition which have been selected for applying Generalized Association Rules. Since the Tuition modules are updated daily, it is not possible to apply the generalized association rules because these modules are based on URI query and access to data is from separate database. Due to this problem, only Question Banks were selected for mining the patterns using Generalized Association Rules. Small simulator program was written using Active Server Pages in order to mine the rules and to calculate the support and confidence.

Generalized association rules were applied to mine the useful patterns using support and confidence counting. From the server logs, hierarchy of the websites is determined. To perform this task, generalized association rules is applied until level 3. Comparing with the standard association rules, generalized association rules allow rules at different levels (Dunham, 2002). Generalized association rules were also used to tackle the data diversity problems (Xue et al. 2002).

To perform generalized association rules, server logs must be cleaned or filtered. Many entries are considered uninteresting for mining were removed. In the entries such as images (e.g. * ${ }^{*}$ if, $\left.{ }^{*} . j p g\right)$ and scripts $\left({ }^{*}\right.$. .css, $\left.{ }^{*} . j s\right)$ are removed.

An algorithm for cleaning the entries of server logs is presented below:

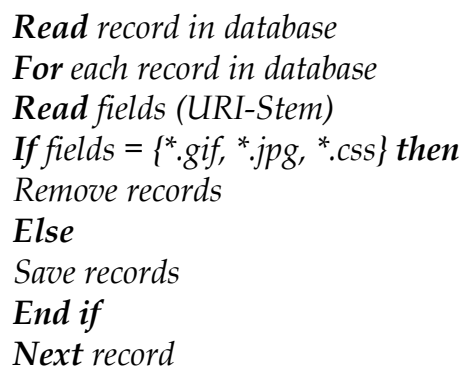

Fig. 3. Algorithm for data cleaning

To generate the rules, the simulator program is executed and the page will prompt the server logs (see Figure 4)

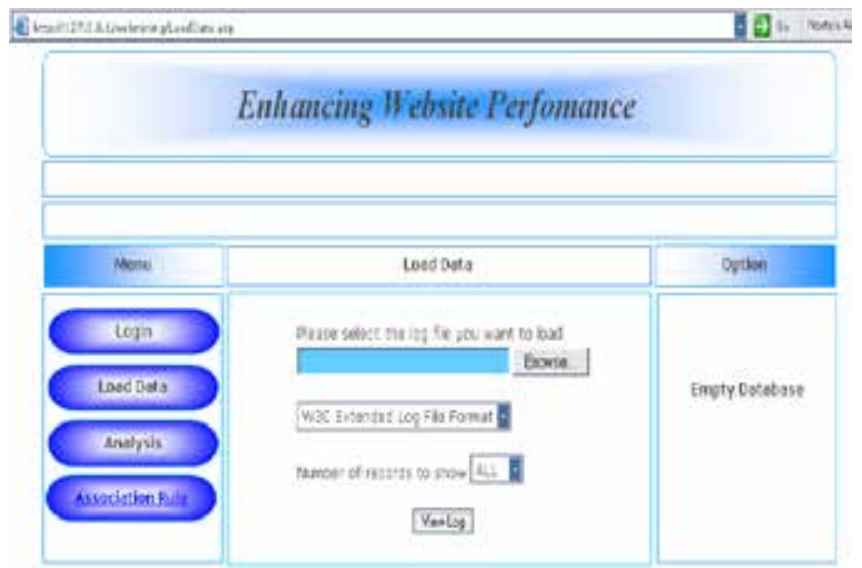

Fig. 4. Starter Prompt for Log File Simulator 
Figure 4 illustrates the interface for input the log file, in this simulator; log file will be prompted before the analysis begun. This simulator performs the generalized association rules only. After loading the files generalized association rules can be executed. Rule produced from this simulator is shown in Figure 5.

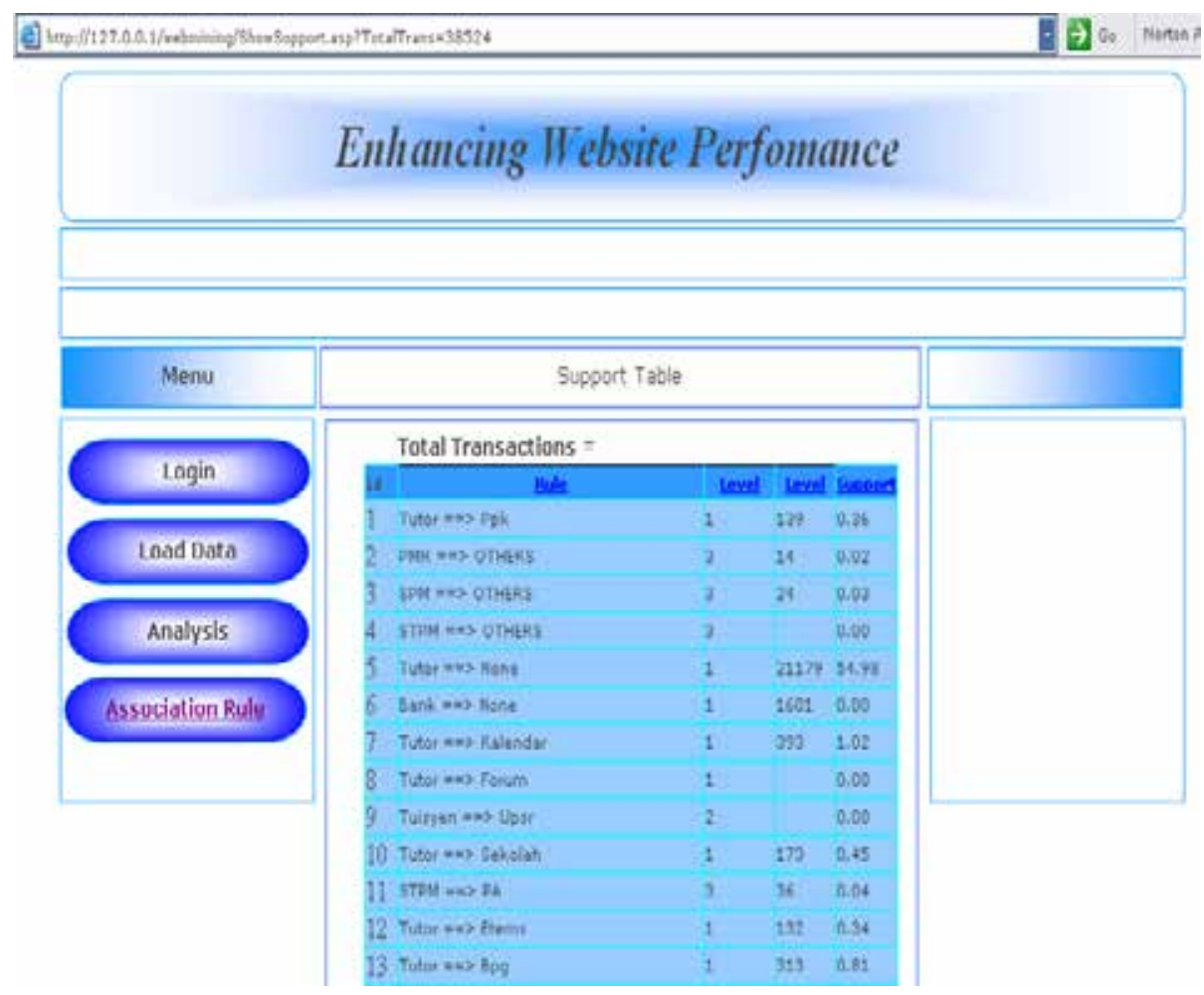

Fig. 5. Rule produced by the simulator

Figure 5 illustrates the rule produced by the simulator. In this study, the total transaction before cleaning is 82683 records and after cleaning total transaction becomes 38524 .

\subsubsection{Counting Occurrence Algorithm}

Before support and confidence for each rules is determined, number of occurrence for each rules must be calculated. An algorithm for counting the number of occurrence is shown below:

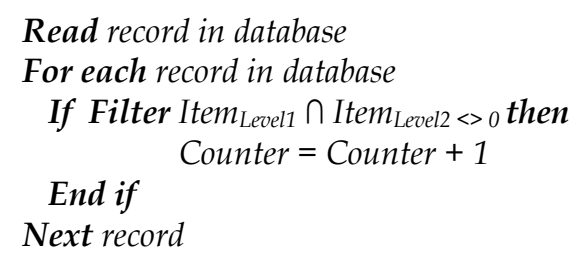

Fig. 6. Counting Occurrences Algorithm 
Figure 6 illustrates an algorithm for calculating the number of occurrence based on the rules for each record and level. The implementation of this algorithm is as follows (see Figure 7).

From Level 1:

Identify child from parent

For example: Tutor $\rightarrow$ QuestionBank

Tutor (parent node)

QuestionBank (child node in level 1)

Combine parent and node using AND operator then fit in Filter Function

Fig. 7. Implementation of counting occurrences

\subsubsection{Algorithm for Support}

Support measures how often the rules occur in database. To determine the support for each rules produced, several arguments have been identified in calculating the support such as Total Transaction in database and number of occurrences for each rules. The formula for support is shown in Figure 8.

Input :-

Support $=$
Total Transaction in DB

No. of occurrences each item $\{x, y\}$

Number of occurrences $\{x, y\}$

Total Transaction in DB

Fig. 8. Support calculation formula

Based on the Figure 8 below, an example of calculation of support from the mined rules is shown below (see Figure 9).

$$
\begin{aligned}
& \text { Total Transaction in Database }=38524 \text { (After cleaning process) } \\
& \text { Total occurrence for Tutor } \rightarrow \text { Estidotmy }=310 \\
& \text { Support }(\text { Tutor } \rightarrow \text { Estidotmy })=(310 / 38524) * 100 \\
& \text { Support }(\text { Tutor } \rightarrow \text { Estidotmy })=0.80
\end{aligned}
$$

Fig. 9. Implementation of Support Counting

Based on the Figure 9, the support for rules Tutor $\rightarrow$ Estidotmy has a value 0.80 percent. This means the probability the Tutor $\rightarrow$ Estidotmy occur in database is $0.80 \%$.

\subsubsection{Algorithm for Confidence}

Based on the examples, support measures how often the rules occur in database while confidence measures strength of the rules. Typically, large confidence values and a smaller support are used (Dunham, 2002). Formula for calculating the confidence value is shown in Figure 10. 
Input :-

Total occurrence for item $X$

Total occurrence for item $X$ and $Y$

Confidence $=$

Total occurrence for item $X$ and $Y$

\section{Total occurrence for item $X$}

Fig. 10. Confidence calculation formula

Based on Figure 10, the example of calculation of confidence value from rules produced by simulator (see Figure 11).

Calculating confidence for rules Tutor $\rightarrow$ Estidotmy

Number of occurrence for Tutor $=21179$

Number of occurrence for Tutor $\rightarrow$ Estidotmy $=310$

Confidence $($ Tutor $\rightarrow$ Estidotmy $)=(310 / 21179) * 100$

Confidence $($ Tutor $\rightarrow$ Estidotmy $)=1.46$

Fig. 11. Implementation of Confidence Counting

Since the confidence from the above calculation shows that Confidence > Support, it means the rules for Tutor $\rightarrow$ Estidotmy are interesting rules.

When applying generalized association rules, any unused transaction from the database such as images access (e.g. .gif, .jpg), scripts (e.g. .css, .js) were removed.

\section{Results and Analysis}

After generating the rules from the system, the result will be illustrated using graphical representation in order to perform analysis. In addition, the support and confidences of different levels of server portal accessed will be illustrated using bar charts. The system administrator could make a decision from the result illustrated in order to improve or enhance the content, link, site navigation and facilities.

\subsection{Support and Confidence for Structure Level 1}

The first level of Portal Pendidikan Utusan or Tutor.com consists of eleven elements option as shown in Figure 12. However, the table for support and confidence for Level 1 one can be referred in Table 2.

\begin{tabular}{|l|c|c|c|c|}
\hline Rules & Level & Occurrences & Support & Confidence \\
\hline Tutor --> Kalendar & 1 & 393 & 1.02 & 1.86 \\
\hline Tutor --> Forum & 1 & 0 & 0.00 & 0.00 \\
\hline Tutor --> ContentRM & 1 & 79 & 0.21 & 0.37 \\
\hline Tutor --> Sekolah & 1 & 173 & 0.45 & 0.82 \\
\hline Tutor --> Ppk & 1 & 139 & 0.36 & 0.66 \\
\hline
\end{tabular}




\begin{tabular}{|l|l|c|l|l|}
\hline Tutor -->Etems & 1 & 132 & 0.34 & 0.62 \\
\hline Tutor --> Bank & 1 & 313 & 0.81 & 1.48 \\
\hline Tutor --> Estidotmy & 1 & 310 & 0.80 & 1.46 \\
\hline Tutor --> Motivasi & 1 & 120 & 0.31 & 0.57 \\
\hline Tutor --> Tuisyen & 1 & 124 & 0.32 & 0.59 \\
\hline Tutor --> Games & 1 & 1118 & 2.90 & 5.28 \\
\hline
\end{tabular}

Table 2. Rule Structure level 1

The bar charts shown in Figure 12 reveal that Game option has the highest support, which score $2.90 \%$, and confidence, which score $5.28 \%$. This implies that the most popular option is the entertainment option. The second popular is the Calendar option, which scores $1.02 \%$ for support and $1.86 \%$ for confidence. The result illustrated in Figure 12 also indicates that Tuisyen is one of the least popular options. This might be due to the fact that user's access to Tuisyen option based on users' queries. The query session was stored in a separate database. Therefore this session cannot be accessed through the server logs. Another option that has been emphasized in this study is the question bank. The analysis of this option is further explained in Figure 13

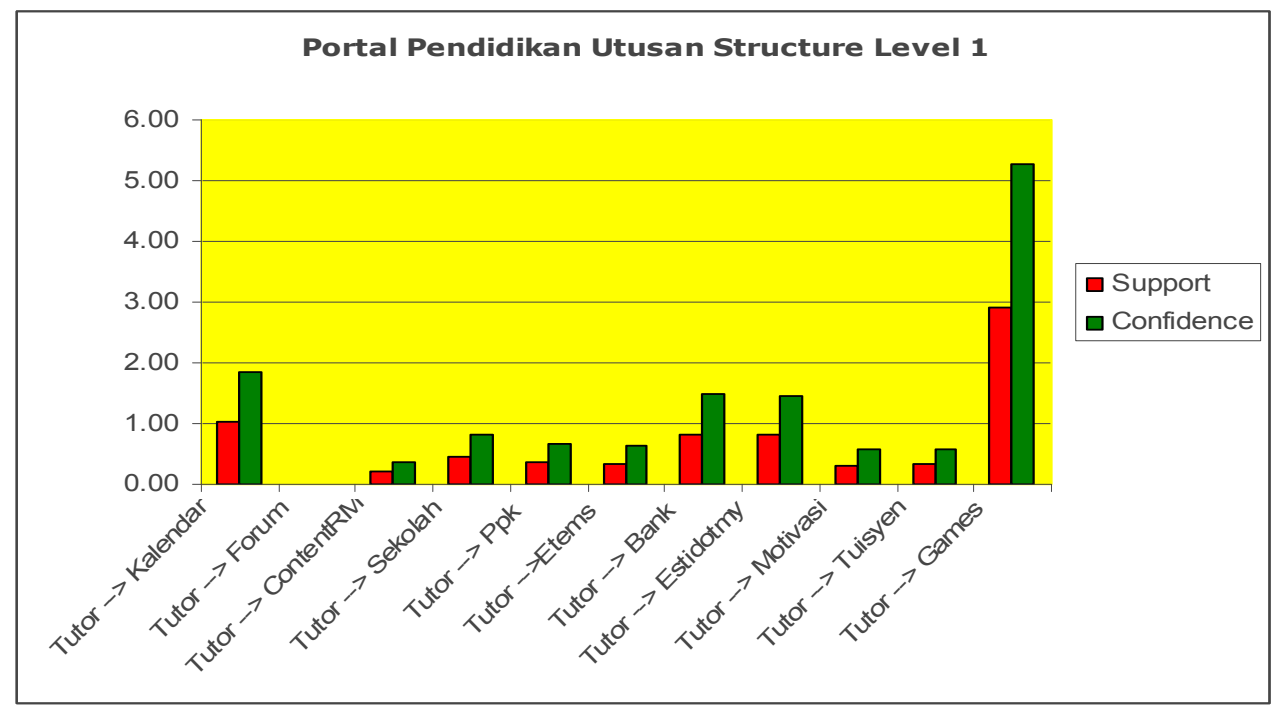

Fig. 12. Support and Confidence for level 1 for Portal Tutor.com

\subsection{Support and Confidence for Structure Level 2}

Fig 13 shows the graph in the Question Banks. It is interesting to note that SPM question bank scores the highest confidence, which score $48.34 \%$ compared to other examination in question banks. The least confidence is shown by STPM question bank, which score $1.31 \%$. The graph in Figure 13 also indicates that UPSR question bank is also a popular option. Since the server logs were processed, this implies that both SPM and UPSR question bank are still popular even after the UPSR and SPM examinations. Table contains the support and confidence can be seen on Table 3 . 


\begin{tabular}{|l|c|c|c|c|}
\hline Rules & Level & Occurrences & Support & Confidence \\
\hline Bank --> Upsr & 2 & 537 & 1.39 & 33.54 \\
\hline Bank --> Pmr & 2 & 194 & 0.50 & 12.12 \\
\hline Bank --> Spm & 2 & 774 & 2.01 & 48.34 \\
\hline Bank --> Stpm & 2 & 21 & 0.05 & 1.31 \\
\hline
\end{tabular}

Table 3. Rule Structure level 2

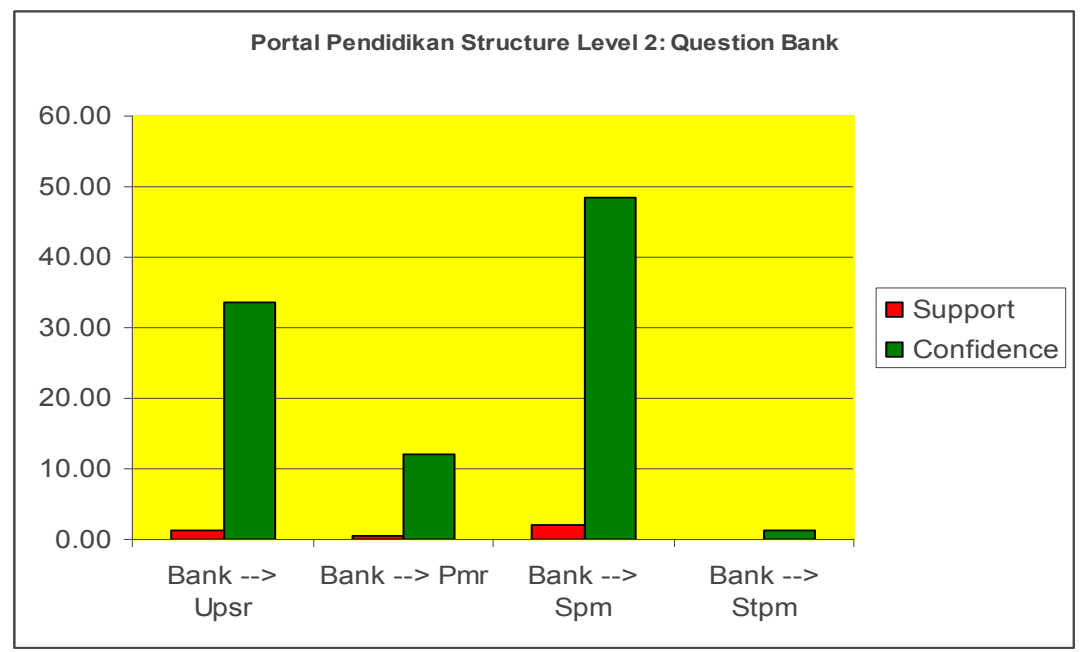

Fig. 13. Support and Confidence for Question Banks

\subsection{Support and Confidence for Structure Level 3}

Structure of level 3 contains the support and confidence can be seen on Table 4 .

\begin{tabular}{|l|l|l|l|l|}
\hline Rules & Level & Occurrences & Support & Confidence \\
\hline Upsr --> BM & 3 & 178 & 0.46 & 32.13 \\
\hline Upsr --> BI & 3 & 94 & 0.24 & 16.97 \\
\hline Upsr --> Sains & 3 & 82 & 0.21 & 14.80 \\
\hline Upsr --> Mat & 3 & 159 & 0.41 & 28.70 \\
\hline
\end{tabular}

\begin{tabular}{|l|l|l|l|l|}
\hline Rules & Level & Occurrences & Support & Confidence \\
\hline Pmr --> BM & 3 & 58 & 0.15 & 25.66 \\
\hline Pmr --> BI & 3 & 25 & 0.06 & 11.06 \\
\hline Pmr --> Mat & 3 & 83 & 0.22 & 36.73 \\
\hline Pmr --> Sains & 3 & 45 & 0.12 & 19.91 \\
\hline Pmr --> Sej & 3 & 0 & 0.00 & 0.00 \\
\hline Pmr --> Geo & 3 & 1 & 0.00 & 0.44 \\
\hline Pmr --> Others & 3 & 14 & 0.04 & 6.19 \\
& & & & \\
\hline
\end{tabular}




\begin{tabular}{|l|l|l|l|l|}
\hline Rules & Level & Occurrences & Support & Confidence \\
\hline Spm --> BM & 3 & 62 & 0.16 & 7.69 \\
\hline Spm --> BI & 3 & 46 & 0.12 & 5.71 \\
\hline Spm --> Mat & 3 & 134 & 0.35 & 16.63 \\
\hline Spm --> ADM & 3 & 157 & 0.41 & 19.48 \\
\hline Spm --> Sains & 3 & 64 & 0.17 & 7.94 \\
\hline Spm --> Sej & 3 & 26 & 0.07 & 3.23 \\
\hline Spm --> Geo & 3 & 0 & 0.00 & 0.00 \\
\hline Spm --> Fizik & 3 & 84 & 0.22 & 10.42 \\
\hline Spm --> Bio & 3 & 64 & 0.17 & 7.94 \\
\hline Spm --> Kim & 3 & 138 & 0.36 & 17.12 \\
\hline Spm --> Others & 3 & 24 & 0.06 & 2.98 \\
\hline
\end{tabular}

\begin{tabular}{|l|l|l|l|l|}
\hline Rules & Level & Occurrences & Support & Confidence \\
\hline Stpm --> BM & 3 & 3 & 0.01 & 1.95 \\
\hline Stpm --> PA & 3 & 27 & 0.07 & 17.53 \\
\hline Stpm --> Ekon & 3 & 0 & 0.00 & 0.00 \\
\hline Stpm --> Others & 3 & 0 & 0.00 & 0.00 \\
\hline
\end{tabular}

Table 4. Rule Structure level 3

For UPSR's access as illustrated in Figure 14, Bahasa Melayu (32.13\%) subject is more popular followed by Mathematics $(28.70 \%)$ and Science $(14.80 \%)$. One possible explanation for Bahasa Melayu preferences may be due the fact that there are two Bahasa Melayu's papers in UPSR examination.

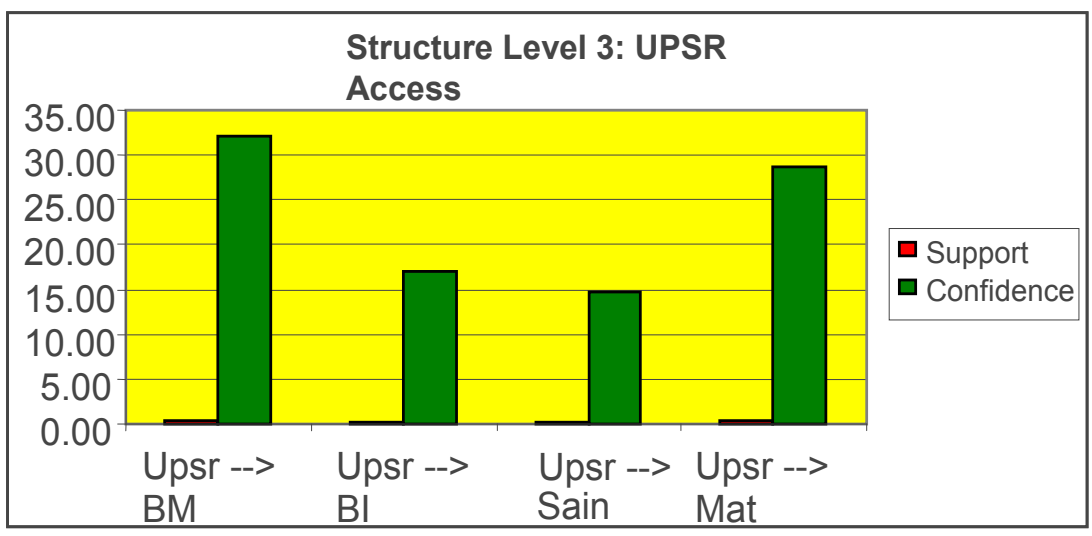

Fig. 14. Support and Confidence for UPSR

The PMR access results shown in Figure 15 also indicate that Mathematics (36.73\%) is the most popular subject among PMR's examination subjects. The next popular subject at PMR level is a Bahasa Melayu (25.66\%) subject. Comparing Figure 14 and Figure 15, the results exhibit that Mathematics and Bahasa Melayu subjects are more popular than other subjects. 


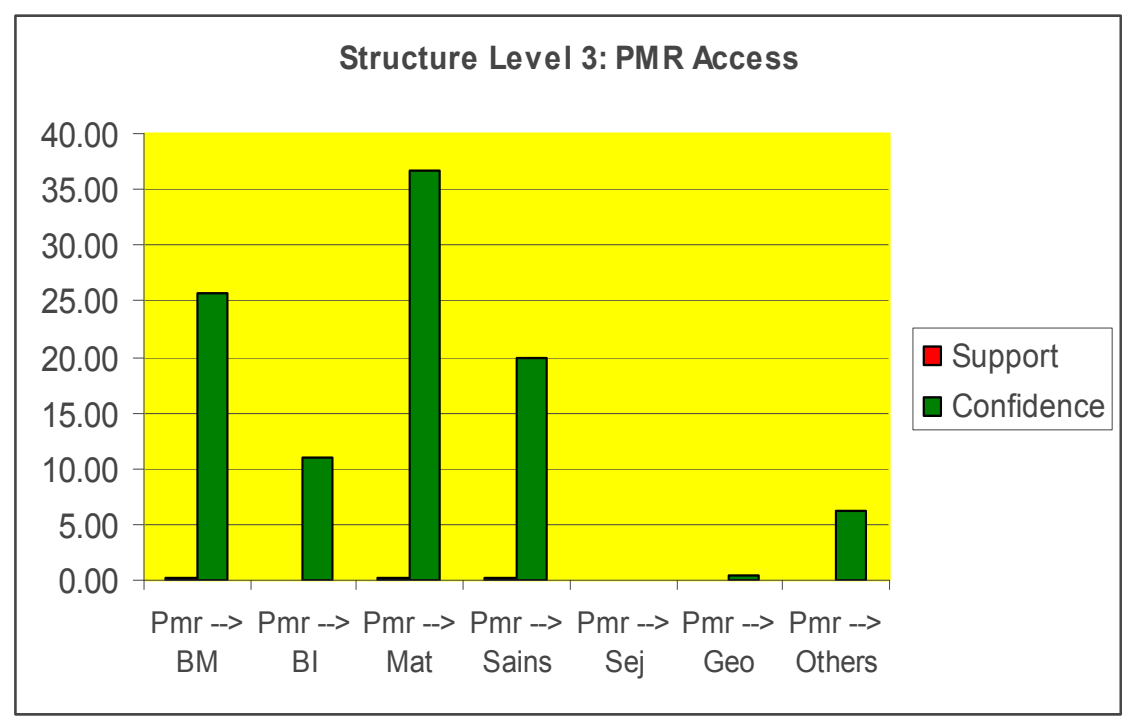

Fig. 15. Support and Confidence for PMR

To identify which SPM subject are more popular to users, further association rules mining was performed on SPM access (see Figure 16). The results indicate that Additional Mathematics question bank has been accessed more frequent which score $19.48 \%$ compared with other subjects. The next popular subject is Chemistry, followed by Mathematic. The least popular subject is History. Hence, the findings in Figure 16 show that science stream subject question bank at Tutor.com has been visited more frequently than arts stream subjects.

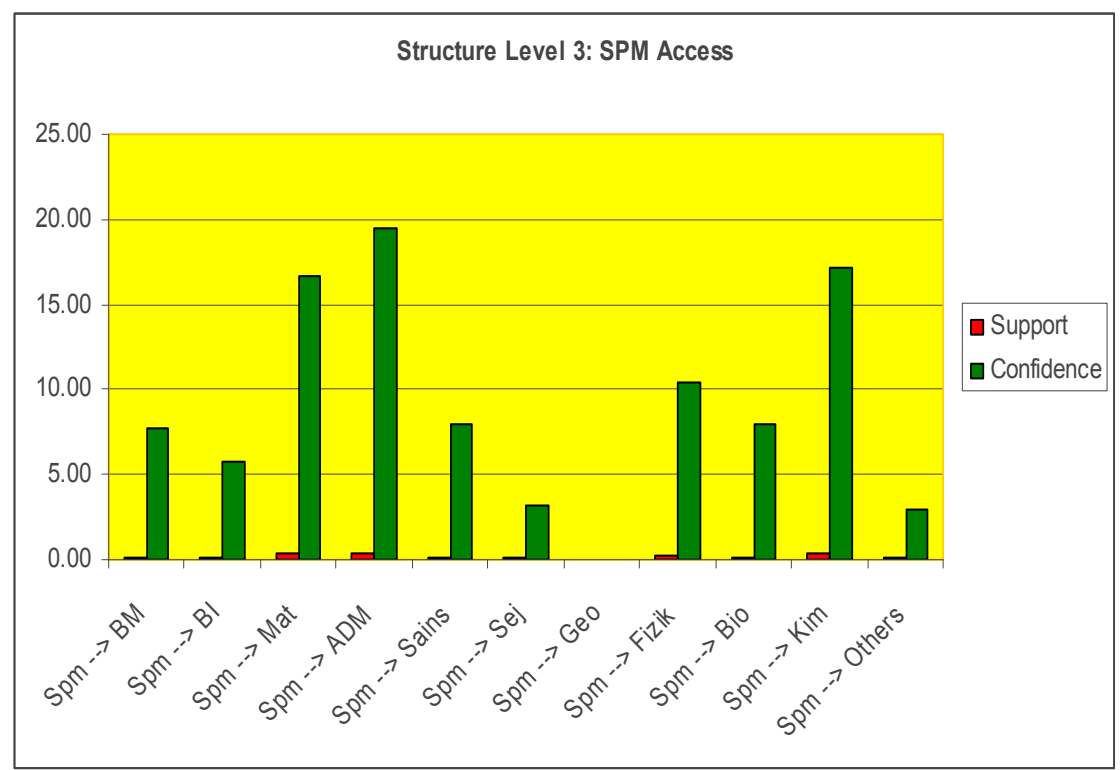

Fig. 16. Support and Confidence for SPM 
Results exhibited in Figure 17 indicate that STPM question bank was the least popular. Further analysis on STPM's access reveals that only one subject that is General Paper (PA) question bank was referred to by Tutor.com's users and the least subject referred is Bahasa Melayu subject.

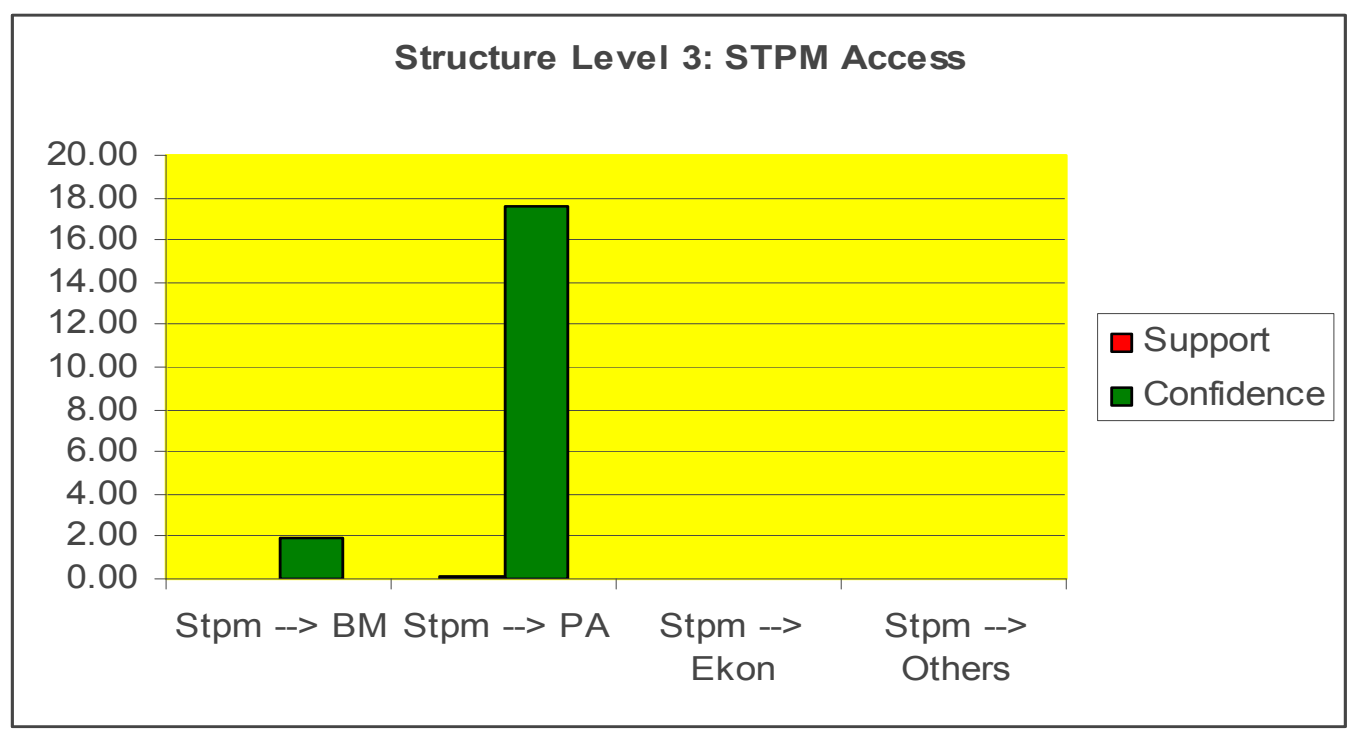

Fig. 17. Support and Confidence for STPM

\section{Conclusion}

Web Usage Mining is an aspect of data mining that has received a lot of attention in recent year (Kerkhofs et al., 2001). Commercial companies as well as academic researchers have developed an extensive array of tools that perform several data mining algorithms on log files coming from web servers in order to identify user behaviour on a particular web site. Performing this kind of investigation on the web site can provide information that can be used to better accommodate the user's needs.

Web usage mining has been applied to several applications such as business and finance (Lee and Liu, 2001), E-commerce (Srivastava, 2000), information retrieval (Pal, 2002) and Academic and Industry (Srivastava et al., 2000). In this study, generalized association rules have been applied to web server log from Tutor.com.

It is important to mention that the most efforts have relied on relatively simple techniques which can be inadequate for real user profile data since noise in the data has to be firstly tackled. Thus, there is a need for robust methods that integrates different intelligent techniques that are free of any assumptions about the noise contamination rate. 


\section{References}

Agrawal, R. and Srikant, R. (1994). Fast algorithms for mining association rules. Proc. of the 20th VLDB Conference. pp 487 - 499.

Borgelt, C. and Kruse, R. (2002). Induction of Association Rules: Apriori Implementation. $15^{\text {th }}$ Conference on Computational Statistics (CompStat 2002)

Castellano, G., Fanelli, A. M., and Torsello, M. A. (2007). Log Data Preparation for Mining Web Usage Patterns. In Proceeding of IADIS International Conference on Applied Computing 2007. Pp. 371 - 378.

Chakrabarti, S., Dom, B., Gibson, D., Klienberg, J., Kumar, S., Raghavan, P., Rajagopalan, S., and Tomkins, A. (1999). Mining the Link Structure of The World Wide Web. IEEE Computer. Vol. 32. No. 8. pp. 60 - 67.

Desikan, P., Srivastava, J., Kumar, V., Tan, P. N. (2002). Hyperlink Analysis - Techniques \& Applications. Army High Performance Computing Center Technical Report.

Drott, M. C. (1998). Using Web Server Logs to Improve Site Design. Association for Computing Machinery (ACM) Proceeding of the Sixteenth Annual International Conference on Computer Documentation. pp. 43 - 50.

Dunham, M. H. (2002). Data Mining: Introductory and Advanced Analysis. New Jersey: Prenhall.

Fayyad, U. M., Piatetsky-Shapiro, G., Smyth, P., and Uthurusamy, R. (1996). Advances in Knowledge Discovery and Data Mining. AAAI Press/ The MIT Press.

Kerkhofs, J., Vanhoof, K., and Pannemas, D. (2001). Web Usage Mining on Proxy Server: A Case Study. Technical Report. Limburg University Centre.

Kosala, R. and Blockeel, H. (2000). Web Mining Research: A Survey. ACM SIGKDD. Vol. 2. Issue 1.pp. 1 - 14.

Lee, R. S. T. and Liu, J. N. K. (2001). iJADE eMiner: A Web-Based Mining Agent Based on Intelligent Java Agent Development Environment (iJADE) on Internet Shopping. PAKDD 2001. LNAI 2035. pp. $28-41$.

Madria, S., Bhowmick, S. S., Ng, W. K., and Lim, E. P. (1999). Research Issue in Web Data Mining. Data Warehousing and Knowledge Discovery.

Mobasher, B., Jain, E., Han, E., and Srivastava, J. (1996). Web mining: Pattern discovery from World Wide Web Transactions. Technical Report TR 96-050.

Moh, C-H., Lim, E-P., Ng, W. K. (2000). DTD-Miner: A Tool for Mining DTD from XML Documents. WECWIS 2000. pp. $144-151$.

Mohammadian, M. (2001). Intelligent Data Mining and Information Retrieval from World Wide Web for E-Business Applications. http://www.ssgrr.it/en/ssgrr2002w/papers/230.pdf

Mohd Helmy Abd Wahab, Mohd Norzali Haji Mohd, Hafizul Fahri Hanafi, and Mohamad Farhan Mohamad Mohsin. (2008). Data Preprocessing for Web Server Logs for Generalized Associations Rule Algorithm. In Proceeding of World Academy of Science, Engineering and Technology. Vol. 36. pp. 970 - 978.

Nakayama, T., Kato, H., and Yamane, Y. (2000). Discovering the Gaps Between Web Site Designers' Expectations and Users' Behaviour. Proc. Of the Ninth Int'l World Wide Web Conference. 
Novak and Hoffman. (1996). New Metrics for New Media: Toward the Development of Web Measurement Standards

http://www2000.ogsm.vanderbilt.edu/novak/web.standards/webstand.html [Date Accessed: 28 February 2009].

Pramudiono, I. (2004). Parallel Platform for Large Scale Web Usage Mining. Phd Thesis. Department of Computer Science, University of Tokyo.

Pei, J., Han, J., Asl, B. M., and Zhu, H. (2000). Mining Access Patterns Efficiently from Web Logs.

Perkowitz, M. and Etzioni, O. (1998). Adaptive sites: Automatically Synthesizing WebPages. Proceedings of the fifteenth National Conference on Artificial Intelligence. pp. 727 - 732.

Perotti, V. (2003). Techniques for Visualizing Website Usage Patterns With an Adaptive Neural Network. The ACM Digital Library. Pp $35-40$.

Rosenfeld, L. and Morville, P. (1998). Information Architecture for the World Wide Web. $\mathrm{O}^{\prime}$ Reilly, Cambridge.

Srikant, R., Vu, Q., and Agrawal, R. (1997). Mining Association Rules with Item Constraints. American Association of Artificial Intelligence (AAAI).

Srivastava, J., Desikan, P., and Kumar, V. (2002). Web Mining: Accomplishments and Future Directions.

Srivastava, J., Cooley, R., Tan, P. -N. (2000). Web Usage Mining: Discovery and Applications of Usage Patterns from Web Data. SIGKDD Explorations. Vol. 1. No. 2. pp. 12 - 33.

Stout, R. (1997). Web Site Stats: tracking hits and analyzing traffic. Osborne McGraw-Hill: Berkeley.

Tao, F., Murtagh, F., and Farid, M. (2003). Weighted Association Rule Mining using Weighted Support and Significant Framework. SIGKDD 2003.

Tanasa, D. and Trousse, B. (2004). Advanced Data Preprocessing for Intersites Web Usage Mining. IEEE Intelligent System, pp. 59 - 65.

Wang and Liu, H. (1998). Discovering Typical Structures of Documents: A Roadmap Approach. Proceeding of the ACM SIGIR Symposium on Information Retrieval.

Xue, G. R., Zeng, H. J., Chen, Z., Ma, W. Y., and Lu, C. J. (2002). Log Mining to Improve the performance of Site Search. Third Int. Conf. of WISEw '02.

Yang, Q. (2002). Building Association Rule-Based Sequential Classifiers for Web Document Prediction. Journal of Data Mining and Knowledge Discovery. 


\title{
Fuzzy modeling by Active Learning Method
}

\author{
Hamid Taheri Shahraiyni \\ Civil Engineering Department, Faculty of Engineering, Tarbiat Modares University \\ Tehran, Iran
}

\section{Introduction}

The constructions of different modeling methods are similar. The models are consisted of the following major stages:

1- Recognizing the true or most effective inputs.

2- Finding the numerical relationship between inputs and output.

3- Explaining the numerical relationship mathematically.

4- Utilizing the mathematical expressions to calculate the output using different inputs.

5- Comparing the calculated and actual outputs and calculating the error.

6- Modifying the mathematical expressions based on the calculated error.

These stages seem to be complicated. This complexity seems to be due to the quantitative and exact definitions of the mentioned stages (Bagheri Shouraki and Honda, 1998). There are some demonstrations that the mentioned stages are performed qualitative with nonexact concepts in the human brain (Schmidt, 1985), therefore any effort toward of expressing them using exact expressions (such as mathematics) are expected to have some differences with human thinking or modeling method. In the other words, the utilizing of exact mathematics in modeling has contradiction with human abilities (Bagheri Shouraki and Honda, 1999).

Fuzzy concepts (e.g. Zadeh 1965) and related inferences (e.g. Mamdani 1974) proposed a new approach to human modeling and calculation methods. Although, different powerful fuzzy modeling methods have been developed up to now, but some of these methods are different with real human modeling method, because of utilized mathematics and exact calculations in their constructions (Bagheri Shouraki and Honda, 1999). The construction of human modeling is similar to the above stages, but avoids of mathematical complexities. Active Learning Method (ALM) is one of the fuzzy modeling methods Which uses basic level of mathematics. ALM was innovated by Bagheri Shouraki and Honda (1997). ALM has very simple algorithm that avoids of mathematical complexity and its accuracy and exactness increase unlimitedly by increasing the number of iterations of its algorithm.

It is very difficult for human to memorize the numerical data points but tries to memorize the general behavior function of data points. In addition, for modeling, the human converts a MIMO (Multi Inputs - Multi Outputs) system to some SISO (Single Input - Single Output) systems and then human tries to find the general behavior function in each SISO system and the effects of other inputs are considered as the deviation of data points around of the general behavior function. In addition, human can save the data points on a continuous path 
which means the general behavior function, but usually can not save the randomly distributed data points in the space of variable. ALM algorithm uses all of these mentioned constructions of human modeling method.

Taheri Shahraiyni (2007) developed new heuristic search, fuzzification and defuzzification methods for ALM algorithm. In the next sections of this chapter, ALM algorithm with these modifications is explained and the ALM abilities and applications are illustrated.

\section{ALM algorithm}

The ALM algorithm has been presented in figure 1.

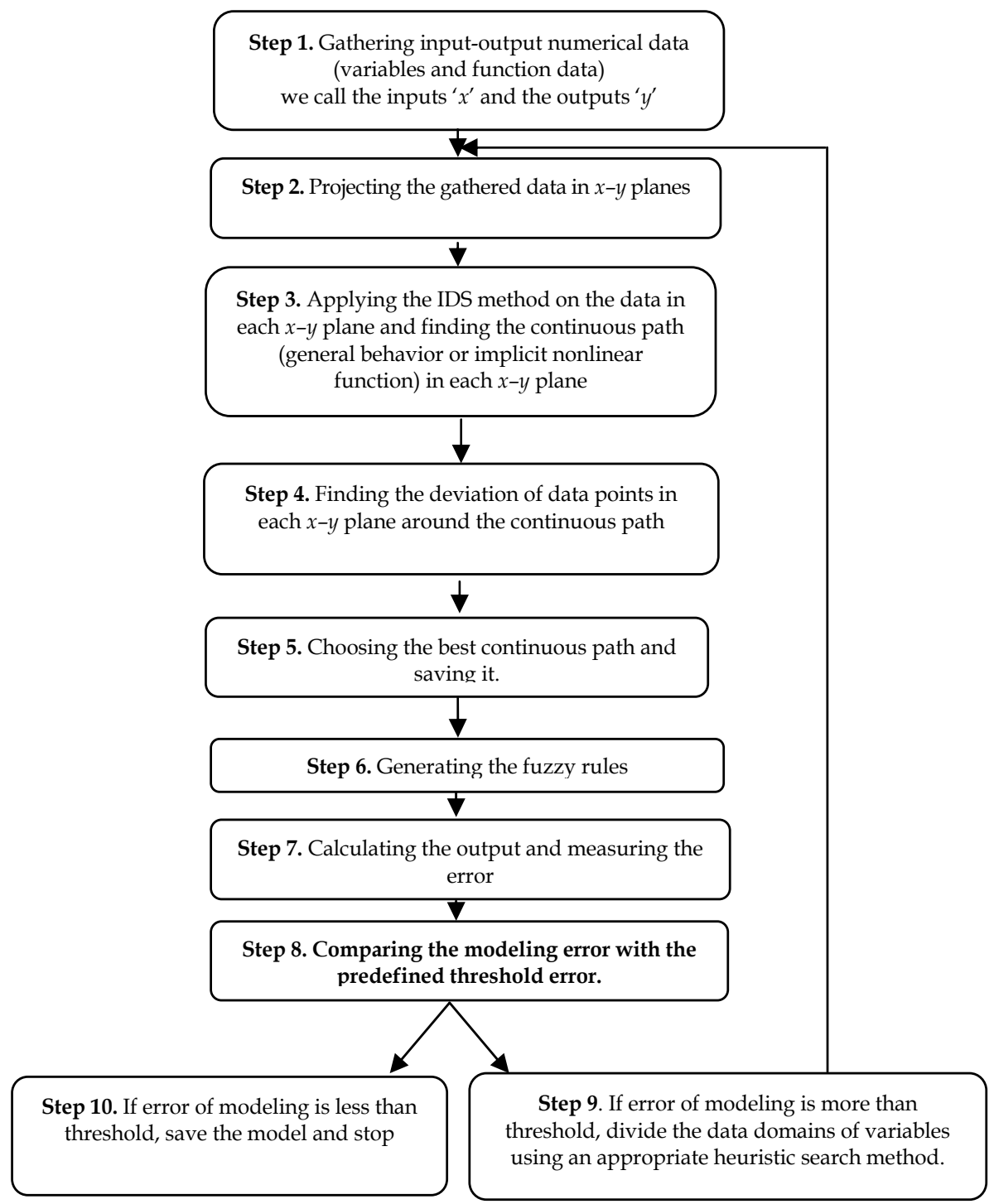

Fig. 1. Proposed algorithm for Active Learning Method. 
For the purpose of explaining the ALM algorithm, the Sugeno and Yasukawa (1993) dummy non-linear static problem (equation (1)) with two input variables $\left(x_{1}\right.$ and $\left.x_{2}\right)$ and one output $(y)$ is solved by this method.

$$
y=\left(1+x_{1}^{-2}+x_{2}^{-1.5}\right)^{2}, \quad 1 \leq x_{1}, x_{2} \leq 5
$$

First, some data are extracted from equation 1 and some random noises are added to data (step 1). Then the data are projected on $x-y$ plane (figures $2 \mathrm{a}$ and $2 \mathrm{~b}$ ) (step 2).

Step 3: The heart of calculation in ALM is a fuzzy interpolation and curve fitting method which is entitled IDS (Ink Drop Spread). The IDS searches fuzzily for continuous possible paths on data planes. Assume that each data point on each $x-y$ plane is a light source with a cone or pyramid shape illumination pattern. Therefore, with increase of distance of each data point, the intensity of light source decreases and goes toward zero. Also the illuminated patterns of different data points on each $x-y$ plane are combined and new bright areas are formed. The IDS is exerted to each data point (pixel) on the normalized and discretized $x-y$ planes. The radius of the base of cone or pyramid shape illumination pattern in each $x-y$ plane is related to the positions of data in it. The radius increases until the all of the domain of variable in $x-y$ plane be illuminated. Figures $2 \mathrm{c}$ and $2 \mathrm{~d}$ show the created illumination pattern (IL values) after the combination of the illumination patterns of different points in $x_{1}-y$ and $x_{2}-y$ planes, respectively. Here, pyramid shape illumination pattern has been used.

Now, the paths, general behaviour, or implicit nonlinear functions are determined by applying the center of gravity on $y$ direction. The center of gravity is calculated using this equation: $y\left(x_{i}\right)=\frac{\sum_{j=1}^{M}\left[y_{j} \times I L\left(x_{i}, y_{j}\right)\right]}{\sum_{j=1}^{M} I L\left(x_{i}, y_{j}\right)}$, Where $j: 1 \ldots M, M$ is the resolution of $y$ domain, $y_{j}$ is the output value in $j$ th position, $\operatorname{IL}\left(x_{i}, y_{j}\right)$ is the illumination value on $x-y$ plane at the $\left(x_{i}, y_{j}\right)$ point or pixel and $y\left(x_{i}\right)$ is the corresponding function (path) value to $x_{i}$.

Hence, by applying the centre of gravity method on figures $2 \mathrm{c}$ and $2 \mathrm{~d}$, continuous paths are extracted (figures 2e and 2f).

Subsequently, the deviation of data points around each continuous path can be calculated by various methods such as coefficient of determination $\left(R^{2}\right)$, Root Mean Square Error (RMSE) or Percent of Absolute Error (PAE). The PAE values of continuous paths on $x_{1}-y$ and $x_{2}-y$ planes (figures 2e and 2f) are 20.4 and $13.5 \%$, respectively (Step 4).

The results show that the path of figure $2 \mathrm{f}$ is better than the path of figure2e. The selected paths should be saved because these are implicit non-linear functions. The paths can be saved as a look-up table, heteroassociative neural network memory (Fausset, 1994) or fuzzy curve expressions such as Takagi and Sugeno method (TSM) (Takagi and Sugeno, 1985). Look up tables are most convenient method and it is used for path saving in this example (Step 5).

We have no rules in the first iteration of ALM algorithm, hence we go to step 7. 


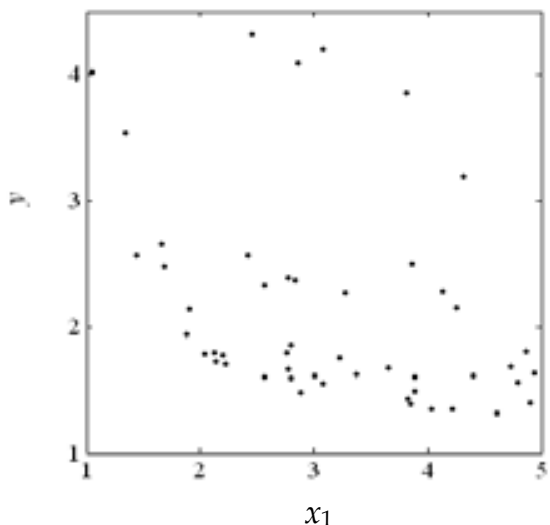

(a)

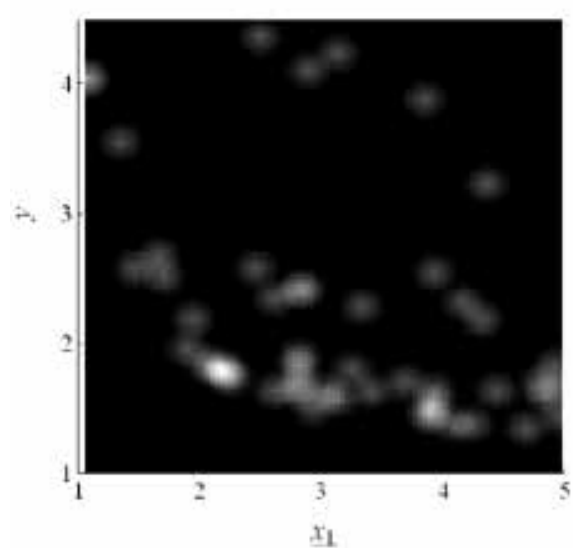

(c)

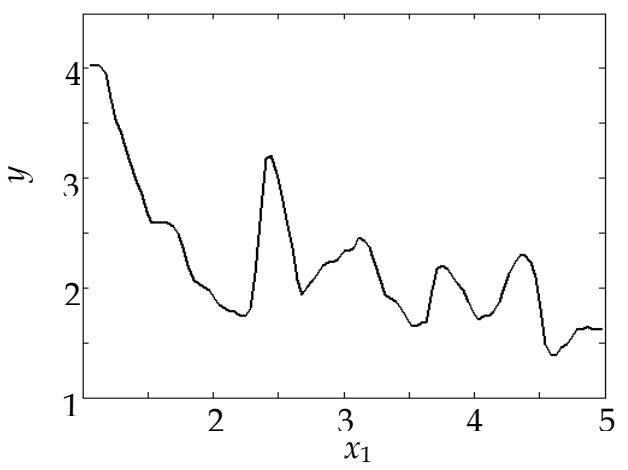

(e)

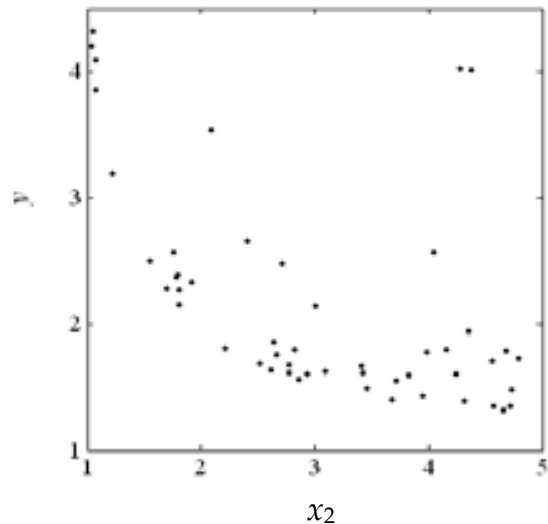

(b)

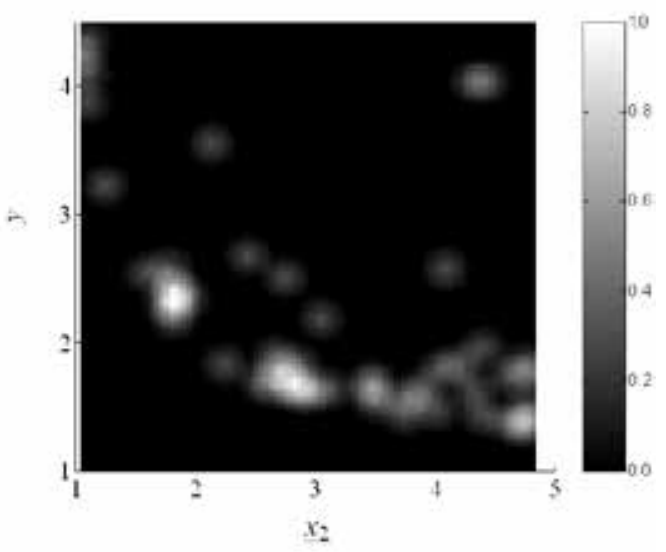

(d)

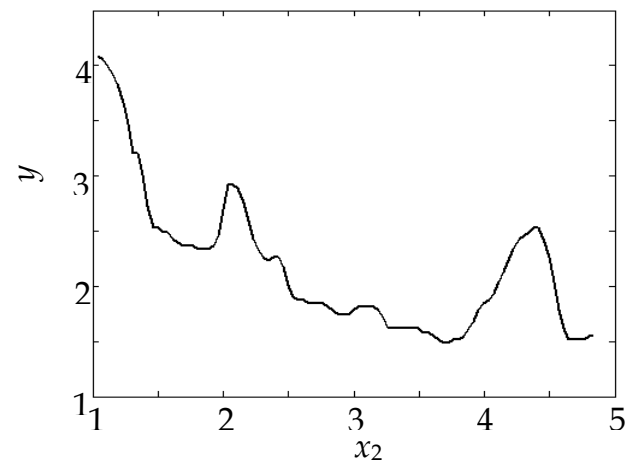

(f)

Fig. 2. (a) Projected data on $x_{1}-y$ plane, (b) projected data on $x_{2}-y$ plane; (c) Results of applying IDS method on the data points in $x_{1}-y$ plane, $(d)$ results of applying IDS on the data points in $x_{2}-y$ plane; (e) Extracted continuous path by applying center of gravity method on figure $2 c,(f)$ extracted continuous path by applying center of gravity method on figure $2 d$. 
The PAE of chosen path is more than a predefined threshold PAE value $(5 \%)$. Hence, the error is more than predefined error (Steps $7 \& 8$ ) and we divide each space in two by using only one variable (Step 9) and go to the step 2 of figure 1. Dividing can be performed crisply or fuzzily, but for simplicity, a crisp dividing method is used here and the fuzzy dividing will be illustrated later. The results of ALM modeling after crisp division of space to four subspaces using a heuristic search method has been presented in figure 3 . According to figure 3, the following rules are generated (Step6):

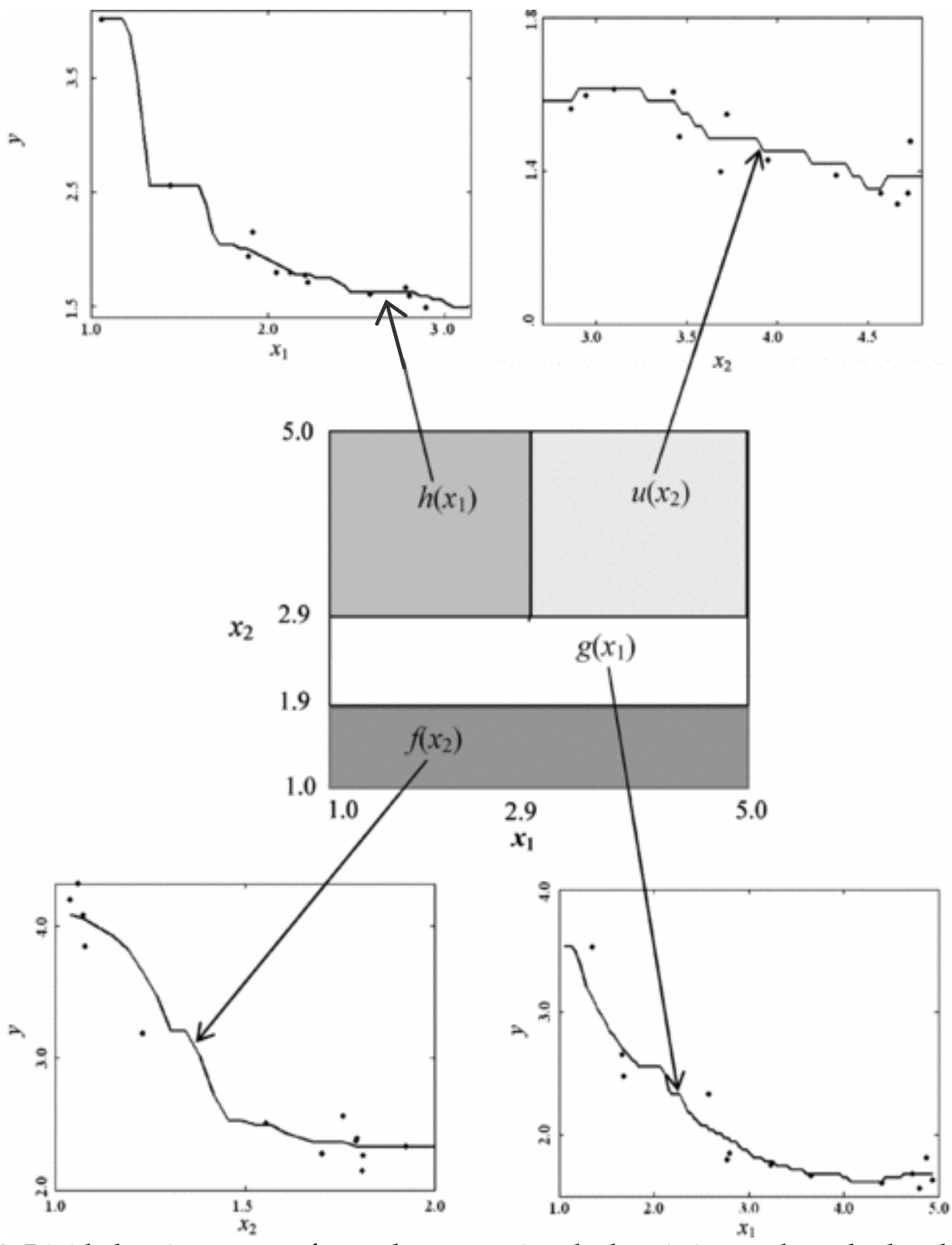

Fig. 3. Divided entire space to four subspaces using the heuristic search method and the best continuous path (implicit non-linear function), extracted for each subspace (the data points in each subspace have been shown by black circles). 
If $\left(x_{2} \geq 1.0 \& x_{2}<1.9\right)$ then $y=f\left(x_{2}\right)$

If $\left(x_{2} \geq 1.9 \& x_{2}<2.9\right)$ then $y=g\left(x_{1}\right)$

If $\left(x_{2} \geq 2.9 \& x_{1}<2.9\right)$ then $y=h\left(x_{1}\right)$

If $\left(x_{2} \geq 2.9 \& x_{1}>2.9\right)$ then $y=u\left(x_{2}\right)$

Whenever the PAE value of the above rules is less than the threshold of $5 \%$, the procedure of ALM modeling is stopped. Here, using four rules, a PAE of $3.8 \%$ is achieved.

\subsection{The new heuristic search method}

In this section, a new heuristic search method is introduced for dividing space in the ALM algorithm.

Partitioning of multi-dimensional space is a combinatorial problem. There is no theoretical approach for it; therefore, heuristic search methods are used (Takagi and Sugeno 1985).

The heuristic search is a guided search and it does not guarantee an optimal solution. However, it can often find satisfactory solutions (Abbass et al. 2002).

Consider $k$ inputs $\left(x_{1}, x_{2}, \ldots, x_{k}\right)$, and a single output $(y)$ system. The algorithm of the new heuristic search method for this system is depicted in figure 4.

Step 1. The domain of $x_{1}$ is divided into two parts (small and big). Using the ALM algorithm, the best continuous path is determined for each part of the $x_{1}$ domain. Assume these paths are $f_{11}\left(x_{j}\right)$ and $f_{12}\left(x_{m}\right)$, which are the best paths for the first dividing step and for the small and big parts of the divided variable that are the functions of the $j$ th and $m$ th variables, respectively. Here, the rules for modeling are:

If ( $x_{1}$ is small) then $y=f_{11}\left(x_{j}\right)$

If $\left(x_{1}\right.$ is big) then $y=f_{12}\left(x_{m}\right)$

Then, the modeling error $\left(e_{11}\right)$ is calculated for the above rules. Similarly, the domain of other variables are divided and their modeling errors are calculated and a set of $k$ errors $\left(e_{11}\right.$, $\left.e_{12}, \ldots, e_{1 k}\right)$ are generated. For example, $e_{1 k}$ shows the minimum modeling error after dividing the domain of $k$ th variable in the first step of dividing. The variable corresponding to the minimum error is the best one for dividing of space. Suppose $e_{1 s}$ is the minimum error and it is correspond to $x_{\mathrm{s}}$, then, the $x_{\mathrm{s}}$ domain is divided into small and big values. If $e_{1 \mathrm{~s}}$ is more than the threshold error, the dividing algorithm should continue.

Step 2. Consider all possible combinations of $x_{\mathrm{s}}-x_{\mathrm{j}}(j=1,2, \ldots, k)$ for each part of $x_{\mathrm{s}}$ and then divide the domain of $x_{j}$ again into two parts. Thus, $2 k$ combinations are generated $(k$ combinations of $x_{s(\mathrm{small})}-x_{j}$ and k combinations of $\left.x_{s(\mathrm{big})}-x_{j}\right)$ where each combination has two parts. For example, $x_{s(\mathrm{big})}-x_{j}$ means that when $x_{s}$ has a big value, the domain of $x_{j}$ is divided into small and big parts. Similarly, the ALM algorithm is applied to each part and the minimum modeling error is calculated for each $k$-combinations. Suppose these are $e_{2 m}$ and $e^{\prime}{ }_{2 n}$. They imply that the minimum modeling errors in the second step of dividing the space of variables is related to dividing of $m$ th and $n$th variables for the small and big parts of $x_{\mathrm{s}}$, respectively. Based on minimum errors, $x_{m}$ and $x_{n}$ are divided and the rules for modeling after dividing are: 
If $\left(x_{s}\right.$ is small \& $x_{m}$ is small $)$ then $\ldots$

If $\left(x_{s}\right.$ is small \& $x_{m}$ is big) then ...

If $\left(x_{s}\right.$ is big \& $x_{n}$ is small $) \quad$ then ...

If $\left(x_{s}\right.$ is big \& $x_{n}$ is big) then ...

$e_{2 m}$ and $e_{2 n}^{\prime}$ are the local minimum errors. The appropriate global error $\left(e_{2}\right)$ can be calculated using minimum local errors $\left(e_{2 m}\right.$ and $\left.e_{2 n}^{\prime}\right)$. Dividing continues until the global error is less than the threshold error. In this heuristic search method, the global error decreases simultaneously by decreasing the local errors.

Figure 4 depicts the next step of dividing algorithm which is step 3.

This heuristic search method uses an appropriate criterion to select a variable for dividing and the median of data is used as the boundary for crisp dividing. Hence, the number of data points in the subspaces are equal.

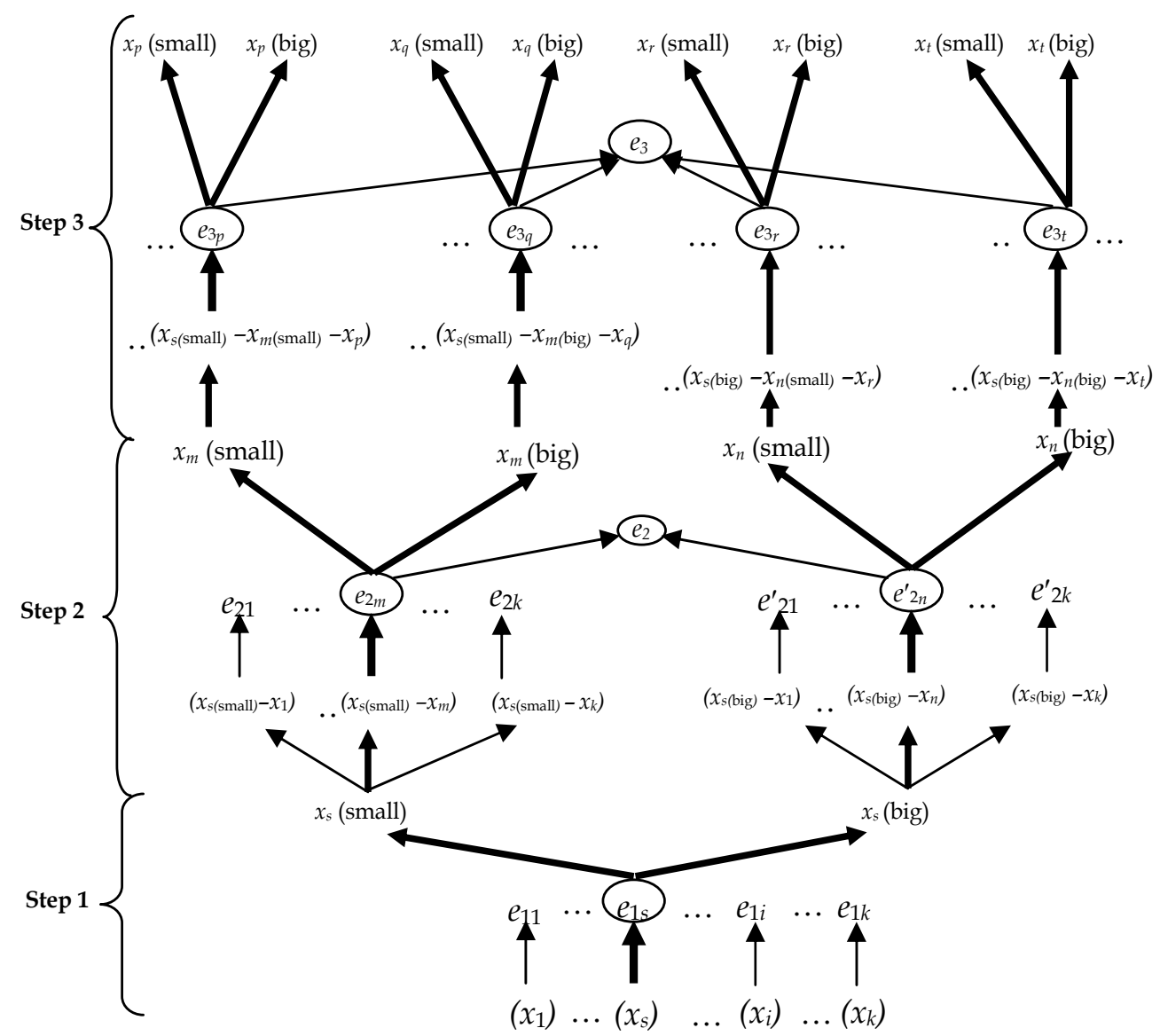

Fig. 4. Algorithm of the new heuristic search method for dividing the space. 


\subsection{Fuzzy dividing}

Although, ALM implements crisp or fuzzy dividing methods, but fuzzy dividing and modeling methods can improve the ALM performance by:

1- Satisfaction of continuity condition,

2- Better knowledge extraction of multi-variable non-linear systems,

3- Decrease of ALM sensitivity to noise.

Fuzzy dividing is similar to crisp dividing. In crisp dividing, the dividing point of a variable is the median as shown in figure 5a. But in fuzzy dividing, the boundary of small values of a variable is bigger than the median (figure $5 b$ ) and vice versa (figure $5 c$ ). Hence, the regions of small and big values of a variable can overlap.

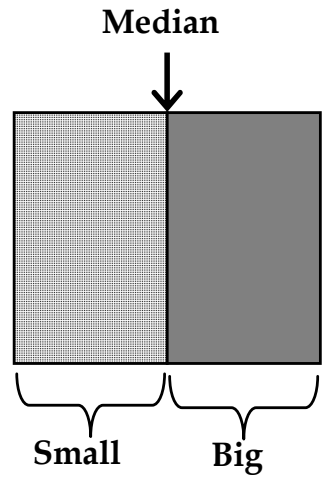

(a)

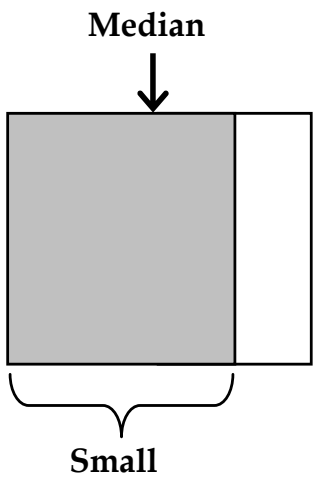

(b)

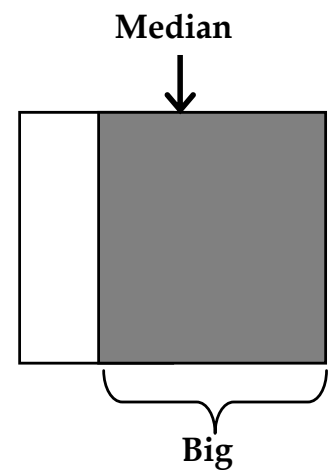

(c)

Fig. 5. Schematic view of different dividing methods, (a) crisp dividing, (b) small part of variable domain in fuzzy dividing, $(c)$ big part of variable domain in fuzzy dividing.

The fuzzy systems are not too sensitive to the dividing points. Therefore, the appropriate points for fuzzy dividing can be calculated by investigating various alternatives to select the most appropriate one.

\subsection{Fuzzy modeling in ALM}

Since the presented new heuristic method (section 2.1) utilizes a complicated dividing method, the typical fuzzification methods are not compatible with it. Here, a new simple fuzzy modeling method is presented which is attuned to the heuristic search method. This fuzzy modeling method has been developed by Taheri Shahraiyni (2007).

We denote the membership function of a fuzzy set as $A_{i j}^{k s}\left(x_{k}^{m}\right)$ in which $i$ is the dividing step, $j$ is the number of dividing in each $i$ which has a value between 1 and $2^{i-1} . s$ is the membership function that is related to small $(s=1)$ and big parts $(s=2)$ of a variable domain. $k$ denotes the divided variable number and $x_{k}^{m}$ is the $m$ th member of the $k$ th variable $\left(X_{k}\right)$ $\left(x_{k}^{m} \in X_{k}\right)$ and $X_{k} \in X . X=\left\{X_{1}, \ldots, X_{n}\right\}$ is a set of $n$ variables. ALM can be implemented by fuzzy modeling with miscellaneous shapes of membership functions and the performance of ALM 
as a fuzzy modeling method is not sensitive to the shape of membership function. Trapezoidal membership functions are one of the most used membership functions. In addition, implementation of a fuzzy modeling method using trapezoidal membership functions is very straightforward. Hence, trapezoidal membership functions are applied here.

The truth value of a proposition is calculated by a combination of membership degrees. For example, the truth value of ' $x_{1}^{1}$ is $A_{11}^{11}$ and $x_{2}^{1}$ is $A_{21}^{22}$ ' is expressed as:

$$
\left(x_{1}^{1} \text { is } A_{11}^{11} \text { and } x_{2}^{1} \text { is } A_{21}^{22}\right)=\left(A_{11}^{11}\left(x_{1}^{1}\right) \wedge A_{21}^{22}\left(x_{2}^{1}\right)\right)=\left(A_{11}^{11}\left(x_{1}^{1}\right) \times A_{21}^{22}\left(x_{2}^{1}\right)\right) \text {. }
$$

In this fuzzy method, the general fuzzy rules are defined as below:

$$
\mathrm{R} p: \quad \text { If } \quad\left(x_{k_{1}}^{m} \text { is } A_{1 j_{1}}^{k_{1} s_{1}} \& x_{k_{2}}^{m} \text { is } A_{2 j_{2}}^{k_{2} s_{2}} \& \ldots\right) \text { then } y_{p}^{m}=f_{p}\left(x_{k_{3}}^{m}\right)
$$

Where $p$ is the rule number and has a value between 1 and $h$ ( $h$ is total number of fuzzy rules), $\mathrm{R}^{p}$ is the $p$ th rule and $f_{p}$ is the $p$ th one-variable non-linear function for the $p$ th subspace $(p$ th rule).

$1 / P\left(f_{p}\right)$ is considered as the weight of the $p$ th rule $\left(W_{r p}\right)$ where $P\left(f_{p}\right)$ is PAE of $f_{p}$ (continuous path in the $p$ th rule). Fire strength or membership degree of the $p$ th rule, $W_{f p}^{m}$ is equal to the truth value of the proposition which is:

$$
W_{f p}^{m}=A_{1 j_{1}}^{k_{1} s_{1}}\left(x_{k_{1}}^{m}\right) \times A_{2 j_{2}}^{k_{2} s_{2}}\left(x_{k_{2}}^{m}\right) \times \ldots
$$

Obviously, the summation of truth values of all of the propositions should be equal to 1

$$
\left(\sum_{p=1}^{h} W_{f p}^{m}=1\right) .
$$

Finally, the corresponding output $\left(y^{m}\right)$ to $m$ th set of input dataset $\left(x_{1}^{m}, \ldots x_{k}^{m}, \ldots x_{n}^{m}\right)$ is calculated as:

$$
y^{m}=\frac{\sum_{p=1}^{h}\left(y_{p}^{m} \times W_{f p}^{m} \times W_{r p}\right)}{\sum_{p=1}^{h}\left(W_{f p}^{m} \times W_{r p}\right)}
$$

\subsection{An example for fuzzy modeling in ALM}

Now, we present a small example to show the simplicity of the described fuzzy modeling method. 
Consider a system with two variables $\left(X=\left\{X_{1}, X_{2}\right\}\right)$ and $x_{1}^{m} \in X_{1}, x_{2}^{m} \in X_{2}$ that its fuzzy rules are:

$\mathrm{R}^{1}: \quad$ If $\quad\left(x_{1}^{m}\right.$ is small \& $x_{1}^{m}$ is very small $)$

then $\quad y_{1}^{m}=f_{1}\left(x_{1}^{m}\right)$

$\mathrm{R}^{2}: \quad$ If $\quad\left(x_{1}^{m}\right.$ is small $\& x_{1}^{m}$ is moderate small $)$

then $\quad y_{2}^{m}=f_{2}\left(x_{2}^{m}\right)$

R3: If $\quad\left(x_{1}^{m}\right.$ is big $\& x_{2}^{m}$ is small $)$

then $\quad y_{3}^{m}=f_{3}\left(x_{2}^{m}\right)$

R4: If $\quad\left(x_{1}^{m}\right.$ is big \& $x_{2}^{m}$ is big)

then $\quad y_{4}^{m}=f_{4}\left(x_{1}^{m}\right)$

The above linguistic propositions are expressed by the following rules:

\begin{tabular}{|c|c|c|c|c|}
\hline $\mathrm{R}^{1}:$ & If & $\left(x_{1}^{m}\right.$ is $A_{11}^{11} \& x_{1}^{m}$ is $\left.A_{21}^{11}\right)$ & then & $y_{1}^{m}=f_{1}\left(x_{1}^{m}\right)$ \\
\hline $\mathrm{R}^{2}:$ & If & $\left(x_{1}^{m}\right.$ is $A_{11}^{11} \& x_{1}^{m}$ is $\left.A_{22}^{12}\right)$ & then & $y_{2}^{m}=f_{2}\left(x_{2}^{m}\right)$ \\
\hline R3: & If & $\left(x_{1}^{m}\right.$ is $A_{11}^{12} \& x_{2}^{m}$ is $\left.A_{23}^{21}\right)$ & then & $y_{3}^{m}=f_{3}\left(x_{2}^{m}\right)$ \\
\hline & If & $\left(x_{1}^{m}\right.$ is $A_{11}^{12} \& x_{2}^{m}$ is $\left.A_{24}^{22}\right)$ & then & $y_{4}^{m}=f_{4}\left(x_{1}^{m}\right)$ \\
\hline
\end{tabular}

Suppose the PAE of $f_{1}, f_{2}, f_{3}$ and $f_{4}$ are $5,10,5$ and 20. Then, $W_{r 1}, W_{r 2}, W_{r 3}$ and $W_{r 4}$ are equal to $0.2,0.1,0.2$ and 0.05 , respectively.

Figure 6 shows the fuzzy dividing and the membership functions for this example

As seen in figure 6 , the membership degrees for $x^{1}\left(x_{1}^{1}, x_{2}^{1}\right)$ are:

$A_{11}^{11}\left(x_{1}^{1}\right)=0.34, A_{12}^{11}\left(x_{1}^{1}\right)=0.66, A_{21}^{11}\left(x_{1}^{1}\right)=0, A_{22}^{12}\left(x_{1}^{1}\right)=1, A_{23}^{21}\left(x_{2}^{1}\right)=0.1$,

$A_{24}^{22}\left(x_{2}^{1}\right)=0.9$,

In accordance with the membership degrees of different membership functions, the truth values of different rules $\left(W_{f p}^{m}\right)$ can be determined using the following calculations.

$$
\begin{aligned}
& W_{f 1}^{1}=A_{11}^{11}\left(x_{1}^{1}\right) \times A_{21}^{11}\left(x_{1}^{1}\right)=0.34 \times 0=0 \\
& W_{f 2}^{1}=A_{11}^{11}\left(x_{1}^{1}\right) \times A_{22}^{12}\left(x_{1}^{1}\right)=0.34 \times 1=0.34 \\
& W_{f 3}^{1}=A_{11}^{12}\left(x_{1}^{1}\right) \times A_{23}^{21}\left(x_{2}^{1}\right)=0.66 \times 0.1=0.066 \\
& W_{f 4}^{1}=A_{11}^{12}\left(x_{1}^{1}\right) \times A_{24}^{22}\left(x_{2}^{1}\right)=0.66 \times 0.9=0.594
\end{aligned}
$$

Assume the rule functions $\left(y_{p}^{m}\right)$ values for point $x^{1}$ are:

$y_{1}^{1}=f_{1}\left(x_{1}^{1}\right)=2.0, y_{2}^{1}=f_{2}\left(x_{2}^{1}\right)=1.8, y_{3}^{1}=f_{3}\left(x_{2}^{1}\right)=2.2$ and $y_{4}^{1}=f_{4}\left(x_{1}^{1}\right)=1.7$. 
According to the equation 3 , the output $\left(y^{1}\right)$ can be calculated as below:

$$
\begin{gathered}
y^{1}=\frac{\sum_{p=1}^{4}\left(y_{p}^{1} \times W_{f p}^{1} \times W_{r p}\right)}{\sum_{p=1}^{4}\left(W_{f p}^{1} \times W_{r p}\right)}=\frac{\left(y_{1}^{1} \times W_{f 1}^{1} \times W_{r 1}\right)+\left(y_{2}^{1} \times W_{f 2}^{1} \times W_{r 2}\right)+\left(y_{3}^{1} \times W_{f 3}^{1} \times W_{r 3}\right)+\left(y_{4}^{1} \times W_{f 4}^{1} \times W_{r 4}\right)}{\left(W_{f 1}^{1} \times W_{r 1}\right)+\left(W_{f 2}^{1} \times W_{r 2}\right)+\left(W_{f 3}^{1} \times W_{r 3}\right)+\left(W_{f 4}^{1} \times W_{r 4}\right)} \\
=\frac{(2.0 \times 0 \times 0.2)+(1.8 \times 0.34 \times 0.1)+(2.2 \times 0.066 \times 0.2)+(1.7 \times 0.594 \times 0.05)}{(0 \times 0.2)+(0.34 \times 0.1)+(0.066 \times 0.2)+(0.594 \times 0.05)}=1.83
\end{gathered}
$$

It could be observed that the calculations in this fuzzy modeling method are simple and straightforward.

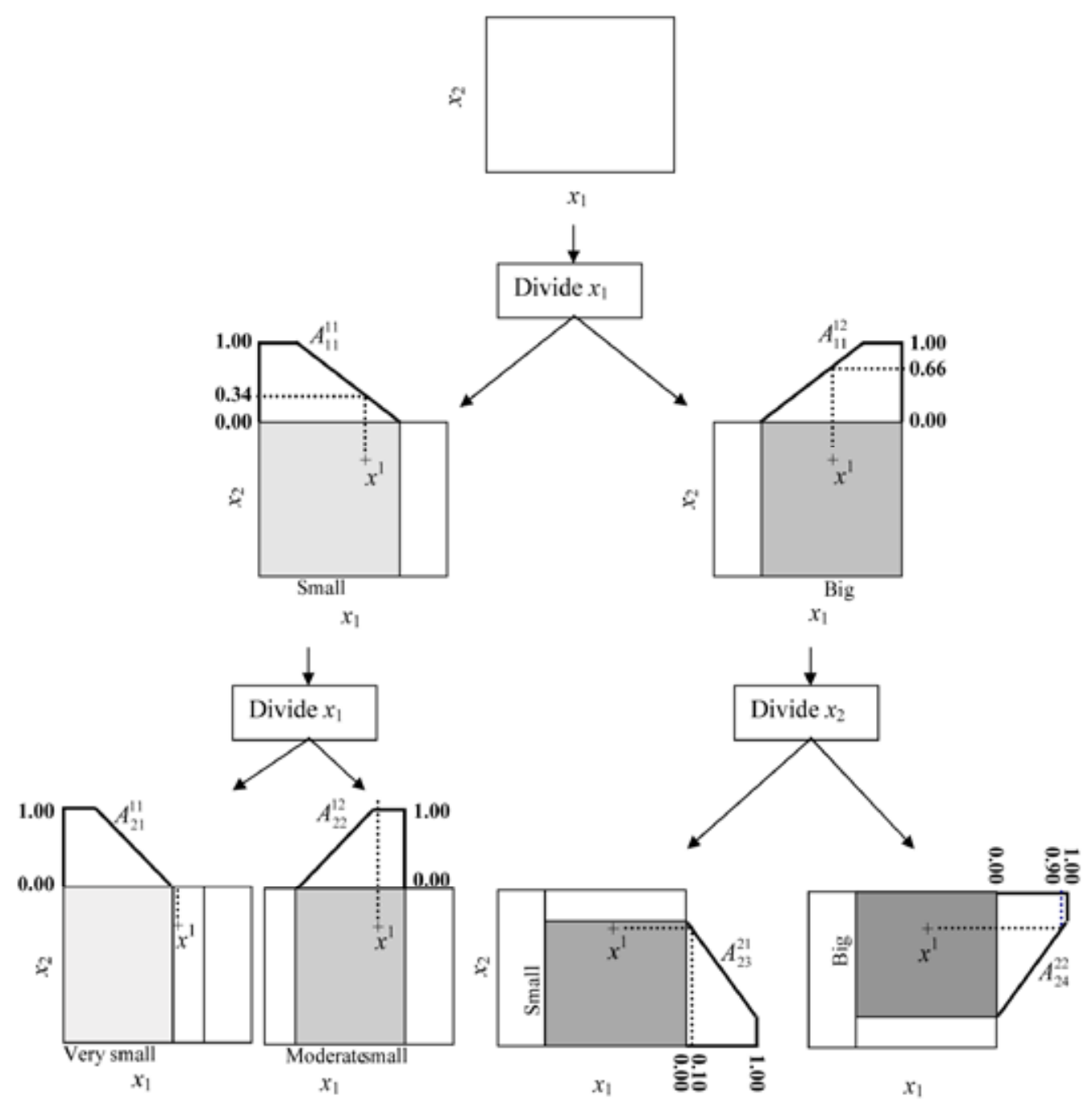

Fig. 6. Fuzzy dividing, trapezoidal membership functions and membership degrees for point $x^{1}\left(x_{1}^{1}, x_{2}^{1}\right)$. 


\section{The specific abilities of ALM}

\subsection{Initial parameters for training}

In spite of many other well-known modeling methods (e.g. Neural Networks), ALM does not need initial parameters to start the training and thus it does not repeat the training, hence ALM training is very easy and straightforward and it is not time consuming.

\subsection{Finding and ranking the effective variables}

Although, the appropriate inputs to models are often determined before the beginning of modeling by physical or empirical based methods, but ALM can determine and rank the important input variables. Because it is very easy for ALM to find the important or divided variables and one-variable function in each step of modeling. Then, the variables can be ranked according to their roles in modeling easily. Suppose that the ranking criterion for used variables in one-variable functions is the number of subspaces which have been estimated using each variable. Similarly, suppose that the ranking criterion for the used variables for dividing is dividing times of each variable. Hence, ALM similar to human can find and rank the effective variables in a system.

\subsection{Sensitivity to noise}

In general, ALM has low sensitivity to noise. The main factor of robustness of ALM is IDS operator and it is similar to adding a cone shaped distribution noise to the inputs and output data using cone shaped illumination (distribution) pattern (solid line in figure 7). As shown in figure 7 , the cone shaped noise which is added automatically by IDS is very similar to a normal distribution (solid line) noise.

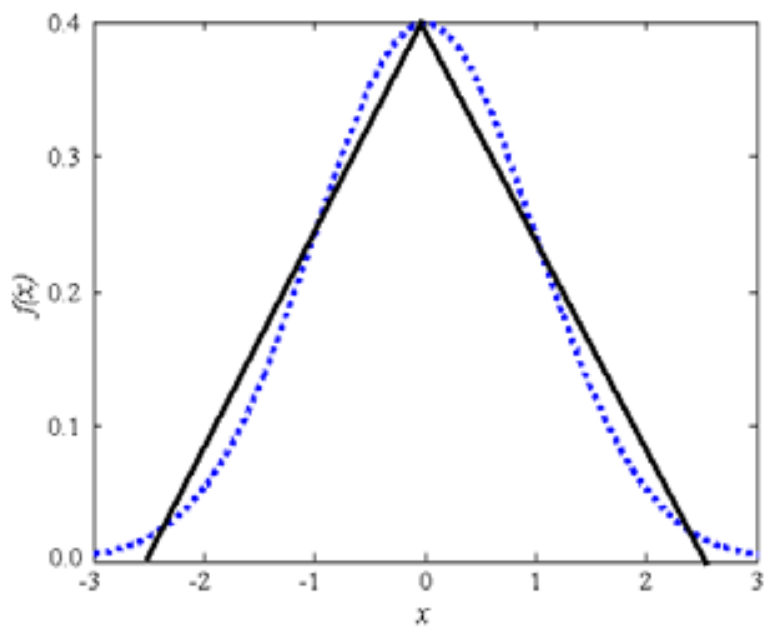

Fig. 7. Schematic view of normal distribution function $\left(f(x)=\frac{1}{\sqrt{2 \pi}} e^{-\frac{x^{2}}{2}}\right)$ (dotted line) and cone shape illumination (distribution) pattern of IDS (solid line). 
For the low sensitivity to noise (robust) modeling, it is necessary to add some noises to data and then train the model with noisy data. In the crisp modeling methods (e.g. neural networks), adding noise to data should be performed by repeating the dataset (for example 100 times) and adding noise (often normal distribution noise) to the repeated dataset. It is time consuming to repeat the dataset, add random noise to data and train the model using the large repeated dataset. Therefore robust modeling is time consuming. But ALM does not need to repeat the dataset nor add noise to it. Because the cone shaped noise which is added automatically by IDS operator is very similar to a normal distribution (dotted line) noise (see figure 7). Thus, ALM is more rapid than any other crisp modeling method in training stage.

In the crisp modeling methods, because of lack of necessary knowledge about the noise level in the system (e.g. noise level for different variables and functions), a constant level of noise is added to the functions and variables. But ALM determines the appropriate noise level for different variables by calculation of the optimum slopes of cone shaped illumination (distribution) patterns in the IDS operator for variables. Also ALM is able to add different levels of noise to the function and variables data by changing the resolution in $\mathrm{x}$ and $\mathrm{y}$ axes.

For more details about the ALM abilities and demonstration of the mentioned abilities, refer to Taheri Shahraiyni (2007) and Taheri Shahraiyni et al. (2009).

\section{An application of ALM modeling}

Here, ALM is used for modeling of a very important problem (Algal bloom in marine environment) and its abilities and performances are investigated.

Algal bloom is the result of interaction between physiological and ecological characteristics of the species as well as physiochemical processes in the water column. Algal bloom detection in the water bodies and the monitoring of its spatiotemporal changes is necessary for water quality or marine ecosystem modeling and management. Chlorophyll-a is an indicator for the phytoplanktonic biomass, hence the retrieved spatial distribution of chlorophyll concentration in a water body can be used for the monitoring of spatiotemporal changes of algal bloom.

Remote sensing can provide a complementary tool for the chlorophyll retrieval in varying water bodies. Due to the very high level inherent noises in satellite images, the low sensitivity to noise method is needed for the extraction of chlorophyll concentration from satellite images. ALM is promising to be low sensitive to noise, hence used for solving of this problem. The reflected radiations of sea surface, measured in different wavelengths by satellite, are the input variables to ALM and the output is the corresponding chlorophyll concentration.

We need to a dataset for training of ALM. The training dataset was generated by numerical solution of radiative transfer equation. Radiative transfer equation simulates the radiative transfer in atmosphere-ocean system.

ALM was trained by this dataset and tested by satellite images and concurrent in-situ measurements of chlorophyll in Caspian Sea.

The Caspian Sea is a land-locked sea between Asia and Europe. It is the largest inland water body in the world. It covers a surface area more than $370,000 \mathrm{~km}^{2}$, reaches a maximum depth of about $1000 \mathrm{~m}$. An algal bloom happened in the Caspian Sea on August 2005 in the southern part of Caspian Sea and it disappeared in October 2005. The sampling and analysis of species in the algal bloom showed that the bloom is related to toxic specie of the Cyanobacteria (Bluegreen algae) which is named Nodularia. 
The utilized satellite images in the study were MERIS images. MERIS has been located on board of the ENVISAT satellite (European Space Agency). MERIS takes 15 images in different wavelengths between 412 and $900 \mathrm{~nm}$. For details about MERIS refer to Rast et al. (1999).

The results of test of trained ALM showed that it can derive the chlorophyll concentration with appropriate accuracy (percent of absolute error $=44 \%$ ). This shows that ALM is very robust to noise. The developed ALM model had only 8 rules. The processing time of fuzzy models is highly related to the number of rules and this number of rules (8 rules) is small enough to allow for an appropriate processing time in operational applications. In addition, it could find that $\{490,510,560,685$, and $885 \mathrm{~nm}\}$ are important and necessary wavelengths for chlorophyll modeling and remove the other ones. For details about this Chlorophyll estimation by ALM modeling refer to Taheri Shahraiyni et al. (2007).

Finally, the satellite images were used as input to developed ALM model and the output chlorophyll concentration maps in Caspian Sea were extracted. Figure 8 shows these results from August to October.

As you see in figure 8 and explanations on the figure 8 , ALM has been successful for the appropriate monitoring of change of chlorophyll concentration in the Caspian Sea.

The results demonstrated that ALM model is an appropriate and useful method for modeling of chlorophyll concentration and consequently, detection and monitoring of algal bloom in marine environment.

\section{Other applications}

Although we focused on the ALM abilities for modeling, but according to the structure of ALM, its application is not limited to modeling and it is applicable in different fields of engineering. Here we hint to some of ALM applications.

\subsection{Large scale optimization problems}

The large scale optimization problems are important in different fields of science, engineering and operation research. Unfortunately most of them are NP (Non-Polynomial) problems and finding their optimum solution in reasonable time is almost impossible (Garey and Johnson, 1976). ALM can be used for solution of these problems and it presents satisfactory results.

An example: Bin-Packing problem is a NP problem (Garey and Johnson, 1976) and it has many different applications such as loading trucks subject to weight limitations. Lotfi and Bagheri Shouraki (2004) used ALM for solution of Bin-packing problem. They showed, in spite of very simple construction of ALM, it can obtain very good results for solving Bin Packing problem.

\subsection{Control problems}

Human modeling method has low sensitivity to noise. ALM is similar to human modeling methods and it is very robust to noise. Therefore it is very useful method for control problems. Up to now, several researches has been performed on the application of ALM in control problems. Some of these control researches are as below:

Bagheri Shouraki and Honda (1998) showed the ability of ALM for stable controlling of dynamic systems such as invert pendulum.

Shahdi and Bagheri Shouraki (2002) used of ALM for design of controller for Beam and Ball problem and showed the ability of ALM for control of this system. 
Shahdi and Bagheri Shouraki (2003) used of ALM for design of an intelligent control system in an automated vehicle. The problem was the control of a truck which is moving at the back of another truck in one line. The automated truck doesn't contact to another truck. The ALM has been used to extract driver's behavior and control rules for control system. Also, the effective parameters of controller were derived using ALM.

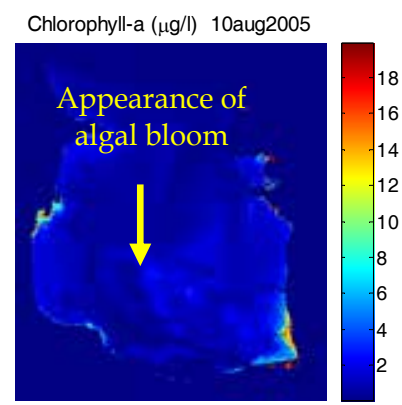

(a)

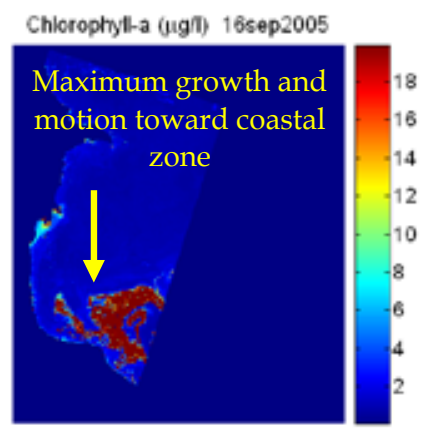

(d)

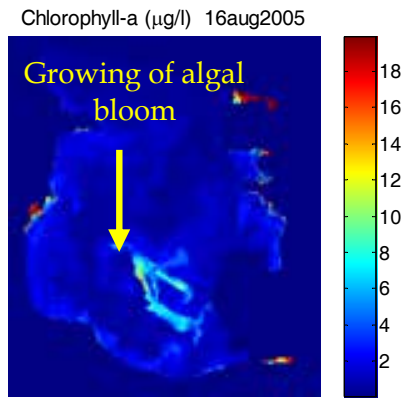

(b)

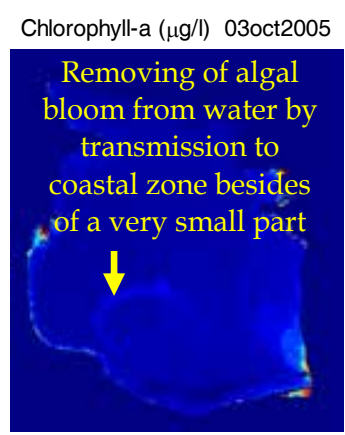

(e)

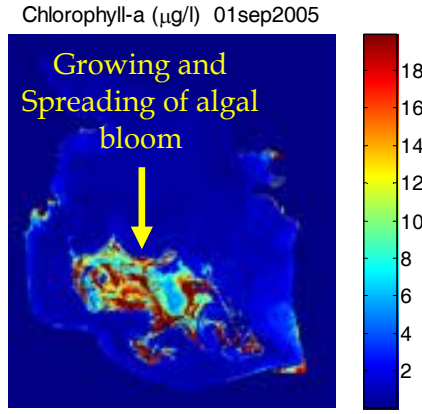

(c)

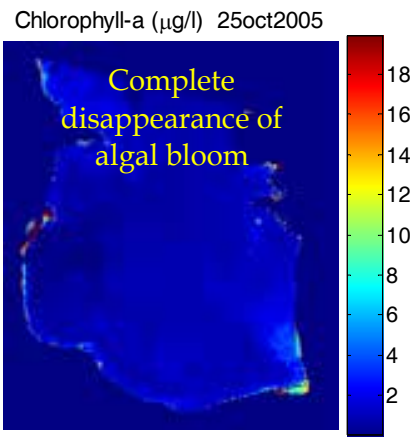

(f)

Fig. 8. Chlorophyll concentration ( $\mu \mathrm{g} /$ lit) maps in Caspian Sea, derived by developed ALM model which shows the algal bloom appearance on August and its disappearance until October, 2005

\section{References}

AbBASs, H.A., SARKER, R.A. and NeWTON, C.S., 2002, Data mining: A heuristic approach (Idea Group Publishing).

BAgHeri SHOURAKI, S. and HondA, N., 1997, A new method for establishing and saving fuzzy membership functions. In 13 th Fuzzy Symposium, 4-6 June 1997, Toyama, Japan, pp. 91-94.

BAGHERI SHOURAKI, S. and HONDA, N., 1998, Fuzzy controller design by an active learning method. In 31 th Symposium of Intelligent Control, 26 August 1998, Japan, Tokyo, pp. 1-10. 
BAGHERI SHOURAKI, S. and HONDA, N., 1999, Recursive fuzzy modeling based on fuzzy interpolation. Journal of Advanced Computational Intelligence, 3, pp. 114-125.

FAUSSET, L., 1994, Fundamentals of neural networks: Architectures, algorithms and applications., Prentice Hall, 461 p.

GAREY, M. R. and JOHNSON, D.S., 1979, Computer and interactability: A guide to the theory of NPcompleteness., W. H. Freeman.

Lotfi, T. and BAGHeri SHOURAKI, S., 2004, Active learning method to solve Bin Packing problem. In Proc. of Neural Networks and Computational Intelligence (NCI 2004), 23-25 February 2004, Grindelwald, Switzerland, Paper No. 413-009.

MAMDANI, E.A., 1976, Advances in the linguistic synthesis of fuzzy controllers. International Journal of Man-Machine Studies, 8, pp.669-678.

RAST, M., BEZY, J.L. and BRUZZI S., 1999, The ESA medium resolution imaging spectrometer (MERIS)-a review of the instrument and its mission. International Journal of Remote Sensing, 20, pp. 1681-1702.

SCHMIDT, R.F., 1985, Fundamentals of Neurophysiology (Springer-Verlag).

SHAHDI, S.A. and BAGHERI SHOURAKI S., 2003, Use of active learning method to develop an intelligent stop and go cruise control. In Proc. of Intelligent Control and Systems (ISC 2003), 25-27 June 2003, Salzburg, Austria, Paper No. 388-157.

SHAHDI, S.A. and BAGHERI SHOURAKI S., 2002, Supervised active learning method as an intelligent linguistic controller and its hardware implementation. In Proc. of International Conference on Artificial Intelligent and Applications, 9-12 Sep., Malaga, Spain.

SugENO, M. and YASUKAWA, T., 1993, A fuzzy-logic based approach to qualitative modeling. IEEE Transactions on Fuzzy Systems, 1, pp. 7-31.

TAHERI SHAHRAIYNI, H., 2007, Extraction of water quality parameters using the satellite data and inverse modeling of radiative transfer equation by active learning method, Phd Thesis, Civil. Eng. Dept., Sharif University of Technology, Tehran, Iran, 167 p. (In Persian).

TAHERI Shahraiyni, H., Bagheri Shouraki, S., Fell, F., SchaAle, M., Fischer, J., TAVAKOli, A., Preusker, R., Tajrishy, M., Vatandoust, M. And Khodaparast, H., 2009, Application of Active Learning Method to retrieve pigment from spectral remote sensing reflectance data. International Journal of Remote Sensing, 30, pp. 1045-1065.

Taheri Shahraiyni, H., Schaale, M., Fell, F., Fischer, J., Preusker, R., Vatandoust, M., BAgheri SHOURAKI, S., TAJRISHY, M., KHODAPARAST, H., AND TAVAKOlI, A., 2007, Application of Active Learning Method for the estimation of geophysical variables in the Caspian Sea from satellite ocean colour observations. International Journal of Remote Sensing, 28, pp. 4677-4683.

TAKAGI, T. and SugENO, M., 1985, Fuzzy identification of systems and its applications to modeling and control. IEEE Transactions on Systems, Man, and Cybernetics, SMC-15, pp. 116-132.

ZADEH, L. A., 1965, Fuzzy sets. Information and Control, 8, pp. 338-353. 


\title{
Generic Framework for Collaborative Work Environments
}

\author{
Cosmin Porumb, Sanda Porumb, Bogdan Orza and Aurel Vlaicu \\ Technical University of Cluj-Napoca \\ Romania
}

\section{Introduction}

The "i2010 - An European Information Society for growth and employment" initiative was launched by the European Commission on $1^{\text {st }}$ of June 2005as a framework for addressing the main challenges and developments in the information society and media sectors up to 2010 . It promotes an open and competitive digital economy and emphasizes ICT as a driver of inclusion and quality of life. The initiative contains a range of EU policy instruments to encourage the development of the digital economy such as regulatory instruments, research and partnerships with stakeholders. According to the European Union initiatives, this chapter proposes a generic framework that illustrates the advances of collaborative work environments where the attendees from different domains of interest can share ideas, concepts, studies, digital content and resources, in an interactive manner. The approach highlights the importance of the advanced technologies in E-Service platforms: video telephony, video conferencing, online focus group, virtual shared space, media streaming or video capture/recording functionalities. The presented prototype is developed according to the extended LAMP architecture and consists of the following blocks: Apache web server, MySQL database server, Red5 media server, OpenOffice 2.0 and GostScript libraries.

The generic framework can be applied to different domains such as business, education, telemedicine or administration and security. The chapter contains sections dedicated to collaborative work capabilities for electronic commerce and negotiating or customer live support, open and distance/blended learning, administrative real-time decission in different situations (calamities, vandalism acts), remote consulting and surgery acts. Some examples and suggestive demonstration films are also presented in the chapter in order to illustrate the advantages of the computer supported collaborative work framwork as the core of any virtual community.

\section{Generic Framework for computer-supported collaborative work}

The proposed framework can be considered as a multimedia distributed system that complies with the extended LAMP architecture (Figure 1) and consists of the following blocks: Apache web server that allows the end-users to access the collaborative work services, MySQL database server that stores the information regarding the end-users, 
resources, innovative products and services, suppliers, customers and virtual community, and Red5 media server that provides with video telephony/conferencing capabilities, media streaming and video capture functionalities and supports the remote shared objects. The OpenOffice 2.0 and GostScript libraries are involved in order to implement the conversion block fuctionalities and allow the service handler component to organize the digital content in an unified manner. The Business intelligence block consists of a multi-agent platform able to learn the behavior of the actors and elaborate statistics, analytics reports and scenarios based on activity tracking. The client block allows the end-user to access the system via web or from his/her Pocket Pc.

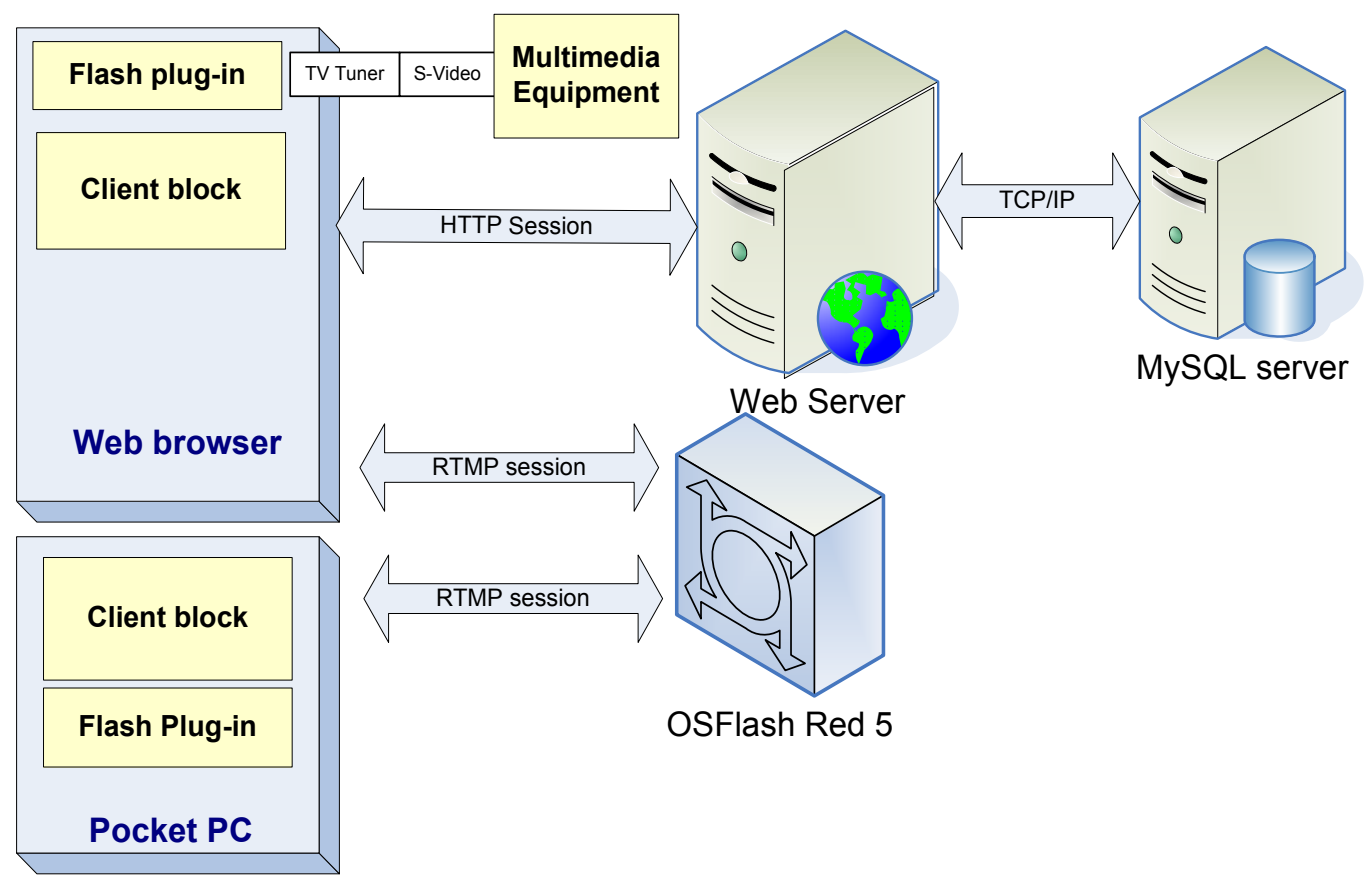

Fig. 1. Framework Architecture

\subsection{LAMP-based Computer-Supported Collaborative Work}

A large number of web sites are built using PHP and MySQL on Linux platforms with Apache as the web server. This combination is known as the LAMP architecture. Apache, PHP and MySQL are also available on Windows platforms giving rise to combinations like WAMP (Windows, Apache, MySQL and PHP) and WIMP (where the IIS web server is used instead of Apache). Even though LAMP is a very popular architecture there has been little work to characterize and benchmark the architecture, especially at an application level.

In reality LAMP describes any architecture that relies on an open source operating system, open source web server, open source database, and open source programming language in the implementation process. In fact, open architecture means cost effectiveness but also high performance.

The LAMP architecture guarantees several advantages such as cost effectiveness, flexibility, and scalability. The cost is the most obvious advantage of the LAMP architecture. Since each 
layer in the stack is based on an open source solution, the entire stack can be implemented for the cost of development plus the cost of hardware. No piece of the stack requires software licenses. The LAMP stack is arguably easier to maintain and expand, and quicker to adjust to business requirements. If mobility is required, the end-user accesses the services using the mobile device (Pocket PC, PDA device) without any other deployment actions, just using a Pocket PC-based client application. The LAMP architecture is horizontally scalable, meaning it grows as you add hardware to it. No single piece of the architecture is a bottleneck because each piece of the stack grows on its own, and is loosely coupled to the other pieces in the stack. In that case, the system specifications highlighted the importance of the multimedia capabilities such as: video communication, media recording/streaming, virtual shared space, knowledge and application sharing. The system architect easily introduced an open source media server (OSFlash Red5) in the classic LAMP architecture in order to support the needed functionalities.

\subsection{Media server block}

OSFlash Red5 media server provides developers with an elegant and powerful set of mechanisms to invoke methods remotely. The client block can invoke methods on the server and the server can invoke methods in the client-side components. Method calls can be broadcast to each client component and application instance connected to a shared object or stream, or they can be sent to and from individual client component. An application instance can even create proxies of methods belonging to another application instance.

The stream and shared object send( ) methods both provide a mechanism to send method invocation requests to multiple client components at the same time. The call( ) methods provide a way to invoke a method on one recipient at a time. Unlike send( ), call( ) makes it possible to receive the return value of a remote method. The call( ) method can invoke methods on numerous objects attached to the client component or NetConnection objects. The call( ) method provides fine-grained messaging control from client to server to clients, if necessary, and has advantages and disadvantages in securing an application.

\subsection{Client bloc}

The scalability is an important aspect when designing multimedia distributed systems and the proposed framework is developed according to the rules that define the scalability. The differences between the client components for the web access and the components that allow the access from the desktop/Pocket PC are insignificant. They are developed as extensions of the client-side generic components that implement basic functionalities such as: interface between the application and the peripherals (access control to sound and video blasters, or TV tuner), audio/video data manipulation (data transmission/retrieval to/from the media server), audio rendering, video playback, and handwriting/drawing features.

The client block diagram in the Figure 2 illustrates the client-side functionalities. The client block controls the microphone and web/video camera connected to the client machine, captures the multimedia data and sends data packages over a stream to the server-side component. In the same manner, the video sequences captured from the multimedia peripherals such as multimedia equipments, using the TV tuner, can be broadcasted within the shared space component. 


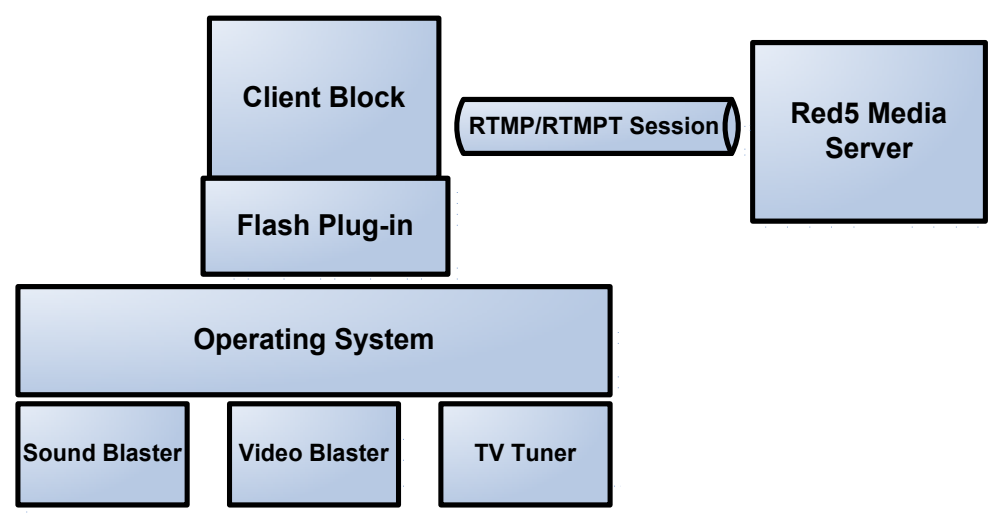

Fig. 2. Client Block Diagram

Video data transmissions can require a significant bandwidth that depends on the video parameters such as frame rate, resolution and quality (compression). If talking about a virtual community built around VPN/LAN (high-speed intranet in which bandwidth is not an issue) higher-quality transmission can be setup. Even in such cases,, there are limits to what a PC can reasonably capture, for example, the application shouldn't exceed $80 \%$ of CPU capacity on Windows. Most applications are deployed over the Internet and the bandwidth requirements can profoundly affect the application's functioning and the user experience. The proposed framework considers those aspects, that way the smallest appropriate dimensions and lowest acceptable frame rate is selected in a dynamic manner (within the bandwidth management function).

\section{Computer-Supported Collaborative Work framework within virtual organizations}

(Crispim \& Pinho de Sousa, 2007) illustrated that business environments, especially the virtual enterprises, respect several important rules. Most of them regard the manner of creating the partnership, which is the trustworthy partner, how to search for this kind of partners or what resources, customers and suppliers to be shared within the virtual enterprise. The product manager in a virtual enterprise is able to manage the resources, the customer/supplier relationships and to analyze the value chain in a dynamically and secure manner from anywhere in the world. The tasks can be performed from the working station in the office or even from the PDA/Pocket PC, thanks to the high degree of scalability provided by the prototype described in the article. Due to the sharing capabilities and collaborative work features, the marketing functions evidently increase in a virtual enterprise. The sales can be focused on the new products and services created in the virtual enterprise or even enlarge the product categories and deliver all the products the partners usually distribute, if the partnership allows that.

The authors consider the scenario above as really important and propose a prototype for the virtual enterprises as the basis of a large scale virtual community. The prototype presents several advantages the multimodal interfaces guarantee, and combines the speech and ink formats to facilitate the creation of robust and efficient multimodal mobile EBusiness systems. 


\subsection{Virtual enterprises and electronic market}

Recent advances in communications and distributed information technologies have changed the way that business is conducted. Enabled by technologies such as software agents and ecommerce, enterprises have gone beyond the geographical and socio-cultural boundaries and have become entities that not only compete in the global market, but also draw their resources from an international market. The trend of outsourcing seems to be replaced by strategic alliances, where enterprises or individuals work together towards a common goal and share their responsibilities as well as their profits. The concept of a virtual enterprise (VE) has emerged as a means of dealing with this new type of alliance. The approach consists of the individual entities, human beings, software agents or organizations that come together as a team to achieve a specific goal. The partners co-operate to complete a set of tasks, share their skills, costs, profits, risks and markets and then move on to join another virtual enterprise.

(Camarinha-Matos et al., 2007) focused on the most important stages in the lifecycle of the virtual enterprise, formation of the virtual organization. Since virtual enterprises have a limited lifetime, they need to be formed very quickly in order to meet the deadlines of the goals. An important part of the formation of the virtual enterprise is the selection of its partners. They are selected on the basis of their ability to fulfill the requirements of the virtual enterprise. The partners have to work as a team and these requirements must address not only the individual partners of the virtual organization, but also how the partners fit into a team.

The main idea of electronic market is to create competition among buyers/sellers while allowing them to adjust all the aspects of the deal that are typically only dealt within a negotiation. This creates a problem of "comparing apples and oranges": bids may be quite different in many dimensions and therefore cannot easily be compared. Apart from the dimension of price these could include pre-negotiated discounts (e.g. for loyalty), specific qualities, combinations of goods and services with conditional pricing, freight differentials, contract fulfillment timing, payment terms, or deliberate constraints such as market share limits.

Many suppliers do not know the potential benefits that electronic market can offer and they do not believe their off-line relationships with a buyer/supplier could be transferred to the digital environment. Thus, buyers need to convince their suppliers that electronic market is not just a price based marketplace but instead a business model where mutual benefits can be achieved based on existing trust relationships between a buyer and limited number of qualified suppliers.

\subsection{Actors and virtual assistance}

Four types of real actors can be defined in the use case scenario: product manager, sales agent, customer and service engineer. Each one has allocated his own virtual assistant that implements presentation, notification or collaborative functionalities.

The product manager performs management tasks related to the value chain, human resources, or specific suppliers and customers of the partnership. The product manager also controls the partnership and takes important decisions based on the suggestions the business intelligence components provides with. The virtual assistant allows the actor to perform the specified activities, notifies the product manager, via SMS/email/multimedia messaging, about new economic agents in the system searching for partners, about 
problems in the existing partnership, elaborates statistics about the sales process and value chain, allocated resources, and present production plans or cost approaches.

The customer usually wants to buy products/services, find more information about a specific product/service or fix a problem the bought product/service generated. In the real market, the sales agent meets the customer from the entrance with a welcome message and presents the product/service categories. In the presented approach, that role is played by the customer's virtual assistant. If the customer needs more details about a product/service, an interactive catalogue or booklet is browsed by the virtual assistant in front of the customer. There are situations when the customer wants to talk to a real agent from the sales department, so, the own virtual assistant establishes a secured communication session between the customer and the sales agent, using the Live Customer Support module.

There are situations when the customer needs technical support and his/her personal assistant must contact the technical support specialist's virtual assistant. The technical support specialist is able to offer remote assistance, so, the innovative products/services the virtual enterprise provides with are created based on that approach: the customer's desktop can be shared, a web camera can be easily integrated in the system, if necessary, or a small but useful control panel can be activated in order to allow the technical support specialist to remotely fix the problem on the customer's end.

\subsection{Collaborative work capabilities for virtual assistance}

The product manager's virtual assistant typically implements the secretary concept and deals with the business intelligence component in order to get the information regarding the partnerships, value chain, human resources, suppliers, customers and product lifecycle. It also presents the information to the product manager as statistics and notification messages. A high degree of interactivity is provided, that way, the product manager is able to dynamically control the virtual enterprise.

The customer's virtual assistant represents the system in the dialogue with the customer and implements the multimodal interface concept for both e-commerce and technical support sections. The role of the multimodal interface is to make the virtual market close to the real world: the virtual assistant and the real vendor in the sales process are similar. The customer's virtual assistance also implements the call center capabilities: it queries the business intelligence component that decides the type of problem the customer has, calls the technical support and establish the communication between the customer and one of the technical support specialists.

The sales agent's virtual assistant informs the sales agent about a new customer interested in buying products/services, his/her options, what product/service is selected, or different suggestions about the customer. The virtual assistant also provides the sales agent with demo films and booklets, statistics or other information during the collaborative commerce sessions. These capabilities increase the efficiency of the sales process and help the sales agent to easily do the own job. On the other side, the customer receives not just the information from the sales agent but he/she can view the selected product/service in different situations according to the demo films, how it works or state conditions and parameters it reaches during the lifecycle. The virtual assistant deals with the business intelligence component in order to get the information for the sales statistics and presents the statistics to the agent in an interactive manner: speech-based user interaction, interactive 2D graphics, animations, macros, sound, text or video. 
The service engineer's virtual assistant is notified by the business intelligence component about the problems one customer met when using the product/service he/she bought from the virtual. Its main task is to establish the communication between the customer and the service engineer and allows him/her to fix the problem remotely. The decision regarding the specialist is the due of the business intelligence and it is elaborated according to the activity tracking approach. That aspect allows the customer to talk about the problems he/she met to a specialist able to fix the issues during a simple video call or collaborative session.

\subsection{Product manager module}

The product manager in a virtual enterprise handles the partnership, manages the value chain, human resources, suppliers, customers or the product lifecycle. He/she has an updated status of the virtual organization, can access complex statistics regarding the value chain, human resources, partnership or sales. The figure 4 illustrates the sales statistics provided to the product manager in HTML format.

The product manager's virtual assistant can easily establish communication sessions with sales agents or another person involved in the virtual enterprise processes. When the product manager and one of the sales agents use a PC2mobile private link the video communication is allowed for improving the communication features. If using a mobile2mobile communication link the system enables an audio link because of the bandwidth economy.

\section{Fig. 3. Sales statistics}

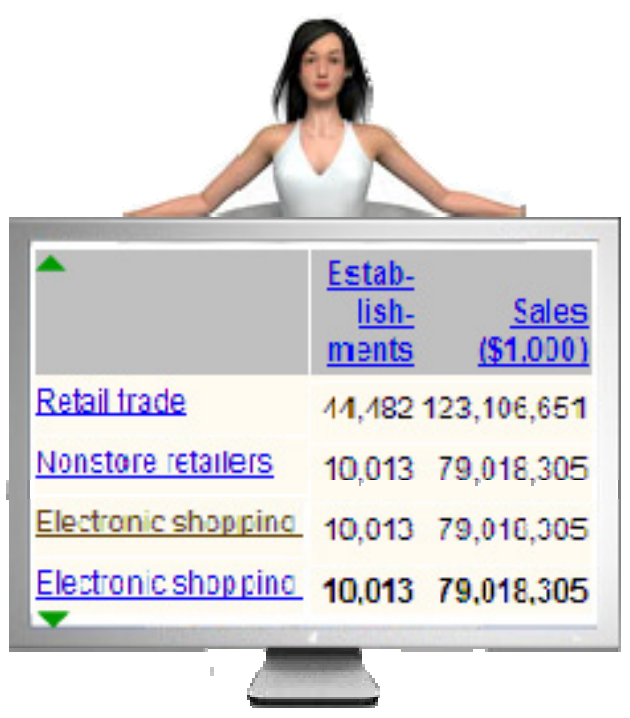

The audio and text are considered as high priority and the bandwidth needs do not raise problems. The video can be supported just when using the Wireless LAN/UMTS networks that allow big amount of data to be transferred. If the product manager invites one or more specialists in a collaborative session, the shared space component will be enabled. It allows the product manager to share resources such as documents, booklets, demo films, with the 
invited specialists. In figure 5, the attendees to the collaborative sessions share the statistics that illustrates important aspects regarding the virtual organization.

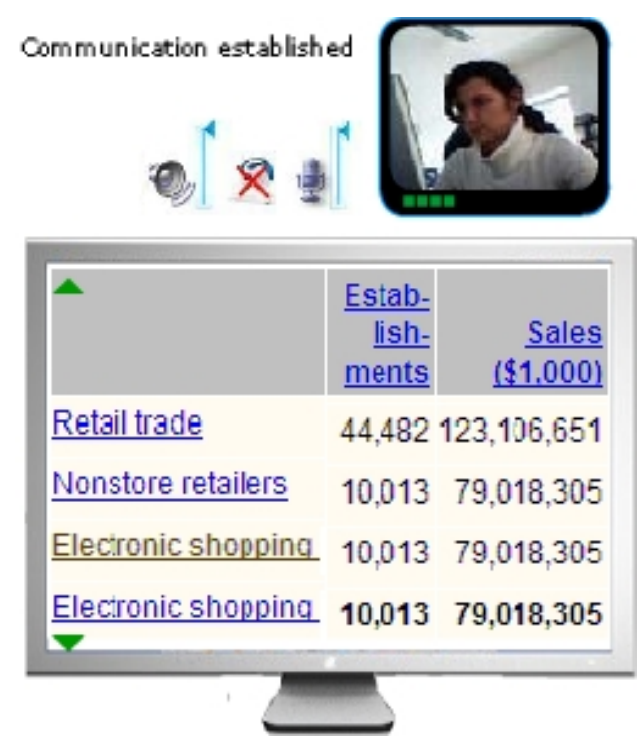

Fig. 4. Video link and shared space

\subsection{Customer module}

The client component in the customer module offers a user friendly interface for both ecommerce and technical support scenarios. Two different sections will be implemented: the virtual store and the live customer support.

The virtual store supports speech-based user interaction and 2D interactive graphics. The multimodal interface allows the customer to browse the catalogues, select a product and buy it using the own voice. The figure 6 illustrates the virtual assistant presenting a selected product to the customer.
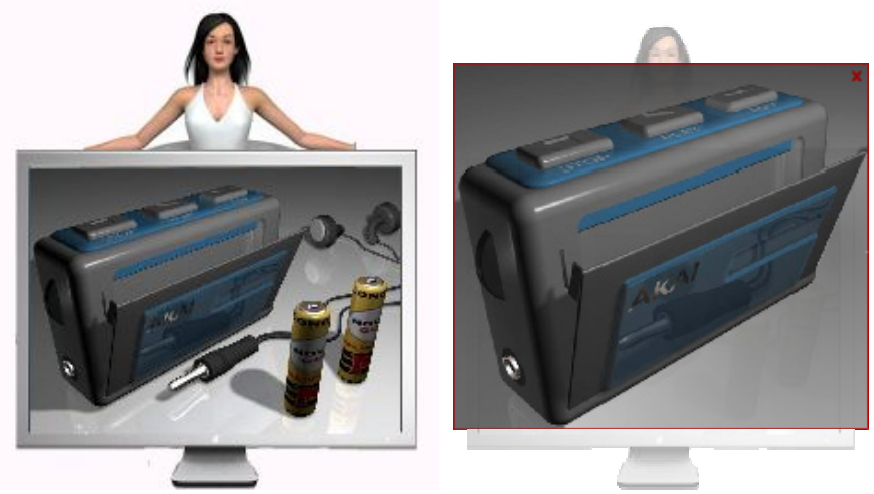

Fig. 5. Product presentation

When the customer needs technical support, the own virtual assistant schedules a communication session with one of the services engineers. The system setup a collaborative session and the virtual assistant of the service engineer prepares some suggestive demos, if 
necessary. The real-time communication increases the efficiency and the customer can be sure of the high quality services.

\subsection{Sales agent module}

The notifications about a customer interested in buying products/services include the customer's options, some information about the navigation history, what product/service is selected and several suggestions. The communication session is established and the sales agent's virtual assistant provides with demo films and booklets, statistics or other information.

Fig. 6. Live customer service

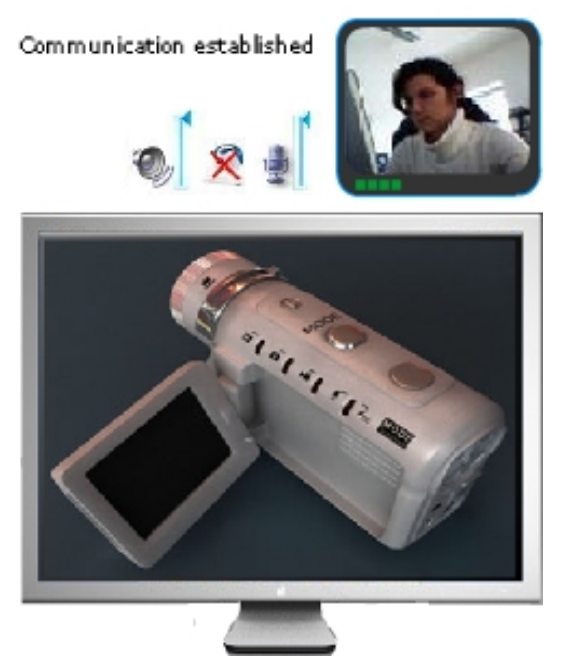

The multimedia capabilities increase the efficiency of the sales process and help the sales agent to easily do the own job. The customer also receives not just the information from the sales agent but he/she can also view the selected product/service in different situations according to the demo films, how it works or state conditions and parameters it reaches during the lifecycle.

\subsection{Technical support}

The technical support scenario is based on the call center concept: the service engineer helps the customer to fix a problem with a product/service the customer bought from the virtual store. If talking about a software product/service, the customer is able to shared his/her desktop and allow the service engineer to remotely manage the situation. The product/service can contain a control panel that allows the service engineer to remotely access it. If talking about another type of product, for example, the service engineer can use the web cam from the customer's machine and see what happened to the product, in that case a video camera.

There are situations when the service engineer meets problems when assisting the customer and must invite one of his/her colleagues to the video communication session. That way the collaborative session will allow the third attendee to connect and share the communication channel and resources. 


\section{Low cost computer-supported collaborative learning prototype}

A lot of research has been done in the field of learning processes and learning activities. This has resulted in various theories such as cognitive learning theories, constructivist theories, and social-historic theories. (Shaozi et al., 2003) demonstrated that each of these theories is associated with a number of specific design principles and prescriptions. In parallel to the development of theories there was also an explosion of network-based technologies, mainly Internet and Web-based, enabling traditional and non-traditional distance learning approaches. Most of the developments have been learner and teacher centered. Group centered designs consider that learning is achieved through constructivism and collaboration.

Due to the changing nature of work and study under knowledge-based economy of this century, as society participants, the teachers and students need to develop ways to deal with complex issues that require new kind of knowledge. (Tan \& Chan, 2008) presented the students and teachers to work, collaborate and learn new things from a variety of resources and people, to investigate questions and bring their learning back to their dynamic learning communities. The number of learning communities grows up but just some of them have the expected success. Often new collaboration tools focus on a specific solution or collaboration task only, without considering the integration of this process into a large but easy to use and very suggestive environment. Moreover, new collaboration technologies require a change in human interaction. Thus the uptake is mainly driven by the benefit. If users do not experience an immediate personal benefit new applications are not applied even if the new tools are properly introduced and training.

Leaded by social and technological problems in the third generation of E-Learning systems the authors propose a low cost prototype based on a Web 2.0 Collaboration Architecture that provides the teachers and students with educational services in different domains such as mathematics, physics, engineering, social sciences or foreign languages. The authors review common groupware problems and reflect the changes in both education and training. The article highlights the Web 2.0 approach in the eService environments, especially eLearning area, it describes the architecture proposed adapted to the requirements of a generic collaborative learning framework that works properly in different domains. It also illustrates how Computer-Supported Collaborative Learning (CSCL) tools improve the knowledge building process and what kind of solutions the designer of a CSCL environment should consider in order to avoid the CSCL weaknesses.

\subsection{Fixing problems in CSCL environments}

(Williams \& Roberts, 2002) illustrated strengths and weaknesses of the computer-supported collaborative work such as effects on academic stuff, repositioning of the responsibility of learning, educational content, interaction time management, communication application of workplace skills, computer competency, or class geography and size. After some years when both hardware and software technologies are more powerful and stable, there are still problems when talking about the collaborative tools in education and training and they have different causes. The prototype allows the E-Learning services providers to fix a list of problems in computer-supported collaborative learning area. It can be easily adapted to the requirements in the fields of primary and secondary education but it is focused on challenges of virtual universities and professional training. 


\subsection{Issues regarding the academic staff}

The educational process is conducted by teachers without experience with many of the CSCL techniques, neither blended learning capabilities, they did not have any training in delivering collaborative classes via a computer and they do not realize that sometime they have been collaborating between themselves by the use of the emails, ISLs and phone conversations between lecturers and faculties. The proposal highlights both user- and group-centered learning concepts that allow the teachers to prepare the educational content to be shared between students and schedule/conduct the virtual classroom sessions and the students to actively participate to the educational process. The framework is designed using the Web 2.0 principles, so, the application should be easy-to-use, the graphic components are simple but very suggestive and comprehensive and the server-side components are powerful, affordable and stable. There are four main features that characterize the Web 2.0 applications and the authors focused on these when proposing the system architecture. These four features just match with social software application problems: Decentralization as a consequence of being a real network. Each node has the ability to act as emitter and receptor of information; Openness - using standards in communication, free licenses on content, promotes collaboration; Dynamic - applications are developed and deployed quickly. User suggestions are attended and supported; User orientation - easier and better user interfaces facilitates participation.

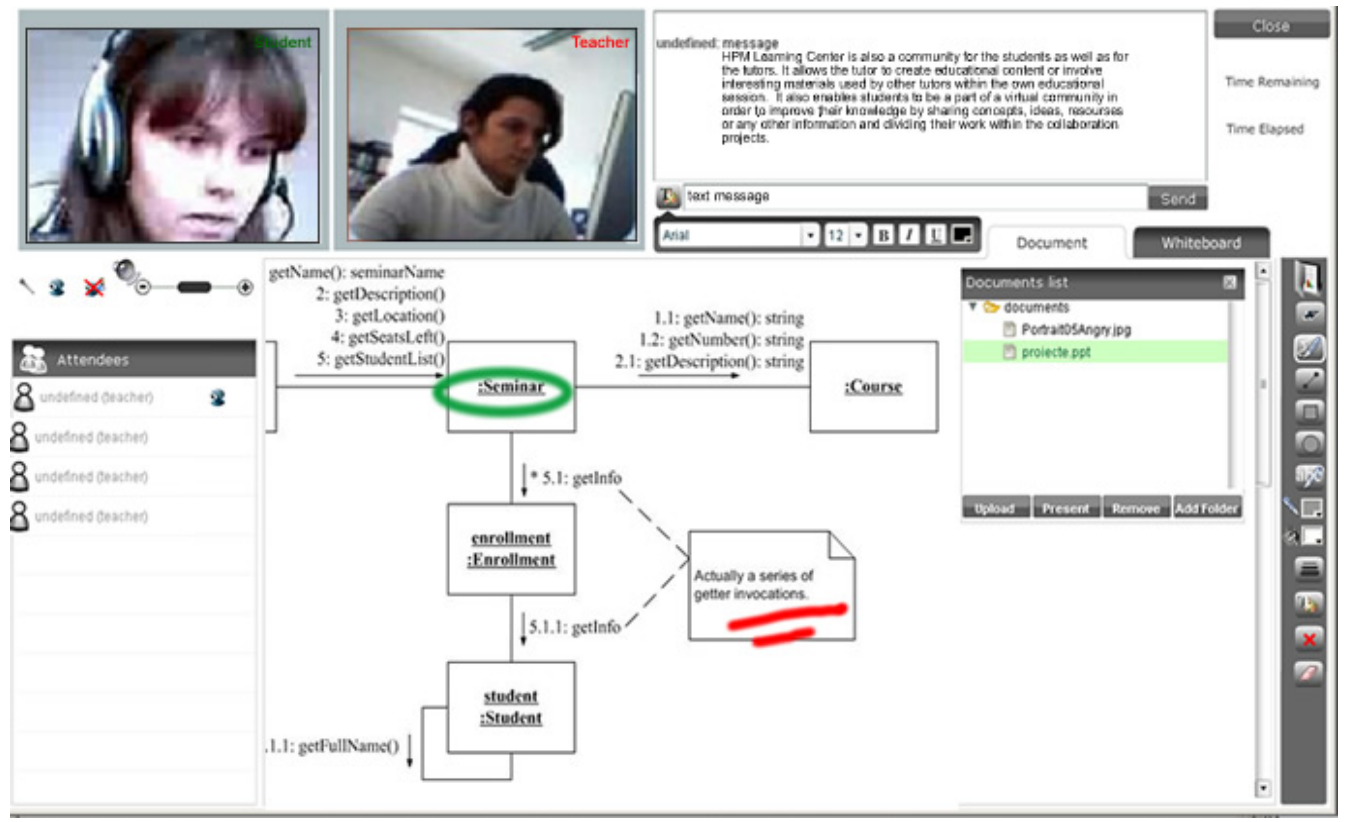

Fig. 7. Collaborative Tool. Teacher module

The system architect included to those Web 2.0 advantages other important aspects that help the teacher within the educational process. The teacher always prefers to present to the class the own materials such as scanned copies of notes, Word/RTF/PDF documents, PPT presentations, even web resources. The prototype allows the teacher to schedule the collaborative session, prepare the content for the class by updating the media library, start 
the class and share the educational content with all the students attending the session. Usually, the teacher needs annotations for explaining more about the content and that aspect is considered as an important feature. The virtual shared space consists of two different tabs, document and whiteboard. The first one allows the teacher to share the resources with the students and make annotations on the document surface using the handwriting capabilities, drawing lines/circles/rectangles or adding text boxes). The whiteboard looks like an empty white page used by the teacher when adding more explanations to the educational material. The whiteboard can be also used in a collaborative session if the teacher decides to lively assess the knowledge of the class by enabling one of the students to have the whiteboard control for a short exercise. The figure 2 illustrates the functionalities implemented by the teacher module.

\subsection{Responsibilities of learning}

The traditional education system as encouraging competition and individual responsibility between students and discourages any interaction, whereas the collaborative learning environments redefine the relationship between students and teachers by creating a supporting environment versus a competitive one. In the traditional system the teacher presents the course materials to the class but the asynchronous collaboration transfers the lecturer's role to that of facilitator and resource guide as CSCL requires that the student takes a more active role in his/her own learning.

The proposed technology supports both traditional and collaborative learning and combines those two concepts in order to allow the teacher to make face-to-face presentations of the educational materials (video communication link and annotation tools), to have full control of the class, monitor students, control project activities (project teams monitoring), etc. The framework also allows the student to actively participate to the course presentation, communicate to the teacher in a real-time manner and collaborate with his/her colleagues for achieving the project goals. An intelligent component dynamically controls the system configuration, elaborates the statistics and analytical reports, bandwidth measurements, server overloading or media library setup.

\subsection{Educational content}

Many of the current handbooks and textbooks are not designed to promote group activities and provide minimal suggestions on how to promote these activities, although a few publishers are now tailoring their books to cater at least partially for collaborated work. CSCL techniques still require the formulation of handouts but these handouts are designed more to create a relationship among the students and provide a basis for them to work together. Since there is very little supporting information, the lecturers themselves may often have to develop this information in the forms of worksheets and any other appropriate materials.

The system architect considers the lecturer's effort and designs a complementary tool that allows the lecturer to dynamically handle the educational content. Two types of educational content are stored into the E-Learning platform: public and private content. If the tutor considers one of his/her materials as really important for the public interest, that material will be uploaded on the server, convert to an internal format (slideshow, e.g.) and stored into the media library as a public material. If the material is private or the tutor has no rights 
to make it public, it will be converted to the slideshow format and then stored into the media library as private. The tutor is able to browse the media library, load it on the shared space (whiteboard) and share it between the students during the educational session. The content is presented within the document surface as in the original editor, see the Figure 2, and both teacher and active student can use the scrolling bar for browsing it, if necessary. The scrolling action automatically generates updates of the remote shared objects and the result is displayed on the document surface of every instance of the virtual shared space, so, it can be viewed by all the participants to the collaborative session.

\subsection{Interaction and integration}

One of the major components of CSCL is the interaction that takes place between students. Teamwork is vital to a successful CSCL environment. The interactivity that takes place such as the giving and receiving of help and feedback, the exchanging of resources and information and being able to challenge and encourage other students is materialized in many research studies elaborated in the last years. There are four main types of interaction: interaction with resources, interaction with teachers, interaction with peers and interaction with both teacher and learner through an interface.

The prototype supports the interactive learning concept and highlights the aspects related to the web presence, "face-to-face" interaction, real-time collaboration, knowledge sharing and multimodal interactivity. Technically, the web presence is illustrated in the student list where all the students registered to the course appear, each one with his/her status (off/online), "face-to-face" interaction happens when the student and lecturer share opinions using the virtual classroom tool, real-time collaboration and knowledge sharing means that tutor and learners share educational content via virtual shared space component and interact with the system that supports drawing and handwriting capabilities. The collaborative session is stored in a multimedia format and the attendees are able to review the educational process using the media on demand functionalities. Interacting with the resources is an important feature when talking about collaborative learning in foreign language. Here, the learners need dictionary or text-to-speech capabilities. The framework allows the student to access five languages dictionaries (English, French, Italian, Portuguese and German) and the TTS component is integrated with the vocabulary and dictionary components see the Figure 8. That way, learners can easily translate words from a language to another one and then render the correct pronunciation in the speakers.

These features help the learner to improve the pronunciation and the communication skills. Drawing and handwriting functionalities make the virtual tool closer to the real teaching process, they allow the tutor to present the educational content like he/she does in the classroom. A project section is developed in order to allow the students to work as a team during the project development period of time. The project supervisor monitors and controls the project development, views the students' activities and provides each student with an objective feedback based on the student's performed tasks. 


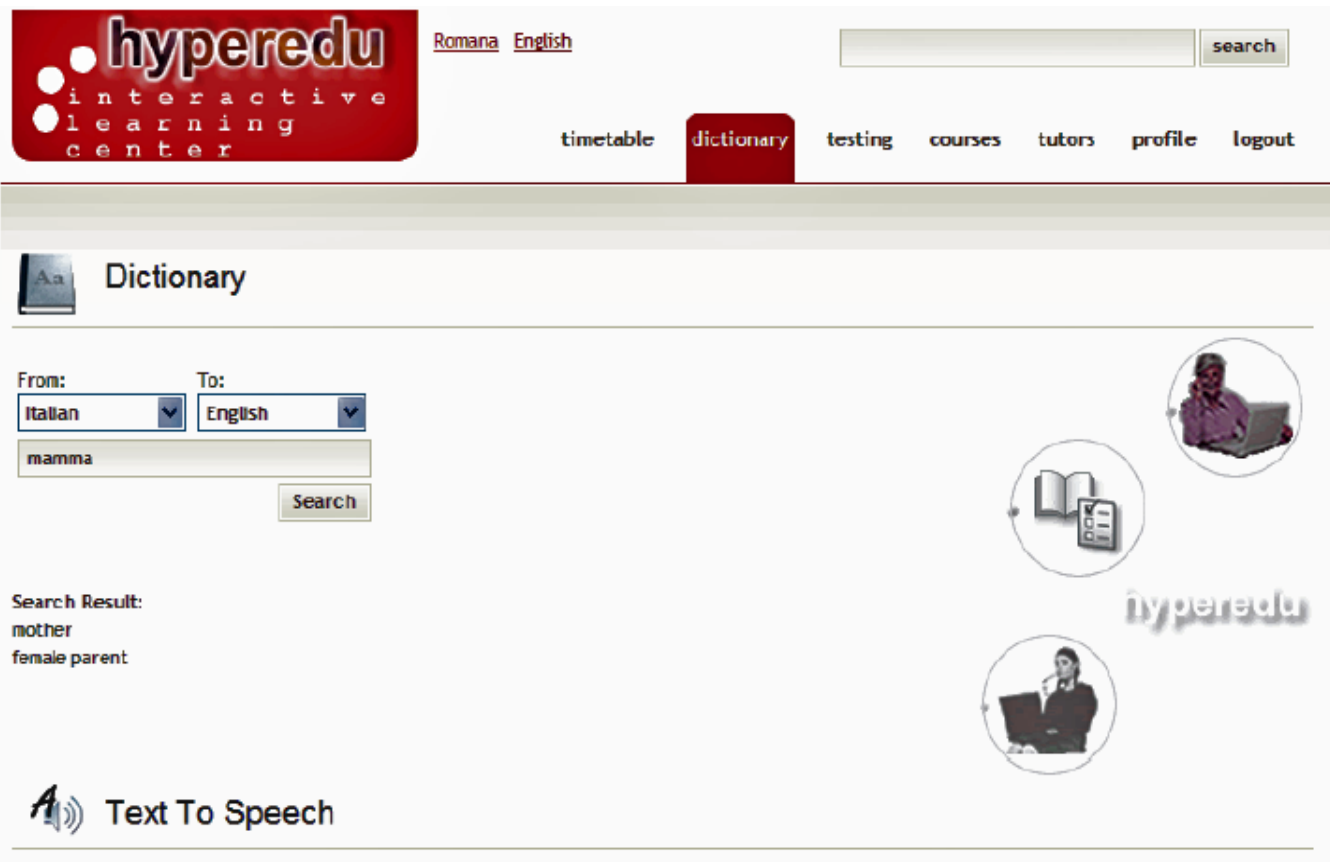

\begin{tabular}{|c|c|}
\hline mother & 슬 \\
\hline START TextToSpeech \\
\hline STA \\
\hline
\end{tabular}

Fig. 8. Student Module - Dictionary and Text-to-Speech Components

Teaching signal processing courses, as (Ambikairajah et al., 2007) illustrated, at any level presents challenges in conveying complex mathematical concepts, retaining the students' attention, and addressing individual student needs in a large classroom, so, only a small percentage of students are able to grasp the key concepts at the time of the live lecture delivery. The other students have to understand the topics presented in their own time and with whatever assistance is available. That way, the recording of rich multimedia information combining video and lecture or dynamically annotated tutorial slides can be used to improve self-directed student learning and offer new possibilities for course delivery. In practice, most annotations serve as attention marks, providing critical linkage between the slide content and spoken context.

The prototype should be able to integrate advanced teaching technologies such as interactive whiteboards, tablet PCs, digital tablet pens, video projectors, etc. within a stable and powerful educational environment that supports blended learning services. The teacher is able to manage the educational process using two different components integrated each other. The virtual classroom component enables the teacher to remotely conduct the class and the whiteboard component that allows the lecturer to present the educational content in a traditional way. The students can opt for both learning services being in the classroom to be able to lively attend the teacher's exposure or to virtually participate to the learning process via web. 


\section{Virtual Medical Community}

Each physician can share the knowledge and experience with other colleagues in the own country or abroad and the proposed telecommunication infrastructure provides with real advantages in that way. The physician in a small hospital or medical studio that investigates a patient can meet difficulties and he/she needs a specialist's opinion (the opinion of his/her professor in the university or even the opinion of his/her colleague from another department) before the conclusion. The young physician on guard has to investigate an unusual case and he/she needs the opinion of his/her professor/head of department but a simple phone call is not enough for offering all the medical information related to the patient.

The multimedia capabilities allow the physicians to build their virtual community as a subnet where the friends and colleagues with the same interests share ideas, concepts, methodologies and experiences or cases in an interactive manner. For example, the live streaming from the echograph when diagnosing the patient can be shared between the physicians in order to increase the investigation efficiency. Video telephony online focus group functionalities are implemented for improving the communication capabilities.

\subsection{Physician's point of view}

The physicians use the interactive environment as a simple tool not a sophisticated one. The physician is focused on the patient not on the interactive tool. He/she needs the communication tool if meeting a strange case, the physician is in crisis and the patient can be scared. For that reason, the communication has to be transparent but not scaring the patient. The communication sessions between the physicians can be realized in the front of the patient or not and the system must support both of the video capture and live streaming capabilities. When the physician seems to have problems when diagnosing the patient, the video capture component will be used. The system captures the video data from the S-Video output of the echograph and stores the data on the media server. The video capture will be shared within the communication session (on the shared space) between the physicians but the specialist can ask the physician for a new echography and the live streaming component will be used.

\subsection{Specialist's point of view}

The specialist can be located in the same hospital the physician performs his/her activity, in another hospital, in the university, at home, or abroad. The specialist is notified by the system about one colleague/friend in his/her community requires assistance. The notification can be via email or SMS if the specialist is off-line or using the internal messaging if he/she is on-line. The specialist can access the system using the PC or Pocket PC and he/she can also invite another specialist to assist if necessary. When assisting the physician, the specialist asks for the video sequence captured from the echograph. If this video sequence is not enough for the diagnosis a new echography can be done. That way the specialist seems to remotely investigate the patient asking him/her a few details about his/her status during the live echography. After the new echography the physicians can share the captured video sequences of the new echography and shared their opinions. When the specialist is not in the front of his/her PC and accesses the system via GSM/UMTS/WLAN network the Pocket PC is used. The quality of the new echography 
will not be the best one and sometimes the specialist cannot grant his/her solution is the best because of the live video streaming quality: view component for the Pocket PCs is $240 \mathrm{x}$ 240 pixels and the details are not visible. The opinion of another specialist is helpful in this kind of situations and the small group meeting component allows the physicians to communicate and exchange information in an interactive manner.

\subsection{Multimedia capabilitie within the virtual medical community}

The physician meets the problem when diagnosing the patient, launch the application, connect the echograph to the client module and record the video sequences of the echography. The client block dynamically setup the video capture parameters according to the bandwidth between the client and server machines and starts capturing till the end of the echography. At the end of the echography, the physician is able to review the frame sequence, mark the important zones using the marker or the handwriting and save the media file on the server.

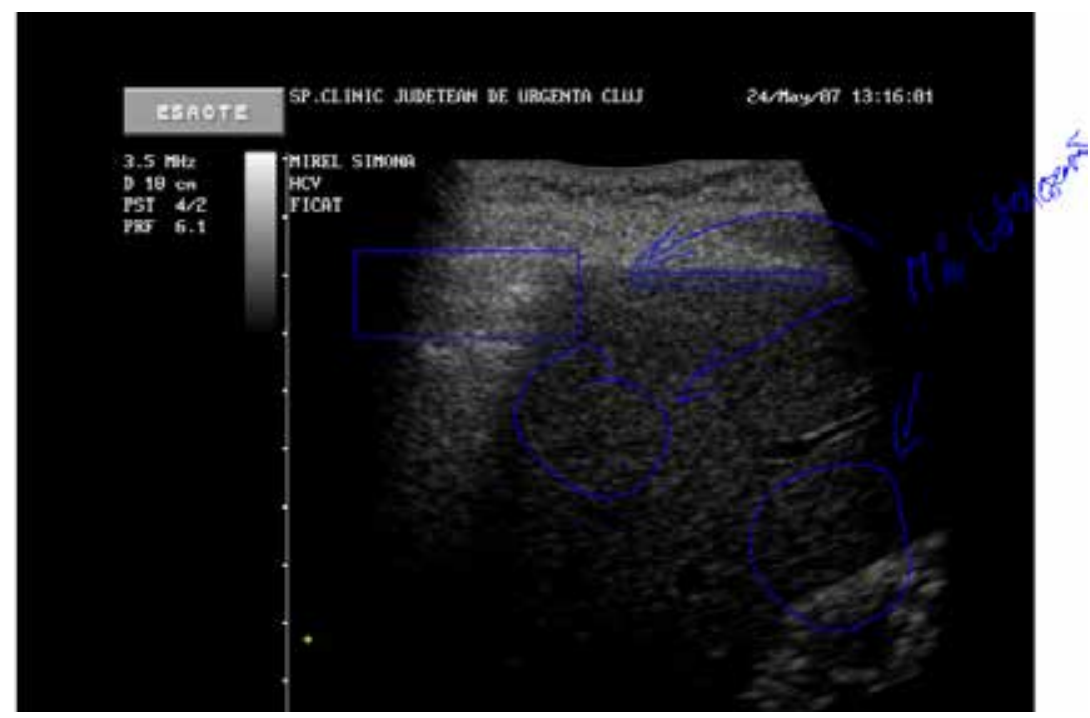

Fig. 9. Collaborative work during the liver echography

The remote shared objects concept is used in order to implement the shared space component. One client component in the system changes a property of its copy of an remote shared object, the updates are sent automatically to all the other client blocks sharing the same object.

\section{Advances in Administrative and Security Services using Collaborative Tools}

The prototype highlights the most important advantages of a heterogeneous telecommunication system designed for improving the administrative and security services by using the media capabilities such as collaborative work, image and video capture from the mobile devices, server-side real-time collaboration, media content retrieving and 
delivery. The concept proposes some of the most popular technologies for implementing the client- and server-side components of a simple but very useful collaborative work environment: Java/J2ME, Adobe and PHP technologies.

The collaborative tools are dedicated to the administrative and security domains and allow the end-users to synchronously/asynchronously communicate each other or in group for achieving their goals (find solutions for complex situations). As a short scenario, the security agent in area takes photo(s), using the mobile phone and the media content will be automatically uploaded on the media server. The person in charge is notified about a possible problem in area, so, the system allows him/her to have a real image of what is happening there providing with new elements (images or video sequences) accessible from the media library. If necessary, the person in charge can contact the commander for a suggestion/solution related to the situation illustrated by real images/video sequences. The digital resources stored into the virtual library are editable, that way the person in charge can easily confirm with the solution just annotating the part of the image/video frame being interesting. The system automatically sends the marked picture back to the agent in area in order to help him/her for taking the correct decision.

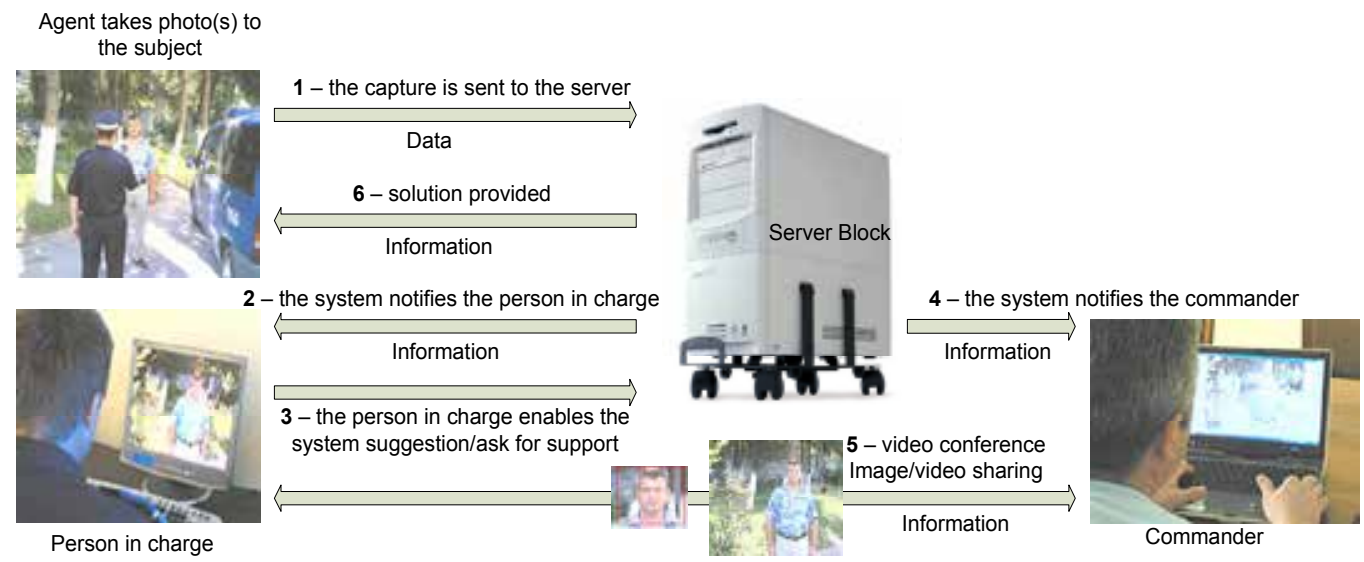

Fig. 10. Collaborative tools in administrative and security areas. Flow chart.

The multi-channel access is considered the only one solution for implementing the functionalities described in the scenarios from the Introduction. The explanation is very simple: the agent in the area uses the mobile device in order to communicate to headquarter and the persons in charge has to share the processing results using the web browser, a desktop application or a mobile device.

This heterogeneous system involves some of the most popular technologies: J2ME for the mobile area, PHP and Flash for the web components, MySQL as the database server. In fact, the authors have been constrained to use those technologies that offer the following advantages: J2ME MMAPI/AMMS API provides the developer with video capture capabilities and MIDP I/O package offers networking features that extend the resources available on a network into the mobile space; PHP components implement the tool management, the authentication functionalities and invoke the processing block; Flash offers excellent multimedia capabilities: 2D shared graphics, audio/video support, etc. 


\subsection{MIDP-based client application}

The agent has to prove the situation to headquarter by taking photos and recording short video sequences related to the status in area. When using a mobile device, the agent, in different situations, has the advantages of the mobility and real-time processing. The MIDPbased client application must assure an image/video quality necessary for the processing but it is also responsible for the data communication - the best quality of the video sequence or image means big amount of data transmitted via GPRS/EDGE. This fact will be avoided and, for the first stage, the image snapshot will be realized using a normal resolution about $640 \times 480$ pixels. That way, the upload is done in some seconds and the space allocated into the media library will be about $50 \mathrm{~KB}$. More, if trying to record a video sequence related to the situation in area, the agent will actually take snapshots about 1 frame per second and the application automatically uploads them onto the server. The server-side components receive the frames, one by one, and then put them together as a video sequence at $1 \mathrm{fps}$. The system architect opted for that approach because a video sequence at 320x240 is not relevant and a bigger resolution means big amount of data impossible to upload in real time.

From the users' point of view, the system is transparent - the agent takes the snapshots and presses the button OK, then puts the mobile device in his/her pocket and waits for the feedback. In fact, the client application manages the data transmission until receiving the acknowledgement. An HTTPConnection instance will be used for opening a connection to the PHP upload component and sends the data packets.

\subsection{Advantages of the proposed solution}

The prototype provides with the following advantages. An asynchronous communication link is established between the headquarter and the agent in area. That way the agent is able to illustrate a real image of the situation created. The agents have the video control and monitoring of important objectives and the multimedia insformation illustrating the real image of the objectives can be stored into the virtual library. The decision making is easier to be realized when the headquarter has a real image of a special situation in area without going there and losing time. The system supports low cost equipments (normal mobile devices, web cams and headsets) and provides with high quality of work (high results/investments report). The telecommunication infrastructure respects a low cost architecture: normal internet connection and workstations/laptops for accessing the services via web and GPRS/EDGE mobile devices for the mobile area. Virtual library contains proofs that consist of authentication elements and the time they have been realized. All of those can be used for future researches, if necessary.

\section{Conclusion}

In the context of the crisis and time and cost constrains, teleconferencing and web-based meetings increased with $40 \%$ but the travel costs decreases with $31 \%$, in the last months, accoding to the IEEE Spectrum, February 2009. So, there are interests in using collaborative tools but the technology is not very friendly. More, the professional conferencing systems (POLYCOM, e.g.) are really expensive but limited. The limitations especially regard the service access - the end-user needs a conference room, TV/access equipments in each access place, etc. Other issues are related to the resource sharing because usually the conferencing attendees are talking about real aspects such as management issues, marketing aspects, 
commerce (negotiation) problems, so the resource sharing capabilities are very important. The developers need a generic framework that allows them to easily build the own collaborative environment and customize it according to the domain rules.

The presented framework is dedicated to the business, education and administrative/security domains and enables the web masters to integrate the collaborative tools within the own web portals and sites as widgets. It helps the E-Service providers to fix a set of issues met in the different domains such as: IT\&C experience, time management, responsibilities in E-Services, interaction or interactivity. The goals for real-time collaboration technologies are straightforward, for that reason, the authors must add some other important aspects to the ones already enumerated.

As described in detail in that chapter, Internet-Supported Collaborative Work solutions must be affordable, available, scalable, supportable, and manageable.

Affordable: Real-time collaboration total cost ownership (TCO) tends to exceed projections due to the high costs of maintaining a globally accessible real-time communications infrastructure. Linux offers a really high uptime and the system components work properly on a Linux server machine. In conclusion, the framework can be considered affordable.

Available: The TCO of real-time collaboration must include the cost of downtime. As virtual collaboration becomes more strategic to the business, educational, or administrative processes, the cost of downtime increases. A cost effective collaboration solution must therefore be highly available.

Scalable and high-performance: The end-users increasingly demand sophisticated real-time collaboration functions such as rich multimedia. These video/ voice/data requirements, coupled with performance and reliability challenges posed by the variability of Internet access (including dial-up links, ISDN, Wi-Fi, satellite, and other bottlenecks) and the need to support large numbers of attendees, place stringent architectural requirements on collaboration solutions. The novel methods of virtual collaboration discussed in that chapter have gained excellent acceptance from attendees and have shown potential for increasing their understanding beyond the level provided by traditional methods.

\section{References}

Crispim, J.A. \& Pinho de Sousa, J. (2007). Multiple criteria partner selection in virtual enterprise, Proceedings of the 8th IFIP Working Conference on Virtual Enterprise, Guimaraes, Portugal, September 2007

Camarinha-Matos, L.M.; Oliveira, A. I.; Ratti, R.; Demsar, D.; Baldo, F. \& Jarimo, T. (2007). A computer-assisted VO creation framework, Proceedings of the 8th IFIP Working Conference on Virtual Enterprise, Guimaraes, Portugal, September 2007

Shaozi, L.; Zhongpan, Q.; Tangqui, L. \& Huowang, C. (2003). Architecture of computer supported collaborative learning based on EJB middleware and it's implementation, Proceedings of the $8^{\text {th }}$ International Conference on Computer Supported Cooperative Work in Design, pp. 142 - 148, Vol.1, 2003

Tan, C. \& Chan Y. Y. (2008). Knowledge Community: A Knowledge-Building System for Global Collaborative Project Learning, Proceedings of the IEEE, Volume 96, Issue 6, pp. 1049 - 1061, 2008 
Williams, S. \& Roberts, T.S. (2002). Computer-Supported Collaborative Learning: Strengths and weaknesses. Proceedings of the International Conference on Computers in Education, pp. $328-331,2002$

Ambikairajah, E.; Epps, J.; Sheng, M. \& Celler B. (2007). Tablet PC and Electronic Whiteboard Use in Signal Processing Education, IEEE SIGNAL PROCESSING MAGAZINE. pp. 130 - 133, 2007

Tapiador, A.; Fumero, A.; Salvachua, J. \& Aguirre, S. (2006). A Web Collaboration Architecture,

Proceedings of the CollaborateCom 2006 International Conference on Collaborative Computing: Networking, Applications and Worksharing, pp. 1-4, 2006

Miller, L.D.; Eck, A.; Soh, L.-K. \& Hong J. (2007). Statistics and analysis tools for a computersupported collaborative learning system, Proceedings of the $37^{\text {th }}$ ASEE/IEEE Frontiers in Education Conference, pp. F3J-1 - F3J-6, 2007

Yao, Q.; Cui, L. \& Wang, H. (2007). Toward Cooperative Designing of Customized Business Process in Web Service Environment, Proceedings of the CSCWD 2007 11th International Conference on Computer Supported Cooperative Work in Design, pp. $258-263,2007$

Wang, H.; Zhang, H. \& Liao, L. (2007). A Service Oriented Paradigm to Support Collaborative Product development Proceedings of the CSCWD 2007 11th International Conference on Computer Supported Cooperative Work in Design, pp. $240-245,2007$

Ader, M. (2001). Technologies for the Virtual Enterprise, Excellence in Practice, Volume III Innovation and Excellence in Workflow/Process Management and Document/Knowledge Management, Fischer L. 360 pages. E-Workflow Pub House.

Laso-Ballesteros, I. \& Prinz, W. (2006). New Collaborative Working Environments 2020, Report on industry-led FP7 consultations and $3^{\text {rd }}$ report of the Experts Group on Collaboration@Work. European Commission Information Society and Media. Bruxelles, 2006

Fain, Y.; Rasputins, V. \& Tartakovsky, A. (2007). Rich Internet Applications with Adoble Flex and Java. Secrets of the Masters. $1^{\text {st }}$ Edition. 736 pages. SYS-CON Books 


\title{
Integrated medical system using DICOM and HL7 standards
}

\author{
Orza Bogdan ${ }^{1}$, Cordos Alin², Vlaicu Aurel ${ }^{1}$ and Meza Serban ${ }^{1}$ \\ Technical University of Cluj Napoca ${ }^{1}$ \\ Pixeldata ${ }^{2}$ \\ Romania
}

\section{Introduction}

Informational systems and the standardization of data transfer protocols have become an important part of nowadays medical world. Software applications transformed themselves into an indispensable tool for the specialist engaged in the medical act. In this sense, the use of information and communication technology has influenced greatly the fast and reliable (also secure) transmission of medical data throughout informational systems, shortening the required processing times.

The increasing use of IT\&C in the medical world also demanded for new protocols that would facilitate data transfer (between different software application) and data storage in a common format. Likewise, several organizations have undertaken this difficult task of medical digital data standardization, among which the National Electrical Manufacturers Association (NEMA) and American College of Radiology (ACR) are the most important ones. Their efforts concentrate mostly in the development of the DICOM (Digital Imaging and Communications in Medicine) standard. It has all started in the 1970's with the increasing use of CT scanners and other image-based diagnostic devices that opened the way for large - scale deployment of software applications in the medical field and thus the need to interface machines and applications manufactured/produced by various companies was born. This translated into the need to specify a common image format for all medical imaging devices.

In 1983, ACR and NEMA delegated a committee to solve this problem and propose a standard that would allow the following:

- Data exchange concerning generated digital medical images between devices produced by different manufactures;

- The development of PACS (Picture Archiving and Communication Systems);

- The development of medical databases able to be interrogated using (geographically) distributed software applications;

In 1985 version 1.0 of the DICOM standard was published by ACR and NEMA, followed by 2 revisions in October 1986 and January 1988. Version 2.0 of the standard was issued also in 1988 and added a set of commands for displays, by introducing a new image identification scheme based on "data-elements" for a better characterization of the image parameters (NEAC, 2008). 
The last published version, 3.0, issued in 2000, contains a large number of changes and additions with respect to the previous ones. The DICOM standard facilitates medical imaging equipments inter-operability by specifying:

- A set of protocols that compliant devices must withstand;

- The commands' semantics and syntax as well as the associated information format that can be transmitted using the protocol.

In a heterogeneous system, in order to integrate medical equipments supplied by various manufactures, the use of DICOM and HL7 standards is compulsory. DICOM, as previously presented, is mainly dedicated to medical imaging, whereas HL7 (Health Level Seven) covers more general aspects of medical digital data processing and management. HL7 is used for transmitting data related to patient charts, files and other associated documents and audio recordings. The number " 7 " refers to the "application" layer, the 7 th one from the OSI (Open Systems Interconnection) model system representation (HL7, 2008).

The HL7 standard was first published in 1987 by a group of companies manufacturing medical equipment. Version 2.0 succeeded in 1988, followed by versions 2.1, 2.2, 2.3 and 2.3.1 in 1990 to 1999. In 1994 ANSI (American National Standards Institute) officially recognized it as an industry standard. Currently, version 3.0 is on its way, with a draft already released.

The main objective of the HL7 standard is to produce a set of specifications that allows free communication and exchange of data between medical software applications in order to eliminate or reduce incompatibility among different applications. To achieve this, the following measures have been proposed:

- the standard must support information exchange between systems implemented in a large variety of development environments (technical) and communication environments. Its implementation must be possible in all the major existing programming languages.

- immediate, single transaction, transfer must be available in the same time as file sharing/transfer based on multiple-transactions;

- the highest degree of standardization must be obtained when compared to the most often encountered cases of elements formatting; the standard must comply with the specific necessities of each medical field. Accordingly, the standard comprises situation specific tables, definitions and segments that can be customized (Z-segments)

- the standard must cope with variations suffered in time due to inherent technical progress and evolution;

- the standard evolution must be based on the experience already gained and on already existing and well known industrial protocols. Favors given to certain producers or companies must be avoided by all means.

- HL7 makes no presumptions related to the architecture of the medical informatics system, and does not try to resolve the architectural differences present in medical informatics systems. Due to this reason, HL7 cannot have a "plug and play" interface.

- a first interest of the HL7 workgroup was to use the standard as soon as possible. Once published, HL7 was voted and recognized as a standard by the American National Standards Institute (ANSI) and the Accredited Standards Organization (ASO). 
- currently, the cooperation with other standardizing organization from the medical field (ACR/NEMA DICOM, ASC X12, ASTM, IEEE/MEDIX, NCPDP, etc.) has become a priority for HL7 and focus on a better development of medical informational systems has contributed to the group's joining to the ANSI HISPP (Health Information Systems Planning Panel) process, ever since its debut, in 1992. The two standards, DICOM and HL7, form the basis of the informational integration of software based medical processes. In November 1998, Healthcare Information and Management Systems Society (HIMSS) and Radiological Society of North America (RSNA) founded the Integrating the Healthcare Enterprise (IHE) forum, with the declared goal of helping the integration of software application from various medical fields and domains. Its main objective is to ensure that in the course of the medical act all the information necessary in the decision making and taking processes are accurate and available in time for the medical specialist. Its purpose is not to define new standards, but to promote the use of the existing ones, namely DICOM and HL7. Currently the main focus is on radiology. The DICOM and HL7 standards provide the necessary means and technology for developing software applications, while IHE supervises their adoption into real-life medical world. IHE provides support for the medical software applications users by ensuring a better access to information and eliminating, as much as possible, confusions or misunderstanding when acquiring such applications.

From the medical software application development point of view, the IHE specifications facilitate fast and safe releases of new products as well as simple mechanisms for implementing interfacing options with other, already existing ones.

\section{Using the HL7 and DICOM standards in medical informatics}

\subsection{General overview of the HL7 standards}

The HL7 standard addresses software developers and medical equipments manufactures with the declared goal of unifying the way the information present in medical units and institutions is transmitted, exchanged and/or stored, based on a common format, agreed by all the parties involved. There are other standards dedicated to the medical sector, each having a very well defined domain and focus: pharmacy, medical devices, medical imaging, and insurances. HL7 is, on this matter, dedicated to the processing and management of administrative and clinical data (HL7, 2008). The HL7 focuses on the following fields/domains:

- Patient management - admit, discharge, transfer patient (ADT);

- Queries, resources (rooms, beds, devices, etc.), patient scheduling;

- Scheduling of medical procedures, results, clinical trials;

- Financial administration;

- Medical documents;

- Medical records;

- Medical treatments;

Taking into considerations the great variety of applications involved in the process of the medical act and the requirement that these have to exchange information/data between them, it is obvious that many of such communication interfaces would greatly benefit from using a standardizes approach. The HL7 standards comes exactly to solve this problem and ease the burden of message passing and data exchange between various applications by providing a very precise structure under which this must happen. 


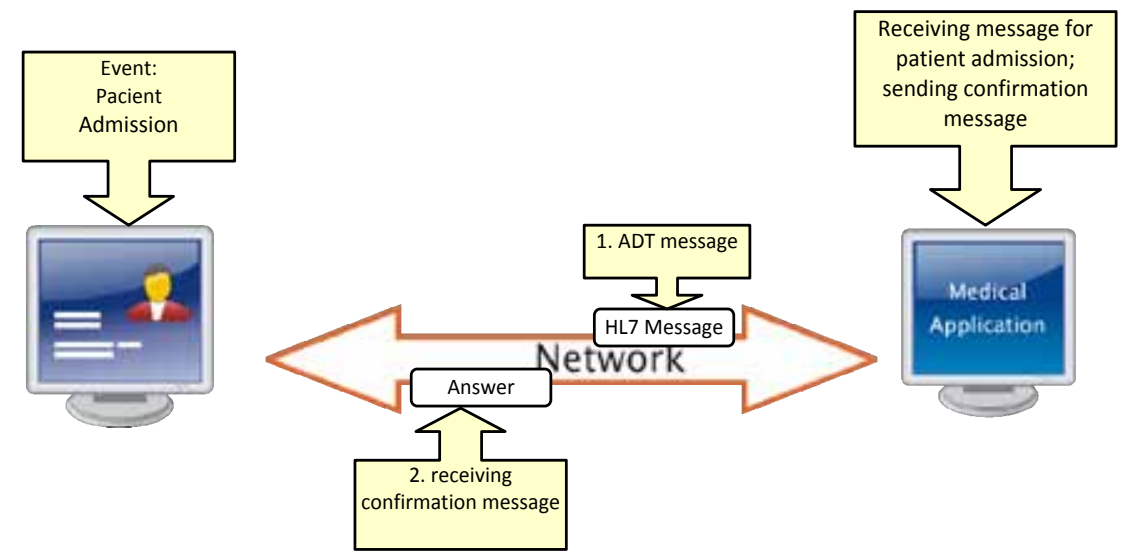

Fig. 1. Example of using HL7 messages

A medical application that uses the HL7 standard sends to another application a "HL7 type" message generated as a result of some medical event occurring in the current activity: admit patient -A01, transfer patient - A02, discharge patient - A03, etc. If the application receiving the messages also complies with HL7 regulations, then it can be certain that there will be no missing information, all information received will be interpreted the right way and a proper response will be issued back. Thus, the exchange of information made is coherent and efficient (HL7, 2008).

The HL7 standards define a number of messages that cover all activities specific to medical units. A HL7 message is made up of segments, fields, components and sub-components and is characterised by the message type (a 3 character code). Message types are organized by different domains/fields (e.g. admit, discharge, transfer, clinical trials scheduling, etc).

\subsection{HL7 scenarios}

Figure 2 presents various scenarios that make use of the HL7 standards inside a single hospital/medical unit and also among several hospitals. In a hospital, information is managed by an application generically named HIS (Hospital Information System). Using this all other activities taking place can be monitored and controlled. The application thus has different interfaces that allow communication with all the existing sub-systems, like the imaging center (RIS - Radiology Information System), financial department, human resources, pharmacy, etc. In order to connect to the "outside world" a web-based application is used. This allows reading and writing of information in the hospital data base. As it can be seen in figure 2, HL7 enables the communication among different software applications existing in the hospital, each developed by various/different companies.

For a better communication between the hospital and other different medical institutions, the HL7 standard can be used to perform data exchanges. It is enough for the informational systems in these institutions to have a HL7 compatible interface.

The HL7 standard is used for exchanging information related to patients, doctors, resources and documents between hospitals. At this level there are also data synchronization protocols that ensure data persistence among applications. By using the HL7 standard, this data exchange can be performed independently of the software solutions, operating systems 
or data base management systems running at each institution. What is important is the information contained by the HL7 message.

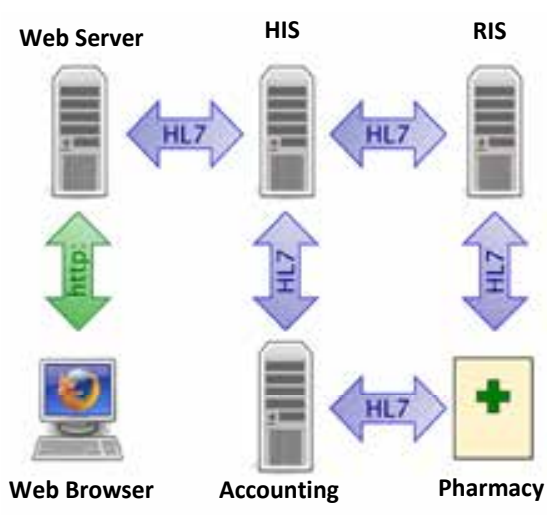

a)

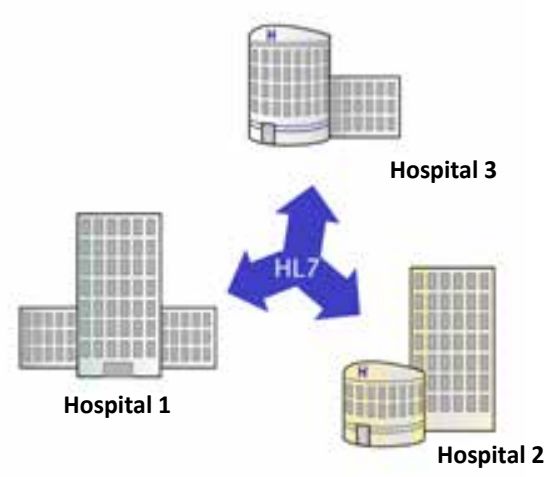

b)

Fig. 2. Example of using HL7 standard: a) inside a hospital b) between hospitals

The major benefit of standardization is represented by the informational integration. The benefits that HL7 brings to the medical system are that of having the possibility of accessing the medical information/data and process them independently on the particular solution chosen by each institution. Thus, it has no importance who the provider for a certain software application is, as long as this is compliant with the standards' specification, and it limits by no means the choices existing in acquiring other systems as well (they do not necessarily need to be developed by the same company in order to work well together).

\subsection{HL7 - Clinical Document Architecture CDA}

HL7 CDA is an XML-based standard that specifies the structure and semantics of clinical documents having in mind the exchange of such data. Previously known as PRA (Patient Record Architecture), CDA provides a model for clinical document interchanging, such as "epacris", "evolutions", etc. and makes it possible for the integration of the information technology in the medical field. CDA allows for the clinical documents to be compatible to the informatics field by using XML, HL7 RIM (Reference Information Model) and medical informatics codes but also tries to maintain a high degree of understanding and assimilation by the medical staff. The content of CDA documents can be viewed using web browsers (due to the fact that they are based on XML).

The CDA standard is based on the following concepts:

- CDA documents are based on the XML standard (Extensible Markup Language);

- The structure of the CDA documents is based on the HL7 reference model named RIM (Reference Information Model) and the HL7 data types;

- The CDA specification are very flexible and are structured on 3 levels:

- Document level;

- Section level

- Entity level

- A CDA document is a completely defined object and can include text, images, sound recording and other multimedia components; 
HL7 CDA clinical documents contain observations and services and have the following characteristics/attributes:

- Persistency: continues to exist in an unaltered state for a well-defined period of time:

- Organization: maintained by an organization entrusted with this task;

- Possibility to certify: contains a set of information that can be certified;

- Context: sets a predefined value;

- Integrity: the authentication of a clinical document is made as a whole and is not applied to parts of the document without taking into account the whole context;

- Readability: the possibility of being read. Guaranties that the obtained CDA document can be easily viewing using a web-browser.

\subsection{The DICOM standard}

The DICOM (Digital Imaging and Communications in Medicine) standard was developed with the purpose of helping the distribution, display and storage of medical images (CT, MRI, US) in mind. The standard is more than just a file/image format, being in fact a set of standards that describe the way digital data used in medicine can be transferred, stored and displayed.

The DICOM standard has a series of advantages (Oleg S. Pianykh, 2008):

- Is a universal standard. Generally, all medical equipments acquiring medical images support this standard and communicate among them using it;

- Images are stored at a higher quality. DICOM supports:

○ JPEG compressed images: lossless JPEG compression for 8, 10, 12, 16 bits gray levels, or 24 bit color images, or lossy JPEG compression for 8, 12 bits gray levels or 24 bits color images;

- JPEG 2000 compressed images: lossless or lossy JPEG 2000 image compression for 8-16 bits gray level or 24 bits color images

- RL (run-length) compressed images

- Uncompressed images (bitmap)

- Allows the storage of additional information related to the image acquisition parameters. DICOM can store besides the actual 2D image additional information, such as: the patients 3D position, physical size of the objects present in the image, slice thickness, exposure parameters, and others. These are used for a better later processing and interpretation.

- Support for full storage of medical data. The DICOM files and messages support more than 2000 standardized attributes that maintain patient's medical data and images

- Images are acquired and stored using parameters that are device independent. Likewise, DICOM images can be processed without taking into account the actual device used in the acquisition process. 


\subsection{IHE (Integrating the Healthcare Enterprise) specifications}

IHE is an initiative dedicated to stimulating the integration of informational systems present in modern health care institutions. Its main objective is to guarantee the fact that all the information about the patient and its state necessary in the treatment and health care process are correct, accurate and available in time for the medical staff. With more than 450 billion people ensured in 27 countries, Europe represents a unique challenge for IHE (www.ihe-europe.net, 2008). IHE functions as a forum that aims at integrated all the existing informational systems present in the medical field. The IHE objectives are (O. Pianykh,2008):

- To describe the problems and their solutions concerning software application integration;

- To instrument best-practices cases and manuals for the deployment of the existing standards deployment

- To deliver an optimal support for the informational flux

- To integrate the IT-based solutions in the medical field.

IHE identifies a subset of functional components from a medical informatics system and describes its interactions using a set of transactions. The actors and the transactions described in the IHE specifications are abstractions of the medical system present in a hospital. The reason behind introducing these actors and transactions is to be able to define the interactions between the entities forming a medical informatics system.

The actor - represents the role assumed by an entity of the system. The main actors defined according to the IHE specifications are: image acquisition devices, patient registration systems, cost calculation systems for billing the performed medical procedures, medical examination management systems, image, medical reports and associated information visualization systems, image storage management and archiving systems, medical reports management systems, medical reports visualization systems, medical images printing servers (DICOM Print Server) and the financial administration systems.

Transactions are interactions between actors that transfer the required information through standards-based messages.

The integrating profiles offer a common language that healthcare professionals and vendors may use in communicating requirements for the integration of products. Integration Profiles describe real-world scenarios or specific sets of capabilities of integrated systems. An Integration Profile applies to a specified set of actors and for each actor specifies the transactions necessary to support those capabilities.

The DICOM and HL7 standards provide the necessary means and technology for the medical software applications development, while the IHE ensures the specifications needed for implementing these into the real-world. IHE specifications include the following equipments and medical software applications:

- Informatics systems that ensure patient and resource management for medical centers (HIS, RIS);

- Imaging and investigation equipments from the medical field (MR, CT, US, CR, DX, etc.);

- Software applications from the field of medical imaging (PACS, Review Stations, Reporting Systems);

- Other equipments from the medical field (Printers, Imagers, etc.). 


\section{Patient management in medical imaging centers}

Patient and resource management in a medical imaging center is provided by informatics systems generically named RIS (Radiology Information System). From the software point of view these can be classified as ERP (Enterprise Resource Planning) application.

The main functions of RIS informatics systems are: patients registration, examination scheduling, automated distribution of documents, the possibility to operate on more imaging centers in the same time, data base access management, document scanning and storing, radiological scanning library management, imaging center resource management (medical equipment, cameras, available procedures), automated cost calculation and billing associated to the medical act, patients medical insurance policy management, management of the individual procedures undertaken by each patient, patient history management, patients allergy evidence management, avoiding procedure incompatibilities (warnings).

One of the main components of such a system is the PACS server (Picture Archiving and Communication Systems). The PACS is a system (comprised of both hardware and software) meant for storing digital medical images using the DICOM standard. All PACS must be compliant with the DICOM standard. All medical image acquisition devices (CT, RMN, US) store the generated DICOM images in a PACS, from where are than later on available for display using DICOM viewers.

Communication interfaces are used for exchanging data between the RIS informatic system and adjacent software applications. They are compatible with the HL7 standard and use the TCP/IP communication protocol. All interfaces are compliant with the HIPAA (Health Insurance Portability and Accountability Act) regulations. Some of these interfaces are designed based on the IHE specifications. Figure 3 describes the connection between the RIS server and other medical informatics systems (HIMSS and RSNA, 2005).

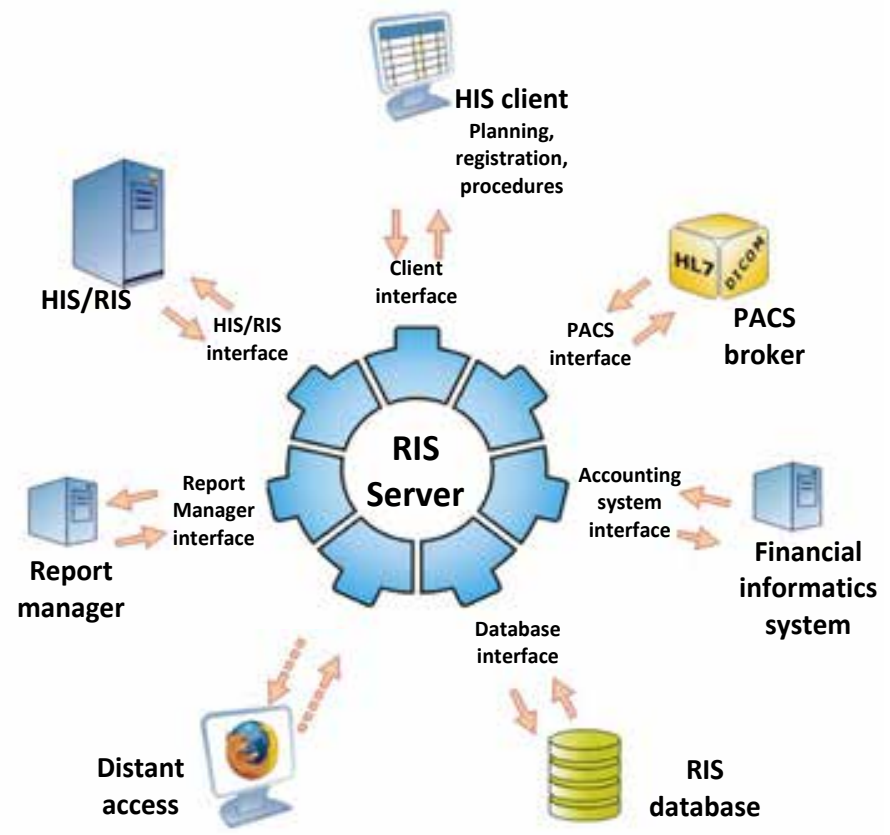

Fig. 3. Communication interfaces of the RIS server 


\subsection{The communication interface with the HIS application}

This is an interface compatible with the HL7 standard and the IHE (Integrating the Healthcare Enterprise) recommendations and is bidirectional. Through this interface information regarding the imaging center like: admission of new patients, patients relocations in beds, patient discharges, treatment changes, calls for further investigations, information regarding the medical staff, the degree of deployment of the medical resources and equipments, etc. can be exchanged. The HL7 messages exchanged by this interface are:

- Messages referring to patient admission, discharge or transfer;

- Messages referring to medical examinations

- Medical reports - for this type of action one single type of message is used, which includes the original medical rapport as well as latter additions to it;

\subsection{The communication interface between the RIS and the report management application}

This is a HL7 and IHE compatible interface and is used by the report server application to send the results for the procedures that have been made by the equipments in the imaging center to the RIS. Also through this interface the report server updates the information regarding patients and procedures. The RIS informs the report server that there are procedures that have ended and a report is required for them.

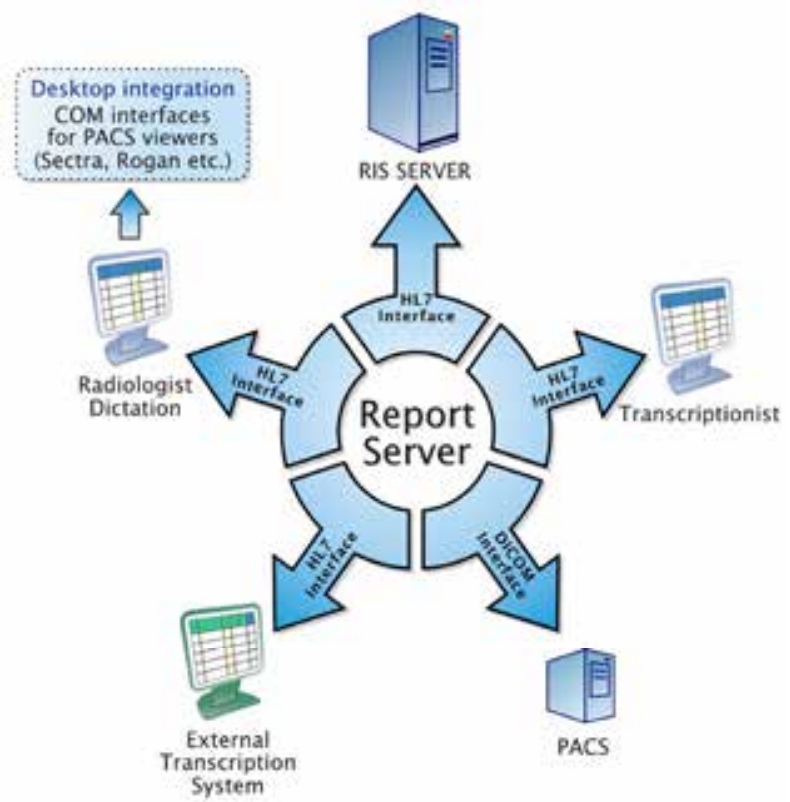

Fig. 4. System for management of medical reports

The management of reports in an imaging center is ensured by a suite of software applications made up from a server and two client applications (fig 4.). All communication between the 3 is based on HL7 messages. The three applications forming the report management system are (note that the medical report is the result of an image based investigation performed by the radiologist): 
a) The Report server - ensures the interfacing between the clients and the data base as well as the security and integrity of the data stored in the data base. The automated and manual distribution of reports is made with the help of the server application. This also maintains a permanent connection to the RIS server, updating continuously (as quickly as possible) the information referring to patients, allergies, diagnostics, procedures and the medical staff.

b) The Radiologist client - is used by the radiologist in order to record or write the medical report based on the examination of the images retrieved from the PACS server. This application can call other COM interfaces belonging to other software programs dedicated to medical images visualization (Alin Cordoş et al., 2008)

c) The Translating client - is an application dedicated to the translation of the spoken medical reports, stored as .wav files, to written form (text file). After formatting, the report is send back to the radiologist for verification and signing.

\subsection{The WEB server interface}

This is again a HL7 compatible interface destined to connect the WEB servers with the RIS. A special WEB application running inside an internet browser can receive the information from the RIS through the WEB server. Also, through this application one can inquire for a new investigation for a patient, find out available time slots in the centers' schedule, and in the same time check the status of a selected procedure.

\subsection{The RIS client communication interface}

Through this HL7 compatible interface the RIS client can perform the following tasks:

- Patient recording;

- Patient admission/discharge/transfer

- New investigation inquiry

- Manual or automatic patient scheduling

- Patient rescheduling

- Information regarding costs/prices

- Patient history

- Information regarding consumable items in the imaging center;

- Radiological film management;

\subsection{The communication interface with the PACS informatics system}

Through this interface the exchange of messages between the RIS and the PACS Broker software application is made. The PACS Broker converts HL7 messages into DICOM messages and the other way around. The following HL7 messages are used:

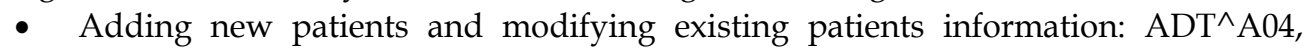
$\mathrm{ADT}^{\wedge} \mathrm{A} 08$.

- $\quad$ Adding / modifying medical examinations: ORM^O01.

- $\quad$ Adding / modifying medical reports: ORU^R01. 


\subsection{The communication interface with the imaging acquisition equipment}

This interface refers to the communication between the software applications responsible for the creation, storage and management of images (medical equipment, image servers (PACS) and any electric device having a communication interface compatible with the DICOM standard) as well as those responsible for patient and resource management (RIS and/or HIS servers).

The conversion interface between the DICOM and HL7 standards is based on the requestreply principle. The entities that initiate the protocol are medical imaging equipments or PACS servers. The interface has the following characteristics:

- Works as an interface between the PACS or the medical equipment and RIS

- Allows multiple TCP/IP simultaneous connections

- Is based on the communication standards used in the medical field (DICOM, HL7)

- Implements "Modality Work list Information Model - MWLIM" from the DICOM - SOP Class UID 1.2.840.10008.5.1.4.31 standard

- Is compliant with the IHE specifications

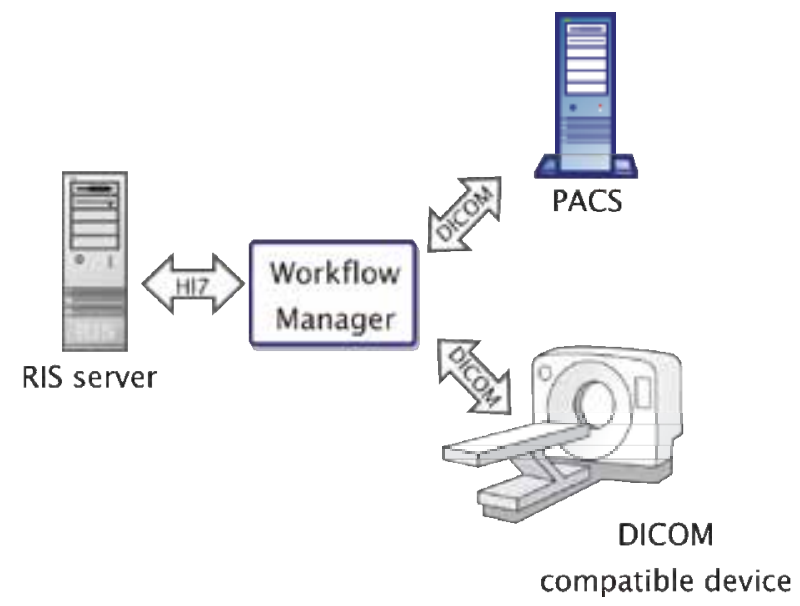

Fig. 5. Workflow manager

The interface works in the following way:

Step 1: the PACS initiates a query to the MWL - Modality Work list - this is a software application that stands between the PACS, RIS and medical imaging equipment. This is the application that informs in real time the equipment operator about the patients that must be investigated and the type of procedure to be undertaken.

Step 2: Using the filter requested by PACS, MWL initiate a HL7 type query message to the RIS server.

Step 3: Based on the answer received from the RIS server, MWL fills in all the DICOM fields requested by the PACS and sends answers to the entity that initiated the communication.

\section{Remote and distant access to information using WEB technologies}

In order to remotely access the information stored in the healthcare unit informational system a system based on web technologies has been developed. This technology was used 
because it allows the users (mostly doctors) to deploy the system even if they are not inside the health care unit.

A client/server application that is able to run inside an internet browser allows the users to access information from the imaging center like: patients, procedures, reports, images associated to certain procedures.

- The server is implemented under the form of a .dll using ISAPI (Internet Server API) and runs inside the IIS (Internet Information Services) server;

- The client is implemented using the Active $X$ concept and scripting languages such as VBScript and JavaScript

The architecture of the web application is presented in the figure below:

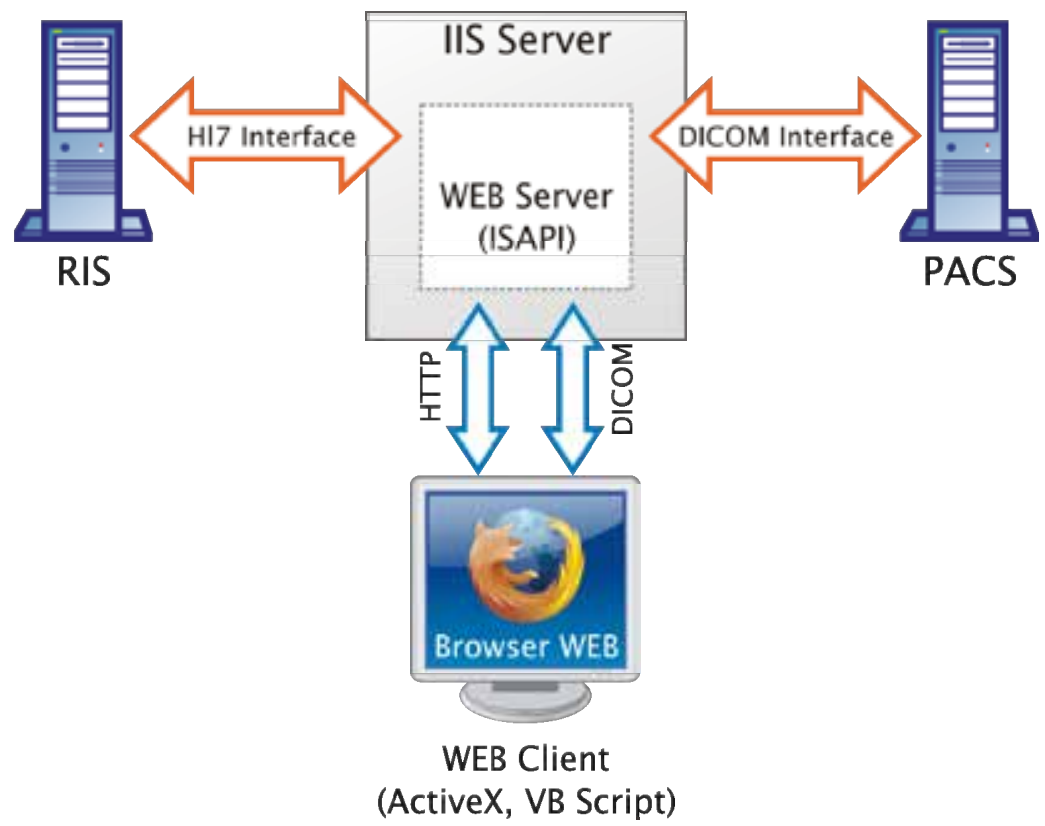

Fig. 6. Web application architecture

The servers take the requests coming from the client running in the web browser and send them to the RIS or PACS server. From the RIS server information regarding patients, medical examinations, procedures and reports are retrieved. In order to transfer the images in the shortest time possible inside the browser, the web server has an image buffer implemented. When the user asks for an image, after visualization the server stores it for a certain period of time, in case the user wants to review the image again. Moreover, to further obtain a decrease in the time necessary for an image to be available for the viewer, a JPEG compression of the images coming from the PACS server is made prior to sending them to the web client.

Usually the web servers are installed outside the "firewall" security type systems that are present in imaging centers; due to this, a careful planning and implementation of the communication interfaces is necessary. In this manner, the communication between the RIS server and the web server is made using encryption algorithms and secure transmission protocols like SSL (Secure Sockets Layer) (Alin Cordos et al., 2004). 


\section{Integrated Management System for Medical Information Using HL7 Standard - SIMIMED}

The theoretical and practical knowledge gained by the authors of this chapter in the field of medical software development were used in the PNII-D11019/2007 research project funded under the Romanian national research program scheme. The project aimed at developing a pilot HIS integrated system using the latest standards available (HL7 and DICOM), adapted to the particular case of the Romanian National Health System and compliant with the EU requirements. The SIMIMED project contains a packet of software modules that provide 4 service categories:

- The HIS integrated system: is made up from the server application and the client applications distributed by sections/departments. The system uses the 3 tier architecture and the communication between the clients and the server is based on the http/https protocol. The HIS is used for saving information in the database, client application management and database querying. The server connects to the Microsoft SQL Server database using an ADO connection and uses the web service principle loaded by the IIS server; each service is workflow - oriented. The HIS client applications offers the client a friendly and intuitive graphical user interface that allows for the user to input or retrieve data into/from the SIMIMED system. It is implemented in C\# and communicates with the server using the http/https protocol.

- The Datacenter - is a server application that manages the data stored in the common data base by all the HIS applications running in different hospitals/institutions. This stores medical data to be used by researchers. The storage center was developed based on the Microsoft SQL server technology. The HIS servers running at the medical institutions involved in the project send data to the storage center. In the data exchange process between the HIS servers and the Datacenter the HL7 together with the TCP/IP protocol are used. Due to the fact that the data stored is dedicated mainly to research purposes, no information that would allow patient identification is stored.

- The web application - is a web-client application that has/gives/allows access to the data stored in the Datacenter. It can be used by researchers, students and PhD students to study different cases classified by the Datacenter. The access is controlled by the data center administrator, who is entitled to issue access accounts based on username/password pairs.

- The AdminTool application - is a web application used for remote administration of the HIS server, and allows for quick and in time support in case of malfunctions. 


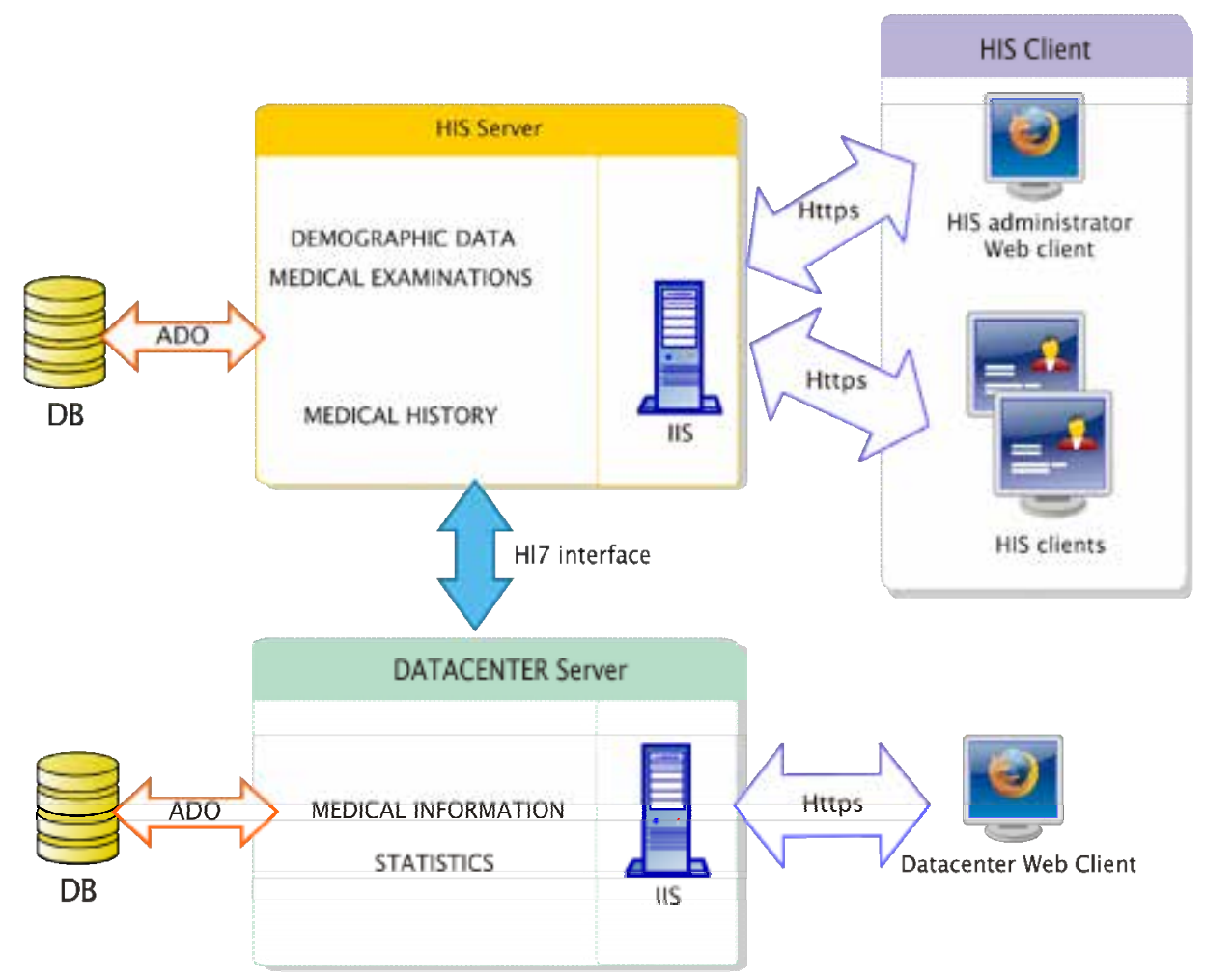

Fig. 7. SIMIMED system architecture

The SIMIMED HIS client contains five important modules: Patient Demographic, Examinations, Surgery, Patient History and Schedule.

The Patient Demographic module contains the graphical controls to add/edit/search demographic data of a patient. Using this module one can add/modify visits, document upload/download documents, add/modify transfers or other type of information (allergies, antecedents, and medical history), health insurance, and patient discharge.

The Examinations module allows the editing of information regarding examinations taken on a patient, and contains a visit list and 4 tab-controls: general clinical examinations - for general information (height, weight, general condition), examinations - for surgery procedures, specialty examinations (ex. oncology, radiology), and evolution and treatment. Data in each tab-control is loaded from the server based on the selected visit, and can be modified if the selected visit is the current one.

The Surgery module contains components regarding surgery intervention. We can edit information related to intervention date, operating physician, assistants, medical equipments or tools used and the list of surgery interventions in the selected visit.

The Patient History module contains in a single tab-control all the relevant information regarding patient visits: the list of visits, list of surgery interventions, medications, documents, allergies, transfers.

The Schedule module is used by physician to schedule meetings with patients, interventions and other events. This module contains a reminder for upcoming and important events. 


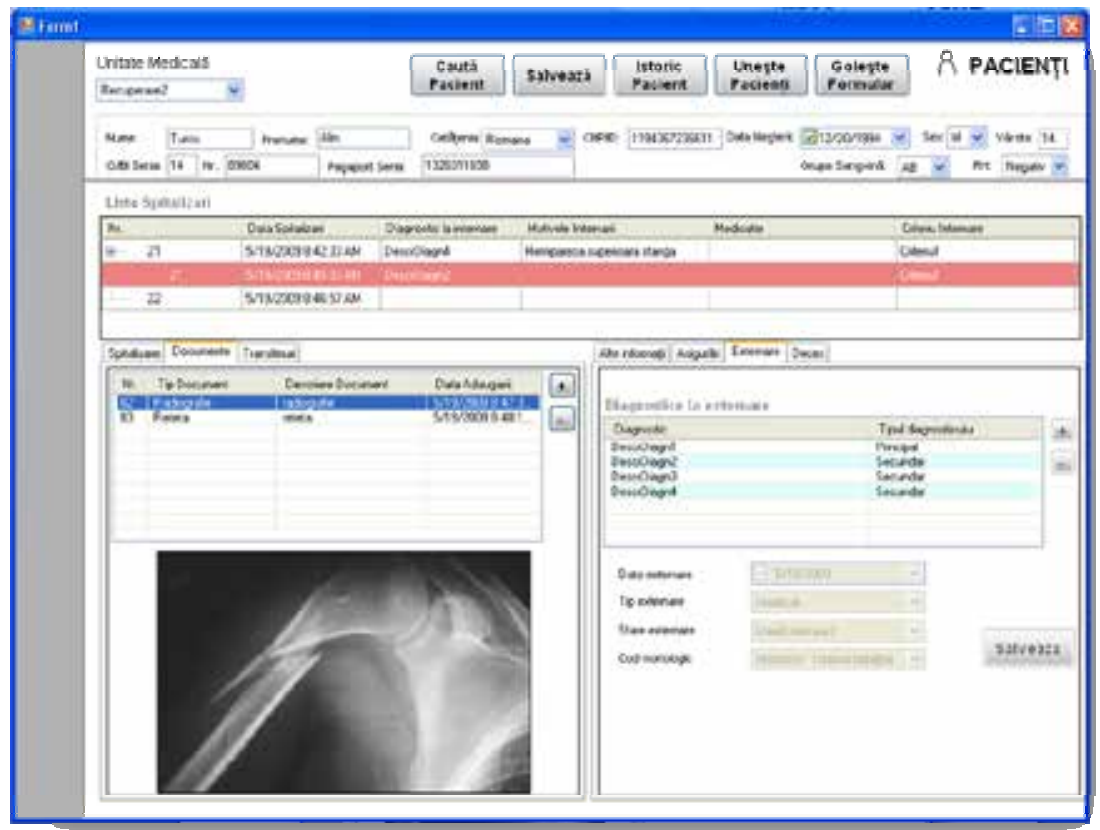

Fig. 8. Patient Demographic module

\section{Conclusions}

Software applications used in medical centers must be modular and scalable in order to meet the needs of the beneficiaries. To ensure the proper integration of various software modules it is necessary to use HL7 and DICOM standards which provides a set of rules and algorithms specific tothe medical field.

\section{References}

NEAC (2008) DICOM standard, http:/ / medical.nema.org/

HL7 (2008) HL7 standard, http:/ / www.hl7.org

Oleg S. Pianykh (2008). Digital Imaging and Communications in Medicine (DICOM), Springer-Verlag Berlin, page numbers (3-7, 301-309), Heidelberg

HIMSS and RSNA, Integrating the Healthcare Enterprise, Revision 5.3 August 15, 2005

Alin Cordos, Mihaela Hedesiu, Bogdan Feldrihan, Marius Rotariu, Transferring medical records to CD/DVD using DICOM standard, 2008 IEEE International Conference on Automation, Quality and Testing, Robotics AQTR 2008, Cluj-Napoca, Romania, May 2008, Poster Session, page numbers (275 - 279)

Cordos, A. Vlaicu, Security implementation in medical network communication, Cost 276/2004, 2004, Ankara, Turkey 


\title{
Modelling Embedded Systems with AADL: A Practical Study
}

\author{
Naeem Muhammad ${ }^{1}$, Yves Vandewoude 1 , Yolande Berbers ${ }^{1}$ and Sjir van Loo ${ }^{2}$ \\ ${ }^{1}$ Departement of Computer Science, Katholieke Universiteit Leuven, Belgium \\ ${ }^{2}$ Embedded Systems Institute, The Netherlands
}

\section{Introduction}

In today's world, embedded systems can be seen everywhere around us. These systems range from consumer electronics such as mobile phones, cameras and portable music players to sophisticated devices such as planes and satellite systems. In either form embedded systems are designed to perform specific tasks with constraints on their qualities and available resources. These constraints can either be soft or hard depending on the nature of the system: a satellite system, for example, has hard safety constraints. Some of the major constraints for embedded systems are high reliability, performance, safety and dependability, small memory size, low power and low processing capabilities. Designing systems with such constraints is a challenge.

Developing system architectures during system development has gained importance as it helps in analyzing the system before its implementation. A system architecture is a formal description of a system that describes its building blocks, their properties and the interactions among them. System architectures can be used to analyze various properties of a system such as memory consumption and system safety. For embedded systems, this is of extreme importance since a well described system architecture allows us to predict whether any of the previously mentioned constraints can be met, without requiring the construction of an often expensive prototype implementation.

Description of system architectures can be achieved using the formal notations offered by Architecture Description Languages (ADLs). Such ADLs often also provide tool support for the modelling and analysis of the system architecture. Many ADLs for embedded systems are available in both academic and industrial communities, such as Rapide, MetaH, AADL and Wright. Among the available ADLs, the best known and most actively used language is the Architecture Analysis and Design Language (AADL). Standardized by the Society of Automotive Engineers, AADL was originally developed for modelling and analysis of systems in the domain of avionics. However, because of its rich modelling and analysis capabilities, it is widely used for embedded systems in other domains as well. AADL provides a modelling formalism accompanied by a toolset to support modelling activities and system analyses. AADL models can be used to perform various analyses such as flow latency, resource consumption, real-time schedulability, security and safety analysis. Because of its history in the avionics domain, AADL does not address each and every 
modelling and analysis requirement of other embedded domains. However, during its design, it was foreseen that use of AADL in other domains could require additional modelling concepts and analyses. To meet potential needs AADL was designed as an extensible ADL.

This chapter is intended to provide insight into the design needs of embedded systems and the formalisms available to address those needs; we discuss them in section 2 and 3 respectively. We will explain the suitability of AADL and will present its architectural elements for modelling embedded systems in section 4 . We will also highlight the shortcomings currently present in AADL and describe its extension mechanism by addressing one of the shortcomings with the help of an example, in section 5 .

\section{Embedded Systems}

There is no standard, universally accepted definition of embedded systems. Some recognize embedded systems as a computer inside a product; some view them as computing systems embedded inside electronic devices; some refer them as electronic programmable devices integrated in a larger heterogeneous system. Despite the lack of a standard definition, a general consensus about embedded systems is that they are computer systems designed to perform dedicated functions. Today we can see many such systems ranging from very simple single-chip systems to complex and highly distributed embedded systems. They are present around us in various forms at various places such as digital cameras, home appliances, elevators, planes and medical devices. The role of embedded systems in human life has increased drastically over the last decade as human dependency on electronic systems has surged. In past, embedded systems were mainly used to address needs of the mission-critical systems. Recently however, they are used in devices such as mobile phones and PDAs as well. One example of a very common embedded system is a digital camera, which is a composition of hardware and software components. The hardware is mainly responsible for capturing objects through sensors and storing images whereas the software facilitates image processing functions. Digital cameras usually have constraints such as low computation power, small size and low power consumption.

Physical constrains, such as size and weight, and cost are reasons why embedded systems, such as mobile phones or digital cameras are forced to perform their functions with considerably fewer resources than conventional systems. Other systems, such as flightcomputers or transportation systems have high safety and reliability requirements. It is the presence of these non-functional constraints that makes the design and development of such systems a daunting task.

Embedded systems can be divided in to real-time and non-real time embedded systems. Real-time embedded systems have various timing constraints on their behavior, they are required to react and respond to such constraints. Correctness of their behavior depends on the ability to perform it in the given time frame or before a certain deadline. Whereas, nonreal time embedded systems do not have time obligations.

Real-time embedded systems can further be categorized in hard and soft real-time embedded systems. Hard real-time systems have tight timing constrains with nearly zerotolerance level. Failure to meet the deadlines causes the operations to stop and is considered an anomaly. Mission critical systems usually fall in hard real-time embedded systems category. Soft real-time constrains can be found in video streaming applications for 
example. Failure to achieve required deadlines or throughput is annoying but does not necessarily invalidate the results. An embedded system may have both hard and soft realtime constraints for different functional and non-functional requirements.

The affordability of embedded systems is an indicator for a further increase of their use in the near future. Not only they are more widely used, embedded systems also provide more and more features and grow increasingly more complex each year. In a study, in 2008, about 30 embedded microprocessors were found per person in developed countries, with at least 2.5 million function points in the associated embedded software (Christof \& Capers, 2009). The number of embedded devices in automobiles is an important growth rate indicator. Modern cars contain 20 - 70 electronic control units with up to 1Gigabyte total size of the accompanied software. A similar growth rate is predicted for embedded systems in other domains too. Embedded systems with complex specifications require sophisticated development methodologies in general and especially for modelling their architecture (Bouyssounouse \& Sifakis, 2005).

\section{Embedded Systems Specification and Modelling Needs}

Embedded systems consist of both functional and non-functional properties. Although the nature of their functional properties differs between different domains, embedded systems often have similar non-functional properties known as Quality Attributes (QA). A camera for instance has different functional properties than a mobile phone but both of them are required to work with a small amount of memory and less computation power. QAs are system requirements used to describe quality aspects of the system. The quality aspects may include performance, usability, security, portability, availability, robustness and testability. There is no standard list of such QAs that define system quality, however a general criteria that distinguishes them from other requirements is that they only describe non functional aspects of system. The quality of a system is analyzed against these requirements; importantly they are used during architecture level analysis to find and fix any discrepancy to system requirements early in the development life cycle. Trudy Sherman in his research work identified QAs for embedded systems by examining eleven architectural designs of embedded systems developed by three different organizations. He produced a list of 30 QAs that are used to define various quality aspects of embedded systems (Sherman, 2007). Following are some commonly used QAs for embedded systems given in the list he produced:

\begin{tabular}{|l|l|}
\hline \multicolumn{1}{|c|}{ Quality Attributes } & \multicolumn{1}{c|}{ Description } \\
\hline Reliability & $\begin{array}{l}\text { The ability of a system to perform desired behavior under } \\
\text { previously specified circumstances, and recover from } \\
\text { undesired states if occurred. }\end{array}$ \\
\hline Safety & $\begin{array}{l}\text { The ability of a system to avoid potential hazards to itself, } \\
\text { its users and the environment in which it is used. }\end{array}$ \\
\hline Security & The ability of a system to resist any unauthorized usage. \\
\hline Memory Usage & The capability to work with a limited amount of memory. \\
\hline Performance & $\begin{array}{l}\text { The degree to which a system or component accomplishes } \\
\text { its designated functions within given constraints, such as } \\
\text { speed and accuracy. }\end{array}$ \\
\hline Usability & The system must be easy to use, operate and handle. \\
\hline
\end{tabular}

Table 1. Embedded System Quality Attributes 
The magnitude of constraints on QAs defines the nature of a system as either a non-real time, soft or hard real-time embedded system. This magnitude must be explicitly defined and is based on the domain for which the system is developed. Furthermore, it serves as an important factor during QAs' tradeoff.

Because of the high pressure to produce embedded systems with previously mentioned characteristics in a low-cost and short time-to-market setting, embedded systems design methodologies are required to well address functional and non-functional constraints, and resolve potential issues before implementation. According to Talarico et al. they must provide support mainly to (Talarico et al., 2005)

1. Describe the interactions between the system and the external environment

2. Describe the system architecture

3. Model the behavior of hardware and software components that make up the system

4. Describe the system constraints and requirements

5. Describe the test scenarios used to simulate the system

6. Define a set of gauges to measure various performance metrics during simulation execution

Although the criteria of Talarico et al. are valid for every software system a number of aspects are especially different for embedded systems. For example, the forth requirement support for a cost-effective method to formally specify system constrains is difficult for embedded systems, since the exact nature of all constraints is often not yet known in the early stages of development.

In addition, embedded systems are composed of software and hardware components which require that the modeling needs of both domains are well addressed. These needs include the specification of all types of software elements (such as processes, threads, communication among software elements and shared data), hardware elements ( for instance processors, memory and physical communication channels), and most importantly the mapping of software elements to hardware elements. Additionally support is required for describing system dynamics. These dynamics may include system flows, system states and run-time interactions among system components.

It is also important that the support offered by the design methodology is not restricted to the modelling only but that it also facilitates system designers to analyze designs for issues and fix them early. Therefore, for modern system development methodologies the presence of design artifacts is extremely important. They not only serve as input to the next phase but are used for early system verification and validation $(\mathrm{V} \& \mathrm{~V})$. They can be analyzed to find potential performance deficiencies, security leaks and safety hazards. As previously discussed fixing these issues at early stage is cost effective. Any unaddressed performance deficiency or security leak that propagates to later stages of development may lead to a major refactoring and cost afterwards. With this additional perspective design techniques and tools are required to support $\mathrm{V} \& \mathrm{~V}$ activities.

Moreover, it is important to assist design activities with rich set of toolsets to make these activities quick and correct. Although, many tools are available for modeling and analysis of functional and non-functional behaviours, some non-functional aspects remain unaddressed which are of primary focus for embedded systems. Tool support should be enhanced not only to deal with unaddressed areas but to tackle future design complexities. 


\section{Architecture Description Languages}

Architecture Description Languages (ADLs) are modelling formalisms that provide support for describing system architectures through their formal notations. These are considered as modelling-language-plus as they can model more than conventional modelling languages. ADLs can model not only static but dynamic properties of systems as well. There is no consensus on a standard definition of ADL yet, for our discussions we will however use the one provided by Medvidovic and Taylor (Medvidovic \& Taylor, 2000) "an ADL must explicitly model components, connectors, and their configurations; furthermore, to be truly usable and useful, it must provide tool support for architecture-based development and evolution".

The definition identifies three essential requirements an AADL must fulfil. Firstly, it should provide support for modelling a static structure in the form of components and connectors. Secondly, ADLs should provide support for modelling configurations of components and connectors, which usually define system's dynamic behaviour. The third pivotal requirement for an ADL is to provide tool support to assist modelling and analysis activities. There are several ADLs available to date; some of them are given in the following table.

\begin{tabular}{|l|l|}
\hline \multicolumn{1}{|c|}{ ADL } & \multicolumn{1}{c|}{ Application Domain } \\
\hline AADL & $\begin{array}{l}\text { Is used to model real time embedded systems particularly in the avionics } \\
\text { domain. }\end{array}$ \\
\hline Acme & Interchanges architecture description information between ADLs. \\
\hline Aesop & $\begin{array}{l}\text { Is used to model style-specific architecture descriptions, also provides } \\
\text { support for designing custom architecture styles. }\end{array}$ \\
\hline ArchC & Is a SystemC based language used to describe hardware elements. \\
\hline ArchJava & $\begin{array}{l}\text { Checks conformance of an architecture of a software system to its } \\
\text { implementation, and keeps architecture and code consistent during their } \\
\text { evolution. }\end{array}$ \\
\hline ControlH & $\begin{array}{l}\text { Is used to develop the architecture specification and code generation for } \\
\text { control and navigation systems. }\end{array}$ \\
\hline C2 & $\begin{array}{l}\text { Supports architecture description of highly-distributed, evolvable, and } \\
\text { dynamic systems. }\end{array}$ \\
\hline Darwin & $\begin{array}{l}\text { Is used to describe architectures of dynamically changing highly- } \\
\text { distributed systems. }\end{array}$ \\
\hline EAST-ADL & Addresses modelling and analysis needs of automotive electronic systems. \\
\hline MetaH & $\begin{array}{l}\text { Supports modelling of real-time systems in the domain of guidance, } \\
\text { navigation and control. }\end{array}$ \\
\hline Modechart & Is used to describe architecture for hard real-time embedded systems. \\
\hline Rapide & $\begin{array}{l}\text { Provides support for developing event based simulations for distributed } \\
\text { event-extensive systems. }\end{array}$ \\
\hline SADL & $\begin{array}{l}\text { Is designed to simulate real-time properties for hard real-time systems } \\
\text { from the avionics domain. }\end{array}$ \\
\hline Weaves & $\begin{array}{l}\text { Is used for describing architecture for data-flow-extensive systems with } \\
\text { real-time processing on a high volume of data. }\end{array}$ \\
\hline Wright & Is used for describing communication behaviour of concurrent systems. \\
\hline
\end{tabular}

Table 2. Architecture Description Languages 
The ADLs given in the table clearly show that most of them are domain specific description languages addressing needs of systems for particular domains. They vary widely in their supported abstractions and analysis capabilities. Their incapability to be applicable for every domain is resulting in new ADLs. With a large number of ADLs available and most of them domain specific, and considering the fact that a single ADL does not address every modelling requirement particularly in the case of multidisciplinary systems, it is difficult to choose the right language. Some work however is being done in establishing a contact point among ADLs where multiple languages may be used together. Acme is serving as such a contact point, it is an ADL whose core purpose is to support the mapping of an architecture description of one ADL to another (Medvidovic \& Taylor, 2000).

The role of architecture for early system validation certainly is of great importance. However, the effectiveness of the validation activities can only be increased by a formal description of the architecture and assisting the activities with tool support. ADLs with their support for formal description and accompanied toolset for architecture modelling and analysis can serve for this purpose.

The QAs of an embedded system are difficult to model and analyze because they are associated with a system's dynamic behavior that is only available during the last phases of its development. It is nevertheless cost effective to resolve issues related to them as early as possible. ADLs' support for modelling dynamic behavior and tools for analyzing architecture for quality attributes can be utilized for embedded systems.

Although a large number of ADLs is available, most of those are present only in the research community, and are not applied in industry. In addition, some of them are no longer in use. In a survey, which we conducted to find a suitable ADL for modelling performance for an electron microscope embedded system we found that most of the candidate languages are no longer active. Among MetaH, Rapide, AADL and Wright, only AADL is active and being used in industry. In addition, the amount of literature published between 2000 and 2008 for AADL magnitudes greater than for others, suggesting a wide acceptance in the research community as well.

Besides its wide acceptance, AADL distinguishes itself from other ADLs by its extensible nature. Although initially designed for the avionics industry it can be applied to other domains by extending its core concepts where required (Feiler et al., 2006). This will help in generalizing an ADL for various domains, previously an issue with many ADLs.

In the following section we will discuss AADL in detail; we will highlight its modelling and analysis capabilities and discuss its toolset. Moreover, in section 5 we will explain how AADL can be extended to meet custom modelling needs using an example.

\section{Architecture Analysis and Design Language (AADL)}

In compliance to the definition of ADL, AADL provides a modelling formalism accompanied by a toolset to support modelling activities and analysis. Originally developed for modelling and analysis of systems in the domain of avionics, it has been standardized by the Society of Automotive Engineers. Because of its rich modelling and analysis capabilities it is widely used for embedded systems in other domains as well, especially suitable for model-based analysis and specification of complex real-time embed systems (Feiler et al., 2006). In this section a brief introduction is given on the AADL architectural notations, its analysis and its tool support. 


\subsection{Modelling}

AADL consists of a rich set of architectural elements for modelling components, their interactions and their configurations. Architectural elements and the core concepts of the language are given in figure 1.

Components in AADL are used in terms of component types and component implementations. A component type defines the externally visible characteristics of a component usually by using features, flows and properties. Whereas, a component implementation models the internal structure of the component. An internal structure may consist of subcomponents, connection among them, flows across them and their operational behaviour. AADL distinguishes between three types of components: software components, hardware components and composite (system) components:

\section{Software Components:}

Thread: Represents a unit of sequential execution through source code.

Thread group: Represents a logical grouping of threads.

Process: Represents a protected address space.

Data: Represents static data and data types.

Subprogram: Represents a callable part of a source code.

\section{Hardware Components:}

Processor: Hardware that is responsible for executing threads.

Device: Hardware that interacts with the external environment.

Bus: Hardware that provides access to the other execution platform components.

Memory: Hardware that stores digital data.

Composite (System) Component: A component composed of software and hardware or even system components.

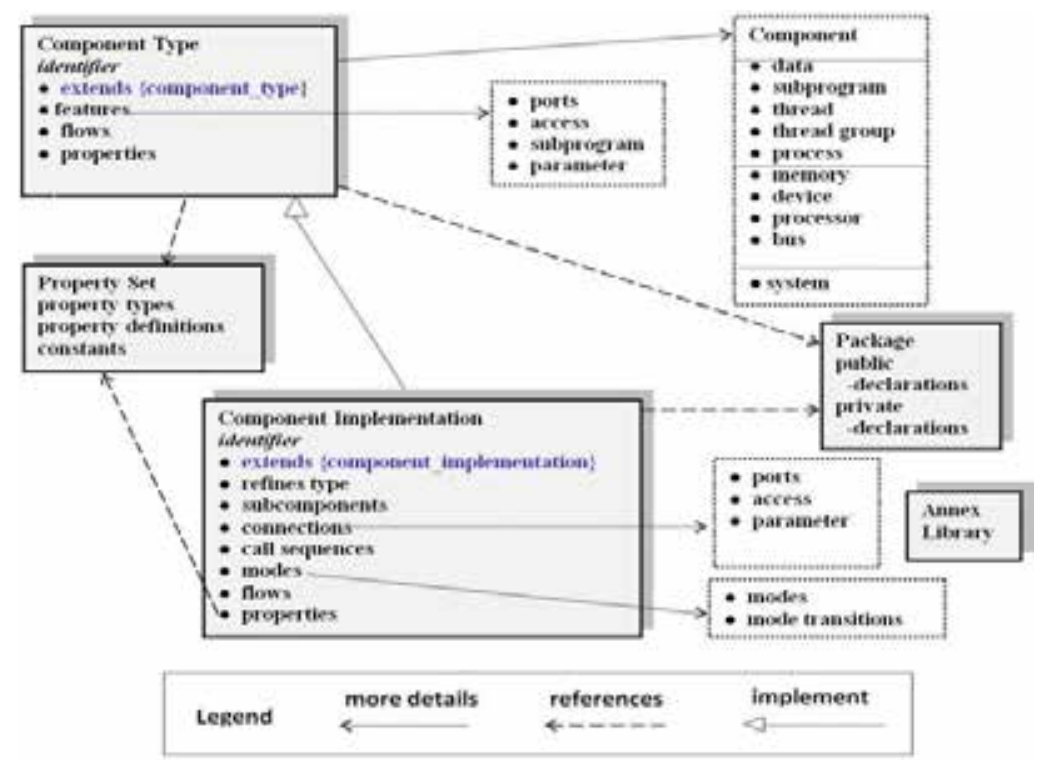

Fig. 1. AADL Architectural Elements (Feiler et al., 2006) 
Interactions between components can be realized by using features and connections. Features are interaction points of components, which are used for communication. Features are classified into ports, component access, subprogram calls and parameters. A port is a communication interface of components used to exchange data and events. AADL categorizes ports as data ports, event ports and event data ports. Moreover, multiple ports can be grouped together in a port group. Component access enables components to access shared data and bus. For access, components are required to explicitly use provides access and requires access declarations. Subprogram calls are used for synchronous calls to subprogram components, and parameters are used to represent data values passing in and out of a subprogram. Connectors are used to connect interaction points of components. AADL provides data, event, eventdata, dataaccess, bussaccess and portgroup connectors.

\subsection{Analysis and Tool Support}

In its conformity to the ADL definition, AADL provides support for various kinds of analyses along with conventional modelling. A few of the supported analysis are:

\section{Flow Latency Analysis}

Understand the amount of time consumed for information flows within a system, particularly the end-to-end time consumed from a starting point to a destination.

\section{Resource Consumption Analysis}

Allows system architects to perform resource allocation for processors, memory, and network bandwidth and analyze the requirements against the available resources.

\section{Real-Time Schedulability Analysis}

AADL models bind software elements such as threads to hardware elements like processors. Schedulability analysis helps in examining such bindings and scheduling policies.

\section{Safety Analysis}

Checks the safety criticality level of system components and highlights potential safety

hazards that may occur because of communication among components with different safety levels.

\section{Security Analysis}

Like safety levels, AADL components can be assigned various security levels. The analysis helps in identifying the security loopholes that may happen because of mismatches in security levels between a component and its subcomponents, and communication among components with different security levels.

Various tools are available to perform these analyses:

OSATE (Open Source AADL Tool Environment) developed by SEI is a set of Eclipse plugins for front-end processing and various analyses of AADL models (Feiler \& Hansson, 2007). ADeS (Architecture Description Simulation) by Axlog simulates various aspects of the system behaviour, such as scheduling of processes and threads by processors (AXLOG, 2009).

Cheddar (Singhoff \& Plantec, 2007), developed by LISyC Team, is a real-time scheduling tool which provides support for quick prototyping of real-time schedulers and schedulability analysis. Like ADeS, Cheddar also supports simulation of scheduling properties of a system.

ANDES (ANalysis-based DEsign tool for wireless Sensor networks) (Prasad et al., 2007) was developed for modelling and analysis of wireless sensor network systems. It provides support for real-time communication schedulability, target tracking and real-time capacity analyses. 


\section{Extension to AADL}

Initially designed as a language for modelling avionic systems, AADL includes core modelling concepts and certain analyses essential for real-time systems in the aerospace domain. However, during its design, it was foreseen that use of AADL in other domains could require additional modelling concepts and analyses. To meet potential needs AADL was designed as an extensible ADL.

It is possible to extend the AADL concepts either by introducing new properties to the modelling elements, by addition of new modelling notations, or by developing a sublanguage as annex to the AADL standard (Frana et al., 2007). The latter technique is mainly used for large-scale extensions and was considered out of scope for our own purpose. Since, for our research work we extended AADL by using the property based extension technique, the scope of the example we present here will be restricted to this technique only. In this ecample, we will extend AADL End-to-End (EtE) flows to provide support for Composite EtE flow modelling and latency analysis.

EtE flow latency is the amount of time consumed by the contributing components for a specific flow of information from a source to a destination. Currently, AADL requires that flow specifications of the contributing components are connected through the AADL connector element and does not provide support for composite EtE flows: flows that themselves consist of multiple discrete EtE flows. We will describe how this issue can be overcome with an extension to AADL, by introducing a new property for the AADL EtE flow element. Latter we will discuss results of the extended EtE flow analysis with the help of an analysis tool that we developed for this purpose.

We will apply this technique on an electron microscope embedded system. An electron microscope is a sophisticated microscope used to examine minute specimens by creating highly-magnified images.

\subsection{AADL EtE Flow Extension}

Flows in AADL describe the different sequences of an information flow through a set of contributing components. The description of this flow is subsequently used in certain analyses such as a flow latency analysis. In AADL, flows are defined with a flow specification and a flow implementation. A flow specification represents the externally visible flow of information in a component; it is specified within the component type declarations using flow sources, flow paths and flow sinks (Feiler \& Hansson,2007). A flow source represents the originator of the flow, the flow sink represents the end consumer of the flow information, and the flow path embodies the link between incoming and outgoing ports involved in the flow. A flow implementation on the other hand represents the actual realization of a flow within a component; it is specified within the component implementation declarations. 


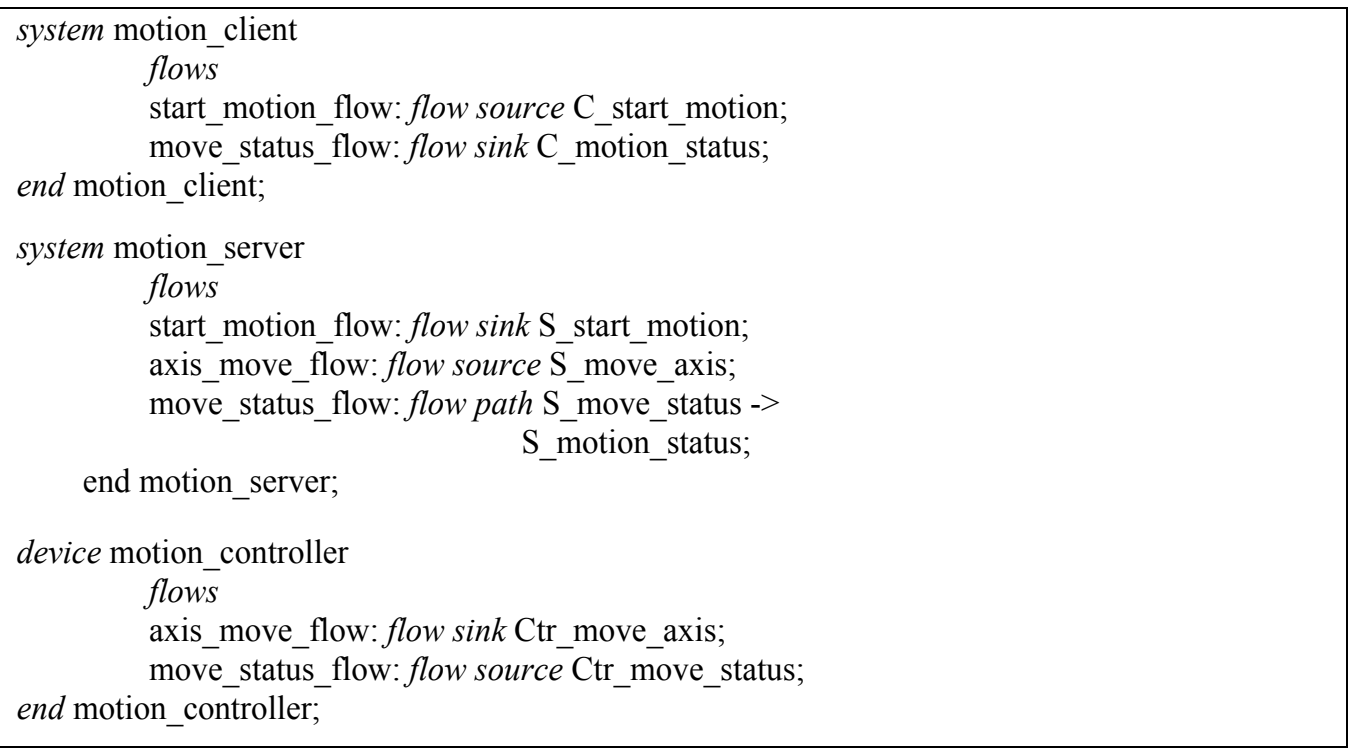

Fig. 2. AADL Flow Specification

An example of both a flow specification and a flow implementation is given in Figures 2 and 4. The excerpts are taken from the electron microscope's motion-subsystem that is responsible for moving the specimen holder. The system consists of three major components working in a client-server environment: the motion client, the motion server and the motion controller. The motion server receives its stage movement commands from a client application, processes it and moves the motion controller to the desired position. The externally visible flow of the move command is shown in Figure 3, which corresponds with the textual AADL representation in Figure 2.

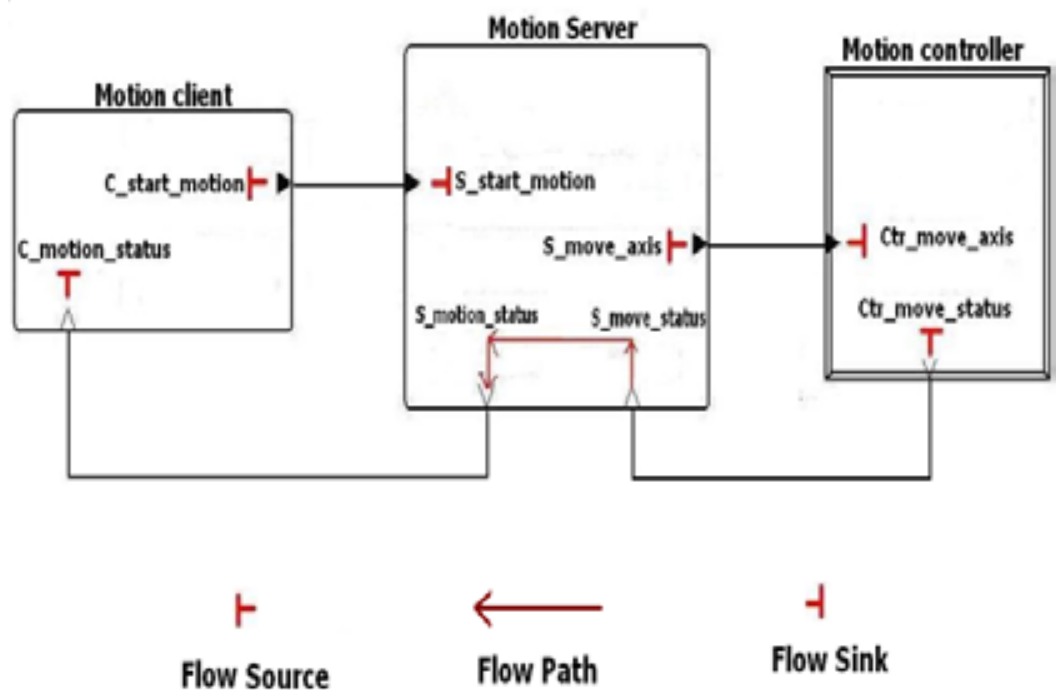

Fig. 3. Motion-Subsystem Move Command Flow 


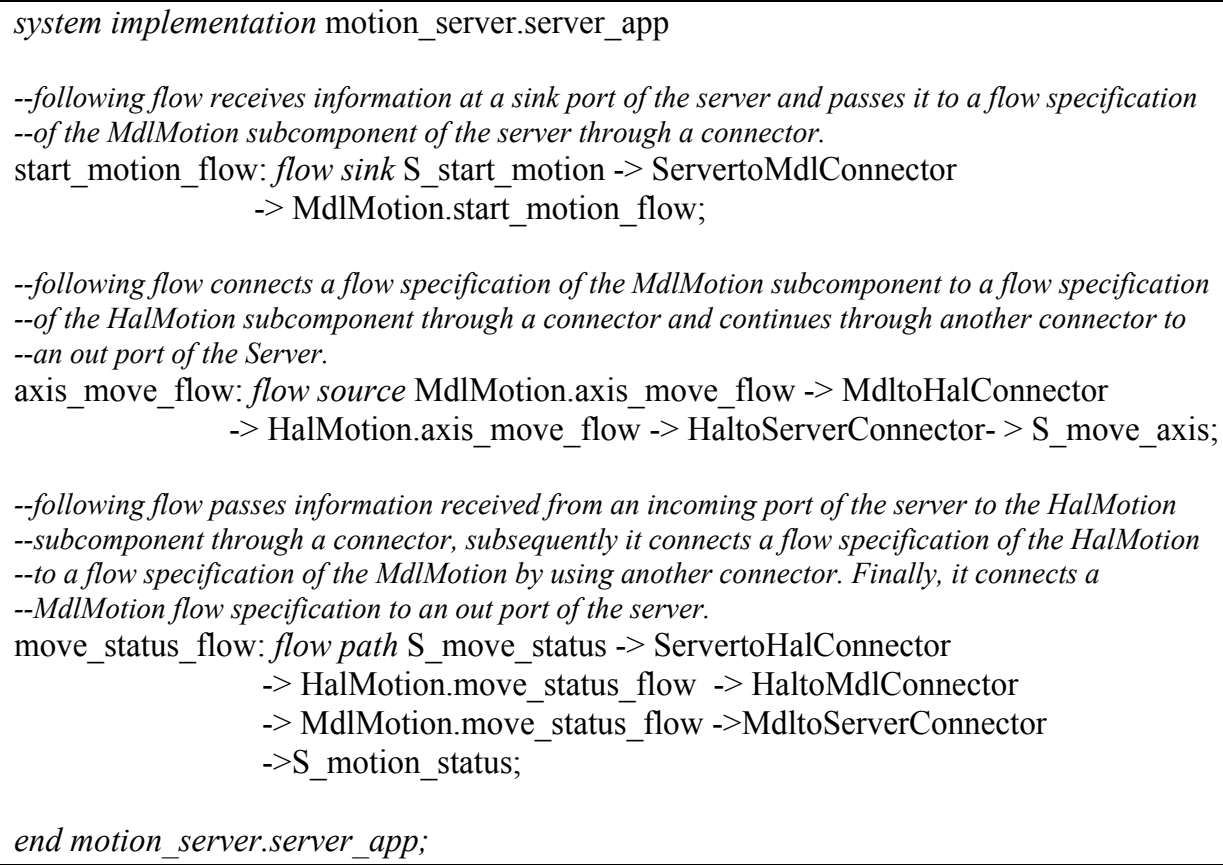

Fig. 4. AADL Flow Implementation

An EtE flow latency analysis requires the specifications of EtE flows. An EtE flow represents a logical flow of information from a source to destination passing through various system components. It is defined in the component implementation (typically in the top level component in the system hierarchy) and refers to the specifications of other flows in the system.

An EtE flow specification consists of the flow specifications of the contributing components connected through the AADL connector, Figure 5 contains the standard syntax for EtE flow specification.

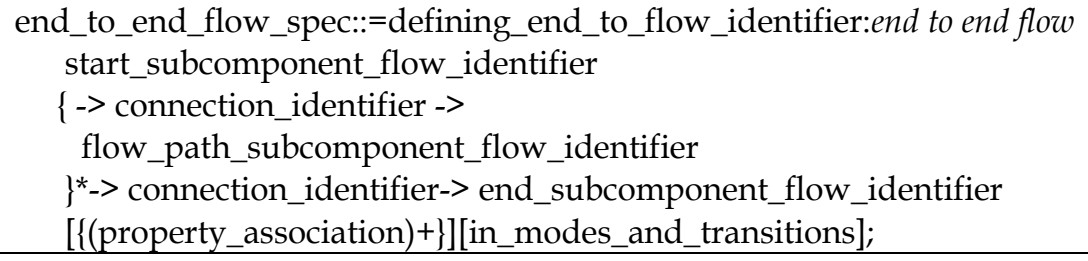

According to the standard specification, an EtE flow starts with a flow specification of the starting subcomponent, connects it to a flow specification of the subsequent subcomponent and so on, and finally connects to a flow specification of the last component in a flow. A notable point in the EtE flow modelling is that each contributing flow specification is connected to its adjacent flow specifications. The starting and ending flows are connected to one flow each, all intermediate flows are connected to both the predecessor and successor flows. This concept restricts EtE flow analysis only to those flow specifications that are 
linked. AADL does not provide support for more abstract flows whose internal flows are not linked. For clarity, we distinguish in our discussion between discrete EtE flows (in which all sub-flows are connected) and composite EtE flows (that consists of unlinked subflows).

In case of our motion-subsystem example, Move_Stage is a composite EtE flow: the flow starts when a client application sends a move request to the server and ends when client gets acknowledgement that stage has been moved to a desire position. Internally, Move_Stage consists of three consecutive discrete (disconnected) EtE flows Start_motion_flow, Axis_move_flow and Move_status_flow.

\section{Start_motion_flow:}

The flow starts with a move request from the client application and ends with the server. Internally, the server simply places the command in a queue, therefore this flow ends here. A part of the AADL textual representation given in Figure 2 specifies this behaviour of the flow.

Axis_move_flow:

As soon as a move command is available in the queue, a component of the motion server processes it and generates a new instruction for the motion controller. Upon receipt of the instruction, the motion controller moves the stage to the desired position. The server component involved in this task is different from the component involved in previous EtE flow. More importantly, both are not connected with each other for this particular task. Since the flow specifications of both components are not linked with each other, according to the AADL specification they can not be a part of a single EtE flow. Hence, this results in another EtE flow starting from the motion server and ending with the motion controller. The specification of this flow can be seen in Figure 2.

\section{Move_status_flow:}

As soon as the stage is moved to its position, the motion controller sends a motion completion acknowledgement back to the server which subsequently dispatches it to the client application. Internally, two different subcomponents of the motion controller are responsible for stage movement and acknowledgement generation. As such, the flow specifications of both components are not connected with each other. Therefore, sending acknowledgement back to the server is the start of a separate EtE flow with the motion controller as its starting point and the client as its ending point. The specification of this flow is shown in Figure 2 as well.

AADL's incapability to model composite EtE flows exists at any level of abstraction in AADL models, although chances of having such flow specifications increase with higher level of abstraction. Therefore, need for modelling and analysis of such EtE flows is significant at higher abstraction (system architecture) levels. Providing modelling support for composite EtE flows will also enhance flow latency analysis. The capabilities will enable system architects to analyse system flows at higher abstraction.

The incapability, as we described earlier, exists because AADL does not provide any support for linking disconnected flow specifications. Bridging such flow specifications can enable modelling and analysis of composite EtE flows. We will introduce a new property that will serve as a bridge between disconnected flow specifications.

\section{Property Based Extenstion}

An AADL property provides descriptive information about components, subcomponents, features, connections, modes, subprogram calls and flows (Feiler et al., 2006). A property consists of an associated value and type; the AADL standard consists of a set of predefined 
properties. However, new properties can be introduced in order to add additional information about the above mentioned architectural elements. The standard properties for EtE flows are:

\section{Expected_Latency: Time \\ Actual_Latency: Time \\ Expected_Throughput:Data_Volume \\ Actual_Throughput:Data_Volume}

We introduce a new property called Link_Flow to the existing properties. The new property holds a string value representing the identifier of the EtE flow that is to be linked. As new properties are defined in the AADL property sets, we declare the new property in the FLOWCONN property set. The declaration of the property is:

property set FLOWCONN is

Link_Flow: aadlstring applies to (flow);

end FLOWCONN;

Afterwards, the Link_Flow property can be used in the EtE flow declaration, in which it is assigned the identifier of the EtE flow to be linked.

While linking discrete EtE flows by using a property we assume that the delay between adjacent EtE flows is zero. Although this is the case in the motion subsystem (information is passed on through shared memory), many scenarios can be thought of in which this is not the case (such as the presence of a queue). Although not addressed in depth here, the solution remains applicable for those cases as well by including the delay in the model as a connector-like construct and applying a statistical model to them. The resulting latency of the EtE flow will also be a statistical distribution.

The introduction of properties allows us to attach additional information to the different elements of the AADL model. Subsequently this information can be inspected and/or manipulated by the accompanying tools that carry out the EtE flow latency analysis.

\subsection{Applying Our Solution}

In the previous section we defined a new property Link_Flow to connect discrete EtE flows. Now, we apply the proposed solution on the motion-subsystem, by using the property to connect internal discrete EtE flows of the Move_Stage flow. The use of our property within the standard AADL syntax is shown in Figure 6.

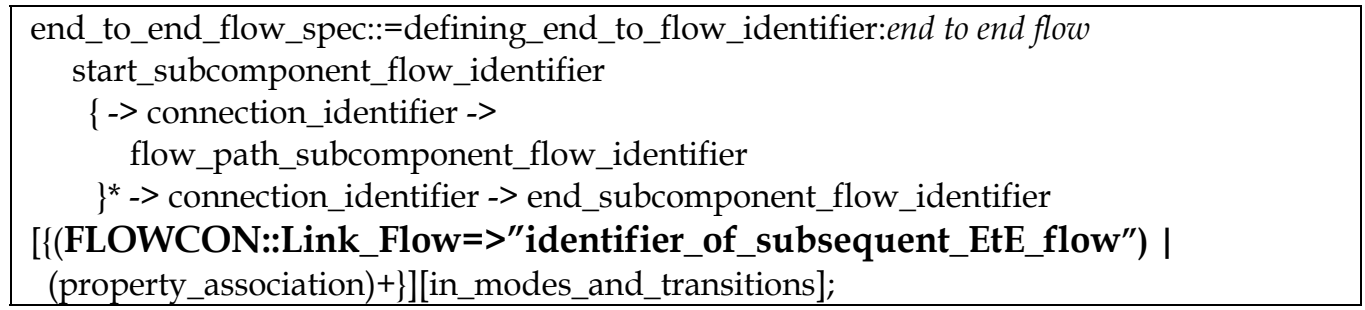

Fig. 6. EtE Flow Syntax with Link_Flow Property 
As stated earlier the Move_Stage EtE flow is composed of Start_motion_flow, Axis_move_flow and Move_status_flow in sequence. By using the Link_Flow property we will link Start_motion_flow to Axis_move_flow, and Axis_move_flow to Move_status_flow. An AADL textual description of the linkage is given in subfigures 7(a) and 7(b).

\section{Start_motion_flow:end to end flow \\ MotionClient.move_request_flow -> \\ ClienttoServerMotionConnection -> \\ MotionServer.move_request_flow \\ $\{$ FLOWCONN::Link_Flow $=>$ "Axis_move_flow"; $\}$;}

(a)

Axis_move_flow: end to end flow

MotionServer.axis_request_flow ->

ServertoControllerAxisConnection ->

MotionController.axis_request_flow

$\{$ FLOWCONN::Link_Flow =>"Move_status_flow"; $\}$;

(b)

Move_status_flow: end to end flow

MotionController.status_flow ->

ControllertoServerStatusConnection ->

MotionServer.status_flow ->

ServertoClientStatusConnection ->

MotionClient.status_flow;

(c)

Fig. 7. Link_Flow Property for Move_Stage Composite EtE flow

Since Move_status_flow is the last EtE flow in the composition it is not linked further, as shown in subfigure 7(c).

Using our technique, any number of EtE flows in a composition can be linked. Afterwards, the value of the defined property can be used during analysis to calculate the total flow latency of the Move_stage EtE flow. The calculation starts with the latency of the first flow, subsequently adding the latency of the EtE flow given in the Link_Flow property value. The addition continues until an EtE flow without Link_Flow property (or with empty value) is found.

We developed an OSATE-plugin that analyzes AADL models for composite EtE flows. OSATE is built on top of the Eclipse platform and is very well suited as a basis for the development of tools that operate on AADL models. OSATE's extensible plug-in architecture provides a wide range of methods and services generated from the AADL meta-model that can be used by plug-ins to manipulate AADL models.

By using the proposed property Link_Flow the developed plug-in differentiates composite flows from distinct flows, counts them, identifies their compositions (list of the contributing discrete EtE flows) and calculates their latencies (total time consumed by the composite EtE flow).The results of the analysis on the motion-subsystem is shown in Figure 8. 


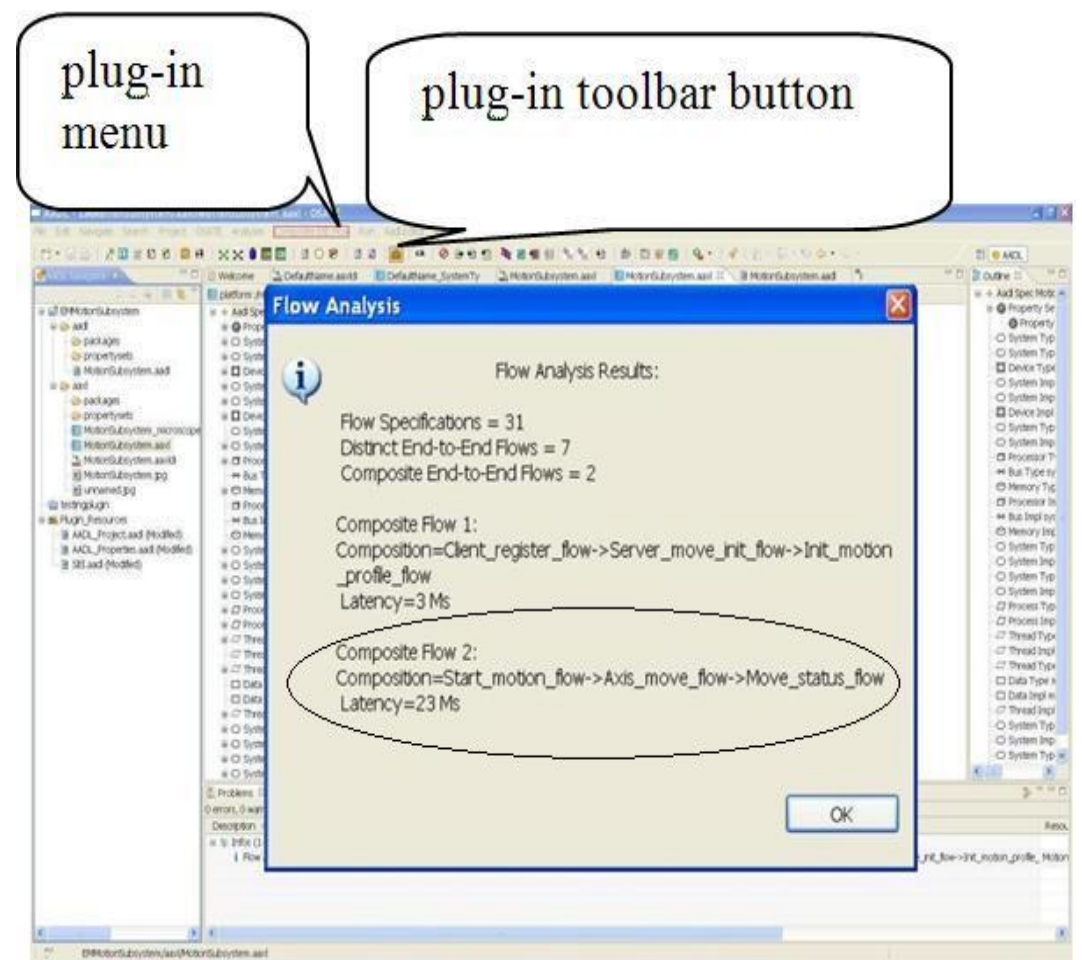

Fig. 8. Composite End-to-End Flow Analysis

The analysis shows that the motions-subsystem contains 31 flow specifications, 7 discrete and 2 composite EtE flows. The Move_stage composite EtE flow that we are discussing in our example is among the two identified by the tool. The encircled part of the Figure contains its composition and latency.

\section{Summary}

In this chapter we highlighted the design needs for embedded systems and discussed the formalisms available to address those needs. We found that development of embedded systems is more complex than that of general IT systems because of the constraints associated to them. In spite of the development complexity their use is increasing significantly and their rapid growth imposes challenges to the current development methodologies. Sophisticated and formal approaches are required to tackle their growing complexity throughout their development life cycle in general and for their design in particular.

We also discussed how the magnitude of constraints on requirements of embedded systems categorizes them in non-real time, hard and soft real-time embedded systems.

Quality attributes are the driving force for embedded system design as they have great impact on design decisions. During our discussions we identified some quality attributes specific for embedded systems and highlighted the needs for modelling those attributes.

Architecture description languages with their formal notations and analysis capabilities can reduce design complexities for embedded systems. In this chapter we gave a brief overview 
of some of the existing ADLs with a brief description of their scope. Although a significant number of ADLs exists, many of those only operate in an academic setting or are no longer actively used and maintained. AADL is one of the best known and most actively used architecture description language for embedded systems. With a rich set of architectural elements for describing both software and hardware elements of embedded systems it is quite useful for designing their soft and hard real-time properties. Furthermore AADL provides support for analyzing and fixing those properties. Since most ADLs are domain specific, no single ADL can serve for all domains. AADL, although designed for the avionics domain, is an extensible language that makes it possible to enhance its applicability to other domains. In this chapter we presented an introduction to AADL and showed with an example how it can be extended for custom needs. We extended its EtE flows to include support for modelling and analysis of composite flows. We applied the proposed solution to an industrial case of the motion-control subsystem of an electron microscope. The application included modelling a composite EtE flow of the motion-subsystem, which is composed of three discrete EtE flows. With the help of a new AADL property we successfully linked the discrete EtE flows to model them as a single abstract EtE flow. In addition, we presented the results of the extended EtE flow analysis which we performed with the help of an analysis tool (an OSATE plug-in) that we developed for this purpose.

\section{Acknowledgment}

This work has been carried out as a part of the Condor project (http://www.esi.nl/ Projects->Condor) at FEI company under the responsibilities of the Embedded Systems Institute (ESI). This project is partially supported by the Dutch Ministry of Economic Affairs under the BSIK program

\section{References}

Bouyssounouse, B. and Sifakis, J. 2005 Embedded Systems Design: the ARTIST Roadmap for Research and Development (Lecture Notes in Computer Science). Springer-Verlag New York, Inc.

Christof Ebert, Capers Jones, "Embedded Software: Facts, Figures, and Future," Computer, vol. 42, no. 4, pp. 42-52, Apr. 2009, doi:10.1109/MC.2009.118

Feiler, P.H.; J. Hansson, "Flow Latency Analysis with the Architecture Analysis and Design Language (AADL)," Technical Note CMU/SEI-2007-TN-010, Software Engineering Institute, 2007

Feiler, P.H.; Lewis, B.A.; Vestal, S., "The SAE Architecture Analysis \& Design Language (AADL) A Standard for Engineering Performance Critical Systems," ComputerAided Control Systems Design, 2006 IEEE International Symposium on, pp.12061211, 4-6 Oct. 2006

Feiler, P.H., Gluch, D.P., and Hudak, J.J., "The architecture analysis \& design language (AADL): An introduction," Technical Report CMU/SEI-2006-TN-011, Software Engineering Institute, 2006

Frana, R.B.; Bodeveix, J.-P.; Filali, M.; Rolland, J.-F., "The AADL behaviour annex -experiments and roadmap," Engineering Complex Computer Systems, 2007. 12th IEEE International Conference on, pp.377-382, 11-14 July 2007 
Medvidovic, N.; Taylor, R.N., "A classification and comparison framework for software architecture description languages," Software Engineering, IEEE Transactions on , vol.26, no.1, pp.70-93, Jan 2000

Prasad, V.; Ting Yan; Jayachandran, P.; Zengzhong Li; Son, S.H.; Stankovic, J.A.; Hansson, J.; Abdelzaher, T., "ANDES: An ANalysis-Based DEsign Tool for Wireless Sensor Networks," Real-Time Systems Symposium, 2007. RTSS 2007. 28th IEEE International , pp.203-213, 3-6 Dec. 2007

Singhoff, F. and Plantec, A., "AADL modeling and analysis of hierarchical schedulers," In Proceedings of the 2007 ACM international Conference on Sigada Annual international Conference, pp. 41-50, 4- 8 Nov. 2007

Sherman, Trudy: Quality Attributes for Embedded Systems. Springer (2007), S. 536-539.

Talarico, C.; Aseem Gupta; Peter, E.; Rozenblit, J.W., "Embedded system engineering using $\mathrm{C} / \mathrm{C}++$ based design methodologies," Engineering of Computer-Based Systems, 2005. ECBS '05. 12th IEEE International Conference and Workshops on the, vol., no., pp. 81-88, 4-7 April 2005

AXLOG. ADeS: A Simulator for AADL http://www.axlog.fr/aadl/ades_en.html, 2009 


\title{
QuadR-Tree Indexing Selection Engine for Tuning Spatial Database System using Mobile Geographical Information System Technology
}

\author{
${ }^{1}$ Maruto Masserie Sardadi, ${ }^{2}$ Daut Daman, ${ }^{3}$ Mohd Shafry and ${ }^{4}$ Zahabidin Jupri \\ Faculty of Computer Science and Information System \\ University Technology of Malaysia, \\ 81310 Skudai, Malaysia
}

\section{Introduction}

The latest Mobile GIS technology is probable to manage the spatial components of daily business project, in the corporate database, and to apply proper geographic analysis efficiently in a desktop-focused application. This technology uses wireless internet for transferring process of spatial data from server to Mobile GIS or vice versa. However, the problem in wireless Internet is system bottlenecks that can slow down in the process of transferring data. One of the reasons why system bottlenecks could happen in this situation is because the spatial data is too large. The database design therefore is the most important part that must be considered. Database systems are now expected to be self-managing, self healing, and always-up. Another parts which also contribute in this problem are network, application server and web server (Kwan \& Shi, 2002).

Most of previous researchers have experimented to change the structure of R-tree and Quadtree spatial data indexing method that could give better performance. However, it still leaves some issues and problems that need to solving. One of the most common issues and problems is the method only applicable to some of applications such as data arrangement, but it needs speed improvement in the transfer process and data retrieval. This chapter gives different methodology that concentrates on fine tuning of a spatial database system using R-tree and Quadtree spatial data indexing method, without changing the structure of those spatial data indexing method.

This chapter introduces the creation of indexing engine selection to tune the spatial database system. This indexing engine selection, as it call QuadRtree Engine Selection, uses Rule Base - Knowledge Base Expert System (KBES) to select between R-tree and Quadtree spatial data indexing method. Those spatial data indexing methods have different advantages and disadvantages based on the condition of the data. This engineering innovation is one of the challenges in actual global technology. The objectives of this chapter are to make an optimization in transferring data and thus to provide the latest accurate information for mobile users with a limited communication bandwidth. 


\section{R-tree Spatial Data Indexing}

R-trees are tree data structures that derived from B-trees. It is used for representing multidimensional point data, but is used for spatial process methods for indexing multidimensional information. Each node in the tree communicates to the smallest d-dimensional rectangle that surrounds its son nodes. The leaf nodes include cursor to the real geometric entity in the database, as an alternative of childs. The entity are represented by the smallest associated rectangle in which they are enclosed (Guttman, 1984).

Frequently the nodes communicate to disk leaf, and therefore the constraint defining the tree is selected so that a small amount of nodes is appointed during a spatial query. Note that rectangles matches to different nodes may be related. Also a rectangle may be spatially controlled in numerous nodes; so far it can be correlated with only one node. This indicates that a spatial query may regularly necessitate a number of nodes to be visited before discovering the existence or absence of a particular rectangle.

R-tree spatial data indexing method uses Minimum Bounding Rectangle (MBR) in the algorithm method. The following figure denotes the rectangle part of an index entry E by EI, and the tuple-identifier or child-pointer part by EP. Give an R-tree whose root node is T, and find all index records whose rectangles overlap a search for rectangle $S$.

- $\quad$ Sl [Search sub trees] If T is not a leaf, check each entry E to determine whether EI Overlaps S. For all overlapping entries, invoke Search from the tree whose root node is pointed to by Ep.

- $\quad$ S2 [Search leaf node] If $\mathrm{T}$ is a leaf, check all entries $\mathrm{E}$ to determine whether $\mathrm{EI}$ overlaps S. If so, E is a qualifying record.

Fig. 1. R-tree algorithm (Guttman, 1974)

In Mobile GIS technology, R-tree has been chose for further explanation in this chapter. The reason for choosing $\mathrm{R}$-tree is that the application dealing with data containing and several overlap like what can be seen in Mobile GIS technology will bring better results, and the application dealing with data containing "whole Earth objects", bounding-box based methods will not work properly. In addition, Mobile GIS technology, will store, retrieve and process spatial data. Spatial data in GIS is a mandatory thing that needs to be solved for most all of the problems that might come.

\section{Quadtree Spatial Data Indexing}

A Quadtree is a tree data composition used to exploit a set hierarchical data compositions. The common property is that they are based on the principle of recursive decomposition in which each internal node has up to four children. Generally, Quadtree separate two dimension space through divide it into four sub part or region. The part could be in rectangular, arbitrary or square shape. Raphael Finkel and J.L. Bentley gave a name Quadtree for this data structure in 1974. This spatial data indexing has similar partitioning method with Q-tree. Quadtree has general decomposition approach, it decompose the space into flexible cell which has maximum capacity. The region split, then directory tree chase Quadtree spatial decomposition when meet the optimum capacity (Finkel \& Bentley, 1974). 
According to Spatial Concept in Oracle 10g white paper, "Quad tree spatial data indexing method uses fixed-size tiles to cover geometry. Fixed-size tiles are controlled by tile resolution. If the resolution is the sole controlling factor, then tessellation terminates when the coordinate space has been decomposed a specific number of times. Therefore, each tile is of a fixed size and shape". Fig 2. shows the process of Quadtree.

- $\quad$ The quadtree begins with a square in the plane; this is the root of the quadtree.
- This large square can be broken into four smaller squares of half the perimeter and
- $\quad$ a quarter the area each; these are the four children of the root.

Fig. 2. Quadtree algorithm (Finkel \& Bentley, 1974)

Currently Quadtree is used for point data, curves, surfaces, areas and volumes. It decomposes to the same parts on each level, or it may be manage by the input. This peculiarity, in computer graphics is often expressed in terms of image space ladder against object space ladder. The decomposition resolution may be fixed in advance, or it may be manage by materials of input data. In some of the applications the data formation on the origin of whether they state the restrictions of sections also can be distinguished.

\section{Rule-based Knowledge Base Expert System}

Expert system contain a memory evoke utility and a conjecture mechanism. This formation is like a human brain. It develops the data in the same way as expert people illustrates on the skill, knowledge and judgment expanded through understanding to achieve conclusion about a problem (Chow et al., 1995). For accuracy and sensibility, the effort's result of knowledge base expert system problem solving must be evaluated. The output should always examine as advisory (Uzel, 1987). An expert system approach process well when the problem to be solved is ill-defined or multifarious, and when decision and familiarity are helpful tools in finding the solution (Norman \& Lesser, 1994).

Ruled-based systems are a comparatively simple form that may be adjusted to some of difficult problems. With Artificial Intelligence (AI), rule-based system has its strength as well as limitation that must be reflect on before deciding if it's the appropriate procedure to use for a given problem. Generally, rule-based systems are only viable for problems in which any and all knowledge in the predicament area can be written in the outline of if-then rules and for which this problem area is not large (Zhang et al., 2008). Another advantage of using Rule-Based is that it can represent in a natural and understandable way the knowledge that summarize and explain the data.

Base on the previous exploration knowledge, it is need to setting up the weight of the rules to get the order of importance. All of the knowledge and weight has been set up in the ruled-based data set. This data set is used to decide whether it is need to use R-tree or Quadtree spatial data indexing in the Mobile GIS system. This research tries to give the weight from all of the knowledge above regarding the effect that can be affected to the system. The highest weight of the rule is the highest effect that can be affected to the system, thus the priority to chose is highest. Some of the rules are compulsory to be applied without considering another rules, because these rules are depend to the condition of the data and requirement of the system, otherwise our system will perform slower. This knowledge and 
dataset are dynamic, which can be updated again if the new knowledge has been explored. Here is the rule-based data set with the order of importance base on our research.

\begin{tabular}{|c|c|c|c|}
\hline No & R-Tree Indexing & Quadtree Indexing & Order of Importance \\
\hline 1 & $\begin{array}{l}\text { An R-tree index is required } \\
\text { for a whole-Earth index. }\end{array}$ & $\begin{array}{l}\text { A quadtree index cannot be } \\
\text { used for a whole-Earth index. }\end{array}$ & $\begin{array}{l}\text { First Choice. } \\
\text { Use R-tree }\end{array}$ \\
\hline 2 & $\begin{array}{l}\text { The approximation of } \\
\text { geometries cannot be fine- } \\
\text { tuned. (Spatial uses the } \\
\text { MBR) }\end{array}$ & $\begin{array}{l}\text { The approximation of } \\
\text { geometries can be fine-tuned } \\
\text { by setting the tiling level and } \\
\text { number of tiles. }\end{array}$ & $\begin{array}{l}\text { Second Choice. } \\
\text { Use Quadtree }\end{array}$ \\
\hline 3 & $\begin{array}{l}\text { You can index up to four } \\
\text { dimensions. }\end{array}$ & $\begin{array}{l}\text { You can index only two } \\
\text { dimensions. If LRS (Linear } \\
\text { Referencing System) data is } \\
\text { indexed using a spatial } \\
\text { quadtree index, only the first } \\
\text { two dimensions are indexed; } \\
\text { the measure dimension and } \\
\text { its values are not indexed. }\end{array}$ & $\begin{array}{l}\text { Third Choice. } \\
\text { Use R-tree }\end{array}$ \\
\hline 4 & $\begin{array}{l}\text { Heavy update activity to the } \\
\text { spatial column may } \\
\text { decrease the R-tree index } \\
\text { performance until the index } \\
\text { is rebuilt. }\end{array}$ & $\begin{array}{l}\text { Heavy update activity does } \\
\text { not affect the performance of a } \\
\text { quadtree index. }\end{array}$ & $\begin{array}{l}\text { Weight:15. } \\
\text { Use Quadtree }\end{array}$ \\
\hline 5 & $\begin{array}{l}\text { If your application } \\
\text { workload includes nearest- } \\
\text { neighbor queries (SDO_NN } \\
\text { operator), R-tree indexes are } \\
\text { faster, and you can use the } \\
\text { sdo_batch_size keyword. }\end{array}$ & $\begin{array}{l}\text { If your application workload } \\
\text { includes nearest-neighbor } \\
\text { queries (SDO_NN operator), } \\
\text { quadtree indexes are slower, } \\
\text { and you cannot use the } \\
\text { sdo_batch_size keyword. } \\
\end{array}$ & $\begin{array}{l}\text { Weight: } 13 . \\
\text { Use R-tree }\end{array}$ \\
\hline 6 & $\begin{array}{l}\text { An R-tree index is } \\
\text { recommended for indexing } \\
\text { geodetic data if } \\
\text { SDO_WITHIN_DISTANCE } \\
\text { queries will be used on it. } \\
\end{array}$ & $\begin{array}{l}\text { A quadtree index is not } \\
\text { recommended for } \\
\text { Indexing geodetic data if } \\
\text { SDO_WITHIN_DISTANCE } \\
\text { queries will be used on it. } \\
\end{array}$ & $\begin{array}{l}\text { Weight: } 9 . \\
\text { Use R-tree }\end{array}$ \\
\hline 7 & Less storage is required. & More storage is required. & $\begin{array}{l}\text { Weight: } 4 . \\
\text { Use R-tree }\end{array}$ \\
\hline 8 & $\begin{array}{l}\text { Index creation and tuning } \\
\text { are easier. }\end{array}$ & $\begin{array}{l}\text { Tuning is more complex, and } \\
\text { setting the appropriate tuning } \\
\text { parameter values can affect } \\
\text { performance significantly. }\end{array}$ & $\begin{array}{l}\text { Weight: } 3 . \\
\text { Use R-tree }\end{array}$ \\
\hline
\end{tabular}

Table 1. R-tree and Quadtree Rule-based data set

The knowledge expert from R-tree and Quadtree Rule-based data set above has been applied to geographical map, which is Pasir Gudang Map (one of the location in Malaysia). This map was used as GIS data case. It is needs to apply the Rule-Base data set to all of the tables in Pasir Gudang database. It also need exploration to recognized whether those tables suitable to use the R-tree, Quadtree or without spatial data indexing method. 


\section{QuadRtree Engine Selection}

QuadR-tree Engine Selection is a technique of selecting an R-tree and Quadtree spatial data indexing method in terms of data condition. There are some considerations when choosing R-tree or Quadtree spatial data indexing. The approach is how to make a decision to choose between R-tree or Quad-tree spatial data indexing that is suitable for different cases of geographical data based on spatial data indexing knowledge. Thus there would be one or two spatial data indexing method in one single spatial database system. The following figure is the algorithm of QuadRtree engine selection.

QuadRtree spatial data indexing Engine Selection is based on rule-based, Knowledge Base Expert System (KBES). The rule-based approach contains all of the proper encoded knowledge; generally written in If-Then rules. It also puts in working memory which holds some data or information. Firstly it needs to explore the knowledge base of R-tree and Quadtree spatial data indexing with the advantages and disadvantages comparison of those spatial data indexing methods. Here is the QuadRtree Engine Selection algorithm:

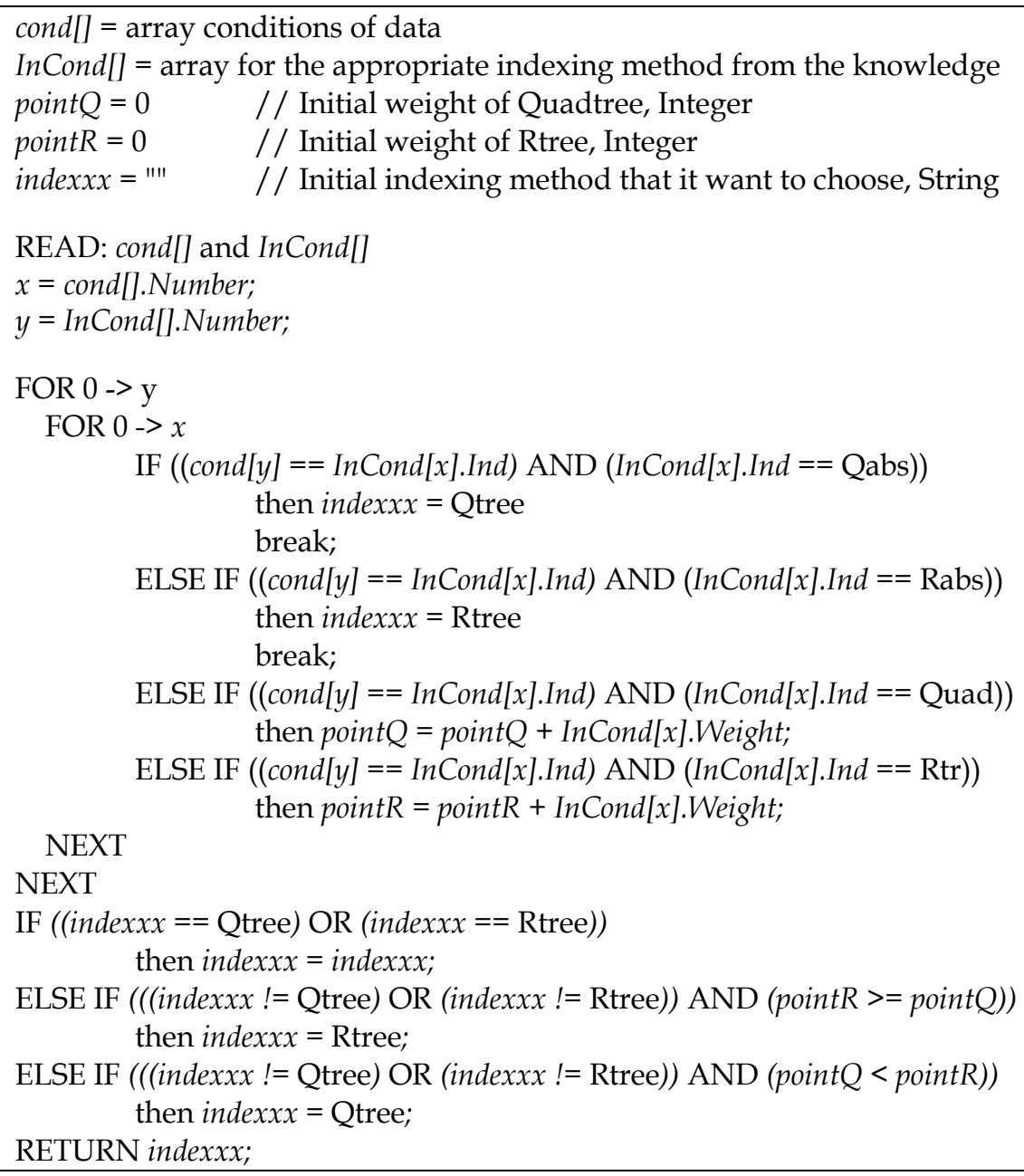

Fig. 3. QuadRtree selection engine algorithm 
All of the exploration of knowledge and weight would be put in the InCond[] array as it called rule set. This rule set will be used to compare with the input data cond[] array to match and get the appropriate spatial data indexing method. Through a set of assertions, which collectively form the 'working memory', and a set of rules that specify how to act on the assertion set, a rule-based system will be created. This rule set is dynamic; it could be added or updated again from the new knowledge's exploration of those spatial data indexing comparisons.

Before reading the set of data input, it is important to create the weight initialization for Rtree (pointR) and Quadtree (pointQ) spatial data indexing method that will be used in the selection engine's algorithm. These pointQ and pointR are used to decide whether R-tree or Quadtree spatial data indexing method which will be used based on the weight of data condition (cond[]). The initial indexing method (indexxx) also needs to create that be change to the appropriate spatial data indexing method.

The loop will be created to read and match all of the input data conditions cond[] and the rule set provided knowledge InCond[]. There are two methods in the InCond[] array they are InCond[].Ind to get the condition from the rule set knowledge and InCond[x].Weight to get the priority weight. Next, that there are two conditions from the knowledge they are absolute condition and the priority condition. The absolute condition is the condition that should use the proposed indexing method; R-tree (Rabs) or Quadtree (Qabs) without concerning to another condition and knowledge. If there is more than one similar proposed indexing method, it can use R-tree or Quadtree spatial data indexing method. The priority condition is the condition that needs to calculate the proposed amount of the weight from Rtree (Rtr) and Quadtree (Quad) spatial data indexing method. Thus it should choose the highest result of the weight amount either from R-tree or Quadtree spatial data indexing method.

\section{Testing \& Evaluation}

In order to evaluate the selection engine algorithm, this chapter presents the result of Mobile GIS application development. The selection engine algorithm has been putted in that application and could measures the speed of geographical map process over Mobile GIS network. The proposed indexing selection engine algorithm has tested using Pasir Gudang map. The developed Mobile GIS application has common GIS function, but it has additional function which is searching feature to measure the selection engine algorithm performance. The model used for testing and evaluation in this research has been simplified with the following assumptions:

- One dimension time

- Oracle $10 \mathrm{~g}$ spatial databases are used to develop the GIS Map data;

- Hyper Text Transfer Protocol (HTTP) utilized with data transfer protocol was used for connection from server to client or vice versa. Data retrieval process uses an active $\mathrm{TCP} / \mathrm{IP}$ connection with providing mobile user initial position.

Simplified mobile GIS equipment has demonstrated how process retrieval and transfer data works. A mobile user access data's from server that uses Oracle 10g Spatial Extension as a SDBMS to show the geographical map. The following way is to characterize the performance of this function; The average response time from the mobile client is measured as the time spent (in seconds) from the moment of the query is issued to the moment of the 
query is generated. The equipments and applications used in this research are summarized in the following table.

\begin{tabular}{|l|l|l|}
\hline Web Server \& GIS Server: & CPU & Intel Pentium Core 2 Duo \\
\cline { 2 - 3 } & RAM & 2 GHz \\
\cline { 2 - 3 } & Web Server & IIS \\
\cline { 2 - 3 } & GIS Server & $\begin{array}{l}\text { Oracle Application Server, } \\
\text { MapViewer and MapGuide Open } \\
\text { Source }\end{array}$ \\
\cline { 2 - 3 } & Database & Oracle 10g Spatial \\
\hline Mobile Device Equipment: & Model & Tablet PC \\
\cline { 2 - 3 } & OS & Windows XP \\
\cline { 2 - 3 } & Web Browser & Internet Explorer \\
\hline
\end{tabular}

Table 2. Setting of the experiments

This chapter tried to evaluate the geographical data using R-tree, Quadtree, QuadRtree and without using spatial data indexing method. This evaluation has been tried to Pasir Gudang Map. The total number of records used in the spatial database is around 35.000 . Some steps are needed to measure the retrieve and transfer processing time. It was tested from the number of record 1000, 5000, 10000, 15000, 20000, 25000, 30000 until 35000. Here is the evaluation process to Pasir Gudang geographical maps.

The coordinate system used in the geographical map is XY-M (Arbitrary X-Y Coordinates (Meters)) with lower left coordinates $\mathrm{X}$ : 37,478.171281 Y: -7,091.537339 and upper right coordinates X: $39,341.018451$ Y: $-66,250.168600$. The initial view of the map is center $X$ : 38,409.594866 Y: $-66,670.852970$ with zoom to 1: 32,965.970000. Our map has also built some of the general GIS map functions, like: Select Mode, Clear Selection, Zoom in, Zoom Out, Help, Pan Mode, Buffer, Select Polygon, Select Radius, Select Within, Measure, Print, and Search.

The Pasir Gudang Map spatial database use layers to organize the map and make easier to access. These layers also used to develop the map order which pins down one layer to another. There are four layers used in this spatial database. The first one is Default Layer which has BANGUNAN_ELLIPSE, BANGUNAN_POLYLINE, BANGUNAN_REGION, BANGUNAN_TEXT, MINIMUM_TROUGHPUT_REGION, and SEMPADAN_KAWASAN_ REGION tables. The second one is LOT Layer which has LOT_LUAR_REGION and LOT_ REGION tables. The third one is JALAN Layer which has JALAN_POLYLINE table. And the last layer is ZONING Layer which has ZONING_REGION table. The order for all of those layers is start from Default Layer, LOT Layer, JALAN Layer and ZONING Layer. The order of layers will take the effect in the layers position.

Figure 4. show the process of searching data or location. This searching method can choose the data or location by LOT, ZONING or REGION. The QuadR-tree indexing engine selection run in this process. Start from find the data or location until the data will be shown and zoom like in Figure 5. Furthermore, this process measure the speed of using indexing or not over Mobile GIS network. 


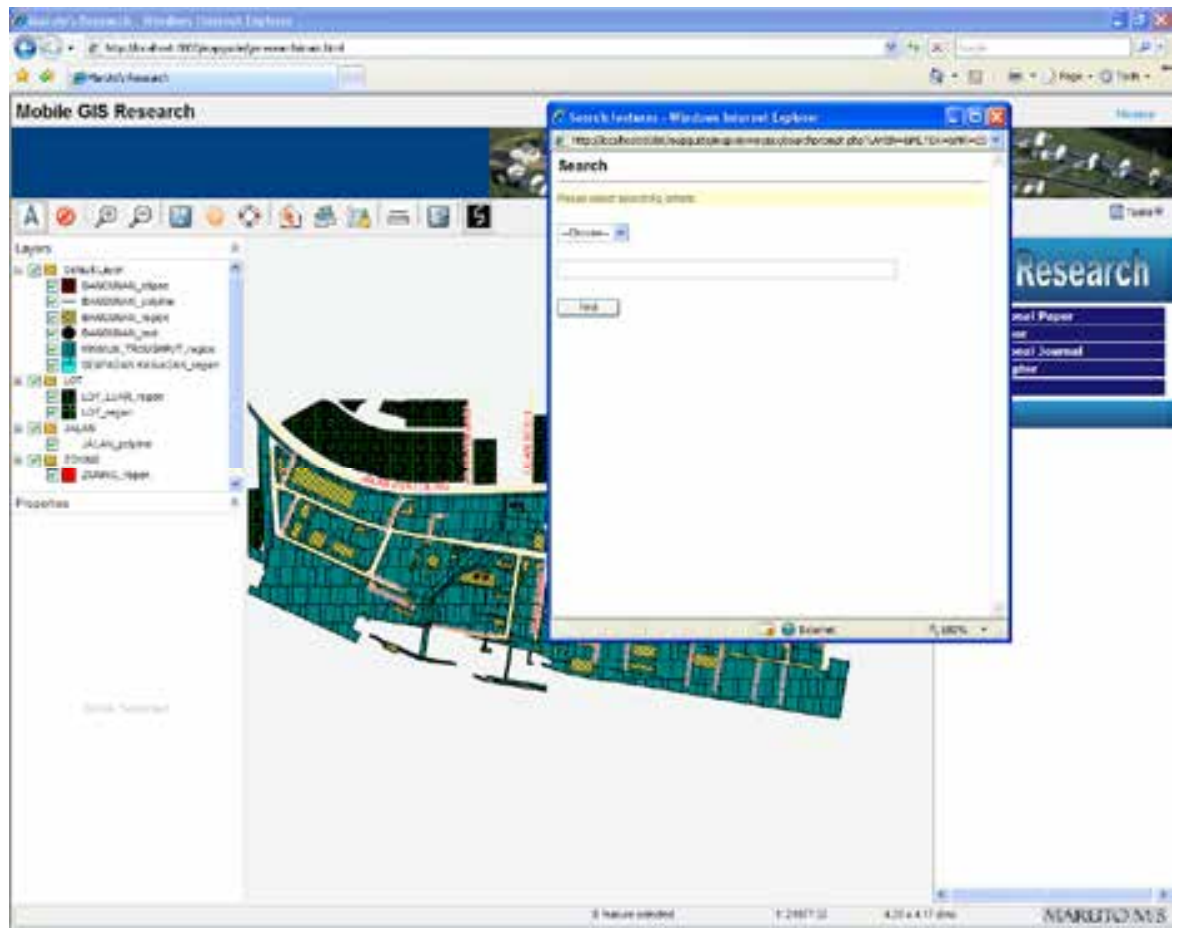

Fig. 4. Search the data or location.

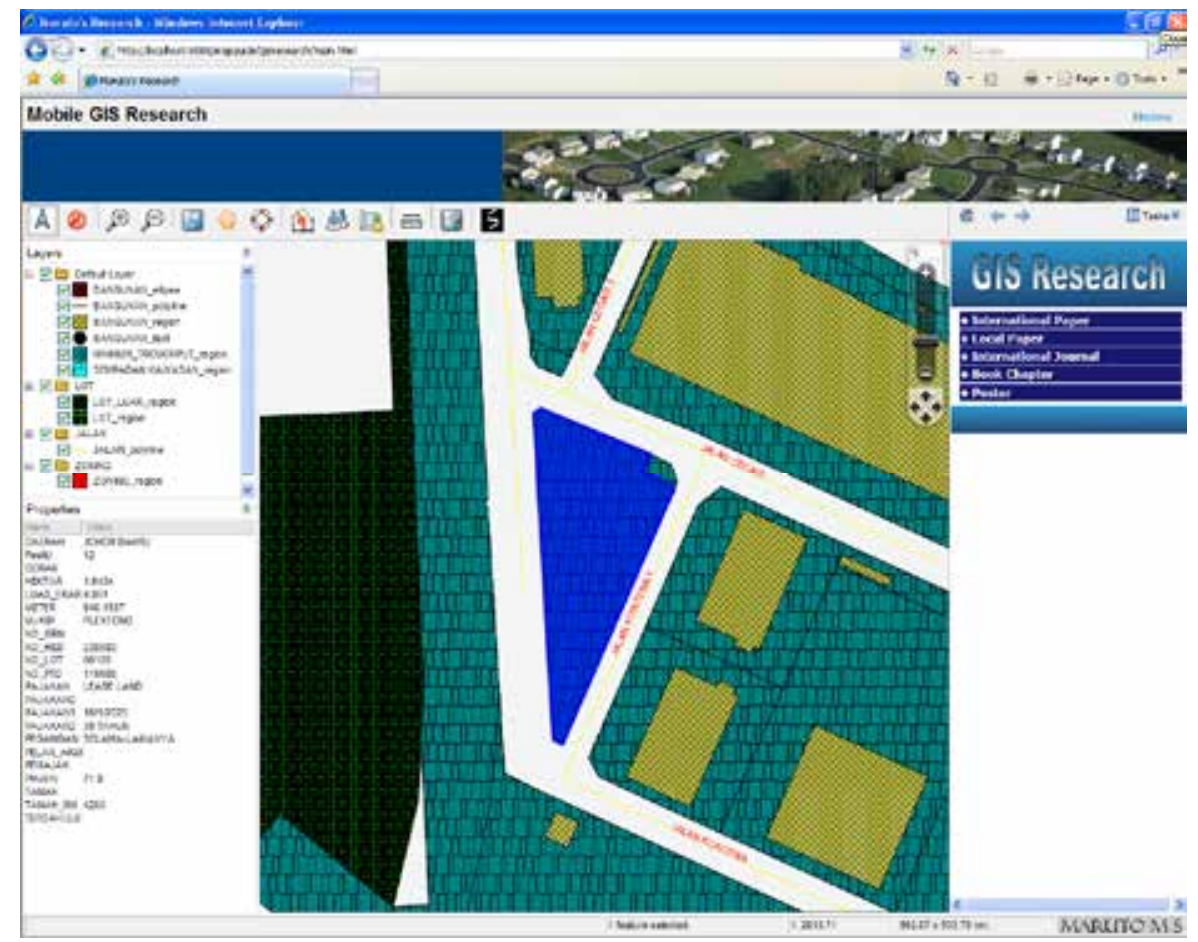

Fig. 5. Zoom the searched data. 
The Pasir Gudang Map application result is depicted in Figure 6., which is plotted as a twodimensional graph to illustrate the results of average response time using Mobile GIS application. The line with square point corresponds to the time measurement from the data use an R-tree spatial data indexing. The time measurement for the data that use Quadtree spatial data indexing has plotted with circle point. While the line with triangle point represents the time measurement for the data that doesn't use spatial data indexing. This research observes that the time saved increases as the database size expands. For the number of records, over 30,000, the time required in the data that uses an R-tree as well as Quadtree spatial data indexing are almost two times more than the data that doesn't use spatial data indexing. It also can be seen from the result, the used of QuadRtree indexing selection engine algorithm can make faster the process. The QuadRtree indexing is plotted in the line with plus point. It is noted, however, that this is related to the actual time of the query, since the database volume is varied as the record changes. The response time is slightly different to the result shown that figure. Nevertheless, it can easily discover that the response time on the data that uses an R-tree as well as Quadtree spatial indexing are faster than the data that doesn't use spatial indexing, moreover if the system use QuadRtree indexing selection engine algorithm.

\section{Seconds}

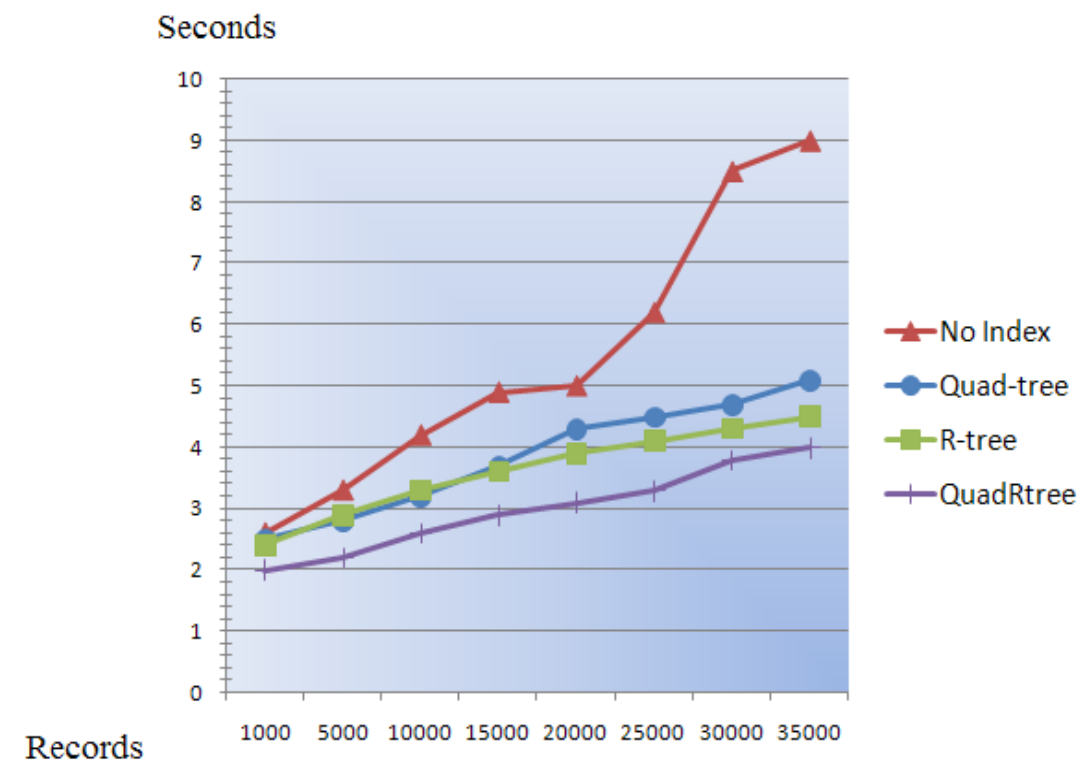

Fig. 6. The average response time.

\section{Conclusion}

The use of QuadR-tree indexing engine selection can improve the speed of transfer and retrieve data over Mobile GIS network. However, this engine still not automatically input the spatial data and condition of the data. This research also can be extended to explore the new knowledge of R-tree and Quadtree spatial data indexing that will be putted in the dynamic Rule-based data set. This knowledge will be used for selection consideration in QuadR-tree indexing engine selection algorithm. The Knowledge could be advantages or disadvantages of those spatial data indexing method. 


\section{References}

Chow, P. K. O., Yeung, D. S. (1995). Multidimensional Knowledge Structure. Expert Systems with Applications. Vol. 9, No. 2, 1995, pp. 177-187.

Finkel, R., \& Bentley, J. L. (1974). Quad Trees: A Data Structure for Retrieval on Composite Keys. Acta Informatica 4 (1): 1- 9.

Guttman, A. (1984). R-Trees: A Dynamic Index Structure for Spatial Searching. Proc. 1984 ACM SIGMOD International Conference on Management of Data, pp. 47- 57.

Kwan, K., \& Shi, W. (2002). A Study of Dynamic Database in Mobile GIS.

Norman, C. \& Lesser, V. (1994). The Evolution of Blackboard Control Architectures. Expert Systems with Applications, Vol. 7, No. 1, 1994, pp. 1-30.

Oracle Spatial 10g White Paper (2006). Oracle Spatial Quadtree Indexing, $10 \mathrm{~g}$ Release 1.

Uzel, A. R. (1987). Guidelines for Expert System Applications. CEM, Feb. 1987, pp. 40-45.

Zhang, L. \& Xi, L. F. (2007). A Novel Fractal Image Coding Based on Quadtree Partition of the Adaptive Threshold Value. Theoretical Advances and Applications of Fuzzy Logic and Soft Computing, 504-512. 


\title{
Quran Vibrations in Braille Codes Using the Finite State Machine Technique
}

\author{
Abdallah M Abualkishik and Khairuddin Omar \\ University Kebangsaan Malaysia \\ Malaysia
}

\section{Introduction}

How will blind people participate in a literate culture? How will they continue their education? What will make them feel as normal people? What about the Muslim blind people? Do they have the ability to read the Quran? What is a Braille system and how it was created? From these questions, the idea of Braille system has evolved.

Braille is a system of writing that uses patterns of raised dots to inscribe characters on paper. Therefore, it allows visually-impaired people to read and write by touching instead of seeing. Also it is a way for blind people to participate in a literate culture. The idea of Braille was started by Louis Braille, who was a blind person himself as a consequence of an accident that occurred when he was three years old. He needed a new way to learn. Although he had stayed at his old school for more than two years, he couldn't learn everything by listening. In 1821, a soldier named Charles Barbier visited the school. He introduced his invention, called "night writing," which was a code of 12 raised dots that had enabled soldiers to share top-secret information on the battlefield without having to speak. Louis had trimmed Barbier's 12 dots into 6 and then published the first Braille book in 1869 (Kim, 2009).

Braille is the system of touch reading and writing, which utilizes raised dots to represent the print alphabet letters for persons, who are blind. This system includes symbols to represent punctuations, mathematic and scientific characters, music and computer notations, and foreign languages. The blind are able to review and study written words using the Braille language, which provides a vehicle for literacy, giving the blind the ability to become familiar with spelling, punctuation, paragraphing, footnotes, bibliographies and other formatting considerations. A Braille cell, which consists of 6 dots - 2 across and 3 down, is considered as the basic unit for all Braille symbols. For easier identification, these dots are numbered downward 1, 2, 3 on the left, and 4, 5, 6 on the right, as shown in Figure 1.

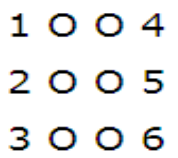

Fig. 1. Braille Code Cell 
Sixty-four probabilities are possible from these six dots, including the space symbol. These sixty four patterns are represented by equation 1

$$
\begin{array}{ll}
\mathrm{P}=2^{\wedge} \mathrm{N} & \\
& \mathrm{P}: \text { number of Braille symbols } \\
\mathrm{N}: \text { number of Dots }
\end{array}
$$

With the huge growth of technology nowadays, Braille systems have become widely adopted in most natural languages such as English, Germany, French, and so on. The Arabic language has also been translated to the Braille system. The Arabic Braille symbols were created based on the English characters as the translation process from any language to Braille must match the English characters first. Table 1 shows the Arabic characters, the matched English characters and the Braille symbols.

This research deals with the Al-Quran, that has its own limitations and rules for Muslims or for those who need to read about Islam as GOD holy book. The Quran recitation is different from the Arabic language reading in that it has special vibrations that must be adhered to, as any change in the way of reading can give another meaning. 'Allah said:

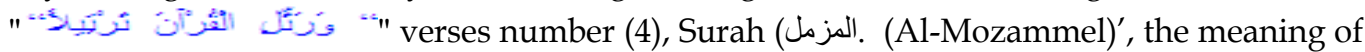
which is 'you should take care reading Quran verses, which Allah has ordered Muslims not to fall in the reading mistake'. The Arabic Holy Quran was translated to Braille code in Roy (2004). However this system is not complete as it does not include special vibrations required in reciting Al-Quran, which is different from reciting the Arabic language. In the study of Omar \& Kishik (2008), they have concentrated on the Noon + Scoon vibrations only, whereas this study has included more vibrations left out by them.

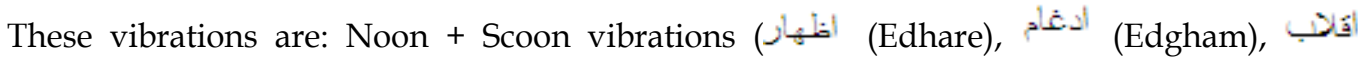

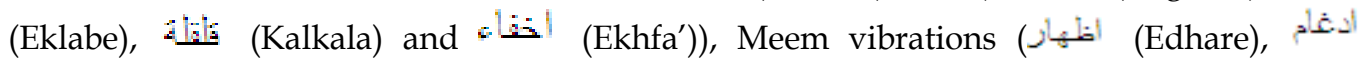
(Edgham), اخفاء (Ekhfa')); Lam vibrations (أنغان (Edhare), أناز (Edgham)) and Mud vibrations. Therefore there is a need to develop a system for translating these special vibrations into Braille symbols for the Quran verses.

In this research, the Decision table controls the operation of the finite state machine and provides more flexibility for it. A new grade has been created for the Arabic Quran Braille, covering the vibrations, absent in existing systems. A suitable finite state machine has also been developed to handle (i) a new grade Braille for the same language, allowing a single set of rules to double in the translation to Braille and (ii) a simple list of character translation rules that can be edited directly by non-technical users.

\section{Related Research}

There were many researches done to translate natural languages to Braille symbols. In this section, there are two research examples using finite state machine - decision table and rule 
base system to translate the English language to Braille symbols, and to apply some rules at the grade 1 of Braille symbols to become grade 2 .

\subsection{Text and Braille computer translation (King, 2000)}

This was concerned with the translation of texts from and to Braille using the matching of left and right contexts of the translation windows with the finite state machine to handle grades of Braille within the same language and to allow a single set of rules to duple as translation from and to Braille. The decision table controls the operations of the finite state machine and simple list of character translation rules that can be edited directly by nontechnical users.

\subsection{A system for converting print into Braille (Blenkhorn, 1997)}

In recent years, the UMIST translation system has been one of the few published works on text and Braille translation. The engine state is controlled by a finite state machine using the contents of the decision table, which regulates which subset of the language translation rules can be used. The translation engine can use any language rules table. So, any language can be translated to or from Braille code if the language rules table is constructed.

\section{The Existing Braille Systems}

\subsection{Duxbury Braille Translation software}

Duxbury Braille Translation (DBT) software is a window-based software system that automates the process of conversion from regular prints to Braille (Holladay, 2001) providing translation and formatting facilities. It also provides word-processing facilities directly in the Braille mode, where the user can treat the keyboard as Perkins machine to enter Braille text as well as using the software for ordinary word processing tasks. In addition, the software can translate Arabic text to Braille. With this supplementary function, one can create and edit natural Arabic texts using Microsoft Word (Arabic version), and then import and translate the file into Arabic Braille using the DBT. English texts can also be intermixed, and both languages may or may not be contracted, or in combination. Although DBT supports all the above features, there was still a small bug. The translation of Arabic characters in DBT was not $100 \%$ equivalent to the Arabic character set supported by Windows 95/98/NT/2000. Perhaps this was due to the difference in character code page used by DBT and Windows 95/98/NT/2000. Moreover, DBT does not support any type of sound, essential for the blind to interact with computers.

\subsection{IBSAR}

A Braille translate system (IBSAR) was developed by Al Alamiah, KSA (2004). The software was designed for the sighted users, having the ability to translate Arabic and English texts into Braille without contractions. It uses MS-Word as its platform.

\subsection{Printing system with Braille software}

The Kuwait institution for scientific research has developed software called printing system with Braille (Roy, 2000). It is a window-based application oriented for the sighted people to 
convert Arabic texts to Braille (one way translation). The software supports multi-level contractions as well as the conversion of Holy Quran files into Braille, but it doesn't involve the vibrations of the Holy Quran.

\subsection{Sensus Braille software}

Sensus Braille was developed by Sensus ApS, a co-operative effort of Danish and International Braille authorities. It is window-based software that automates the translation of English and Danish texts into Braille and vice-versa. However it does not support the Arabic language (Sensus, 1999).

\section{Theory Building}

Theory Building is the construction of a conceptual framework that declares the truth, formulates a concept, designs a method and develops a theory that is used during the prototype development.

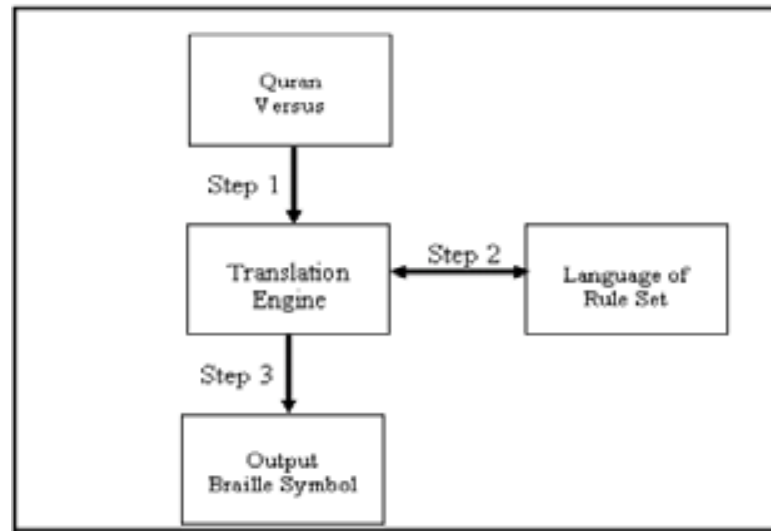

Fig. 2. Methodology approach for the translation algorithm

During the translation process the engine works along the input texts, character by character. It attempts to match a window of input text starting with the current character with one of the translation rules in the language rules table. Finite state machine involves the complications of state, control tables and many rules. An alternative approach using only the matching of left and right contexts of the translation window was developed. The former contains the translation algorithms and functions, while the latter has all of the translation information for translation of one language in one direction. The translation engine can use any language rules table, so any language can be translated to or from Braille code if the language rules table is constructed.

The language rules table consists of a set of translation rules and a decision table. A successful match with a translation rule must match a segment of text, the context - the text to the left and right of the window and the state of the engine. The engine state is controlled by the finite state machine, using the contents of the decision table, and regulates which subset of the language translation rules can be used. The translation rule provides the 
translation for that window of input text, which is appended to the growing output text, and the engine moves along the input text to the next unmatched character.

The standard world Braille is represented by the English character, so that the developers of the Arabic Braille systems are able to use the English characters to represent the Arabic Braille symbols. Therefore, the process to create the new vibrations to be used in Quran

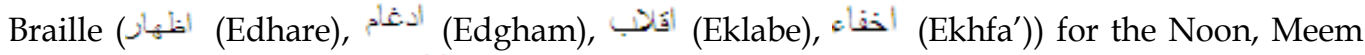
and lam characters; and (Kalkala) for the Scoon, entails finding all the possible probability symbols, that do not duplicate with existing Arabic symbols, thus constituting the 5 new vibrations.

\subsection{Develop the Rule set}

1) Create New Vibration

The recitation for the Quran verses is not similar to the Arabic language reading, as the Quran verses have special vibrations that help the Quran reader to get the right meaning for the verses. This research is concerned with the creation 8 new vibrations that did not exist in previous systems.

As shown in Table 1, the Noon Sakenah, Meem Sakenah, and Lam Sakenah have similar tow vibrations (Edhare and Edgham); the Ekhfa' vibration occurs with the Noon Sakenah and Meem Sakenah; the Eklabe occurs with the Noon Sakenah, and the Kalkala occurs with the Scoon. The Noon Sakenah, Meem Sakenah, Lam Sakenah and Scoon have different rules for each vibration detected.

\begin{tabular}{|c|c|c|c|c|c|c|}
\hline $\begin{array}{c}\text { Noon and } \\
\text { Scoon }\end{array}$ & $\begin{array}{c}\text { Meem and } \\
\text { Scoon }\end{array}$ & $\begin{array}{c}\text { Lam and } \\
\text { Scoon }\end{array}$ & Scoon & $\begin{array}{c}\text { English } \\
\text { Characters }\end{array}$ & $\begin{array}{c}\text { Braille } \\
\text { Symbols }\end{array}$ & $\begin{array}{c}\text { Number } \\
\text { of Dots }\end{array}$ \\
\hline Edhare & Edhare & Edhare & & 0 & \pm & 356 \\
\hline Edgham & Edgham & Edgham & & 8 & $=$ & 236 \\
\hline Ekhfa' & Ekhfa' & & & 7 & $=$ & 2356 \\
\hline Eklabe & & & & 6 & $=$ & 235 \\
\hline & & & Kalkala & $\mathrm{P}$ & $\mathbb{E}$ & 1234 \\
\hline
\end{tabular}

Table 1. New Quran Vibration Symbols

The Mud vibrations have many types - Mud for two characters (the reader should recite the character that have the Mud as two characters), Mud for four characters and Mud for six characters. 


\begin{tabular}{|c|c|c|c|}
\hline Mud Type & English Characters & Braille Symbols & Number Of Dots \\
\hline Mud 2 Char & B & : : : & $456+12$ \\
\hline Mud 4 Char & -D & :ः: & $456+145$ \\
\hline Mud 6 Char & -F & 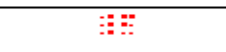 & $456+124$ \\
\hline
\end{tabular}

Table 2. Mud Braille symbols

2) Character Rules
a) Arabic characters that need to translate.
b) Identifying all the Arabic characters to be translated.
c) Identifying all the new vibrations to be translated.
d) Identifying the English characters that meet the Arabic characters.
e) Identifying the new created symbols.
f) Identifying the world standard Braille symbols for the English characters

3) Vibration Rules

There are around 20 reciting styles for the Holy Quran verses, but the most widespread reciting in the Islamic World is the Huffs reciting style, as mentioned in Al-kari' (1998), followed by Ali bin Abi-Talib "Allah blesses him". The rules set for the Quran vibrations applied are consistent with the Huffs style reciting, and this is explained comprehensively in this section.

The system will check the verses, word by word and if it finds any vibration, it will put its symbol under the vibration in a separate line to maintain the reader's attention to the vibration for the word that he/she reads it before he/she falls in default.

\section{a) Noon Sakenah Vibrations}

This vibration occur, if the characters in each vibration comes after Tanween or Noon Sakenah as presented in Tables 3, 4, 5 and 6.

\begin{tabular}{|c|c|c|c|c|c|c|c|c|c|}
\hline$\dot{C}$ & $\varepsilon$ & $C$ & $\varepsilon$ & $-s$ & $c$ & i & $!$ & 3 & 6 \\
\hline $\mathrm{X}$ & $>$ & : & ) & $\mathrm{H}$ & ^ & 1 & . & 1 & $\mathrm{Y}$ \\
\hline$\ddot{Z}$ & $\because:$ & : & : & $:$ & $\ddot{:}$ & $\ddot{z}$ & $\ddot{\vdots}$ & $\vdots$ & $\because$ \\
\hline
\end{tabular}

Table 3. أظهباد (Edhare Vibration)

\begin{tabular}{|l|l|l|l|l|}
\hline$j$ & $j$ & $i$ & $j$ & $\xi$ \\
\hline $\mathbf{N}$ & L & M & R & I \\
\hline $\mathbf{Z}$ & $\mathbf{E}$ & $\mathbf{Z}$ & $\mathbf{E}$ & $\vdots$ \\
\hline
\end{tabular}

Table 4. ادغام (Edgham Vibration) 


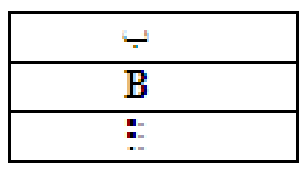

Table 5. أفَلن (Eklabe Vibration)

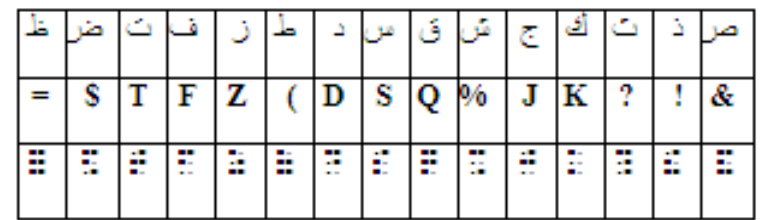

Table 6. اخفاء (Ekhfa' Vibration)

b) Meem Sakenah Vibrations

The vibrations that will occur, if the characters come after Meem + Scoon, are shown in Tables 7, 8 and 9 .

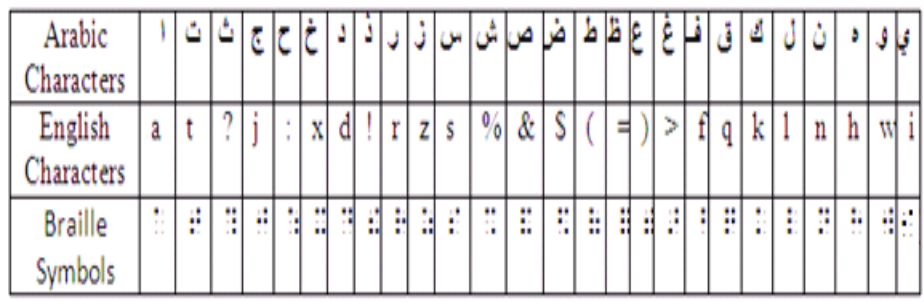

Table 7. إذهبر (Edhare Vibration)

\begin{tabular}{|l|c|}
\hline Arabic & $*$ \\
Character & \\
\hline English & \\
\hline $\begin{array}{l}\text { Braille } \\
\text { Symber }\end{array}$ & $\mathrm{m}$ \\
\hline
\end{tabular}

Table 8. ادغام (Edgham Vibration)

\begin{tabular}{|l|c|}
\hline $\begin{array}{l}\text { Arabic } \\
\text { Character }\end{array}$ & + \\
\hline $\begin{array}{l}\text { English } \\
\text { Character }\end{array}$ & b \\
\hline $\begin{array}{l}\text { Braille } \\
\text { Symbol }\end{array}$ & $=$ \\
\hline
\end{tabular}

Table 9. اخفاء (Ekhfa' Vibration) 
c) Lam Sakenah Vibrations

The vibrations that will occur, if the characters come after Lam + Scoon, are given in Tables 10 and 11.

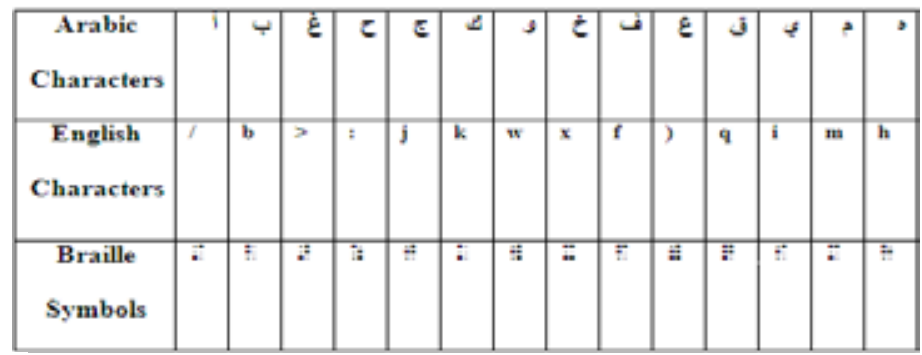

Table 10. اظظهبار (Edhare Vibration)

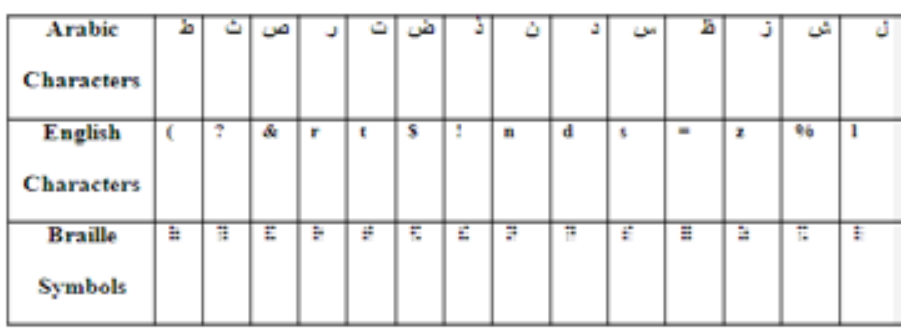

Table 11. ادنغا (Edgham Vibration)

d) Kalkala Vibrations

The vibrations that will occur, if the characters come after Scoon, are given in Table 12.

\begin{tabular}{|c|c|c|c|c|}
\hline$\lrcorner$ & $e$ & + & $\downarrow$ & $\xi$ \\
\hline$d$ & $j$ & b & ( & q \\
\hline$:$ & $:$ & $\vdots$ & $\vdots$ & $:$ \\
\hline
\end{tabular}

Table 12. فُّأ (Kalkala)

e) Mud Vibrations

The vibrations for Mud occur in three different conditions as presented in sub-sections below with the associated tables.

1. Mud for two characters

\begin{tabular}{|c|c|c|c|c|c|c|}
\hline Arabic Character & 1 & $9+$ & ئ & $1+\xi$ & $9+6$ & + \\
\hline English Character & $\mathrm{a}$ & $u+w+3$ & $e+i+3$ & $+a$ & $+w+3$ & $6+i+3$ \\
\hline Braille Symbols & $\because:$ & $\because+\vdots+: \because$ & $\because:+:+:$ & $\because \vdots+\because:$ & $\because:+:+::$ & $::+::+:$ \\
\hline
\end{tabular}

Table 13. Mud rules for two characters 
2. Mud for four characters

\begin{tabular}{|c|c|c|c|}
\hline Arabic Character & $a+1$ & $5+9$ & $4+\xi$ \\
\hline English Character & $a+\sqrt{ }$ & $w+3+i$ & $i+3+$ \\
\hline Braille Symbols & $\because \vdots+\vdots$ & $\because \vdots+\vdots+\vdots$ & $\because+\because+\because+$ \\
\hline
\end{tabular}

Table 14. Mud rules for four characters

3. Mud for six characters

\begin{tabular}{|c|c|c|c|}
\hline Arabic Character & + & g + (حرن) & ئي +(حرن) + \\
\hline English Character & $a+($ char +3$)$ & $(w+3)+($ char +3$)$ & $(1+3)+($ char +3$)$ \\
\hline Braille Symbols & $\begin{array}{c}(: \because+:) \\
+ \\
(\text { char }+: \because)\end{array}$ & $\begin{array}{c}(: \vdots+:) \\
+ \\
(\text { char }+: \because)\end{array}$ & $\begin{array}{c}(: \vdots+:) \\
+ \\
(\text { char }+: \because)\end{array}$ \\
\hline
\end{tabular}

Table 15. Mud rules for six characters

\begin{tabular}{|c|c|c|c|}
\hline Arabic Character & +1 & g + و (حرن) & ئي +(حرن) + \\
\hline English Character & $a+($ char,+$)$ & $(w+3)+($ char,+$)$ & $(I+3)+($ char,+$)$ \\
\hline Braille Symbols & $\begin{array}{c}(:+: \because) \\
+ \\
(\text { char }+::)\end{array}$ & $\begin{array}{c}(::+:) \\
+ \\
(\text { char }+: \because)\end{array}$ & $\begin{array}{c}(::+:) \\
+ \\
(\text { char }+::)\end{array}$ \\
\hline
\end{tabular}

Table 16. Mud rules for six characters

Table 17 shows that if the listed Arabic characters are found separately at the beginning of the surah (Quran Text), it will have Mud for six characters.

\begin{tabular}{|c|c|c|c|c|c|c|c|c|c|c|c|c|}
\hline $\begin{array}{l}\text { Arabic } \\
\text { Character }\end{array}$ & $\dot{0}$ & ق & صن & يسن & طظن & حم & ائر & |أن & |'نمر | & ط"ينب & انمضن & كون \\
\hline $\begin{array}{l}\text { English } \\
\text { Character }\end{array}$ & $\mathbf{n}$ & $q$ & $d$ & $\mathrm{i}+\mathrm{s}$ & $j+s$ & $:+\mathrm{m}$ & $a+L+r$ & $a+L+m$ & $a+L+m+r$ & $\mathrm{j}+\mathrm{s}+\mathrm{m}$ & $\mathrm{a}+\mathrm{L}+\mathrm{m}+\mathrm{d}$ & $\mathbf{k}+\mathbf{h}+\mathbf{i}+(+\&$ \\
\hline $\begin{array}{l}\text { Braille } \\
\text { Symbols }\end{array}$ & $\because$ & $\mathbf{Z}$ & : & $:: \vdots:$ & : : : & 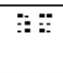 & $\because:: \vdots$ & $::::$ & : : : : : : : & :\#: :: & $\because::::$ & : : : : : : : \\
\hline
\end{tabular}

Table 17. Mud rules for six characters

4) Develop the Decision Table

Decision table is a powerful documentation tool for understanding and maintaining documents and is also useful in verifying the rules. Table 18 is regarded as the main table that will manage the process of applying the vibrations rule for the Holy Quran text. 


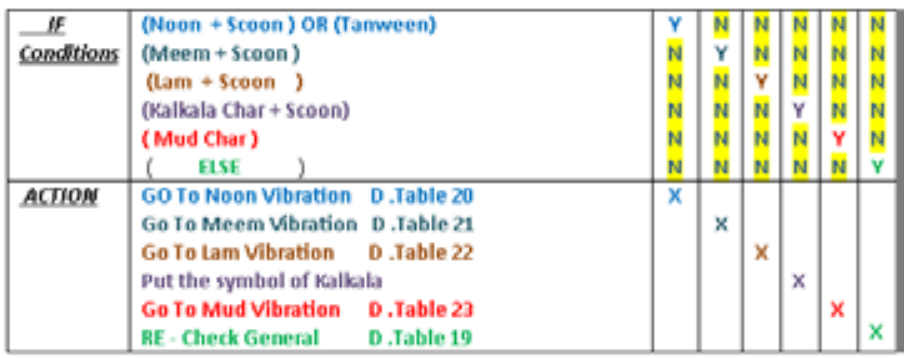

Table 18. General Table

Tables 19, 20, 21 and 22 represent the actions that occur after applying the conditions shown in Table 18.

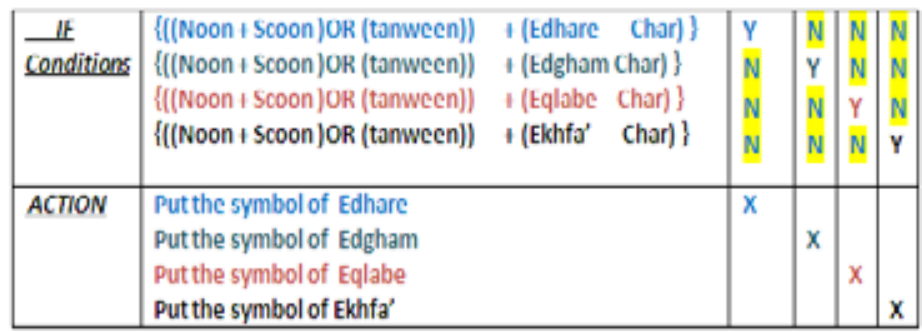

Table 19. Noon vibrations

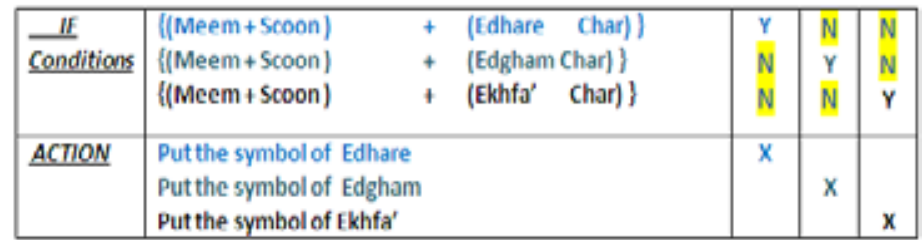

Table 20. Meem vibrations

\begin{tabular}{|l|l|c|c|}
\hline \multicolumn{1}{|l|}{} & $\begin{array}{l}(\text { Lam }+ \text { Scoon })+((\text { Edhare Char })+(\text { Not Shudah)) } \\
\text { Conditions }\end{array}$ & $\begin{array}{c}\mathrm{Y} \\
(\text { Lam }+ \text { Scoon })+((\text { Edgham Char })+(\text { Shudah }))\end{array}$ & $\mathrm{N}$ \\
\hline$\underline{\text { ACTION }}$ & $\begin{array}{l}\text { Put the symbol of Edhare } \\
\text { Put the symbol of Edgham }\end{array}$ & $\mathrm{X}$ & $\mathrm{X}$ \\
\hline
\end{tabular}

Table 21. Lam vibrations

\begin{tabular}{|c|c|c|c|c|}
\hline$\frac{\text { Cf }}{\text { Conditions }}$ & $\begin{array}{l}(\text { MUD 2) } \\
\text { (MUD 4) } \\
\text { (MUD 6) }\end{array}$ & $\begin{array}{l}\mathrm{Y} \\
\mathrm{N} \\
\mathrm{N}\end{array}$ & $\begin{array}{l}\mathrm{N} \\
\mathrm{Y} \\
\mathrm{N}\end{array}$ & $\begin{array}{l}N \\
N \\
Y\end{array}$ \\
\hline ACTION & $\begin{array}{l}\text { Put the symbol of MUD } 2 \\
\text { Put the symbol of MUD } 4 \\
\text { Put the symbol of MUD } 6\end{array}$ & $x$ & $x$ & $x$ \\
\hline
\end{tabular}

Table 22. Mud vibrations 


\section{5) Develop the Finite State Machine}

Finite state machine (FSM) is a useful data structure to express actions with a given sequence of events. FSM concepts are used for pattern recognition, artificial intelligence studies, and language. The basic concepts are easy to understand and immensely powerful. The idea behind FSM is that a system such as a machine with electronic controls can only be operational in a limited (finite) number of states. Consider some simple systems that you encounter every day - a door may be open or closed; a light may be on or off; a light bulb may be on, off or broken; a cassette player may be playing, stopped, rewinding or fast forwarding, (Gibson, 2000).

For each pair of state and input symbol there is one and only one transition to a next state, or each input symbol will then be transitioned to a state governed by a transition function. The first step to develop the FSM is determining the states needed to do the translation process (five main states in this study). Then determine the transition function needed to make the transition from state to state.

Figure 3 depicts the FSM method that was used in the system to monitor the translating processes.

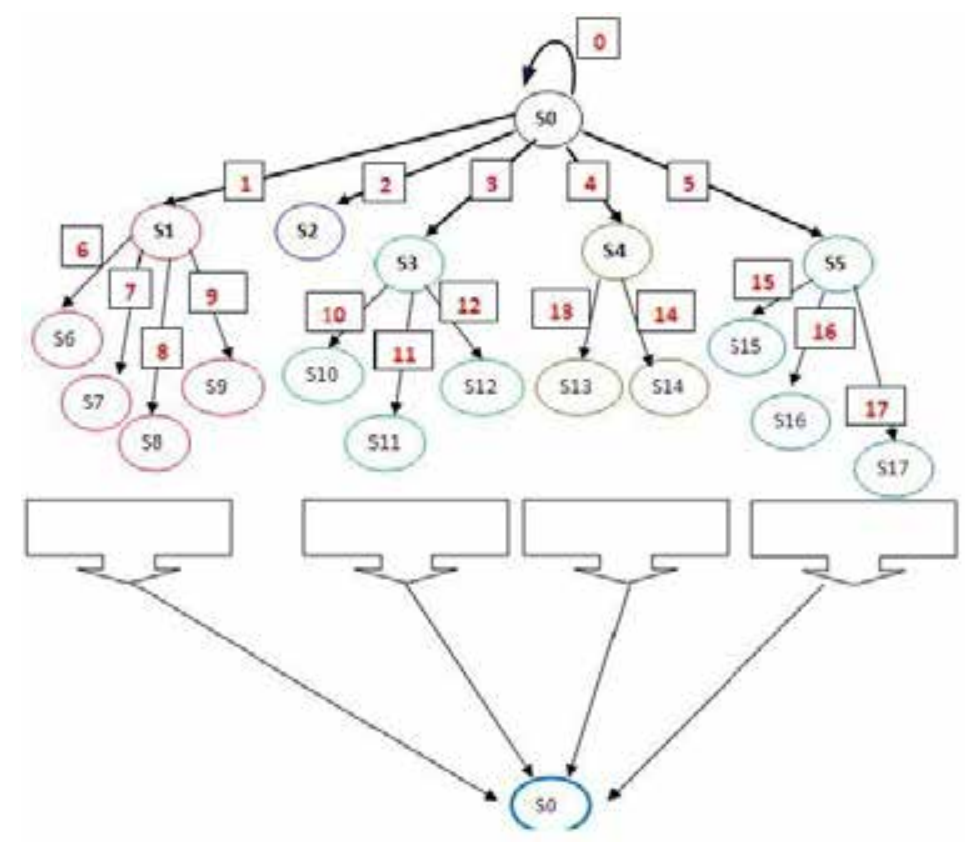

Fig. 3. Finite state Machine Diagram

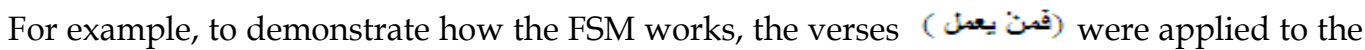
technique. At the initial state S1, the cursor reads the first character in the verses and because it does not have any vibrations, the transaction function is (0), translating the character to the corresponding Braille symbol, and the current state for the algorithm 
becomes S0. The cursor then moves to the second character, and as it also does not have vibration, the transaction function is (0) as well, with the current state still at S0. Next, the cursor moves to the third character and finds - Noon followed by Scoon, and then applies the transaction function (1). Then it checks the character after the - Noon followed by Scoon to determine the special vibration for the character, and subsequently moves to S1. Finally, the cursor checks the next character to determine the type of vibration present, then translating it to an appropriate symbol of the special vibration at this vibration location. In the previous example, the character that followed the Noon Sakenah was (Ya), where it was then considered as one of the Edgham vibration, so the cursor moves to S6 after translating the Edgham vibration. After that, the current state was back at $\mathrm{S} 0$ to continue the translation and detecting process for the rest of the verses and so on.

Table 23 represents the truth table for the FSM status for each state and each possible input from the user.

\begin{tabular}{|c|c|c|c|c|c|c|c|c|c|c|c|c|c|c|c|c|c|c|}
\hline Status & \multicolumn{18}{|c|}{ Transition Function } \\
\hline & 0 & 1 & 2 & 3 & 4 & 5 & 6 & 7 & 8 & 9 & 10 & 11 & 12 & 13 & 14 & 15 & 16 & 17 \\
\hline 50 & 50 & 51 & 52 & 53 & 54 & 55 & 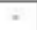 & $=$ & 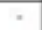 & $=$ & - & $=$ & $=$ & 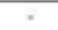 & $=$ &. &. & $\bar{\sigma}$ \\
\hline 51 & $\bar{*}$ & - & $\bar{*}$ & 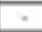 & $\cdot$ & $\cdot$ & 56 & 57 & 58 & 59 & & 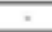 & 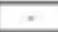 & 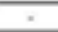 & $\overline{-}$ & . & $\bar{s}$ & $\bar{*}$ \\
\hline 52 & $\cdot$ & $\cdot$ &. & 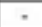 & $\cdot$ &. & $\cdot$ &. &. &. & - & - & - & $\overline{5}$ & $\bar{\sigma}$ & - & - & $\bar{\sigma}$ \\
\hline 53 & - & 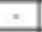 & - & - & $\cdot$ & 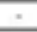 & - & 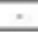 & 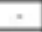 & - & $\mathbf{5 1 0}$ & 511 & 512 & & 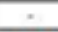 &. & - & - \\
\hline$\$ 4$ & $\cdot$ & $\cdot$ & $\cdot$ & $\cdot$ & $\cdot$ & $\cdot$ & $\cdot$ & $\cdot$ & 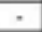 & $\cdot$ & $\cdot$ & $\overline{-}$ & $\cdot$ & 513 & $\$ 14$ & & $\cdot$ & $\cdot$ \\
\hline 55 & - & $=$ & 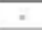 & 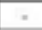 & $\cdot$ & $\cdot$ & 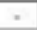 & 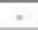 & $=$ & - & $=$ & 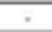 & 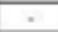 & 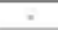 & - & 515 & $\mathbf{5 1 6}$ & 517 \\
\hline
\end{tabular}

Table 23. Truth Table

Table 24 describes the transition for each state. The transition function is to determine the next state based on the current input and state.

\begin{tabular}{|l|l|}
\hline \multicolumn{1}{|c|}{ Transition Function } & \multicolumn{1}{c|}{ Description } \\
\hline 0 & Normal character \\
\hline 1 & (Noon+Scoon)OR(Tanween) \\
\hline 2 & Kalkala \\
\hline 3 & Meem + Scoon \\
\hline 4 & Lam + Scoon \\
\hline 5 & Mud \\
\hline
\end{tabular}

Table 24. Transition Function Description 
6) Design and developing the translation engine

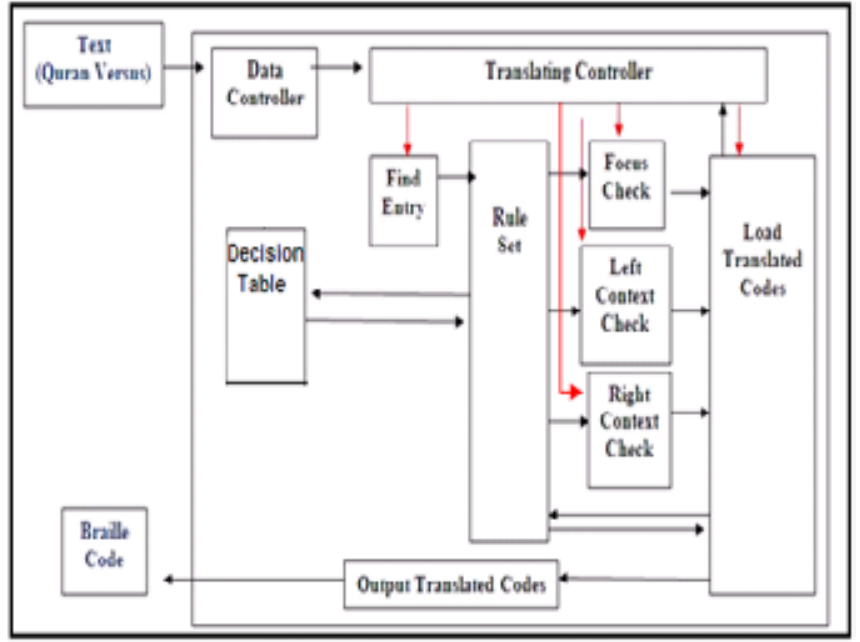

Fig. 4. Translation Block

Figure 4 depicts the translation block. The find-entry block receives one entry character from the translating-controller block and outputs a particular address to the rule-set block, where two operations are kept running. One reads rules from the Decision table block, while the other sends every single rule to focus-check, right-context-check, left context-check, and load-translated-codes blocks. The rule-set block receives signals from the find-entry block obtaining addresses, and signals from the load-translated-codes block, that indicates if the output rule can be used. The rule-set block sends an address to the Decision table to read one rule at a time and sends it separately to the focus-check, right context-check and leftcontext-check blocks. If the rule does not find a match, then a signal is generated and the rule-set block gets the next rule and sends it. This process continues until a match is found and the focus is successfully translated. The focus-check and right-context-check blocks receive not only the rules from rule-set block, but also the whole group of words to be translated from the translating controller because more than one letter of focus and right context may need to be checked. Each block generates signals for the load-translated-codes block indicating if the focus, the right context or the left context were successfully matched. If one of the three fails, then a signal is sent back to the rule-set block requesting the next rule. If the focus, right context and left context match one of the rules, then the load translated-codes block sends the translated codes to the output translated-codes block, and informs the translating-controller block how many characters were translated. After one group of characters has been translated, the output-translated-codes block transmits the corresponding Braille ASCII characters one by one. Then the translation of a new set of characters begins. 


\section{Conclusion}

In this study, the Quran Braille System was developed. It provides blind Muslims an easy way to read and understand the Holy Quran as well as the chance for other blind people to learn about Islam.

The experiments have produced a full translation prototype for the Quran verses including associated vibrations. The result of the experiment will be printed out using a Braille printer to introduce the usefulness of this study particularly to researcher and society at large. This study will adhere to its objectives, which are:

- $\quad$ To reorganize appropriate Braille symbols for the Quran vibrations.

- To translate Quran verses to Braille symbols.

- To propose accurate Quran Braille prototype for the translating process and the vibrations that will be detected from the Quran verses.

In order for this study to achieve the quality of outstanding systems, contributions from many institutes and research centres nationwide are required. Also, there is a need to standardize the Braille symbols to the Quran vibrations, because they have individual contraction rules.

\section{Reference}

Alamiah (2004), Al Alamiah Group \& Sakhr Co, http://www.ameinfo.com/42316.html , retrieve at $(13 / 11 / 2008)$.

Al-kari'. A. (1998), “Quran Reciting Rules-Haffes Style”, Islamic University, KSA, Vol.1, No.1, pp24- 44 .

Blenkhorn. P. (1997), "A System for Converting Print into Braille", IEEE Transactions on Rehabilitation Engineering, Vol 5, No 2, pp121 - 129.

Gibson (2000), "Making simple work of complex functions. PDF", SPLat Controls Pty.

Holladay. D. (2001), Mega Dot Product Manager, http://www.duxburysystems.com, retrieve at $(28 / 02 / 2009)$.

Kim, B. (2009). History of Reading Codes for the Blind, Blindness Resource Centre, http:/ / www.nyise.org/blind/barbier2.htm, Retrieve at (01/03/2009)

King. A. (2000), Keio University Access Research Group, Keio University ARG website, http:/ / buri.sfc.keio.ac.jp/access/arc/nabcc.html, retrieve at (02/01/2009).

Omar. KH. \& Kishik. A. (2008), "Quranic Braille System. PDF”. in Proceedings of the World Academy of Science, Engineering and Technology.

Roy. W. (2000), Braille Computer Facilities for Kuwait Special Schools, The Kuwait institute for scientific research. Printing system with Braille, user manual. Kuwait. (In Arabic), <http:/ / science.kisr.edu.kw/kisr_society.asp >, retrieve at (01/07/2008).

Sensus ApS. Sensus, (1999), http://www.sensus.dk/indexuk.htm, retrieve at (08/09/2008). 


\title{
Residential Noise Control Requirements for Powerline Communications Channel
}

\author{
Edward Guillen, Julian Lopez and Daniel Padilla \\ Telecommunications Engineering Department \\ "Nueva Granada"Military University Bogota, \\ Colombia
}

\section{Introduction}

Power line communications -PLC- is a technology that uses the electric grid for data transmission. Although the electrical channels were not designed to carry data, the implementation of PLC allows the using of a single channel for both electric and information transmission.

Even tough the PLC technology initially aimed at transferring low rate control data between electrical stations, the communications over electric channels have been widely implemented not only for control data transmission but also for establishing high rate data, voice and video communications.

Communications by power lines, begun as a slow analog communications technology, and recently and recently has become in a wide band technology that has been compared with wireless LAN solutions [1]. High-speed home network solutions have been developed to provide connectivity by wireless or wired mediums including 802.11 -WLAN-, 802.15 -WPAN- and high speed 1394. One of PLC's greatest advantages is the use of low power residential lines with non impact over the electrical circuits, sharing the medium for both power and communications solutions. Electric companies invest on wired Internet access to achieve low cost solution for fixed Internet access as last mile solution and they can use the system to gain control over energy meters and it can be used for demotic applications using electrical appliances networks.

The initial PLC standard for PLC home networking is HomePlug 1.0 [2] for LAN connectivity at every power outlet, and it has been implemented in a wide variety of commercial equipments such as routers, bridges, wireless access points, audio end points, speakers, VoIP phones and security cameras. HomePlug AV, supports entertainment applications such as HDTV and home theater throughout the home without new wires and provides this capabilities at competitive costs [3]. Recently, Telecommunications Industry Association -TIAhas adopted HomePlug 1.0 to be published in its standard TIA 1113, as the first multi-megabit power line communications standard approved by American National Standards Institute [4]. The first an only -ANSI- PLC standard defines operations, functions and interface characteristics of a system for medium speed networking using the medium of power line wiring based on Orthogonal Frequency-Division Multiplexing -OFDM-. 
For PLC implementations on electrical Latin-American environments, most of the noise control regulations are difficult to complain or simply regulations do not exist, that's why this chapter tries to analyze the effects of noisy electrical wired channels over the network throughput in common scenarios, as well as define the residential noise control requirements for PLC implementation. The network model on noisy channels is described in section $\mathrm{V}$.

\section{PLC Technology Overview}

This section is going to show the use of OFMD technology in PLC, a model of an electrical low power channel in a communications environment and a mathematical model of a PLC channel.

A. Orthogonal Frequency-Division Multiplexing -OFDM

In OFDM, a whole channel is divided into many narrow sub-channels, which are transmitted in parallel. With OFDM technique, the duration of a symbol is increased and the Inter Symbol Interference -ISI- is reduced [7]. HomePlug 1.0 uses 84 OFDM sub-carriers equally spaced as the physical layer. The first subcarrier start at $4.49 \mathrm{MHz}$ and the last one is at $21 \mathrm{MHz}$, in a bandwidth of $16.21 \mathrm{MHz}$. To avoid intersymbol interference in the timedomain and intercarrier interference in the frequency domain, a cyclic prefix comprising the last 172 samples from the inverse fast Fourier transform (IFFT) interval of 256 samples is added to the beginning of the IFFT interval to form a 428-sample OFDM symbol. HomePlug 1.0 used the Robust Mode of OFDM when the channel is degraded and in this mode all subcarriers are activated [8]. The 84 subcarriers distribution is shown in Figure 1.

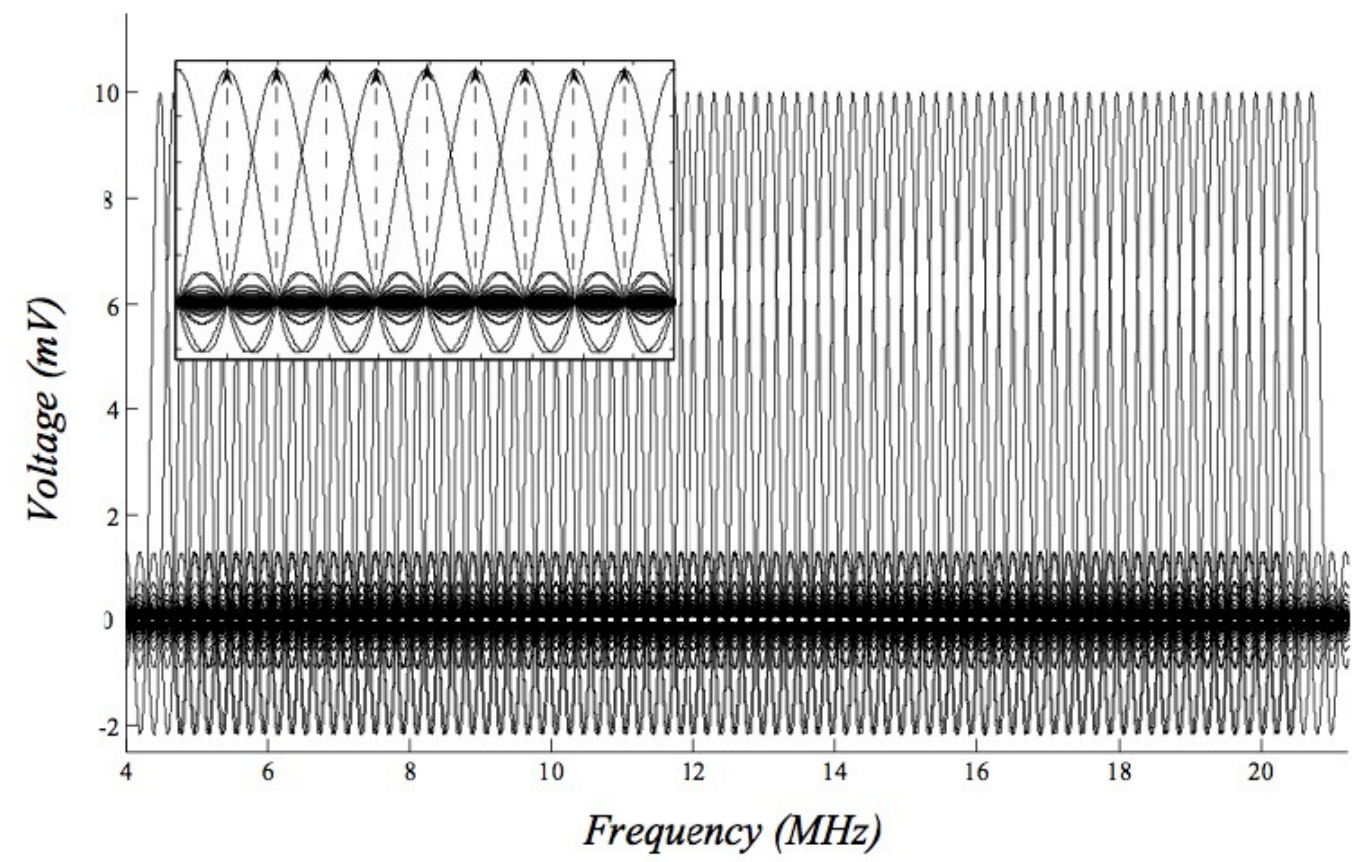

Fig. 1. OFDM spectral distribution 


\section{B. Features of PLC Transmission Channel}

An electric transmission line has been designed to transmit power in low frequency and causes a variance of the impedance because of the fact that a wide variety of appliances are connected to the outlet. Some statistical analysis and achieved measurements has shown a medium impedance of 100 to $150 \mathrm{ohms}$. However those values tend to decrease with frequencies bellow to $2 \mathrm{MHz}$. The attenuation of a powerline increases with the distance according to the impedance components that are composed by resistance per unit length, $-R^{\prime}-$, inductance per unit length, $-L^{\prime}-$, conductance per unit length, $-G^{\prime}-$, and capacitance, $-C^{\prime}-$, per unit length. The parameters have a closed relationship with the frequency and the line impedance can be described as can be seen on equation (1) [9].

$$
Z_{L}=\sqrt{R^{\prime}(f)+\frac{j 2 \pi f \cdot L^{\prime}(f)}{G^{\prime}(f)}+j 2 \pi f \cdot C^{\prime}(f)}
$$

Where $\omega=2 \pi f$. Another component to analyze is the propagation constant $\gamma$, whose equation is formulated in (2).

$$
\gamma(f)=\sqrt{\left[R^{\prime}(f)+j 2 \pi f \cdot L^{\prime}(f)\right] \cdot\left[G^{\prime}(f)+2 \pi f \cdot C^{\prime}(f)\right]}
$$

It's also usual to describe the propagation constant as a function of the next complex equation.

$$
\gamma(f)=\alpha(f)+j \beta(f)
$$

Where $\alpha(f)$ as the real part, represents the line attenuation factor and $\beta(f)$, as the imaginary part, is the line phase factor and the angle variation between the transmitted and received signals.

C. Mathematical Model of a PLC Channel

In [9], a PLC channel is described as a discrete-time impulse response in a path $i$ with a certain delay $\tau_{i}$ and certain attenuation factor $\mathrm{G}$ as is shown in (4)

$$
h(t)=\sum_{i=1}^{N} C_{i} \cdot \delta\left(t-\tau_{i}\right)
$$

In PLC channels the equation (4) is defined for frequency ranges between $500 \mathrm{KHz}$ to 30 $\mathrm{MHz}$ [10]. The transfer function in the frequency domain can be described as follows.

$$
H(f)=\sum_{i=1}^{N} g_{i} \cdot \exp \left[-\left(a_{0}+a_{1} f^{k}\right) \cdot l_{i}\right] \cdot \exp \left(-j 2 \pi f \tau_{i}\right)
$$

Where $g_{i}$ is a weighting factor representing the product of the reflection and transmission factors along the path; $l_{i}$ is the path length. The adjustable parameters $a_{0}, a_{i}$ and $k$ are used to 
show the attenuation of the channel with $k$ values from 0,5 to 1 and $N$ paths from 5 to 50 for the above range of frequencies.

\section{Noise on PLC Channels}

It is not possible to analyze power line channels as a traditional noisy channel with additive white Gaussian noise -AWGN-. Zimmermann [5, 6] classifies PLC noise into five classes according to Figure 2.

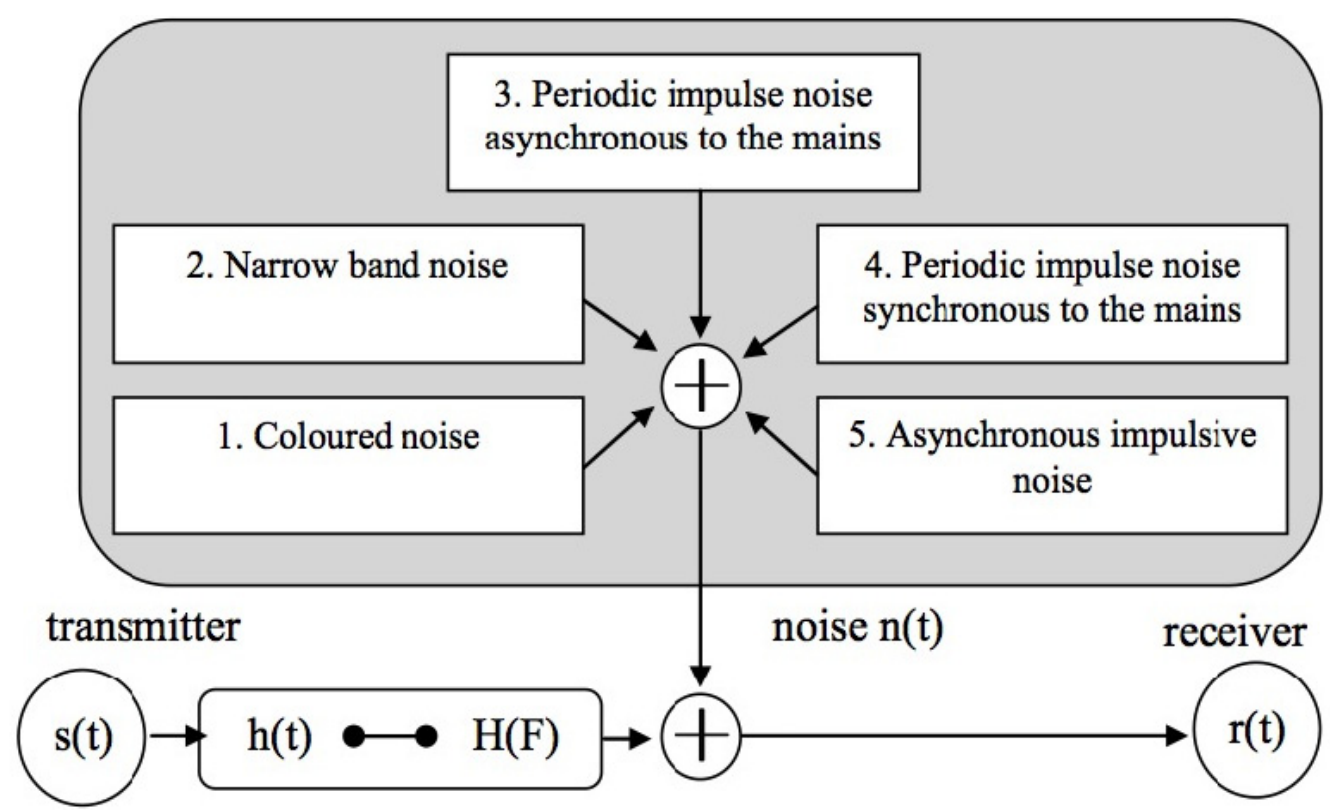

Fig. 2. Classification of Noise on PLC Channels

Coloured noise, narrow band noise and periodic impulsive noise are usually modeled as background noise because they remain stationary from seconds to even hours. Periodic impulsive noise synchronous to the mains and asynchronous impulsive noise may cause bit or burst errors over the transmission, although they are time variant. A complete theoretical analysis on shown noises in Figure 2 can be found on $[5,6,11,12,13]$.

\section{Noise and Throughput on PLC}

In order to probe the throughput variations caused by noisy channels in electrical LatinAmerican environments, five scenarios were created with a PLC network, in a common electrical distribution home network, with $120 \mathrm{AC}$ volts and 60Hz. Figure 3 shows the basic implementation. The electric noise source is connected in the outlet over the same electrical circuit using common wall sockets depending upon the scenario. In a first stage, the noise source is connected at the Tx host side, the measurements are taken and the noise source is connected at the receptor side for finally measurements. 


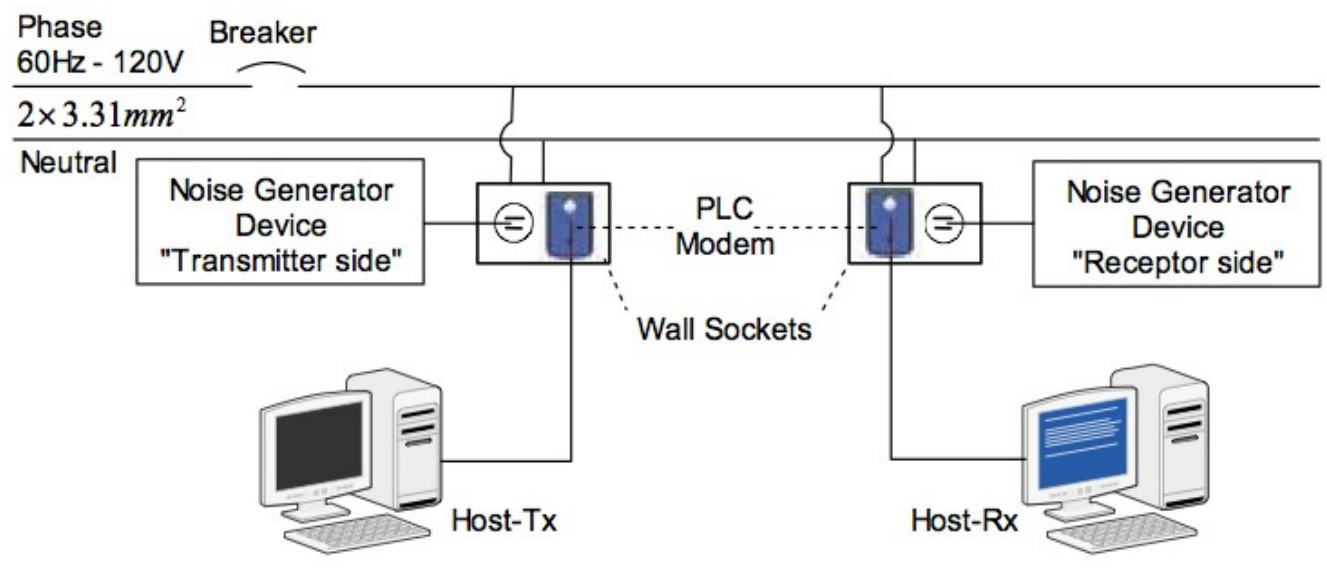

Fig. 3. Basic Networking Model

The probed scenarios are five. In the scenario 1, Base Scenario, traffic is sent over a free electrical generated noise by sending files between $20 \mathrm{MB}$ to $100 \mathrm{MB}$, in steps of $20 \mathrm{MB}$ incremented once every 50 samples. The collected data is: delay, bit length on the frames and throughput. This scenario is intended to evaluate transmission on normal conditions, and to compare results in noisy scenarios. The same files and variations are going to be used in every single scenario in order to collect data.

In the scenario 2, the channel is exposed to a 500 watts inductive load in the $90 \%$ of the transmission time that was taken in scenario 1. At scenario 3, an astable inductive load is connected to the electrical network. The periods of the generation are $2.5 \mathrm{sg}, 5 \mathrm{sg}$ and $10 \mathrm{sg}$ and are called tiAT. Every single period has the same value during the whole transmission. In the fourth scenario, an inductive and resistive load of 40 watts is connected to the electric channel. The load is attached during all transmission time. At scenario 5, an electronic and resistive load is connected along the channel during the transmission time.

The effects of the proved noise sources over the low voltage home network and the scenarios results comparison are shown in part A. In the second results part, the noises that affected the probed channel are analyzed with the statistical resources.

A. PLC Throughput Results

In a protocol analyzer installed on the transmitter equipment, the total time for the transference was analyzed for the transmission path between the hosts. The total bit length was also measured and the throughput was calculated, in Mbps, with the scenarios described before. The obtained throughput is compared with the base scenario and the noise effect over the channel is obtained.

1) Scenario1 vs. Scenario 2

Figure 4 shows the throughput for: scenario without noise generation (scenario1), scenario2 with the noise source connected at the Tx side and scenario 2 with the noise source attached at the Rx Host side. 


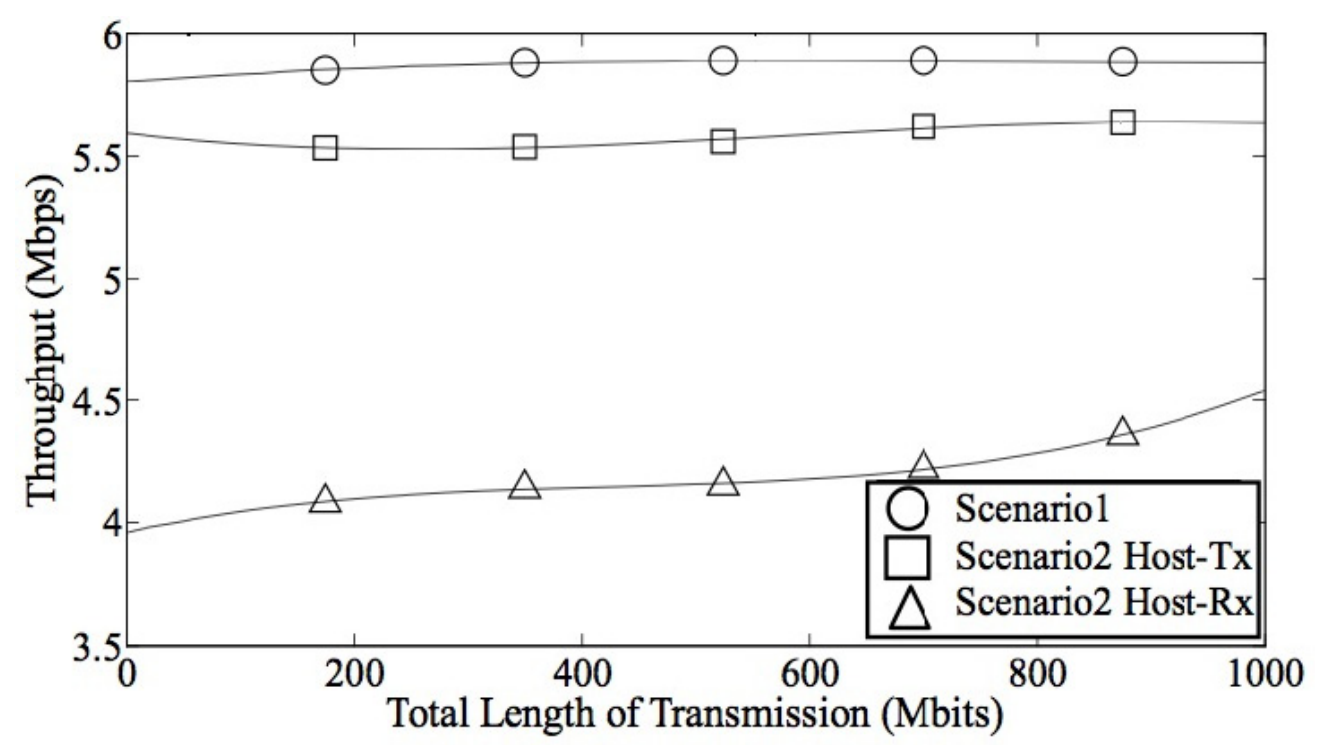

Fig. 4. Throughput, Scenario 1 Vs Scenario 2

When the noise is generated at the transmission side, it is possible to see a throughput reduction of $5 \%$, compared with the throughput obtained in the scenario 1 , but when the noise generation occurs in $\mathrm{Rx}$ side, as probed scenario 2, throughput decreases in $29 \%$ compared with the base scenario. In the last case, the $58 \%$ of the transmitted bits are transferred during the noise generation, afterwards, the throughput increases and time decreases as it is shown in Figure 5, with files of $20 \mathrm{MB}, 40 \mathrm{MB}, 60 \mathrm{MB}, 80 \mathrm{MB}$ and $100 \mathrm{MB}$. Figure $5 \mathrm{a}), \mathrm{b}), \mathrm{c}), \mathrm{d}$ ) and e) shows the instant in which the inductive load is turned off. In Figure $5 \mathrm{f}$ ), when the transmitted file is $100 \mathrm{MB}$ of length, the throughput is constant along the communication, as shown in Figure $5 \mathrm{f}$ ).

2) Scenario3 vs. Scenario 2

As explained for scenario 3, three comparisons had been made according to the times of $t_{I A T}$. In the Figure $6 \mathrm{a}$ ), the throughput for $t_{I A T}=10 \mathrm{sg}$ is shown. The throughput decreases when the astable load is connected at the Tx-host side in $0.82 \%$ and the throughput diminish in $0.96 \%$ when the load is attached at the Rx side.

For $t_{I A T}=5 \mathrm{sg}$, the throughput has a higher alteration for lower bit-lengths as it is shown in Figure $6 \mathrm{~b}$ ). The decrease for this compared scenario is $1.28 \%$ and $1.89 \%$ for the astable inductive load connected at the Tx-side and at the Rx-side, respectively. 


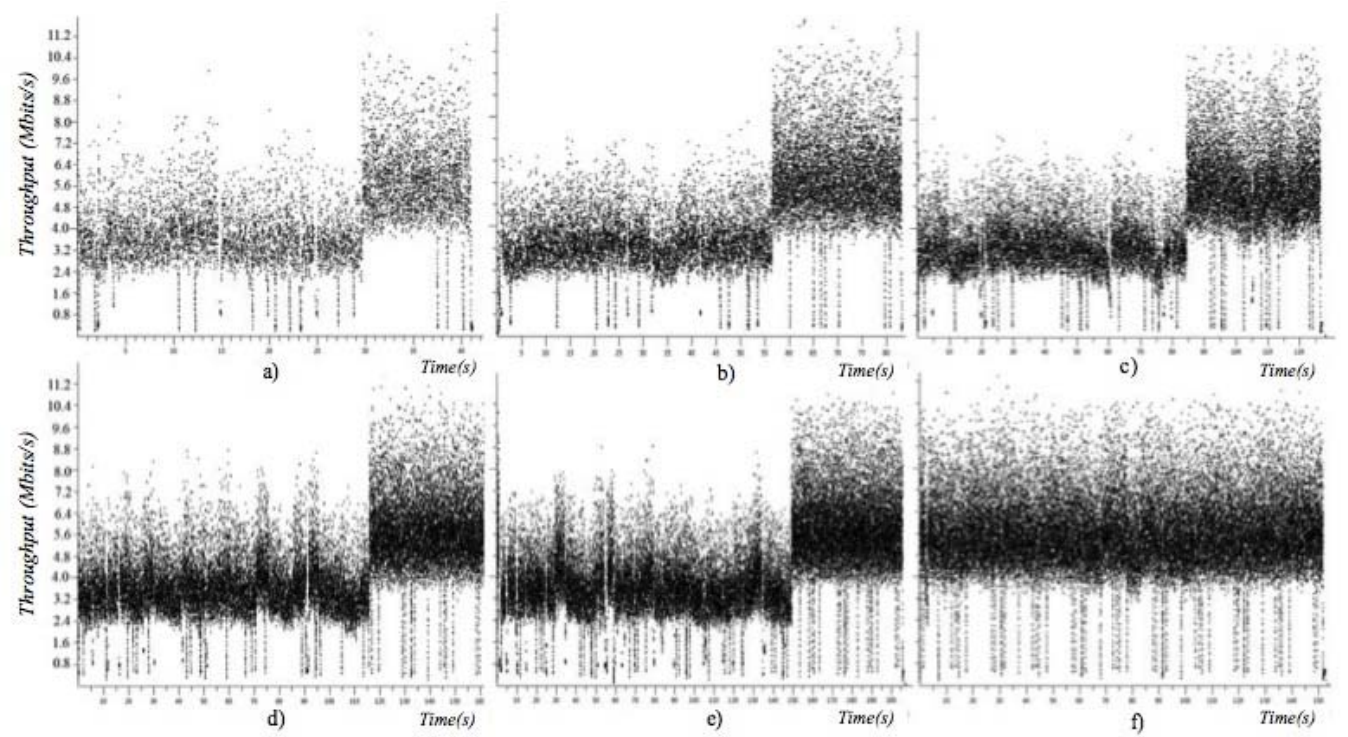

Fig. 5. Scenario 2 at Rx side, with files of a) $20 \mathrm{MB}$, b) $40 \mathrm{MB}$, c) $60 \mathrm{MB}$, d) $80 \mathrm{MB}$ and e) $100 \mathrm{MB}$. f) At the Tx-side with $100 \mathrm{MB}$

\section{3) Scenario3 vs. Scenario1}

As explained for scenario 3, three comparisons had been made according to the times of $t_{\text {IAT }}$.

In the Figure $6 \mathrm{a}$ ), the throughput for $t_{I A T}=10 \mathrm{sg}$ is shown. The throughput decreases when the astable load is connected at the Tx-host side in $0.82 \%$ and the throughput diminish in $0.96 \%$ when the load is attached at the Rx side.

For $t_{I A T}=5 \mathrm{sg}$, the throughput has a higher alteration for lower bit-lengths as it is shows in Figure $6 \mathrm{~b}$ ). The decrease for this compared scenario is $1.28 \%$ and $1.89 \%$ for the astable inductive load connected at the Tx-side and at the Rx- side, respectively.

The last compared scenario, with $t_{I A T}=2.5 \mathrm{sg}$, shows throughput differences of $1.45 \%$ with the load at the Tx-side and of 5\% with the load at the Rx-side. These differences can be seen in Figure $6 \mathrm{c}$ ), always compared with the scenario 1.

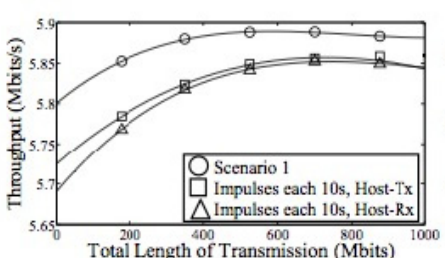

a)

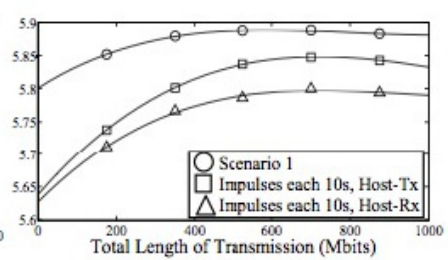

b)

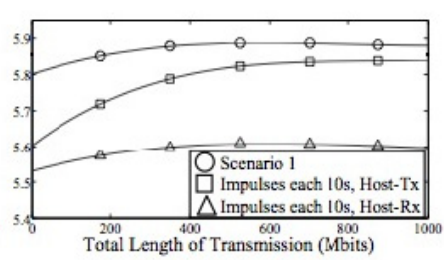

c)

Fig. 6. Throughput for scenario3 for astable period times of a) $10 \mathrm{sg}$, b) $5 \mathrm{sg}$, c) $2.5 \mathrm{sg}$

It is also possible to analyze the transmission time with the throughput for each period of the scenario 3 with the transmitted file of $60 \mathrm{MB}$; this case is shown in Figure 7. 

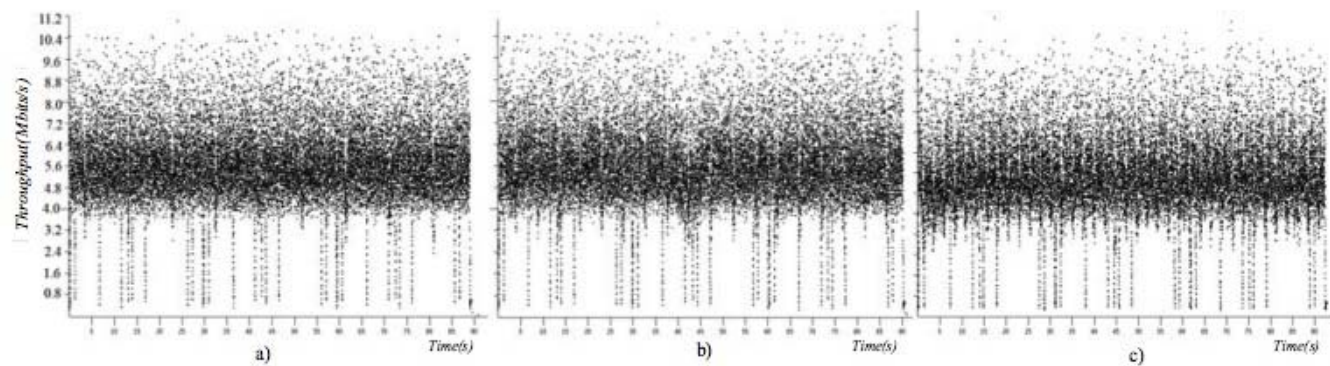

Fig. 7. Scenario 3 at the Rx-side with a transmission of $60 \mathrm{MB}$ with r.. of a) 10sg, b) 5sg and c) $2.5 \mathrm{sg}$

It is possible to see, on each case, white spaces on the graph that occurs in the edge of the load activation at the $t_{I T}$ period, as seen on Figure 7. When the load is attached at the Tx-side those spaces are not possible to distinguish. Although most of the graphs and data are not available for this paper we can provide them upon an email request.

4) Scenario4 vs. Scenario1:

The throughput result shows that there is a decrease of $0.33 \%$ when this kind of load is connected at the Tx-side and there is a decrease of $5.66 \%$ at the Rx-side. As it occurs in most of the cases, the throughput is minimal affected when the noise is generated at the Tx-side, but it is meaningfully affected when the noise is connected at the Rx-side.

The throughput results for the scenario 4 are shown in Figure 8. It is interesting to see that the maximum loss occurs with bit-lengths transmission of less than $400 \mathrm{Mbits}$ and with noise generated at the Rx-side.

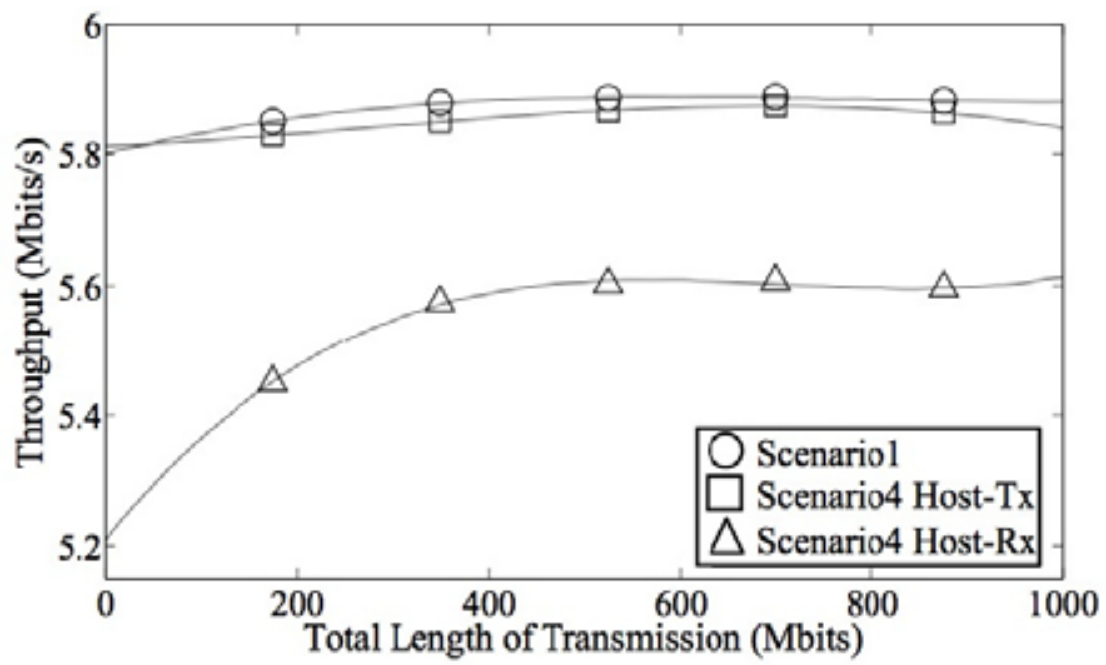

Fig. 8. Throughput for scenario 4

5) Scenario5 vs. Scenario1:

In the scenario 5 the differences are very small compared with scenario 1 only a decrease of $0.24 \%$ and $0.26 \%$ with the load attached at the Rx-side and at the Tx-side respectively. The results can be seen on Figure 9 


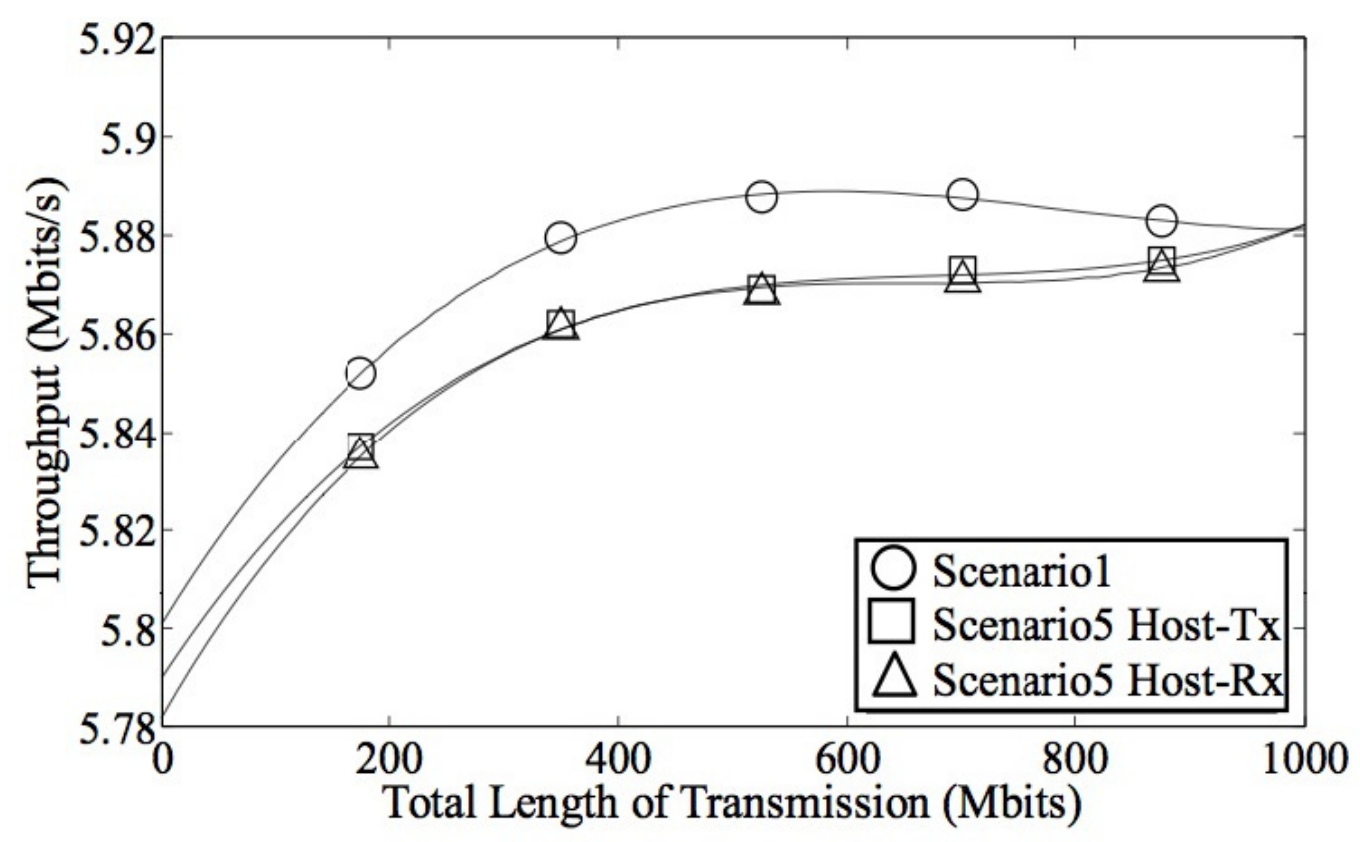

Fig. 9. Throughput for Scenario5

A summary of the throughput affectation in all the scenarios compared with scenario 1 for PLC is shown in Table 1. The results show a major throughput decrease with the characterized impulsive noise.

\begin{tabular}{|c|c|c|c|c|}
\hline \multirow{2}{*}{\multicolumn{2}{|c|}{$\begin{array}{c}\text { Scenario } 1 \\
\text { compared with: }\end{array}$}} & \multicolumn{2}{|c|}{ Load Connected at: } & \multirow{2}{*}{$\begin{array}{c}\text { Represented } \\
\text { Noise Set }\end{array}$} \\
\hline & & Host-Tx (\%) & Host-Rx (\%) & \\
\hline \multicolumn{2}{|c|}{ Scenario2 } & 5,00 & 29,0 & \multirow{4}{*}{$\begin{array}{l}\text { Impulsive } \\
\text { Noise }\end{array}$} \\
\hline \multirow{3}{*}{ Scenario 3} & $10 \mathrm{~s}$ & 0,82 & 0,96 & \\
\hline & $5.0 \mathrm{~s}$ & 1,28 & 1,89 & \\
\hline & $2.5 \mathrm{~s}$ & 1,45 & 5,00 & \\
\hline \multicolumn{2}{|c|}{ Scenario 4} & 0,33 & 5,66 & Background \\
\hline \multicolumn{2}{|c|}{ Scenario 5} & 0,24 & 0,26 & Noise \\
\hline
\end{tabular}

Table 1. Throughput Reduction for Every Single Scenario Compared with Base Scenario

\section{B. Noise affecting PLC channels}

The noises that are affecting the PLC channel during the probed scenarios, can be analyzed as impulsive noise and as generalized background noise, both of them are specified as follows:

1) Impulsive Noise

In the generated noise within the scenario 3, the waveforms that are showed in Figure 10 was obtained. 


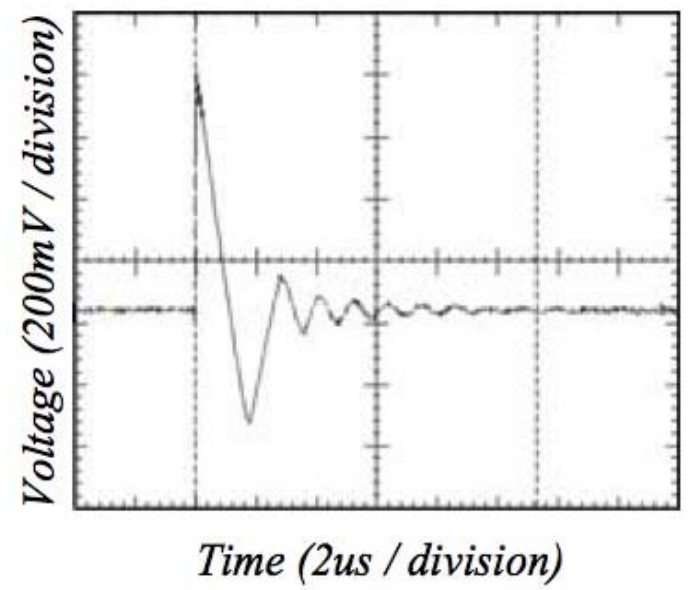

a)

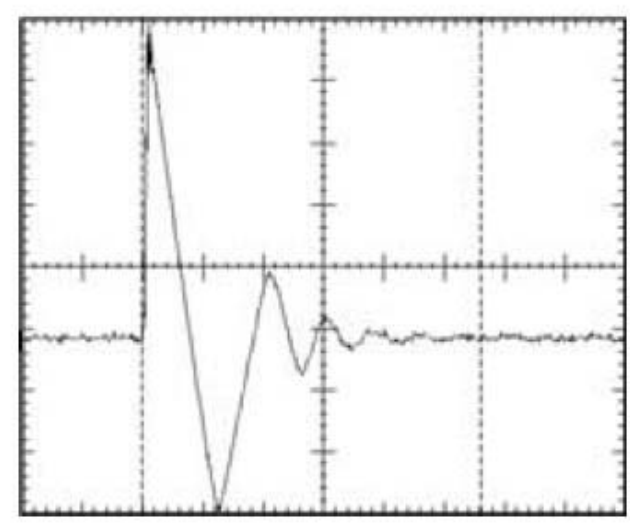

Time (2us / division)

b)

Fig. 10. Impulsive noise obtained within the scenario 3 a) casel, b) case2 The impulsive noise can be modeled by the equation (6)

$$
n_{s}(t)=\sum_{i=1}^{I_{s}} A_{i} \sin \left[2 \pi f_{i}\left(t-t_{a r r, s}\right)+\alpha_{i}\right] e^{\left(\frac{t-t_{a r r, s}}{\tau_{i}}\right) \cdot u\left(\frac{t-t_{a r r, s}}{t_{\omega, s}}\right)}
$$

Where $A_{i}$ defines the amplitude of the impulse, $f_{i}$ and $\alpha_{i} \mathrm{i}$, the frequency and phase of the iesime impulse, $t_{a r r, s}$ specifies the time where the impulses starts, $t_{\omega, s}$ the time on seconds of the signal $u(t)$ that is the shape of the pulse. In order to calculate the power of the impulses, the relation of Zimmermann and Dostert [5, 6], is written in the equation (7)

$$
P_{n_{s}(t)}=\frac{1}{t_{w, s}} \int_{t_{a r r, i}}^{t_{a r r, i}+t_{w, s}} n_{s}(t)^{2} d t
$$

The results of the analysis can be seen in the Table 2

\begin{tabular}{ccccc}
\hline \hline Impulse & $\begin{array}{c}\text { Duration } \\
t_{w, s}(\mu s)\end{array}$ & $\begin{array}{c}\text { Amplitude } \\
A_{i}(m V)\end{array}$ & $\begin{array}{c}\text { Frequency } \\
f_{i}(\mathrm{Khz})\end{array}$ & $\begin{array}{c}\text { Power } \\
\mathrm{dBm}\end{array}$ \\
\hline Case 1 & 11.27 & 766 & 778 & 14.69 \\
Case 2 & 11.10 & 975 & 403 & 16.53 \\
\hline \hline
\end{tabular}

Table 2. Results of the scenario 3

In the scenario 2, the impulses waveforms were analyzed in time domain and frequency domain as it is shown in the Figure 11 


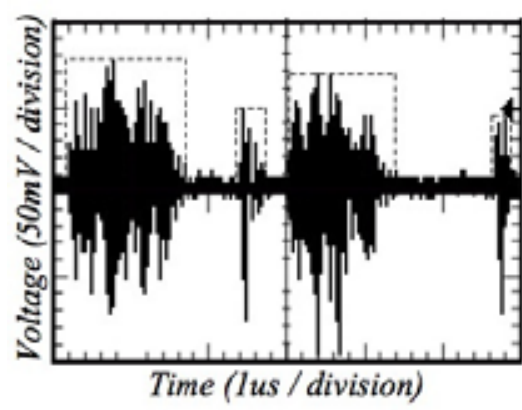

a)

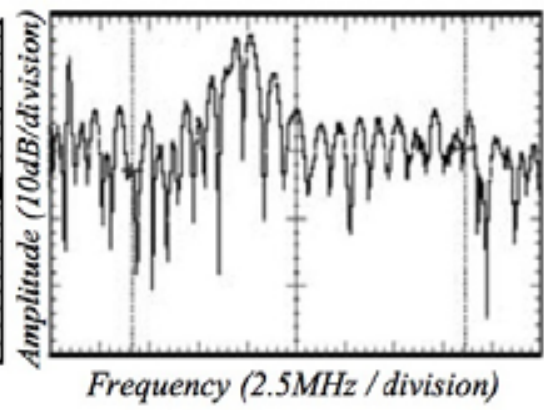

b)

Fig. 11. Impulsive noise measured for scenario 2

The noise presented in scenario 2 has the duration of $t_{w, s}$, with a time between impulses of $t_{a r r, s}$ and an amplitude $A_{i}$. This noise is presented during the complete period in which the inductive load is connected. In the spectral waveform of the Figure $11(b)$, the reference level is $-45 \mathrm{dBm}$, spam of $25 \mathrm{MHz}$, and the range limited by the cursors lines is the bandwidth of the PLC technology.

In order to generalize the noise 200 samples of the noises are taken and analyzed with a statistical tool. The amplitudes obtained are modeled by a Weibull Probability Density Function (PDF), as it is suggested for similar noises on ADSL analysis $[15,16]$.

The PDF Weibull can be described by equation (8) where $a$ is the continuous variable of amplitude, $a$ is a scale factor and $\beta$ is a shape parameter, with all positive values.

$$
f(a)=\frac{\beta}{\alpha} a^{\beta-1} \exp \left[-\left(\frac{a}{\alpha}\right)^{\beta}\right], a>0
$$

The PDF obtained with the Weibull approach appears in the Figure 12 a), with the calculated parameters.

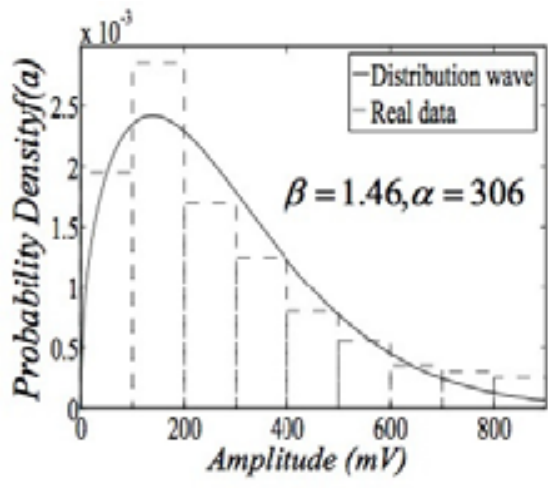

a)

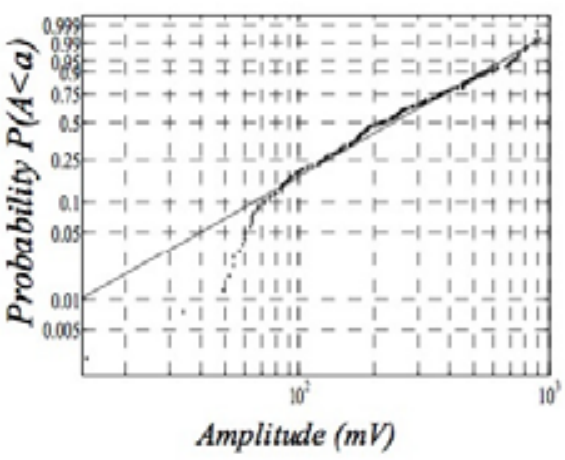

b)

Fig. 12. Weibull Distribution a) PDF, b) Amplitude function 
The Fig 12. b) shows the cumulative theoretical probability for Weibull distribution.

The next two variables are represented for an Exponential PDF that is symbolized by the equation (9), where $x$ represents the continuous variable of the distribution and is equivalent to the impulses duration $\mathrm{w}$, while the inter-arrival times is expressed by $\mathrm{r}$.

$$
f(x)=\lambda e^{(-\lambda x)}, x \geq 0
$$

The representation of the probability density function of $w$ is shown in Figure 13 a), where a higher probability of impulses duration can be found before of $16 \mu \mathrm{sg}$.

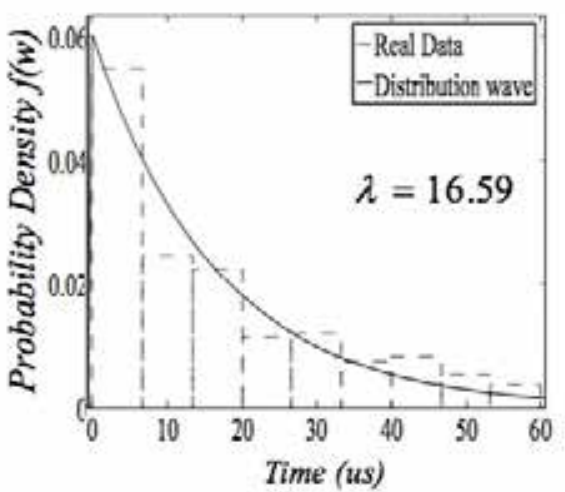

a)

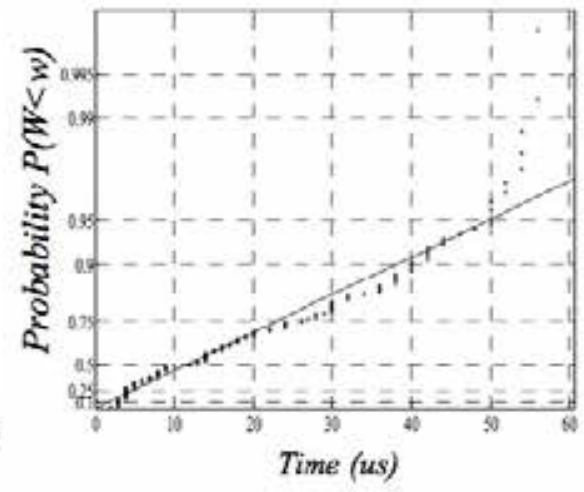

b)

Fig. 13. Exponential function for the impulses duration a) PDF b) Distribution Function

The inter-arrival time between impulses has a probability density function $f(r)$ as it is shown in Figure $14 \mathrm{a}$ ). There are not impulses for times below of $40 \mu \mathrm{sg}$. The plotted data fitted the theoretical function.

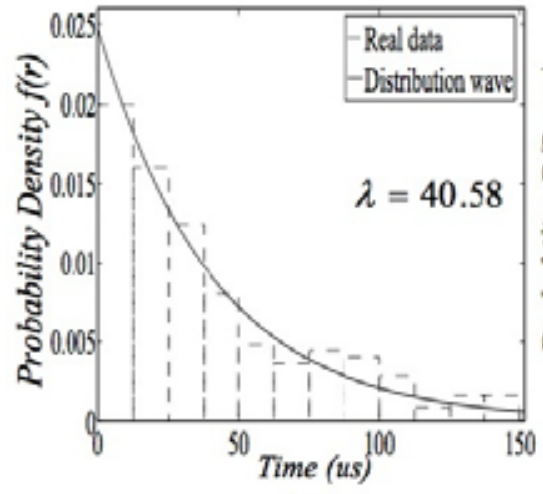

a)

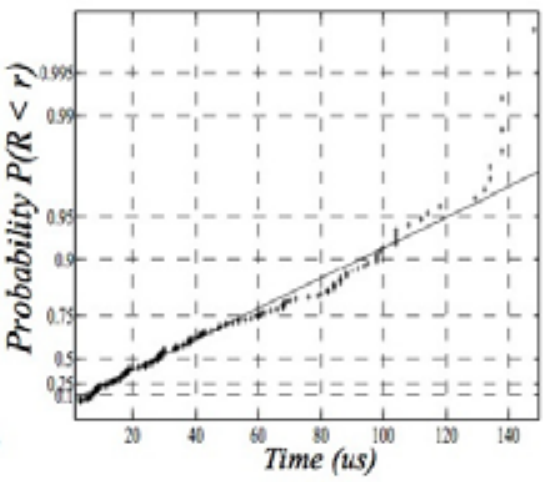

b)

Fig. 14. Exponential function distribution for inter-arrival times between impulses a) PDF b) Exponential probability 
As a summary of the analyzed variables, the Table 3 shows the distributions and its parameters.

\begin{tabular}{|llll|}
\hline \multicolumn{1}{|c}{ Variable } & \multicolumn{1}{c}{ Distribution } & \multicolumn{1}{c|}{ Mean } & \multicolumn{1}{c|}{ Variance } \\
\hline Amplitude (a) & Weibull & $277.2 \mathrm{mV}$ & $37244 \mathrm{~m} V^{2}$ \\
Duration (w) & Exponential & $16.595 \mu \mathrm{s}$ & $275.39 \mu \mathrm{s}^{2}$ \\
Inter-arrival & Exponential & $40.58 \mu \mathrm{s}$ & $1646.5 \mu \mathrm{s}^{2}$ \\
times (r) & & & \\
\hline \hline
\end{tabular}

Table 3. Mean and variance for the analyzed

\section{2) Generalized Background Noise}

The set of noise belong to the background noise do not show an important affectation to the throughput on the communication network when the load is connected at the Tx side. The phenomenon is explained by the low power density of this kind of noises in the range of frequencies used by PLC, see Figure 15.

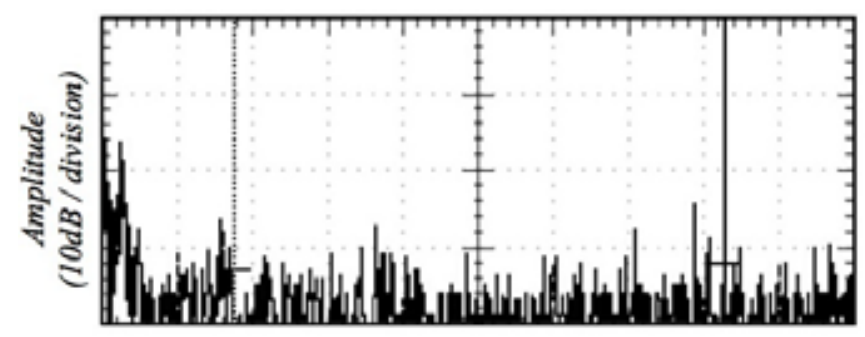

Frequency $(2.5 \mathrm{MHz} /$ division)

Fig. 15. Spectral Plot for the Background Generalized Noise. Spam 25MHz, Center $12.5 \mathrm{MHz}$, Reference $-47 \mathrm{~dB}$

\section{Network model on noisy channels}

The control requirements for the previously described coloured noise, narrow band noise, periodic impulsive noise and asynchronous impulsive noise, can be separately analyzed in two different scenarios.

\section{A. In-home Scenario}

In-home network model controls the noises on electrical channels within a local area network, -LAN-, by implementing band-pass and band-stop filters as given in Figure 16. The communication signals within the powerline infrastructure is bandpass filtered at each PLC client connection point by installing PLC splitters. The noise control requirement isolates the noisy signals on communication channels.

The application in-home network requires bandwidth control affected by the analyzed noises. The scenarios showed enough control with filters applied not at the noise source but at the electric connection in the data equipment. The neighbors' electric networks are not expected to be affected by the noises in the PLC LAN but they could be affected by external noises even though their implications are filtered by the suggested appliances and it is not necessary to install special filters at the main electrical panel. 


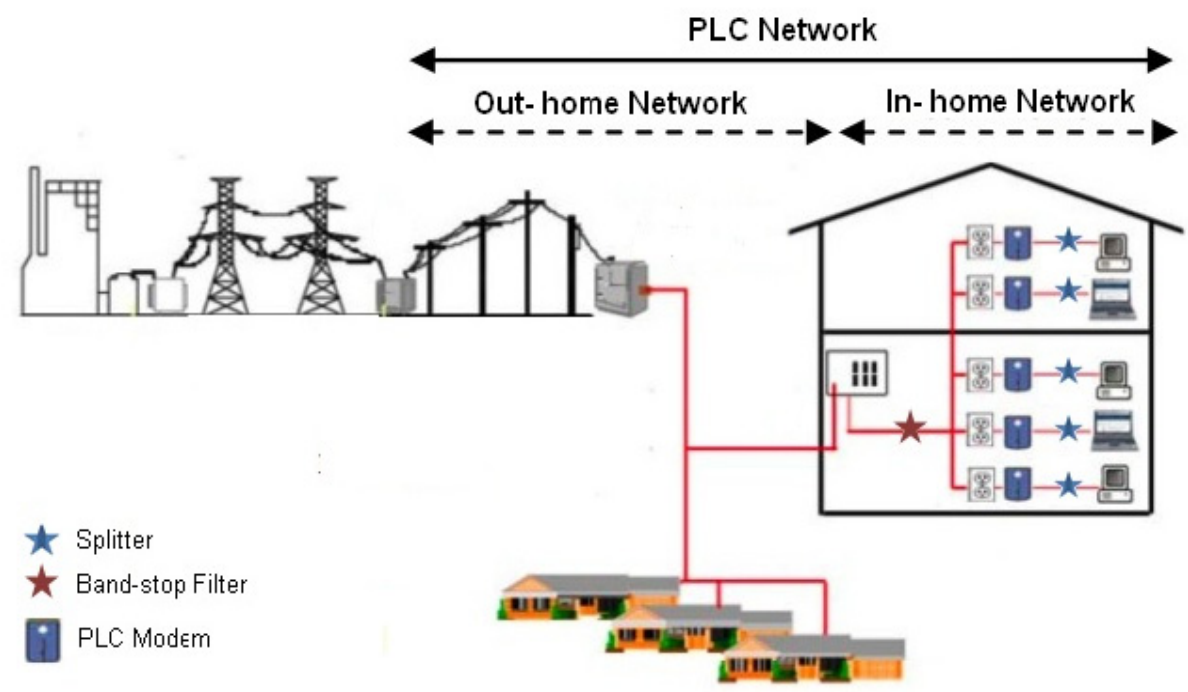

Fig. 16. In-home Network Model on Noisy Channels

On the other hand, a band-stop filter is implemented at the in-home electric provision point. The proposed noise control filters the not desired outgoing and incoming communication signals of the house.

B. Powerline ISP Scenario

PLC Networks can be used by electric distributors to offer internet access as add value. The analyzed noise can affect the throughput in the distribution and it is necessary to install filters not only at the power source per PLC connection but also the filter must be installed at the main electrical panel. The general scheme can be seen in Figure 17.

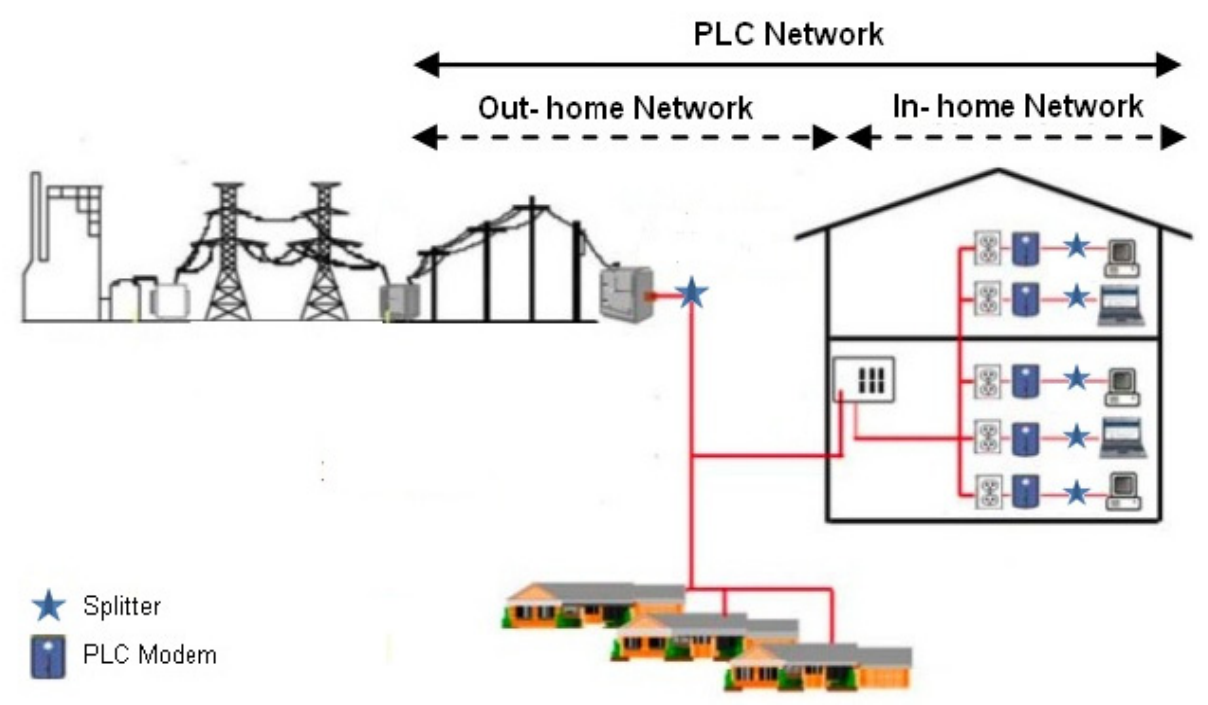

Fig. 17. In-home Network Model on Noisy Channels 


\section{Conclusion}

Noises on powerline communication channels such as coloured noise, narrow band noise, periodic impulsive noise and asynchronous impulsive noise affect PLC network throughput. However, these noises can be successfully controlled by band-pass and bandstop filters installed on strategic PLC network connection points.

It's necessary to establish a model to probe if the noise measurements on this work can be applied on industrial environments and if the noise can affect PLC networks installed on near locations to PLC ISP distributors.

\section{References}

[1] Lee, M.K.; Latchman, H.A.; Newman, R.E.; Katar, S.; Yonge,L.;., "Field Performance Comparison of IEEE 802.11b and HomePlug 1.0" Proceedings. LCN 2002. 27th Annual IEEE Conference on Local Computer Networks, 2002, PP: 598 -599.

[2] M. K. Lee, R. Newman, H. A. Latchman, S. Katar, and L. Yonge, "HomePlug 1.0 Powerline Communication LANs-Protocol Description and Comparative Performance Results," International Journal on Communication Systems on Powerline Communications, pp. 447-473, 2003.

[3] D. Webgner, O. Logvinov, "HomePlug Alliance position statement", IEEE BoPL meeting, July 2004

[4] American National Standard Institute, ANSI. "TIA 1113: Medium-Speed (up to $14 \mathrm{Mbps)}$ Power Line Communications (PLC) Modems using Windowed OFDM", Telecommunications Industry Association, TIA, May 2008.

[5] Zimmermann, M.; Dostert, K.;, "An Analysis of the Broadband Noise Scenario in Powerline Networks," Proceedings of the 4th International Symposium on Powerline Communications and Its Applications. Limerick (Ireland), vol. 138, 2000.

[6] Zimmermann, M.; Dostert, K.; "Analysis and modeling of impulsive noise in broad-band powerline communications", IEEE Transactions on Electromagnetic Compatibility, Volume 44, Issue 1, Feb. 2002, pp 249 -258

[7] Li, Y.; Cimini, L.J., Jr.; Sollenberger, N.R.; "Robust Channel Estimation for OFDM Systems with Rapid Dispersive Fading Channels", IEEE Transactions on Communications, Volume 46, Issue 7, July 1998 pp 902- 915.

[8] Hsu, C.; Neng Wang; Wai-Yip Chan; Jain, P.; "Improving HomePlug Power Line Communications with LDPC Coded OFDM", 28th Annual International Telecommunications Energy Conference, 2006. INTELEC '06. Sept. 2006 pp 1 - 7 [9] H. Hrasnica, A. Haidine, and R. Lehnert, Broadband Powerline Communications: Network Design: Wiley \& Sons, 2004, pp. 52-55

[10] T. Sartenaer, Multiuser communications over frequency selective wired channels and applications to the powerline access network. PhD Thesis, Département d'électricité, Université Catholique de Louvain, Septembre, 2004

[11] R. Pighi and R. Raheli, "Linear Predictive Detection for Power Line Communications Impaired by Colored Noise," EURASIP Journal on Advances in Signal Processing, vol. 2007, pp. 1-12, 2007.

[12] P. Scherz, "Switching Regulator Supplies (Switches)," in PracticalElectronics for Inventors: McGraw-Hill/TAB Electronics, 2000, pp. 292-293. 
[13] M. Babic, M. Hagenau, K. Dostert, and J. Bausch, "Theoretical postulation of PLC channel model," IST Integrated Project Deliverable D4v2. 0, The OPERA Consortium, March, 2005

[14] R. Boden, "Realistic modeling spectral and amplitude distribution characteristics of impulsive noise in ADSL broadband access networks," Proc. 10 thInternational OFDM Workshop, Hamburg, pp. 46-50, 2005

[15] D. Umehara, S. Hirata, S. Denno, and Y. Morihiro, "Modeling of Impulse Noise for Indoor Broadband Power Line Communications," International Symposium of Information Theory and its Applications, ISITA, (Seoul, Korea), 2006

[16] E. Griful and G. Ponsati, "Tasa de fallo no constante, el modelo de Weibull y otros," in Fiabilidad industrial: Edicions UPC, 2003, pp. 41 


\title{
Robot Navigation with Speech Commands
}

\author{
Anupam Shukla and Ritu Tiwari \\ $A B V$-Indian Institute of Information Technology and Management, Gwalior \\ India
}

\section{Introduction}

The field of Robotics draws on a multitude of engineering disciplines. There are mechanical, electrical and software considerations but the interaction among these and other disciplines is quite complex. Today's robots are equipped with wheels, wings, legs and also camera and laser/sonar range finders to perceive its neighborhood world. Vision-based robots are obviously smarter, but a mobile robot needs an online facility for scene analysis, which is a formidable task. AI tools may be used to model its neighborhood world using visual input and range information. Such model of perception enhances the robot's capability in coordinating complex actions pertaining to motion, visual tracking and avoidance of obstacle (Corke Peter, 2005; Corke Peter, 2003; Ragavan S. Veera \& Ganapathy V, 2007). The field of neural networks can be thought of as being related to AI, machine learning, parallel processing, statistics, and other fields. The attraction of neural networks is that they are best suited to solving the problems that are the most difficult to solve by traditional computational methods (Rich, E. and Knight, K, 1991). ANNs were inspired by models of biological neural networks since much of the motivation came from the desire to produce artificial systems capable of sophisticated, perhaps "intelligent", computations similar to those that the human. The network presented here learns with the Back Propagation (BP) algorithm (Basheer, I.A \& Hajmeer, M, 2000; E. D. Karnin, 1990; R. Hecht-Nielsen, 1989; Yegnanarayana, B, 2004).

This chapter deals with robot navigation avoiding static and dynamic obstacles in the path of the mobile robot, planning the future path of the mobile robot with the help of Artificial Neural Networks (ANNs) and also accepting the speech commands to traverse the directed path and accordingly to reach the destination.

Three ANN models has been discussed one each for speech signal inputs ( ANN-1), static and dynamic input ( ANN-2)and decision making (ANN-3). The robot can move left, right, forward left, forward and forward right along with start and stop commands based on speech signals. The speed of the robot is constant but it can be varied to have the advantage of providing the acceleration at the start and de-acceleration to avoid any damage to robot or obstacle.The direction of movement can be controlled speech commands. 
The uttuances of 50 speakers were recorded for seven words namely start, stop, TL, TR, FL, FW and FR are recorded and sampling is done at $16 \mathrm{kHz}$. The speech data bank is developed by cutting the word and storing in the seperate files. The signal processing tool box in MATLAB is usede to extact the speech feature namely,formant frequency,Peak power spectral density, cepstrul magnitude and number of zero crossing. The mean values for all the words are calculated to get speech feature for command control of the Robot. The ANN Model is trained for the command signals and use to drive the robot.

\section{Methodology of Robot Navigation}

The methodology of navigation of mobile robot using ANN with speech commands is represented in the figure No-1. After reading the positions of source, destination and obstacles (static and dynamic), the mobile robot makes decision for two aspects mainly; the avoidance of collisions with obstacles and secondly reaching the destination through optimized path in minimum time. The decision making is accomplished by ANN technique. In the mean time if the robot gets the speech command, it pause from whatever is doing and takes the new instructions for further movements and decision making.

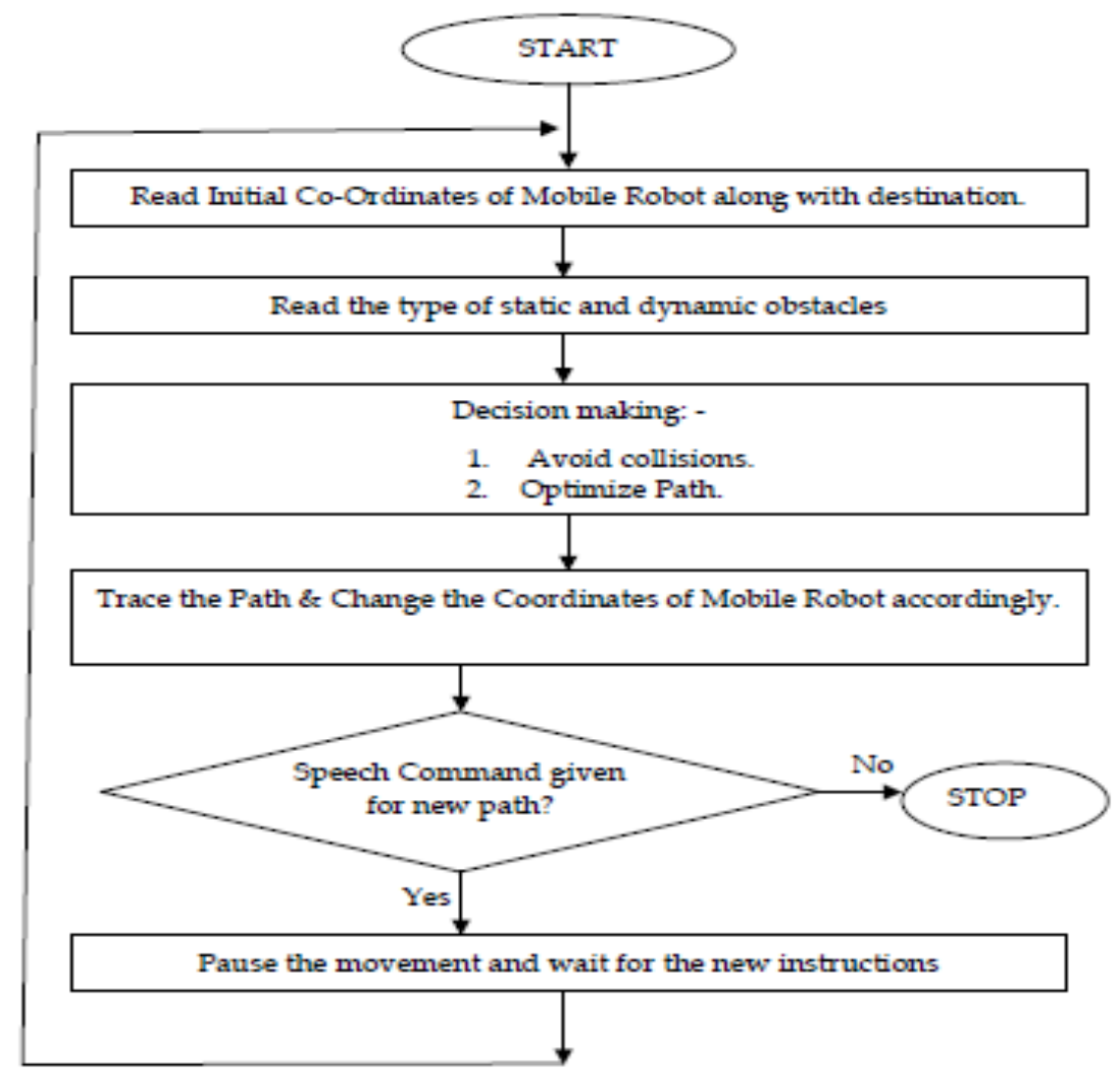

Fig. 1. Flow diagram for robot path planning using speech command 


\subsection{The Neural Network Based Local Guidance Scheme}

The navigation of the mobile robot is based on the steering decision based on a local grid. The local grid is used to collect the local information. The speech command is issued to direct the robot to avoid the obstacles and move towards the current sub-goal. The ANN consist of 3-layers structure (Figure 2) with two hidden layers of 10 neurons each and the network is trained by the Back-propagation algorithm.

The learning rate is 0.01 and momentum is 0.9 .The $\mathrm{NN}$ is traned and tested on the ANN Toolbox in MATLAB 7.0.1.

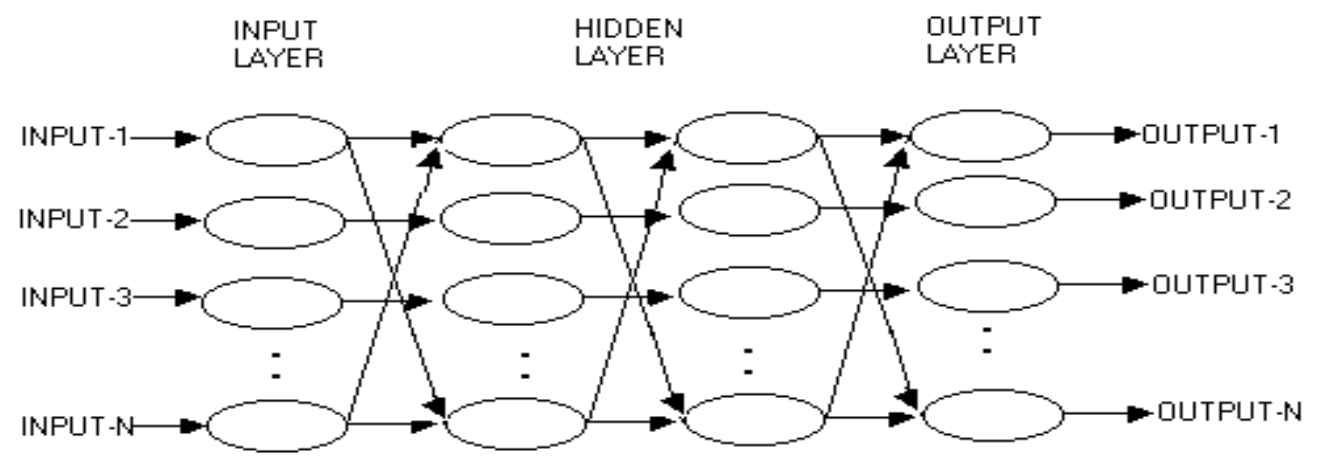

Fig. 2. Multi-Layer Neural Network

\subsection{Local Grid}

The local information is represented as 15 equal squares (Figure 3), which are used to indicate the occupation state of the immediate neighborhood of the robot. A binary value is assigned to each square. The value ' 1 ' implies that the square is full (presence of obstacles), ' 0 ' means that the square is empty (absence of obstacles) and for speech commands binary value ' 0 ' means no speech command is given during the motion and binary value ' 1 ' means speech command STOP is given and so mobile robot shall stop at that very moment to get the new instructions. After getting the new destination the robot again plans the path and reaches the new destination avoiding the obstacles. MR represents the Mobile Robot. There are five steering commands, which define the following moving action:

$\mathrm{FW}=$ Forward (MR to12),

$\mathrm{FL}=$ Forward left (MR to11),

$\mathrm{FR}=$ Forward right (MR to13),

$\mathrm{TL}=$ Turn left (MR to10),

$\mathrm{TR}=$ Turn right (MR to14) \&

STOP $=$ Pause the motion .

START=to start the Robot 
Fig. 3. Local Grid

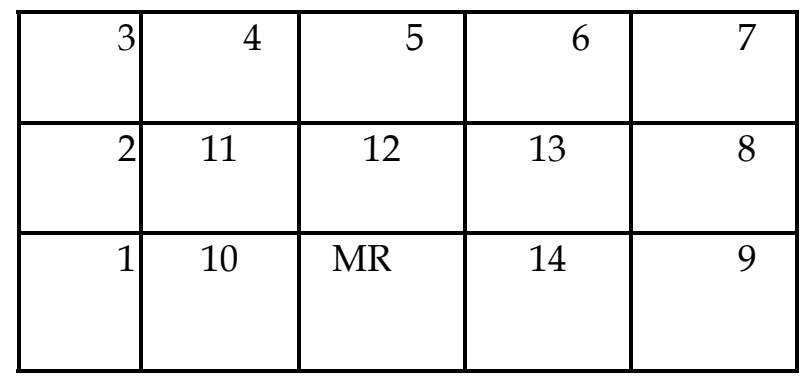

\subsection{Observations \& Results}

The sample training patterns for the static and dynamic and Path planning nets are presented in tabular form in Table No. 1, 2 and 3 respectively. The entries under inputs, denotes the cell numbers surrounding the Robot.

Inputs are position of obstacles at respective cells of the grid $(1=$ presence and $0=$ absence of obstacle) and the speech command SP $(0=$ no speech command and $1=$ speech command STOP). Outputs are five action commands ( $\mathrm{L}=\mathrm{Left} ; \mathrm{FL}=$ Forward Left; F = Forward; FR = Forward Right; $\mathrm{R}=$ Right) and two-direction indicator $(\mathrm{TR}=$ Turn Right and $\mathrm{TL}=$ Turn Left).

Outputs of Table No. 1 and 2 are AND ed and given as input to Table 3. The output of the DM net gives command to the robot i.e. YES to go and NO to stop. The user menu is made fully interactive and is equipped with the stunning graphic effects. The current location of the robot is indicated on the screen, whenever the robot moves. After giving the destination the work of the operator is over and robot starts moving towards the destination, recognizing the obstacles and also uses the past knowledge about obstacle to decide the appropriate path.

In case the operator wants to change the destination while the robot is traversing the path, the operator gives the speech command STOP and robot stops the motion it is traversing, and waits for the new instructions. After the operator gives the new instructions (i.e. new destination) the robot traverses the path accordingly avoiding the static and dynamic obstacles.

\begin{tabular}{|l|l|l|l|l|l|l|l|l|l|l|l|l|l|l|l|l|}
\hline \multicolumn{1}{|l|}{ INPUTS (Square Values) } \\
\hline 1 & 2 & 7 & 8 & 9 & 10 & 11 & 12 & 13 & 14 & L & TL & FL & F & FR & TR & R \\
\hline 1 & 1 & 0 & 1 & 1 & 0 & 0 & 0 & 0 & 1 & 1 & 1 & 1 & 1 & 1 & 1 & 0 \\
\hline 1 & 1 & 0 & 1 & 0 & 0 & 1 & 0 & 0 & 0 & 1 & 1 & 0 & 1 & 1 & 1 & 1 \\
\hline. &. &. &. &. &. &. &. &. &. &. &. &. &. &. &. &. \\
\hline 0 & 0 & 0 & 0 & 0 & 0 & 0 & 0 & 0 & 0 & 1 & 1 & 1 & 1 & 1 & 1 & 1 \\
\hline
\end{tabular}

Table 1. Training Pattern Samples of staticNetwork 


\begin{tabular}{|l|l|l|l|l|l|l|l|l|l|l|l|l|l|l|l|l|}
\hline \multicolumn{1}{|l|}{ INPUTS (Square Values) } \\
\hline 1 & 2 & 3 & 8 & 9 & 10 & 11 & 12 & 13 & 14 & L & TL & FL & F & FR & TR & R \\
\hline 0 & 0 & 0 & 0 & 0 & 0 & 0 & 0 & 0 & 1 & 1 & 1 & 1 & 0 & 0 & 1 & 0 \\
\hline 0 & 0 & 1 & 1 & 0 & 0 & 0 & 0 & 0 & 0 & 1 & 1 & 0 & 1 & 0 & 1 & 0 \\
\hline. &. &. &. &. &. &. &. &. &. &. &. &. &. &. &. &. \\
\hline 0 & 0 & 0 & 0 & 0 & 0 & 0 & 0 & 0 & 0 & 1 & 1 & 1 & 1 & 1 & 1 & 1 \\
\hline
\end{tabular}

Table 2. Training Patterns Samples of Dynamic Networks.

\begin{tabular}{|l|l|l|l|l|l|l|l|l|l|}
\hline \multicolumn{1}{|l}{ INPUTS } \\
\hline L & TL & FL & F & FR & TR & R & SP & Y & N \\
\hline 1 & 1 & 1 & 0 & 0 & 1 & 0 & 0 & 1 & 0 \\
\hline 1 & 1 & 1 & 0 & 0 & 1 & 0 & 1 & 0 & 1 \\
\hline 1 & 1 & 0 & 1 & 0 & 1 & 0 & 0 & 1 & 0 \\
\hline 1 & 1 & 0 & 1 & 0 & 1 & 0 & 1 & 0 & 1 \\
\hline. &. &. &. &. &. &. &. &. &. \\
\hline. &. &. &. &. &. &. &. &. &. \\
\hline 1 & 1 & 1 & 1 & 1 & 1 & 1 & 0 & 1 & 0 \\
\hline 1 & 1 & 1 & 1 & 1 & 1 & 1 & 1 & 0 & 1 \\
\hline
\end{tabular}

Table 3. Training Pattern Samples of Path planning Network.

\section{Conclusions}

The navigation planning and guidance of the mobile robot is successfully implemented using ANN in a planner workspace by finding the collision free path to lead the robot from the initial critical configuration to the goal among the set of obstacles, other wise every possible input is tried and there is no way to be certain for the precise output. The path planning algorithm may be expected to make several prediction regarding probable paths to destination by making linked queue and whenever it encounters obstacle it should follow the other nearest queue having the least possibility of the obstacles. The speed of the robot is constant but it can be varied to have the advantage of providing the acceleration at the start and de-acceleration when the obstacle is sensed for stopping the robot or slowing down to avoid any damage to robot or obstacle. This work can be extended to real time path planning and hence can be effectively applied for commercial purpose.

\section{References}

Amit Konar AI and SC Behavioral and Coqnitive modelling of the human brain CRC Publication

Ayala-Ramirez V, Perez-Garcia A, Montecillo-Puente E J, Sanchez-Yanez R E. (2004). Path planning using genetic algorithms for mini-robotic tasks, 2004 IEEE International Conference on Systems, Man and Cybernetics, pp. 3746- 3750, vol.4, ISBN: 0-78038566-7, F.I.M.E.E., Universidad de Guanajuato, 10-13 Oct. 2004, IEEE, Mexico. 
Basheer, I.A., and Hajmeer, M. (2000). Artificial neural networks: fundamentals, computing, design, and application, J. Microbiol. Methods, vol. 43, No. 1 (2000), pp. 3-31.

Corke Peter, Peterson Ron, Rus Daniela (2005), Localization and Navigation Assisted by Networked Cooperating Sensors and Robots, International Journal of Robotics Research, Vol. 24, Issue 9, pp. 771 - 786 (Sept. 2005), Sage Publications, Inc. Thousand Oaks, CA, USA.

Corke Peter, Peterson Ron, Rus Daniela. (2003), Networked Robots: Flying Robot Navigation usinga Sensor Net", April 18, 2003.

http:/ / cmc.cs.dartmouth.edu/papers/corke:flying.pdf

E. D. Karnin (1990). A simple procedure for pruning back-propagation trained neural networks. IEEE Transactions on Neural Networks, vol. 1, No. 2, pp. $239-242$ (1990).

M.F. Selekwa and E. G. Collins (2003) A centralized fuzzy behavior control for robot navigation, Proceedings of IEEE International Symposium on Intelligent control, pp. 602-607 (October, 2003), Houston, Texas.

M.F. Selekwa, D. D. Dunlap \& E. G. Collins (2005), Implementation of multi-valued fuzzy behavior control for robot navigation in cluttered, Proceedings of IEEE International Conference on Robotics and Automation, pp. 3699-3706 (April, 2005), Barcelona, Spain

Matlin, W. Margaret (2004), Cognition, John Wiley \& Sons, ISBN: 0471450073, ISBN-13: 9780471450078, 978-0471450078

Na Yong-Kyun and Oh Se-Young (2004), Hybrid Control for Autonomous Mobile Robot Navigation Using Neural Network Based Behavior Modules and Environment Classification, Autonomous Robots, Vol. 15, No. 2, pp. 193-206, (Nov. 2004) Springer Netherlands. ISSN 0929-5593 (Print) 1573-7527 (Online).

P. Rusu, E. M. Petriu, T. E. Whalen, A. Cornell \& H. J. W. Spoelder (2003), Behavior-based neurofuzzy controller for mobile robot navigation, IEEE Trans. Instrum. Measur. vol.52, No. 4, pp.1335-1340, (Aug, 2003).

Pagac, D., Nebot, E. M. and Durrant. W., H. (1998), An evidential approach to map building for autonomous robots, IEEE Trans. On Robotics and Automation, vol.14, no.2, pp. 623-629, (Aug. 1998).

R. Hecht-Nielsen (1989). Theory of the Backpropagation. Neural Network, Proceedings of the International Joint Conference on Neural Networks, 1: pp. 593-606. IEEE, New York.

Ragavan S. Veera, and Ganapathy V (2007), A Unified Framework for a Robust ConflictFree Robot Navigation, Proceeding of World Academy of Science, Engineering and Technology, pp.1307-6884, (21 January 2007).

Rich, E. and Knight, K. (1991.), Artificial Intelligence, pp. 29-98. McGraw-Hill, New York,

S.K. Pradhan, D. R. Parhi and A. K. Panda (2006), Navigation of multiple mobile robots using rule based- neuro-fuzzy technique, International Journal of Computational Intelligence, Vol. 3, No. 2, pp.142-151.

Shaygan K. and T. James S. (1998), Real-time Control of Robot Manipulators in the presence of Obstacles", IEEE Journal of RA, Vol.4, No. 6 (Dec. 1988).

Yegnanarayana, B. (2004), Artificial Neural Networks, Prentice Hall of India. ISBN:8120312538 


\title{
Ti indiffused Lithium Niobate $\left(\mathrm{Ti}: \mathrm{LiNbO}_{3}\right)$ Mach-Zehnder interferometer all optical switches: A review
}

\author{
G.Singh, R.P.Yadav and V.Janyani \\ Department of Electronics \& Communication Engineering \\ Malaviya National Institute of Technology Jaipur-India \\ E-mail: gschoudhary75@gmail.com
}

\section{Introduction}

Appropriate switching technology plays a major role in deciding the workable layout of any network and can improve accuracy, speed, scalability and flexibility efficiently, if chosen and implemented in best possible way. All-optical switches (O-O-O) do not require optoelectronic conversion and regeneration steps, as in case of mixed switches (O-E-O), where switching is done in electronic form. The development of all optical switches becomes necessary to fit them with the fast networks to make use of WDM technology, which incorporates hundreds of wavelength channels over a single fiber link. The speed and sensitivity of optical switches necessarily has to match with new generation photonic networks requirement, in which light not only carries the data and routing information, but also controls the switches in its path directly. Various optoelectronic materials, with MMI, MZI and other structures, have been used to design all optical switches like Si, SOI, InP, $\mathrm{InP} / \mathrm{InGaAsP}$, InGaAs-AlGaInAs, $\mathrm{LiNbO}_{3}$, photonic crystals etc. InP/lnGaAsP optical integrated MMI switches (Shintaro et al., 2008), InGaAsP-InP MZI optical space switch (Agrawal et al., 1995) and monolithically integrated InGaAs-AlGaInAs machzehnder interferometer optical switch using quantum-well intermixing (Wong et al., 2005) are some of examples. The electro-optic coefficient of lithium niobate is well suited for modulation and switching of fast optical communication systems.

A lithium niobate with a Ti diffused waveguides are useful for optical fiber communication and signal processing. Ti: $\mathrm{LiNbO}_{3}$ technology has been used to fabricate switch/modulators, acousto-optic deflectors, tunable wavelength filters, polarization controllers and splitters, frequency shifters and doublers (Alferness, 1986). The optical loss of a conventional Ti: $\mathrm{LiNbO}_{3}$ waveguide modulator is usually $0.2 \mathrm{~dB} / \mathrm{cm}$, and posses a graded index profile (Xiaobo, 2004). Ti diffused LN had also been used to fabricate phase modulators, by placing it in the middle of a travelling wave structure (Binh et al., 2007). The chapter is organized in the following sections. Section 2 summarizes the lithium niobate and Ti diffused lithium niobate material properties and characteristics. Section 3 gives a brief introduction to all optical switches and their advantages for using them with fast communication systems and 
networks. Section 4; explain the principle of operation of MZI based switches and their applications. In Section 5, the design of all optical switches using MZI structures on Ti doped lithium niobate are elaborated. This section also discusses the effect of other parameters on the performance of the designed switch. Section 6 contains the discussion and concluding remarks.

\section{Lithium Niobate- A crystal for optical processing}

Lithium Niobate $\left(\mathrm{LiNbO}_{3}\right)$ is a colorless, insoluble with water and ferroelectric material suitable for a variety of applications. Its electro-optical, acousto-optical and non linear optical properties attract designers to use it in integrated optics and have been proved as excellent material for optical waveguides manufacturing. The property of high intrinsic modulation bandwidth makes it a suitable candidate for communication technology. Table 1 gives list of various specifications for lithium niobate crystal (courtesy: Almaz optics, Inc.). The lithium niobate is a versatile material \& used for various applications in guided wave optics, electro-optics, acousto-optics and nonlinear optics.

\begin{tabular}{|c|c|}
\hline Chemical Formula & $\mathrm{LiNbO}_{3}$ \\
\hline Crystal Class & $\begin{array}{l}\text { Trigonal, } \\
3 \mathrm{~m}\end{array}$ \\
\hline Solubility in water & None \\
\hline Color & None \\
\hline Molecular Weight & 147.9 \\
\hline Density, $\mathrm{g} / \mathrm{cm}^{3}$ at $293 \mathrm{~K}$ & 4.644 \\
\hline Transmittance Range, nm & $350-5500$ \\
\hline $\begin{array}{l}\text { Dielectric Constant at } 100 \mathrm{KHz} \mathrm{e}_{\mathrm{a}} \\
\text { (perpendicular), } \mathrm{e}_{\mathrm{c}} \text { (parallel) }\end{array}$ & 85,29 \\
\hline Melting Temperature, $\mathrm{K}$ & 1530 \\
\hline Curie Temperature, $\mathrm{K}$ & 1415 \\
\hline $\begin{array}{l}\text { Thermal Conductivity, W/(m K) at } \\
300 \mathrm{~K}\end{array}$ & 5.6 \\
\hline Bandgap, eV & 4.0 \\
\hline $\begin{array}{l}\text { Optical Damage Threshold at } 1064 \\
\mathrm{~nm}, \mathrm{t} \sim 10 \mathrm{nsec}, \mathrm{MW} / \mathrm{cm}^{2}\end{array}$ & 250 \\
\hline
\end{tabular}

Table 1. $\mathrm{LiNbO}_{3}$ crystal spec. (courtesy: almaz optics, Inc.)

Its piezo-electric and photo-elastic properties had been used for various technical developments. Lithium niobate has been used to fabricate integrated waveguides because of its high electro-optic coefficient, optical damage resistance and low losses (Kalabin et al., 2004). Their Strain-optic effects are used to implement wavelength-tunable polarization converters (Wang \& Chung, 2004). The use of $\mathrm{LiNbO}_{3}$ modulators, with bulky diode pumped YAG lasers and cavity RF filters to construct Optoelectronic oscillators (OEO's) with ultra-low phase noise capability is explained by (Yao \& Maleki, 1999). The use of lithium niobate to make QPM structure by reversing the spontaneous polarization under the influence of a sufficiently large electric field has been reported in (Xiuping, 2006). The lithium niobate material has been extensively used to design switches with low loss, but 
their polarization dependency is the major concern (Suzuki et al., 2008). The choice of LN based switches is proved as best for high and low speed systems (Thylen, 1989). In recent years, $\mathrm{LiNbO}_{3}$ devices have successfully addressed the modulation requirements in digital fiber-optic time-domain-multiplexed (TDM) and wavelength-division-multiplexed (WDM) systems (Wooten et al., 2000).

\subsection{Ti-diffused Lithium Niobate}

$\mathrm{LiNbO}_{3}$ waveguides can be fabricated to design switches and modulators using either titanium in-diffusion or annealed proton exchange processes. To fabricate planner waveguides, the entire $\mathrm{LiNbO}_{3}$ is subjected to while for channel waveguides, photolithography process is used to defined masks for selected regions on it with these processes. In some cases, $\mathrm{Mg}$ oxide is doped with $\mathrm{LiNbO}_{3}$ to control optical damages. The proton-exchanged lithium niobate waveguides is easy to fabricate and can operate with low temperature. Ti diffused lithium niobate waveguides are useful for various communication, signal processing and sensor systems. Ti doping in the lithium niobate crystal increases refraction indexes, and which allow both TE and TM modes to propagate along the waveguides, which satisfies desired conditions for optical signal processing.

\section{All optical switches}

An optical switch routes an optical signal from one or more input ports to one or more output ports. Theoretically, all optical switches $(\mathrm{O}-\mathrm{O}-\mathrm{O})$ are independent of bit rate and protocols with unlimited scalability, which leads to more flexibility in the network. The choice of technology, switch architectures and size as well, plays an important role in performance of all optical switches. Following figure 1 shows a basic all optical switch with two different possible switching states named as bar \& cross state. In this, inputs \& outputs are in optical form and the switching is also performed in optical domain within the device.

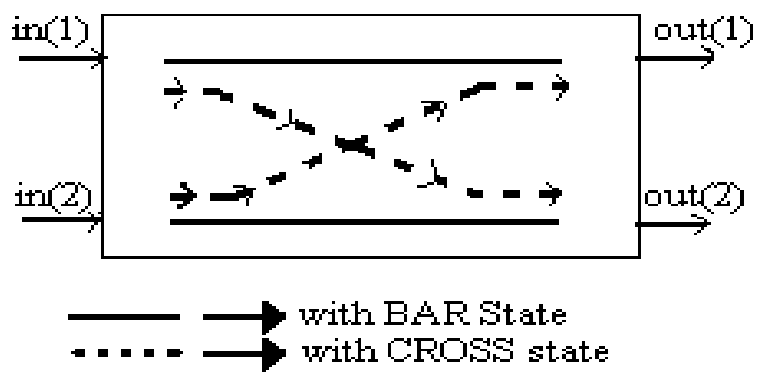

Fig. 1. All optical switch

The use of all optical switches are now taking over the use of O-E-O switches, as the O-E-O switches requires complex circuitry and have their own limitation while performing smooth \& lossless switching. The most common applications of these are WDM optical add-drop multiplexing (OADM), cross connects and protection switching. Yet the use of all optical 
switches are to cover up O-E-O switches uses in optical switching because of their difficulty in accommodating within the circuit with the use of VLSI technology, while fabrication with low fabric losses and to use the same to realize higher order switches.

\section{MZI structures \& their switching behavior}

Among different topologies, MZI structures are most efficient, converts a phase modulation into an intensity modulation, which is widely used for many optical applications e.g. modulators etc. Monolithically integrated MZI switches represent the most promising solution due to their compact size, thermal stability and low power (few fJ for input signal and few hundred fJ for control pulse) operation. Basic 2x2 MZI switch structure can be viewed, as shown in figure 2 , as two interferometric arms of equal length connecting two $3 \mathrm{db}$ couplers. The first coupler is used to split the signals in two beams, which when passed through the interferometric arms experiences phase difference. This phase difference is due to voltage variations across electrodes covering interferometric arms that in turn changes refractive indices. Finally, both the beam with different phases are put together again into a single signal by the second $-3 \mathrm{db}$ coupler, and the outputs are observed as per constructive or destructive interference (Papadimitriou et al., 2003).

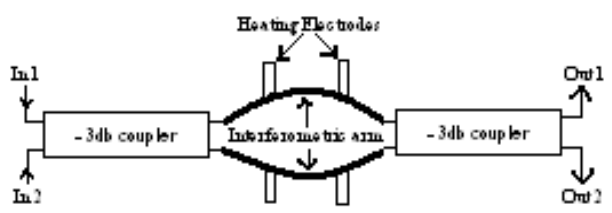

Fig. 2. Basic 2x2 MZI switch structure, ref. [10]

The LN has been used to design modulators and switches based on machzehnder interferometer or directional coupler efficiently. The symmetric machzehnder interferometer (MZI) has shown the better flexibility and shortest switching windows as compared to various configurations (Toliver et al., 2000). Improvement in all optical switching performance and high-speed capability of the MZI switches are investigated in (Schreieck et al., 2001), (Song et al., 2005) respectively. The integration of SOA's with in the MZI structures also the useful technology to design polarization insensitive switches (Gupta et al., 2005). Recently the MZI structures are fabricated using the photonic crystal to achieve the goals of VLSI photonics (Han et al., 2008). The MZI can be configured as an intensity modulator or a switch, but its periodical response can be a drawback since it requires an accurately controlled driving voltage

\section{Design of MZI all optical switches}

\subsection{Design steps:}

In this part, we have elaborated the design of Machzehnder interferometer based $2 \times 2$ all optical switch. Figure 3 shows the switch layout, which has been designed and simulated with the opti bpm layout designer. 


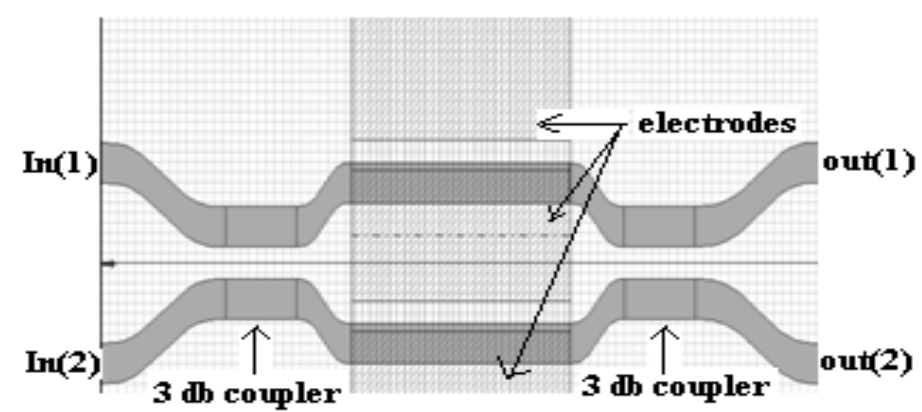

Fig. 3. 2 × 2 MZI switch layout

We assume that the integrated switch is created on a z-cut wafer of Lithium Niobate and is surrounded by air cladding. The device is oriented along Y-optical axis of the Lithium Niobate. Therefore, we define a diffused material for the substrate and a dielectric material for cladding. The crystal lithium niobate has a crystal cut along $\mathrm{z}$ axis and propagation direction along y axis. The dielectric material selected is air with refractive index 1.0. Traditionally, $\mathrm{LiNbO}_{3}$ waveguides are fabricated using either titanium in-diffusion or annealed proton exchange processes (APE). The main advantage of proton-exchanged waveguides is the low (approximately $240^{\circ} \mathrm{C}$ ) temperature, less than Curie point, and easy fabrication process (Korkishko et al., 2003). The diffusion of Titanium in Lithium Niobate substrate is being used to design the machzehnder interferometer waveguide. Ti doping in the lithium niobate crystal increases refractive indices, and allow both TE and TM modes to propagate along the waveguides. Only one diffused profile is needed $\left(\mathrm{TiLiNbO}_{3}\right)$. The insertion losses of the devices can be minimized by controlling the profile of in diffusion process. Using this technique an integrated $1 \times 2$ Mach-Zehnder linear analog modulator with $3 \mathrm{~dB}$ on chip losses is reported at (Arrioja et al., 2008). Other design parameters used are listed in table 2 and table 3 (courtesy: BPM layout designer, Optiwave Inc.)

\begin{tabular}{|l|l|}
\hline Diffused material & Dielectric material \\
\hline Crystal: $\mathrm{LiNbO}_{3}$ & Material: Air \\
Crystal cut: $\mathrm{Z}$ & Refractive index: 1.00 \\
Propagation direction: $\mathrm{Y}$ & \\
\hline \multicolumn{2}{|c|}{ Diffused profile: Ti $\mathrm{LiNbO}_{3}$ PRO 1} \\
\hline \multicolumn{2}{|c|}{ Waveguide properties } \\
\hline $\begin{array}{l}\text { Wafer profile: Ti LiNbO } \\
\text { Wafer dimension (all in } \mu \mathrm{mts})\end{array}$ \\
Length =33000, Width $=8.0$, Breadth $=100$ \\
\hline \multicolumn{2}{|c|}{$3 \mathrm{D}$ Wafer properties } \\
\hline Cladding & Substrate \\
\hline Material: Air & Material: $\mathrm{LiNbO}_{3}$ \\
Thickness $(\mu \mathrm{mts}): 2.0$ & Thickness $(\mu \mathrm{mts}): 10.0$ \\
\hline
\end{tabular}

Table 2. MZI design Specifications 


\begin{tabular}{|c|c|c|c|c|c|c|c|}
\hline \multicolumn{2}{|c|}{ Electrode 1} & \multicolumn{2}{|c|}{ Electrode 2} & \multicolumn{2}{|c|}{ Electrode 3} & $\begin{array}{l}\text { Gap } \\
1-2\end{array}$ & $\begin{array}{c}\text { Gap } \\
2-3 \\
\end{array}$ \\
\hline W & $\mathrm{V}$ & W & $\mathrm{V}$ & $\mathrm{W}$ & $\mathrm{V}$ & W & W \\
\hline 50 & 0.0 & 26 & 0.0 & 40 & 0.0 & 6.0 & 6.0 \\
\hline 50 & 0.0 & 26 & 6.75 & 40 & 1.5 & 6.0 & 6.0 \\
\hline \multicolumn{8}{|c|}{ Electrode 2 center position : 5.5} \\
\hline \multicolumn{6}{|c|}{ Stripe thickness before diffusion } & \multicolumn{2}{|c|}{$0.05 \mu \mathrm{m}$} \\
\hline \multicolumn{6}{|c|}{ Dopant constant } & \multicolumn{2}{|c|}{$5.67 \mathrm{e}+022$} \\
\hline \multicolumn{6}{|c|}{ Lateral diffusion length $\left(\mathrm{D}_{\mathrm{H}}\right)$} & \multicolumn{2}{|c|}{$4 \mu \mathrm{m}$} \\
\hline \multicolumn{6}{|c|}{ Diffusion length in-depth( $\left.\mathrm{D}_{\mathrm{v}}\right)$} & \multicolumn{2}{|c|}{$3.5 \mu \mathrm{m}$} \\
\hline
\end{tabular}

Table 3. Specifications for electrode \& other regions. $\{\mathrm{W}=$ width $(\mu \mathrm{mts}) \mathrm{V}=$ voltage (volts) $\}$

\subsection{Observations}

We checked the RI profile of the XY slice. We also defined an electrode region on the substrate and built up electrodes on the top of a buffer layer. The input plane has been selected with MODE as the starting field and 0.0 as $\mathrm{Z}$-offset. After the input plane has been defined the global data is set with refractive index MODAL and wavelength 1.3 micrometer. We calculated the 2D isotropic simulation. We varied the electrode voltages for region 2 . More detailed investigations of the electro-optic switch have been performed using the scripting language. We scanned the voltage on the central electrode and observed the overlap integral of the output ports with the waveguide mode. The switch is found to have better switching from CROSS to BAR state for electrode voltage value in between 6.6 and 6.8 volt with negligible coupling losses. States (cross and bar) of the switch are shown in figure 4 and figure 5 with their corresponding optical field propagation in 3D views.

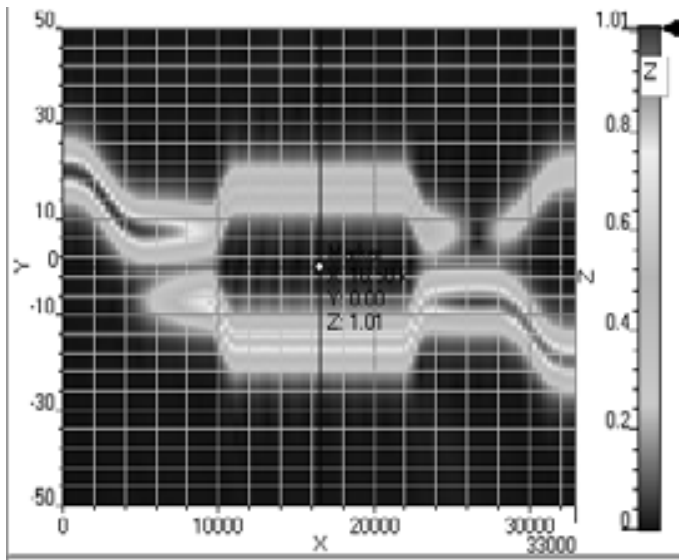

(a)

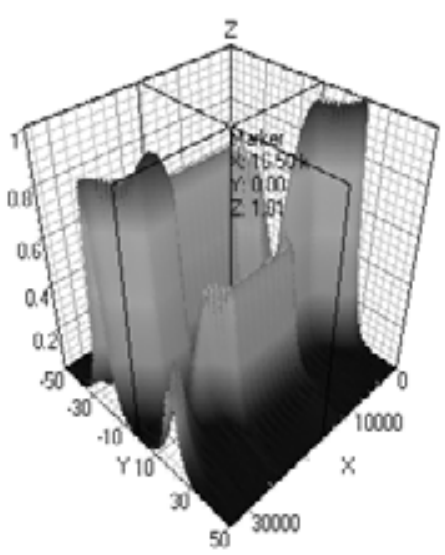

(b)

Fig. 4. (a) Switch with CROSS state (b) Optical field propagation for CROSS state (3D View) 


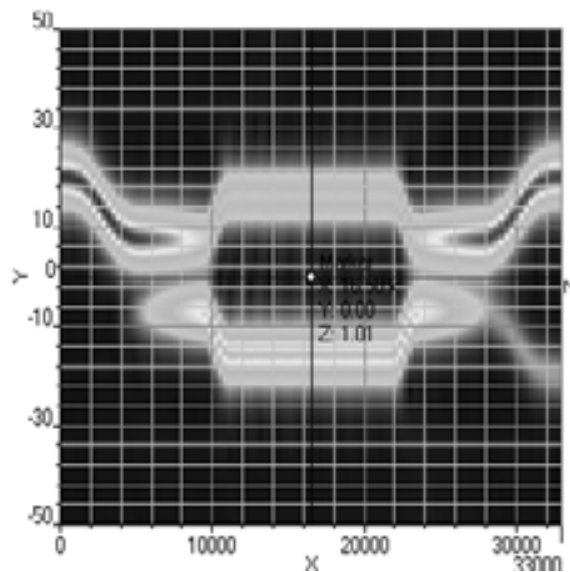

(a)

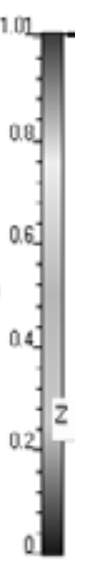

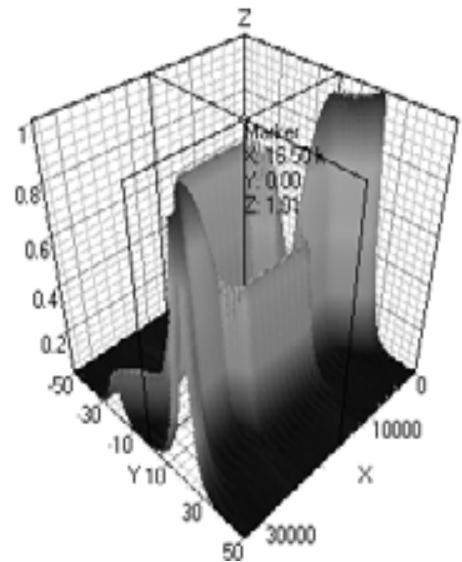

(b)

Fig. 5. (a) Switch with BAR state (b) Optical field propagation for BAR state (3D view)

\subsection{Discussion: effects of electrode shape \& size:}

In this section, we have discussed the effect of electrode dimension especially in terms of its length and spacing with with each other, to perform desired switching for signals with wavelength of $1.3 \mu \mathrm{m}$ and $1.55 \mu \mathrm{m}$. We performed different iterations with changes in the electrode voltages, incrementing it by 0.8 volts per iteration and found the switching voltages for different length of electrode region. For this, we have taken refractive index of the electrode buffer part as 1.47, buffer layer thickness $0.3 \mu \mathrm{m}$, horizontal permittivity 4 , vertical permittivity 4 and electrode thickness $4 \mu \mathrm{m}$. Also, the width of electrodes 1 and 3 is kept at $50 \mu \mathrm{m}$ initially, while for electrode 2 , width is $26 \mu \mathrm{m}$ \& positioned at 5.5 , centrally. The electrode gap (spacing) is kept at $6.0 \mu \mathrm{m}$ initially. Figure 6 indicate the $d_{1} \& d_{2}$, the spacing considered for these effects.

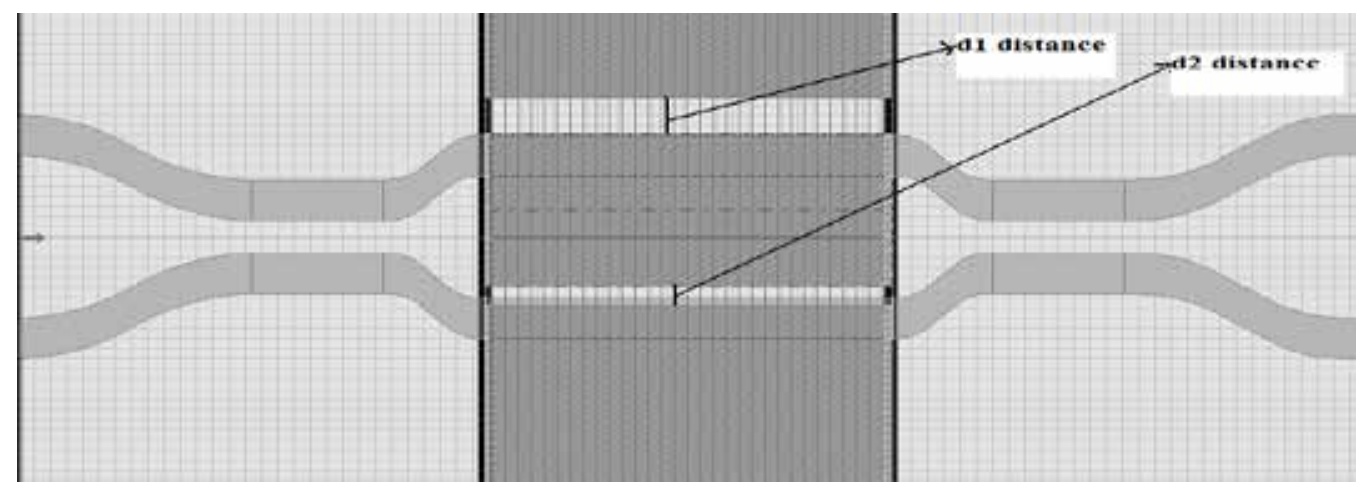

Fig. 6. Layout of the MZI switch with parameters given above.

\begin{tabular}{|l|l|c|c|c|}
\hline $\begin{array}{l}\text { Observation } \\
\text { no: }\end{array}$ & $\begin{array}{l}\text { Electrodes } \\
\text { dimension }(\mu \mathrm{m})\end{array}$ & $\begin{array}{l}\text { Length of the } \\
\text { electrode }(\mu \mathrm{m})\end{array}$ & $\begin{array}{l}\text { Voltage for } \\
\text { bar state }(\mathrm{V})\end{array}$ & $\begin{array}{l}\text { Voltage for } \\
\text { cross state }(\mathrm{V})\end{array}$ \\
\hline 1 & $11500-21500$ & 10000 & 0 & 6.75 \\
\hline 2 & $11550-21450$ & 9900 & 0 & 9.0 \\
\hline
\end{tabular}




\begin{tabular}{|l|l|l|l|l|}
\hline 3 & $11600-21400$ & 9800 & 0 & 14 \\
\hline 4 & $11650-21350$ & 9700 & 0 & 16 \\
\hline 5 & $11700-21300$ & 9600 & 0 & 18.5 \\
\hline 6 & $11750-21250$ & 9500 & 0 & - \\
\hline
\end{tabular}

Table 4. Electrodes dimension variations \& respective switching voltages

From the observation as depicted in table 4, it has been found that when we decrease the length of electrode region the switching voltage for the MZI switch increases, which is required to change its bar state to the cross state and on the further decrease of the electrode region result into distortion of the switching ability. When we decrease it further, it has been found that MZI is not able to perform proper switching at this stage.

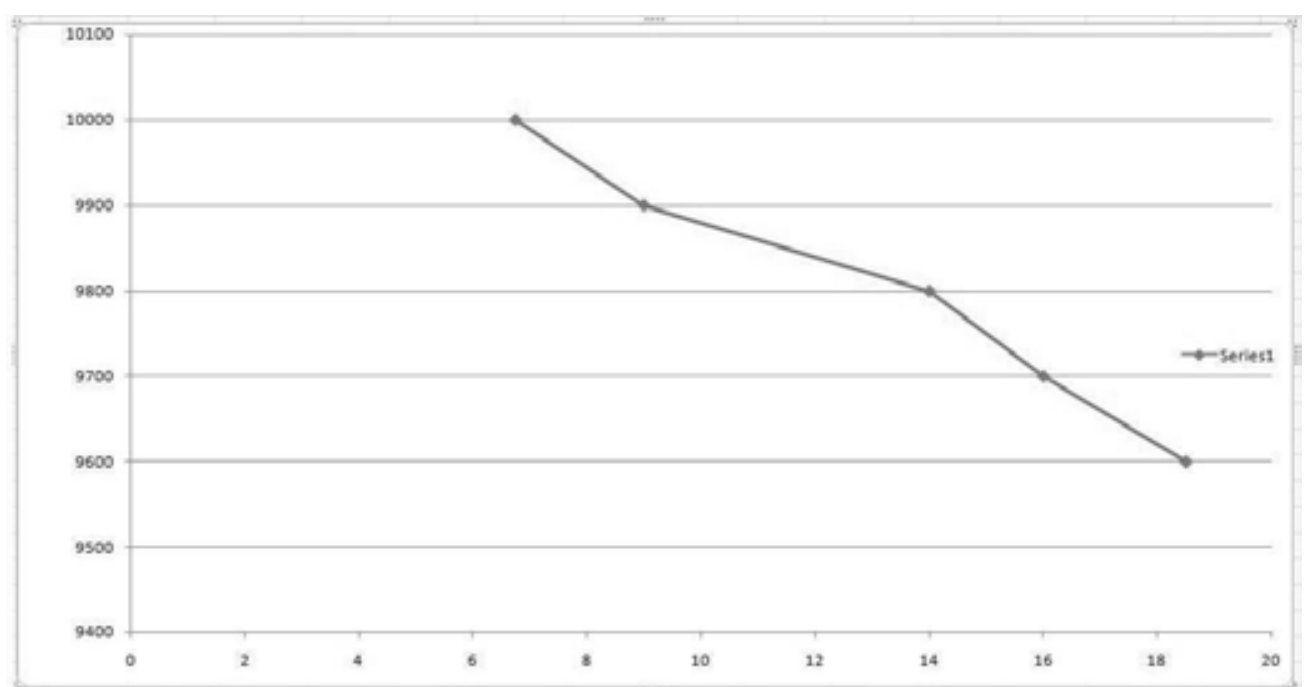

Fig. 7. Switching voltage against the electrode length variations

Following table 5 shows the driving voltage Vs different value of electrodes gap in $\mu \mathrm{m}$. The driving voltage varies as we change the width of the electrode of the electrode 2 . This table shows the effect of the width of the change of the electrode on driving voltage. The effect of electrode length is depicted graphically also, shown in figure 7.

\begin{tabular}{|c|c|c|c|c|c|c|c|}
\hline $\mathrm{d}_{2}(\mu \mathrm{m})$ & 1 & 2 & 3 & 4 & 5 & 6 & 7 \\
\hline 1 & 12.5 & 13.5 & 14.0 & 14.0 & 14.0 & 14.5 & 12.0 \\
\hline 2 & 13.5 & 12.5 & 13.5 & 12.5 & 12.5 & 12.0 & 9.50 \\
\hline 3 & 14.0 & 13.5 & 12.5 & 10.5 & 10.5 & 10.0 & 9.25 \\
\hline 4 & 14.5 & 13.0 & 10.0 & 8.80 & 7.00 & 8.00 & 9.00 \\
\hline 5 & 14.5 & 12.0 & 11.5 & 8.75 & 8.00 & 8.50 & 7.50 \\
\hline 6 & 15.0 & 10.0 & 11.0 & 9.50 & 8.25 & 6.75 & 7.75 \\
\hline 7 & 16.0 & 15.0 & 14.0 & 12.0 & 11.7 & 11.5 & 8.00 \\
\hline
\end{tabular}

Table 5. Switching voltage matrix representation w.r.t different values of $d_{1}$ and $d_{2}$ (electrodes spacing). 


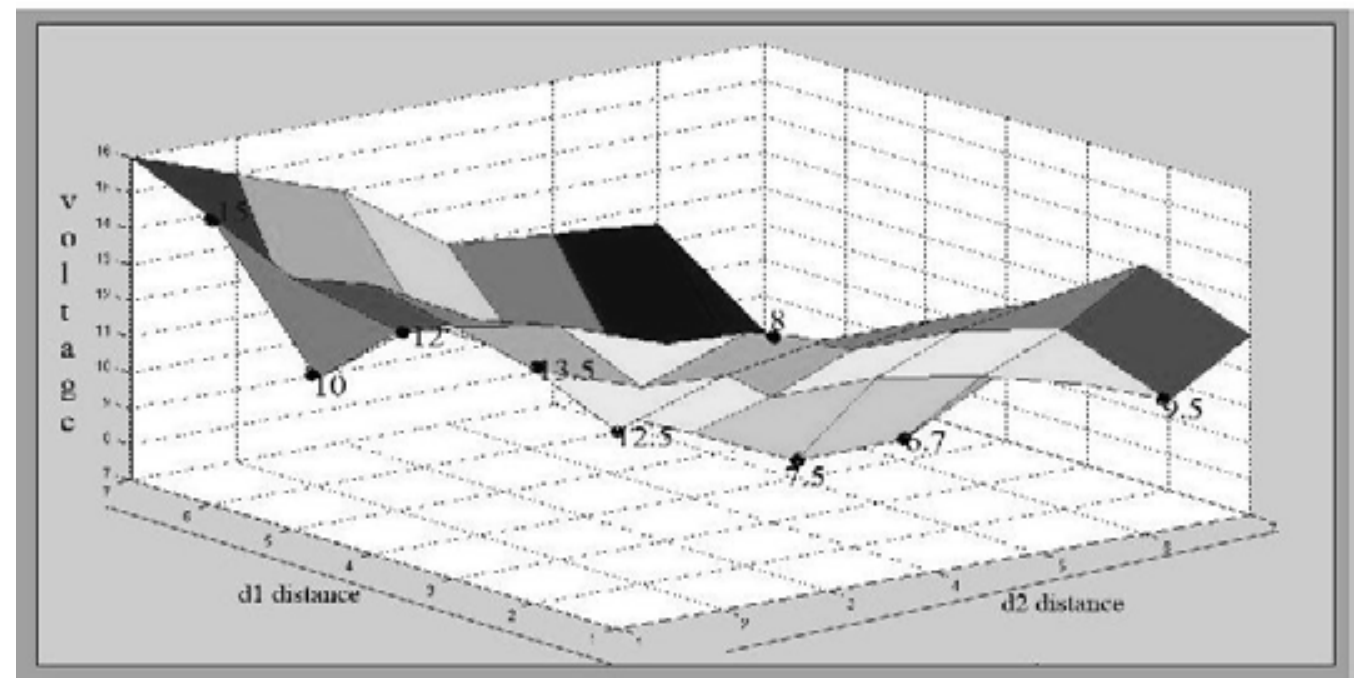

Fig. 8. Graphical (3D view) representation of the switching voltage v/s $d_{1} \& d_{2}$

From table 5 and figure 8 we observed that when the hot electrode is placed exactly on both the waveguide, this results minimum coupling in the MZI arms. Even when one of the gaps between the electrodes is more i.e. when hot electrode isn't on the waveguide then this also results minimum coupling and needs higher voltage for the switching. When the width of the electrode region is changed, the switching voltage also changes depends width and alignments, as shown in figure 8.

Effect of the change of the coupling region of the $3 \mathrm{~dB}$ coupler: when we changed the coupling region of the $3 \mathrm{~dB}$ coupler this results in the distortion in the output of the $1^{\text {st }}$ coupler, as a result of which the characteristics of the MZI switch looses. It can't act as the switch in this condition. Figure 9 and figure 10 shows the effect of variation of electrode voltage with respect to unit output power across bar \& cross states and switching in between these states for input signals with wavelength of $1.3 \mu \mathrm{m}$ and $1.55 \mu \mathrm{m}$ respectively.

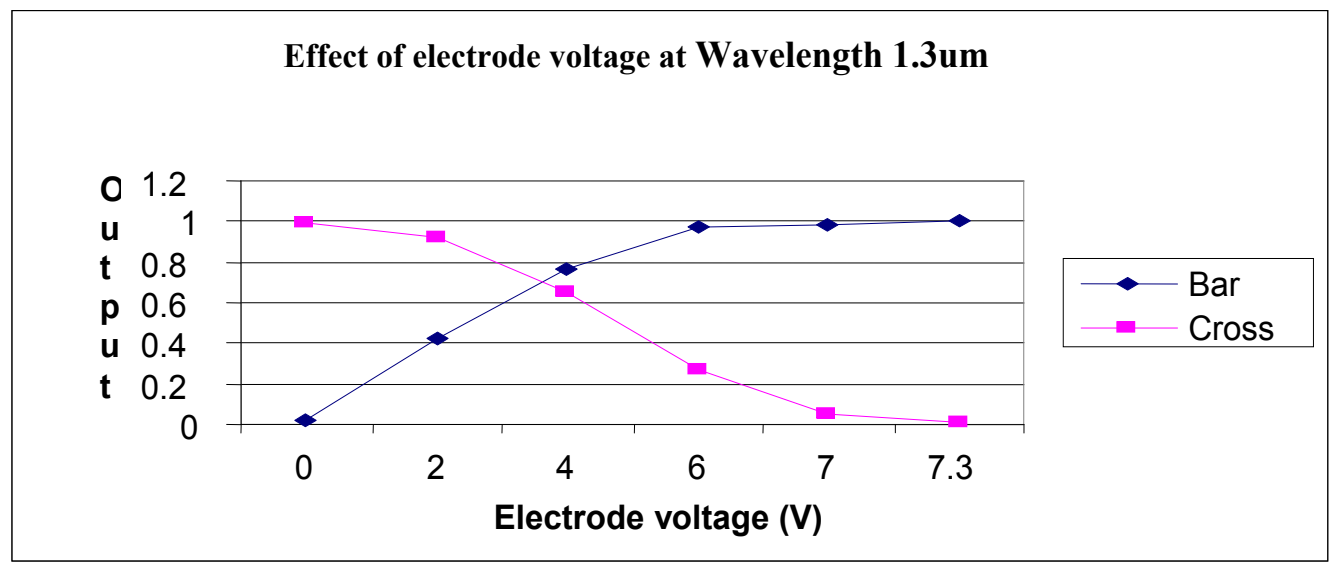

Fig. 9. Variation of output power with electrode voltage $(\lambda=1.3 \mu \mathrm{m})$ 


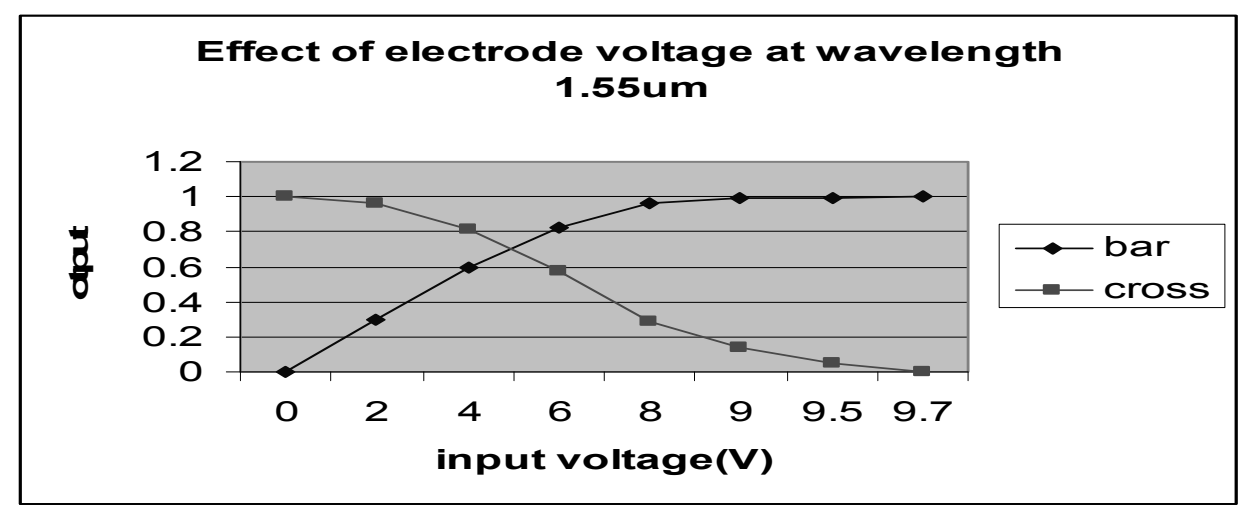

Fig. 10. Variation of output power with electrode voltage $(\lambda=1.55 \mu \mathrm{m})$

\section{Conclusion}

This chapter reviewed the Machzehnder interferometric (MZI) structure and their applications for optical technology, especially in design of all optical switches. In this, we have demonstrated the design and performance of basic MZI switching element $(2 \times 2$ all optical switch) Ti indiffused lithium niobate waveguide, while considering the optimum design parameters. The effect of electrodes shapes and size on the switching driving voltage is also discussed. Application of Lithium Niobate and when doped with Titanium, are discussed. The uses of MZI structures to design all optical switches are reviewed in short and the design of basic 2x2 all optical switch on Ti doped lithium niobate substrate discussed in brief. This work is expected to prove as a useful informative document for current and future researchers to begin with for high end design of Ti indiffused LN based large all optical MZI switches. Losses within the switches have greater impact on their performance, and a detailed investigation on these will add useful suggestion to design better ones. Inclusion of semiconductor amplifiers (SOA's) within or outside these structures has shown enhanced switching characteristics. Ultrafast all-optical signal processing using SOA based symmetric mach-zehnder switch (SMZs) is demonstrated at (Tajima et al., 2001). (Zhujun Wan et al. 2007) reported a fast (response time less then 3ms) $2 \times 2$ integrated thermo optic Machzehnder interferometer (MZI) switch based on multimode interference (MMI) couplers, with $3.40 \mathrm{~dB}$ insertion loss, $0.47 \mathrm{~dB}$ polarization dependent loss and extinction ratio (ER) at bar state and cross are $32.01 \mathrm{~dB}, 16.42 \mathrm{~dB}$ respectively. MZI with SOA based tree architecture, have been used to design integrated all optical logic and arithmetic operations. This architecture enables all optical processing of signals, including two input logic operations, half-adder, full-adder, full-subtractor, one-bit data comparator, etc (Roy, 2007).

\section{References}

Agrawal. N, C. M. Weinert, H.4. Ehrke, G. G. Mekonnen, D. Franke, C. Bornholdt, and R. Langenhorst (1995) "Fast 2 × 2 Mach-Zehnder optical space switches using InGaAsP-InP multiquantum- well structures" IEEE Photonics technology letters, Vol. 7, No. 6, page no. 644-645, June 1995. 
Alferness R. C. (1986) “Ti-diffused lithium niobate waveguide devices” ieeexplorer, CH 2358O/86/0000-0001, 1986.

Arrioja D.A. May, P. LiKamWa, I. Shubin, P.K.L. Yu (2008) “Integrated InGaAsP MQW MachZehnder modulator" Microelectronics Journal, Volume 39, Issues 3-4, March-April 2008, Pages 660-663.

Binh LN, TL Huynh and A. Rahman (2007) "Raman optically amplified MSK multi-span transmission Systems: simulation", (c) Monash University, 2007.

Gupta S., M. Presi, N. Calabretta, G. Contestabile, E. Ciaramella (2007) “Operational equivalence of self-switching effect in SOA-based nonlinear polarization and MZI switches" ieeexplorer, 1-4244-0925-X, page no. 810-811,2007.

Han Tae Yong, Hyun-Shik Lee and El-Hang Lee (2008) "Design of compact silicon optical modulator using photonic crystal MZI structure" ieeexplorer, page no. 308-310, 978-14244-1768-1/2008.

Kalabin Ivan E., Tatiana I. Grigorieva, Lev D. Pokrovsky and Victor V. Atuchin (2004) “High quality double doped polarizing insertion in titanium diffused lithium niobate waveguides" $5^{\text {th }}$ International siberian workshop and tutorial EDM'2004, session I, July 1-5, ERLAGOL, page no. 48-50, ISBN 5-7782-0463-9.

Korkishko Yu.N., V.A. Fedorov, S.M. Kostritskii, A.N. Alkaev, E.I. Maslennikov, E.M. Paderin, D.V. Apraksin, F. Laurell (2003) , Microelectronic Engeneering, 69 (2003) 228-236.

Papadimitriou, Georgios I., Chrisoula Papazoglou, and Andreas S. Pomportsis (2003) “Optical Switching: Switch Fabrics,Techniques, and Architectures" Journal of Lightwave Technology, Vol. 21, No. 2, page no. 384-405, February 2003.

Roy Jitendra Nath (2007) "Mach-Zehnder interferometer-based tree architecture for all-optical logic and arithmetic operations" Optik - International Journal for Light and Electron Optics, In Press, available online 26 November 2007.

Schreieck Roland, Martin Kwakernaak, Heinz Jäckel, Emil Gamper, Emilio Gini, Werner Vogt, Hans Melchior (2001) "Ultrafast Switching Dynamics of Mach-Zehnder Interferometer Switches" IEEE Photonics Technology Letters, Vol. 13, NO. 6, page no. 603-605, JUNE 2001.

Shintaro Niwal, Shinji Matsuo,Takaaki Kakitsuka, Kenichi Kitayama (2008) “Experimental demonstration of $1 \times 4 \mathrm{InP} / \operatorname{lnGaAsP}$ optical integrated multimode interference waveguide switch" 20th International Conference on Indium Phosphide and Related Materials, IPRM 2008, Page(s):1 - 4, 25-29 May 2008.

Song Xueliang, Naoki Futakuchi, Foo Cheong Yit, Zhenrui Zhang,Yoshiaki Nakano (2005) "28ps switching window with a selective area MOVPE all-optical MZI switch" IEEE Photonics Technology Letters, Vol. 17, No. 7, July 2005.

Suzuki Kenya, Takashi Yamada, Osamu Moriwaki, Hiroshi Takahashi and Masayuki Okuno (2008) "Polarization-insensitive MZI switch composed of an in phase shifter array and silica-based plc-integrated polarization beam splitter" OFC/NFOEC 2008, ieeexplore.ieee.org/iel5/4512186/4528018/04528714.

Tajima K. , S. Nakamura and Y. Ueno (2001) “Ultrafast all-optical signal processing with Symmetric Mach-Zehnder type all-optical switches" Optical and Quantum Electronics, Volume 33, Numbers 7-10 / July, 2001.

Thylen, L. (1989) "Lithium Niobate Devices in Switching and Multiplexing" Philosophical Transactions of the Royal Society of London. Series A, Mathematical and Physical Sciences, Volume 329, Issue 1603, pp. 83-92, Sept., 1989. 
Toliver P., R. J. Runser, I. Glesk, and P. R. Prucnal (2000) “Comparison of three nonlinear optical switch geometries," in Proc. Conf. on Lasers and Electro-Optics (CLEO 2000), San Francisco, CA, May 7-12, pp.254-255.

Wang T.-J., Chung J.-S. (2004), IEEE photon. Technol. Lett. 16 (2004), 2275.

Wong H. Y., M. Sorel,A. C. Bryce,J. H. Marsh, J. M. Arnold (2005) "Monolithically integrated InGaAs-AlGaInAs Mach-zehnder interferometer optical switch using quantumwell intermixing" IEEE Photonics Technology Letters, Vol. 17, No. 4, page no. 783785, April 2005.

Wooten Ed L., Karl M. Kissa, Alfredo Yi-Yan, Edmond J. Murphy, Donald A. Lafaw, Peter F. Hallemeier, David Maack, Daniel V. Attanasio, Daniel J. Fritz, Gregory J. McBrien, Donald E. Bossi (2000) "A Review of Lithium Niobate Modulators for Fiber-Optic Communications Systems" IEEE Journal of selected topics in Quantum Electronics, Vol. 6, No. 1, page no. 69-80, January/February 2000.

Xiaobo Hou (2004) "A leaky waveguide all-optical analog-to-digital converter" A PhD thesis, Drexel University, December 2004.

Xiuping Xie (2006) " High gain parametric processes in quasi-phase matching protonexchange lithium niobate waveguides" Ph.D dissertation, the department of applied physics, Stanford University, September 2006.

Yao Yu Ji, X.S, Maleki L. (1999) “ Compact optoelectronic oscillator with ultra-low phase noise performance" electronics letters, 1554, vol. 35, No. 18, 2nd September, 1999.

Zhujun Wan, Yaming Wu, Sihua Li (2007) “Experimental research on an integrated thermo optic switch based on multimode interference couplers" Frontiers of Electrical and Electronic Engineering in China, Volume 2, Number 1, March, 2007.

\section{Authors:}

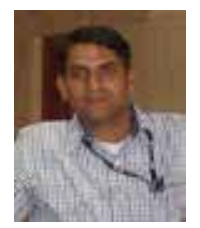

Mr. Ghanshyam Singh. He has completed B.E from REC Silchar (1997), M.Tech from MNIT Jaipur (2003) and is currently pursuing PhD. His areas of interest include Optical Communication, Optical Switches, Optical digital logics \& Interconnects.

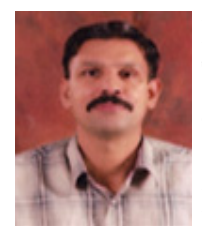

Prof. R.P.Yadav. He has completed his Master of Science and Master of Technology from IIT Delhi and Ph.D. degree from MNIT Jaipur. He is currently working as the head of Dept. of ECE, MNIT. His areas of interest are Digital and Wireless Communication and Coding, Antennas, Optics and Networking.

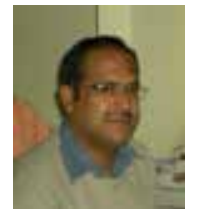

Dr. Vijay Janyani. He has completed B.E. and M.E. from MREC Jaipur and PhD from Nottingham University, UK. He is currently working as Asso. Prof., Dept.of ECE, MNIT. His areas of interest include Optoelectronics, Nonlinear Optic and Numerical Modeling. 


\title{
An Interval Metric
}

\author{
Roque Mendes Prado Trindade \\ Universidade Estadual do Sudoeste da Bahia - UESB \\ Brazil
}

\begin{abstract}
Benjamín René Callejas Bedregal, Adrião Duarte Dória Neto
Universidade Federal do Rio Grande do Norte - UFRN

Brazil

Benedito Melo Acióly

Universidade Estadual do Sudoeste da Bahia - UESB

Brazil
\end{abstract}

\section{Introduction}

The measurement of distance has become very important due to significant applications in various fields such as remote sensing, data mining, pattern recognition and multivariate data analysis. Researchers in these areas often face problems that have essentially noise distance, but no real metric capture this problem model. Accordingly, we propose a distance that extends the concept of generic metric of real numbers to an interval metric, where the distance between two elements of a set can be some interval measure. We see the use of distances in several areas, such as cluster algorithms for automatic classification of data of high dimensionality in the work of Fu \& Huang (2007), and in problems of segmentation for audio by Sundaram \& Narayanan (2007). Both use the K-means algorithm in their work.

We have the concepts of acoustic distance and phonemic distance in the work by Lin \& Lee (2007). The proposed distance notion can be used also to compare histograms used as feature indexing of images in databases in environments of content-based image retrieval, CBIR, because the traditional metric distance between two histograms of two similar images can be as large as the distance of two histograms of two very different images and that is unwanted in a CBIR system.

Girard \& Pappas (2007) propose the use of metric for approximating discrete and continuous systems, a comparison of languages accepted by automata. Many works in the area of fuzzy numbers deal or propose metrics, such as Fono et al. (2007) which use metric in Fuzzy sets, but it does not preserve uncertainties. Balopoulos et al. (2007) suggest a family of distances and similarity fuzzy measures based on the matrice norm. In the image processing area we have several works by Yu et al. (2006) which propose a robust method for distances metrics for estimation of similarity. Manouvrier et al. (2005) propose a distance between images recursively partitioned into structures of trees, which is effective in CBIR techniques. Georgiou et al. (2007) propose a metric distance between distributions in the image segmentation process. 
One of the pioneers in the fuzzy distance approach that preserves uncertainty was Voxman (1998) which addresses principles of distance on the fuzzy point of view and treats the principle of convergence on the view of the Cauchy sequences. He was the first to propose a fuzzy distance between fuzzy numbers.

In this work we propose an extension of the concept of real metric for a concept of interval metric. In this sense the distance that we propose is a generalisation of the Euclidean distance. In our approach the distance between two intervals is an interval, without losing the characteristics of the Euclidean metric when it leads with real numbers or degenerated intervals. The metric proposed here, beyond to providing the needs of the areas mentioned above, it preserves the monotonic inclusion of the Moore arithmetic Moore (1966), since this does not include the main feature of their arithmetic which is the property of monotonic inclusion, beyond it is not strictly interval because the distance between two intervals of uncertainty is a real number.

Of all works cited here that more close to our idea is the work by Chakraborty \& Chakraborty (2006) which proposes a fuzzy distance for fuzzy numbers, which preserves a distance uncertainties. The authors put a natural question: " if we do not know the numbers exactly how can the distance between them be an exact value? ". At the same time, they criticise the use of the supreme, of the minimal or of any other candidate as absolute representative of the distance between two fuzzy numbers. They also consider the distance between two fuzzy numbers as a fuzzy number, saying that the distance between two numbers with uncertainties must be a number with uncertainty. In addition, for fuzzy sets, Grzegorzewski (2004) uses a Hausdorff metric in the construction of a fuzzy metric, that unfortunately it does not preserve uncertainty. In their works they use metric spaces and topological spaces.

The proposed metric beyond of being a generalisation of the Euclidean metric in the reals it includes both the logic part of fuzzy logic as well the numerical part. This metric opens many possibilities for research and it possible to say that it creates a new paradigm of metrics. Much of mathematics is based on the notion of distance. This notion is fundamental to building the principles of calculus such as limit and continuity. As mathematical is a tool for many areas of knowledge, the importance of distance metric impact several areas such as signal processing, robust control systems, neural network that deal directly or indirectly with this notion. Often it represents characteristics of systems objects with uncertainties, where these uncertainties can be generated by the following factors: lack of precision of sensors, precision in mathematical representation of the system, the limitation of implementation in machine arithmetic. Therefore these areas need a model of distance that captures the uncertainties inherent in their processes. Algorithms for classification of patterns such as K-means, the SOM, support vector machine (SVM), algorithms for retrieval (CBIR), genetic algorithms and others using the very notion of distance in the separation of inaccurate data noisy. Often these area researchers are faced with problems that is essentially a distance noisy, but no real metric captures this type of problem.

According we propose an extension of the real metric to an "interval metric ", where a distance between two intervals is an interval, without losing the characteristics of the Euclidean metric when it manipulates real numbers or degenerated intervals. We see the need for this metric because the one proposed by Moore Moore (1966) does not include the main feature of the arithmetic that is the property of inclusion monotony, and it is not strictly interval, because the distance between two intervals of uncertainty is a real number. We propose this metric in order to increase the power of representation of interval mathematics. With this metric it is possible to formulate new concepts of interval sequences, interval limits, thereby reshaping 
the concepts of interval integral, derivative, complex variables, analysis of convergence and stability of LTI(Linear Time Invariant) systems. We present the definition of distance and we built versions of some results with this distance. In this work the distance has the role of supporting the definition of a module that preserves uncertainty for application in the convergence analysis of LTI systems.

\section{Interval Mathematics}

Mathematics has been successful as a language in knowledge construction. It allows us to create language from abstractions of real entities. Like any language it has its limitations, one of which is not to have an algorithmic representation for real numbers. This representation has been a topic of research since Pythagoras to nowadays. In the 50's Sunaga and Moore proposed an interval to control errors for handling real numbers. In their work they describe the interval arithmetic that is, in some way, an extension of real arithmetic. One use this approach to develop several branches applications such as linear systems to model real systems for digital signal processing. Here, we use the interval set IR as discussed by Vaccaro (2001). Accuracy of mathematical calculations in various areas of science and technology has been the subject of scientific work, always seeking the development of arithmetic algorithms, aiming to achieve the best possible accuracy in the processing of numerical data as seen in Marciniak (2003) and Popova (1994). This is not always possible due some factors such as: lack of precision of input data, imprecision of floating-point arithmetic and the physical limitations of the machines. As mentioned earlier, we can see that it is not a simple problem and that it covers the entire computer system, including its logical representation, mathematical modelling, memory capacity, size of words, floating-point arithmetic and so on. We focus mainly on mathematical representation by interval approach, because here the arithmetic operations on real numbers is invariant by interval arithmetics. The pioneers works that marked the beginning of the development of interval arithmetic was Moore (1966) and Sunaga (1958). This research area of mathematics is mainly interested in solving the mathematical expressions that can be performed by computers. Therefore, it is crucial that this approach responds to the questions of accuracy and efficiency which arises in the practice of scientific computing. Despite the success of interval mathematics in the field of computing science, the interval analysis has been not a similar success as, for exemplo, the theory of complex variables as an extension of real analysis. More seriously was the fact that the interval analysis was not successful as a basis for interval computation. Perhaps this was mainly because the researchers in this area have insisted on a metric which does not capture the interval approach.

Nowadays, one has observed that the classical binary logic is not so much adequate for the conversion of the real world to a virtual world of representation that makes the fuzzy logic a so good alternative to account this problem. The operations of fuzzy logic in the interval $[0,1]$ can be bijectively mapped with the interval $-\infty,+\infty$, on which one can work with the operations of interval arithmetic. In this way we can see that the interval arithmetic is more suited than the fuzzy logic to approach the traditional real arithmetic. We can also imagine the same situation when the range $[0,1]$ represents probability space, which is also home to a well interval arithmetic.

Definition 1 (Interval Representation). A function $F: \mathbb{I} \mathbb{R}^{m} \rightarrow \mathbb{I} \mathbb{R}^{n}$ is called an interval representation of a real function $f: \mathbb{R}^{m} \rightarrow \mathbb{R}^{n}$ if, for each $\vec{X} \in \mathbb{I}^{m}$ and $\vec{x} \in \vec{X}, f(\vec{x}) \in F(\vec{X})$. 
Definition 2 (Interval). Let $a$ and $b \in \mathbb{R}$ be such that $a \leq b$. The set $X=\{x \in \mathbb{R}: a \leq x \leq b\}$ is called an interval and will be denoted by $X=[a ; b]$. The set $X=\{x: x \in \mathbb{R}$ and $a \leq x \leq b\}$. The set of all intervals will be represented by $\mathbb{I} \mathbb{R}$.

Each interval has associated to it two projections, $\pi_{1}$ and $\pi_{2}$, defined by $\pi_{1}([a ; b])=a$ and $\pi_{2}([a ; b])=b$. In order to simplifying notation we will use $\underline{X}$ for representing $\pi_{1}(X)$ and $\bar{X}$ for $\pi_{2}(X)$. Let $F: \mathbb{I} \mathbb{R} \rightarrow \mathbb{I} \mathbb{R}$ be an interval function. The lower limit of $F(X)$ is the semiinterval function $\underline{F}(X): \mathbb{I} \mathbb{R} \rightarrow \mathbb{R}$, where $\underline{F}(X)=\pi_{1}(F(X))$ and the upper limit of $F(X)$ is the semi-interval function $\bar{F}(X): \mathbb{I} \mathbb{R} \rightarrow \mathbb{R}$, where $\bar{F}(X)=\pi_{2}(F(X))$.

Definition 3 (Diameter of Interval). Let $X \in \mathbb{I} \mathbb{R}$ be an interval. The diameter of the interval $X$ is defined by the non-negative real number $\operatorname{Diam}(X)=\bar{X}-\underline{X}$.

The radius of an interval, $X$, is defined as the half diameter of $X$, as it is shown in the following equation:

$$
\operatorname{raio}(X)=\frac{\operatorname{Diam}(X)}{2}
$$

Definition 4 (Inclusion Order). Let $X$ and $Y \in \mathbb{I} \mathbb{R}$. We say that $X \subseteq Y$ if only if $\underline{Y} \leq \underline{X}$ and $\bar{X} \leq \bar{Y}$.

Definition 5 ( Kulisch-Miranker Order). Let $X$ and $Y \in \mathbb{I R}$. $X$ is least or equal to $Y$, denoted by $X \leq Y$, if $\underline{X} \leq \underline{Y}$ and $\bar{X} \leq \bar{Y}$.

If $X \leq Y$ and $X \cap Y=\varnothing$. Then we say that $X \prec Y$, which is equivalent to say that $Y \succ X$.

An interval, $X$, is said to be positive if $\underline{X}>0$ and negative if $\bar{X}<0$.

Definition 6 (Middle Point of an Interval). Given $X \in \mathbb{I} \mathbb{R}$ we define the middle point of $X$ as the real number given by

$$
p m(X)=\frac{X}{2} \bar{X}
$$

Definition 7 (The greatest lower bound of set of intervals or infimum). Let $M \subseteq \mathbb{I}$. The greatest lower bound or infimum of $M$, with regard to the order $\leq$, denoted by $\operatorname{Infm}(M)$, is the interval $Y$ such that $Y \leq X, \forall X \in M$ and given any interval $Z \leq X \forall X \in M$, then $Z \leq Y$.

Proposition 1. Let $M \subseteq \mathbb{I} \mathbb{R}$. The infimum of $M$, with regard the order $\leq$, is given by the equation (2).

$$
\operatorname{Infm}(M)=[\inf \{\underline{X}: X \in M\} ; \inf \{\bar{X}: X \in M\}] .
$$

Proof. By the definition 7 we have that $\operatorname{Infm}(M) \leq X, \forall X \in M$. Thus by definition 5 $\underline{\operatorname{Infm}}(M) \leq \underline{X}, \forall X \in M$ and $\overline{\operatorname{Infm}}(M) \leq \bar{X}, \forall X \in M$. Therefore $\operatorname{Infm}(M)=\inf \{\underline{X}: X \in$

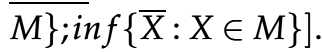

The infimum is greatest of the lesser bounds of the set and when it is applied to a degenerate interval set it coincides with the notions of infimum of the real number set.

Definition 8 (Minimum of an Interval). Let $M \subseteq \mathbb{I} \mathbb{R}$ and $X \in \mathbb{I} \mathbb{R}$, we say that $X$ is the minimum of $M$, with regard to the order $\leq$, denoted by $\operatorname{Min}(M)$, if $X$ is the infimum of $M$, with regard to the order $\leq$ and $X \in M$. 


\section{Semantics of Intervals}

There are several semantics to $X \subseteq \mathbb{I} \mathbb{R}$. Here we emphasize only two of them, that which treats the interval as a real envelopment and that which sees the interval as a numeric entity. Each one of them has its advantages and disadvantages.

\subsection{Intervals as Envelopment of Reals}

Researchers who take this semantic for the set $\mathbb{I} \mathbb{R}$ see each interval as a wrapper that has information of a real number. From this point of view the multiplication of an interval $X \in$ $\mathbb{I} \mathbb{R}$ by itself not always has the same result that the multiplication proposed by Moore. In this approach $X^{2}$ is always a nonnegative interval, since it represents the same real number. This interpretation is usually accepted in the context of interval arithmetic. According to this semantics an interval of real numbers is a real subject to uncertainties, ie

$$
\forall x \in \mathbb{R}, X \text { represents } x \leftrightarrow x \in X .
$$

Thus, any real number belonging to the envelopment is a possible representative of the real number which the envelopment represents. The effect of envelopment is modelling the propagation of error in numerical calculation in floating point Vaccaro (2001).

\subsection{Intervals as Interval-numbers}

In this approach an interval is seen as a mathematical entity that represents the real numbers and intervals belonging in it. It constitutes a different type of information from that it conveys, it is a new type of number whose semantics can be defined as follows:

$$
\forall X \in \mathbb{R}, Y \text { represents } X \leftrightarrow X \subseteq Y .
$$

In words, an interval represents all the real intervals it contains, in particular, the real numbers regarded as degenerate intervals.

Thus, an interval-number represents all the subsets and not only the real numbers individually selected within a field of uncertainty. The concept of interval exceeds the trichotomic law of the real numbers because intervals can contain numbers both positive and negative.

The basic concepts and notations presented here can be found in Oliveira et al. (1997), Vaccaro (2001), Trindade (2002), Santiago et al. (2006)

\subsubsection{Moore Interval Arithmetic}

Definition 9 (Arithmetic Operations in $\mathbb{I} \mathbb{R}$ ). Let $X, Y \in \mathbb{I} \mathbb{R}$ be two real intervals. The operations of addition, subtraction, multiplication and division in $\mathbb{I} \mathbb{R}$ are defined by $X * Y=\{x * y: x \in X, y \in Y\}$, where $* \in\{+,-, \times, \div\}$ is one of the four arithmetic operations. If $\omega$ is an unary arithmetic operation, then $\omega(X)$ is defined by $\omega(X)=\{\omega(x): x \in X\}$.

Note: It should be noted that for the operation of division, it is necessary to assume that $0 \notin Y$, because, otherwise, the operation is not well defined.

The following proposition will be not proved, because it is a basic concept already available in the literature, but its proof can be found eg in Oliveira et al. (1997) in the form of several theorems.

Proposition 2. If $X, Y \in \mathbb{I} \mathbb{R}$ are two real intervals, then

1. Interval Addition $X+Y=[\underline{X}+\underline{Y}, \bar{X}+\bar{Y}]$. 
2. Interval Pseudo Inverse Additive $-X=[-\bar{X}-\underline{X}]$.

3. Interval Subtraction $X-Y=X+(-Y)=[\underline{X}-\bar{Y}, \bar{X}-\underline{Y}]$.

4. Interval Multiplication $=X . Y=[\min \{\underline{X} \cdot \underline{Y}, \underline{X} \cdot \bar{Y}, \bar{X} \cdot \underline{Y}, \bar{X} \cdot \bar{Y}\} ; \operatorname{Max}\{\underline{X} \cdot \underline{Y}, \underline{X} \cdot \bar{Y}, \bar{X} \cdot$ $\underline{Y}, \bar{X} \cdot \bar{Y}\}]$.

5. Interval Pseudo inverse Multiplicative $X^{-1}=1 / X=\left[\frac{1}{\bar{X}}, \underline{\frac{1}{X}}\right]$ and $0 \notin X$.

6. Interval Division $\frac{X}{Y}=X \cdot Y^{-1}=\left[\min \left\{\frac{\underline{X}}{\underline{\underline{Y}}}, \underline{\underline{\underline{X}}}, \frac{\bar{X}}{\underline{Y}}, \frac{\bar{X}}{\bar{Y}}\right\} ; \operatorname{Max}\left\{\frac{\underline{X}}{\underline{\underline{Y}}}, \underline{\underline{\bar{X}}}, \frac{\bar{X}}{\underline{Y}}, \frac{\bar{X}}{\bar{Y}}\right\}\right]$ with $0 \notin[\underline{Y}, \bar{Y}]$.

Proposition 3. If $X, Y$ and $Z \in \mathbb{I} \mathbb{R}$ are real intervals, then

1. Algebraic Properties of Addition in $\mathbb{I} \mathbb{R}$

- Closeness: If $X \in I R$ and $Y \in I R$ then $X+Y \in I R$;

- Associativity: $(X+Y)+Z=X+(Y+Z)$;

- Commutativity: $X+Y=Y+X$;

- Neutral Element: $\exists$ ! $\quad \mathbf{0}=[0 ; 0] \in \mathbb{I} \mathbb{R}$ such that: $X+\mathbf{0}=\mathbf{0}+X=X$

Proposition 4. If, $X, Y$ and $Z \in \mathbb{I} \mathbb{R}$ are real intervals, then

1. Algebraic Properties of the Multiplication in $\mathbb{I} \mathbb{R}$

- Closeness: If $X \in \mathbb{I} \mathbb{R}$ and $Y \in \mathbb{I} \mathbb{R}$, then $X \cdot Y \in \mathbb{I}$;

- Associativity: $(X \cdot Y) \cdot Z=X \cdot(Y \cdot Z)$;

- Commutativity: $X \cdot Y=Y \cdot X$;

- Neutral Element: $\exists$ ! $\quad \mathbf{1}=[1 ; 1] \in \mathbb{I} \mathbb{R}$ such that: $X \cdot \mathbf{1}=\mathbf{1} \cdot X=X$;

- Subdistributivity: $X \cdot Y+Z \subseteq X \cdot Y+X \cdot Z$;

2. Consequence of the Pseudo Inverse Multiplicative: Let $X$ be an interval such that $0 \notin X$. Then $1 \in X / X$

One of the main properties of the Moore arithmetic is the monotonic property. It guarantees the interval correctness and the error inclusion.

Definition 10 (Interval Canonical Representation - CIR). Let $f: \mathbb{R}^{m} \rightarrow \mathbb{R}$ be a non-asymptotic function ${ }^{1}$ The function $C I R(f): \mathbb{I R}^{m} \rightarrow \mathbb{I R}^{n}$ is a canonical representation of the function $f:$ $\mathbb{R}^{m} \rightarrow \mathbb{R}$ if $C I R$ is defined by

$$
C I R(f)(\vec{X})=[\inf \{f(\vec{x}): \vec{x} \in \vec{X}\} ; \sup \{f(\vec{x}): \vec{x} \in \vec{X}\}] .
$$

In other words, $C I R(f)$ is an interval function that maps an m-tuple $\vec{X}$ in the lower n-tuple of intervals that contain $f(\vec{X})$..

Proposition 5. Let $f: \mathbb{R}^{m} \rightarrow \mathbb{R}$ be not asymptotic. Then $\forall \vec{X} \in \mathbb{I}^{m}$ and $\vec{x} \in \vec{X}$ we have $f(\vec{x}) \in$ $\operatorname{CIR}(f)(\vec{X})$.

Proof. If $f: \mathbb{R}^{m} \rightarrow \mathbb{R}$ is a total function and it is not asymptotic and $\vec{x} \in \vec{X}$, then $\inf \{f(\vec{a})$ : $\vec{a} \in \vec{X}\} \leq \vec{x} \leq \sup \{f(\vec{a}): \vec{a} \in \vec{X}\}$. Therefore, by definition 10, we have $f(\vec{x}) \in$ $\operatorname{CIR}(f)(\vec{X})$.

${ }^{1}$ A real function $f$ is called asymptotic if for a given interval $X$ the set $\{f(a): \underline{X}<a<\bar{X}\}$ not have the smallest element or not have the largest element. 


\section{Topology of $\mathbb{I I R}$}

In this section we will present some topological properties of the space $\mathbb{I} \mathbb{R}$ as a metric space. The properties presented here are based on the notion of proximity and limit as it is the case of distance. Because only it will be shown some topological properties of $\mathbb{I} \mathbb{R}$ the proof will be omitted. The objective here is to compare properties in the following sections. Anyway the interested readers can found the proofs in Moore (1966), Moore (1979), Oliveira et al. (1997) and Trindade (2002).

\subsection{Basic Definitions}

In the following will be presented some definitions on the topology of $\mathbb{I} \mathbb{R}$.

\subsection{Distance}

A function $d_{e}: \mathbb{R}^{m} \times \mathbb{R}^{m} \rightarrow \mathbb{R}$, defined by $d_{e}(\vec{x}, \vec{y})=\|\vec{x}-\vec{y}\|$, is called Euclidian distance from $\vec{x}$ to $\vec{y}$ in $\mathbb{R}^{m}$. It has the following properties:

$$
\begin{aligned}
& \left(D_{1}\right) d_{e}(\vec{x}, \vec{y}) \geq 0 \text { and } d_{e}(\vec{x}, \vec{y})=0 \Leftrightarrow \vec{x}=\vec{y} \text { (positive definite); } \\
& \left(D_{2}\right) d_{e}(\vec{x}, \vec{y})=d_{e}(\vec{y}, \vec{x}) \text { (symmetrical); } \\
& \left(D_{3}\right) d_{e}(\vec{x}, \vec{y}) \leq d_{e}(\vec{x}, \vec{z})+d_{e}(\vec{z}, \vec{y}) \text { (triangular inequality). }
\end{aligned}
$$

Definition 11. Let $A$ be any set. A function $d: A \times A \rightarrow \mathbb{R}$ is called a metric on $A$ if it satisfies the following properties:

1. reflexivity: $d(x, x)=0$;

2. triangular inequality: $d(x, z) \leq d(x, y)+d(y, z)$;

3. symmetry: $d(x, y)=d(y, x)$;

4. indiscernible identity: if $d(x, y)=0$ then $x=y$.

Definition 12. A distance $d: A \times A \rightarrow \mathbb{R}$ is called quasi-metric if it satisfies the following properties:

1. reflexivity: $d(x, x)=0$;

2. triangular inequality: $d(x, z) \leq d(x, y)+d(y, z)$;

3. indiscernible symmetrical identity: if $d(x, y)=d(y, x)=0$, then $x=y$.

Definition 13 ( Moore Distance). The Moore distance, $D_{M}: \mathbb{I R}^{2} \rightarrow \mathbb{R}$, between $X$ and $Y \in \mathbb{I} \mathbb{R}$, is given by

$$
D_{M}(X, Y)=\max \{|\underline{X}-\underline{Y}|,|\bar{X}-\bar{Y}|\} .
$$

Theorem 1. The Moore distance, above defined, is a metric on $\mathbb{I} \mathbb{R}$.

Proof. See Moore (1962).

Geometrically, a distance between two interval is the length of the largest segment that separates the extremes of the intervals.

Definition 14 (Moore Interval Module Moore (1979)). Let $X \in \mathbb{I} \mathbb{R}$ be an interval. The module or norm of the interval $X$ is defined as the non-negative real number, $|X|_{M}=D_{M}(X, 0)$, which corresponds to the distance from $X$ to zero.

In other words, $|X|_{M}=D_{M}(X, \mathbf{0})=\max \{|\underline{X}|,|\bar{X}|\} \geq 0$. 
Theorem 2 ( Moore Interval Module properties).

1. $|X|_{M}=0 \Leftrightarrow X=0$;

2. $|X+Y|_{M} \leq|X|_{M}+|Y|_{M}$;

3. $|X \cdot Y|_{M}=|X|_{M} \cdot|Y|_{M}$.

Proof. It follows immediately from the module definition.

The figure 1 gives a geometric interpretation to the module of an interval.

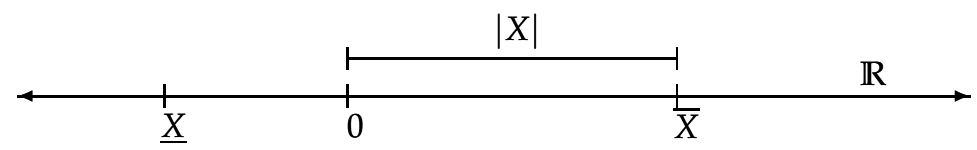

Fig. 1. Geometric interpretation to the module of an interval

Geometrically, the module of an interval is the length of the largest segment which joins the extremes of the interval to the origin.

Theorem 3. Let $X, Y, Z, W \in \mathbb{I} \mathbb{R}$ be intervals. Then, the following properties are true.

1. $D_{M}(X+Y, X+Z)=D_{M}(Y, Z)$;

2. $D_{M}(X . Y, X . Z) \leq|X|_{M} \cdot D_{M}(Y, Z)$;

3. $D_{M}(X+Y, Z+W) \leq D_{M}(X, Z)+D_{M}(Y, W)$;

4. $D_{M}(X . Y, Z . W) \leq|Y|_{M} \cdot D_{M}(X, Z)+|Z|_{M} \cdot D_{M}(Y, W)$;

5. $D_{M}(X, Y) \leq|X|_{M \cdot}|Y|_{M} \cdot D_{M}\left(\frac{1}{X}, \frac{1}{Y}\right)$, if $0 \notin X, 0 \notin Y$;

6. $X \subseteq Y \Rightarrow|X|_{M} \leq|Y|_{M}$;

Proof. See Oliveira et al. (1997).

Geometrically, the diameter of an interval is the length of the segment which joins the extremes of the interval.

Definition 15. Let $M$ be any set. A function $d: M \times M \rightarrow \mathbb{I R}$, is said an interval metric if it satisfies the following properties:

1. reflexivity: $0 \in d(X, X)$,

2. triangular inequality $|d(X, Y)|_{M} \leq|d(X, Z)|_{M}+|d(Z, Y)|_{M}$

3. symmetry: $d(X, Y)=d(Y, X)$

4. indiscernible identity: if $0 \in d(X, Y)=d(X, X)=d(Y, Y)$ then $X=Y$.

Definition 16. [An Interval Distance] Let $X$ and $Y \in \mathbb{I} R$. An Interval distance between $X$ and $Y$, denoted by $m_{e i}(X, Y)$, is defined by

$$
m_{e i}(X, Y)=\left[\inf \left\{d_{e}(x, y): x \in X \text { and } y \in Y\right\}, \sup \left\{d_{e}(x, y): x \in X \text { and } y \in Y\right\}\right] \text {. }
$$


Proposition 6. Let $X$ and $Y$ be two intervals, with $X \leq Y$ and $X \cap Y=\varnothing$. Then

$$
m_{e i}(X, Y)=[\underline{Y}-\bar{X}, \bar{Y}-\underline{X}]
$$

Proof. If $x \in X$ and $y \in Y$, then $x \leq \bar{X}$ and $y \geq \underline{Y}$. As $X \leq Y$ and $X \cap Y=\varnothing$, then $|x-y|=y-x$. Therefore, $y-x \geq \underline{Y}-\bar{X}$ and, so, $\underline{Y}-\bar{X}=\min \{|x-y|: x \in X$ and $y \in Y\}$. Similarly, it is possible to prove that $\bar{Y}-\underline{X}=\max \{|x-y|: x \in X$ and $y \in Y\}$.

Proposition 7. Let $X$ and $Y$ be two interval, with $X \leq Y$ and $X \cap Y \neq \varnothing$. Then

$$
m_{e i}(X, Y)=[0, \bar{Y}-\underline{X}] \text {. }
$$

Proof. As $X \cap Y \neq \varnothing, \exists z \in X \cap Y$. Therefore, $0 \in\left\{d_{e}(x, y): x \in X\right.$ and $\left.y \in Y\right\}$. Then, $\inf \left\{d_{e}(x, y) x \in X\right.$ and $\left.y \in Y\right\}=0$. Because $X \leq Y, \underline{X}$ is the least element of $X$ and $\bar{Y}$ is the greatest element of $Y$, it follows that $\bar{Y}-\underline{X}=|\bar{Y}-\underline{X}|=\max \left\{d_{e}(x, y): x \in X\right.$ and $\left.y \in Y\right\}$. Therefore, $m_{e i}(X, Y)=[0, \bar{Y}-\underline{X}]$.

Proposition 8. Let $X$ and $Y$ be two intervals, with $X \subseteq Y$. Then

$$
m_{e i}(X, Y)=[0, \max \{\bar{X}-\underline{Y}, \bar{Y}-\underline{X}\}] .
$$

Proof. As $X \subseteq Y, X \cap Y \neq \varnothing$ and $\min \left\{d_{e}(x, y): x \in X\right.$ and $\left.y \in Y\right\}=0$. If $X \subseteq Y$, then $\underline{Y} \leq \underline{X} \leq$ $\bar{X} \leq \bar{Y}$. Let $x \in X$ and $y \in Y$. If $y \leq \bar{X}$, then $|x-y| \leq \bar{X}-\underline{Y}$. If $y>\bar{X}$, then $|x-y|=y-x \leq \bar{Y}-\underline{X}$ . So $|x-y| \leq \max \{\bar{X}-\underline{Y}, \bar{Y}-\underline{X}\}$. Therefore, $m_{e i}(X, Y)=[0, \max \{\bar{X}-\underline{Y}, \bar{Y}-\underline{X}\}]$.

Corollary 1. Let $X \cap Y \neq \varnothing$. Then $m_{e i}(X, Y)=[0, \max \{\bar{X}-\underline{Y}, \bar{Y}-\underline{X}\}]$.

Proof. It is a direct application of the propositions 7 and 8.

Proposition 9. The distance $m_{e i}$ coincides with the Euclidian distance, $d_{e}$, when it is applied to degenerate intervals. So, if $X=[x, x]$ and $Y=[y, y]$, then

$$
m_{e i}(X, Y)=\left[d_{e}(x, y), d_{e}(x, y)\right] .
$$

Proof. As $X=[x, x]$ and $Y=[y, y]$, it follows that

$$
\begin{aligned}
m_{e i}(X, Y) & =\left[\min \left\{d_{e}(x, y): x \in X \text { and } y \in Y\right\}, \max \left\{d_{e}(x, y): x \in X \text { and } y \in Y\right\}\right] \\
& =\left[\min \left\{d_{e}(x, y): x \in\{x\} \text { and } y \in\{y\}\right\}, \max \left\{d_{e}(x, y): x \in\{x\} \text { and } y \in\{y\}\right\}\right] \\
& =\left[\min \left\{d_{e}(x, y)\right\}, \max \left\{d_{e}(x, y)\right\}\right] \\
& =\left[d_{e}(x, y), d_{e}(x, y)\right] .
\end{aligned}
$$

Corollary 2. The distance $m_{e i}$, restricted to degenerate intervals, is an interval metric.

Proof. It follows directly from proposition 9.

Corollary 3. If $X \cap Y \neq \varnothing$. Then $m_{e i}(X, Y)=[0, \max \{\bar{X}-\underline{Y}, \bar{Y}-\underline{X}\}]$.

Proof. It follows directly from propositions 7 and 8.

Proposition 10. The distance $m_{e i}$ is the CIR of the Euclidian distance.

Proof. It is a direct consequence of the definitions 10 and 16. 
Theorem 4 ( CIR of a metric is an interval metric). Let $A$ be a set and $d$ a metric. Then $C I R(d)$ is an interval metric.

Proof. $C I R(d)(X, Y)$ satisfies the four proprieties of interval metric.

- Reflexivity: $0 \in C I R(d)(X, X)$, because $0=d(x, x) \in\{d(x, y): x$ and $y \in X\}$.

- Triangular inequality: $|C I R(d)(X, Y)|_{M} \leq|C I R(d)(X, Z)|_{M}+|C I R(d)(Z, Y)|_{M}$, since $\max \{d(x, y): x \in X$ and $y \in Y\} \leq \max \{d(x, z): x \in X$ and $z \in Z\}+\max \{d(z, y): z \in$ $Z$ and $y \in Y\}$

- Symmetry: $\operatorname{CIR}(d)(X, Y)=C I R(d)(X, Y)$, because $\min \{d(x, y): x \in X$ and $y \in Y\}=$ $\min \{d(y, x): x \in X$ and $y \in Y\}$ and $\max \{d(x, y): x \in X$ and $y \in Y\}=\max \{d(y, x): x \in$ $X$ and $y \in Y\}$.

- indiscernible Identity: We intend to prove that if $0 \in C I R(d)(X, Y)=$ $C I R(d)(X, X)=C I R(d)(Y, Y)$, then $X=Y$. For that, suppose that $X \neq Y$. If $X \cap Y=$ $\varnothing$, then $0 \notin C I R(d)(X, Y)$ absurd, by the hypothesis. Case $X \cap Y \neq \varnothing$ we have four possible cases. Case $X \subset Y$ or $Y \subset X C I R(d)(X, X) \neq C I R(d)(Y, Y)$ also it absurd from the hypothesis. The others two cases $X \leq Y$ or $Y \leq X$ both cases or $C I R(d)(X, Y) \neq$ $C I R(d)(X, X)$ or $C I R(d)(X, Y)=C I R(d)(Y, Y)$ which also is absurd by hyphotesis. So, $C I R(d)$ satisfies the indiscernible property.

We believe that in the extension of real representation to interval representation there is a shift of paradigm, by which we mean that the most theorems valid for real numbers need to be adapted for interval version.

The same we can say about the properties of definition 11, because if we do an analysis of the reflexive property we observe that it is good for real numbers since they are non dimensional when they are seen as point of real line, by the impossibility of physical existence. In this case the distance from it to itself is zero. However if the entity is an extensive body it reasonable that a least distance from it to itself be zero and the largest distance be the measure of the extension of its body. We observe that in this case the distance would be an interval whose extremes is zero and the measure of the extension of the body. So, we can see that the distance from an interval to itself may zero when one measures from a point of the interval to itself or its diameter when one measures the distance between its extremes.

We can observe one more inconsistency in the use of a propriety of a set whose elements are dimensionless when extended to those with extensive features, as the Mooore distance, whose distance is a real number. In a semantic field where the interval are used to represent uncertainties it reasonable to expect that given two intervals $X$ and $Y$ the distance between them be an interval of uncertainties that varies between $\min \left\{d_{e}(x, y): x \in X\right.$ and $\left.y \in Y\right\}$ and $\max \left\{d_{e}(x, y): x \in X\right.$ and $\left.y \in Y\right\}$.

Proposition 11. The distance $m_{e i}$ is an interval metric.

Proof. It is a direct consequence of proposition 10 and theorem 4.

Proposition 12. Let $X$ and $Y \in \mathbb{I} R, m_{e i}(X, Y) \leq[0, \operatorname{Diam}(Y)]$ if only if $X \subseteq Y$. 
Proof. $\Leftarrow)$ Suppose that $X \subseteq Y$, then it satisfies the definition 4 and the proposition 8 , so we have

$$
m_{e i}(X, Y)=[0, \max \{\bar{X}-\underline{Y}, \bar{Y}-\underline{X}\}] .
$$

By the hypothesis that $X \subseteq Y$ and by the definition 4 we conclude that

$$
\begin{aligned}
\max \{\bar{X}-\underline{Y}, \bar{Y}-\underline{X}\} & \leq \bar{Y}-\underline{Y} \\
& =\operatorname{Diam}(Y) .
\end{aligned}
$$

By the equation (4) and the definition 5 we have

$$
m_{e i}(X, Y) \leq[0, \operatorname{Diam}(Y)]
$$

$\Rightarrow)$ Now, suppose that $m_{e i}(X, Y) \leq[0, \operatorname{Diam}(Y)]$ is within the three possible cases and its symmetric $m_{e i}(X, Y)$ satisfies at least one of the propositions 6, 7 ou 8. Assuming that $m_{e i}(X, Y)$ satisfies the conditions of proposition 6 we have

$$
m_{e i}(X, Y)=[\underline{Y}-\bar{X}, \bar{Y}-\underline{X}] .
$$

By the hypothesis that $m_{e i}(X, Y)$ satisfies the conditions of the proposition 6 and by the equation (6) we have

$$
\begin{array}{ll}
\underline{Y}-\bar{X} \quad \geq 0 \\
\overline{\mathrm{e}} \\
\bar{Y}-\underline{X} \quad \geq \bar{Y}-\underline{Y} .
\end{array}
$$

By the equation (7) and the definition 5 we conclude that

$$
[0, \operatorname{Diam}(Y)] \leq m_{e i}(X, Y)
$$

By the equation (8) we conclude if $m_{e i}(X, Y)$ satisfies the conditions of the proposition 6 , then it does not satisfies the conditions of the proposition 12.

Suppose that $m_{e i}(X, Y)$ satisfies the conditions of proposition 7 then

$$
m_{e i}(X, Y)=[0, \bar{Y}-\underline{X}] .
$$

By the hypothesis that $m_{e i}(X, Y)$ satisfies the conditions of the propositions 7 and by the equation (9) we have

$$
\begin{aligned}
0 & \geq 0 \\
& \text { and } \\
(\bar{Y}-\underline{X}) & \geq(\bar{Y}-\underline{Y}) .
\end{aligned}
$$

By the equation (10) and the definition 5 we conclude that

$$
\begin{aligned}
m_{e i}(X, Y) & \leq[0, \operatorname{Diam}(Y)] \\
& \text { only if } \\
& \underline{X}=\underline{Y} .
\end{aligned}
$$

By the equation (11) we conclude that if $m_{e i}(X, Y)$ satisfies the conditions of proposition 7 , it satisfies also the conditions of proposition 12 only if the equality $\underline{X}=\underline{Y}$ is satisfied. Thus $X \subseteq Y$.

Suppose, now, that $m_{e i}(X, Y)$ satisfies the conditions of the proposition 8 , then we get

$$
m_{e i}(X, Y)=[0, \max \{\bar{X}-\underline{Y}, \bar{Y}-\underline{X}\}] .
$$


By the hypothesis that $m_{e i}(X, Y)$ satisfies the condition 8 and by the equation (12) we have

$$
\begin{aligned}
& 0 \leq 0 \\
& \text { and } \\
&(\bar{Y}-\underline{Y}) \quad \leq \max \{(\bar{X}-\underline{Y}),(\bar{Y}-\underline{X})\} .
\end{aligned}
$$

By the equation (13) and the definition 4 we conclude that

$$
\begin{aligned}
m_{e i}(X, Y) & \leq[0, \operatorname{Diam}(Y)] \\
& \text { and } \\
& X \subseteq Y .
\end{aligned}
$$

Finally, by the equations (8), (11) and (14), we conclude that if $m_{e i}(X, Y) \leq[0, \operatorname{Diam}(Y)]$, then $X \subseteq Y$.

Proposition 13. Let $X$ and $Y \in \mathbb{I} \mathbb{R}$ be such that $X \neq Y$. Then, $m_{e i}(X, Y) \leq[0, \operatorname{Diam}(Y)+\operatorname{Diam}(X)]$ if only if $X \cap Y \neq \varnothing$.

Proof. The case where $X \subseteq Y$ was proved in the proposition 12. For the other cases we analyse the three possible cases for $m_{e i}(X, Y)$, which are the cases of the propositions 6,7 and 8 .

Suppose that $m_{e i}(X, Y)$ satisfies the conditions of the proposition 6 , then we get

$$
m_{e i}(X, Y)=[\underline{Y}-\bar{X}, \bar{Y}-\underline{X}] .
$$

By the hypothesis that $m_{e i}(X, Y)$ satisfies the conditions of the proposition 6 and the equation (15) we conclude

$$
\begin{aligned}
{[0, \bar{Y}-\underline{Y}+\bar{X}-\underline{X}] } & \leq[\underline{Y}-\bar{X}, \bar{Y}-\underline{X}] \\
{[0, \operatorname{Diam}(Y)+\operatorname{Diam}(X)] } & \leq m_{e i}(X, Y) .
\end{aligned}
$$

By the equation (16), we conclude that if $m_{e i}(X, Y)$ satisfies the conditions of the proposition 6 it does not satisfies the conditions of proposition 13.

Suppose that $m_{e i}(X, Y)$ satisfies the conditions of proposition 7 then we get

$$
m_{e i}(X, Y)=[0, \bar{Y}-\underline{X}] .
$$

By the hypothesis that $m_{e i}(X, Y)$ satisfies the conditions of the proposition 7 and by the equation (17) we have

$$
\begin{aligned}
& {[0, \bar{Y}-\underline{X}] \leq[0, \bar{Y}-\underline{Y}+\bar{X}-\underline{X}]} \\
& m_{e i}(X, \bar{Y}) \leq[0, \operatorname{Diam}(Y)+\operatorname{Diam}(X)]
\end{aligned}
$$

By the equation (18) we conclude that if $m_{e i}(X, Y)$ satisfies the conditions of proposition 7 then it satisfies also the conditions of proposition 13.

Suppose that $m_{e i}(X, Y)$ satisfies the conditions of the proposition 8 then we get

$$
m_{e i}(X, Y)=[0, \max \{(\underline{Y}-\bar{X}),(\bar{Y}-\underline{X})\}] .
$$

By the hypothesis that $m_{e i}(X, Y)$ satisfies the conditions of proposition 8 and by the equation (19) we have

$$
\begin{aligned}
{[0, \max \{(\underline{Y}-\bar{X}),(\bar{Y}-\underline{X})\}] } & \leq[0, \bar{Y}-\underline{Y}+\bar{X}-\underline{X}] \\
m_{e i}(X, Y) & \leq[0, \operatorname{Diam}(Y)+\operatorname{Diam}(X)]
\end{aligned}
$$


By the equation (20) we conclude that if $m_{e i}(X, Y)$ satisfies the conditions of proposition 8 , then it also satisfies the conditions of proposition 13.

By the equations (16), (18) and (20) we conclude that $m_{e i}(X, Y) \leq[0, \operatorname{Diam}(Y)+\operatorname{Diam}(X)]$ if only if $X \cap Y \neq \varnothing$.

Proposition 14. Let $X$ and $Y \in \mathbb{I} R$. Then $[0, \operatorname{Diam}(Y)+\operatorname{Diam}(X)] \leq m_{e i}(X, Y)$ if only if $X \cap Y=$ $\varnothing$.

Proof. By the equations (16), (18) and (20) we conclude only in the case that $m_{e i}(X, Y)$ satisfies the conditions of propositions 6 and 14. Then $[0, \operatorname{Diam}(Y)+\operatorname{Diam}(X)] \leq m_{e i}(X, Y)$ if only if $X \cap Y=\varnothing$.

If we associate the uncertainty degree of an interval to its diameter, we can observe that the metric $m_{e i}$ preserves the uncertainties, since a distance between two accurate intervals(null diameter) is an accurate measurement also of null diameter and the distance between two inaccurate intervals (diameter $\neq 0$ ) is also an inaccurate measurement, as it is shown in the following proposition.

Proposition 15. Let $X$ and $Y \in \mathbb{I} \mathbb{R}$, then we have $\operatorname{Diam}\left(m_{e i}(X, Y)\right) \leq \operatorname{Diam}(X)+\operatorname{Diam}(Y)$.

Proof. We split this proof in two parts. The first one, in the case that $m_{e i}(X, Y)$ satisfies the conditions of proposition 6 and the second, the cases in which it satisfies the conditions of proposition 7 or the proposition 8 , which can be directly inferred from propositions 12 and 13 . For the first part, suppose that $m_{e i}(X, Y)$ satisfies the conditions of proposition 6 , then we get

$$
m_{e i}(X, Y)=[\underline{Y}-\bar{X}, \bar{Y}-\underline{X}]
$$

Therefore,

$$
\begin{aligned}
\operatorname{Diam}\left(m_{e i}(X, Y)\right) & =(\bar{Y}-\underline{X})-(\underline{Y}-\bar{X}) \\
& =\bar{Y}-\underline{X}-\underline{Y}+\bar{X} \\
& =(\bar{X}-\underline{X})+(\bar{Y}-\underline{Y}) \\
& =\operatorname{Diam}(X)+\operatorname{Diam}(Y) .
\end{aligned}
$$

With the proposed metric the module notion can redefined as following.

Definition 17. Let $X$ be an interval. The interval module, denoted by $|X|_{I}$, is defined by the distance $m_{e i}(X,[0 ; 0])$.

Theorem 5 ( Interval Module Properties ).

1. $|X|_{I}=\mathbf{0} \Leftrightarrow X=\mathbf{0}$;

2. $|X+Y|_{I} \leq|X|_{I}+|Y|_{I}$;

3. $|X \cdot Y|_{I}=|X|_{I} \cdot|Y|_{I}$. 


\section{Proof. Property 1}

Suppose that $X \neq \mathbf{0}$, then $\sup \left\{d_{e}(x, 0): x \in X\right\}$ is greater than zero. Therefore, $|X|_{I} \neq \mathbf{0}$.

\section{Property 2}

For that we need to prove that the inferior limit of $|X+Y|_{I}$ is always lesser than or equal to the inferior limit of $|X|_{I}+|Y|_{I}$ and that the superior limit of $|X+Y|_{I}$ is always lesser than or equal to the superior limit of $|X|_{I}+|Y|_{I}$. Thus we have

$$
|X+Y|_{I}=|[\underline{X}+\underline{Y} ; \bar{X}+\bar{Y}]|_{I}
$$

If $0 \in X+Y$, then

$$
|X+Y|_{I}=[0 ; \max \{|\underline{X}+\underline{Y}|,|\bar{X}+\bar{Y}|\}]
$$

Case $0 \notin X+Y$, then

$$
|X+Y|_{I}=[\min \{|\underline{X}+\underline{Y}|,|\bar{X}+\bar{Y}|\} ; \max \{|\underline{X}+\underline{Y}|,|\bar{X}+\bar{Y}|\}],
$$

and for $|X|_{I}+|Y|_{I}$ we get

$$
\left.|X|_{I}+|Y|_{I}=\underline{\left[|X|_{I}\right.}+\underline{|Y|_{I}} ; \overline{|X|_{I}}+\overline{|Y|_{I}}\right]
$$

If $0 \in X$ or $Y$, and not both. By assuming that $0 \in Y$, the other case is symmetrical, and thus, we have

$$
|X|_{I}+|Y|_{I}=[\min \{\underline{X}, \bar{X}\} ; \max \{|\underline{X}|+|\underline{Y}|,|\underline{Y}|+|\bar{X}|,|\underline{X}|+|\bar{Y}|,|\bar{X}|+|\bar{Y}|\}] .
$$

If $0 \in X$ and $Y$, then

$$
|X|_{I}+|Y|_{I}=[0 ; \max \{|\underline{X}|+|\underline{Y}|,|\underline{Y}|+|\bar{X}|,|\underline{X}|+|\bar{Y}|,|\bar{X}|+|\bar{Y}|\}] .
$$

Case $0 \notin X$ and $Y$, then

$$
\begin{aligned}
& |X|_{I}+|Y|_{I}= \\
& {[\min \{|\underline{X}|+|\underline{Y}|,|\underline{Y}|+|\bar{X}|,|\underline{X}|+|\bar{Y}|,|\bar{X}|+|\bar{Y}|\} ;} \\
& \max \{|\underline{X}|+|\underline{Y}|,|\underline{Y}|+|\bar{X}|,|\underline{X}|+|\bar{Y}|,|\bar{X}|+|\bar{Y}|\}] .
\end{aligned}
$$

For the cases where $0 \in X+Y$ by the positive definition of the module it follows the inferior limit. It remains to prove the superior limit. For that it is enough to prove that

$$
\max \{|\underline{X}+\underline{Y}|,|\bar{X}+\bar{Y}|\} \leq \max \{|\underline{X}|+|\underline{Y}|,|\underline{Y}|+|\bar{X}|,|\underline{X}|+|\bar{Y}|,|\bar{X}|+|\bar{Y}|\} .
$$

For that suppose that $\max \{|\underline{X}+\underline{Y}|,|\bar{X}+\bar{Y}|\}=|\underline{X}+\underline{Y}|$ then we have

$$
|\underline{X}+\underline{Y}| \leq|\underline{X}|+|\underline{Y}| \text { as the real analysis. }
$$

Now, assuming that $\max \{|\underline{X}+\underline{Y}|,|\bar{X}+\bar{Y}|\}=|\bar{X}+\bar{Y}|$ we get

$$
|\bar{X}+\bar{Y}| \leq|\bar{X}|+|\bar{Y}| \text {, as real analysis. }
$$

So, when $0 \in X+Y$ we have $|X+Y|_{I} \leq|X|_{I}+|Y|_{I}$.

Now we analyse the case where $0 \notin X+Y$. In particular, for the case that $X$ or $Y$ contains zero and not both. As we see before, we chose $Y$ containing the zeros and the other cases are symmetrical. We prove first for the inferior limit. For that we have to prove that

$$
\min \{|\underline{X}+\underline{Y}|,|\bar{X}+\bar{Y}|\} \leq \min \{|\underline{X}|,|\bar{X}|\} .
$$


Suppose that $\min \{|\underline{X}+\underline{Y}|,|\bar{X}+\bar{Y}|\}=|\underline{X}+\underline{Y}|$, then $0 \leq X+Y$ and $\min \{|\underline{X}|,|\bar{X}|\}=|\underline{X}|$. As $\underline{Y} \leq 0$, we have $|\underline{X}+\underline{Y}| \leq|\underline{X}|$.

Now suppose that $\min \{|\underline{X}+\underline{Y}|,|\bar{X}+\bar{Y}|\}=|\bar{X}+\bar{Y}|$. Then $X+Y \leq \mathbf{0}$ and $\min \{|\underline{X}|,|\bar{X}|\}=|\bar{X}|$. As $0 \leq \bar{Y}$, it follows $|\bar{X}+\bar{Y}| \leq|\bar{X}|$, which proves the case for the inferior limit. The superior we cant get from the equations (30),(31) and (32).

In the case where $0 \notin X$ and $Y$, we will prove only for the inferior limit, since the superior limit follows from the equations (30), (31) and (32).

Suppose that $0 \leq X+Y$, then we have

$$
\min \{|\underline{X}+\underline{Y}|,|\bar{X}+\bar{Y}|\}=|\underline{X}+\underline{Y}|
$$

and

$$
\min \{|\underline{X}|+|\underline{Y}|,|\underline{Y}|+|\bar{X}|,|\underline{X}|+|\bar{Y}|,|\bar{X}|+|\bar{Y}|\}= \begin{cases}|\underline{X}|+|\underline{Y}| & \text { if } \mathbf{0} X X \text { and } Y . \\ |\underline{X}|+|\underline{X}| & \text { if } X \leq \mathbf{0} \text { and } \mathbf{0} \leq Y \\ |\underline{X}|+|\bar{Y}| & \text { if } Y \leq \mathbf{0} \text { and } \mathbf{0} \leq X\end{cases}
$$

By the hypothesis that $0 \leq X+Y$ and by the conditions of the equation (35) we get

$$
|\underline{X}+\underline{Y}| \leq \begin{cases}|\underline{X}|+|\underline{Y}| & \text { if } \mathbf{0} \leq X \text { and } Y \\ |\underline{Y}|+|\bar{X}| & \text { if } X \leq \mathbf{0} \text { and } \mathbf{0} \leq Y \\ |\underline{X}|+|\bar{Y}| & \text { if } Y \leq \mathbf{0} \text { and } \mathbf{0} \leq X\end{cases}
$$

So, we conclude the prove of the property 2 , leaving the case where $X+Y \leq 0$ because it is similar to the cases where $0 \leq X+Y$.

Property 3

If $X$ or $Y$ contains zero we have

$$
|X \cdot Y|_{I}=[0 ; \max \{|\underline{X} \cdot \underline{Y}|,|\underline{X} \cdot \bar{Y}|,|\bar{X} \cdot \underline{Y}|,|\bar{X} \cdot \bar{Y}|\}]
$$

and

$$
|X|_{I} \cdot|Y|_{I}=[0 ; \max \{|\underline{X}| \cdot|\underline{Y}|,|\underline{X}| \cdot|\bar{Y}|,|\bar{X}| \cdot|\underline{Y}|,|\bar{X}| \cdot|\bar{Y}|\}] .
$$

As in the real analysis where the product of the module of two real numbers is equal to the module of the product of these numbers, in the interval module it is similar as it shown by the equations (37) and (38). So, given two intervals where at least one of them contains the zero, the interval module of the product is equal to the product of the modules of these numbers. Now, we will prove the property in discussion for the case where neither of the intervals contains zero.

If $X$ and $Y$ does not contain zero, we have

$$
|X \cdot Y|_{I}=[\min \{|\underline{X} \cdot \underline{Y}|,|\underline{X} \cdot \bar{Y}|,|\bar{X} \cdot \underline{Y}|,|\bar{X} \cdot \bar{Y}|\} ; \max \{|\underline{X} \cdot \underline{Y}|,|\underline{X} \cdot \bar{Y}|,|\bar{X} \cdot \underline{Y}|,|\bar{X} \cdot \bar{Y}|\}]
$$

and

$$
|X|_{I} \cdot|Y|_{I}=[\min \{|\underline{X}| \cdot|\underline{Y}|,|\underline{X}| \cdot|\bar{Y}|,|\bar{X}| \cdot|\underline{Y}|,|\bar{X}| \cdot|\bar{Y}|\} ; \max \{|\underline{X}| \cdot|\underline{Y}|,|\underline{X}| \cdot|\bar{Y}|,|\bar{X}| \cdot|\underline{Y}|,|\bar{X}| \cdot|\bar{Y}|\}] .
$$

The property 3 follows directly from the equivalence between the equations (39) and (40).

The figure 2 gives a geometric interpretation for the module of a Moore-interval. 


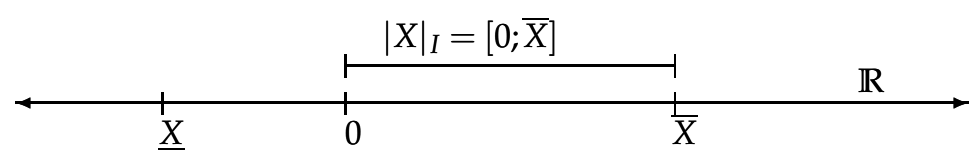

Fig. 2. Geometric interpretation of the interval module of an interval

Theorem 6. Let $X, Y, Z \in \mathbb{I} \mathbb{R}$ be intervals. Then, the following properties are true:

1. $m_{e i}(X+Y, X+Z)=m_{e i}(Y, Z)+m_{e i}(X, X)$ or $m_{e i}(Y, Z)+[-\operatorname{Diam}(X) ; \operatorname{Diam}(X)]$

2. $X \subseteq Y \Rightarrow|X|_{I} \subseteq|Y|_{I}$;

Proof. Item 1 If $m_{e i}(X+Y, X+Z)$ satisfies the conditions of proposition 6 we have

$$
\begin{aligned}
m_{e i}(X+Y, X+Z) & =[(\underline{X}+\underline{Z})-(\bar{X}+\bar{Y}) ;(\bar{X}+\bar{Z})-(\underline{X}+\underline{Y})] \\
& =[(\underline{\underline{Z}}-\overline{\bar{Y}})+(\underline{X}-\bar{X}) ;(\bar{Z}-\underline{Y})+(\bar{X}-\underline{X})] \\
& =[(\underline{Z}-\bar{Y})-\operatorname{Diam}(X) ;(\bar{Z}-\underline{Y})+\operatorname{Diam}(X)] \\
& =[(\underline{Z}-\bar{Y}) ;(\bar{Z}-\underline{Y})]+[-\operatorname{Diam}(X) ; \operatorname{Diam}(X)] \\
& =m_{e i}(Y, Z)+[-\operatorname{Diam}(X) ; \operatorname{Diam}(X)] .
\end{aligned}
$$

If $m_{e i}(X+Y, X+Z)$ satisfies the conditions of corollary 3 we get

$$
\begin{aligned}
m_{e i}(X+Y, X+Z) & =[0 ; \max \{(\bar{X}+\bar{Y})-(\underline{X}+\underline{Z}),(\bar{X}+\bar{Z})-(\underline{X}+\underline{Y})\}] \\
& =[0 ; \max \{(\bar{Y}-\underline{Z})+(\bar{X}-\underline{X}),(\bar{Z}-\underline{Y})+(\bar{X}-\underline{X})\}] \\
& =[0 ; \max \{(\bar{Y}-\underline{Z})+\operatorname{Diam}(X),(\bar{Z}-\underline{Y})+\operatorname{Diam}(X)\}] \\
& =[0 ; \max \{(\bar{Y}-\underline{Z}),(\bar{Z}-\underline{Y})\}]+[0 ; \operatorname{Diam}(X)] \\
& =m_{e i}(Y, Z)+[0 ; \operatorname{Diam}(X)] \\
& =m_{e i}(Y, Z)+m_{e i}(X, X) .
\end{aligned}
$$

Item 2 It follows directly from propositions 16 and 17.

The notion of circumference, limit and continuity can be constructed by this metric which will appear in another work.

Finally, about the Moore metric can be said that in the implementation of the absolute value function for intervals of real numbers X, by Moore Moore (1979), in programming language, for example

$$
|X|=\max [|\underline{X}|,|\bar{X}|]
$$

gives a single scalar value, and not an interval.

When passing intervals to an algorithm that was originally written with other numerical types in mind some users have expressed surprise at the result returned by the $|X|$ function. They would expect, for instance, that the absolute value of the interval $[-3,2]$ would return $[0,3]$, which is a reasonable expectation, and would allow many more algorithms to work without modification. In some interval mathematics applications, such as non-smooth optimisation, you want "| |" to be the range of the absolute value, whereas, in other implementations, you want it to be the "magnitude," that is, the largest absolute value, rounded up, of any point in the argument interval. Additionally, the "magnitude," or the smallest absolute value of 
any point in the interval, rounded down, is usually included, for a triad of extensions to the absolute value. The magnitude is useful, for example, in proving diagonal dominance of a matrix with uncertain entries.

\section{Conclusion}

By it was said above the distance notion is ubiquitous to mainly research areas and for system modelling with uncertainties. The usual metrics fail to accomplish those necessities, because it is not indeed an interval metric. By other hand the proposed metric is not only a generalisation of the usual Euclidean metric but also it is an essentially interval metric, as it is was shown above. With the proposed metric one can redefine the notions of limit, continuity, neighbourhood, convergence etc. Most basic properties of mathematics can be redefined with the proposed notion of distance.

The proposed metric accomplishes the numeric aspect if one takes an interval as an approximation of a real number and the logic aspects if one takes an interval as a fuzzy information.

\section{References}

Balopoulos, V., Hatzimichailidis, A. G. \& Papadoupoulos, B. K. (2007). Distance and similarity measures for fuzzy operators, Science Direct-Information Sciences (177): 2336-2348.

Chakraborty, C. \& Chakraborty, D. (2006). A theoretical development on a fuzzy distance measure for fuzzy numbers, Mathematical and Computer Modelling (43): 254-261.

Fono, L. A., Gwet, H. \& Bouchon-Meunier, B. (2007). Fuzzy implication operators for difference operations for fuzzy sets and cardinality-based measures of comparison, European Journal of Operational Research (183): 314-326.

$\mathrm{Fu}$, Y. \& Huang, T. S. (2007). Unsupervised locally embedded clustering for automatic highdimensional data labeling, IEEE pp. 1057-1060. ICASSP2007.

Georgiou, T., Michailovich, O., Rathi, Y., Malcolm, J. \& Tannenbaum, A. (2007). Distribution metrics and image segmentation, Linear Algebra and Its Applications (425): 663-672.

Girard, A. \& Pappas, G. J. (2007). Approximation metrics for discrete and continuous systems, IEEE Transactions on Automatic Control 52(5): 782-798.

Grzegorzewski, P. (2004). Distances between intuitionistic fuzzy sets and/or interval-valued fuzzy sets based on the hausdorff metric, Fuzzy Sets and Systems (148): 319-328.

Lin, C.-K. \& Lee, L.-S. (2007). Pronunciation modeling for spontaneous speech recognition using latent pronunciation analysis (lpa) and prior knowledge, IEEE . ICASSP2007.

Manouvrier, M., Rukoz, M. \& Jomier, G. (2005). A generalized metric distance between hierarchically partitioned images, $M D M / K D D^{\prime} 05$, Chicago,IL, USA.

Marciniak, A. (2003). On multistep interval methods for solving the initial value problem, Poznan University of Technology - Institute of Computing Science - Piotrowo 3a. 60-965 Poznan Poland .

Moore, R. E. (1962). Interval arithmetic and automatic error analysis in digital computing, Technical Report 25, Department of Mathematics, Stanford University, Stanford, California. NR-0440211.

Moore, R. E. (1966). Interval analysis, Prentice Hall, New Jersey .

Moore, R. E. (1979). Methods and Applications of Interval Analysis, Studies in Applied Mathematics - SIAM, Philadelfia.

Oliveira, P. W., Diverio, T. A. \& Cludio, D. M. (1997). Fundamentos da matemtica intervalar, Sagra Luzzato. 
Popova, E. D. (1994). Extended interval arithmetic in ieee floating-point envioronment, Interval Computation pp. 100-129.

Santiago, R. H. N., Bedregal, B. R. C. \& Acily, B. M. (2006). Formal aspects of correctness and optimality of interval computations, Formal Aspects of Computing 18: 231-243.

Sunaga, T. (1958). Theory of an interval algebra and its application to numerical analysis, RAAG MEMOIRS.

Sundaram, S. \& Narayanan, S. (2007). Analysis of audio clustering using word descriptions, in IEEE (ed.), ICASSP2007, pp. 769-772.

Trindade, R. M. P. (2002). Uma anlise intervalar de scott, Master's thesis, Departamento de Informtica da UFPE.

Vaccaro, G. L. R. (2001). Soluo de equaes intervalares, PhD thesis, Instituto de Informtica da Universidade Federal do Rio Grande do Sul - UFRGS.

Voxman, W. (1998). Some remarks on distances between fuzzy numbers, Fuzzy Sets and Systems (100): 353-365.

Yu, J., Amores, J., Sebe, N. \& Tian, Q. (2006). Toward robust distance metric analysis for similarity estimation, IEEE Computer Society Conference on Computer Vision and Pattern Recognition . 


\title{
Walking Hexapod Robot in Disaster Recovery: Developing Algorithm for Terrain Negotiation and Navigation
}

\author{
Mohiuddin Ahmed, M. R. Khan, Md. Masum Billah and Soheli Farhana \\ International Islamic University Malaysia (IIUM) \\ Malaysia
}

\section{Introduction}

The catastrophic cyclone Sidr that wrecked havoc Bangladesh in 2007, tsunami hit most of the countries of Asia around Indian ocean in 2004, Katrina hit Arkansas in 2005, and the terrorist attacks on the World Trade Centers in 2001 are clear indication that we are not prepared for disaster recovery at all. In all cases the infrastructure could not withstand the fury of nature, even in the case of WTC the NYPD was not prepared for such gigantic task of rescue mission. The conventional reaction to such disaster is not adequate; a new paradigm shift is needed to address such calamities utilizing all resources at hand. Disaster recovery is defined to be the emergency response function which deals with the collapse of man made structures (G. Nejat, 2006). In any disaster either man made or due to Mother nature, the elementary tasks at hand are: (i) reach the affected hazardous field (ii) find and get information about victims, and (iii) rescue as many of them as possible.

It is possible for robot to reach any hazardous field unlike who have limited mobility in such missions. Nowadays legged and wheeled robots are involved in such mission (Habib, 2000), (Y. Mori, 2005), (Rizo J, 2003), (Y. Baudoin, 1999). In terms of hazardous field navigation for disaster recovery mission, legged robots have advantages over wheeled robots.

Wheeled robots are the simplest and cheapest and tracked robots are very good for moving, but not over almost all kinds of terrain. Manned wheeled vehicles (Habib, 2000), (Y. Mori, 2005) or robotic systems (Rizo J, 2003), (Y. Baudoin, 1999), have already been tested. Navigate over obstacles and ditches and even on stairs one of the foremost advantages legged robots hold over their wheeled or tracked counterparts. It shows that legged robots can operate in both even and rough terrain. Some general-purpose robots were tested for this kind of application, nowadays specific prototypes having special features are being built and tested for specific mission. The TITAN VIII walking robot, a four-legged robot was developed as a general-purpose walking robot at the Tokyo Institute of Technology, Japan (Hirose S, 1998). COMET-I maybe the first legged robot purposefully developed for rescue missions. It is a six-legged robot developed at Chiba University, Japan, and incorporates different sensors and location systems (Nonami K, 2000), (Q.J. Huang, 2003). This robot weighs about $120 \mathrm{~kg}$. The Chiba University group has developed the fourth version of this 
robot, COMET-IV, which weighs about one ton. ARIEL is another hexapod robot, developed by the robots Company, for mine-recovery operations. The Defense Advanced Research Projects Agency (DARPA) and the US Office of Naval Research (ONR) are exploring methods for using this robot for underwater missions (D. Voth, 2002). This robot uses six 2DOF legs with limited mobility to perform omni directional motions. The legged robots working group at the Industrial Automation Institute (IAI-CSIC) has long experience in the development of walking robots (P. Gonzalez, 1995, 2000, 2003) and since 1999 it has been working on the application of the RIMHO-2 walking robot for detecting and locating unexploded ordnance. Hexapod robot could also be used in agricultural fields, though wheel robots already are being used in this field (Philip J., 2005). The wheel robot can move only directed even terrain, when hexapod robot can navigate even and uneven terrain. In navigating hexapod considers different gaits namely adaptive gaits (C.-L. Shih, 1993), FTL gaits (F. Ozguner, 1984), graph search method (P. K. Pal, 1991), free gaits (R. B. McGhee, 1979). Hexapod robots are appropriate for disaster recovery mission for its versatile properties.

The main focus of this research is to develop an efficient terrain negotiation \& locomotion for a spider like hexapod robot. The focus is to negotiate of even and uneven terrain efficiently.

\section{Hexapod Configuration}

\subsection{Hexapod Body Configuration}

A hexapod robot is a perfunctory medium that walks on six legs. Since a robot can be statically secure on three or more legs, a hexapod robot has a great deal of suppleness in how it can move. If legs become disabled, the robot may still be able to walk. Furthermore, all of the legs are not needed for stability. A hexapod, can achieve higher speed than a quadruped, and it is well known that a hexapod achieves its highest speed when using a statically stable gait. However, the robot's static stability margin is not optimum when using gaits, for instance, five-leg support. Nevertheless, a hexapod configuration using alternating tripods has been chosen just to try to increase the machine's speed, albeit at the cost of slightly jeopardizing stability. To navigate in hazardous field, it will rotate in any direction; heavy legs with powerful servo motors are chosen over other types so that they can withstand heavy loads. It contains the required subsystems, such as an onboard computer, electronics, drivers, a PIC Microcontroller, LCD and batteries. Walking robots body shape can determine key features such as static stability, as studied in (Schneider A, 2000). Figure 1 depicts the spider like hexapod configuration.

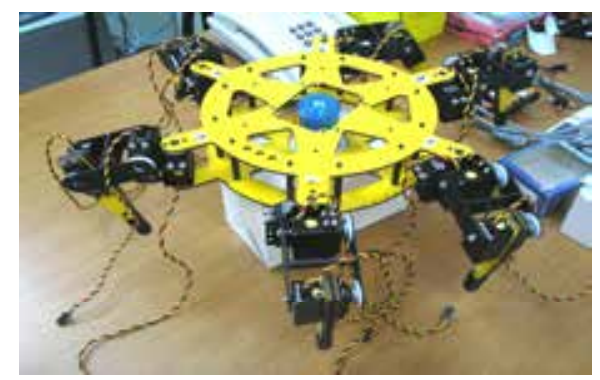

Fig. 1. Body of Hexapod agent 


\subsection{Hexapod Leg Configuration}

Hexapod robots require legs that touch the ground only at certain contact points. Legs have Legs have been designed taking into consideration weight of the robot along with its subsystems as well as the weight of the legs. Spider-like leg configurations are typical for walking robots. It is known that a spider-like leg configuration is the most efficient one from the energy point of view and it requires less torques. However, it is not very efficient in terms of stability. Energy efficiency being a very important factor for outdoor mobile robots, spider-like leg configuration is chosen for accomplishing its job with both stability and energy efficiency. Three servomotors are used in each leg as shown in Fig. 2. The servomotors at joints 2 and 3 are able to rotate about two horizontal axes; however, the third one used at joint 1 is able of rotating about a vertical axis only.

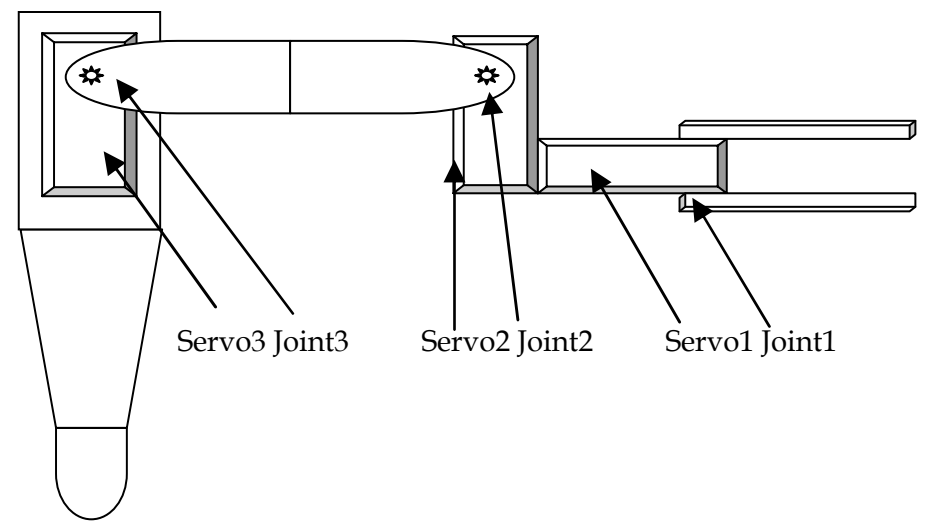

Fig. 2. Leg configuration

\section{Hexapod Locomotion}

For the hexapod locomotion, we would like to configure it by using two types of gait system. As this robot is able to navigate all kind of terrain in the hazard field, so it can move faster when it will get into even terrain, and in the event of uneven terrain, it will navigate very leisurely. Bump sensor helps it to detect any obstacle like hill or ditch which it may encounter in the hazardous field. This obstacle refers to uneven terrain. Thus we have used two types of gaits for different terrain as shown in Figure 3.
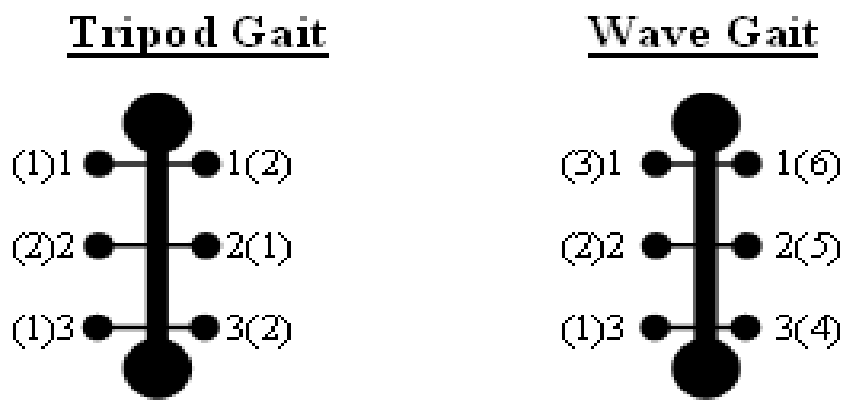

Fig. 3. Different types of gait 


\subsection{Tripod Gait for Even Terrain}

The Tripod Gait is the best-known hexapod gait. A tripod consists of the front-back legs on one side and the middle leg on the opposite side. For each tripod, the legs are lifted, lowered, and moved forwards and backwards in unison.

(1)

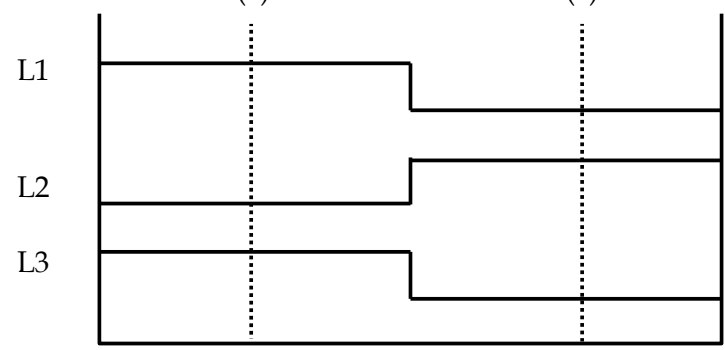

R1

Fig. 4. Stepping patterns of swing movement

During walking, a hexapod uses its 2 tripods not unlike a bi-pad stepping from one foot to the other - the weight is simply shifted alternately from one tripod to the other. Since 3 legs are on the ground at all times, this gait is both "statically" and "dynamically" stable. The movement scheme is easily visualized by examining the Figure 4; the numbers adjacent to the legs in the body diagram correspond to time points on the graph. The leg coordination of walking spiders appears to be quite regular too, and is described by the so-called tripod gait. In the tripod gait three legs, front and rear leg of one side and the middle leg of the other side, perform their swing movements at the same time. The tripod gait appears when the animals walk fast and the load is small. The occurrence of this gait may indicate that leg movement is controlled by a hierarchical superior system which determines the temporal sequence of the movements of the different legs (V. Holst).

\subsection{Wave Gait for Uneven Terrain}

In the Wave Gait, all legs on one side are moved forward in succession, starting with the rear most leg. This is then repeated on the other side. Since only one leg is ever lifted at a time, with the other five being down, the animal is always in a highly-stable posture. One conjecture is that the wave gait cannot be speeded up very much. Wave gaits are a group of gaits in which a wave motion of foot falls and foot lifting's on either side of the body move from the rear to the front, one after another with constant intervals, and in which the laterally opposing legs differ in phase exactly half of a leg cycle. Each wave gait is characterized by a forward wave of stepping actions from the back to the front on each side of the body. The wave gait pattern is chosen in this system because it provides the maximum stability margin for uneven terrain navigation. The control algorithm is used for the control action of wave gait locomotion with an angular position input and torque command output (Katsuhiko I.). The foot is commanded to move forward a constant length as viewed from the main body at each integration time interval. The numbers adjacent to the legs in the body diagram correspond to timepoints for the wave gait is showing in the Figure 5. 


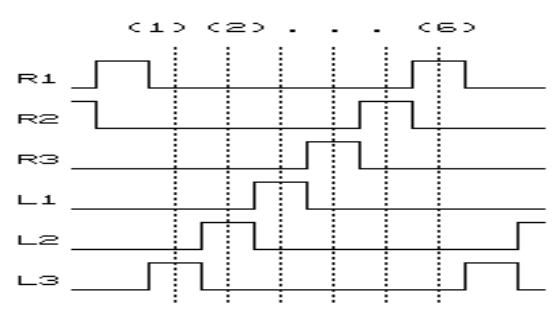

Fig. 5. Stepping patterns of swing movements for wave gait (Oricom T.)

\section{Methodology}

\subsection{Movement of Hexapod}

General behaviour of the hexapod is shown in Table. 1.

\begin{tabular}{|c|c|}
\hline Behavior 1 & Go forward \\
\hline Behavior 2 & Rotate right \\
\hline Behavior 3 & Rotate left \\
\hline Behavior 4 & Go backward \\
\hline
\end{tabular}

Table 1. Set of elementary reactions

From table 1, the steps of hexapod movement control system with elementary behaviors are summed up as follows-

Steps of progress:

1. the hexapod stands for forward movement with tripod gait until detects obstacle.

2. if perceive obstacles then wave gait with

a) timer on for $t$ seconds

b) terrain consider as uneven

c) go backward in terms of behavior4, then uses behavior 2 or 3 to rotate right (90 angle) if the obstacle is in the left or right.

d) use behavior1 until detect obstacle

3. else tripod gait with behavior 1 .

\subsection{Sensors Edifice}

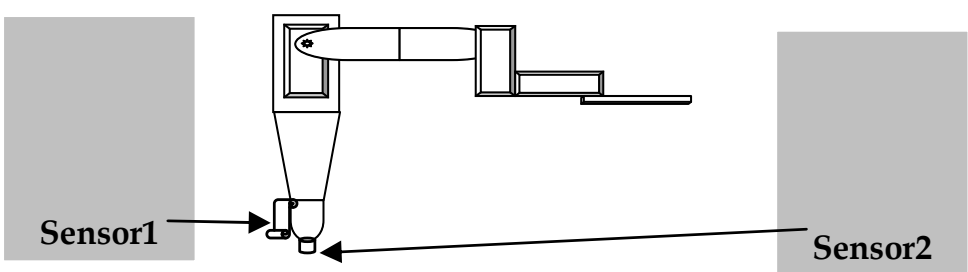

Fig. 6. Construction of the Sensors

The hexapod contains 8 bump sensors. Two sensors are attached to each of the front legs. Figure 6 shows one front leg with sensors. Each of the rest 4 sensors is attached with each of the 4 legs as sensor2. 


\subsection{Obstacle and Collision Avoidance}

We have designed two types of obstacle for the hexapod robot, such as 'hill' \& 'ditch'. Thus the hexapod robot will detect these obstacles during the navigation period. Sensor1 is used for the detecting of 'hill 'obstacles and sensor2 for 'ditch' obstacles. Table 2 shows different actions and scenario.

\begin{tabular}{|c|c|}
\hline Behavior 1 & No obstacle \\
\hline Behavior 2 & Left obstacle \\
\hline Behavior 3 & Right obstacle \\
\hline Behavior 4 & Front obstacle \\
\hline
\end{tabular}

Table 2. Different operation

\subsection{Terrain Negotiation}

The hexapod will negotiate two different types of terrain: even terrain \& uneven terrain.

Terrain negotiation always comprises safety aspects on the motion execution in order to protect robot's hardware. Especially rough terrain capabilities require the robot to distinguish traversable from hostile locations. Obstacles classification done through the sensors attach to the front two legs, obstacle classification algorithm is given in Appendix A.

\subsection{Scenarios of Hexapod}

Figure 7 shows the state diagram of the developed algorithm of the hexapod robot. We have a state table in Appendix A, and we have constructed the state diagram from this state table. We need to examine it further and construct a stable state table. In all the states, the actions to get to the robot and the action performed by it. For example, when hexapod turns on, it puts itself in the turned on state. From here, it is allowed to conduct various movements.

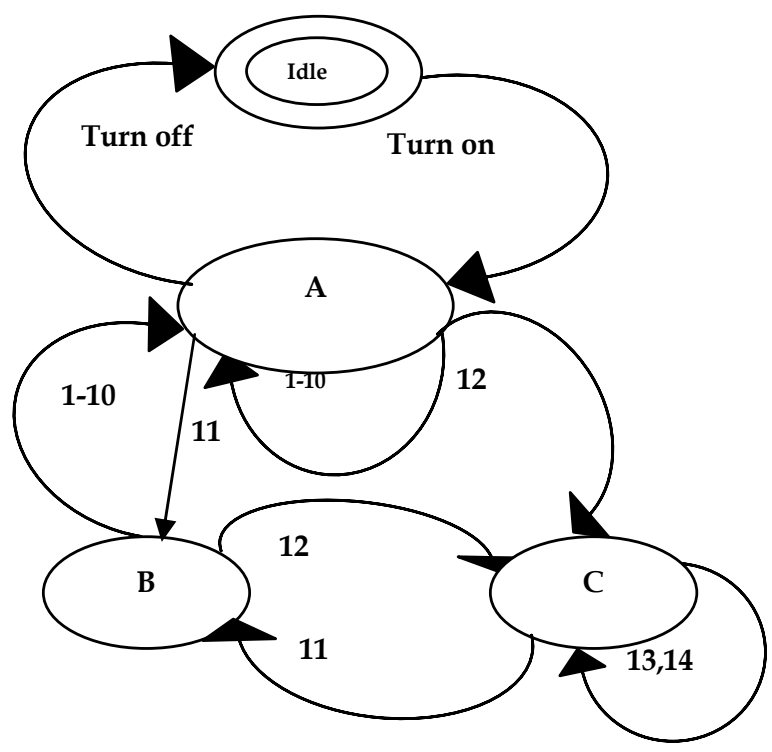

Fig. 7. State diagram for Random Scenarios 
Steps of Algorithm:

1. idle mode

2. call turn_on procedure.

3. Active state: able to do all operation sequentially
(a). move_forward
(b). move_backward
(c). turn_left
(d). turn_right
(e). sensor_1(on/off)
(f). sensor_2(on/off)
(g). t_gait
(h). w_gait
(i). timer_on

2. Uneven terrain state: if sensor_1(on) or sensor_2(off) then
(a) timer_on
(b) w_gait
(c). If hill type obstacle-
move_backward then rotate opposite direction of the obstacle.
(d). If ditch type obstacle-
Stop \& check again for depth;
If depth more than 1 " move_backward then rotate opposite direction of the ditch.
Else move_forward.

\section{(e). Gait Transition}

3. Even terrain state: else
(a). t_gait
(b). move_forward

\subsection{Control System}

Figure 8 shows the control environment currently implemented. The user interfaces have control over the running of the microcontroller and are fed back information about the status of the robot. The onboard controller is a PIC Microcontroller system. In test phase, communication between operator station and onboard computer is performed by a data cable. The microcontroller reads the information and controls the movements of the robot. The drive system consists of servo motors. The motor is driven by a high powered PWM (Pulse Width Modulation) controller board; it gets the signal from the microcontroller. The servo motor controller has its own soft start and stop facilities allowing smooth stopping and starting with no processing time required for the microcontroller. The $0-5 \mathrm{v}$ analogue signal input also allows the microcontroller to control the speed, acceleration and declaration. Computer module shows the user relevant information on the robots status and allows the user to control the robot directly with ease. 


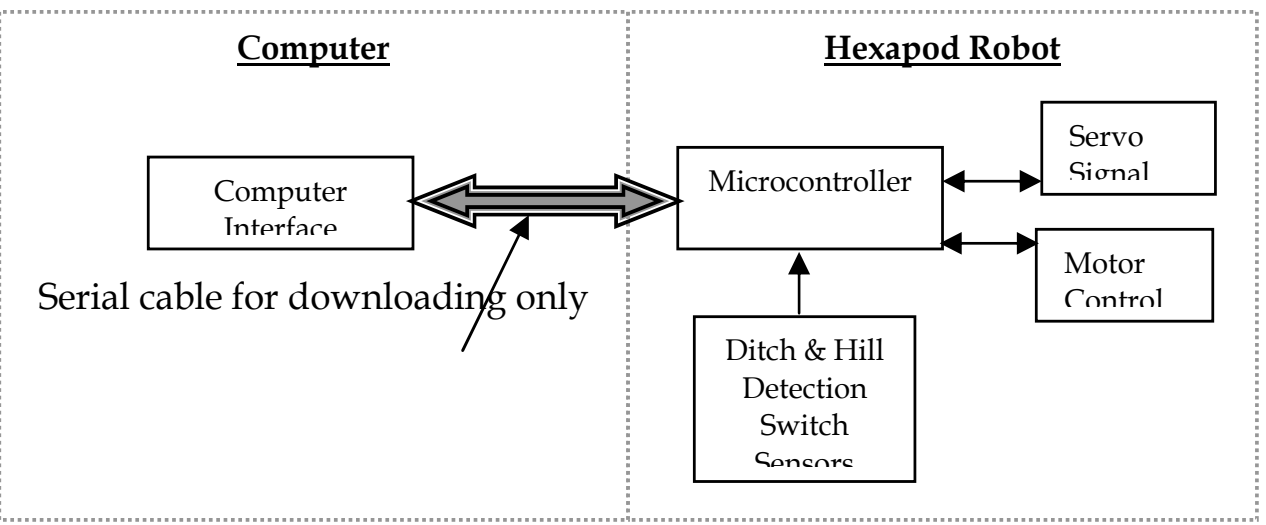

Fig. 8. Control environment of the developed system

\section{Conclusion}

We have developed a method to perform obstacle avoidance terrain negotiation in a static uncertain environment by using different gait of locomotion. The novelty of the method we have developed consists of the explicit consideration of uncertainty in the perception system, to negotiate the terrain of the environment from bump sensors. This method is able to take decision in accordance with the terrain. The case of hexapod robot's different navigation system in the hazardous field has been tested successfully. Using this algorithm hexapod robot will be overcome many of the shortcomings of previous legged robots developed for hazardous environment.

\section{Appendix A}

State Table for Random Scenarios

\begin{tabular}{|c|c|c|c|c|c|}
\hline State & Event & Actions & Comment & \begin{tabular}{|l|} 
Caused \\
By
\end{tabular} & $\begin{array}{l}\text { Will } \\
\text { Effect }\end{array}$ \\
\hline \multirow[t]{6}{*}{$\begin{array}{l}\text { A. } \\
\text { Active }\end{array}$} & 1.turnOn & Boot Up & Hexapod is turning on & & $2-10$ \\
\hline & 2.bootUp & Activity & Allow various activities & 1 & $3-8$ \\
\hline & $\begin{array}{l}\text { 3.forward } \\
\text { movement }\end{array}$ & Walk Forward & $\begin{array}{l}\text { Hexapod will begin to walk } \\
\text { forward }\end{array}$ & 2 & 11,12 \\
\hline & $\begin{array}{l}\text { 4. backward } \\
\text { movement }\end{array}$ & Walk Backward & $\begin{array}{l}\text { If obstacle is in left/right front, } \\
\text { hexapod will begin to walk } \\
\text { backward }\end{array}$ & & 12 \\
\hline & 5. right rotation & $\begin{array}{l}\text { Rotating in right } \\
\text { side }\end{array}$ & $\begin{array}{l}\text { If obstacle is left side, hexapod } \\
\text { will turn by } 90^{*} \text { angle in right } \\
\text { direction }\end{array}$ & & 12 \\
\hline & 6. left rotation & $\begin{array}{l}\text { Rotating in left } \\
\text { side }\end{array}$ & $\begin{array}{l}\text { If obstacle is right side, hexapod } \\
\text { will turn by } 90^{*} \text { angle in left } \\
\text { direction }\end{array}$ & & 12 \\
\hline
\end{tabular}




\begin{tabular}{|l|l|l|l|l|l|}
\hline & 7. sensor2 press & Button on & No ditch obstacle & 11 \\
\hline & $\begin{array}{l}\text { 8. sensor2 not } \\
\text { press }\end{array}$ & Button off & Ditch obstacle found & 12 \\
\hline & $\begin{array}{l}\text { 9. sensor1 press } \\
\text { 10. sensor1 not } \\
\text { press }\end{array}$ & Button on & Hill obstacle found & 12 \\
\hline $\begin{array}{l}\text { B. } \\
\text { Even } \\
\text { terrain }\end{array}$ & \begin{tabular}{l|l|l|l|} 
11. tripod gait \\
\hline $\begin{array}{l}\text { C. } \\
\text { Uneven } \\
\text { terrain }\end{array}$
\end{tabular} & Fast movement & No obstacle & Hexapod will walk fast forward \\
until it gets an obstacle & 7,10 & 3 \\
\hline & $\begin{array}{l}\text { 13. stop \& wave } \\
\text { backward }\end{array}$ & No surface & $\begin{array}{l}\text { Hexapod will walk or rotate at a } \\
\text { rotate } \\
\text { snail's pace for t seconds. }\end{array}$ & 8,9 & $3,4,5,6$ \\
\hline $\begin{array}{l}\text { 14. stop \& wave } \\
\text { forward }\end{array}$ & $\begin{array}{l}\text { Surface available } \\
\text { with 1“ dich }\end{array}$ & $\begin{array}{l}\text { Hexapod will walk forward } \\
\text { slowly }\end{array}$ & 7,8 & 3,12 \\
\hline
\end{tabular}

\section{References}

C.-L. Shih and C. A. Klein, "An adaptive gait for legged walking machines over rough terrain," IEEE Trans. Syst. Man Cybem., vol. SMC-23, no.4, pp. 1150-1 155, July/Aug. 1993.

F. Ozguner, S. I. Tsai and R. B. McGhee, "An approach to the use of terrain-preview information in rough-terrain locomotion by a hexapod walkin, achine," Int. J. Robotics Res., vol. 3, no. 2, pp. 134- 146, Summer 1984.

G. Nejat and Z. Zhang, "The Hunt for Survivors: Identifying Landmarks for 3D Mapping of Urban Search and Rescue Environments," The World Multi-Conference on Systemics, Cybernetics and Informatics (WMSCI 2006), 2006.

P. K. Pal and K. Jayarajan, "Generation of free gait graph search approach," IEEE Trans. Robot. Automat., vol. 7, no. 3, pp. 299-305, June. 1991.

R. B. McGhee and G. I. Iswandhi, "Adaptive locomotion of a multilegged robot over rough terrain," IEEE Trans. Syst. Man Cybem., vol. SMC-9, no.4, pp. 176-182, Apr. 1979.

Habib Mechanical mine clearance technologies and humanitarian demining applicability and effectiveness; 2000.

Y. Mori, K. Takayama, T. Adachi, S. Omote and T. Nakamura, Feasibility study on an excavation-type demining robot, Auton Robot 18 (2005), pp. 263-274.

Rizo J, Coronado J, Campo C, Forero A, Otalora C, Devy M, et al. URSULA: robotic demining system. In: Proceedings of the 11th international conference on advanced robotics; 2003. p. 538-43.

Y. Baudoin, M. Acheroy, M. Piette and J.P. Salmon, Humanitarian demining and robotics, Mine Action Inform Center J 3 (2) (1999).

Hirose S, Kato K. Quadruped walking robot to perform mine detection and removal task. In: Proceedings of the first international conference on climbing and walking robots; 1998. p. 261-6. 
Nonami K, Huang QJ, Komizo D, Shimoi N, Uchida H. Humanitarian mine detection sixlegged walking robot. In: Proceedings of the third international conference on climbing and walking robots; 2000. p. 861-8.

Q.J. Huang and K. Nonami, Humanitarian mine detecting six-legged walking robot and hybrid neuro walking control with position/force control, Mechatronics 13 (2003), pp. 773-790.

D. Voth, Nature's guide to robot design, IEEE Intell Syst (2002), pp. 4-7.

P. Gonzalez de Santos and M.A. Jimenez, Generation of discontinuous gaits for quadruped walking machines, J Robot Syst 12 (9) (1995), pp. 599-611.

P. Gonzalez de Santos, M.A. Armada and M.A. Jimenez, Ship building with ROWER, IEEE Robot Autom Mag 7 (4) (2000), pp. 35-43

P. Gonzalez de Santos, J.A. Galvez, J. Estremera and E. Garcia, SILO4 - A true walking robot for the comparative study of walking machine techniques, IEEE Robot Autom Mag 10 (4) (2003), pp. 23-32.

P. J. Sammons, Tomonari Furukawa and Andrew Bulgin, Autonomous Pesticide Spraying Robot for use in a Greenhouse, ARC Centre of Excellence for Autonomous Systems,School of Mechanical and Manufacturing Engineering. The University of New South Wales, Australia, September 9, 2005.

G. Mor S., Ronen B., Kazaz I., Josef S., Bilanki Y. (1997), Guidance for Automatic Vehicle for Greenhouse Transportation", ACTA Horticulture, Vol 443, pp. 99-104.

Sezen, B. (2003), Modelling Automated Guided Ve- hicle Systems in material Handling", Dogus Univer- sitesi Dergisi, Vol 4, No. 3, pp. 207-216.

Schneider A, Zeidis I, Zimmermann K. Comparison of body shapes of walking machines in regards to stability margins. In: Proceedings of the third international conference on climbing and walking robots; 2000. p. 275-81.

V. Holst "Leg Coordination" - tripod gait.

Katsuhiko I. and Hisato K., "Adaptive Wave Gait for Hexapod Synchronized Walking "Hosei University, Kaj ino-cho, Koganei, Tokyo 184, JAPAN.

Oricom Technologies, Hexapod Robot Gait, www.oricomtech.com. 



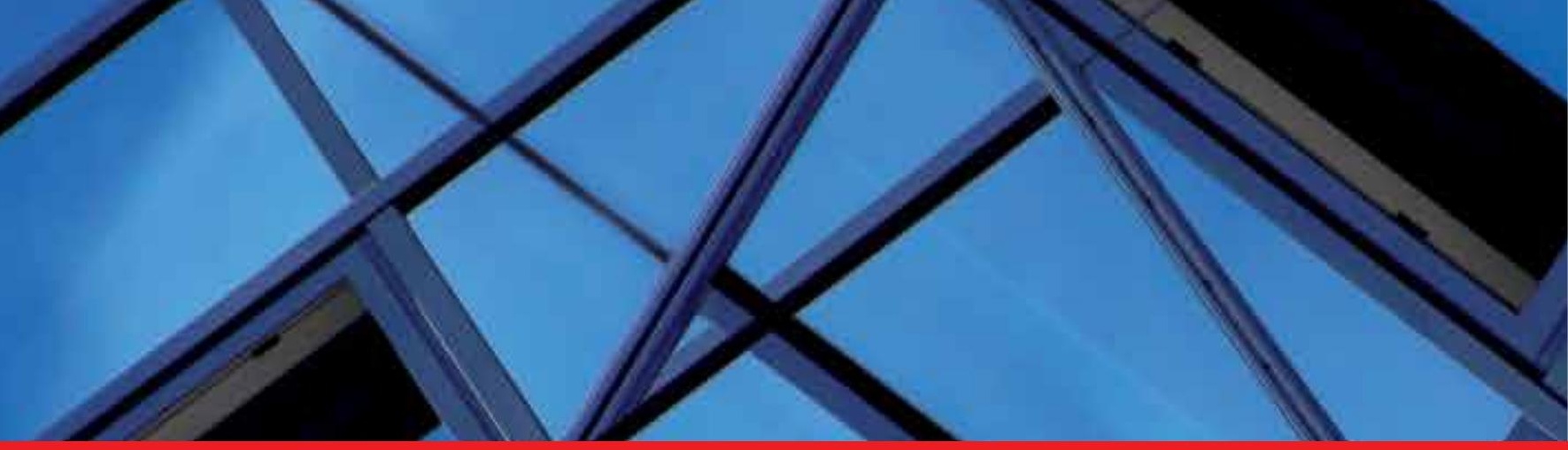

\section{Edited by Aleksandar Lazinica}

This book collects original and innovative research studies concerning advanced technologies in a very wide range of applications. The book is compiled of 22 chapters written by researchers from different areas and different parts of the world. The book will therefore have an international readership of a wide spectrum. 